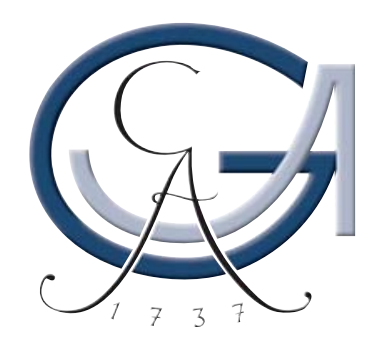

\title{
Risikosport - Interdisziplinäre Annäherung, empirische Befunde und Anwendungsbezüge
}

\author{
Dissertation \\ zur Erlangung des sozialwissenschaftlichen Doktorgrades \\ der Sozialwissenschaftlichen Fakultät der Universität Göttingen
}

vorgelegt von

Arne Göring

aus Wolfenbüttel

Göttingen 2006 
1. Gutachter: Prof. Dr. Arnd Krüger

2. Gutachter: Prof. Dr. Axel Dreyer

Tag der mündlichen Prüfung: 27.03.2006 


\section{Zusammenfassung: \\ Risikosport - interdisziplinäre Annäherung, empirische Befunde und Anwednungsbezüge}

Ausgehend von einer geringen wissenschaftlichen Fundierung des Forschungsgegenstandes wird in der Arbeit eine interdisziplinäre Theorie des Risikosports entworfen, sowie eine theoretisch-empirische Verknüpfung im Rahmen einer sicherheitspsychologischen Untersuchung vorgenommen. Die Arbeit beinhaltet dabei zwei Herausforderungen, die - aufeinander bezogen - unterschiedliche Herangehensweisen und Forschungszugänge erfordern: Im ersten Teil wird eine inhaltliche Bestimmung des Risikosportphänomens vorgenommen und Erklärungszusammenhänge im Rahmen eines interdisziplinären Erkenntnisprozesses exploriert. Mittels kritischer Interpretation und Abduktion werden psychologische, historische, soziologische, ethische und pädagogische Fragestellungen thematisiert und zu einer integrativen Theorie verknüpft. Im zweiten Teil der Arbeit werden die theoretischen Kontexte des Risikosports im Rahmen einer empirischen Untersuchung in ihrem Erklärungsgehalt deduziert und für mögliche Anwendungsfelder transformiert.

Das Verständnis des Risikosports erschließt sich in der vorliegenden Dissertation insbesondere durch ein den gesellschaftlichen Konventionen bisweilen widersprechendes Verständnis von Risiko, Sicherheit und Gefahr. Der Mensch so wird im Risikosport transparent - ist demnach kein monokausales Sicherheitswesen, sondern im Sinne einer erhöhten Handlungsgratifikation auch an Risikosituationen interessiert. Die vorgenommene wissenschaftliche Auseinandersetzung mit dem Forschungsgegenstand Risikosport verlangt, von gebräuchlichen Sicherheitskonventionen abzurücken und eine Differenzierung von Risiko, Sicherheit und Gefahr voranzutreiben, die über den wissenschaftlichen Status Quo hinausgeht. 


\section{Précis: \\ Extreme sports - an interdisciplinary approach; empiric data and applied samples.}

This dissertation develops an interdisciplinary theory concerning extreme sports. As a foundation, a less scientific approach is used. It will then approach a theoretical and empirical relation in the context of psychological safety. This includes two challenges, which require separate scientific approaches in order to succeed. The first part probes deeper into the content and characteristics of extreme sports followed by an attempt to find an interdisciplinary explanation of why people choose to perform extreme sports. Critical interpretation and analysis determine the questions used to form a central theme that is then integrated into the theory. These questions include psychological, historical, sociological, ethical and pedagogical. In the second part, theories are deduced as to why extreme sports are worthwhile and the possible applications they may hold. This conclusion is embedded in a framework of empirical research.

In this dissertation, the understanding of risk is controversially unlike that of current social conventions. Extreme sports prove that humans are not close-minded to activities that concern their well-being. Rather they are quite willing to expose themselves to hazardous situations. From this dissertation, it can be concluded that the concept of safety is outdated. Therefore, an unconventional understanding of safety is required, one that differentiates risk, safety and danger from one another which will ultimately improve the current scientific status quo. 


\title{
INHALT:
}

\section{Abbildungsverzeichnis}

\author{
Tabellenverzeichnis
}

1 EINLEITUNG 1

\section{RISIKO-RISIKOVERHALTEN-RISIKOSPORT: BEGRIFFSBESTIMMUNGEN UND THEORETISCHE GRUNDLAGEN}

$2.1 \quad$ Risiko - ein multidisziplinärer Forschungsgegenstand 9

2.2 Das Wortfeld „Risiko“ $\quad 11$

2.2.1 Inhaltliche Bestimmung und Abgrenzung des Risikobegriffs 11

2.2.2 Inhaltsorientierte Analyse des sportlichen Risikos als Handlungs- und Erlebnisfeld. 14

$\begin{array}{ll}\text { 2.2.2.1 Risikoarten } & 15\end{array}$

$\begin{array}{ll}\text { 2.2.2.2 Das Risikoverhalten } & 15\end{array}$

2.2.2.3 Risikobewusstsein, Risikoentscheidung und Risikobereitschaft 17

$\begin{array}{ll}\text { 2.2.2.4 Die Risikosituation } & 17\end{array}$

2.3 Risikosport - Einordnung, Definition und Eingrenzung 18

$\begin{array}{llr}\text { 2.3.1 Die Entwicklung des Freizeitsports } & 20\end{array}$

2.3.2 Risikosport - Überblick über den Forschungsstand 32

2.3.3 Entwurf einer eigene Definition von Risikosport 42

2.3.3.1 Das Risiko im Sport - ein subjektzentrierter Ansatz 42

2.3.3.2 Risikosport als Handlungsfeld - Definition und Abgrenzung 43

2.3.3.3 Charakteristika des Risikosports 48

2.3.3.4 Klassifizierungsvorschlag von Risikosportarten 51

2.3.3.5 Exemplarische Analyse des Risikomoments am Beispiel des Kanu-Wildwasserfahrens und des alpinen Bergsteigens $\quad 54$

$\begin{array}{llr}2.4 & \text { Zusammenfassung und Einordnung der Befunde } & 57\end{array}$

\section{ZUR GESCHICHTE DES RISIKOSPORT - HISTORISCHE WURZELN UND IDEOLOGISCHE GRUNDLAGEN RISIKOSPORTLICHER AKTIVITÄTEN.}

3.1 Die Geburt des Risikos - Unsicherheitswahrnehmung im historischen Rückblick $\quad 60$

3.1.1 Begriffsgeschichte oder: Unsicherheit zwischen Fortuna und Aventiure 60

$\begin{array}{lll}3.1 .2 & \text { Risiko und Sicherheit } & 70\end{array}$

3.2 Das Risiko im Sport - Kulturhistorische Entwicklung und Zusammenhänge $\quad 74$

3.2.1 Das sportliche Risiko als Herausforderung - die Geschichte des Alpinismus 76

$\begin{array}{lll}\text { 3.2.1.1 Der Präalpinismus } & 78\end{array}$

3.2.1.2 Der Alpinismus als zweckfreie Handlung und Sport $\quad 79$

3.2.1.3 Die Gründung der alpinen Vereine $\quad 83$

$\begin{array}{lll}\text { 3.2.1.4 Der Schwierigkeits- und Gefahrenalpinismus } & 84\end{array}$

3.2.1.5 Der Alpinismus als Massenphänomen $\quad 86$

3.2.1.6 Konklusion: Der Alpinismus als historische Wurzel des Risikosports 88 
3.2.2 EXKURS: Risiko und Abenteuer bei der sportlichen Großwildjagd des 19. Jahrhunderts

3.2.3 Das sportliche Risiko als Erlebnis - Die ideologischen Hintergründe des Risikosports

Die natürliche Erziehung bei Rousseau

3.2.3.2 Das Naturerlebnis als Selbsterfahrung bei Thoreau

3.2.3.3 Das Erlebnis als Voraussetzung des Erkenntnisprozesses (Dilthey)

3.2.3.4

3.2.3.5

Die Ambivalenz des Abenteuers bei Simmel

Erziehung zur Verantwortung (Hahn)

3.2.3.6 Konklusion: Das Risiko als Sinnfindung

3.2.4 EXKURS: Das sportliche Risiko als Abhärtung - historische Gedanken zum Risikosport als

Kriegsvorbereitung und Kriegsersatz

$\begin{array}{ll}\text { 3.2.4.1 } & \text { Risikosportliche Aktivitäten als Kriegsvorbereitung } \\ \text { 3.2.4.2 } & \text { Männlichkeitsideale im kriegsvorbereitenden Risikosport }\end{array}$

3.2.4.3 Risikosportliche Aktivitäten als Kriegsersatz

\section{WAS MOTIVIERT MENSCHEN ZUM RISIKOSPORT? ANTHROPOLOGISCHE ÜBERLEGUNGEN UND PSYCHOLOGISCHE ERKLÄRUNGSANSÄTZE}

4.1 Motivationspsychologische Ansätze als theoretischer Bezugsrahmen 112

4.1.1 Motiv - Anreiz - Motivation: Motivationspsychologische Grundbegriffe 112

$\begin{array}{ll}\text { 4.1.2 Forschungszugänge der Motivationspsychologie } & 114\end{array}$

4.2 Der Mensch und das Risiko - Anthropologische Annahmen 119

$\begin{array}{ll}\text { 4.2.1 Der Mensch als ,riskiertes’ Wesen } & 120\end{array}$

4.2.2 Die Anthropologie des menschlichen Handelns 121

4.2.3 Neugierverhalten - ein Sicherheitstrieb 123

4.3 Risikosport und Motivation: Erklärungsversuche der Psychologie 125

4.3.1 Aktivationstheoretische und persönlichkeits-psychologische Ansätze: Risikosport als Erregungssuche

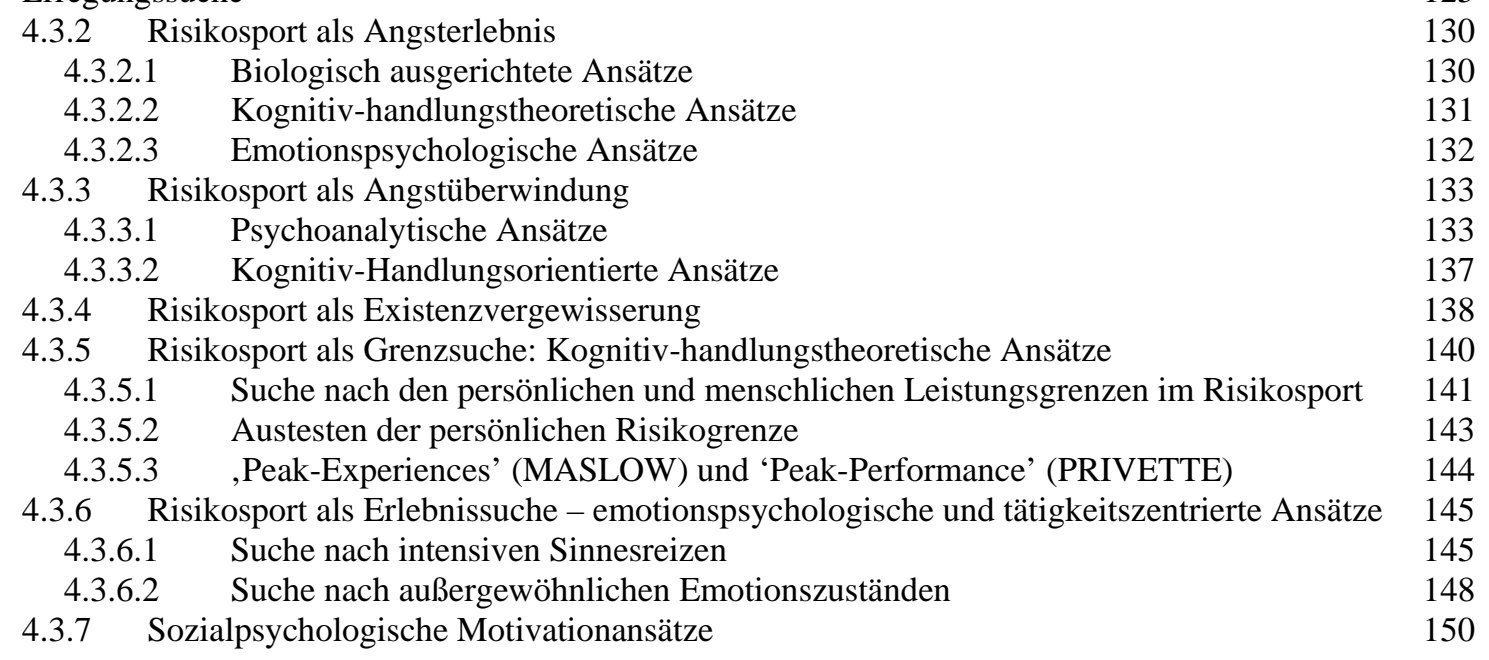

4.4 Zusammenfassung und Einordnung

151

\section{RISIKOSPORT UND GESELLSCHAFT - SOZIOLOGISCHE, PÄDAGOGISCHE UND ETHISCHE PERSPEKTIVEN}

5.1 (Post-)Modernisierung als theoretischer Bezugsrahmen des Risikosports 156

$\begin{array}{ll}5.1 .1 & 158\end{array}$

$\begin{array}{ll}\text { 5.1.2 Der Wandel der Existenzformen } & 162\end{array}$

$\begin{array}{ll}\text { 5.1.3 Moderne Sozialstrukturanalyse } & 163\end{array}$ 
5.1.4 Pluralisierung und Differenzierung 166

$\begin{array}{llr}5.1 .5 & \text { Wertewandel } & 168\end{array}$

$\begin{array}{llr}5.2 & \text { Soziologie des Risikosports } & 170\end{array}$

5.2.1 Sinnsuche und Subjektivtätspflege $\quad 170$

5.2.2 Risikosport als Identitätsarbeit und Distinktion 172

5.2.3 Risikosport als Gegenwelt einer defizitären Gesellschaft 174

$\begin{array}{llr}\text { 5.3 Sicherheit und Risiko aus soziologischer Perspektive } & 178\end{array}$

5.3.1 Risiko, Unsicherheit, Gefahr - eine Annäherung aus soziologischer Perspektive 179

5.3.2 Die gesellschaftliche Konstruktion von Sicherheit 181

5.3.3 Moderne Sicherungsstrategien 183

5.3.4 Risikosport im Kontext einer ,sicheren Gefahrengesellschaft’ 184

$\begin{array}{llr}5.4 & \text { Risikosport im Lebenslauf } & 187\end{array}$

$\begin{array}{llr}5.5 & \text { Risikosport und Geschlecht } & 189\end{array}$

5.6 Das Risiko im Sport - ein sportpädagogisches Fazit 195

5.7 Ethische Betrachtung des risikosuchenden Verhaltens im Sport 200

$\begin{array}{llr}5.8 & \text { Zusammenfassung und Einordnung } & 202\end{array}$

\section{6 ÜBER DEN UMGANG MIT RISIKO - RISIKOSPORT UND HANDLUNGSTHEORIE}

6.1 Risikoverhalten und Handlungsregulation 205

6.1.1 Individuelle Schwierigkeitsanforderungen am Beispiel des Kanu-Wildwasserfahrens 206

$\begin{array}{ll}\text { 6.1.2 Handlungsregulation und Phasenstruktur } & 208\end{array}$

$\begin{array}{ll}\text { 6.1.3 Kognition und Risikoverhalten } & 212\end{array}$

$\begin{array}{ll}\text { 6.1.4 Emotion und Risikoverhalten } & 217\end{array}$

6.1.5 Das ,Adventure Experience Paradigm’ 220

6.1.6 ,Risiko-Regulierung’ im Risikosport 222

6.2 Einflüsse auf Risikoverhalten, Gefahrenkognition und Risikobeurteilung - Ansätze und Befunde der Verhaltenspsychologie

6.2.1 Persönlich bedingte Variablen $\quad 225$

$\begin{array}{ll}\text { 6.2.2 Heuristiken und Urteilsfehler } & 226\end{array}$

$\begin{array}{lll}\text { 6.2.3 Lerntheoretische Einflüsse } & 228\end{array}$

6.2.4 Sozialpsychologische Annahmen 230

6.2.5 Risikoverhalten unter Bedingungen subjektiver Sicherheit 231

6.2.5.1 Der psychologische Sicherheitsrahmen 232

6.2.5.2 Das Sicherheits-Risiko-Gesetz 233

6.2.5.3 Die Theorie der Risikokompensation 233

6.2.5.4 Das Modell der subjektiven und objektiven Sicherheit 235

6.3 Handeln im Risikosport - ein transaktionales Modell 236

6.3.1 Grundlegende Annahmen des Modells 237

6.3.2 Die internen Modellkomponenten und ihre Interaktion 238

$\begin{array}{ll}\text { 6.4 Zusammenfassung und Einordnung } & 242\end{array}$

7 ZWISCHENFAZIT 245 


\section{THEORETISCHE GRUNDLAGEN DER UNFALL- UND SICHERHEITSFORSCHUNG}

8.1 Unfallforschung in der Sportwissenschaft

8.2 Der Unfall: Terminologisch-konzeptionelle Auseinandersetzung mit dem Gegenstand der Unfall- und Sicherheitsforschung

8.3 Theoretische Ansätze der Unfallforschung 255

8.3.1 Persönlichkeitszentrierte Ansätze 255

$\begin{array}{lll}\text { 8.3.2 Interaktionistische Ansätze } & 256\end{array}$

$\begin{array}{ll}\text { 8.3.3 Systemtheoretische Ansätze } & 257\end{array}$

8.3.4 Handlungstheoretische Ansätze 258

8.3.5 Ansätze einer erweiterten Sicherheitsforschung 259

8.3.6 Das Modell der Gefahrenkognition aus der Arbeitssicherheit 262

\section{EMPIRIE: UNFÄLLE UND GEFAHRENKOGNITION IM RISIKOSPORT - EINE SICHERHEITSPSYCHOLOGISCHE STUDIE}

9.1 Grundlagen, Ziele und Fragestellung der Studie $\quad 268$

9.1.1 Gefahrenkognition als zugrunde liegendes Modell 269

$\begin{array}{ll}\text { 9.1.2 Aufgaben, Fragestellungen und Hypothesen } & 271\end{array}$

$\begin{array}{llr}9.2 & 274\end{array}$

$\begin{array}{lll}\text { 9.2.1 Objektives Datenmaterial } & 276\end{array}$

9.2.1.1 Abgrenzung und Definition der Aktivitäten 276

$\begin{array}{lll}\text { 9.2.1.2 } & \text { Reanalyse der Unfalldaten } & 278\end{array}$

$\begin{array}{ll}\text { 9.2.2 Subjektives Datenmaterial } & 280\end{array}$

$\begin{array}{lll}\text { 9.2.2.1 Teilnehmer der Befragung } & 280\end{array}$

9.2.2.2 Exkurs: Internetbasierte Befragungen - eine kritische Diskussion 281

9.2.2.3 Untersuchungsmaterial: der Fragebogen zur subjektiven Gefährlichkeit im alpinen Berg-

und Kanusport. 285

9.2.3 Z-Wert-Transformation als statistisches Verfahren zur Konversion der objektiven und subjektiven Datensätze $\quad 286$

$\begin{array}{lll}\text { 9.2.4 Differenzberechnung } & 288\end{array}$

9.3 Ergebnisse $\quad 289$

9.3.1 Unfälle und Gefahrenkognition im alpinen Bergsport 289

9.3.1.1 Die objektive Datenbasis: die Ergebnisse der Unfallanalyse 290

9.3.1.2 Die subjektive Datenbasis: die Ergebnisse der internetbasierten Befragung 297

9.3.1.3 Differenzbildung: objektive und subjektive Daten im Vergleich 300

9.3.1.4 Ergebnisse der Ergänzungsuntersuchung: Unterschiede im Bezug auf Geschlecht, Alter,

Erfahrung und Übungsleiterlizenzen. $\quad 303$

9.3.1.4.1 Geschlechtsspezifische Unterschiede 303

9.3.1.4.2 Altersspezifische Unterschiede 305

9.3.1.4.3 Gefahrenkognition und alpine Erfahrung: 307

9.3.1.4.4 Gefahrenkognition bei Übungsleitern 309

9.3.2 Unfälle und Gefahrenkognition im Kanusport 311

9.3.2.1 Die objektive Datenbasis: Die Ergebnisse der Unfallanalyse 311

9.3.2.2 Die subjektive Datenbasis: die Ergebnisse der internetbasierten Befragung 315

9.3.2.3 Differenzbildung: objektive und subjektive Daten im Vergleich 317

9.3.2.4 Ergebnisse der Ergänzungsuntersuchung: Unterschiede im Bezug auf Geschlecht, Alter,

Erfahrung und Übungsleiterlizenzen 319

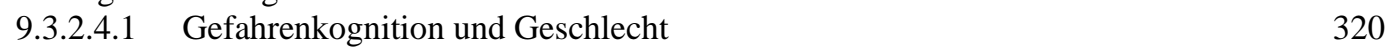

9.3.2.4.2 Gefahrenkognition und Alter 321 
9.3.2.4.3 Gefahrenkognition und Erfahrung 322

9.3.2.4.4 Gefahrenkognition von Übungsleitern 324

9.4 Diskussion der Untersuchungsergebnisse $\quad 325$

9.4.1 Aufgabenstellung der Untersuchung 325

9.4.2 Hauptbefunde 326

9.4.2.1 Anwendbarkeit des gewählten Verfahrens 326

9.4.2.2 Replikabilität der Befunde 329

9.4.2.3 Weiterführende Zusammenhänge zwischen Gefahrenkognition und Unfalldaten im Risikosport 331

9.4.3 Theoretische Einordnung der Befunde 333

$\begin{array}{ll}\text { 9.4.4 Offene Forschungsfragen } & 334\end{array}$

$\begin{array}{ll}9.5 & \text { Fazit der Untersuchung }\end{array}$

$\begin{array}{lll}9.6 & \text { Zusammenfassung und Einordnung } & 336\end{array}$

\section{RISIKOMANAGEMENT ALS ANWENDUNGSBEZOGENE INTERVENTION IM RISIKOSPORT}

\begin{tabular}{ll}
10.1 & Gedanken zum Interventionsziel \\
\hline
\end{tabular}

10.2 Risikomanagement als Paradigmenwechsel 340

10.3 Eckpfeiler eines Risikomanagement-Konzeptes im Risikosport 343

10.3.1 Information: Erkenntnis objektiver Gefahren und alternativer Handlungsstrategien 344

$\begin{array}{ll}\text { 10.3.2 Reflexion auf eigenes Verhalten } & 345\end{array}$

10.3.3 Offene Kommunikation 346

10.4 Risikomanagement als Vermittlungsprinzip: Pädagogische Schlussfolgerung 346

10.5 Beispiele für die Anwendung des Risikomanagements in der Praxis 350

10.5.1 3x3 Zoom-System und Risikoreduktionsmethode (MUNTER 1999) 351

10.5.2 Stop or go (LARCHER 1999)

10.5.3 SnowCard (ENGLER \& MERSCH 2000) 354

10.5.4 Nivo Test (BOLOGNESI 2000) 354

10.5.5 3x3-Methode (GRAU 2004) 354

10.6 Risikobewusstsein und Restrisiko: Eine kritische Schlussbetrachtung 355

11 RESÜMEE UND AUSBLICK 357

LITERATUR $\quad 361$

Anhang 


\section{Abbildungsverzeichnis:}

ABBILDUNG 2.1: BEREICHE DES RISIKOVERHALTENS (IN ANLEHNUNG AN KUHN \& TODT 2003, 11)

ABBILDUNG 2.2: TRADITIONELLES VERSUS MODERNES VERHÄLTNIS ZWISCHEN ARBEIT UND FREIZEIT (NACH DIGEL 1986, 34).

TABELLE 3.1: ABENTEUERSUCHE ALS DEFINITION DES WAHREN MENSCHSEINS IM AUSGEHENDEN 12. JAHRHUNDERT (ZIT. NACH NERLICH 1998, 85).....

ABBILDUNG 4.1:GRUNDMODELL DER KLASSISCHEN MOTIVATIONSPSYCHOLOGIE (RHEINBERG 2002, 72).

ABBILDUNG 4.2: DIE SICHERHEITSBILANZ: AUSGLEICH VON ENTWICKLUNG UND STABILISATION (MODIFIZIERT NACH HALLER 1998, 225).

ABBILDUNG 4.3: FLOW-ERLEBEN IN ABHÄNGIGKEIT VON KOMPETENZ UND SITUATIVEN HERAUSFORDERUNGEN (MODIFIZIERT NACH CSIKSZENTMIHALYI \& RATHUNDE 1993, 75)

ABBILDUNG 6.1: BESTIMMUNG VON SUBJEKTIVEN SCHWIERIGKEITSANFORDERUNGEN ANHAND SUBJEKTIVER FÄHIGKEITEN UND OBJEKTIVER FAKTOREN IM KANUWILDWASSER (IN ANLEHNUNG AN BAUR ET AL. 1977, 377).....

ABBILDUNG 6.2: PHASENSTRUKTUR DES HANDLUNGSPROZESSES IM RISIKOSPORT (ERWEITERT NACH NITSCH $(1986,230)$.

ABBILDUNG 6.3: RISIKOVERHALTEN ALS KOGNITIVER PROZESS( MOD. N. BOYES 1991, 16).

ABBILDUNG 6.4: DAS ,ADVENTURE EXPERIENCE PARADIGM“ (MOD. N. PRIEST \& GASS 1997, 46).

ABBILDUNG 6.5: DISKREPANZ ZWISCHEN SUBJEKTIVER WAHRNEHMUNG UND OBJEKTIVEN HANDLUNGSBEDINGUNGEN (MOD. N. PRIEST \& GASS 1997, 47).....

ABBILDUNG 6.6: SCHEMATISCHE DARSTELLUNG DER THEORIE DER GELERNTEN SORGLOSIGKEIT (IN ANLEHNUNG AN SCHULZ-HARDT ET AL. 1996, 470).

ABBILDUNG 6.7: HANDELN IM RISIKOSPORT - EIN INTEGRATIVES MODELL (EIGENE DARSTELLUNG)

ABBILDUNG 8.1: RISIKOSPORT ALS SYSTEM AM BEISPIEL DES KANU-FAHRENS. IN ANLEHNUNG AN DIE 'ACCIDENT THEORY'; VERÄNDERT UND ERWEITERT NACH PRIEST \& GASS 1997, 89. LDUNG 8.2: VERANSCHAULICHUNG DER BEZIEHUNG ZWISCHEN DEM BEREICH OBJEKTIVER GEFAHR UND DEM BEREICH, DER ALS SUBJEKTIV GEFÄHRLICH ERKANNT WIRD (IN ANLEHNUNG AN RINKE 2002, 33; MOD. NACH MUSHAL 1997, 30).

ABBILDUNG 8.3: GEGENÜBERSTELLUNG DER GEFAHRENKOGNITION UND DER TATSÄCHLICHEN UNFÄLLE BEI ARBEITSTÄTIGKEITEN IN EINEM WALZWERK (IN RINKE 2002, 35).

ABBILDUNG 8.4: UNFÄLLE UND SUBJEKTIVES GEFAHRENWISSEN BEI TURNTÄTIGKEIT IN ALLGEMEINBILDENDEN SCHULEN (NACH RINKE 2002, 80)........................................266

ABBILDUNG 9.1: SICHERHEITSPSYCHOLOGISCHES MODELL DES UNFALLGESCHEHENS IM RISIKOSPORT AM BEISPIEL DES UNFALLSYSTEMS KANUSPORT. (EIGENE DARSTELLUNG IN ANLEHNUNG AN HOYOS 1980, 13).....

ABBILDUNG 9.2: SCHADENSPYRAMIDE NACH HOWLAND (MOD. N. BÄNI 1991A, 3),...........280

ABBILDUNG 9.3: EXEMPLARISCHE DARSTELLUNG DER ITEMS AM BEISPIEL DES BERGWANDERNS.

ABBILDUNG 9.4: UNFÄLLE IM ALPINEN BERGSPORT FÜR DIE AKTIVITÄTEN BERGWANDERN, HOCHTOUREN, FELSKLETTERN, SKI-TOUREN UND SKI-PISTEN \& VARIANTE FÜR DIE JAHRE 2000 - 2003.

ABBILDUNG 9.5: ABSOLUTE UNFALLZAHLEN DER EINZELNEN AKTIVITÄTEN FÜR DIE JAHRE 2000 - 2003.

ABBILDUNG 9.6: UNFÄLLE IM ALPINEN BERGSPORT NACH ANTEILEN DER UNFALLFOLGEN (ANGABEN IN PROZENT).

ABBILDUNG 9.7: UNFALLZAHLEN IM ALPINEN BERGSPORT (ABSOLUT UND EXPOSITIONSBEREINIGT AUF DER BASIS DER EINSCHÄTZUNG DER AUSÜBUNGSHÄUFIGKEITEN DER PROBANDEN).

ABBILDUNG 9.8: Z-WERTE DER ABSOLUTEN UNFALLZAHLEN IM ALPINEN BERGSPORT AUF DER BASIS DER ANALYSIERTEN UNFALLDATEN DES DAV (FÜR DIE JAHRE 2000 2003). 
ABBILDUNG 9.9: Z-WERTE DER EXPOSITIONSBEREINIGTEN UNFALLZAHLEN IM ALPINEN BERGSPORT AUF DER BASIS DER ANALYSIERTEN UNFALLDATEN DES DAV (FÜR DIE JAHRE 2000 - 2003).

ABBILDUNG 9.10: UNFALLZAHLEN ABSOLUT UND ANZAHL DER UNFÄLLE MIT TÖDLICHEN ODER SEHR SCHWEREN VERLETZUNGSFOLGEN IM ALPINEN BERGSPORT (VGL. AUCH RANDELZHOFER 2003)...

ABBILDUNG 9.11: Z-WERTE DER AUF IHRE SCHWERE RELATIVIERTEN UNFALLZAHLEN IM ALPINEN BERGSPORT.

ABBILDUNG 9.12: EINSCHÄTZUNGEN DER UNFALLHÄUFIGKEIT BEI DEN VORGEGEBENEN AKTIVITÄTEN DES ALPINEN BERGSPORTS. .298

ABBILDUNG 9.13: EINSCHÄTZUNGEN DER UNFALLFOLGEN BEI DEN VORGEGEBENEN AKTIVITÄTEN IM ALPINEN BERGSPORT..

ABBILDUNG 9.14: Z-WERT DIFFERENZ ZWISCHEN DEN SUBJEKTIVEN EINSCHÄTZUNGEN ÜBER DIE UNFALLHÄUFIGKEIT UND DEN TATSÄCHLICHEN ABSOLUTEN UNFALLZAHLEN IM ALPINEN BERGSPORT.

ABBILDUNG 9.15: Z-WERT DIFFERENZ ZWISCHEN DEN SUBJEKTIVEN EINSCHÄTZUNGEN ÜBER DIE UNFALLHÄUFIGKEIT UND DEN EXPOSITIONSBEREINIGTEN UNFALLZAHLEN IM ALPINEN BERGSPORT

ABBILDUNG 9.16: Z-WERT DIFFERENZEN ZWISCHEN DER SUBJEKTIVEN EINSCHÄTZUNG DER UNFALLFOLGEN UND DER OBJEKTIVEN AUF DIE SCHWERE RELATIVIERTEN UNFALLZAHLEN IM ALPINEN BERGSPORT 302

ABBILDUNG 9.17: Z-WERT DIFFERENZ ZWISCHEN MÄNNERN UND FRAUEN BEZOGEN AUF DIE SUBJEKTIVE EINSCHÄTZUNG DER UNFALLHÄUFIGKEIT UND DEN TATSÄCHLICHEN UNFALLZAHLEN IM BERGSPORT 304

ABBILDUNG 9.18: Z-WERT DIFFERENZ ZWISCHEN MÄNNERN UND FRAUEN BEZOGEN AUF DIE SUBJEKTIVE EINSCHÄTZUNG DER UNFALLFOLGEN UND DEN AUF DIE SCHWERE RELATIVIERTEN UNFALLZAHLEN IM BERGSPORT. 305

ABBILDUNG 9.19: Z-WERT DIFFERENZ ZWISCHEN VERSCHIEDENEN ALTERSGRUPPEN BEZOGEN AUF DIE SUBJEKTIVE EINSCHÄTZUNG DER UNFALLHÄUFIGKEIT UND DEN TATSÄCHLICHEN UNFALLZAHLEN IM BERGSPORT 306

ABBILDUNG 9.20: Z-WERT DIFFERENZ ZWISCHEN VERSCHIEDENEN ALTERSGRUPPEN BEZOGEN AUF DIE SUBJEKTIVE EINSCHÄTZUNG DER UNFALLFOLGEN UND DEN AUF DIE SCHWERE RELATIVIERTEN UNFALLZAHLEN IM BERGSPORT ...................................306

ABBILDUNG 9.21: Z-WERT-DIFFERENZEN ZWISCHEN DER SUBJEKTIVEN EINSCHÄTZUNG DER UNFALLHÄUFIGKEIT UND DEN TATSÄCHLICHEN UNFALLZAHLEN IM ALPINEN BERGSPORT IN ABHÄNGIGKEIT DER ALPINEN ERFAHRUNG 308

ABBILDUNG 9.22: Z-WERT-DIFFERENZEN ZWISCHEN DER SUBJEKTIVEN EINSCHÄTZUNG DER UNFALLFOLGEN UND DEN AUF DIE SCHWERE RELATIVIERTEN UNFALLZAHLEN IM ALPINEN BERGSPORT IN ABHÄNGIGKEIT DER ALPINEN ERFAHRUNG.

ABBILDUNG 9.23: Z-WERT-DIFFERENZEN ZWISCHEN DER SUBJEKTIVEN EINSCHÄTZUNG DER UNFALLHÄUFIGKEIT UND DEN TATSÄCHLICHEN UNFALLZAHLEN IM ALPINEN BERGSPORT IM VERGLEICH ZWISCHEN AUSGEWÄHLTEN ÜBUNGSLEITER-LIZENZEN UND PROBANDEN OHNE LIZENZ.

ABBILDUNG 9.24: Z-WERT-DIFFERENZEN ZWISCHEN DER SUBJEKTIVEN EINSCHÄTZUNG DER UNFALLHFOLGEN UND DEN AUF IHRE SCHWERE RELATIVIERTEN UNFALLZAHLEN IM ALPINEN BERGSPORT IM VERGLEICH ZWISCHEN AUSGEWÄHLTEN ÜBUNGSLEITER-LIZENZEN UND PROBANDEN OHNE LIZENZ

ABBILDUNG 9.25: ABSOLUTE UNFALLZAHLEN IM KANUSPORT FÜR DIE EINZELNEN KANUSPORTLICHE DISZIPLINEN MIT UNFALLAUFKOMMEN FÜR DIE JAHRE 19982003..

ABBILDUNG 9.26: SUMME DER UNFÄLLE IM KANUSPORT FÜR DIE JAHRE 1998 - 2003 BEZOGEN AUF DIE EINZELNEN KANUSPORTLICHEN DISZIPLINEN.

ABBILDUNG 9.27: ABSOLUTE UND EXPOSITIONSBEREINIGTE UNFALLZAHLEN IM KANUSPORT ALS SUMME DER JAHRE 1998 - 2003..

ABBILDUNG 9.28: Z-WERTE DER TATSÄCHLICHEN UNFALLZAHLEN IM KANUSPORT........ 314 ABBILDUNG 9.29: Z-WERTE DER EXPOSITIONSBEREINIGTEN UNFALLZAHLEN IM KANUSPORT.

ABBILDUNG 9.30: Z-WERTE DER SUBJEKTIVEN EINSCHÄTZUNG DER UNFALLHÄUFIGKEIT IM KANUSPORT 
ABBILDUNG 9.32: Z-WERT DIFFERENZ ZWISCHEN DER SUBJEKTIVEN EINSCHÄTZUNG DER UNFALLHÄUFIGKEIT UND DEN TATSÄCHLICHEN UNFALLZAHLEN IM KANUSPORT.

ABBILDUNG 9.33: Z-WERT DIFFERENZ ZWISCHEN DER SUBJEKTIVEN EINSCHÄTZUNG DER UNFALLHÄUFIGKEIT UND DEN EXPOSITIONSBEREINIGTEN UNFALLZAHLEN IM KANUSPORT.

ABBILDUNG 9.34: Z-WERTE DER GESCHLECHTSSPEZIFISCHEN EINSCHÄTZUNG DER UNFALLHÄUFIGKEIT IM KANUSPORT.

ABBILDUNG 9.35: GESCHLECHTSSPEZIFISCHE Z-WERT DIFFERENZEN ZWISCHEN DER SUBJEKTIVEN EINSCHÄTZUNG DER UNFALLZAHLEN UND DEN TATSÄCHLICHEN UNFALLZAHLEN IM KANUSPORT. DATENBASIS SIND 83 UNFÄLLE IN DEN JAHREN 1998 - 2003 SOWIE DIE EINSCHÄTZUNGEN VON BEFRAGTEN FRAUEN.

ABBILDUNG 9.36: ALTERSSPEZIFISCHE Z-WERT-DIFFERENZEN ZWISCHEN DER SUBJEKTIVEN EINSCHÄTZUNG DER UNFALLHÄUFIGKEIT UND DEN TATSÄCHLICHEN UNFALLZAHLEN IM KANUSPORT 322

ABBILDUNG 9.37: Z-WERTE DER SUBJEKTIVEN EINSCHÄTZUNG DER UNFALLHÄUFIGKEIT IN ABHÄNGIGKEIT DER ERFAHRUNG.

ABBILDUNG 9.38: ERFAHRUNGSSPEZIFISCHE Z-WERT-DIFFERENZEN ZWISCHEN DER SUBJEKTIVEN EINSCHÄTZUNG DER UNFALLHÄUFIGKEIT UND DEN TATSÄCHLICHEN UNFALLZAHLEN.

ABBILDUNG 9.39: Z-WERT DIFFERENZEN ZWISCHEN DER SUBJEKTIVEN EINSCHÄTZUNG DER UNFALLZAHLEN UND DEN TATSÄCHLICHEN UNFALLZAHLEN BEI FACHÜBUNGSLEITERN MIT DEM SCHWERPUNKT WILDWASSER

ABBILDUNG 10.1: DIE ECKPFEILER DES SPORTLICHEN RISIKOMANAGEMENTS 344

\section{Tabellenverzeichnis:}

TABELLE 2.1: BEGRIFFABGRENZUNG UND INHALTLICHE DIFFERENZIERUNG DES RISIKOSPORTS (EIGENE DARSTELLUNG).

TABELLE 2.2: KONSTITUTIVE ELEMENTE UND KENNZEICHNENDE MERKMALE DES RISIKOSPORTS ( EIGENE DARSTELLUNG) …………....................................................... 51

TABELLE 2.3: KLASSIFIZIERUNG DES RISIKOSPORTS (EIGENE DARSTELLUNG) ......................53

TABELLE 2.4: EVALUATION DES SPEZIFISCHEN RISIKOMOMENTS AM BEISPIEL KANUWILDWASSEFAHREN (EIGENE DARSTELLUNG).

TABELLE 2.5: EVALUATION DES SPEZIFISCHEN RISIKOMOMENTS AM BEISPIEL ALPINES HOCHTOURENBERGSTEIGEN (EIGENE DARSTELLUNG) ...................................................56

TABELLE 3.1: ABENTEUERSUCHE ALS DEFINITION DES WAHREN MENSCHSEINS IM AUSGEHENDEN 12. JAHRHUNDERT (ZIT. NACH NERLICH 1998, 85).

TABELLE 4.1: VIER KOMPONENTEN DES SENSATION SEEKING NACH ZUCKERMANN (1978); LEICHT MODIFIZIERT NACH RHEINBERG $(2002,170)$. 128

TABELLE 9.1: VERFAHRENSSCHRITTE ZUR EMPIRISCHEN BESTIMMUNG DER GEFAHRENKOGNITION ANHAND DER RELATIVEN ÜBEREINSTIMMUNG ZWISCHEN SUBJEKTIVEN URTEILEN ÜBER DIE GEFÄHRLICHKEIT VERTRAUTER AKTIVITÄTEN UND DEREN OBJEKTIVEN ENTSPRECHUNG IN FORM VON UNFALLDATEN (IN ANLEHNUNG AN MUSHAL $(1997,234)$.

TABELLE 9.2: ZUSAMMENSTELLUNG DER ABGRENZBAREN AKTIVITÄTEN FÜR DEN BEREICH DES ALPINEN BERGSPORTS UND DES KANUSPORTS

TABELLE 9.3: EIGENSCHAFTSVERGLEICH VON INTERNETBASIERTEN, MÜNDLICHEN, TELEFONISCHEN UND SCHRIFTLICHEN BEFRAGUNGEN (AUS THEOBALD 2000, 25).. 282

TABELLE 9.4: DIFFERENZEN IM EINSCHÄTZUNGSVERHALTEN AM BEISPIEL DES ALPINEN SKIFAHRENS AUF PISTEN UND VARIANTEN (N=724). 300

TABELLE 9.5: ZUSAMMENFASSUNG DER SCHÄTZUNGSDIFFERENZEN ZWISCHEN SUBJEKTIVER EINSCHÄTZUNG DER UNFALLHÄUFIGKEIT UND EXPOSITIONSBEREINIGTEN UNFALLZAHLEN UNTERTEILT NACH UNTERSCHÄTZTEN AKTIVITÄTEN $\left(\mathrm{Z}_{\in \mathrm{G}}<-1,0\right)$, REALISTISCH EINGESCHÄTZTEN AKTIVITÄTEN $\left(-1,0<\mathrm{Z}_{\in \mathrm{G}}>\right.$ $1,0)$ UND ÜBERSCHÄTZTEN AKTIVITÄTEN $\left(Z_{\in G}>1,0\right)$. 


\section{Einleitung}

Und die Stärke des Fadens liegt nicht darin, dass irgendeine Faser durch seine ganze Länge läuft, sondern darin, dass viele Fasern einander übergreifen.

(Ludwig Wittgenstein, Philosophische Untersuchungen, \67)

Zweifelsfrei gibt es wissenschaftliche Arbeiten, die von der ersten Formulierung einer Hypothese und der daraus abgeleiteten Konzeption eines Forschungsvorhabens, in methodisch unbestrittener Vorgehensweise, systematische und umfassende Ergebnisse liefern. Die vorliegende Arbeit kann und will diesen Eindruck nicht erwecken, denn zu vielfältig waren die Anstöße und zu fruchtbar die sich daraus entwickelten ,Umwege', die zum Abschluss dieser Arbeit geführt haben. Zu umstritten ist auch die Frage nach den Methoden und Erkenntniszielen innerhalb einer Sportwissenschaft, die sich im Spannungsfeld zwischen Legitimationskrise und theoretischen Diskursen der ,Mutterwissenschaften' bewegt. Zu vielschichtig und komplex erscheint auch der Forschungsgegenstand, als dass ein disziplinäres und stringent abgeschlossenes Forschungsvorhaben zu einem komplexen Erkenntnisgewinn führen könnte. Zugegeben: neben diesen äußeren Bedingungen liegt es sicherlich auch an den persönlichen Arbeitsbedingungen des Autors und den damit verbundenen Anregungen, die zu der gewählten Perspektivierung des Forschungsgegenstands geführt haben.

Dabei behandelt diese Arbeit ein vermeintlich hochaktuelles Thema: Risiken sind nicht nur allgegenwärtig, sie sind längst zum Normalfall des Alltags geworden. Vom Eintritt in die Risiko- und die Rückversicherungsgesellschaft ist die Rede, diskutiert wird über die Risiken der Gentechnik und der zukünftigen Energieversorgung, Risikokommunikation und Technikfolgeabschätzung wird als Schlüssel zur Risikominimierung gepriesen und immer wieder erschallt der Ruf nach mehr Achtsamkeit und Verantwortung. Unsicherheit - so hat es den Anschein - ist das einzige, dessen man sich heute 
sicher sein kann. In einer Gesellschaft, in der zunehmend administriert und reglementiert, ritualisiert und romantisiert, sichert und versichert wird, erhält Risiko einen festen Platz im alltäglichen Leben. Nicht nur Experten und Politiker, auch Laien und Wissenschaftler aller Fachrichtungen sind ,auf der Suche nach der verlorenen Sicherheit" (FISCHER 1991).

Vor diesem Hintergrund erscheint es fast schon paradox, eine Parallelentwicklung zu identifizieren und als Forschungsgegenstand zu defnieren, die sich im gesellschaftlichen Teilsystems des Sports beschreiben lässt: Immer mehr Menschen betreiben hier Aktivitäten, in denen konventionelle Sicherheitsvorstellung scheinbar nur eine untergeordnete Rolle spielen. Während ganze Teilbereiche des gesellschaftlichen Zusammenlebens von DINSchutznormen, Gesundheitsbestrebungen und Sicherheitsvorkehrungen geprägt sind, bewirbt die Industrie ihre Produkte mit waghalsigen Klippenspringern und Snowboardern: ,No risk - no fun' wird zum vermeintlichen Dogma einer modernen Sportkultur, die sich immer mehr als eigenständiges Handlungsfeld etabliert. Allein von ihrem Charakter her - durch die Ausübung in ,fremden' Elementen - beinhalten diese Sportarten das Risiko einer Verletzung, dass von den Protagonisten anscheinend bewusst in Kauf genommen wird. Manche Aktivitäten, die diesem Sportbereich zugeordnet werden - wie beispielsweise das Klettern oder das Mountainbiken - haben sich regelrecht zu Massenphänomenen ausgeweitet.

Die teilnahmslose Öffentlichkeit nimmt die Begeisterung einer derartigen Risikofreudigkeit im Sport indessen mit einer eigentümlichen Ambivalenz zur Kenntnis. Zum einen erscheint der Risikosport - gemessen an den oft spektakulären Bildern illustrierter Medienberichte, die die Gefahr scheinbar verdeutlichen - auch Außenstehende zu faszinieren. Zum anderen wird nach gravierenden Unfällen wie z.B. im Sommer 1999 bei einer Canyoning-Tour im schweizerischen Interlaken allgemeines Unverständnis und der Ruf nach Verboten derartiger Betätigungen laut. In Anbetracht der realen Gefährlichkeit wird an der Sinnhaftigkeit risikosportlicher Aktivitäten gezweifelt: In einer Gesellschaft der,Vollkasko-Angebote', gesundheitsorientierten Präventionsprogrammen und ,Rundum-Schutz-Pakete' müssten solche Aktivitäten - in denen man die eigene körperliche Unversehrtheit vermeintlich ganz freiwillig aufs Spiel setzt - doch weitestgehend gemieden werden, sind sie doch scheinbar unvereinbar mit ,[...] der allgemeinen Vorstellung sinnhaften Verhaltens“ (AUFMUTH 1989, 126) und werden ,[...] als Angriff auf die Integrität der alltäglichen Sinnwelt aufgefasst“" (ebd., 122).

Mit Risikosport als Forschungsgegenstand wird im Rahmen dieser Arbeit also ein Thema aufgegriffen, welches den Beobachter von Grund auf mit unterschiedlichsten Fragen konfrontiert und allein aus dem Alltagsverständnis heraus eine Problemstellung entwickelt: Ist Sicherheit nicht ein Urbedürfnis, bei dem zweifellos eine biologische Fundierung anzunehmen ist, da die Vorteile für die Selbst- und Arterhaltung offensichtlich sind? Was treibt Menschen dazu, sich solchen Risiken im Sport freiwillig auszusetzen? Hat die Menschheit während ihrer bisherigen Geschichte nicht unter Einsatz aller Fähigkeiten versucht, die konkreteste Form der Gefahr, die Bedrohung 
von Leib und Leben durch Naturgewalten aus dem Alltag zu verbannen? Und warum sind diese Sportbetätigungen gerade in unserer Zeit und in jenen Gesellschaften scheinbar am stärksten ausgeprägt, in denen dieses Aussperren unmittelbarer Gefahren aus dem Alltag - vom Terrorismus einmal abgesehen - am besten gelungen ist? Was bringt Menschen letztlich dazu unter großem Einsatz zeitlicher, finanzieller und physischer Ressourcen genau diese elementare Bedrohungen wieder aufzusuchen? Hinzu kommt ein weiterer Sachverhalt: Spektakuläre Sportunfälle in den verschiedensten Aktivitäten im Umfeld des Risikosport, wie beispielsweise beim Klettern oder Drachenfliegen, nähren den Verdacht, dass die Entwicklung dieses Sportbereiches mit einer Reihe erheblicher gesundheitsökonomischer Kosten verbunden ist. Gefordert werden Interventionskonzepte, die zu mehr Sicherheit im Risikosport führen sollen. Aber wie ist, Sicherheitsintervention' in einem Praxisfeld möglich, welches Risiko zu einem expliziten Thema macht?

Angesichts dieser sich aufdrängenden Fragen ist es erstaunlich, dass das Phänomen Risikosport bis dato nur eine geringe wissenschaftliche Rezeption erfahren hat. Zwar ist die Diskussion um „Das Wagnis im Sport“ (NEUMANN 1999) insbesondere in der Sportpädagogik deutlich vorangekommen und auch aus Reihen anderer sportwissenschaftlicher Disziplinen sind in den letzten Jahren verschiedene Beiträge zum Thema Risikosport erschienen ${ }^{1}$, eine systematische und vor allem interdisziplinäre Forschungsleistung steht aber weiterhin aus. Dabei findet sich in den Forschungsdisziplinen der Bezugswissenschaften ein reichhaltiger Fundus an wissenschaftlichen Abhandlungen, der zwar nicht explizit auf das Handlungsfeld Sport verweist, durch den aber überaus fruchtbare Anschlussleistungen ermöglicht werden. Im Gegensatz zu anderen disziplinären Forschungsvorhaben richtet sich das forschungsleitende Interesse dieser Arbeit demnach nicht nach einer konkreten Forschungsfrage, sondern nach dem Ziel möglichst viele unterschiedliche Ansätze zur Erklärung des Phänomens Risikosport zu explorieren und - aufeinander bezogen - zu evaluieren. Zwar vermag auch die vorliegende Arbeit keine endgültigen Antworten auf die oben angedeuteten Fragen liefern, denn es ist zu bezweifeln, dass es in diesem Zusammenhang überhaupt endgültige Antworten geben kann. Wohl aber eröffnet diese Arbeit durch den interdisziplinären Forschungszugang Perspektiven, die dazu beitragen können, das Phänomen Risikosport zu verstehen und in seinen interdependenten Zusammenhängen zu erklären. Die methodologische Schwäche, die mit einem interdisziplinären Forschungsansatz verbunden ist, wird dabei bewusst in Kauf genommen, um die Vielfalt der Beiträge und Forschungsansätze zu erfassen und im Bezug auf risikosportliche Aktivitäten systematisch zu entfalten. Angeregt durch die Diskussion um transdisziplinäre Forschungszugänge in den Sozialwissenschaften (BRAND et al.

\footnotetext{
${ }^{1}$ Hierbei handelt es sich in der Mehrzahl um disziplinäre Beiträge z.B. der Sportpädagogik (SCHLESKE 1977; NEUMANN 1999 \& 2003; WARWITZ 2001), der Sportpsychologie (EPSTEIN 1977; AUFMUTH 1983; ERDMANN 1990; APTER 1994; SEMMLER 1994; ALLMER 1998; OPASCHOWSKI 2000; BEIER 2001; SCHIEDEK 2003), der Sportsoziologie (LE BRETON 1995; BETTE 1999 \& 2004; RITTNER 2001; STERN 2003; WHEATON 2004), der Sportphilosophie (ELSÄSSER 1979; CLAUSEN 2003), der Physiologie (ZUCKEMAN 1978; FABRY 1990).
} 
2004) wird eine wissenschaftliche Forschung intendiert, die disziplinübergreifend zur sportwissenschaftlichen Problemlösung führen soll.

„Dabei wird das Gesamtproblem so in Teilbereiche eingeteilt (Problemzerle-
gung), dass in den Teilbereichen Methoden aus unterschiedlichen Dis₹iplinen
angewendet und dafür auch kombiniert und abgewandelt werden können
(Freibeit in der Methodenwabl). Gleichzeitig ist jeder Teilbereich auf die übri-
gen Teilbereiche - und somit auf das Gesamtproblem - ausgerichtet"
(JAEGER \& SCHERINGER 1998, 15).

Für eine integrierende Sportwissenschaft, die die menschliche Bewegung zum Forschungsgegenstand macht, ist aber nicht allein die theoretische Deduktion von erkenntnistheoretischem Interesse, denn Sport als Forschungsgegenstand impliziert allein durch seine gesellschaftliche Bedeutung einen konkreten Anwendungsbezug theoretischer Erkenntnisse. Die gesellschaftliche Forderung nach Interventionskonzepten zur Unfallprävention im Risikosport, die vor allem nach Unfallsituationen wie in Interlaken 1999 laut werden (vgl. RITTNER 2001, 218), wird im Rahmen dieser Arbeit daher als pragmatische Aufgabe begriffen, einen sinnhaften Anwendungsbezug der theoretischen Erkenntnisse herzustellen.

Genaugenommen beinhaltet die Arbeit damit zwei Herausforderungen, die - aufeinander bezogen - unterschiedliche Herangehensweisen und Forschungszugänge erfordern. Zum einen muss das Phänomen Risikosport in seinen inhaltlichen Bezügen und strukturellen Bedingungen untersucht werden, um - im Sinne einer sportwissenschaftlichen Grundlagenforschung Erklärungszusammenhänge dieses Handlungsfeldes darzustellen. Auf der anderen Seite erfordert die pragmatische Aufgabenstellung eine intensive Auseinandersetzung mit dem spezifischen Unfallgeschehen im Risikoport. Aus diesen beiden Aufgaben ergibt sich auch die Struktur dieser Arbeit: Im ersten Teil wird eine inhaltliche Bestimmung des Risikosportphänomens vorgenommen und Erklärungszusammenhänge im Rahmen eines interdisziplinären Erkenntnisprozesses exploriert. Da es weder größere Theoriearbeiten noch themenbezogene empirische Studien zum Unfallgeschehen im Risikosport und ihren sportwissenschaftlichen Erklärungszusammenhängen gibt, wird im Rahmen der anwendungsbezogenen Aufgabenstellung im zweiten Teil der Arbeit ein deduktiv-empirischer Forschungszugang gewählt. Dabei lässt sich das Interventionsziel der Arbeit nur unter Berücksichtigung beider Teile hinreichend verwirklichen, denn in ein System intervenieren kann man wirksam nur dann, wenn man eine grundlegende Vorstellung vom Zustand des Systems ${ }^{2}$, seinen Funktionsprinizipien und Wechselwirkungen hat (vgl. MUSAHL 1997). Während also im ersten Teil der Arbeit sozusagen das Basiswissen für das Interventionsziel bereitgestellt wird, erfährt es durch die empirische Studie im zweiten Teil eine anwendungsrelevante Transformation.

\footnotetext{
${ }^{2}$ Ein System bezeichnet in Sinne LUHMANNs ,[...] einen ganzheitlichen Zusammenhang von Teilen, deren Beziehungen untereinander quantitativ intensiver und qualitative produktiver sind als ihre Beziehungen zu anderen Elementen“ (WILKE 1993, 19).
} 
Zum Aufbau dieser Arbeit: Im Anschluss an diese Einleitung wird im Hinblick auf die grundsätzliche Definitionsschwäche sportwissenschaftlicher Risikoterminologien zunächst eine Begriffsbestimmung vorgenommen (Kap. 2). Hierbei werden Unsicherheitsbegriffe vor dem Hintergrund ihrer inhaltlichen und konzeptionellen Bedeutung im Rückgriff auf forschungsrelevante Ansätze vorgestellt (2.1). Es schließt sich eine inhaltsorientierte Analyse der Wortfeldes Risiko an, die im konkreten Bezug zum Risiko im Sport über die Abgrenzung und Bestimmung risikoaffiner Begriffe erfolgt (2.2). Die Einordnung und Definition des Risikosports als sportwissenschaftliche Terminologie (2.3) wird darauf folgend über die Einordnung in die gegenwärtige Entwicklung des Freizeitsports und einen Überblick über den Forschungstand vollzogen. Daraus resultiert schließlich ein eigener Definitionsentwurf von Risikosport, bei dem ein subjektzentrierter Bestimmungsansatz im Mittelpunkt steht.

In Kapitel 3 wird ein geschichtswissenschaftlicher Zugang gewählt, um historische Entstehungszusammenhänge des Risikos im Sport aufzuspüren und grundlegende Erkenntnisse über die Funktionszusammenhänge zu gewinnen. Da sporthistorische Forschungsansätze zum Risikosport bis auf wenige Ausnahmen nicht existent sind, werden kultur- und sozialhistorische Forschungsleistungen zum Erkenntnisprozess herangezogen. Dabei werden zuerst begriffsgeschichtliche Zusammenhänge konkretisiert und Risiko als historischer Unsicherheitstopos deduziert (3.1). Darauf folgend werden kulturhistorische Verknüpfungen risikosportlicher Aktivitäten expliziert (3.2). Hierbei findet sich ein erster historischer Zugang zum sportlichen Risiko in der Geschichte des Alpinismus, der in seinen historischen Dimensionen auf konkrete Entwicklungszusammenhänge des Risikosport verweisen kann. Aber auch die klassischen Schriften der Erlebnispädagogik (ROUSSEAU; THOREAU; DILTHEY; SIMMEL; HAHN) sind für den Erkenntnisprozess von herausragender Bedeutung, da sie für risikosportliche Aktivitäten eine Art ideologischen Nährboden darstellen.

Eine Bestandsaufnahme psychologisch-anthroplogischer Forschungsbeiträge zum Risikosport wird Gegenstand des 4. Kapitels. Dabei werden zunächst motivationstheoretische Erklärungsansätze als theoretischer Bezugsrahmen psychologischer Motivationsforschung dargestellt (4.1). Darauf aufbauend wird mit den anthropologischen Annahmen ein grundsätzliches Erklärungsmodell für Risikoverhalten deduziert (4.2). Aus der Vielfalt motivationspsychologischer Theorien und Untersuchungen werden anschließend die für den Risikosport bedeutsamen Befunde dargestellt und im Bezug auf ihre Forschungsrelevanz evaluiert (4.3). Hierbei werden neben aktivationstheoretischen und kogitiv-handlungstheoretischen Forschungsbeiträgen auch biologisch ausgerichtete Studien sowie psychoanalytische Ansätze vorgestellt.

Kapitel 5 greift die im Kontext des geschichtswissenschaftlichen Forschungszugangs deduzierten Ergebnisse über die kulturhistorischen Entstehungszusammenhänge des Risikosports erneut auf. Aus soziologischer Perspektive wird hierbei nach den gesellschaftstheoretischen Funktionszusam- 
menhängen des Risikosports gefragt und eine Verknüpfung der zuvor dargelegten Motivationselementen auf makrosoziologischen Ebene vorgenommen. Als Bezugspunkt der soziologischen Deduktion werden zunächst zentrale Gesellschaftsdiagnosen für die (Post-) Moderne aufgegriffen (5.1) und in einen forschungsrelevanten Kontext transformiert (5.2). Da Sicherheit und Risiko im Sinne gesellschaftlicher Erfahrungswirklichkeiten relevante Kategorien für den Risikosport darstellen, schließt sich eine Annäherung aus soziologische Perspektive an (5.3). In Kapitel 5.4 werden darauf folgend Wirkungszusammenhängen im Bezug auf Risikosport im Lebenslauf eruiert. Ähnlich verhält es sich auch in Abschnitt 5.5, in dem auf der Basis sozialisationstheoretischer Forschungsansätze nach risikosportlichen Geschlechtsdivergenzen gefragt wird. Um die phänomenologische Betrachtung des Risikosports abzuschließen folgt abschließend eine sportpädagogische (5.6) und sportphilosophische (5.7) Evaluation des Risikosports und seiner pädagogisch-ethischen Implikation.

In Kapitel 6 wird dem handelnden Risikosportler erneut die Mirkoebene des Risikosports fokussiert und der Frage nach spezifischen Handlungsregulativa des Risikoverhaltens im Sport nachgegangen (6.1). Auf den Erkenntnissen der modernen Handlungstheorie aufbauend wird anschließend nach beeinflussenden Variablen im Bezug auf Wahrnehmungs- und Beurteilungsvorgänge im Risikosport gesucht (6.2). Hierbei werden Erkenntnisse der Kognitionspsychologie und Verhaltenspsychologie für den Kontext des Risikosports deduziert. Abschließend wird das Handeln im Risikosport zusammenfassend in der Form eines transaktionalen Modells expliziert (6.3).

Mit einem Zwischenfazit in Kapitel 7 wird der ersten Teil der Arbeit abgeschlossen und die Tragfähigkeit der im Rahmen der interdisziplinären Analyse aufgestellten komplexen Ergebnisse diskutiert. Dabei wird eine Überleitung auf den zweiten Teil der Arbeit vorgenommen und das Forschungsvorhaben auf der Basis der bisherigen Erkenntnisse entwickelt.

Mit Kapitel 8 wird der zweite Teil der Arbeit erschlossen. Hier werden auf den Erkenntnissen des ersten Teils aufbauend - die theoretischen Grundlagen der empirischen Studie vorgestellt, die im Rahmen der gestellten anwendungsorientierten Aufgabe dieser Arbeit durchgeführt wurde. Da die Untersuchung auf grundlegenden Paradigmen der Unfallforschung aufbaut, werden zunächst die theoretischen Grundlagen der sportwissenschaftlichen Unfallforschung dargestellt (8.1). Mit einer konzeptionellen Bestimmung des Unfalls wird anschließend der kategoriale Rahmen der Untersuchung expliziert (8.2). Abschließend werden verschiedene Forschungszugänge der Unfall- und Sicherheitsforschung deduziert und auf ihre spezielle Forschungsrelevanz für die Aufgabenstellung dieser Arbeit hin untersucht (8.3).

Die empirische Studie wird schließlich in Kapitel 9 vorgestellt. Zuerst wird mit der Transformation des Gefahrenkognitionsmodells von MUSAHL (1997) ein konkretes Untersuchungsmodell für den Kontext des Risikosport deduziert und die Frage- und Aufgabenstellung der Untersuchung verdeutlicht (9.1). In Abschnitt 9.2 wird schließlich das methodische Vorgehen dargestellt. Das gewählte empirische Verfahren, entstammt der 
klassischen Sicherheitspsychologie in Anlehnung an MUSAHL (1997) und kombiniert Unfallanalysen mit subjektiven Erhebungsverfahren. Die im Rahmen der vorliegenden Arbeit durchgeführte empirische Erhebung beschreitet dabei methodisch - für die wissenschaftliche Forschung im allgemeinen sowie für die Sportwissenschaften im Besonderen - einen relativ neuen Weg: Die Datenerhebung der subjektiven Daten erfolgte mittels eines internetbasierten Fragebogens. Da onlinebasierte Befragungen in den Sozialwissenschaften allgemein noch wenig evaluiert sind, wird diesem innovativen Erhebungsinstrument eine Sonderstellung im Rahmen eines Exkurses eingeräumt. Anschließend werden die Untersuchungsergebnisse vorgestellt (9.3) und schließlich im Bezug auf die Aufgabenstellung der Studie diskutiert (9.4). In einem Fazit über die Untersuchung werden die relevanten Ergebnisse nochmals zusammengefasst und hinsichtlich ihrer Anwendungsrelevanz bewertet (9.5).

Mit Kaptitel 10 erfolgt schließlich der Versuch ein wissenschaftlich fundiertes Interventionskonzept für den Risikosport mit dem Ziel einer pragmatischen Unfallprävention zu entwickeln. In 10.1 werden zunächst grundlegende Gedanken zum Interventionsziel ,Risikomanagement' dargestellt. Anschließend wird der für ein Interventionskonzept im Risikosport notwendige fundamentale Paradigmenwechsel, der eine Abkehr von herkömmlichen Sicherheitskonzeptionen fordert, deduziert (10.2). Die Eckpfeiler des Interventionskonzeptes ,Risikomanagement' stellen die Ableitung der theoretischen Erkenntnisse und empirischen Befunde dar und werden in $\mathrm{Ab}$ schnitt 10.3 dargestellt. In Abschnitt 10.4 werden darauf folgend systematische Gedanken zum Vermittlungsprozess in Risikoportarten expliziert und abschließend schon bestehende Interventionskonzepte in ihrer Bedeutung als Risikomanagementinstrumente analysiert (10.5). Das Kapitel schließt mit einer kritischen Schlussbetrachtung (10.6).

Aufgabe der Schlussbemerkung in Kapitel 11 ist, den wissenschaftlichen Erkenntnisgewinn der Arbeit abschließend zusammenzufassen und den Ertrag der Arbeit vor dem Hintergrund der bestehenden Diskussion zu reflektieren. Risikosport wird in seinen Erklärungszusammenhängen schließlich pointiert hervorgehoben. Es wird zudem abschließend kritisch diskutiert, inwieweit die Ergebnisse der Arbeit Anregungen für weitere Forschungsvorhaben darstellen können.

Eine Anmerkung zum Schluss: Aus Gründen der Lesbarkeit wird in dieser Arbeit die männliche Anredeform benutzt, wobei die weibliche selbstverständlich mit eingeschlossen ist.

Außerdem wird versucht, die Regeln der neuen Rechtschreibung zu verwenden. An einigen Stellen, vor allem bei Zitaten, wird aber noch die alte Rechtschreibung benutzt, was unvermeidbar ist und nicht extra gekennzeichnet wird. 


\section{Risiko-Risikoverhalten-Risikosport: Begriffs- bestimmungen und theoretische Grundlagen}

Nichts geschieht obne Risiko, aber obne Risiko gescbiebt auch nichts. (Walter Scheel (*1919), dt. Politiker)

Wenn es hinsichtlich des Risikosports einer übergeordneten interdisziplinären Forschungsarbeit bedarf, dann ist eine Begriffsklärung und inhaltliche Einordnung in den Kontext des Handlungsfeldes Sports und seiner zugeordneten Begriffe notwendig. Der Versuch einer Evaluation wichtiger Zusammenhänge kann im Bezug auf einen Forschungsstand letztlich erst dann unternommen werden, wenn deutlich ist, was sich hinter dem Begriff des Gegenstandes verbirgt. Gefordert ist hier also eine möglichst konkrete Operationalisierung von relevanten Begriffe im Rahmen des hier angestrebten Forschungsdesigns.

Zunächst ist eine allgemeine Klärung der Wortbedeutungen von ,Risiko', ,Wagnis', ,Abenteuer' und ,Gefahr' erforderlich, da diesen Begriffen im alltäglichen Sprachgebrauch oft eine diffuse Bedeutungsgleichheit unterstellt wird (vgl. NEUMANN 1999, 9). Zudem ist es notwendig, das sportliche Risiko von anderen Risikobereichen, wie wirtschaftlichen oder technischen Risiken, zu unterscheiden und damit das Phänomen Risikosport einzugrenzen. Einen ersten klärenden Zugang zu den Begriffsbestimmungen bietet daher eine etymologische Annäherung an den Begriff ,Risiko', um diesen von ,Wagnis', ,Abenteuer' und ,Gefahr' abzugrenzen. Darüber hinaus wird das sportliche Risiko als Erlebnis- und Handlungsfeld anhand inhaltlicher Differenzierungen des Risikobegriffs (Risikoarten, Risikoverbalten, Risikosituation) analysiert und dargestellt.

Im Anschluss ist eine strukturelle und inhaltliche Analyse des Begriffs und Phänomens ,Risikosport' in der Darstellung des Forschungstandes er- 
forderlich, die im Bezug auf ausgewählte Sportarten überprüft wird. Der Begriff ,Risikosport' ist bisweilen im Kanon der sportwissenschaftlichen Beiträgen nicht eindeutig definiert worden und wird mit anderen Teilbereichen des modernen Sports wie z.B. Trendsport, Extremsport oder Funsport vermischt. Eine Begriffsbestimmung wird hierbei aus einer vergleichenden Darstellung des Forschungsstandes deduziert und in Bezug auf die inhaltliche Bestimmung des Risikobegriffes expliziert.

\subsection{Risiko - ein multidisziplinärer Forschungsgegens- tand}

Risiko als Begriff wird in den verschiedensten wissenschaftlichen Forschungsgebieten in Abhängigkeit des untersuchten Kontextes definiert und verwendet. Dabei ist die Begriffsdefinition kein wissenschaftlicher Wert an sich, sondern sie dient der präzisen Beschreibung dessen, wovon zu sprechen ist. Die Qualität der Definition richtet sich nach dem Ausmaß, in dem sie diese pragmatische Funktion erfüllen kann.

Risiko als multidisziplinäres Forschungsfeld bedeutet also eine Vielfalt ganz unterschiedlicher Definitionen, die sich aus dem entsprechenden Forschungskontext ergeben. GLENDON (1987) erklärt die Multidisziplinarität des Risikos als Forschungsgegenstand aus der Tatsache, dass die unterschiedlichen Fachdisziplinen ineinander verschachtelt sind und aufeinander aufbauen. In ganz ähnlicher Weise beschreibt auch DODERLEIN (1987) Risiko als ein Forschungsfeld mit ganz unterschiedlichen Ebenen und betont die Notwendigkeit einer strikten Trennung zwischen den Zuständigkeiten der verschiedenen Disziplinen.

Die wissenschaftliche Risikodiskussion wird dabei vor allem in den Bereichen der Wirtschaftstheorie, dem Versicherungswesen (Mathematik), der Rechtswissenschaften sowie in Diskurses der Soziologie, der Politikwissenschaft, der Philosophie, der Kulturanthropologie und der Psychologie geführt (vgl. CAPPEL 1993, 5; dazu auch JAPP 2000; BANSE \& BECHMANN 1998).

In der Mathematischen Risikoforschung, die auch in der Versicherungsbranche von Bedeutung ist steht Risiko für Unbestimmtheit künftiger Situationen und Ereignisse, die beim handelnden Subjekt sowohl zu Gewinnen als auch zu Verlusten führen können. Die Kalkulation des Risikos mit Hilfe systematischer Berechnungssystemen steht diesbezüglich im Vordergrund. In einem engen Zusammenhang stehen natur- und technikwissenschaftliche Forschungsrichtungen, die als Hauptgegenstand die Versagensfälle im Bereich der Technik und Umwelt betrachten und sich damit vor allem als Sicherheitsforschung verstehen. In den Wirtschaftswissenschaften steht die Risikobetrachtung im Kontext der Ziele und Zielerreichungsmöglichkeiten von Unternehmen. Hierbei stehen vor allem unsichere Unternehmungen und ihre Machbarkeit im Fokus der wissenschaftlichen Beiträge. In den Rechtwissenschaften existiert derzeit noch keine eigenständige Risikodebatte, obwohl 
Risiko und Sicherheit zu zentralen Themen auch in der Rechtswissenschaft zählen. Die bis dato geführte Diskussion hat die Risikoverwaltung sowie die Risikoabschätzung in technologischen Bereichen zum Gegenstand. In der Soziologie hat sich Risiko als Konzept erst relativ spät zu einem gesellschaftlich relevanten Thema etabliert. Gegenstand der soziologischen Risikoforschung ist die Bedeutung von Risiko- und Sicherheit für Vergesellschaftungsprozesse sowie die makrosoziologische Bedeutung des Risikos für die Gesellschaftskonstituierung. Der Schwerpunkt politikwissenschaftlicher Analysen liegt auf der Ausarbeitung von institutionellen Verarbeitungs- und Regulationsprinzipien im Bezug auf politisch-administrative Entscheidungen. Die politikwissenschaftliche Debatte thematisiert darüber hinaus das RisikoManagement und Fragen politischer Strategien im Bezug auf unsichere Themen. Die Kulturtheorie untersucht die Wahrnehmung von Unsicherheit unter differenzierten kulturellen Bedingungen. Risiko wird hier als Bestandteil eines zusammenhängenden Interpretationsrahmens und Weltdeutungssysteme verstanden. Der philosophische Ansatz zur Risikoforschung greift kognitive, methodologische und normative Probleme der Risikothematik auf und versteht sich in ihrer Auslegung dieser Probleme als Lebens- und Entscheidungshilfe. Die Psychologie zeichnet sich durch unterschiedliche Begrifflichkeiten und konzeptionelle Ansätze im Bezug auf die inhaltliche Deutung von Risiko aus. Im Mittelpunkt der publizierten Forschungsbeiträge steht in der Mehrzahl das Individuum und seine für Risikoentscheidungen relevanten kognitiven und emotionalen Prozesse sowie die sozialpsychologische Bedeutung des Risikos für Vergemeinschaftungen und Identitätskonzepte (vgl. BANSE 1996b; BANSE \& BECHMANN 1998). In der Sportwissenschaft ist Risiko als Forschungsgegenstand noch ein eindeutiges Forschungsdesiderat auch wenn Anzahl und Qualität der wissenschaftlichen Beiträge in den letzten Jahren deutlich zugenommen haben (vgl. NEUMANN 1999; BETTE 2004).

Risiko, so kann zusammenfassend bestätigt werden, wird also von den beteiligten Wissenschaften in unterschiedlicher Weise und mit der jeweiligen methodologischen ,Forschungsbrille' ihrer Paradigmen betrachtet. Für den interdisziplinären Ansatz dieser Arbeit ist der Risikobegriff in mannigfacher Weise konzeptuell bedeutsam. Der Rückgriff auf verschiedene fachwissenschaftliche Konzepte der Risikoforschung und damit die unterschiedliche sprachliche sowie inhaltliche Begriffsverwendungen wird im Rahmen eines solches Ansatzes berücksichtigt.

Die vorliegende Arbeit vollzieht sich an der Schnittstelle von Psychologie, Soziologie, Geschichte und Philosophie, wobei ein interdisziplinärer Ansatz mit sportwissenschaftlichem Interesse intendiert wird. Da eine einheitliche Begriffsbestimmung und Differenzierung in der Sportwissenschaft zur Zeit noch aussteht wird ein kumulativ-integrierender Ansatz bei der Begriffsfindung verfolgt. 


\subsection{Das Wortfeld „Risiko“}

Der Begriff Risiko (von lat. risicare) bedeutet ursprünglich ,Gefahr laufen’ oder ,Klippen umschiffen'. Er lässt sich ursprünglich aus dem Seemannsmilieu ableiten und steht dort für Ereignisse und Tätigkeiten, die mit kritischen Situationen verknüpft sind (WARWITZ 2001, 15). Abgeleitet aus dem italienischen Wort, riscio' oder, risco', wurde der Begriff im 16. Jahrhundert als kaufmännischer Terminus benutzt und im Rahmen des individuellen Tauschhandels gebraucht (vgl. JAPP 2000, 6f). Der Ursprung des deutschen Begriffs ,Risiko' liegt im Wirtschafts- und Versicherungswesen und wird mit einer allgemeinen ,Verlustgefahr' definiert und mit, Wagnis', ,Abenteuer' und ,Gefahr' übersetzt (ebd.).

Die Schwierigkeit einer Begriffsbestimmung besteht einerseits in der Überschneidung mit ähnlich verwendeten Begriffen zu denen eine eindeutige Abgrenzung erforderlich ist und andererseits in der oben erwähnten stark divergierenden Begriffsverwendung in den unterschiedlichen wissenschaftlichen Disziplinen. MUSHAHL beispielsweise lehnt den Begriff Risiko aus Gründen ,[...] der notwendigen Trennung von Konzepten zur Beschreibung und zur Erklärung, also im Sinne ,begrifflicher Hygiene' [..]" (MUHSAHL 1997, 122) grundsätzlich ab und plädiert für Gefahr als übergreifenden Begriff.

Wie im folgenden gezeigt wird, eröffnet aber gerade die Differenzierung zwischen Risiko und Gefahr - die im Rückgriff auf LUHMANN erfolgen wird - eine fruchtbare Anschlussleistung. Die folgende Begriffsbestimmung erfolgt daher zunächst in Abgrenzung zu risiko-affinen Begriffen und der Deutung der wichtigsten kompositorischen Wortbedeutungen.

\subsubsection{Inhaltliche Bestimmung und Abgrenzung des Risikobegriffs}

PRIEST beschreibt das Risiko als „the potential to lose something of value“ (PRIEST 1990, 157). Diese allgemeine Definition kann aber auch auf die Bedeutung des Begriffs ,Gefahr' zutreffen, der als ,[...] die menschliche Sicherheit bedrohendes Unheil" (BROCKHAUS 1983 Bd. 2, 175) beschrieben wird. Als Opponent zur Sicherheit beschreibt die Psychologie das Risiko als „[...] eine Situation, die durch eine mangelnde Voraussehbarkeit des Kommenden bestimmt ist und zudem den Situationspartner bedroht [...]“ (DORSCH (Psychologisches Wörterbuch) 1998, 743).

LUHMANN (1988; 1990; 1991) setzt Risiko nicht in Opposition zu Sicherheit sondern nimmt eine Abgrenzung zur Gefahr vor ${ }^{3}$. Im Vergleich zur Gefahr besitzt das Risiko einen immanenten dynamischen Aspekt von antizipierenden Entscheidungen:

\footnotetext{
${ }^{3}$ LUHMANN führt an, dass die Sicherheit eine doppelte Chance im Gegensatz zur Risiko aufweisen müsste. Nicht nur die Eintrittwahrscheinlichkeit sondern auch die Chance des Risikos müsste im Begriff der Sicherheit berücksichtigt werden (vgl. auch NEUMANN 1999, 10).
} 
„Von Risiken spricht man dann, wenn etwaige künftige Schäden auf die eigene Entscheidung zurückgeführt werden. [...] Bei Gefahren handelt es sich dagegen um von außen kommende Schäden" (LUHMANN 1988, 86).

In Abwägung von möglichen Gewinnen schließt Risiko damit auch eventuelle negative Folgen mit ein:

„Das Risiko wird erst dann zu einem solchen, wenn die Gefahr als Gegenstand und als Folge einer eigenen Entscheidung zu sehen ist. [...] Risiken entstehen nicht durch bloße Unwissenheit, sondern paradox ausgedrückt, durch gewusstes Nichtwissen" (JELDEN 1998, 24).

Vor allem bei technischen und wirtschaftlichen Risiken kann durch eine entsprechende Kalkulation eine konkrete Risikoanalyse durchgeführt werden. Obwohl sich das Risiko z.B. im Sport nicht gegenständlich berechnen lässt, findet auch bei intendierten Bewegungshandlungen ein individueller Bewertungsprozess der Folgen statt, der zwischen der möglichen Schadenswahrscheinlichkeit und den eventuellen Schadensauswirkung abwägt (vgl. BALZ \& NEUMANN 1994, 94).

Das sportliche Risiko wird sowohl im Sprachgebrauch, als auch in der sportwissenschaftlichen Literatur häufig mit den Begriffen Wagnis und Abenteuer synonym verwendet. SHORT (1984) und FREY (1991) bestimmen das Risiko im Bezug auf den Wettkampfsport als Grad der Unsicherheit über den Ausgang des Wettkampfes, bei dem ein signifikanter Gewinn oder Verlust möglich ist (vgl. SHORT 1984, 711; FREY 1991, 137). Wagnis und Abenteuer charakterisieren im unreflektierten Sprachgebrauch eine ganzheitliche Herausforderung, die nur durch den Einsatz der ganzen Person bewältigt werden kann und das Individuum als Entscheidungsträger in den Mittelpunkt rückt (vgl. NEUMANN 1999, 9). Von einem Wagnis wird gesprochen,

„[...] wenn ein Handelnder sich in selbstgefährliche Situationen begibt [...]. Nur ich nebme selber den Reiz, möglicher Gefahren als Herausforderung an und versuche die Situation aktiv zu kontrollieren" BALZ \& NEUMANN 1994, 50).

Auch WARWITZ (2001) differenziert zwischen Wagnis und Risiko unter Einbeziehung der personalen Entscheidungsebene. Während das Risiko als Teilkomponente an jedem Wagnis beteiligt ist, legt der Begriff des Wagnisses seinen Bedeutungsschwerpunkt auf die Vorgänge innerhalb der sich gefährdenden Person (vgl. WARWITZ 2001, 16). Risiko wird demnach eher bei ziel- und zweckgerichteten Fragen Verwendung finden, während der Wagnisbegriff im Zusammenhang mit sinn- und wertgerichteten Problemstellungen erscheint.

„Das Riskieren betrifft lediglich einen Sachaspekt, einen Teil von uns. Das Wagen hebt den Personenaspekt heraus, meint die Persönlichkeit als unteilba- 
res Ganzes. Das Riskieren hat eher die materielle Seite, das Wagen eher mehr die ideelle Seite des Problemfeldes im Blick" (ebd., 17)4.

Um diesen im Wagnisbegriff implizierten Einsatz der ganzen Person im Risikobegriff zu berücksichtigen, definiert KLEBELSBERG das Risiko im Sport als ein ganzheitliches Gefahrenrisiko. Die Gesamtsituation ist demnach nicht durch eine einfache alternative Wahlentscheidung gekennzeichnet, die ein mehr oder weniger an materiellen Gewinnen und Verlusten hervorbringt, sondern durch die antizipierte Risikofolge in Form der Bedrohung der körperlichen Unversehrtheit (vgl. KLEBELSBERG 1969, 174).

RENN (1989; 2002) nähert sich dieser schwierigen Begriffsbestimmung semantisch und differenziert zwischen dem Risiko als Damoklesschwert, dem Risiko als Schicksalsschlag, dem Risiko als Frühindikator für Gefahren, dem Risiko als Glücksspiel und dem Risiko als Herausforderung der eigenen Kräfte. Vor allem im letztgenannten werden die Parallelen zwischen den Begriffen Risiko und Wagnis deutlich, die eine synonyme Begriffsbenutzung erlaubt.

Auch in der frühen wissenschaftlichen Auseinandersetzung um die Begriffe im Kontext des Sports lassen sich wenig Differenzierungsansätze finden. Während BOUET (1969) die Bedingungen und motivierenden Faktoren von Sportarten unter der Perspektive des Risikos diskutiert, spricht BALINT (1994) in seinen Ausführungen über die psychoanalytischen Hintergründe erlebnisreicher Sportarten von Abenteuer, SLUSHER (1967) in „Man, Sport and Existence“ wiederum vom Wagnis. Auch BOLLNOW plädiert für den Wagnisbegriff:

„Man kann durchaus sein Leben für etwas einsetzen, aber das ist etwas ganz. anderes als ein Risiko eingehen, da nehme ich um einer tieferen Verantwortung willen die Gefahr bewusst auf mich. Das ist dann Eingehen gegenüber einem echten Wagnis. Das Wagnis führt wiederum in einen ganz anderen Bereich. Wir können den Unterschied vielleicht am so bestimmen, dass wir sagen: ich riskiere immer etwas, aber ich wage im letzten mich" (BOLLNOW 1959, 137).

Trotz der allgemeinen sprachlichen Bedeutungsgleichheit und synonymen Verwendung bestehen vor allem in der neueren wissenschaftlichen Unterscheidung des Begriffpaares Wagnis / Risiko zum Abenteuer gewichtige Unterschiede. So erscheint das Wagnis / Risiko ,[...] in Form eines Hindernisses, auf das man zugeht, um es zu bewältigen, das Abenteuer in der eines

\footnotetext{
${ }^{4}$ Auch NEUMANN (1999) und STERN (2003a) plädieren für Wagnis als sportwissenschaftliche Kategorie. NEUMANN (ebd., 11) hebt vor allem die Differenzierung zu gesellschaftlichen Risiken hervor, die das Wagnis zum kennzeichnenden Begriff erheben. STERN (2003a, 191) argumentiert semantisch und leitet die Bedeutung des Wagnisses von der Bedeutung des ,abwägens’ ab. Obwohl diesen Argumentationen grundsätzlich zuzustimmen ist, wird in der jeweiligen Begriffsbestimmung nicht deutlich, dass sich das Risiko mit seinem charakteristischen Entscheidungsprozess auf mögliche Folgen eigener Handlungen bezieht und damit Entscheidungsprozess - Handlung und Handlungsfolgen in einen direkten Bezug setzt. Beim Wagnis wird dieser Entscheidungsprozess bisweilen auf die eigenen personalen Entscheidungsvorgänge reduziert. So wage ich z.B. den Besuch einer Achterbahn ohne dabei ein wirkliches Risiko einzugehen. Beim Wagnis steht das ,SichÜberwinden’ im Vordergrund, während beim Risiko aber auch beim Abenteuer das ,Sich-Einlassen’ dominiert.
} 
Hindernisses, das auf uns zukommt, um bewältigt zu werden" (NEUMANN 1998, 7). Da Abenteuer als Begriff auf eine lange Tradition verweist und im Rahmen der historischen Zusammenhänge eine implizite Nähe zu risikosportlichen Handlungen aufweist, soll der Abenteuerbegriff in dieser Arbeit in enger Verwandtschaft zum Risiko im Sinne einer ganzheitlichen Herausforderungen verstanden werden. BEST sieht als ,[...] Grundsynonyme zum Wurzelbegriff Abenteuer" (BEST 1980, 20) das Erlebnis und das Ereignis und verweist damit auf den eigentlichen Charakter des Abenteuers.

SCHLESKE betrachtet das Abenteuer vor allem im Bezug auf Merkmale des Überraschenden und Zufälligen, ,,[...] während beim Wagnis die existenzielle Erfahrung im Vordergrund steht und das Risiko von einer gewissen Kalkulierbarkeit der Handlungsergebnisse gekennzeichnet ist" (SCHLESKE 1977, 45). Im Sport lassen sich letztlich Merkmale aller drei Begriffe ableiten, die die entsprechende sportliche Situation beschreiben und damit Handlungsmerkmale aufweisen. Obgleich NEUMANN als auch WARWITZ den Wagnisbegriff vor allem vor dem Hintergrund pädagogischer Zielsetzungen einleuchtend und hinreichend bestimmen, bleibt es grundsätzlich fraglich, ob durch die gesellschaftliche Diskussion um Risiko und Risikosport durch Einbringung eines neuen Begriffes wirklich definitorische Klarheit erlangt werden kann, insbesondere da sich die angesprochene Differenzierung auch im Risikobegriff wieder findet.

SCHLESKE kommt schließlich zu dem Schluss, dass ,[...] die Begriffe nicht hinreichend scharf unterschieden werden können" (SCHLESKE 1977, 45) und versucht Wagnis, Abenteuer und Risiko im Begriff, Action' zusammen zu führen. Mit Bezug auf GOFFMANN bezeichnet er diejenigen Handlungen als ,action', die folgenreich, ungewiss und um ihrer selbst willen unternommen werden (vgl. ebd.). Obwohl SCHLESKE's Ausführungen durchaus fruchtbar erscheinen, hat sich der Begriff Action bis dato nicht im wissenschaftlichen Diskurs verankert.

Auf Grund der verbreiteten Benutzung des Begriffs und dem im Begriff immanenten Entscheidungsprozess, der sich auf Handlung und Handlungsergebnis bezieht, wird in dieser Arbeit der den Wortbedeutungen zugeschriebene inhaltliche Charakter durch den Begriff Risiko im Verständnis des Gefahrenrisikos bei KLEBELSBERG und des Risikos als Herausforderung der eigenen Kräfte bei RENN ausgedrückt. Da eine Vielzahl von Autoren eine solche Differenzierung nicht konsequent vornehmen, kann es nachfolgend unter Bezug auf unterschiedliche Autoren immer wieder zu synonymen Vermischungen der Begriffe kommen.

\subsubsection{Inhaltsorientierte Analyse des sportlichen Risikos als Hand- lungs- und Erlebnisfeld.}

Um das Handlungsfeld des sportlichen Risikos zu beschreiben, ist es notwendig den Risikobegriff genauer zu differenzieren und inhaltlich zu analysieren. Die geschieht anhand der Erläuterungen zu verschiedenen sportli- 
chen Risikoarten, dem sportlichen Risikoverbalten und der Charakterisierung der Risikosituation.

\subsubsection{Risikoarten}

Im Rahmen sportlicher Handlungen differenziert BÄNI (1991a) für das Wildwasserpaddeln zwischen freiwilligen Risiken, die eine intrinsische Motivation als Grundlage beinhalten und unfreiwilligen Risiken, deren Ursache extern begründet ist (ebd., 3). Da ein unfreiwilliges Risiko nach der angeführten Definition gleichsam eine Gefahr darstellt, erscheint diese Differenzierung als wenig fruchtbar, wenngleich sie in der praktisch-orientierten wissenschaftlichen Literatur Verwendung findet (vgl. MÜCKE 1985).

Zu einer ähnlichen Differenzierung kommt auch MÄGDEFRAU (1980), der zwischen bewussten und unbewussten Risiken unterscheidet. Ersteres wird danach als ,[...] bewusst einkalkuliertes, freiwillig eingegangenes Risiko [...]“" verstanden, welches ,,[...] untrennbar mit dem Erlebniswert verbunden ist" (MÄGDEFRAU 1980, 245). Das unbewusste Risiko hingegen resultiert aus dem ,,...] Unterschätzen des Ausmaßes eines Risikos“ und ist zwar für den Erlebnisgehalt meist ohne Bedeutung, ,[...] nicht aber für die Gesundheit“ (ebd., 246). Auch hier erlangt das bewusste Risiko die Bedeutung eines Wagnisses während das unbewusste Risiko semantisch mit Gefahr gleichzusetzen ist.

Eine für die Diskussion fruchtbare Unterscheidung in Risikoarten nehmen u.a. SCHÜTZ / WIEDEMANN / GREY (2000) vor, die zwischen lifestyle risks und environmental risks unterscheiden. Life-style-risks sind demnach Risiken, die unter der persönlichen Kontrolle des Einzelnen stehen und von der persönlichen Entscheidung und dem individuellen oder kollektiven Handeln abhängen. Environmental risks bezeichnen technische Entwicklungen oder ihre Produkte, die vom einzelnen zwar in ihrer Wirkung und Eintretenswahrscheinlichkeit eingeschätzt werden können, dem einzelnen aber nur wenig Handlungsbeeinflussung zukommt. Risikosportliche Aktivitäten sind demnach in ihrer Modellbildung ausschließlich als life-style-risks einzustufen, die in ihrer Ausübung durchaus von evironmental risks (z.B. Smog, Radioaktivtät) beeinflusst sein können ${ }^{5}$.

\subsubsection{Das Risikoverbalten}

Risikoverhalten vollzieht sich in Situationen, ,[...] in denen es ein erstrebenswertes Ziel gibt und es gleichzeitig an Gewissheit fehlt, dass dieses Ziel erreicht werden kann“ (vgl. DORSCH: (Psychologisches Wörterbuch) 1998, 744). Unsicherheit ist damit ein wesentliches Kennzeichen des Risikoverhaltens. Risikoverhalten kann als ein Typus unsicherheitsbestimmten Handelns mit einer Schädigungsmöglichkeit gegenüber dem eigenen Leben und/oder der Umwelt verstanden werden (vgl. RAITHEL 2004, 27). Im englischspra-

\footnotetext{
${ }^{5}$ Zu einer ähnlichen Differenzierung kommt auch BONB (1995), der zwischen Risikohandlung und Risikosystem unterscheidet (vgl. hierzu Abschnitt 5.3).
} 
chigen Forschungsraum wird diesbezüglich zwischen ,risk behavior' und ,risk taking behavior' unterschieden., wobei ,risk taking behavior' als „,...] explizit risiko-konnotatives Verhalten" (ebd.) beschrieben wird, während ein substanzmittelbezogenens Risikoverhalten (z.B. der Konsum von Drogen, Alkohol etc.) als ,risk behavior' bezeichnet wird (vgl. auch JESSOR 2001).

HALE \& GLANDON (1987) sprechen an der Stelle von Risikoverhalten von ,Verhalten angesichts von Risiko'. Mit KLEBELSBERG ist in diesem Zusammenhang, zusammenfassend zu betonen, dass es sich bei Risikoverhalten nicht um riskantes Verhalten handelt, sondern um Verhalten in Risikosituationen (KLEBELSBERG 1982, 43). Damit lässt sich eine Vielzahl unterschiedlicher Handlungsbereiche differenzieren, in denen es zu Risikoverhalten kommt (vgl. KUHN \& TODT (2003)). Differenziert werden kann das Risikoverhalten bezüglich der Schädigungs- sowie der risikoqualitativen Perspektive in verschiedene Handlungsbereiche (vgl. Abb. 1.1). Der Sport als Handlungsfeld stellt dabei eine Form des Risikoverhaltens dar, wobei es in besonderem Maße zielorientierte Handlungen repräsentiert, die eine Folge freiwilliger Entscheidungen sind. RAITHEL (2004) zählt Risikosport zur Kategorie gesundheitlichen Risikoverhaltens und grenzt es damit von Formen deliquenten und finanziellen Risikoverhaltens ab.

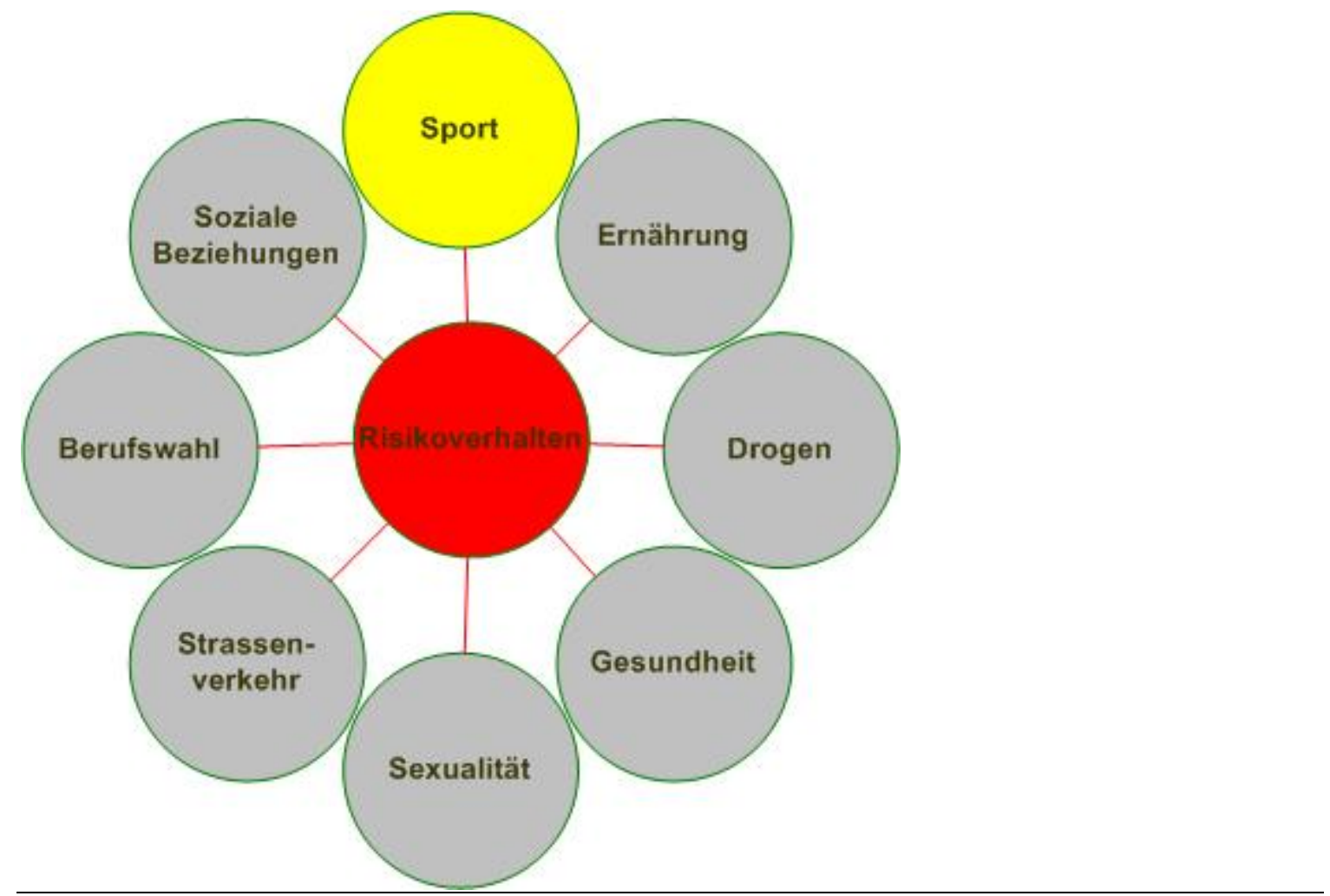

Abbildung 2.1: Bereiche des Risikoverhaltens (in Anlehnung an KUHN \& TODT 2003, 11)

BRENGELMANN (1988) beschreibt Risikoverhalten als ,[...] ein Verhalten, mit dessen Hilfe etwas von Wert in Verlustgefahr gebracht wird“ (BRENGELMANN 1988, 1) und charakterisiert damit das entscheidende 
Merkmal des Risikoverhaltens im Risikosport. In der Antizipation auf ein höheres Ziel, werden beim Risikosport scheinbar auch Situationen in Kauf genommen, die bei Nichterreichung des Handlungsziels unerwünschter als die Ausgangslage sind. Gleichzeitig stellt die Zielposition des durch Risikoverhalten erreichten Ziels eine deutliche Verbesserung gegenüber der Ausgangslage dar. KLEBELSBERG (1982) betont zudem, dass nur dann von Risikoverhalten gesprochen werden kann, wenn eine Entscheidungsmöglichkeit im Sinne verschiedener Handlungsalternativen vorliegt (vgl. ebd., 46). Eine Gefahrensituation, die nicht Folge eines alternativen Entscheidungsprozesses ist, kann damit nicht als Risikoverhalten bezeichnet wer$\operatorname{den}^{6}$.

Um Risikoverhalten im Sport handelt es sich,

„[...] wo im Hinblick auf das Erreichen eines übergeordneten Ziels eine

Leistungs- und Sicherbeitstendenz einen Konflikt herbeifübren, der innerbalb eines begrenaten Zeitraumes durch eine Entscheidung gelöst werden muss" (v. KLEBELSBERG 1969, 174).

Maßgeblich mit dem Risikoverhalten im Sport verbunden ist das Risikoerlebnis als bewusstes Spannungserleben. Risikoverhalten im Kontext sportlicher Betätigungen ist dabei nicht auf Gefahrenrisikos - wie z.B. beim Risikosport - begrenzt. Auch in taktisch-relevanten Sportarten findet Risikoverhalten unter der Prämisse einer Zielerreichung unter Unsicherheit fortwährend statt ${ }^{7}$.

\subsubsection{Risikobewusstsein, Risikoentscheidung und Risikobereitschaft}

Risikobewusstsein beschreibt das Wissen des Handelnden über das Risiko, das er einzugehen bereit ist bzw. dessen er sich aussetzt (vgl. ZIMOLONG 1984). Die Frage nach dem Risikobewusstsein stellt sich als kognitiver Prozess dar, indem im Rahmen einer Risikoentscheidung zwischen Sicherheitstendenzen und Risikobereitschaft abgewogen wird. Das Sicherheitsbewusstsein und damit das Bedürfnis nach körperlicher und geistiger Unversehrtheit steht einer individuellen Risikobereitschaft gegenüber, die bisweilen mit Risikoakzeptanz beschrieben wird. Zu Risikoverhalten kommt es demnach auf Grund einer Risikoentscheidung, wenn entweder das Risikobewusstsein unzureichend ausgeprägt ist, oder das Sicherheitsbedürfnis einer höher favorisierten Risikobereitschaft untergeordnet wird (vgl. MUHSAHL 1997, 117).

\subsubsection{Die Risikosituation}

Da prinzipiell alle sportlichen Situationen die Merkmale des Ungewissen und Spannenden enthalten und damit das Risiko des Misslingens, ist die sportliche Risikosituation von anderen sportlichen Gegebenheiten deutlich zu differenzieren. Im Gegensatz zu vermeintlich handlungssicheren Alltagssituati-

\footnotetext{
${ }^{6}$ Eine detaillierte Analyse des Risikoverhaltens wird in Kapitel 6 vorgenommen.

${ }^{7}$ WANG 1994 hat am Beispiel des Volleyball-Aufschlages das Risikoverhalten bei Spielsportarten analysisiert.
} 
onen ist die Risikosituation im Sport insbesondere durch ein intensives Spannungserlebnis gekennzeichnet. Nach NEUMANN (1999) finden sich Risikosituationen im Sport

„überall dort, wo selbsterzeugte Spannung zum Inhalt des sportlichen Handelns wird, wo die Ungewissheit des Ausgangs zentrale Bedeutung erhält und eine missglückte Handlung bedrohliche Folgen nach sich qiehen kann" (ebd., 120).

Sportliche Risikosituationen sind darüber hinaus weitestgehend zweckfrei, d.h. dass mit der Bewältigung der Situation kein materieller Gewinn auszumachen ist. Dies ist eine u.a. eine wesentliche Unterscheidung zu alltäglichen Risiken, die in der Regel auf ökonomischen Profit ausgerichtet sind. RENN (1989) charakterisiert das Risiko als Herausforderung der eigenen Kräfte durch eine Vielzahl situationsspezifischer Attribute. Neben der Freiwilligkeit und einer temporären Begrenzung charakterisiert vor allem die persönliche Kontrollierbarkeit und Beeinflussbarkeit des Risikos eine derartige Risikosituation.

Nach SCHLESKE (1977) wird in Risikosituationen eine Position der Sicherheit freiwillig aufgegeben, um sich einer Situation der Bedrohung und Ungewissheit auszusetzen. Die Zielerreichung in der Risikosituation ist an eine umfassend unsichere Handlungssituation gebunden, die gerade durch die wirksame Bedrohung mit intensiven Spannungserlebnissen verbunden ist:

„Auf Grund der Offenheit des Ausgangs und der Ungewissheit über Erfolg und Misserfolg des Handelns ergibt sich ein Zukunftsbezug, der als Erwartung und erhoffte Vorwegnahme des Erfolgs eine positive Gespanntheit auf das Ziel hin entstehen lässt" (SCHLESKE 1977, 34).

Entscheidend erscheint aber vor allem die Individualität der Risikosituation, die durch die subjektive Einschätzung des Gefahrenrisikos entsteht. Ob ein Risiko in einer sportlichen Situation als solches wahrgenommen wird, hängt letztlich von den kognitiven und motorischen Fähigkeiten und den entsprechenden Persönlichkeitsdispositionen des Einzelnen ab. Während ein Sportprofi die Befahrung eines Wildbaches als langweilig und sicher einschätzt, kann die Situation für den Anfänger mit einem erheblichen Risiko verbunden sein. Die Suche nach Risiken, die auch als Risikolust (BRENGELMANN 1988, 1) bezeichnet wird, kann damit als das wiederholte aktive Aufsuchen von subjektiven Risikosituationen bezeichnet werden, in denen es zu den beschriebenen Dispositionen von Risikoverhalten kommt.

\subsection{Risikosport - Einordnung, Definition und Eingrenzung}

Die Entwicklung des modernes Freizeitsports steht apodiktisch in enger Verknüpfung zum Phänomen Risikosport, welches seit den 1980er Jahren mehr und mehr in der sportwissenschaftlichen und öffentlichen Diskussion 
steht. Für GEBAUER et al. (2004) stellt das Risiko - im Sinne eines Verlustes des traditionellen Bodenkontaktes - sogar ein zentrales Element einer neuen Bewegungskultur dar:

„In der traditionellen Bewegungskultur des Sports ist die Berührung des Bodens, der sichere Stand, der Abdruck, das Abspringen, die Landung, das Zurückkommen auf die Erde ein wesentliches Merkmal - Bodenständigkeit gab dem Sportler Sicherheit. Kennzeichnend für die neue Entwicklung ist, dass man awischen sich und dem Boden technische Elemente einbaut, Rollen und Kugellager, Räder und Federn, die ein reibungsloses Gleiten ermöglichen und auf diese Weise die gemeinsame Kontaktfläche des eigenen Körpers mit der Erde verändern" (ebd., 12).

Im Rahmen der Ausdifferenzierung des Freizeitsports scheint sich der Risikosport als begriffliches Sammelsurium für die scheinbar unübersichtliche Vielfalt neuer Bewegungspraktiken zu etablieren. ,Thrill' und ,AdrenalinKicks' beherrschen nicht nur die Medien sondern auch weite Teile des bewegungsorientierten Freizeitmarkts. Dabei sind Risiko und Unsicherheit konstitutive Elemente des Spiels und damit auch des traditionellen Sports ${ }^{8}$. So findet beispielsweise auch in Ballsportarten ein ständiger Abwägungsprozess zwischen Handlungskompetenz, Aussicht auf Erfolg und entsprechender Abwägung der Misserfolgsfolgen statt (vgl. WANG 1994). Allein diese Tatsache macht es schwer den Begriff Risikosport eingehend zu bestimmen, da die hierfür notwendige Zuweisung konstitutiver Elemente beschwerlich ist.

Von einer einhelligen und deutlichen Begriffsbestimmung des Risikosports $^{9}$ innerhalb der Sportwissenschaft kann derzeit nicht die Rede sein. So wird die Diskussion um Risiko- und Extremsport bisweilen auf die Frage reduziert, ab welcher ,Risikohöhe' eine körperliche Betätigung zu den Risikosportarten zu zählen ist. Im Rahmen dieser quantitativen Begriffbestimmung differenziert KERR zwischen, safe sports' zu denen er Golf, Yoga und Jogging zählt, und sogenannten ,risk sports', wie Windsurfen oder Motor Sport (vgl. KERR 1991, 613f). Mangels definitorischer Eingrenzung dehnt sich der Begriff damit auf eine Vielzahl von unterschiedlichen Bewegungsaktivitäten aus, die mit Begriffen wie Extremsport und Funsport zum Teil synonym verwendet werden. Darüber hinausgehende Kriterien, die eine derartige Einordnung erlauben, fehlen allerdings in der Mehrzahl der sportwissenschaftlichen Beiträgen.

Auch in der gesellschaftlichen Diskussion findet die Bewertung einer Risikosportart häufig aufgrund der vermeintlichen objektiven Gefahr statt, die sich für den außenstehenden Betrachter offenbart. So werden alpine Sportarten wie Klettern oder Canyoning mit eher neuzeitlichen Bewegungsformen wie dem Bungee-Springen gleichgestellt. Diese Beurteilungen stellen

\footnotetext{
${ }^{8}$ Zur Bedeutung des Risikos im Spiel s. NERLICH (1998).

${ }^{9}$ Neben dem Begriff Risikosport wird in der sportwissenschaftlichen Literatur auch Begriffe wie Wagnissport, Funsport, Trendsport oder Extremsport verwendet.
} 
sich im Wissen um die Individualität einer Risikosituation allerdings als problematisch dar.

Zur Einordnung des Risikosports in den Kontext des heutigen Sports wird zunächst die Entwicklung und Ausdifferenzierung des Freizeitsports in Abschnitt 2.3.1 nachgezeichnet, um einen Einblick in die jüngste Entwicklung des Sports zu geben. Die hier deduzierten Annahmen verstehen sich als ein Versuch, die Vielfalt heutiger Bewegungskultur adäquat zu beschreiben und ersetzt damit eine enge Definition des Sports. In einem Überblick über den Forschungsstand werden anschließend die bisher vorgestellten Definitionen von Risikosport dargestellt (2.3.2). Abschließend wird ein eigener Definitionsansatz expliziert und kennzeichnende Charakteristika von Risikosport vorgestellt (2.3.3).

\subsubsection{Die Entwicklung des Freizeitsports}

Spielerisches Sichbewegen und leistungsbezogenes Kämpfen findet sich in allen Kulturen und historischen Epochen der menschlichen Entwicklung wieder und kann $\mathrm{zu}$ den quasi anthropologischen Konstanten in der menschlichen Entwicklung gezählt werden ${ }^{10}$. Und doch ist das, was wir heute unter Sport verstehen mit dem klassischen Verständnis der vielfältigen Aktivitäten in der Antike und im Mittelalter nur schwerlich vergleichbar. GUTTMANN (1979) hat in diesem Zusammenhang aufgezeigt, dass sich zwar einzelne Merkmale des modernen Sports auch schon in früheren Bewegungsformen wieder finden, dass der Sport in seiner aktuellen Gestalt aber erst seit der Neuzeit im Zuge der Verbreitung einer, Decartschen' Weltanschauung und „Dialektik der Aufklärung“ (ADORNO 1969) seinen gegenwärtigen Charakter erlangte ${ }^{11}$. Im Bezug auf die Ausdifferenzierung des Freizeitsports, in die diese interdisziplinäre Arbeit thematisch fällt, ist sicherlich WOPP $(1995,51)$ zuzustimmen, der den ,zweiten Weg des Sports' [1959] als die Geburtsstunde des heutigen Freizeitsports bezeichnet. Wie andere gesellschaftliche Teilbereiche auch unterliegt der Sport einem kontinuierlichen Prozess des Wandels und der Veränderung, die eng mit gesamtgesellschaftlichen Entwicklungen verknüpft sind. Parallelen zwischen den Veränderungen in den allgemeinen Lebensbedingungen, den historischen Ereignissen und Entwicklungen des Sports sind im Rahmen methodischer Möglichkeiten nur bedingt vollständig nachzuvollziehen. Da diese Arbeit einen stark sportsoziologischen Ansatz verfolgt, werden die folgenden Ausführungen vor allem jene Dimensionen und Entwicklungen betonen, die sich als genuin neuartig verstehen lassen und den Sport in seinen aktuell scheinbar vielfältigen Erscheinungsformen begreifen.

\footnotetext{
${ }^{10}$ Einen Überblick über die Sportentwicklung liefern neben MANDELL (1984) auch KRÜGER (1994); in systemtheoretischer Perspektive CACHAY \& THIEL (2000); im Kontext der Modernisierungsdiskussion NORDEN \& SCHULZ 1988; STICHWEH (1995). Mit besonderer Berücksichtigung des Freizeitsports WOPP (1995) und DIEKERT (2002). HIRN (1936) bietet einen historischen Einstieg.

${ }^{11}$ GUTTMANN (1979) differenziert sieben zentrale Merkmale, die den modernen Sport von seinen Vorläufern unterscheidet: Weltlichkeit, Chancengleichheit, Spezialisierung, Rationalisierung, Bürokratisierung, Quantifizierung sowie die Suche nach Rekorden.
} 
Rein quantitativ lässt sich die neuere Entwicklung des Sportsystems anhand der Sportpartizipation der Bevölkerung nachzeichnen. Trotz erheblicher methodologischer Divergenzen zeigen die Mehrzahl der empirischen Untersuchungen über die Sportpartizipation der Bevölkerung einen stetigen Zuwachs (vgl. hierzu WEBER 1995; AWA 1999; LAMPRECHT \& STAMM 2002). Dies gilt in gleicher Weise für die Mitgliedschaft in Sportvereinen (vgl DSB 2000) als auch im Bereich informeller Sportengagements (vgl. TELCHOW 2000; WOPP 2002). Obwohl beispielsweise das BATFreizeitforschungsinstitut für Westdeutschland im Vergleich von 1987 und 1996 einen leichten Rückgang der Sportaktivenquote verzeichnet (vgl. OPASCHOWSKI 1996), stellen andere Untersuchungen einem deutlichen Anstieg der Sportpartizipation und der subjektiven Bedeutung des Sports in der Freizeit heraus ${ }^{12}$. Diese von GRUPE (1988) als, Versportung der Gesellschaft' etikettierte Entwicklung trifft dabei auf nahezu alle Bevölkerungsgruppen gleichermaßen zu und umfasst verstärkt die gesamte Lebensspanne als auch beide Geschlechter.

Parallel zu dieser quantitativen, Versportung' lassen sich aber auch qualitative Veränderungen konstatieren. So nimmt der Sport in der subjektiven Bedeutung unter den Freizeitaktivitäten heute einen Spitzenplatz ein und rückt zunehmend in der Mittelpunkt auch der alltäglichen Lebensführung (vgl. LAMPRECHT\& STAMM 2002). Als Folge lasten vor allem auf dem Freizeitsport vermehrt Hoffnungen und Wünsche im Bezug auf ,Sinnfindung' und Selbstverwirklichung (vgl. KRIPPENDORF et al. 1984). Analog lässt sich die Durchdringung mit sportiven Symbolen und Zeichen sowie ein gesteigertes Interessen an passiver Sportbeteiligung bei Sportveranstaltungen feststellen (RITTNER 1989; KASCHUBA 1989; SCHWIER 2000). „Sportivität als eine demonstrative Bezugnahme auf Praxisformen, Symbole und Werte des Sports [...] (KASCHUBA $(1989,154)$ weist heute als sozialer Leitwert über die Ebene des Sichbewegens oder der Körpererfahrung hinaus und bezieht Images eines neuen „,...] kulturellen Habitus“ (ebd., 162) ein. Diese alltagsrelevante Durchdringung wird ergänzt durch eine weit verbreitete positive Grundhaltung im Rahmen öffentlicher und/oder wissenschaftlicher Diskussionen. So wird Sport vor allem in den Diskursen der Medizin, Psychologie und Pädagogik auf breiter Basis befürwortet und mit einer Reihe von Begründungen für eine aktive sportliche Betätigung plädiert (vgl. PFEIFFER-KARABIN 1993, 11). In der Argumentation der unterschiedlichen Fachdisziplinen wird dem Sport ein entscheidender Beitrag zur Gesundheitsförderung sowie zur Steigerung des psychischen Wohlbefindens und des seelischen Ausgleiches attestiert. Darüber hinaus gilt der Sport zunehmend als Ort sozialer Integration und Einbettung (vgl. ABELE \& BREHM 1990, 131; GRUPE 1988, 55; auch RITTNER \& BREUER 2000).

\footnotetext{
12 Zwar schwankt die Sportaktivenquote je nach Untersuchungsdesign zwischen 45\% und 80\%. Längsschnittuntersuchungen, die auf einer kontinuierlichen Datenerhebung basieren liegen in der Bundesrepublik nicht vor. Meist werden Veränderungen der Sportpartizipation durch Gegenüberstellungen von Querschnittsuntersuchungen zu unterschiedlichen Zeitpunkten eruiert (vgl. RÜTTEN 2002, 117).
} 
In Anbetracht dieser allgemeinen Beobachtungen und vor dem Hintergrund der Einordnung des Risikosports in das derzeitige Sportsystem stellt sich die Frage nach den Hintergründen und Ursachen für diesen Expansions- und Differenzierungsprozess.

Ohne zu den historischen Ursprüngen des Sports zurück zu kehren die von verschiedenen Autoren ${ }^{13}$ in ausreichender Form dargestellt wurden, lässt sich das klassische Sportverständnis bis in die 50er und 60er Jahre mit seiner Kombination von Siegescode und Leistungsprinzip und seinen im Vereinswesen fest verankerten Organisationsstrukturen adäquat charakterisieren. Im Verständnis eines fordistischen Regulationsmodells des Kapitalismus nahm der Sport als Teil der Freizeit deutlichen Bezug auf die Arbeitswelt im Sinne der Regeneration oder der Kompensation und stand gleichzeitig in Beziehung auf die tayloristischen Prinzipien der Leistungsgesellschaft ${ }^{14}$. Sport als organisiertes Freizeitverhalten war dabei auf ganz bestimmte Bevölkerungsgruppen explizit zugeschnitten (SCHIMANK 1992; BETTE 1999; ALLMER 2002). Zwar stand der Sport prinzipiell allen Gesellschaftsmitgliedern offen, eine starke Anziehungskraft entwickelte die sportliche Betätigung in seinen unterschiedlichen Ausprägungen vor allem aber auf männliche Mittelstandsangehörige mit höherer Bildung (vgl. GRIESWELLE 1978). In unterschiedlichen Studien konnte zudem eine Verknüpfung zwischen der sportlichen Aktivität und der sozialen Position aufgezeigt werden ${ }^{15}$.

Die Öffnung des Sportsystems seit Mitte der 60er Jahre muss insbesondere im Zusammenhang mit einer allgemeinen wohlfahrtsstaatlichen Entwicklung gesehen werden, die durch eine Anhebung des Wohlstandsniveaus, einer breiten Bildungsexpansion, der Ausdehnung der Freizeit und einer Neudefinition der Geschlechterrolle gekennzeichnet war. Neben dem Leistungsmotiv gewann vor allem die Motivation aus gesundheitlichen Gründen Sport zu treiben an Bedeutung. Die Öffnung der Sportverbände und vereine, die sich in den verschiedenen ,Sport für alle - Aktionen manifestieren, führte zu einer weitgehenden Inklusion unterschiedlichster Personengruppen in das bundesdeutsche Sportsystem. Neben gesundheitlichen Aspekten gewann spätestens seit den 70er Jahren auch ein soziales Phänomen immer mehr an Bedeutung: Sport als Mittel der Integration, der Kommunikation und als Erlebnisraum in einer zunehmend defizitären und affektarmen Gesellschaft ${ }^{16}$. Diese Entwicklung, die in enger Beziehung mit der fortschreitenden Technisierung und Urbanisierung verstanden werden muss, steht dabei in einer engen Wechselbeziehung mit der spätestens seit den

\footnotetext{
${ }^{13}$ Hier sind vor allem DIEM (1923); KRÜGER (1993) zu nennen.

14 In diesem Zusammenhang vgl. HABERMAS 1973.

${ }_{15}$ Neben der sozialen Position erwiesen sich vor allem das Alter, das Geschlecht und mit geringerer Aussagekraft auch die Konfessionszugehörigkeit als entscheidende Variabeln. Vgl. hierzu HEINEMANN 1990; GRIESWELLE 1978; HARTMANN-TEWS \& CACHAY 1998; MÜLLER 1998; zur Bedeutung Geschlecht ABRAHAM 1998.

16 Über die Entwicklung und zunehmende Zivilisierung der Gesellschaft im Zusammenhang mit Sport vgl. ELIAS \& DUNNING (o.J.); ELIAS 1991; DOEHLEMANN 1991; FRANKL 1973a; GIDDENS 1999; im Überblick auch GEBKEN 2001.
} 
80er Jahren beobachtbaren Entfaltung einer alternativen Spiel und Bewegungskultur. Begriffe wie ,Abenteuer', ,Spannung', ,Dramatik' aber auch „Spaß' als Motive sportlichen Handelns erhalten seither Einzug in das Sportsystem.

Die zunehmende Diversifizierung des Sports in der heutigen Gesellschaft erscheint mit Entwicklungen anderer gesellschaftlicher Teilbereiche vergleichbar, die mit Begriffen wie Pluralisierung und Entstrukturierung belegt sind. Bei der Frage nach den Hintergründen dieses Expansion- und Differenzierungsprozesses im Sportsystem muss parallel zu den beschriebenen Veränderungen der Wohlfahrtstaatlichen Entwicklung und neben gesamtgesellschaftlichen Tendenzen wie Individualisierung (BECK 1983; 1986) oder Erlebnisorientierung (SCHULZE 1995) auch der allgemeine gesellschaftliche Wertewandel Berücksichtigung finden. Sowohl die quantitativen als auch die qualitativen Veränderungen der Sportlandschaft stehen diesbezüglich in einem engen Zusammenhang, der sich vor allem über die veränderte Bedeutung der Freizeit im individuellen Alltag manifestiert (vgl. DIGEL 1986 / 1990; GRUPE 1988 / 2000; LAMPRECHT \& STAMM 1994). Neben einer Expansion der zur Verfügung stehenden freien Zeit, Umgestaltungen im Arbeitsleben, allgemeinen demographischen Veränderungen und Haushaltsformen muss vor allem die Erosion konventioneller Werte (wie Disziplin, Leistung, Anpassung, Gehorsam u.a.) Erwähnung finden. Werte „kritisch autonomer Prosozialität" (wie Selbstständigkeit, Hilfsbereitbereitschaft u.a.), hedonistische Werte (wie Lebensgenuss, Abenteuer, Spannung und Abwechselung) sowie individualistische Werte (wie Ungebundensein, Eigenständigkeit u.a.) gewinnen besonders in der Freizeit essentiell an Bedeutung (vgl. KLAGES 1981, 74 ff). Der in der modernen Gesellschaft kontinuierlich zu verzeichnende Zuwachs an arbeitsfreier Zeit ist zwar angesichts längerer Wegstrecken und veränderte Mobilitätsansprüche zu relativieren, unstrittig bleibt aber, dass sich die Erscheinungsformen und die Bedeutung der Freizeit maßgeblich modifiziert haben. Während die Freizeit vormals - wie von HABERMAS (1973) expliziert - als eine von der Arbeit abhängige Variabel im Sinne der Regeneration und Kompensation erfasst wurde, werden der Freizeit heute zunehmend eigene Wertstrukturen zugesprochen. Freizeit im allgemeinen aber auch speziell die Sportbetätigung in freizeitsportlichen Zusammenhängen ist heute Möglichkeitsraum für Selbstbestimmung und Selbstverwirklichung und dient über die Regeneration hinaus der Suche nach Erlebnissen und Stimulation (vgl. DIGEL 1986). 


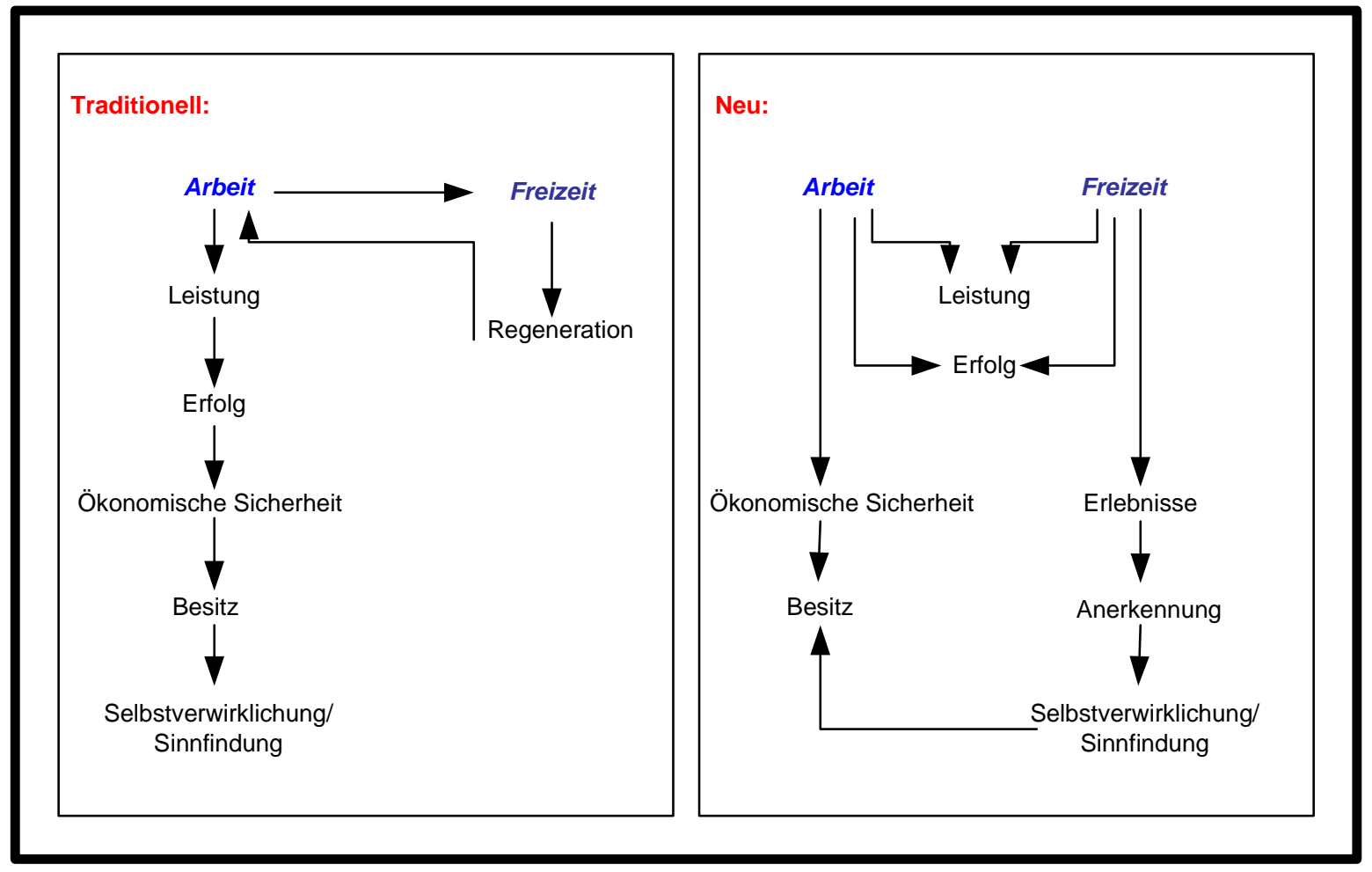

Abbildung 2.2: Traditionelles versus modernes Verhältnis zwischen Arbeit und Freizeit (nach DIGEL 1986, 34).

Der Sport als vormals einheitliches und scheinbar homogenes Gebilde zerfällt dabei in der als ,postindustriell' und ,postmodern' etikettierten Gesellschaft zunehmend in eine Vielzahl unterschiedlicher Formen. An die Stelle eines einheitlichen Sportverständnisses und des Postulats einer Sinnmitte des Sports tritt eine zunehmende Unübersichtlichkeit und Begriffsvielfalt (vgl. PARLEBAS 1986; HEINEMANN 1989; DIETRICH et al. 1990; OPASCHOWSKI 1996/2000; BETTE 1999, 147; SCHWIER 2003, 18). Quantitativ stützt HENKEL (1989/2000) diese These, indem er eine Ausdifferenzierung des Freizeitsports von knapp 80 Sportarten am Ende der 1980er Jahre zu 140 Sportarten im Jahre 2000 konstatiert. HEINEMANN \& SCHUBERT (2001) stellen zu diesem Zeitpunkt bereits über 240 verschiedene Sportangebote in deutschen Sportvereinen fest. ${ }^{17}$ Gleichzeitig bemerkt HEINEMANN (1989), dass insbesondere Freizeitsport immer häufiger auch außerhalb der traditionellen Vereinwelt organisiert und betrieben wird. Diese neue Vielfalt offenbart sich auch durch die Schwierigkeit einer konvergenten Begriffsystematik und Einordnungstypisierung:

„Wo vor wenigen Jahren noch der Hinweis auf die Körper-, Wettkampf- und Leistungsorientierung sportlichen Handelns, die Vereinsgebundenbeit der Akteure und deren Verpflichtung auf bestimmte ethische Werte reichte, um bereits

\footnotetext{
${ }^{17}$ Leider versäumen es die Autoren eine begriffliche Differenzierung zwischen Sportarten, Sportangeboten und Bewegungspraktiken anzuführen, so dass diese Studien lediglich als Indiz für die Ausdifferenzierung angeführt werden können.
} 
Wesentliches über den Sport auszusagen, zeigt sich gegenwärtig ein Sozialbereich, der in seiner Symbolik, Ästhetik, Rollenausprägung und institutioneller Anbindung durch ein bohes Maß an Differenzierung und Pluralisierung geprägt ist" (BETTE 1999, 147).

Diese Einschätzung wird auch anhand der modelltheoretischen Versuche, den gegenwärtigen Sport in seinen Rahmenbedingungen und Strukturen zu erfassen, deutlich. Auch wenn die wissenschaftliche Diskussion in diesem Kontext weitgehend offen bleibt, herrscht doch Einigkeit darüber, dass das noch in den siebziger Jahren etablierte Pyramidenmodell, wonach aus einer breiten Masse von Sporttreibenden (Breitensport) eine sich zunehmend verengende Spitze an Leistungs- und Spitzensportler entsteht, unzeitgemäß ist. Auf DIEKERT (1973) gehen Versuche zurück, einen Dualismus zwischen Freizeitsport auf der einen und Spitzensport auf der anderen Seite modelltheoretisch darzustellen.

Vor allem die starke quantitative Zunahme der Sportpartizipation jenseits des an Wettkampf orientierten Sports, förderte indessen die verstärkende Auseinandersetzung mit pluralen Modellen. HEINEMANN (1983) kommt in seinen Untersuchungen zu den Sportvereinen in Deutschland zu einem viergliedrigen Modell, in welchem er zwischen einem kommerziellen, wettkampforientierten, expressiven und einem funktionalen Sportprogramm unterscheidet. Bei DIGEL (1984) findet sich schließlich eine Differenzierung zwischen Mediensport, Leistungssport, Freizeitsport, instrumentellem Sport und Alternativsport, wobei keine klare Abgrenzung vorgenommen wird. Weitere Ansätze finden sich bei JÜTTING 1987; SCHULKE 1988; KURZ 1988; DIETRICH \& LANDAU 1990; EUERLING 1990a; 1990b; BREUER 2000 und schließlich bei WOPP 1995. Trotz unterschiedlichster methodischer Ansätze und Ergebnisse, herrscht doch weitestgehend Konsens über die Tatsache, dass der explizierte Differenzierungsprozess keine Besonderheit des Sportsystems ${ }^{18}$ offenbart, sondern wie oben dargestellt, an gesamtgesellschaftliche Prozesse rückgekoppelt ist.

Zusammenfassend aus obiger Darstellung, soll im folgenden - im Sinne einer Bestandsaufnahme - herausgearbeitet werden, welche Orientierungen bzw. Sinnbereiche sich im Zuge dieses Differenzierungsprozesses im Freizeitsports bis heute etabliert haben und in welchen Sinnbezügen freizeitsportliches Handeln - jenseits aber auch im Kontext des Risikosports - stattfindet. Obwohl der thematische Rahmen des Risikosports deutliche Vernetzung zu anderen Sportbereichen wie beispielsweise Mediensport oder auch Wettkampfsport ${ }^{19}$ aufweist, wird der Fokus im folgenden insbesondere auf den Freizeitsport gelenkt. Forschungsleitendes Interesse der Arbeit ist das individuelle sportliche Handeln in risikosportlichen Bewegungskontexten und ihre Modellbildung. Obwohl damit die Fokussierung auf einen Teilbe-

\footnotetext{
${ }^{18}$ Der Systembegriff wurde bereits im Bezug auf LUHMANN in Kapitel 1 eingeführt (vgl. auch LUHMANN 1981.

${ }^{19}$ Sowohl in den Medien (z.B. ,Stoked - das Trendsportmagazin') als auch im Wettkampfsport finden sich verschiedene Formen des Risikosports wieder. Inwieweit der Charakter des Risikosports durch Wettkampfsportelemente betroffen ist, wird im Folgenden diskutiert werden.
} 
reich des Freizeitsports gelenkt wird, ist die Einordnung in den Gesamtkontext des Freizeitsports fruchtbar. Im Mittelpunkt des Differenzierungsprozesses steht daher die Vielfalt des aktuellen Sportverständnisses, welche sich in den unterschiedlichen Sinnorientierungen niederschlägt und die Bandbreite des modernen Freizeitsport charakterisiert. Die Unterscheidung der Sinnorientierungen im gegenwärtigen Sportengagement erfolgt dabei in Anlehnung an die Differenzierung von GRUPE (1988, 55f) und KURZ (1986, 46f), die sich in modifizierter Form auch bei DIEKERT \& WOPP (2002, $227 \mathrm{ff}$.) wieder findet.

Fitness und Gesundheit haben sich in den letzten Jahren definitiv zu den zentralen Sinnrichtungen freizeitsportlichen Handelns entwickelt und gelten als entscheidende Größen für die Entwicklung des Freizeitsports ${ }^{20}$. Zweifellos ist die physischen Gesunderhaltung ein vertrautes Argument in der sportwissenschaftlichen Diskussion ${ }^{21}$. In einem weitgehend bewegungsarmen und technisierten Arbeitsalltag erhält der Sport aszendent die Funktion eines präventiven und rehabilitativen Gegenpols. Die Zuwendung zum Sport unter der Prämisse der Gesunderhaltung und des Fitnesstrainings ist dabei mit einer starken Effektorientierung verbunden (HACKFORT 1993). Sportliche Aktivitäten im Kontext Gesunderhaltung und Fitness sind entscheidend mit dem Wunsch nach handlungsfolgenden Zielen verbunden, wie einer allgemeine Fitnessverbesserung, einer individuellen Körperformung oder aber auch einem allgemeinen physischem Wohlbefinden. In unterschiedlichen Untersuchungen zur Motivation des Sporttreibens steht trotz vielfältiger Kombinationen und Überlagerungen - das Gesundheitsund Fitnessmotiv an vorderster Stelle (vgl. OPASCHOWSKI 1987; LAMPRECHT \& STAMM 2002; WOLL 1996; für Trendsportarten BREUER 2000; STUMM 2004). Empirisch lässt sich diese Entwicklung auch anhand der gewachsenen Zahl an kommerziellen Fitness-Studios und einer regelrechten Angebotsexpansion belegen (vgl. RÜTTEN 1998). WOLL \& BÖS (2002, 247) konstatieren hierzu: „Der Boom der FitnessStudios entspricht anscheinend den Bedürfnissen der individualisierten Menschen nach Gesundheit und Fitness, aber auch nach Ästhetik und Selbstdarstellung im Sport". Nachhaltig ist auch die gesundheitsorientierte Inklusion bisher unaktiver Personengruppe in das Sportsystem: Die Altersgruppe über 35 Jahren wird in einer, ergrauenden Gesellschaft' (vgl. LEHR 1994) zu einer bedeutenden Zielgruppe des Sports, die durch gesundheitsorientierte Angebote angesprochen werden. Die Investition in den Körper wird immer häufiger mit der Hoffnung auf Gesundheit verbunden, führt bisweilen aber auch zu neuen Idealbildern eines junggebliebenen Körpers. Körperliches Wohlbefinden wird in der, postmodernen' Gesellschaft zu einem Eigenwert sportlichen Handelns. Verschiedene Autoren (KURZ 1988; BOPP 1987) sehen in diesem Wandel von Sinnmustern daher einen zuneh-

\footnotetext{
20 Auf die inhaltlichen Unschärfen und Definitionsprobleme der Begriffsbestimmung kann im Rahmen dieser Arbeit nicht eingegangen werden. Vgl. hierzu LAASER et al..1993; WOLL 1996; WOLL\& BÖS 2002.

${ }^{21}$ Vgl. im Überblick CACHAY 1988; BALZ 1995; WOLL/BÖS 2000 oder ABELE/BREHM 1990.
} 
menden Narzissmuss und Egozentrismus als Folgen der umfassenden Gesundheits- und Fitnessorientierung im Sport.

Wagnis und Abentener als Sinnorientierungen des Sports zeigen sich besonders deutlich im Wachstum unterschiedlichster Outdoorsportarten wie dem Klettern, Kanufahren, Paragliding oder Fallschirmspringen. Die Suche nach Herausforderungen mit sich selbst und der Natur in natürlichen und urbanen Handlungsräumen wird dabei zunehmend zu einer reduzierten Suche nach Nervenkitzel und Aufregung (vgl. SCHLESKE 1977; APTER 1994). TOMLINSON (1997) listet insgesamt 40 verschiedene - zum Teil neu entstandene - Sportarten auf, die diesem Bereich des Sports zuzuordnen sind. Die Karriere des Wagnisses als Kategorie und Motivstruktur des Sports wird dabei zunehmend im Kontext des Trendsports diskutiert und als postmodernes Phänomen interpretiert (vgl. SIELAND 2003). Obwohl Wagnisund Abenteuer vor allem in informellen Sportengagements zentrale Sinnorientierungen darstellen und quantitative Untersuchungen daher schwierig sind, kommt OPASCHOWSKI (2000, 46f) zu dem Ergebnis, dass sich 14\% der bundesdeutschen Bevölkerung sportliche Wagnisaktivitäten in der Freizeit wünschen. EGNER $(2000,7)$ geht mit Bezug auf LORCH (1995) von einer Vervielfachung wagnisorientierter Sportaktivitäten im letzten Jahrzehnt aus. Kritisch wird diese Entwicklung vor allem vor dem Hintergrund der zunehmenden Kommerzialisierung und Naturnutzung diskutiert (vgl. im Überblick NEUMANN 1999; 2002).

Spaß und Erlebnis sind spätestens seit der kultursoziologischen Arbeit von SCHULZEs (1995) „Die Erlebnisgesellschaft" und POSTMANNs (1988) „Wir amüsieren uns zu Tode“ zu überaus zentralen Kategorien der Freizeitsoziologie und letztlich auch der Sportwissenschaft avanciert. Obwohl die Frage, was Spaß und Erlebnis eigentlich bedeuten, oftmals unklar bleibt, wird auch in der Sportwissenschaft mit den Begriffen gearbeitet ${ }^{22}$ (vgl. DIECKERT 2002). Sportliche Offerten im Umfeld der Erlebnis- und Funsportarten umfassen dabei eine breite Auswahl an unterschiedlichsten Sportangeboten. Während elementare Bewegungsaktivitäten wie das Spielen außerordentlich viel Spaß versprechen, werden auch passive Sinnesreize im Umfeld des Freizeitsports mit außergewöhnlichen Erlebnissen umworben. Analysiert man Untersuchungsergebnisse zu den Beweggründen des Spottreibens, so erscheint es insgesamt nahe liegend zu sein, Spaß als zentrales Motiv zum Sporttreiben zu klassifizieren. Gerade bei Jugendlichen wird ,Spaß haben' und ,etwas erleben' immer wieder als primäre Beweggründe für Sportbetätigungen genannt (vgl. DSB / VELTINS-SPORTSTUDIE 2000). Zwar bleibt es im Rahmen der meisten empirischen Untersuchungen unklar, was mit den Begriffen gemeint ist - ob Spaß semantisch eher Vergnügen im Sinne von Scherz oder Witz meint oder als Ausdruck für die traditionell sinntragenden Begriffe wie Freude, Glück oder auch Wohlbefinden gewählt wird. Insgesamt sind Spaß und Erlebnis aber zu gängigen Grundbegriffe insbesondere des Freizeitsports avanciert. Trotz umfassender Kritik unter-

${ }^{22}$ Zur näheren Begriffsbestimmung und inhaltlichen Differenzierung vgl. BECKER 1991; 1995; BRÄUTIGAMM 1994; RITTNER 1995; ALLMER \& SCHULZ 1998. 
schiedlichster Autoren, die die Existenz eines autonomen Spaßmotivs anzweifeln (vgl. BALZ 1994, 469), bleibt es festzuhalten, dass Spaß als Sammelbegriff eine positiven Bewertung für erfolgte sportliche Aktivitäten impliziert und mit den unterschiedlichsten Sportengagements verbunden ist. Dabei ist es letztlich subjektiv höchst unterschiedlich wie jemand welche Betätigung als freudvoll erlebt und damit Spaß empfindet. Auch der Begriff Erlebnis ist ein eher diffuses Schlagwort im Rahmen sportlicher Betätigungen. Ein Erlebnis meint das aus dem Kontinuum herausspringende Ereignis und hat damit den Charakter des Außergewöhnlichen (vgl. DIECKERT 2002, 232). Die Nähe zu Begriffen wie Spannung, Sensation oder auch Abenteuer die im Wagnis- und Risikosport eine steile Karriere durchlaufen haben, ist immanent. SCHULZE (1995) betont insbesondere die Subjektzentrierung des Erlebnisses, denn „Erlebnisse werden vom Subjekt nicht empfangen, sondern von ihm gemacht. Was von außen kommt, wird erst durch die Verarbeitung zum Erlebnis“ (ebd., 44). Im Gegensatz zur sportlichen Leistung geht es beim Erlebnis in sportlichen Handlungsbezügen damit weniger um das Erringen eines äußeren Ziels, sondern vielmehr um das Erreichen eines inneren Zustandes, wie spannenden oder angenehmbeglückenden Ereignissen. Das Erlebnis wird dabei zu einer regelrechten Leitmaxime der Lebensgestaltung mit besonderen Auswirkungen auf das Freizeitverhalten: „Erlebe Dein Leben ist der kategoriale Imperativ unserer Zeit" (SCHULZE 1995). Auf Grund der zunehmenden Spannungsarmut und Langeweile in alltäglichen Handlungssituation lasten insbesondere auf dem Freizeitsport oft unerfüllte Wünsche und Hoffnung, herrscht teilweise sogar ein Drang nach vielfältigen erlebnisreichen Aktivitäten und Spaß (vgl. KRIPPENDORF et al. 1984; KURZ 1986; DOEHLEMANN 1991). Bei VIRILIO (1989; 1992) werden zudem konkrete Zusammenhände zwischen der zunehmenden Technisierung des Alltags, als eine Form der , [...] künstlichen Domestizierung“ (ebd. 1989, 54) und dem gegenwärtigen Erlebnishunger identifiziert.

Leistung und Wettbewerb sowie die Freude am eigenen Können sind kennzeichnend für den Sport, unabhängig von den Organisationsstrukturen und individuellen Sinnbezügen. GRUPE (1988, 61f) betont, dass es sich bei der Leistung nicht um ein Prädikat des professionellen oder semiprofessionellen Hochleistungssport handelt, sondern in gleichem Masse für den Freizeit- und Breitensport relevant ist. Dieses Begriffsverständnis impliziert eine sehr weite Auslegung des Leistungsbegriffs auf alle Handlungen, die nicht primär auf Zufälle gründen, sondern auf persönlichen Einsatz und subjektive Anstrengung zurückzuführen sind. Leistung orientiert sich damit gegenwärtig weniger an äußeren Gütekriterien als an subjektiven Leistungssteigerungen und individuellen Verbesserungen - er liefert sowohl eine Plattform für ein individuelles Leistungserlebnis und Selbstbestätigung (,ich habe es geschafft'), als auch die Möglichkeit sich durch den Vergleich mit anderen Anerkennung zu verschaffen. KURZ (1986) bringt die Sinnorientierung ,Leistung' anschaulich auf den Punkt: „Man will sich etwas vornehmen, etwas abverlangen, sich an Aufgaben messen und mit anderen vergleichen; 
will seine Möglichkeiten und Grenzen erkennen, die Anerkennung anderer und ein Bewusstsein des eigenen Wertes bekommen" (ebd., 48). Nach GRUPE \& KRÜGER (1997, 270f) ist Leistung und Wettbewerb spätestens seit der ,[...] Individualisierung und Popularisierung des Leistungsprinzip“ auf Basis individueller Maßstäbe auch im Freizeitsport legitimiert. Obwohl die Zielsetzung zwischen Freizeitsportlern und Leistungssportlern im Bezug auf das individuelle Leistungsstreben differieren kann, ist Leistung als Sinngebung auch in informellen und nicht breitensportlich organisierten Sportengagements implizites Element der Sportausübung (vgl. TELCHOW 2000).

Die Sinnorientierung Geselligkeit und Gemeinschaft ist für Menschen aller Altergruppen und sozialen Hintergründen ein zentrales Motiv im Freizeitsport $^{23}$. Dabei stellt Sport zum Zweck der sozialen Interaktion und Integration seit jeher ein probates Mittel dar. Obwohl in der Sportwissenschaft nur eine geringe Erörterung des Geselligkeitsbegriffes zu konstatieren ist, besteht kaum ein Zweifel daran, dass der (Freizeit-)Sport eng mit Formen der Gemeinschaft und Geselligkeit verbunden ist (vgl. GEBKEN 2002, 255). Es wird darauf verwiesen, dass trotz fehlender empirischer Belege, der Freizeitsport in den unterschiedlichsten Einrichtungen und Organisationsformen dazu genutzt wird, Menschen zu treffen und kennen zu lernen. Gleichzeitig wird aber vor dem Hintergrund zunehmender Individualisierungsprozesse eine Veränderung der sozialen Interaktion und Integration im und durch Sport betont. So konstatieren z.B. BAUR \& BRAUN $(2000,93)$ einen Wandel vom eher traditionellen, zeitlich überdauernden Ehrenamt in Sportvereinen zu einem modernisierten und individualisierten Engagement. BETTE (1999, 167) sieht auch im Bezug auf das Sportengagement des Einzelnen gravierende Neuerungen. Er identifiziert als Folge der Individualisierungsprozesse der achtziger und neunziger Jahre einen so genannten Sportnomaden, der , ,[...] ganz im Sinne einer frei flottierenden Bindungsfähigkeit zwischen verschiedenen Körperbetätigungen hin und her wechselt, ohne jene Identifikation zu erreichen, wie sie im überlieferten Sport noch anzutreffen ist" (edb.). Dieser Sportnomade ,[...] ist sozial adäquat durch seine Fähigkeit, vielfältige Bindungsformen mit der entsprechenden Oberflächlichkeit eingehen zu können“" (ebd., 168). Die Diskussion um das ,Ende des Sozialen’ und der ,Krise des Ehrenamts' lassen sich aber nur bedingt eindeutig bestätigen. So stellt TELCHOW $(2000,100 \mathrm{f})$ beispielsweise fest, dass bei Jugendlichen sowohl in informellen Sportengagements als auch in Sportvereinen - wenn auch in unterschiedlichen Strukturierungen - ein hohes Maß an Gemeinschaft und Geselligkeit existiert.

Die Zunahme an Angeboten mit entspannungs- und körpertherapeutischen Inhalten verweisen auf die Sinnrichtungen der Körperzentrierung und Selbsterfahrung, die der Zukunftsforscher HORX $(1999,116)$ zu den bedeu-

${ }^{23}$ Zur näheren Begriffsbestimmung und sportwissenschaftlichen Diskussion vgl. KLEINE/FRITSCH 1990; FRITSCH 1998; SCHILLING 1989; REESE-SCHÄFER 1997. 
tenden Entwicklungen der Zukunft zählt ${ }^{24}$. Das Spektrum an Offerten im Bereich ,Wellness' differenziert sich seit Ende der neunziger von Entspannungsverfahren über asiatische Meditationsformen bis hin zu körpertherapeutischen Verfahren aus. Auch hierbei werden deutliche Bezüge zu Modernisierungsprozessen wie der gesellschaftlichen Individualisierung gezogen:

„Während Fitness noch tief aus der industriellen Logik stammt, richtet sich Wellness [mit der Zentrierung auf Körper und Selbsterfahrung; Anm. d. Verf.] im Herzen auf die Individualkultur (Wablmöglichkeiten, Innenorientierung, Selbstreflexivität) ein." (ebd.).

Über die Zuwendung zum Erleben des eigenen Körpers im Sport aber auch in der Meditation oder der Entspannung wird den Unsicherheits- und Entwurzelungsgefühlen - als Folge des Individualisierungsprozesses nach BECK (1986) - Widerstand entgegengesetzt und Raum für Sinn- wie Kohärenzerfahrungen gewonnen. Die Zentrierung auf den eigenen Körper wird als ein Weg angesehen, wie Individuen in modernen Gesellschaften der Abstraktheit, Routinisierung und Körperferne des Alltags begegnen und den Verlust vormals geltender Sinninstanzen auszugleichen versuchen (vgl. KOLB 2002, 279f). Die Vernetzung zu anderen Sinnorientierungen wie Fitness- und Gesundheit und Erlebnis sind offensichtlich. Dabei vollzieht im Kontext der Körperzentrierung insbesondere der Gesundheitsbegriff eine beachtliche Metamorphose: Gesundheit als Kategorie wird nun von einer Phalanx von Anweisungen und Verhaltenmaßregeln zu einem Balanceakt der Selbstfindung verlagert. Der Körper als Medium der Selbsterfahrung und Identitätsbildung wird aber auch mit Skepsis betrachtet. Kritisch äußern sich vor allem SCHIERZ (1993) und KEUPP (2000), die einen unreflektierten Umgang mit dem Körper im Sinne einer körperbezogenen Sinnsuche als unrealistisch, gar gefährlich erachten:

\section{„Die Zuwendung zum Körper läuft ins Leere, wenn körperzentrierte Verfah- ren unreflektiert als Heilslehre genutat werden, in denen man unbinterfragbare Erkenntnisse einer, ostasiatischen Weisheit' oder einer implizierten ,Weisheit des Körpers' vermutet, die bei der sinnvollen Gestaltung des eigenen Lebens ei- ne Orientierung zu geben vermögen" (KOLB 2002, 278).}

Praxisformen des Freizeitsports mit den Sinnorientierungen Ausdruck und Gestaltung sind in den letzten Jahrzehnten in unterschiedlichen Handlungszusammenhängen aufgeblüht. Neben den traditionellen ,Kompositorischen Sportarten' - wie beispielsweise dem Kunstturnen oder dem Tanzsport - haben sich aus der so genannten expressiven, alternativen Spiel- und Bewegungskultur' der achtziger Jahre unterschiedlichste Bewegungsformen etabliert, die mit Inhalten ästhetischer und mimetischer Bewegungskultur wie Akrobatik, Jonglieren, Zirkuskünsten oder Pantomine verbunden sind. An-

${ }^{24}$ Als sportwissenschaftliche Beiträge zum Thema Körperzentrierung und Selbsterfahrung sind insbesondere BETTE 1993; 1999; TREUTLEIN et al. 1992; KOLB 1994; 2002; SCHNEIDER-WOHLFAHRT \& WACK 1994 zu nennen. 
dererseits finden sich in vielen der gegenwärtigen Trendsportarten wie dem Skate- oder Snowboarden, dem Kanu-Rodeo, dem Inline-Skaten oder BMXFahren vermehrt ausdrucksvolle und symbolische Gestaltungselemente. Aufschlussreich ist hierbei die von MICHELS (2002, 273f) aufgegriffene Differenzierung von Innen- und Außenperspektive. Die Innenperspektive beschreibt die Intention des Akteurs, sich als Darsteller zu inszenieren und das situative Bewegungshandeln bewusst ästhetisch zu beeinflussen. Das Bestreben nach kreativen ,Moves' und ästhetischen Bewegungsformen ist dabei in allen genannten Praxisformen zu konstatieren. Bewegung ist in diesem Zusammenhang individueller Ausdruck und kreative Gestaltung gleichermaßen. Demgegenüber ist der Handlungskontext der Situation im Sinne einer Inszenierungsfolie als Außenperspektive zu thematisieren - sie signalisiert die verstärkte Aufmerksamkeit auf die dramaturgisch-repräsentativen Elemente von Freizeitsportereignissen. Unter diesem Gesichtspunkt werden auch Praxisformen, die an sich keine Ausdrucksbewegungen beinhalten, wie beispielsweise das Fußballspiel zunehmend dramaturgisch inszeniert. Im $\mathrm{Zu}-$ sammenhang mit den Sinnorientierungen Ausdruck und Gestaltung muss auch die 'These der ,Versportung der Gesellschaft' (GRUPE 1988) eingeordnet werden, die einen Funktionswandel der Sportästhetik in der modernen Gesellschaft beschreibt (s.o.). Auch SCHULZE (1995, 33ff) sieht die Ursachen für die Tendenz zum inszenierten Handeln und einer allgemeinen Ästhetisierung im gesellschaftlichen Wandel begründet, die er als „Ästhetisierung des Alltagslebens" in der von ihm beschriebenen Erlebnisgesellschaft bezeichnet. Im Mittelpunkt dieser Argumentation steht die ,Multioptionsgesellschaft' (GROSS 1999), in der das Individuum vor die Herausforderung der selbstgestalteten Lebensführung und Identitätsbildung gestellt ist. Durch die Inszenierung und Symbolisierung des Körpers und der Bewegung können identitätsstabilisierende Ansprüche auf Individualität und Besonderheit untermauert werden. Kritiker beobachten allerdings gleichsam einen marktabhängigen Massenkult, der durch neue Normen und Zwänge physische Überforderung und Freizeit-Stress produziere (vgl. KASCHUBA 1989, 156; RIT'TNER 1989).

Obwohl es sich bei der Darstellung um keine willkürliche, sondern um die in der sportwissenschaftlichen Literatur gängige Darstellungsform handelt, ist eine Kategorisierung und Gliederung von Sinnorientierungen im Freizeitsport zwangsläufig mit Problemen der Ausschließlichkeit und Überlagerungen verbunden. Andererseits gibt sie einen Einblick in aktuelle Entwicklungstendenzen und Schwerpunkte des aktuellen Freizeitsportverständnisses. Zusammenfassend sollen die bedeutendsten Veränderungen im Freizeitsport, die sich in den letzten Jahrzehnten nachvollziehen lassen, im folgenden zusammengefasst werden:

Freizeitsport wird von ganz heterogenen Personengruppen mit ganz unterschiedlichen Wünschen und Ansprüchen betrieben. Ein plurales Sportmodell mit unterschiedlichen Motivstrukturen und Sinnrichtungen tritt an die Stelle der Homogenität und Einheit des Sports im Sinne einer Zentrie- 
rung auf die Sinnorientierungen Leistung und Wettkampf (vgl. GRUPE 1988; DIETRICH et al. 1990; BETTE 1999; WOPP \& DIECKERT 2002).

Im Mittelpunkt der Sportaktivität steht immer mehr ein ungezwungenes freudvolles Erleben anstatt des disziplinierten Trainings, ausgerichtet auf Leistungssteigerung und Zukunftsorientierung (vgl. DIECKERT 2002)

Gesundheit und Wohlbefinden sind innerhalb des Freizeitsport zu „Meta-Kategorien“" avanciert, wie die Entwicklung im Fitness- und Wellnessbereich verdeutlicht. Mit der Zentrierung auf den Körper und dem steigenden Bedürfnis nach Selbsterfahrung werden andere Sinnstiftungen, die sukzessiv brüchiger und leerer gewordenen sind, kompensiert (vgl. BRETTSCHNEIDER \& BRÂUTIGAMM 1990; BETTE 1999; HORX 2000; KOLB 2002)

Die Erscheinungsformen des Sports allgemein und des Freizeitsports im speziellen werden vielfältiger. Differenzierungs- und Expansionsprozesse führen zu einer ,bunten Vielfalt' an Sportarten und Bewegungsangeboten (vgl. CACHAY 1988; DIGEL 1986; HENKEL 1989; WOPP 1995; SCHWIER 2000).

$\mathrm{Zu}$ den Vereinen gesellen sich neue Angebotformen und Anbieter, wie kommerzielle Studios, Hochschulen, Krankenkassen oder Volkshochschulen. Gleichzeitig nimmt die Zahl nicht organisierter Freizeitsportler in informellen Sportarrangements zu (DIETRICH et al. 1990; PALM 1991; DIECKERT \& WOPP 2002).

Mit der von SCHULZE (1995) konstatierten Ästhetisierung des Alltagslebens geht gleichsam eine Ästhetisierung des Sports einher, die den Körper in Bezug auf seine ,Sportivität' thematisiert und im dialogischen Prozess Bewegung und Ausdruck verbindet (RITTNER 1989; KASCHUBA 1989; MICHELS 2002)

Im Zuge der Entwicklung zu einem Massenphänomen ist der Sport zu einem gewichtigen Wirtschaftsfaktor avanciert. Diese Kommerzialisierung im Sport schlägt sich im Freizeitsport vor allem in einem höheren Markenund Qualitätsbewusstsein, sowie einem veränderten Nutzungsverhalten im Bezug auf unterschiedliche Angebotsformen nieder (s.o.) (DIETRICH et al. 1990; LAMPRECHT \& STAMM 2002)

Obwohl die hier aufgezeigten Entwicklungen im Sport auf einer breiten wissenschaftlichen Basis diskutiert werden, zeigt die Forschung im Freizeitsportbereich weiterhin einige Defizite und Desiderata auf (vgl. DIECKERT\& WOPP 2002, 20f.). So fehlt zur Zeit noch eine genauere Theorie des sozialen Wandels im Sport, der es vorbehalten sein wird, im einzelnen zu analysieren, wie sehr der tradierte Sport von gesamtgesellschaftlichen Entwicklungen beeinflusst ist.

\subsubsection{Risikosport - Überblick über den Forschungsstand}

Im Vergleich zu den klassischen Sport-Kompositionen wie Mannschaftssport, Rehabilitations- oder Profisport, lässt das Komposita des Risikosports keine eindeutige Kennzeichnung des Phänomens zu. Dass es sich bei einer 
Individualsport um eine sportliche Aktivität handelt, deren Ausführung hauptsächlich allein ausgeführt wird, lässt sich relativ einfach aus dem $\mathrm{Na}$ men erschließen. Was aber ist das Risiko welches eine Sportaktivität zu einer Risikosportart erhebt?

Zusätzlich erschwert wird die Begriffsfindung zudem durch die uneindeutige Differenzierung und Nomenklatur einer offenbar neuen Bewegungskultur, die zu einer Vielfalt neuartiger Begrifflichkeiten wie Trendsport, Erlebnissport, Funsport, Thrill-Sports, Abenteuersport oder Extremsport geführt hat. Wie der folgende Überblick über den Forschungsstand zeigt, wurde die Frage nach einer Begriffsbestimmung des Risikosports von der Sportwissenschaft von verschiedenen Wissenschaftszweigen aus angegangen, ohne dass sich ein schlüssiges Gesamtmodell etabliert hat.

Um die Vielzahl in der wissenschaftlichen Literatur hervorgebrachten und zum Teil widersprüchlichen Merkmalsbestimmungen und inhaltlichen Zuweisungen aufzuzeigen, werden im folgenden die bedeutsamsten Ansätze der Sportwissenschaft expliziert. Ziel der Darstellung ist eine möglichst konkrete Operationalisierung des Begriffs vor dem Hintergrund der forschungsleitenden Fragestellung. Leider fehlt in einer Vielzahl von Beiträgen zum Thema Risikosport eine eindeutige Definition oder Begriffseingrenzung, so dass bei diesen Beiträgen eine Gegenstandsbeschreibung ausbleibt (z.B. BEIER 2001; BETTE 2004).

SCHLESKE (1977) der sich dem Risikosport unter einer allgemeinen pädagogischen Perspektive nähert, fasst unter der Kategorie Risiko-, Abenteuer und Wagnissportarten alle Abenteuer- und Risikoaktivitäten zusammen, die sich in eine Verbindung mit Sport bringen lassen. Dazu gehören seinen Ausführungen folgend sowohl verschiedene Kampfsportarten wie das Boxen aber auch verschiedene Motorsportarten oder das Bobfahren (vgl. SCHLESKE 1977, 40). In all diesen Sportarten werde eine Position der Sicherheit freiwillig zu Gunsten einer Gefahrensituation aufgegeben, die im Falle des Scheiterns ernsthafte Folgen für die Akteure besitzen können.

„Das Abenteuer als aktive Bewältigung einer dramatischen Situation ist
durch eine charakteristische Handlungsstruktur und Stimmungslage gekenn-
zeichnet: Bewußt und meist freiwillig wird eine Position der Sicherbeit preisge-
geben, man setzt sich einer Situation der Bedrohung, der Ungewißheit und der
Gefahr aus [...] "(ebd., 34)

Charakteristische Handlungsmuster für Risikosportarten sind demnach die Überwindung von Hindernissen - wie beim Bergsteigen -, das Meistern exponierter Raumlagen - wie beim Segelfliegen oder Fallschirmspringen -, die Manipulation und das Beherrschen von Geschwindigkeiten und/oder ungewöhnlichen Arten der Fortbewegung - wie beim Rennfahren, dem Kajak-Wildwasserpaddeln oder den Skifahren - und der Zweikampf mit menschlichen und tierischen Gegner - wie den Kampfsportarten oder beim Stierkampf (vgl. ebd., 107). Lassen sich diese Merkmale noch als Bestimmungs- und Abgrenzungsaspekte zu übrigen Sportkategorien verstehen, können interne Differenzierung zwischen den einzelnen Begriffen bei 
SCHLESKE nur bedingt extrahiert werden. In späteren Veröffentlichungen sieht der Autor im Erlebnissport einen Sammelbegriff, den er mit Hilfe charakteristischer Situations- und Handlungsmerkmalen definiert. Der Sportler gibt demnach freiwillig eine Position der Sicherheit und des festen Standortes auf und setzt sich Risiken aus, die durch die aktive Bewältigung als ,[...] ein Triumph der eigenen Tüchtigkeit, Reaktionsbereitschaft und Leistungsfähigkeit ausgekostet werden“" (SCHLESKE 1991, 85).

AUFMUTH (1983; 1988; 1989) nähert sich dem Phänomen Risikosport aus einer psychologischen Betrachtungsweise. Eine Eingrenzung oder Begriffsbestimmung des Risikosports nimmt der Autor nicht vor, am Beispiel des Bergsteigens entwickelt AUFMUTH aber Situationsmerkmale, die für das psychische Erleben am Berg relevant sind. Neben ,ungeheuren Anstrengungen“ und „hoher Lebensgefahr" bezieht er auch das „,wilde primitive Dasein im Gebirge" als besondere Form der Askese in die Merkmalsbeschreibung ein (vgl. ebd. 1988, 16ff). Eine Übertragung des Risikomoments beim Bergsteigen nimmt der Autor auf das Kanu-Wildwasserfahren und das Drachenfliegen vor. Eine Abgrenzung gegenüber anderen Begrifflichkeiten sowie eine Differenzierung zwischen verschiedenen Formen risikosportlicher Handlungen finden sich dagegen nicht.

Für BAUDRY (1986) sind risikoaffine Bewegungsaktivitäten mit einem Teilverlust von Körperlichkeit im gesellschaftlichen Alltag zu erklären, wobei der Autor keine eindeutige Differenzierung vornimmt, um welche Aktivitäten es sich dabei genau handelt. Die Extremisierung der Körpererfahrung, die der Autor auch als einen Beweggrund für Risikosportarten annimmt, erlaubt - so die zentrale These des Buches - eine authentische Selbsterfahrung, die eine Lebendigkeit des Seins vermittelt, für die im Alltag wenig Platz eingeräumt wird. Als Formen der sportlichen Daseinsvergewisserung („Se Tuer pour ne pas mourir“ (ebd., 177)) führt der Autor eine Vielfalt unterschiedlichster Aktivitäten an, die vom Bodybuilding bis zum Drogenkonsum reicht. Eine Abgrenzung unterschiedlicher Sportaktivitäten nimmt der Autor nicht vor.

PARLEBAS (1986) stellt risikoreiche Sportarten unter die Dimensionen ,domestication / sauvagerie' und konstatiert einen eher fließenden Übergang zwischen, domestizierten Sportarten' und ihren, wilden Varianten' (vgl. PARLEBAS 1986, 131ff). Dabei stellt er am Beispiel des Kayak-Fahrens, des Skifahrens und des Schwimmens dar, inwieweit Modifikationen einzelner Sportarten zu einer veränderten Bestimmung der Aktivitäten führen. Auch für den Bereich der Kampfsportarten werden bei PARLEBAS risikoreichere weil distanzarme Sportarten im Vergleich zu risikoarmen Sporthandlungen expliziert. Die Erweiterung der Diskussion um Bestimmungsfaktoren von Risikosportarten wird bei PARLEBAS um eine subjektive Komponente bereichert, in dem der Autor fließende Grenzen und Vernetzungen einzelner Sportarten aufzeigt. Leider lassen sich aber interne Differenzierungs- und Bestimmungsfaktoren auch hier nicht extrahieren.

Aus einer sportsoziologischen Perspektive nähert sich HARTMANN (1995; 1996) dem Begriff, den er in seinen Texten zum Teil synonym zu 
Begriffen wie ,Funsport' und ,Extremsport' benutzt. Zum Feld dieser Sportarten zählt der Autor subkulturelle Formen der Erlebnissuche, die den klassischen Definitionsrahmen von Sport durchbrechen und in ihren Ausführung distinktive Funktionen erfüllen. Als Risikosport bezeichnet er in diesem Zusammenhang ohne direkte inhaltliche Charakterisierung konkrete Motorsportarten, Luft- und Wassersportarten, sowie subkulturelle Aktivitäten aus dem Bereich des Wintersports und extreme Varianten des Alpinismus (vgl. HARTMANN 1995, 37). In späteren Beiträgen wird der Begriff der ,Extremen Outdoor-Aktivitities' eingeführt, welche sich in verschiedene ,Thrilling-Fields' untergliedern lassen (HARTMANN 1996, 73). In einer 42 Aktivitäten umfassenden Liste werden dabei vielfältige Sportaktivitäten vom Yachtrennen über das Base-Jumping bis hin zum Break-Dancing erwähnt, die auf die „Vielgestaltigkeit“ und „Bizarrerie“ des Phänomens verweisen (vgl. ebd., 68). Ingesamt finden sich auf Grund der weiten Begriffsauslegung ein breites Spektrum an Bestimmungs- und Abgrenzungsakpekten zu übrigen Sportkategorien bzw. Phänomen, die in ihrer Allgemeinheit aber wenig Anknüpfungsmöglichkeiten bieten. Insbesondere die diffuse Vermischung von inhaltlichen Kategorien des ,Fun'-, ,Extrem'- und Risikosports lesen sich eher als ein Versuch das ebenfalls umfangreiche Feld des Trendsports zu charakterisieren und lässt zudem konkrete Anknüpfungspunkte vermissen.

LE BRETON (1995) setzt in seinem Denkmodell der Ordaltheorie Risikosport, Extremsport und Grenzgängertum unter der Perspektive existentieller Bewährungen mit Formen sportlicher Mutproben gleich. Dabei betrachtet er den Risikosport als existenzielle Grenzsuche, die durch den Grad der Todesnähe differenziert wird: „Die Suche nach Grenzen ist ein Spiel ohne Grenzen. Die Grenze herauszufordern ist eine Weise, sich dem Tod zu nähern [...]“ (LE BRETON 1995, 16). Unterschieden werden vier Hauptfiguren des Risikoverhaltens, die sich als Charakteristika von Risikosportarten interpretieren lassen: Die Vertigo-Erfahrung leitet sich aus der Caillois'schen Spielkategorien des ,Inlinx' (Schwindel) und ,Agon' (Wettbewerb) ab und bezeichnet das Erleben von Schwindel und Taumel. Sie ist mit der Auseinandersetzung mit den Umweltkräften verbunden. Als Konfrontation wird die Auseinandersetzung mit sich selbst bezeichnet, die sich im Schlüsselerlebnis des „,[...] Sich-Ausschöpfen [...]“ (ebd., 26) erschließt. Die Entkörperung als dritte Hauptfigur des Risikoverhaltens besitzt im Rahmen sportlicher Aktivitäten nur bedingt Relevanz. Sie kann im Rahmen einer Ersatzsuche nach realen Erlebnissen in Video- und Cyberräumen erfahren werden. Das Überleben und sein Training steht als vierte Hauptfigur zur Charakterisierung des Risikoverhaltens. Dabei wird der temporäre Ausstieg aus der Überflussgesellschaft geprobt und durch asketische Lebensweise Selbsterfahrung erlebt. Die Ordaltheorie selbst leitet Risikosport aus seinem Entstehungszusammenhang ab, den LE BRETON in den gesellschaftlichen Bedingungen der Moderne vermutet.

Obwohl deutlich psychologisch angelegt bringt ALLMER (1998) einen interdisziplinären Ansatz zum Risikosport hervor, in dem der Risiko- und 
Extremsport als ein moderner Komplex innerhalb der Sportlandschaft gedeutet wird. In Anlehnung an AUFMUTH (1989, 125), der seine persönlichen Erfahrungen über das Extrembergsteigen mit den Merkmalen „ungeheuerliche und totale Anstrengung“, ,immenses Maß an Qual“" und „hohe Lebensgefahr" typisiert, kommt ALLMER (1998, 62f) zu einer Charakterisierung des Risiko- und Extremsports anhand der folgenden Merkmale:

1) Außerordentliche körperliche Strapazen - mitunter als qualvolle Anstrengungen und harte körperlichen Prüfungen.

2) Ungewohnte Körperlagen und-zustände: $\mathrm{Zu}$ verstehen in der Tradition CALLOIS' als Gefühl von Beschleunigung und Taumel aber auch als Gefühl von Fallen und schwebenden Zuständen.

3) Ungewisser Handlungsausgang: Charakteristisch für alle Risikosportarten, da sich der Handlungsausgang der Sportarten nicht eindeutig hervorsehen lässt. „Je mehr sich die wahrgenommenen Fähigkeiten und wahrgenommenen Aufgabenschwierigkeiten annähern, desto größer wird die Ungewissheit der Situationsbewältigung“" (ebd., 63).

4) Unvorhersehbare Situationsbedingungen: Ähnlich dem Handlungsausgang lassen sich auch die konkreten Handlungsbedingungen in der Situation nicht konkret hervorsagen. Zwar hängen die unvorsehbaren Situationsbedingungen maßgeblich vom Informationsstand des einzelnen ab, gleichzeitig sind aber die Situationsbedingungen per se nur schwierig vorherzusagen. Explizit hebt ALLMER die natürlichen Handlungselemente von Outdoor-Sportarten hervor, die wie bewegtes Wasser, Wind oder Wetter nur eingeschränkt vorhersehbar sind.

5) Lebensgefährliche Aktionen - als Abgrenzungskriterien zu anderen Sportkategorien. Risikosportarten beinhalten mehr als andere Sportarten die Gefahr das Leben zu verlieren. Risikoaktivitäten finden unmittelbar unter „Ernstfallbedingungen“ statt, in dem ein Scheitern durchaus negative und bedrohliche Folgen für den Akteur haben können.

Als Beispiele für den Extrem- und Risikosport nennt ALLMER eine Reihe von unterschiedlichen Sportarten, die vom „Trans-Sahara-Luft-Marathon“ (ebd., 61) bis zum River-Rafting in alpinen Gebirgsflüssen reichen. Bewusst werden klare Abgrenzungskriterien zugunsten eine graduellen Gewichtungsverschiebung unterlassen. Je nach Handlungszusammenhang können demnach unterschiedliche Kriterien bei der Analyse von Risikosportarten unterschiedlich gewichtet werden. „Notwendig ist, für die vielfältigen Extremund Risikosportaktivitäten eine differentielle Anforderungsanalyse durchzuführen und Extrem- und Risikosportarten mit ähnlichen Anforderungsprofilen zu klassifizieren." (ebd., 64).

NEUMANN25 (1999), der sich dem Phänomen des Risikosports aus einer pädagogischen Fragestellung nähert, bringt einen weiteren Begriff in die Diskussion um risikoreiche Bewegungsaktivitäten ein. Dem Begriff des Risikosports setzt er den Begriff des Wagnissports entgegen, da das Wagnis die ganze Person unter Einsatz aller personalen Bezüge fordert und die Abgren-

${ }^{25}$ NEUMANN's Auseinandersetzung mit pädagogischen Implikationen des Wagnisses sind in verschiedenen Beiträgen publiziert worden (1998; 1999; 2002; 2003). 
zung zu technischen und formalen Risiken verdeutlicht. Das sportliche Wagnis stehe ,[...] für eine ernsthafte Auseinandersetzung, in der sich die individuelle sportbezogene Handlungskompetenz bewähren muss. [...] ob das Wagnis gelingt hängt in erster Linie von seinen Fähigkeiten $a b^{\text {“ }}$ (NEUMANN 1999, 5). Im Verständnis NEUMANN's steht das Wagnis letztlich für eine indivduelle und freiwillige Eintscheidung, sich in eine offene und unsichere Ausgangslage zu begeben (vgl. NEUMANN 2003, 26). Eine Eingrenzung auf einzelne Sportarten oder Aktivitätsbereiche nimmt der Autor nicht vor. Für NEUMANN ist der „pädagogisch relevante Kern“ (ebd.) zu identifizieren und damit der Blick auf die psychischen Prozesse der Handlungsmotivation zu fokussieren. In letzter Konsequenz gibt es bei ihm keinen Wagnissport sondern nur „das Wagnis im Sport“ (ebd. 1999), welches sich in unterschiedlichen Situationen ergeben kann und damit nicht sportartspezifisch gebunden ist.

RUPE (2000) führt in Anlehnung an TRÜMPER (1995) den Begriff der ,Soft-Adventure-Sportarten' als eine Art synergetische Komposition der klassischen Begriffen wie Risiko- oder Extremsport ein. Zu den ,SoftAdventure-Trends' gehören nicht nur Sportarten im engeren Sinne sondern auch Expeditionen, Safaris oder Höhlenerforschungen (vgl. ebd., 43). „Zentrale Momente des Soft-Adventure-Trend sind die Ausübung in der Natur (Outdoor-Sportarten) und das Abenteuer (Abenteuersportarten) und in einigen Fällen ein bestimmtes Risiko (Riskosportarten) und ein Trend zur Extremisierung (Extremsportarten)" (ebd, 46f.). Soft Adventure Trends lassen sich damit als Überbegriff aller sportlichen Aktivitäten verstehen, die das Bedürfnis der Grenzüberschreitung als Motivationsbasis vereinen, dabei immer neue Reizvariationen und Innovationsschübe erfahren, einen wachsenden Zulauf verzeichnen und eine immer neue Verzahnung und Kombination der einzelnen Varianten ermöglichen (vgl. ebd., 48). In der von RUPE veröffentlichte Liste von Soft-Adventure-Sportarten finden sich sowohl traditionelle Sportarten wie das Kanuwandern als auch nicht näher definierte Bewegungsaktivitäten wie das ,Rubber Ducking’ oder ,Bridge Swinging'.

EGNER \& KLEINHANS (2000) nähern sich dem Phänomen des Risikosports unter der übergeordneten Kategorie der Trend- und Natursportarten, wobei ein geographie-soziologischer Ansatz als Basis dient. Zur Strukturierung wählen die Autoren als Abgrenzungskategorien neben dem ,Raumbezug', der ,Raumwirkung', der ,Notwendigkeit von Training und Vorbereitung' sowie dem Kriterium ,Risiko' auch die Kategorie, zentrales Merkmal'. Bei der anschließend vorgestellte Strukturierung wird schließlich zwischen ,Fun Sport', ,Thrill Sport', ,Extreme Sport' und ,Soul Sport' differenziert, wobei insbesondere die Kategorien Raumbezug und Raumwirkung zur Unterscheidung herangezogen werden. Thrill Sports' mit Base-Jumping als Beispiel weisen sich durch den ,[...] Kick durch extreme Situationen“ (ebd., 61) als zentrales Merkmal aus und werden damit ,[...] in der Regel ohne Netz und doppelten Boden ausgeübt, d.h. ohne Sicherheitsreserve" (ebd., 62). ,Soul Sports' zu denen die Autoren Sportarten wie das Mountainbiken, 
Gleitschirmfliegen oder Windsurfen zählen, können durch das zentrale Merkmal eines genussvollen Erlebens, etwas mit dem eigenen Körper zu tun, charakterisiert. Allerdings weisen EGNER \& KLEINHANS darauf hin, dass in der konkreten Sportpraxis durchaus zu Überschneidungen kommen kann.

HLAVAC \& BAUMGARTNER (IITF 2000) stellen Risikosportarten unter der Kategorie des Trendsports vor und versuchen über Systematisierungskategorien zu einer einheitlichen Begriffsbestimmung zu kommen. Im Bezug auf das Risiko differenzieren die Autoren zwischen unterschiedlichen Gefährdungspotentialen, die sie aus den Verletzungsraten ermitteln. Im Bezug auf die Raum-Systematik wird zwischen Natursportarten und infrastrukturgebundenen Sportarten unterschieden. Eine konkrete Definition von Risikosport und Risikosportarten unterbleibt.

Bei OPASCHOWSKI 2000 findet sich kein konkreter Bestimmungsversuch, obwohl der Begriff, Extremsport' im Titel der Arbeit zu finden ist und inhaltlich über motivationale Fragestellungen referiert wird. Anhand eines zweidimensionalen Achsenmodells mit den Dimensionen ,Lifestyle', ,Sport' und 'Thrill' wird lediglich eine Klassifizierung vorgeschlagen, die in die Komplexe ,Trendsport', ,Traditionssport' und ,Risikosport' mündet. Weitere Charakterisierungen und Abgrenzungsversuche zwischen den Begriffen fehlen aber gänzlich. Auch die Auswahl bestimmter Sportarten im Rahmen einer Repräsentativuntersuchung erfolgt ohne Begründung.

In seinen Ausführungen zum „Leben in wachsenden Kreisen“ benutzt WARWITZ 2001 ähnlich wie NEUMANN (1999) den Begriff des Wagnisses und des Wagnissports anstelle des Risikosports. Dabei findet sich bei WARWITZ keine eindeutige Definition von Wagnissportarten. Das Wagen betrifft bei ihm die ,[...] fundamentale Sinneinstellung eines Menschen, seines ethischen Bewusstsein, seiner Verantwortungsfähigkeit, seinen Wertschöpfungswillen“ (ebd., 17). Der Sport stellt demgemäß nur eine von vielfältigen Möglichkeiten dar, ein Wagnis einzugehen. Beim Wagen in sportlichen Handlungszusammenhängen handelt es sich demnach ,[...] um selbst frei entschiedene, aktiv gestaltete, persönlich verantwortete, in ihren Folgen akzeptierte Tätigkeit, die den Wagenden intensiv und ganzheitlich fordern. [...] Das Wagnis gewinnt damit neue Bewährungsräume über die Anforderungen der Grundsportart hinaus" (ebd., 97). Als Grundformen des Wagnis nennt WARWITZ das Rollen / Gleiten / Schweben, das Drehen / Kreisen / Überschlagen, das Rasen, das Klettern, das Fallen und das Fliegen (vgl. ebd.).

CLAUSEN (2003) nähert sich dem Komplex Risiko- und Extremsport unter der Eingrenzung des Begriffes des, Extremsports' im Rahmen seiner ethisch-religiösen Ausführungen über einen inneren Klassifizierungsversuch, bei dem extrem- und risikosportliche Grundmuster kategorisiert werden und schließlich in einem Modellversuch gegenübergestellt werden. Als ersten Komplex identifiziert der Autor den sogenannten ,X-tremsport'. Hier werden postmoderne und kommerzialisierte Bewegungsaktivitäten zusammengefasst, die im Gegensatz zu den extremen Varianten konventioneller Sport- 
arten ihre Genese im Zeitraffer durchlaufen haben und durch die Passivität der Akteure während des Handlungsvollzugs gekennzeichnet sind (vgl. ebd., 26) ${ }^{26}$. Unter der Kategorie ,Modifikationen konventioneller Sportarten' fasst der Autor extrem- und risikosportliche Aktivitäten traditioneller Sportarten zusammen. Diese reichen von extremsportlichen Varianten wie dem UltraMarathon oder dem Langstreckenschwimmen, die unter der Subkategorie des ,Endlos-Extremsports' zusammengefasst werden bis zu risikosportlichen Betätigungen wie dem Kanu-Wildwasserfahren oder dem Skysurfing als Modifikation des Fallschirmspringens (zusammengefasst als High-RiskVarianten konventioneller Sportarten) (ebd., 34f). Als dritte Kategorie führt CLAUSEN den ,Abenteuerhaften Extremsport' an: hier werden die vielfältigen und bisweilen in einer breiten Masse praktizierten Expeditionen zusammengefasst (vgl. ebd., 43) ${ }^{27}$.

Bei KUHN \& TODT (2003) wird die Frage nach der Begriffsbestimmung des Risikosports als Negativ-Definition in Anlehnung an AUFMUTH (1983) beantwortet ${ }^{28}$. Angesichts der breitensportlichen Relevanz des Risikosports erweitern die Autoren die Charakterisierung um eine Differenzierung zwischen Extrem-Risikosport und breiten- und freizeitsportlichen Risikosport. Diese Abgrenzung begründet sich aus der Annahme grundsätzlich unterschiedlicher Handlungskontexte. KUHN / TODT weisen darauf hin, dass Risikosport als Breitenphänomen weitestgehend moderat und ohne subjektiv empfundenes Lebensrisiko betrieben wird, eine differenzierte Charakterisierung des Risikosports wird aber nicht vorgenommen. So wird in ihren Ausführungen nicht deutlich, wodurch sich Risikosportarten beispielsweise von Nicht-Risikosportarten unterscheiden

RINEHART \& SYDNOR (2003) sammeln Risikosportarten in ihrem Beitrag unter den Oberbegriffen 'Extreme Sports' und ,Alternative Sports', zu denen sie eine Vielzahl unterschiedlicher Sportarten wie Inline-Skating, Snowboarding oder Kajakfahren fassen. Ihr qualitatives Verständnis von Risiko- und Extremsport orientiert sich an den Grundannahmen veränderter Vergesellschaftungsprozessen und einer daraus abgeleiteten Sportsozialisation. Aus einer subjektorientierten Perspektive beschreiben sie Risikosportarten unter der Prämisse der Identitätssuche, Distinktion und kulturellen Selbstdefinition (vgl. ebd., 3ff.). So kontstatieren sie den gegenweltlichen Charakter der ,X-Sports' gegenüber traditionellen Sportarten:

„Many extreme sports explode the ,canon' of mainstream sport in several ways. Grassroots extreme sports particpants are not instutionalized with governing bodies; they have no eligible team roster, established practice times/locals, oder coaches." (ebd., 6).

\footnotetext{
${ }^{26} \mathrm{Zu}$ dieser Kategorie zählt der Autor Bewegungsformen wie das ,Bungee-Jumping', ,Zorbing', , Base-Diving' oder ,Heli-Body-Flying' (ebd.).

${ }^{27}$ Zum ,abenteuerhaften Extremsport' zählt der CLAUSEN mehrtägige Bergbesteigungen, Höhlenerforschungen, Durchquerung von Wüsten oder die Überquerung weitläufiger Wasserflächen (ebd.).

${ }^{28}$ Die Strukturierung und Begriffeingrenzung AUFMUTHs findet sich auch bei ALLMER (1998) wieder.
} 
Die von ihnen angeführten Merkmale lassen sich in der deutschsprachigen Literatur in die Begriffsbestimmung des Trendsports - als subkulturelle Bewegungsindentitäten - ansiedeln (z.B. in der Tradition von SCHWIER (1998) oder LAMPRECHT / STAMM (2003)). Welche Differenzierung zwischen modernen Formen des Trendsports (beispielsweise BeachVolleyball) und risikosportlichen Aktivitäten bestehen, bleibt in den Ausführungen der Autoren weitestgehend offen.

STERN (2003a; 2003b) verdeutlicht das strukturelle Anforderungsprofil des Wagnis- und Risikosports anhand der Kontrastierung zum traditionellen Sport. In Abgrenzung zum Wettkampfsport mit der Verengung auf die Pole Sieg und Niederlage und einer allgemeinen objektiven Vergleichslogik wird der Wagnis- und Risikosport demzufolge durch die Tendenz der Entformalisierung gekennzeichnet (vgl. STERN 2003a, 190). Durch die Abwesenheit starrer Regeln und Grenzen ergeben sich im Wagnis- und Risikosport für den Einzelnen individuelle Definitionsfreiheiten bezüglich der Aktivitätsgestaltung. Im Vergleich zum Spitzensport wird das Risiko im Risiko- und Wagnissport um ihrer selbst Willen gesucht und im Rahmen der Bewältigung ein signifikanter Verlust bewusst in Kauf genommen. Den semantischen Rahmen der Aktivitäten beschreibt STERN durch das Spannungsgefüge von Leben und Tod: „Vor diesem Hintergrund zeichnen sich die Aktivitäten durch eine individuelle Gratwanderung zwischen den eigenen Fähigkeiten und Fertigkeiten einerseits und der selbstgewählten Herausforderung andererseits aus" (ebd.). Im Zentrum des Risiko- und Wagnissports ${ }^{29}$ bei STERN stehen folgende Charakteristika:

- Freiwilligkeit: Risiken werden nicht bloß in Kauf genommen sondern bewusst aufgesucht

- Todesrisiko: Fehlverhalten erreicht bei Risiko- und Wagnissportarten die Dimension der vermeintlichen Todesnähe.

- Situationsbegrenzung: Das Risiko ist strikt begrenzt auf eine konkrete Situation, der man sich stellt und besitzt klar abgrenzbare Start- und Endphasen.

- Individualität: Das Risiko bei Risiko- und Wagnissportarten ist individuell angepasst an die Bedürfnisse, Einschätzungen und Fähigkeiten des Einzelnen.

- Selbstkontrolle: Die Risikosituation wird im Bezug auf die Schwierigkeiten und Emotionen sowie die Motivation des Einzelnen kontrolliert und entsprechend ausgewählt (vgl. ebd., 191).

Aus diesem Kontrollmoment des Risikosports leitet STERN schließlich eine Klassifikation von Wagnis- und Risikosportarten ab. Bei Sportarten wie dem Klettern, dem Kajakfahren oder dem Down-Hill-Biken liegt die Kontrollinstanz beim Aktiven selbst. Seine Fähigkeiten und Fertigkeiten entscheiden

${ }^{29}$ Auch STERN (2003a / 2003b) stellt den Begriff des Wagnissports in das Zentrum seiner Ausführungen, da die Etymologie des Begriffs, Wagnis' mit der Bedeutung des ,abwägen' treffend die individuell zu bestimmende Balance ausdrückt, bei der das Wagnis die Person als Ganzes betrifft (vgl. ebd. 2003a, 191). Vor dem Hintergrund der oben angeführten Differenzierung ist der Begriff ,Risikosport' aber durchaus kongruent zu verwenden. 
über Erfolg oder Misserfolg in der Risikosituation. Die zweite Gruppe der Sportarten lässt sich demgegenüber mit der Externalisierung der Kontrolle kennzeichnen. Hierzu gehören Aktivitäten wie das Bungee-Springen, das Fallschirm-Tandemspringen oder in gewissem Ausmaß auch das Wildwasser-Rafting. Mit Beginn der Aktivität besitzt der Protagonist nur wenig bis gar keine aktiven Handlungsmöglichkeiten, der Handlungsausgang ist abhängig vom gewählten Material oder weiteren Personen, die den Handlungsverlauf beeinflussen (vgl. ebd., 192).

WHEATON (2004) versucht die Vielfalt der neuen Sportarten im Outdoorsport auf den Begriff „Lifestyle Sports“ zu vereinen. Die von der Autorin vorgenommene Definition der Sportarten wird unter der Annahme einer existierenden Portmoderne formuliert und geht von einem veränderten Identitätsbedürfnis als Grundlage der verschiedenen Sportarten aus. Risiko als implizite Kategorie wird hierbei nicht abgelehnt, fließt in die inhaltliche Konzeption des Life-Style-Sports aber auch nicht ein, wobei nur wenige Differenzierungen vorgenommen werden (vgl. WHEATON 2004, 4ff).

Ziel dieses zusammenfassenden Überblicks über den Forschungsstandes ist keineswegs die vollständige Beschreibung sämtlicher Aspekte des jeweiligen Verständnisses von Risikosports und synonym verwendeter Begriffe. Vielmehr zeigt sich in der Darstellung die Vielfalt und Uneinheitlichkeit der Begriffsbestimmung und -Benutzung. Es wird deutlich, dass eine einheitliche Betrachtung und Eingrenzung des Risikosport bisher unmöglich erscheint. Bei HARTMANN wird Risikosport zum Teil diffus mit anderen Begriffen wie Funsport vermischt. Bei Le BRETON, EGNER \& KLEINHANS und OPASCHOWSKI wird Risikosport synonym mit Extremsport verwendet ohne klare interne Differenzierungen zu explizieren. Bei CLAUSEN werden zwar inhaltliche Beschreibungen des Risikosports unter dem Etikett des Extremsport ausgeführt und interne Differenzierungsaspekte angeführt, eine Begriffsabgrenzung zum Risikosport unterbleibt aber. Andere Autoren (NEUMANN; WARWITZ) lehnen Risikosport als Begriff ab und plädieren für den Begriff Wagnissport als explizite Kategorie, wobei keine klare inhaltliche Abgrenzung deutlich wird. In der englischen Sprachraum wird der Begriff ,Extreme Sports', Alternative Sports' oder wie in neueren Forschungsbeiträgen ,Lifes-Style Sports' verwendet (RHINEHARDT; WHEATON).

Auch bezüglich der Bestimmungsfaktoren des Risikosports herrscht Uneinheitlichkeit. Wird bei einigen Autoren der Thrillcharakter ${ }^{30}$ hervorgehoben wird (HARTMANN; OPASCHOWSKI), wird in anderen Beiträgen der Raumbezug und der Risikograd der Aktivitäten betont (RUPE; EGNER \& KLEINHANS). Wieder andere Autoren akzentuieren den personalen Bezug des Risikosports unter Einsatz der ganzen Person (NEUMMAN; WARWITZ). Bei Le BRETON werden risikosportliche Aktivitäten durch

\footnotetext{
${ }^{30}$,Thrill' stellt eine der vielfältigen Erlebnisdimensionen des Risikosports dar, die mit dem so genannten ,Adrenalin-Kick' gleichgesetzt werden und die hohe Gelingensspannung der Aktivität beschreibt (s. auch Kapitel 4)
} 
existenzielle Bewährungsproben bestimmt und mit Formen delinquenter Mutproben gleichgesetzt.

Gefordert ist letztlich ein eigener Ansatz, der Risikosport als integrativen Begriff inhaltlich bestimmt, interindividuelle Abgrenzungen vornimmt und als strukturelles Handlungssystem mit entsprechenden Charakteristika kennzeichnet. Hierbei dienen die bis dato explizierten Forschungsbeiträge als Ausgangsbasis mit dem Ziel einer möglichst schlüssigen Integration der einzelnen Ansätze. Darüber hinaus ist eine Differenzierung notwendig, die den Risikosport von zugehörigen Begriffen wie Extremsport und Trendsport abgrenzt.

\subsubsection{Entwurf einer eigene Definition von Risikosport}

Der Überblick über den Forschungsstand offenbart eine Vielzahl unterschiedlichster Definitionsansätze und Bestimmungsversuche für ein als Risiko- oder Wagnissport etikettiertes Handlungsfeld. Dabei sind zwei unterschiedliche Zugänge der Gegenstandsbestimmung prinzipiell zu unterscheiden. Zum einen lässt sich Risikosport über einen subjektzentrierten Ausgangspunkt bestimmen, der das handelnde Individuum und dessen Wahrnehmung von Risiko im Sport in den Mittelpunkt der Gegenstandsdefinition setzt. Andererseits kann aber auch ein systemischer Ansatz gewählt werden, der Risikosport als Handlungsfeld bestimmt und Grundmuster von zugehörigen Aktivitäten und Sportarten deduziert. Nachfolgend wird über die systematische Differenzierung dieser unterschiedlichen Forschungszugänge eine eingrenzende Begriffsbestimmung und Operationalisierung des Risikosports vorgestellt. Zunächst erfolgt eine erste Annäherung an das Risiko im Sport als subjektzentrierter Bestimmungsansatz. Darüber hinaus soll aber auch eine möglichst konkrete Kennzeichnung von Handlungsfeldern durch kennzeichnende Merkmale und abgrenzende Differenzierungen erfolgen. Ziel dieses Definitionsansatzes ist die konkrete Kennzeichnung, Beschreibung und Differenzierung des Risikosports als Handlungsfeld anhand kennzeichnender Handlungskonstitutiva. Dabei werden artverwandte Begriffe der Sportwissenschaft als Kennzeichnung differenter Handlungsfelder abgegrenzt. Abschließend wird der Versuch einer Klassifizierung von Risikosportarten unternommen und am Beispiel des Kanu-Wildwasserfahrens und des alpinen Hochtourenbergsteigens das spezifischen Risikomoments der jeweiligen Aktivitäten in den Kontext des Handlungsfelde Risikosport eingeordnet.

\subsubsection{Das Risiko im Sport - ein subjektzentrierter Ansatz.}

Wenn das zentrale Unterscheidungsmerkmal zwischen Risiko und Gefahr im immanenten Entscheidungsprozess des Risikos liegt, dann erschließt sich auch die Bestimmung des Risikosports zunächst nur über das handelnde Individuum. Hierzu LUHMANN: 


\section{„Die Außenwelt selbst kennt keine Risiken, denn sie kennt weder Unter- scheidungen, noch Erwartungen, noch Einschätzungen noch Wabrscheinlich- keiten […" (LUHMANN 1991, 15).}

Risiko - so muss geschlussfolgert werden - ist ohne die Rückbindung an das Individuum also nicht existent. Damit gilt es auch für eine Bestimmung des Risikohaften im Sport zunächst die Perspektive des handelnden Subjekts einzunehmen: Auch Risikosport ist ohne das handelnde Individuum nicht gegenständlich. Zugegeben, ohne das handelnde Subjekt ist jegliches sportliches Handeln obsolet. Denn wenn Sport ein freiwillig eingegangenes und prinzipiell zweckfrei-intendiertes Bewegungsverhalten in spezifischen kulturellen Kontexten bedeutet ${ }^{31}$, dann wird der Mensch unweigerlich zum expliziten Bezugspunkt von sportwissenschaftlichen Definitionsansätzen. Aber im Gegensatz zu Begriffen wie Trendsport, die eine Begriffsbestimmung außerhalb der Handlungsintention des Subjekts ermöglichen, lässt sich Risikosport ohne konkreten Intentionsbezug inhaltlich nicht bestimmen. Risikosport impliziert demnach, dass das im Sport handelnde Subjekt eine Handlungssituation als Gefahrenrisiko oder Wagnis wahrnimmt, diese als ernsthafte Auseinandersetzung akzeptiert und durch Einsatz einer sportbezogenen Handlungskompetenz freiwillig bewältigt. Die aus dieser Handlungssituation resultierenden negativen oder positiven Folgen betreffen in erster Linie nur den Protagonisten selbst (vgl. NEUMANN 1999, 5).

Risikosport wird unter dieser Perspektive zum Risiko-Sport, denn nur die Handlungsintention des Subjekts entscheidet über eine als RisikoSituation gekennzeichnet Handlungskonstellation. Damit lässt sich prinzipiell jede sportliche Handlung als Risiko-Sport bestimmen, und zwar immer dann, wenn unter Einsatz der eigenen Fähigkeiten und Fertigkeiten eine als Gefahrenrisiko oder Wagnis wahrgenommene Handlungssituation im Sport als Herausforderung angenommen und durch Bewegungshandeln aufgelöst wird. Gleichzeitig lassen sich damit aber auch Handlungen ausgrenzen, die zwar ein vermeintliches Risiko darstellen aber keine Handlungskompetenz zur erfolgreichen Bewältigung erfordern. Das Bungee-Springen oder Tandemfallschirmspringen sind somit nicht als Risiko-Sport zu bezeichnen. Und auch Sporthandlungen, die zwar gefährlich anmuten, der Sportler aber kein Bewusstsein für diese Gefahren entwickelt, können nicht als Risiko-Sport (auch nicht als Risikosport) bezeichnet werden.

\subsubsection{Risikosport als Handlungsfeld - Definition und Abgrenzung}

Zusammenfassend lässt sich Risiko(-Sport) also nur über eine konsequente Berücksichtigung der Wahrnehmung und Intention des handelnden Subjekts bestimmen. Damit kann auch die Definition und Abgrenzung eines Hand-

\footnotetext{
${ }^{31}$ Dieser Definitionsansatzes des Sports stellt sicherlich eine Minimaldefinition dar, vor dem Hintergrund der Vielfalt und Ausdifferenzierung des modernen Sports (vgl. 2.3.1) ist eine nähere Bestimmung aber nur schwer möglich. Soziologische Definitionsansätze wie die von WEIß (1999) sind zwar ebenso allgemeingültig, aber für die wissenschaftliche Perspektive des Risikosports auf soziologisch-psychologische Forschung begrenzt: „Sport ist eine soziale Institution, in der Kommunikation körperlicher Leistung stattfindet“" (WEIß 1999, 10).
} 
lungsfeldes Risikosport als systemischer Zugang nur unter der Annahme erfolgen, dass der Protagonist das Risiko in als solchen charakterisierten Risikosportarten als Herausforderung bewusst eingeht und durch Einsatz seiner sportspezifischen Handlungskompetenz bewältigt. Risikosport als Handlungsfeld ist dann in erster Annäherung also nichts anderes als eine sportliche Handlungssituation, die die handelnden Akteure als Risikosituationen wahrnehmen und sportliche Fähigkeiten zur Bewältigung einsetzen.

Risikosport wird sich demnach in einzelnen oder mehreren Situationen vollziehen, die durch eine gewisse Unsicherheit bezüglich des Handlungsausganges gekennzeichnet sind und mehrheitlich aus einer gewissen situativen Ungewissheit natürlicher und naturnaher Handlungsräume resultieren risikosportliche Handlungssituationen sind damit grundsätzlich offenen hinsichtlich des Handlungsausgangs und der Folgen ${ }^{32}$. Das Kernelement des Risikosports ist die Bewältigung von subjektiven ,Bewährungssituationen', die den Einsatz der ganzen Person erfordern und darüber hinaus spezifisch erlernte Fähigkeiten und Fertigkeiten zur erfolgereichen Bewältigung verlangen. Dieses ,sich-einlassen’ im Risikosport beinhaltet einen expliziten Entscheidungsprozess, der zwischen Handlungsanforderungen und subjektiven Fähigkeiten abgleicht und den Faktor Zufall möglichst zu minimieren versucht. Der Handlungssituation tritt der Sportler reflexiv gegenüber mit dem Ziel eines ,sich-bewährens'. Auch wenn das Spannungsgefüge von Leben und Tod nicht in seiner Urform auf den Risikosport anzuwenden ist, so impliziert ein Scheitern in risikosportlichen Handlungssituationen doch ein durchaus schmerzhaftes oder unangenehmes Handlungsergebnis.

Was aber unterscheidet Risikosport als Handlungsfeld von anderen ,postmodernen' Sportterminologien wie ,Fun-Sport' oder Trendsport? Wie angeführt schließt das grundlegende Moment selbstbestimmter Kontrolle bei Risikosportarten das ,passive' Eingehen von Aktivitäten wie dem Bungee-Springen grundsätzlich aus. Bewegungsaktivitäten, in denen eine Externalisierung der Kontrolle das Handlungsgeschehen kennzeichnet, gehören demzufolge nicht zum Risikosport. Sie werden im folgenden mit, ThrillAktivitäten' bezeichnet. Hierunter werden diejenigen inszenierten spektakulären Bewegungsformen gefasst, die eine fast schockartige Konfrontation mit dem eigenen Körper und dessen Reaktionen beinhalten. Vordergründig findet auch hier eine Überwindung von Hindernissen und scheinbaren Wagnissen statt. Bei genauerer Betrachtung aber findet die Überwindung lediglich im Ich des Akteurs statt. Ein Scheitern in der Situation ist mit dem Abbruch vor der eigentlichen Kernaktivität verbunden. Kernelement der ThrillAktivitäten ist das ,sich-überwinden - spezifische Fähigkeiten und Fertigkeiten werden hierbei nicht gefordert.

Der situative Charakter der risikosportlichen Handlungsbewältigung und die möglichen negativen Handlungsfolgen für den Protagonisten schließen

\footnotetext{
32 Dies gilt grundsätzlich für alle sportlichen Handlungen und bestimmen ihren Charakter. Anders als bei wettkampforientierten Sportarten beziehen sich die Handlungsfolgen bei Erfolg oder Misserfolg aber nicht auf die Pole von Sieg und Niederlage, sondern auf das Spannungsgefüge von Leben oder Tod. Die positiven oder negativen Folgen der Aktivität betreffen in erster Linie nur den Sportler selbst.
} 
zudem extreme Dauer-Aktivitäten aus, die wie Ultra-Triathlons oder mehrfach Marathons vereinzelt unter dem Topos des Risikosports diskutiert werden und die hier unter dem Begriff Extremsport zusammengefasst werden. Charakteristisch für diesen Sportbereich ist die extreme Zeitdauer oder Distanzsteigerung konventioneller Sportarten ${ }^{33}$. Sportler gehen hierbei in den Grenzbereich der Belastbarkeit, die „,[...] intensive Körpererfahrungen ermöglichen und sogar meditative Formen der Selbstbegegnung zulassen “ (SCHLESKE 1998, 104). Der Akteur setzt sich hierbei keinem grundsätzlichen lebensgefährlichen Handlungsrisiko aus, auch wenn der Schmerz als „[...] Kontrapunkt zur sukzessiven Auflösung des Ichs gegeben ist“ (CLAUSEN 2003, 39).

Der Begriff Funsport scheint eher eine kreative Schöpfung der kommerziellen Freizeitindustrie zu sein, denn eine sportwissenschaftliche Kategorie. Auch hier ein subjektiver Zugang gefordert, der ,Spaß' als Bestimmungsfaktor definiert. Eine Abgrenzung von ,Spaß' ist allerdings unklar, besonders da sich die Sportwissenschaft der begrifflichen Schwäche des Spaßbegriffes bisher kaum angenommen hat (vgl. RITTNER 1998, 33ff; DIECKERT 2002). Für RITTNER kennzeichnet der Begriff Funsport die zunehmende Emanzipation des Sporterlebens vom traditionellen Sportverständnis (vgl. ebd.). Bei RUPE (2000, 45) werden unter Funsport jene Sportarten zusammengefasst, denen die Leistungskomponente vollkommen fehlt, die ,Fun' versprechen. Diffus bleibt aber auch hier die Frage nach eindeutigen ,Spaßkriterien' und ihrer Einbettung in den Kontext der Trendsportarten. Der Begriff Funsport wird aus diesen Gründen im Rahmen dieser Arbeit nicht verwendet.

Der Begriff des Erlebnissports ist ebenfalls ein unscharfer Begriff, der sich im Rahmen erlebnispädagogischer Programme etabliert hat. Auch hier ist ein prinzipiell subjektzentrierter notwendig, um zu bestimmen was als Erlebnis wahrgenommen wird. Die von WITTE (2002) vorgenommene Kriterienbestimmung des Erlebnisses verweist nicht zuletzt auf heterogene Vielfalt möglicher Erlebnissportarten. RITTNER definiert Erlebnissport über die Kategorien ,Selbsterleben' und ,Erlebnisintensität', wodurch der Autor eine Nähe zu risikosportlichen Aktivitäten aufbaut. EBERLE (1995) hebt das Grenzerlebnis als zentrale Kategorie des Erlebnissports hervor und zieht dadurch ebenfalls die Verbindung zum Risikosport. Als konkreter Bestimmungsfaktor ist das Erlebnis zur Kennzeichnung eines ,Erlebnissports' letztlich zu ungenau und zu wenig spezifiziert. Auf eine Abgrenzung kann daher verzichtet werden, da keine Eingrenzung des Gegenstands vorgenommen werden kann.

Trendsport stellt als Begriff eine übergeordnete Kategorie dar, die sowohl Extremsport- als auch Risikosportarten und andere alternative Sportarten in

\footnotetext{
${ }^{33}$ Zwar hebt CLAUSEN (2003) hervor, dass auch extreme risikosportliche Aktivitäten wie das Befahren der Rheinfälle bei Schaffhausen mit dem Kajak oder das Durchsteigen der Eiger-Nordwand ohne Sicherheitsseil zum Komplex Extremsport zu zählen sind. Vor dem Hintergrund des subjektiven Charakters der Risikosituation wird diese Ansicht hier nicht weiter vertreten. Zweifelsfrei handelt es sich um extreme Ausführungen von Risikosportarten, die aber nicht zu einer begrifflichen Vermischung führen sollten.
} 
sich vereint. Begriffe wie Trendsport und Trendsportarten implizieren, dass das System Sport als ganzes oder Ausschnitte desselben im ,Trend' liegen ${ }^{34}$. Ein Trend verweist im öffentlichen Verständnis auf das Avangardistische und Neuartige einer Entwicklung und steht in Opposition zum Alltäglichen und Gewohnten. Soziologisch betrachtet, dienen Neuerung dazu, sich vom Mainstream der Gesellschaft abzugrenzen und sich über das Besetzen von Neuheit als Individuum bemerkbar zu machen. Trendsport ließe sich damit in Abgrenzung zur traditionellen Sportausübung als individuelle Differenzbildung verstehen (vgl. DONNELLY 1993; LAMPRECHT \& STAMM 1998, 2002). Trends beschreiben jedoch auch die Sehnsüchte, Vorlieben und Neigungen, die sich in Gesellschaften widerspiegeln und sich zu übergreifenden und vernetzten Entwicklungslinien verdichten (vgl. SCHILDMACHER 1998, 14; auch HORX 1993; SCHWIER 2003; STUMM 2004). HORX (1993, 11f) versteht Trends in diesem Zusammenhang als eine besondere Art der kulturelle Anpassungsübung an veränderte Gegebenheiten. Demnach nimmt die Gesellschaft mit Trends die Spannungen auf, denen sie durch Modernisierung und veränderten Bedingungen ausgesetzt ist und versucht so, mit ihnen umzugehen.

Unter statistischen Gesichtspunkten wird der Trend im allgemeinen als die Daten einer Zeitreihe, die eine Entwicklungstendenz anzeigen, begriffen, wobei Trends stets nur einen bestimmten Ausschnitt darstellen - der eigentliche Geltungsbereich somit also klar begrenzt ist. Trends sind folglich temporär begrenzte Entwicklungen, die in sozialen Kontexten entstehen und sich durch Verdichtung und Ausdifferenzierung zu neuen Entwicklungslinien erhärten. Zwar herrscht zurzeit noch kein Konsens über die zeitliche Beständigkeit von Trends, im Vergleich zu Moden sind Trends aber durch ihre deutlich längere zeitliche Dauer gekennzeichnet. Letztlich ist vor dem Hintergrund der gesellschaftlichen Entwicklungen, aus denen sich Trends speisen nur von solchen zu sprechen, wenn Sie sich über mehrer Jahre im Bewusstsein der Bevölkerung verankern und als Praxis etablieren (vgl. SCHWIER 2003, 21).

Aus dem derzeitigen Forschungsstand zum Trendsport lassen sich zunächst quantitative Ansätze extrahieren (vgl. INSTITUT FÜR SPORTSOZIOLOGIE/KVR 2000; vgl. auch STUMM 2004). Demnach ist eine Trendsportart eine Bewegungsform, die in einem bestimmten Zeitraum eine deutliche Nachfragesteigerung aufweist. In Anwendung eines solches Definitionsansatzes wären beispielweise sowohl das alpine Klettern als auch das Kajak-Wildwasserfahren angesichts der absoluten Nachfragesteigerung der letzten 10 Jahre eine Trendsportart, da beide Sportausübungen deutlich an Anhängern gewonnen hat (vgl. LORCH 1995). Relative Zuwächse, teilweise in exponentiellem Ausmaß bei geringem absoluten Wachstum weisen dagegen andere Sportarten, wie das Wakeboarden oder das Kite-Surfen auf (vgl. BREUER \& MICHELS 2003). Qualitative Definitionsansätze werden z.B. von SCHWIER (1998; 2000; 2003), WOPP (2003) oder LAMPRECHT

34 Vgl. DUDEN (1999, 745): „Eine Trendsportart ist eine Sportart, die im Trend liegt“. 
\& STAMM (1998; 2002), im englischen Sprachraum auch von WHEATON (2004) vertreten. Sie unterstreichen, dass sich die Nachfrage nach Trendsportarten strukturell und inhaltlich von der Nachfrage nach den klassischen Sportarten unterscheidet. Trendsportarten sind demzufolge dadurch gekennzeichnet, dass „,...] sie nicht nur neue Bewegungsformen mit neuen Sportgeräten kreieren, sondern auch ein Sportverständnis propagieren, das teilweise quer zum traditionellen Sportbegriff steht" (LAMPRECHT \& STAMM 1998, 370). Im Zentrum qualitativer Trendsportbetrachtung steht die Annahme eines grundsätzlichen sozialen Wandels, der von den Protagonisten eine erhöhte Identitätsarbeit verlange und dabei über den im Sport vermittelten Stil Zugehörigkeit und Selbstverständnis produziert. Insbesondere die englischsprachigen Forschungsbeiträge weisen darauf hin, dass eine derartige Sportartenentwicklung mit Kommerzialisierungsprozessen verbunden ist, die über das traditionelle Sportverständnis hinaus geht. Inwieweit Risikosportarten diese Kriterien erfüllen, bleibt hinsichtlich mangelnder empirischer Absicherung fraglich. In Kapitel 5 wird der Frage nach den Identitätsprozessen und der Bedeutung von Stil im Risikosport auf der Basis soziologischer Beiträge vertiefend nachgegangen.

Risiko als zentrale Kategorie des Trendsports wird nicht explizit aufgeführt, sowohl bei SCHWIER als auch bei EGNER\& KLEINHANS wird Risiko aber als Teilkategorie angeführt und Risikosport als Teilmenge des Trendsports beschrieben. Unter dem Merkmal ,Extremisierung / Actionorientierung' werden z.B. bei SCHWIER (1998; 2003); EGNER (2000); EGNER/KLEINHANS (2000); SCHILDMACHER (1998); STUMM (2004) und HARTMANN (1996) Sportarten subsumiert, die das spielerische Erleben der eigenen Handlungsfähigkeit auch unter Risikobedingungen als zentrales Handlungsmerkmal identifiziert. Es wird im Rahmen dieser Arbeit angenommen, dass die für Trendsportarten typischen Merkmale auch für Risikosportarten gelten können. Allein darüber lässt sich aber keine konkrete Bestimmung vollziehen, so dass zwar eine generelle Schnittmenge zwischen Trend- und Risikosport angenommen werden kann, eine übergeordnete Verwandtschaft kann aber nicht konstatiert werden.

Die folgende Tabelle soll einen Überblick über die einzelnen Sportkategorien geben und die Differenzierung zwischen den einzelnen Bereichen verdeutlichen: 


\begin{tabular}{|c|c|c|c|}
\hline Kategorie & $\begin{array}{l}\text { Betrachtungspers- } \\
\text { pektive }\end{array}$ & $\begin{array}{l}\text { Bestimmungs- / A- } \\
\text { bgrenzungsaspekt }\end{array}$ & Wiss. Beiträge \\
\hline Trendsport & $\begin{array}{l}\text { Quantitativ-Normativ } \\
\text { Qualitativ- } \\
\text { sozialpsychologisch }\end{array}$ & $\begin{array}{l}\text { Nachfragesteigerung } \\
\text { (absolut / relativ) } \\
\text { Distinktionscharakter } \\
\text { /Abgrenzung zum tradi- } \\
\text { tionellen Sport }\end{array}$ & $\begin{array}{l}\text { SCHWIER 1998; 2003; } \\
\text { LAMPRECHT \& } \\
\text { STAMM 1998; 2003; } \\
\text { SCHILDMACHER } \\
\text { 1998; BREUER } 2003\end{array}$ \\
\hline Risikosport & $\begin{array}{l}\text { Qualitativ- } \\
\text { Handlungsorientiert }\end{array}$ & $\begin{array}{l}\text { Bewusstes Eingehen } \\
\text { von sportlichen Risiko- } \\
\text { situationen mit dem Ziel } \\
\text { der Bewährung durch } \\
\text { aktiven Einsatz eigener } \\
\text { Fähigkeiten. }\end{array}$ & $\begin{array}{l}\text { ALLMER 1998; } \\
\text { NEUMANN 1999; } \\
\text { RUPE 2000; } \\
\text { CLAUSEN 2003; } \\
\text { STERN 2003; }\end{array}$ \\
\hline Extremsport & $\begin{array}{l}\text { Qualitativ- } \\
\text { Handlungsorientiert }\end{array}$ & $\begin{array}{l}\text { Bewährung in physiolo- } \\
\text { gisch-psychologischen } \\
\text { Grenzsituationen durch } \\
\text { extreme Distanzbewäl- } \\
\text { tigung. }\end{array}$ & $\begin{array}{l}\text { ALLMER 1998; } \\
\text { CLAUSEN 2003; } \\
\text { SCHLESKE 1998; }\end{array}$ \\
\hline Thrill-Aktivitäten & $\begin{array}{l}\text { Qualtitativ- } \\
\text { Handlungsorientiert }\end{array}$ & $\begin{array}{l}\text { Passives Sich-Aus- } \\
\text { setzen mit dem Ziel des } \\
\text { Sich-Überwindens vor } \\
\text { der eigentlichen Aktivi- } \\
\text { tät. Vertrauen in techni- } \\
\text { sches Equipment und } \\
\text { führende Personen. }\end{array}$ & $\begin{array}{l}\text { LE BRETON 1995; } \\
\text { CLAUSEN } 2003 ; \\
\text { HARTMANN } 1996\end{array}$ \\
\hline Erlebnissport & $\begin{array}{l}\text { Qualitativ- } \\
\text { Handlungsorientiert }\end{array}$ & $\begin{array}{l}\text { Erleben als zentrale } \\
\text { Kategorie. Bezug zum } \\
\text { Risikosport über Inten- } \\
\text { sitätssteigerung und } \\
\text { Selbsterleben. } \\
\end{array}$ & $\begin{array}{l}\text { EBERLE 1995; WITTE } \\
\text { 2002; }\end{array}$ \\
\hline Funsport & diffus & $\begin{array}{l}\text { Spaß als Leitmaxime } \\
\text { nicht-leistungs- } \\
\text { orientierter Sportaus- } \\
\text { übung }\end{array}$ & $\begin{array}{l}\text { RITTNER 1998; } \\
\text { HARTMANN 1996; } \\
\text { RUPE 2000; }\end{array}$ \\
\hline
\end{tabular}

Tabelle 2.1: Begriffabgrenzung und inhaltliche Differenzierung des Risikosports (eigene Darstellung)

\subsubsection{Charakteristika des Risikosports}

Wenn das zentrale Bestimmungsmerkmal des Risikosports im handelnden Subjekt zu suchen ist, dann können Charakteristika des Risikosports nur unter der Annahme abgeleitet und bestimmt werden, dass diese ein subjektives Risikoverhalten im Sport provozieren. Eine solche Kennzeichnung dient aber nur einer verbesserten Operationalisierung des Begriffs, letztlich entscheidet einzig und allein der handelnde Protagonist, ob er eine Sportart oder bestimmte Handlungssituationen als sportliches Risiko wahrnimmt. Zwar sind die im Folgenden durch kennzeichnende Charakteristika als Risi- 
kosportarten bezeichneten sportlichen Handlungen mit einem bestimmten Gefahrenrisiko verbunden und insofern für die Kennzeichnung als Risikosport prädestiniert, möglich wäre aber auch eine Nicht-Beachtung dieses potentiellen Wagnisses und damit eine Transformation zum Gefahrensport. Betrachtet man das spezifische Risikomoment im Sport, welches Unsicherheit zum Kernelement hat und Bewegung als notwendige Bedingung aufweist, so lassen sich bestimmte Charakteristika von Risikosportarten hervorheben.

In Anlehnung an AUFMUTH $(1989,125)$ erweitert ALLMER (1998) die Kennzeichen einer sportlichen Risikosituation als allgemeinen Kriterienkata$\log$ für Risikosportarten. In Verbindung mit aktuelleren Beiträgen von CLAUSEN (2003) und STERN (2003a) lassen sich danach Risikosportarten „,...] durch außergewöhnliche körperliche Anstrengungen, ungewohnte Körperlagen und - zustände, einen ungewissen Handlungsausgang, unvorbersehbare Situationsbedingungen und lebensgefäbrliche Aktionen" (ALLMER 1998, 64) charakterisieren. SCHLESKE (1977) beobachtet in risikosportlichen Situationen zudem einen ausgeprägten Situationsdruck, der spontane Entscheidungen und improvisierende Aktionen erfordert. In Anlehnung an die Ausführungen von STERN (2003a, 190f) werden zudem konstitutive Elemente des Risikosports wie die Begrenzung auf konkrete Situationen und die Individualität der Risikosituationen sowie die Freiwilligkeit der Aktivität zur Charakterisierung des Risikosports herangezogen. In der folgenden Tabelle werden diese Kennzeichnungen des Risikosports näher erläutert: 


\begin{tabular}{|c|c|}
\hline $\begin{array}{l}\text { Konstitutive Elemente } \\
\text { des Risikosports }\end{array}$ & Beschreibung \\
\hline Individualität & $\begin{array}{l}\text { Das Risiko in Risikosportarten ist eine aus individuellen Handlungsan- } \\
\text { forderungen und Bewältigungsmöglichkeiten resultierende Komponen- } \\
\text { te, die nicht nur individuell unterschiedlich ausfallen kann sonder auch } \\
\text { subjektiv je nach Tagesform und oder Bedingungen anders bewertet } \\
\text { wird. Jedes Risiko ist deshalb ein absolut individuelles und kann nur auf } \\
\text { eine konkrete Situation zu einem bestimmten Tag und eine einzelne } \\
\text { Person bezogen werden. }\end{array}$ \\
\hline Situationsbegrenzung & $\begin{array}{l}\text { Das Risiko ist klar begrenzt auf eine reale Situation, der man sich be- } \\
\text { wusst stellt, um sie zu bewältigen. Bei Risikosportarten gibt es klar be- } \\
\text { grenzbare Anfangs- und Endpunkte, die die Kernaktivität der Risiko- } \\
\text { sportart kennzeichnen und damit das Risikomoment der Sportart defi- } \\
\text { nieren. Abweichend verhält es sich bei abenteuerhaften Risikoaktivitä- } \\
\text { ten, die durch einen langen Zeitraum gekennzeichnet sind und keine } \\
\text { klare Situationsabgrenzung aufweisen können. }\end{array}$ \\
\hline Freiwilligkeit & $\begin{array}{l}\text { Risikoaktivitäten und die darin zu findenden Risikosituationen werden } \\
\text { bewusst und freiwillig aufgesucht um sie zu bewältigen und nicht bloß in } \\
\text { Kauf genommen, um ein übergeordnetes Ziel zu erreichen. }\end{array}$ \\
\hline $\begin{array}{l}\text { Merkmale des } \\
\text { Risikosport }\end{array}$ & Beschreibung \\
\hline $\begin{array}{l}\text { Außergewöhnliche kör- } \\
\text { perliche Anstrengungen }\end{array}$ & $\begin{array}{l}\text { Risikosportarten erfordern den Einsatz der ganzen Person und dabei } \\
\text { nicht selten übermäßige Anstrengungen die sich aus der Konfrontation } \\
\text { mit den Naturkräften und / oder menschlichen oder tierischen Gegnern } \\
\text { ergeben (z.B. Kälte, Hitze etc.). }\end{array}$ \\
\hline $\begin{array}{l}\text { Ungewohnte Körperla- } \\
\text { gen und Zustände }\end{array}$ & $\begin{array}{l}\text { Die Überwindung unüblicher Körperzustände und entsprechender Kör- } \\
\text { perlagen ist in der Mehrzahl immanentes Handlungselement. Hierzu } \\
\text { kann beispielsweise der freie Fall, das ,Schweben' auf dem Wasser } \\
\text { oder das Erfahren hoher Beschleunigungskräfte gezählt werden (vgl. } \\
\text { ALLMER 1998, 62). }\end{array}$ \\
\hline $\begin{array}{l}\text { Ungewisser Handlun- } \\
\text { gsausgang }\end{array}$ & $\begin{array}{l}\text { Bei Risikosportarten ist eine vollständige Einschätzung der Handlungs- } \\
\text { bedingungen unmöglich, da sie in der Mehrheit in Naturräumen oder mit } \\
\text { menschlichen oder tierischen Gegnern praktiziert werden, die sich nur } \\
\text { schwer vollends einschätzen lassen. Der Handlungsausgang bleibt } \\
\text { daher immer bis zu einem gewissen Grad unsicher. }\end{array}$ \\
\hline $\begin{array}{l}\text { Unvorhersehbare Situa- } \\
\text { tionsbedingungen }\end{array}$ & $\begin{array}{l}\text { Verbunden mit den nur schwer einzuschätzenden Handlungsbedingun- } \\
\text { gen sind auch die unvorhersehbaren Situationsbedingungen. Unabseh- } \\
\text { bare Bedingungen sind zum Beispiel die Wetterbedingungen oder der } \\
\text { zeitliche Ablauf, die in ihrer Konsequenz entscheidende Bedeutung für } \\
\text { den Handlungserfolg und die eigene Unversehrtheit besitzen. }\end{array}$ \\
\hline \begin{tabular}{|l} 
Lebensgefährliche A- \\
ktionen
\end{tabular} & $\begin{array}{l}\text { Obwohl Risikosportarten keine explizite Todesnähe aufweisen, beinhal- } \\
\text { ten Risikosportarten mehr als andere sportliche Aktivitäten die Option } \\
\text { der Lebensgefährdung durch die Ausübung in lebensfeindlichen Natur- } \\
\text { räumen (z.B. Wasser; Gebirge) oder lebensgefährdenden Rahmenbe- } \\
\text { dingungen (z.B. der Sturz beim Rodeo-Reiten). Risikosportarten finden } \\
\text { unter ,Ernstfallbedingungen' statt (vgl. SCHNEIDER \& RHEINBERG } \\
\text { 1995, 424). }\end{array}$ \\
\hline
\end{tabular}




\begin{tabular}{||ll||}
\hline Situationsdruck & Risikosportarten sind mehrheitlich mit einem Handlungszwang verbun- \\
& den. Handlungsziele müssen demnach kurzfristig abgewandelt und „[...] \\
& dem sich ständig verändernden Verlauf der Situation angepasst wer- \\
& den“ (SCHLESKE 1977, 34). Ein spontaner Abruch der Aktivität wäh- \\
& rend der eingegangenen Risikosituation ist nicht möglich (z.B. beim \\
& alpinen Bergsteigen oder Kanu-Wildwasserfahren) \\
\hline
\end{tabular}

\section{Tabelle 2.2: konstitutive Elemente und kennzeichnende Merkmale des Risiko- sports ( eigene Darstellung).}

Es ist davon auszugehen, dass diese Kriterien nicht für alle Risikosportarten in gleicher Weise gelten dürften. „Vielmehr sind zur Kennzeichnung der vielfältigen Extrem,- und Risikosportaktivitäten unterschiedliche Gewichtungen der Kriterien vorzunehmen“" (ALLMER 1998, 64). Die dargestellten Charakteristika stellen somit die auf das sportliche Risiko bezogenen Gemeinsamkeiten der verschiedenen Sportarten dar, die unter dem Begriff Risikosport zusammengefasst werden.

\subsubsection{Klassifizierungsvorschlag von Risikosportarten}

Auf Grund der begrifflichen Unschärfe findet auch in der Zuordnung und Klassifizierung einzelner Sportarten zum Komplex des Risikosports keine einheitliche Darstellung in der Literatur statt. Bisweilen vermischen sich auch Risikoaktivitäten mit trendsportlichen Neuentwicklung, die allerdings in ihren Handlungsstrukturen nicht näher erläutert werden ${ }^{35}$ oder es werden Extremsportarten mit Risikosportaktivitäten vermischt (vgl. ALLMER 1998, 61f; CLAUSEN 2003, 25ff).

Eine Einordnung der unterschiedlichen Sportarten zum Phänomen Risikosport ist nur anhand konkreter Definitionsvorgaben und der kennzeichnenden Zuweisung konkreter Merkmale möglich. Um eine detaillierte Klassifizierung der Risikosportaktivitäten vorzunehmen ist allerdings eine differentielle Anforderungs- und Handlungsanalyse der jeweiligen Aktivitäten notwendig, wie sie anschließend exemplarisch für das alpine Bergsteigen und das Kanu-Wildwasserfahren vorgenommen werden. Eine derartige Analyse für alle in Frage kommenden Sportaktivitäten würde den Rahmen dieser Arbeit übersteigen, weshalb anschließend aus den bisher veröffentlichten Beiträgen eine Übersicht über mögliche Risikosportarten mit dem Ziel einer ersten Systematisierung vorgestellt wird. Um den Gegenstand der vorliegenden Arbeit - das Risikoverhalten in sportlichen Risikoaktivitäten - zu analysieren, ist es zudem erforderlich, sich über das risikospezifische Anforderungs- und Situationsprofil einer Sportart im Klaren zu sein. Abschließend werden dazu anhand der konstitutiven Merkmale des KanuWildwasserpaddelns und des alpinen Bergsteigens die spezifischen Risikomomente dieser Sportarten exemplarisch vorgestellt.

\footnotetext{
35 So zählt ALLMER $(1998,61 \mathrm{f})$ u.a. das Sky-Surfen, das River-Raften und das Hydro-Speed-Fahren als aktuelle Beispiele von Risikosportarten auf, wobei unklar bleibt worum es sich bei diesen Sportarten letztlich handelt.
} 
Aus der vergleichenden Analyse des Forschungsstandes und der konkreten Analyse möglicher Risikomomente im Sport lassen sich nun folgende Klassifizierungen extrahieren: Risikosport als Handlungsfeld lässt sich in $\mathrm{Na}$ turbezogene Risikosportarten, geschwindigkeitsorientierte Risikosportarten und schließlich abenteuerhafte Risikosportarten unterteilen. Kontaktsportarten, - auch zwischen Mensch und Tier wie beim Stierkampf - die sich in einem handlungsorientierten Verwandtschaftsverhältnis zum Risikosport befinden, werden auf Grund ihrer divergenten Entwicklungsbedingungen und ihrem Ausschluss von Natur als Handlungsraum nicht zum engeren Kreis der Risikosportarten gezählt ${ }^{36}$. Der Fokus dieser Arbeit wird damit auf natursportliche Risikosportarten gelenkt ohne jedoch angrenzende und im Kontext des Gesamtzusammenhangs bedeutende Verknüpfungen zu vernachlässigen.

Natur als Handlungselement wird im Rahmen dieser Arbeit also als quasi konstitutive Konstante des Risikosports betrachtet, auch wenn es kein implizites Element in der vorgestellten Klassifizierung darstellt. Die Natürlichkeit von verschiedenen Handlungsräumen oder Naturelementen wie Wasser, Luft oder große Höhe steht dem anthropomorphen Handlungsräumen klassischer Sportarten konträr gegenüber und bietet damit ,auf natürliche Weise' ein gewisses Unsicherheitspotential. Zwar bieten auch Kampfsportarten ein sportliches Gefahrenrisiko und das Eingehen von Kampfhandlungen im Sport ist sicherlich mit einer großen Unsicherheit bezüglich des Handlungsverlauf und der Handlungsfolgen verbunden. Anders als in naturbezogenen Risikosportarten ist der Ausgang hierbei aber von der Handlungskompetenz aufeinander bezogener Sportler abhängig. Insofern ist im Vergleich zu anderen sportlichen Risikomomenten der Entscheidungs- und Intentionsbezug verringert. Kampfsportarten stehen deshalb in einem engen Verwandtschaftsverhältnis ohne direkt als Risikosportarten klassifiziert werden zu können. ${ }^{37}$ :

\footnotetext{
36 Zweifelsfrei spielt das Risiko auch in den Kontaktsportarten eine bedeutende Rolle. Dazu ist auch der Stierkampf zu zählen, den z.B. MITCHEL (u.a. 1991) sozialhistorisch evaluiert. Dass Risiko als handlungskonstitutives Element in Kampfsportarten subjektive Bedeutung besitzt wird u.a. auch bei SUDGEN 1998; KATTERMANN 1999 oder KENNEDY 2001 deutlich. Die Erkenntnisse der interdisziplinären Analyse kann letztlich in bestimmten Teilbereichen auch für dieses Handlungsfeld angenommen werden, es wäre im Detail aber einer gesonderten Betrachtung zu unterziehen.

${ }^{37}$ Grundlage der komparativen Analyse sind die Beiträge von ALLMER 1998; CLAUSEN 2003; STERN 2003; OPASCHOWSKI 2000; EGNER \& KLEINHANS 2000; NEUMANN 1999; SCHLESKE 1977; RUPE 2000; HARTMANN 1996; TRÜMPER 1995;
} 


\begin{tabular}{|c|c|c|c|}
\hline Kategorie & $\begin{array}{l}\text { Spez. Handlungskon- } \\
\text { text / Bewegungsvol- } \\
\text { lzug }\end{array}$ & $\begin{array}{l}\text { Spezifisches Risiko- } \\
\text { moment }\end{array}$ & Beispiele \\
\hline $\begin{array}{l}\text { Naturbezogener } \\
\text { Risikosport }\end{array}$ & $\begin{array}{l}\text { Natur als konstitutives } \\
\text { Handlungselement / } \\
\text { Überwinden natürli- } \\
\text { cher Hindernisse und } \\
\text { Raumlagen / Naturge- } \\
\text { walten als Herausfor- } \\
\text { derung / }\end{array}$ & $\begin{array}{l}\text { Unberechenbarkeit der } \\
\text { Naturelemente / kon- } \\
\text { kreter Handlungszwang } \\
\text { und Situationsdruck / } \\
\text { Lebensfeindlicher } \\
\text { Handlungskontext } \\
\text { (Wasser; Schnee; Eis; } \\
\text { Höhe etc.) / }\end{array}$ & $\begin{array}{l}\text { Kanu- } \\
\text { Wildwasserfahren / } \\
\text { alpines Klettern / Wel- } \\
\text { lenreiten / Tourenski / } \\
\text { Drachen- und Gleit- } \\
\text { schirmfliegen / alpines } \\
\text { Mountainbiken / Tief- } \\
\text { seetauchen etc. }\end{array}$ \\
\hline $\begin{array}{l}\text { Geschwindigkeits- } \\
\text { orientierter Risikosport }\end{array}$ & $\begin{array}{l}\text { Eigenmächtige Be- } \\
\text { herrschung und Mani- } \\
\text { pulation von Ge- } \\
\text { schwindigkeit / Erleben } \\
\text { von Beschleunigung / } \\
\text { ungewöhnliche Arten } \\
\text { der Fortbewegung / } \\
\text { hoher Technisierungs- } \\
\text { grad }\end{array}$ & $\begin{array}{l}\text { Kontrollverlust bei ho- } \\
\text { hen Geschwindigkeiten } \\
\text { / Verletzungs- und To- } \\
\text { desnähe bei Misserfolg } \\
\text { / hohe Variablenabhän- } \\
\text { gigkeit }\end{array}$ & $\begin{array}{l}\text { Speedskiing / Speed- } \\
\text { skating / Down-Hill- } \\
\text { Mountainbike / Speed- } \\
\text { biking / Ralley- } \\
\text { Autorennen }\end{array}$ \\
\hline $\begin{array}{l}\text { Abenteuerhafter } \\
\text { Risikosport }\end{array}$ & $\begin{array}{l}\text { Langdauernde Unter- } \\
\text { nehmungen in zumeist } \\
\text { menschen- } \\
\text { unfreundliche Hand- } \\
\text { lungsräume / Grenz- } \\
\text { verschiebung / }\end{array}$ & \begin{tabular}{|l|} 
Unmenschliche Hand- \\
lungsräume / schwer \\
kalkulierbare Hand- \\
lungszusammenhänge / \\
hohes Niveau an Fer- \\
tigkeiten und Kenntnis- \\
sen erforderlich / expli- \\
zite Todesnähe bei \\
Misserfolg /
\end{tabular} & $\begin{array}{l}\text { Alpine Bergbesteigun- } \\
\text { gen / Höhlenexpeditio- } \\
\text { nen / Überquerungen } \\
\text { weiträumiger Wasser- } \\
\text { flächen oder Eisflächen } \\
\text { / Wüstendurchquerun- } \\
\text { gen etc. }\end{array}$ \\
\hline \multicolumn{4}{|l|}{ Enge Verwandtschaft: } \\
\hline Kampfsport & $\begin{array}{l}\text { Zweikampf mit } \\
\text { menschlichen oder } \\
\text { tierischen Gegnern im } \\
\text { Direktkontakt }\end{array}$ & $\begin{array}{l}\text { Verhalten des Gegners } \\
\text { nur schwer einschätz- } \\
\text { bar (vor allem bei Tie- } \\
\text { ren) / hoher Verlet- } \\
\text { zungsgrad }\end{array}$ & $\begin{array}{l}\text { Stierkampf / Rodeo- } \\
\text { Reiten / verschiedene } \\
\text { Varianten des Ultima- } \\
\text { teFighting etc. }\end{array}$ \\
\hline
\end{tabular}

Tabelle 2.3: Klassifizierung des Risikosports (eigene Darstellung)

Natürlich kann es bei derartigen Kategorisierungsversuchen zu einer Vermischung einzelner Sportarten kommen. So lässt sich beispielsweise das Down-Hill-Mountainbiken sowohl unter der Kategorie naturbezogener Risikosport als auch unter der Kategorie des geschwindigkeitsorientierten Risikosports einordnen. Auch sind die verschiedenen Spielarten des Bergsteigens nur graduell zu differenzieren und innerhalb der Kategorisierung in verschiedene Bereiche einzuordnen. Letztlich soll die hier vorgenommene Klassifizierung auch nur als Orientierungshilfe und Strukturierungsleistung ohne Anspruch auf Vollständigkeit verstanden werden.

Die von PARLEBAS erstmals vorgestellte Einteilung von Sportarten anhand eines mehrdimensionalen Raumes verdeutlicht diese graduelle Ab- 
stufung der Aktivitäten und unterstützt - ohne es zu intendieren - die Subjektivität des sportlichen Risikomoments. Der Autor differenziert hierbei zwischen zwei Extrempolen (espace domestique' und espace sauvage'), die das Spektrum von Sportarten beschreiben, die in ihren Ausführungsmöglichkeiten eine große Varianz besitzen. Risikosportarten sind damit nicht unbedingt immer ausschließlich eigene Sportarten sondern können gleichfalls als ,Risiko-Varianten' von risikolosen Sportarten betrachtet werden (vgl. PARLEBAS 1986, 131f).

Risikosport umfasst damit ein breites Spektrum an unterschiedlichsten Aktivitäten, die auf der einen Seite als Modifikationen traditioneller Sportarten gelten können, auf der anderen Seite sich aber auch als neue Bewegungsformen identifiziert lassen, welche in ihrer Genese an die Entwicklungszyklen der Trendsportarten erinnern (vgl. LAMPRECHT \& STAMM 1998). In Anlehnung an PARLEBAS (1986) ist vor allem bei Varianten traditioneller Sportarten - wie dem Wildwassersport als Modifikation des KanuWanderpaddelns - eine graduelle Abstufung des Risikomoments denkbar.

\subsubsection{Exemplarische Analyse des Risikomoments am Beispiel des Kanu- Wildwasserfahrens und des alpinen Bergsteigens}

Aussagen über das Spannungsfeld von Risiko und Sicherheit im Risikosport können nur konkretisiert werden, wenn eine eindeutige Handlungs- und damit auch Erlebnisbeschreibung vorliegt. Die Zuweisung von Sportarten zum Handlungs- und Erlebnisfeld Risikosport erfolgt letztlich anhand subjektiver Selbstzuschreibung. Nur der Sportler selbst entscheidet über die Risikohaftigkeit seiner Sportausübung. Eine Zuweisung von Handlungsprofilen, die ein solches Risikoverhalten aber begünstigen, wurde durch die $\mathrm{Zu}$ weisung kennzeichnender Charakteristika bestimmt. Sie kann in ihrer eigentlichen Form hier nicht für alle Sportarten im Dunstkreis des Risikosports erfolgen. Am Beispiel des Kanu-Wildwasserfahrens und des alpinen Hochtourenbergsteigens soll aber beispielhaft verdeutlicht werden, wie das spezifischen Risikomomente von Risikosportarten exemplarisch zu evaluieren ist. Derartige Analysen bleiben natürlich subjektiv und müssen im Bezug auf die ausübende Person modifizierbar sein. Als Basis der folgenden Darstellungen dient daher zum einen ein introspektiver Zugang, der auf eigenen Erfahrungen aufbaut, sowie Erlebnisberichte aus gängigen Zeitschriften ${ }^{38}$ und die existierende Fachliteratur.

Obwohl das Kanu-Wildwasserfahren als auch das alpine Hochtourenbersteigen werden von verschiedenen Autoren als Risikosportarten bezeichnet werden (z.B. ALLMER 1998, 64; AUFMUTH 1989, 134; KERR 1991, 613; SCHLESKE 1977, 43; WESSELER 1997, 58), liegt eine inhaltliche oder strukturelle Beschreibungen für diese Kennzeichnung liegt allerdings nicht vor. Die folgende Analyse der beiden Risikosportarten soll die Kenn-

\footnotetext{
${ }^{38}$ Erlebnisberichte finden sich für das Kanufahren insbesondere in den Zeitschriften ,KANUSPORT’ und ,KANUMAGAZIN' sowie für das alpine Bergsteigen in ,BERG ALPENVEREINSJAHRBUCH'; „KLET'TERN' und ,OUTDOOR'.
} 
zeichnung von Risikosportarten nach dem Muster der spezifischen Sportartenanalyse plausibilieren, wie es unter anderem ALLMER (1998) fordert. Dass es sich hierbei - trotz der benutzten Fachliteratur um eine subjektive und damit nicht verallgemeinerbare Darstellung handelt, sei hier nochmals betont.

\begin{tabular}{|c|c|}
\hline $\begin{array}{l}\text { Kennzeichnende Mer- } \\
\text { kmale von } \\
\text { Risikosportarten }\end{array}$ & Analyse am Beispiel Kanu-Wildwasserfahren \\
\hline $\begin{array}{l}\text { Außergewöhnliche } \\
\text { körperliche Anstren- } \\
\text { gungen }\end{array}$ & $\begin{array}{l}\text { Obwohl eine Verausgabung bis zur totalen Erschöpfung eher selten ist, kann } \\
\text { es gerade in der Auseinandersetzung mit sehr kalten Wassertemperaturen zu } \\
\text { extremen körperlichen Strapazen kommen. Bei Handlungen unter Wasser } \\
\text { (z.B. Kenterrolle oder Schwimmen nach Verlassen des Bootes) kommt es } \\
\text { außerdem zu kritischen Bedingungen auf Grund des Sauerstoffmangels. }\end{array}$ \\
\hline $\begin{array}{l}\text { Ungewohnte Körperla- } \\
\text { gen und Zustände }\end{array}$ & $\begin{array}{l}\text { Beim Kanu-Wildwasserpaddeln wird eine für den Menschen ,natürliche' Positi- } \\
\text { on an Land für eine ,unnatürliche' Fortbewegung auf bewegtem Wasser auf- } \\
\text { gegeben. Hinzu kommt die relativ unstabile Position des Boot - Fahrer - Sys- } \\
\text { tems } 39 \text {, die mit ihrem Systemschwerpunkt über dem Verdrängungsmittelpunkt } \\
\text { eine aktive Aufrechterhaltung erfordert. Beim Eskimotieren, also dem Durch- } \\
\text { führen einer Kenterrolle kommt der Paddler in eine für den Menschen voll- } \\
\text { kommen ungewohnte Situation, in der er Handlungen entgegen die Schwer- } \\
\text { kraft und mit dem Kopf nach unten durchführen muss. }\end{array}$ \\
\hline $\begin{array}{l}\text { Ungewisser Handlun- } \\
\text { gsausgang }\end{array}$ & $\begin{array}{l}\text { Durch die vielfältigen situativen Bedingungen, die die kanusportlichen Hand- } \\
\text { lungen im Wildwasser beeinflussen, wird eine eindeutige Voraussage der } \\
\text { Handlungssituation nie möglich sein. Die Einschätzung der vielfältigen Einflüs- } \\
\text { se, wie Strömungsgeschwindigkeit, Strömungsart oder Wasserdruck stellen } \\
\text { neben den motorischen Fertigkeiten auch hohe kognitive Ansprüche an den } \\
\text { Paddler. Eine dynamische Auseinandersetzung mit den Situationsbedingun- } \\
\text { gen kann zwar für alle Sportarten postuliert werden, beim Kanu- } \\
\text { Wildwasserfahren besitzt diese aber eine wesentlich größere Bedeutung }\end{array}$ \\
\hline $\begin{array}{l}\text { Unvorhersehbare Situa- } \\
\text { tionsbedingungen }\end{array}$ & $\begin{array}{l}\text { Kanu-Wildwasserfahrten lassen sich nicht im Detail planen oder zeitliche Situ- } \\
\text { ationen vorwegnehmen. Es ist nicht vorhersehbar, welche Situationsbedin- } \\
\text { gungen auftreten werden oder ob die gegebenen weiterhin bestehen. Dies gilt } \\
\text { in besonderer Weise für Wasserstände, zeitliche Vorstellung, Veränderungen } \\
\text { im Flussbett, Baumversperrungen oder Wetterveränderungen, aber auch Ein- } \\
\text { schätzung der eigenen Fähigkeiten und Befindlichkeiten. }\end{array}$ \\
\hline $\begin{array}{l}\text { Lebensgefährliche A- } \\
\text { ktionen }\end{array}$ & $\begin{array}{l}\text { Das lebensbedrohende Element Wildwasser beinhaltet vor allem in höheren } \\
\text { Schwierigkeitsstufen die Möglichkeit, das Leben zu verlieren, sollte eine Hand- } \\
\text { lung scheitern. Langes Schwimmen, rückläufige Walzen, Steckgefahr, Unter- } \\
\text { spülungen und Siphons sind hier nur beispielhaft zu nennen. Aber auch in } \\
\text { niedrigen Schwierigkeitsstufen besteht durch das Element Wildwasser eine } \\
\text { latente Lebensgefahr. Erst der richtige, verantwortungsvolle Umgang führt } \\
\text { dazu, schlimmere Schäden zu vermeiden. }\end{array}$ \\
\hline Situationsdruck & $\begin{array}{l}\text { Entscheidet sich ein Paddler eine bestimmte Stelle im Wildwasser zu paddeln, } \\
\text { so ergibt sich für ihn während der Befahrung ein Handlungszwang. Er kann } \\
\text { die Befahrung zu Ende bringen oder situativ seinen Handlungsplan abändern, } \\
\text { wobei extrem schnelle Reaktionen erforderlich sind. }\end{array}$ \\
\hline
\end{tabular}


Tabelle 2.4: Evaluation des spezifischen Risikomoments am Beispiel KanuWildwassefahren (eigene Darstellung)

\begin{tabular}{|c|c|}
\hline $\begin{array}{l}\text { Kennzeichnende Mer- } \\
\text { kmale von } \\
\text { Risikosportarten }\end{array}$ & Analyse des alpinen Hochtourenbergssteigens \\
\hline $\begin{array}{l}\text { Außergewöhnliche kör- } \\
\text { perliche Anstrengungen }\end{array}$ & $\begin{array}{l}\text { Bei Bergsportarten bringt die Höhe außerordentliche Belastungen bei sportli- } \\
\text { chen Aktivitäten mit sich (Flüssigkeitsverlust; Sauerstoffmangel etc.). Zudem } \\
\text { birgt die Überwindung von Hindernissen in der Vertikalen extreme Strapazen } \\
\text { in sich, die für den alpinen Bergsport immanent sind. }\end{array}$ \\
\hline $\begin{array}{l}\text { Ungewohnte Körperla- } \\
\text { gen und Zustände }\end{array}$ & $\begin{array}{l}\text { Ungewohnt Körperlagen werden beim alpinen Bergssteigen vor allem in der } \\
\text { Vertikalen erlebt. Das Handeln gegen die Schwerkraft bedeutet eine außer- } \\
\text { gewöhnliche Körperlage, die zudem im Zusammenhang mit Naturkräften wie } \\
\text { Kälte, Schnee etc. erlebt wird. }\end{array}$ \\
\hline $\begin{array}{l}\text { Ungewisser Handlun- } \\
\text { gsausgang }\end{array}$ & $\begin{array}{l}\text { Der Handlungsausgang ist beim alpinen Bergsport auf Grund der vielzähligen } \\
\text { situativen Bedingungen hochgradig ungewiss. Dies gilt zum einen hinsichtlich } \\
\text { des Erfolges - z.B. dem Erreichen eines Gipfels - als auch im Bezug auf die } \\
\text { eigene Versehrtheit. }\end{array}$ \\
\hline $\begin{array}{l}\text { Unvorhersehbare Situa- } \\
\text { tionsbedingungen }\end{array}$ & $\begin{array}{l}\text { Die unvorhersehbaren Bedingungen beim alpinen Bergsport sind zum einen } \\
\text { die Wetter- und Klimabedingungen als auch geographische Variablen wie } \\
\text { beispielsweise Gesteinsformationen. Eine dynamische Auseinandersetzung } \\
\text { mit den vielzähligen Variablen ist beim alpinen Bergsteigen unerlässlich. }\end{array}$ \\
\hline $\begin{array}{l}\text { Lebensgefährliche A- } \\
\text { ktionen }\end{array}$ & $\begin{array}{l}\text { Die Höhe besitzt immanente lebensgefährdende Elemente. Das alpine } \\
\text { Bergsteigen mit all seinen Unwägbarkeiten ist schon grundsätzlich als bedro- } \\
\text { hend einzustufen. Steinstürze, das Abrutschen in steilem Gelände oder auch } \\
\text { Lawinen oder Wetterumschwünge bergen eine grundsätzliche Lebensgefahr } \\
\text { in sich, der nur durch entsprechende Planung und Training spezifischer Fä- } \\
\text { higkeiten entgegengetreten werden kann. }\end{array}$ \\
\hline Situationsdruck & $\begin{array}{l}\text { Handlungszwang beim alpinen Bergsteigen ergibt sich aus der Unwegsamkeit } \\
\text { des Geländes sowie der Vertikalen als zentraler Handlungsraum. Der Sportler } \\
\text { kann sobald er in eine Wand eingestiegen ist oder einen Kletterstieg begon- } \\
\text { nen hat diesen zu Ende führen oder aber den ebenso mühseligen Rückzug } \\
\text { antreten. Ein einfaches Abbrechen der Situation ist im alpinen Gelände kaum } \\
\text { möglich. Zudem ist auch der Abbruch einer gesamten Tour mit größeren } \\
\text { Schwierigkeiten verbunden. }\end{array}$ \\
\hline
\end{tabular}

Tabelle 2.5: Evaluation des spezifischen Risikomoments am Beispiel alpines Hochtourenbergsteigen (eigene Darstellung)

Natürlich besitzen sowohl das alpine Hochtourenbergsteigen als auch das Kanu-Wildwasserfahren eine Bandbreite gradueller Abstufung im Bezug auf das spezifische Risikomoment und die spezifischen Handlungsanforderungen. Die graduellen Abstufungen, die sich auch auf das Bergsteigen (vom Spazierengehen bis zur Extrembesteigung) oder das Kajak-Fahren (geruhsame Wanderfahrt bis zur Befahrung von Wasserfällen) anwenden lassen, verweisen auch hier auf das subjektive Moment der Darstellungen.

Nochmals sei hier darauf hingewiesen, dass auf Grund ihres Abstraktionsgrades eine derartige Analyse nicht 1:1 in die Wirklichkeit zu transferie- 
ren ist. Ziel ist lediglich die Operationalisierung von Begriffen und die Validierung gemachter Definitionsleistungen, die für die weitere Darstellung erforderlich ist.

\subsection{Zusammenfassung und Einordnung der Befunde}

Im diesem Kapitel wurde das Begriffsfeld des Risikos eingehend erläutert und von ähnlichen im Sprachgebrauch synonym verwendeten Begriffen abgegrenzt. Im Mittelpunkt des Kapitels steht die Charakterisierung und Definition der sportlichen Risikosituation, des Risikoverbaltens und des Risikosports. Bedeutend erscheint hierbei die Abgrenzung von verschiedenen Unsicherheitsbegriffen, wie sie sich in der Terminologie von Risiko und Gefahr darstellt: Risiko - so kann hier zusammengefasst werden - wird erst dann zu einem solchen, wenn die Gefahr als Gegenstand und als Folge einer eigenen Entscheidung zu sehen ist.

Auf der Basis der grundlegender Begriffbestimmungen und der Einordnung von risikosportlichen Aktivitäten in die neuere Entwicklung des Freizeitsports wurde ein erster Modellbildungsversuch zum Risikosport vorgestellt, der sowohl Definitionsansätze enthält als auch Abgrenzungs- und Klassifizierungsvorschläge. Risikosport wurde hierbei als Handlungsfeld definiert, welches sich durch eine selbstinduzierte, bewegungsvermittelte Bewältigung risikoaffiner Herausforderungen kennzeichnet. Risikosport als Handlungsfeld definiert sich damit zunächst subjektzentriert, denn nur der handelnde Sportler entscheidet letztlich über das subjektive Risiko einer Handlungssituation. Eine Zuweisung von kennzeichnenden Charakteristika ist demnach nur unter der Annahme möglich, dass diese ein sportliches Risikoverhalten begünstigen bzw. fordern. Obwohl sich das Handlungsfeld des Risikosports auf diese Weise als vielfältig und aufgrund unterschiedlicher Vollzugsdauer sowie räumlich-personeller Dispersion als stark variabel darstellt, sind dennoch Generalisierungen aus diesen Aktivitäten ableitbar (vgl. BETTE 2004, 117).

Wenngleich mit den theoretische Darstellungen dieses Kapitels zu einer Abgrenzung und inhaltliche Kategorisierung des Risikosports beigetragen werden konnte, so sind damit doch mehr Fragen aufgeworfen als beantwortet worden. Es wird das forschungsleitende Ziel der nächsten Kapitel sein, Erklärungszusammenhänge für den Risikosport auf phänomenologischer Ebene und das damit implizierte Bewegungshandeln auf individueller Ebene zu liefern. 


\title{
3 Zur Geschichte des Risikosport - historische Wurzeln und ideologische Grundlagen risiko- sportlicher Aktivitäten.
}

\author{
Geschichte ist nicht nur Geschehenes, sondern Geschichtetes - also der Boden, auf \\ dem wir stehen und bauen. (Hans von Keler (*1925), dt. Theologe)
}

Unter einem Kapitel zur Historie des Risikosports vermutet man zunächst die chronologische Aufarbeitung bedeutender Etappen heutiger Risikosportarten. Ein solcher Ansatz erscheint dem soziologisch interessierten Leser wahrscheinlich absurd, werden derartige Sportphänomene doch gerne mit Postulaten einer wie auch immer gearteten Postmoderne in Verbindung gebracht (vgl. OPASCHOWSKI 2000). Wie in Kapitel 5 noch aufgezeigt wird, ist eine solche Diagnose auch nicht ganz verfehlt, dennoch: die Evaluation historischen Kontexte für einen Forschungsgegenstand mit dem $\mathrm{Na}$ men Risikosport birgt einen darüber hinausgehenden Erkenntnisprozess, der neue Perspektiven für das Verständnis von risikoorientierten Sportbetätigungen in der heutigen Gesellschaft eröffnet. Mit LENK (1985) wird das Erkenntnisinteresse dieses Kapitels anschaulich verdeutlicht, denn der Autor betont: „Hohe Berge zu besteigen, die fernen, feindlichen, allein den Göttern vorbehalten - das galt im Altertum und Mittelalter als nahezu undenkbar, als Narretei, Verrücktheit" (ebd., 104). Fragen nach der historischen Bedeutung von Risiko und Sicherheit drängen sich in diesem Zusammenhang auf: Wie sind Menschen früher überhaupt mit Unsicherheiten umgegangen? Was sind die sozialen und individuellen Voraussetzungen, um Bewegungsaktivitäten unter Risikobedingungen bewusst aufzusuchen? Zudem sind aber auch weiterführende Fragen für das Erkenntnisinteresse dieser Arbeit relevant: Wann sind risikosportliche Aktivitäten das ersten Mal ernsthaft 
praktiziert worden und vor allem, in welchem sozial-politischen Zusammenhang stehen diese Tätigkeiten? Eine Beantwortung dieser Fragen ist letztlich nur im Rückgriff auf historische Forschungsansätze möglich, wenngleich eine systematische wissenschaftliche Auseinandersetzung mit dem Thema Risikosport in den Geschichtswissenschaften nicht existiert.

Das Ziel des vorstehenden Kapitels war es, einen kategorialen Rahmen für die verschiedenen interdisziplinäre Fragestellungen sowie für die empirische Studie durch eine einheitliche Begriffsbestimmung und Eingrenzung des Gegenstands vorzunehmen. Der Blick auf die kulturhistorischen Wurzeln und Verknüpfungen des Risikosports wird nun die Einbettung dieser Aktivitäten in den gesellschaftlichen und kulturellen Entstehungszusammenhang ermöglichen. Aus den Entwicklungslinien risikosportlicher Aktivitäten sollen schließlich wichtige Erkenntnisse über die Bedeutung und Wirksamkeit derartiger Sportbetätigungen in der heutigen Gesellschaft gezogen werden und die Grundlage für die soziologische Ansätze zum Risikosport (s. Kap. 5) vorgestellt werden.

Risiko als soziale und individuelle Sonderform im Umgang mit Unsicherheit wird zunächst vor dem Hintergrund der historischen Wahrnehmung von Unsicherheiten in den sozial-historischen Gesamtrahmen eingefügt (Kapitel 3.1). Dabei wird nach den Ursprüngen risikoorientierter Handlungen geforscht und die kulturellen sowie ideologischen Gegebenheiten untersucht, unter denen sich verwandte Aktivitäten in früheren Epochen etabliert haben. Hierzu werden sowohl kulturhistorische Arbeiten als auch etymologische Werke zur Entfaltung einer Entwicklungslinie herangezogen.

Bei dem Versuch zu den konkreten historischen Wurzeln des Risikosports vorzudringen, offerieren sich aus sporthistorischer Sicht nur bedingt fruchtbare Perspektiven. Da die traditionelle Historie des Sports, die sich aus dem deutschen Turnen, der schwedischen Gymnastik und der englischen Spiel- und Wettkampfbewegung entwickelt, kaum Anknüpfungspunkte für eine Geschichte des Risikosports bietet, wird im Folgenden eine eigene Entwicklungslinie entfaltet. Nach ausführlichen Recherchen führt die Beantwortung der Frage nach den historischen Verbindungen zum sportlichen Risiko unausweichlich zum Alpinismus, der in seinem Entstehungszusammenhang auf wichtige Bezüge des Risikosports verweist (Kap. 3.2.1). Aber auch in frühen Jagdpraktiken wie der Großwildjagd finden sich frühe Verweise auf risikosportliche Handlungen (Exkurs 3.2.2). Darüber hinaus lassen sich auch aus der erlebnispädagogischen Tradition ideologische Rückschlüsse und Hinweise auf das Eingehen von Risikosituationen im Kontext sportlicher Aktivitäten ableiten (Kap. 3.2.3). Bei der Auseinandersetzung mit der Geschichte des Risikosport und den verschiedenen historischen Dokumenten fällt darüber hinaus die Verwandtschaft zu militärischen Handlungen auf. Im Rahmen eines Exkurses wird abschließend betrachtet inwieweit und in welcher Form Risikosport als Kriegsvorbereitung und Kriegsersatz einen Einsatz gefunden hat (Exkurs 3.2.4). 


\subsection{Die Geburt des Risikos - Unsicherheitswahrneh- mung im historischen Rückblick}

Unsicherheit und Ungewissheit besitzen zweifelsfrei in allen historischen Gesellschaftsformen eine konstitutive Bedeutung für die Handlungsorientierung der Menschen. Zweifellos war die Möglichkeit von einer Krankheit befallen zu werden, vom Steinschlag getroffen zu werden oder in der Kälte zu erfrieren, in vormodernen Gesellschaften ungleich größer als heute ${ }^{40}$. Das Konzept des beherrschbaren und zweckrationalen Risikos aber auch das freiwillige Eingehen von risikoreichen Handlungssituationen stellen sich jedoch als relativ späte Varianten im Umgang mit und in der Wahrnehmung von Unsicherheit dar. Sucht man nach den Ursprüngen für Aktivitäten, die unsere heutige Auffassung von Unsicherheit als Risiko betreffen, so findet sich bis dato leider nur eine begrenzte Auswahl kulturhistorischer Ansätze, denn „ausführliche und begriffsgeschichtliche Forschungen fehlen [...]“ (LUHMANN 1991, 18.). Der Forschungsstand zur historischen Begutachtung von Unsicherheit und Risiko beschränkt sich hierbei auf Beiträge von BONB; LUHMANN; EVERS \& NOWOTNY; NERLICH; BERNSTEIN ${ }^{41}$ und DOUGLAS \& WILDAVSKY, die mit ihren ethnologischen Beobachtungen zu einer Kulturanthropologie des Risikos beigetragen haben.

Zunächst erfolgt eine historische Einordnung relevanter Begriffs, indem Unsicherheit als gesellschaftliches und individuelles Wahrnehmungskonzept expliziert und im Rückgriff auf vormoderne Begriffe erläutert wird (3.1.1). Anschließend wird die Vernetzung zwischen Risiko und Sicherheit in einem kurzen historischen Abriss vorgestellt (3.2.2). Zugegeben: der in diesem Abschnitt gespannte erkenntnistheoretische Bogen lässt bisweilen direkte Bezüge zum Risikosport von heute vermissen, aber - wie im weiteren Verlauf der Arbeit zu erkennen sein wird - sind auch diese Zusammenhänge von erkenntnistheoretischem Wert für den Fortgang der Arbeit.

\subsubsection{Begriffsgeschichte oder: Unsicherheit zwischen Fortuna und Aventiure}

Die Auseinandersetzung mit Begriffen ist ein seit jeher beliebtes Feld sozialwissenschaftlicher Forschung. Begriffe werden hierbei in ihre Einzelteile zerlegt und auf sinn- und wortverwandte Beziehungen durchleuchtet. Auch hier erlaubt sich bisweilen die Frage nach dem erkenntnistheoretischen Zweck einer solchen Vorgehensweise. Aber: erst Begriffe ermöglichen eine konkrete Anschauung - ohne Begriffe ist alle Wahrnehmung leer. Und auch Wahrnehmung setzt - vice versa - Begriffe im Sinne von Deutungsmustern voraus. Begriffe und die mit ihnen verbundene Deutung - davon ist zumindest auszugehen - lenken das Denken und Handeln der Menschen in allen geschichtlichen Epochen. Über die inhaltliche Bestimmung von Begriffen und deren kontextbezogener sinnhafter Deutung kann sich also ein Stück Wirklichkeitsordnung über Jahrhunderte hinweg erschließen. Oder anders 
formuliert: Nicht die ontologische Struktur ihrer Objekte bestimmt die Realität, sondern vor allem der Sinn unserer Erfahrungen. Wenn Risiko als Kategorie ohne die Rückbindung an subjektive Erwartungen nicht existent ist, dann sind es letztlich genau diese Erwartungen, die inhaltlich bestimmen, was dem Menschen als Risiko erscheint oder erscheinen kann und was nicht. Risiko als konzeptioneller Begriff ist damit eine subjektive Kategorie über dessen kontextbezogene Deutung ein Stück historische Wahrnehmungsrealität transportiert wird. Bereits bei SOMBART (1916) und WEBER (1923) deutlich, dass risikoorientiertes Verhalten im heutigen Sinne also ein spezifisches Selbstverständnis voraussetzt. Auch LUHMANN stellt diesbezüglich fest:

„Die Außenwelt selbst kennt keine Risiken, denn sie kennt weder Unterscheidungen, noch Erwartungen, noch Einschätzungen, noch Wabrscheinlichkeiten [...]" (LUHMANN 1991, 15).

Dieses Verständnis von Unsicherheit ist allerdings nicht für alle Epochen der Menschheitsgeschichte charakteristisch - für die Vormoderne ${ }^{42}$ ist es sogar weitgehend untypisch, wenn nicht sogar befremdlich (vgl. auch BONß 1995, 51). Von Risiko - als spezifisch rationale Wahrnehmung von Unsicherheit - kann demnach frühestens seit der Renaissance gesprochen werden, seitdem nämlich einerseits die Möglichkeiten gezielter Einflussnahme auf Gefährdungs- und Chancenpotentiale vorhanden sind, andererseits diese Potentiale aber an menschliche Entscheidungen rückgebunden werden ${ }^{43}$. Auch die Begriffsgeschichte des Risikos, die sich in der Benutzung des Wortes und der ihm zugewiesenen Bedeutung wieder spiegelt, verweist auf die Bedeutung der Renaissance und Aufklärung ${ }^{44}$ als Ausgangspunkt eines veränderten Umgangs mit Unsicherheit. So konstatiert BANSE:

„Das Bewusstwerden von Risiken, das Begreifen und geistige Verarbeiten von Risikosituationen und das Entwickeln von Strategien für Risikohandeln sind untrennbar mit den weltanschaulichen und lebensweltlichen Veränderungen während der Reformation und Aufklärung, mit dem Entstehen der bürgerlichen, der ,weltlichen' Gesellschaft verbunden" (BANSE 1996b, 23)45.

Ähnlich argumentiert auch GRYMER (1989):

„Von Risiko können wir eigentlich erst sprechen, als im Zuge der Aufklärung der Mensch lernt, dass nicht mehr Gott seine Geschicke lenkt, nicht mehr Gottgewolltes er nur vollzieht, sondern er selbst seinen Weg auf eigenen Beschluss gehen kann"(GRYMER 1989, 137)46

Wie aber ist dieser ,Paradigmen-Wechsel' zu verstehen? Welche Verbindung besteht zwischen einem Unsicherheitsbegriff und der sozialkulturellen Transformationen einer Gesellschaft? Die Beantwortung dieser Fragen ist an eine basale Differenzierung gekoppelt, die sich über die wahrnehmungsspezifische Deutung von Unsicherheit erschließt: Risiko als Unsicherheitswahrnehmung impliziert die systematische Selbstzuweisung von Unsicherheit. Diese Unsicherheit in der Form eines ,zurechenbaren Risikos' setzt - im 
Gegensatz zur ,schicksalhaften Bedrohung' - voraus, dass die Zukunft nicht als Reproduktion einer kosmologischen Ordnung verstanden werden darf, sondern vom eigenen Handeln und damit subjektzentriert beeinflusst werden kann. Es ändert sich also nicht das Objekt, sonder lediglich seine subjektive Wahrnehmung.

Risiko als kategoriale Betrachtung von Unsicherheit setzt damit an gesellschaftlichen Säkularisierungs- und Indiviualisierungsprozessen an und ist eng mit der Ablösung einer Präformiertheit der individuellen Lebensführung verbunden. Denn nur wenn der Mensch in der Lage ist, sich selbst in der Relation zur Zeit und seinem sozialen Umfeld zu setzen, ist Risiko als spezifische Handlungskategorie überhaupt möglich. Die Durchsetzung eines auf einen individuellen Entscheidungsprozess rückgeführten kognitiven Vorgangs, der für das heutige Risikokonzept von Bedeutung ist, vollzieht sich dementsprechend parallel zu den gravierenden geistigen, ökonomischen, politischen und sozialen Veränderungen der Renaissance:

„Der Mensch lebt seit seiner Entstebung mit Risiken, und zwar sowobl mit
natürlichen als auch mit selbstverschuldeten. In ferner Vergangenbeit jedoch
war er nur sehr beschränkt in der Lage, diese Risiken zu kontrollieren. Da er
die kausalen Zusammenhänge nicht durchschaute, konnte er kommendes Un-
heil weder vorhersehen noch sich vor dessen Auswirkungen schützen. Die Fol-
ge davon war eine fatalistische Einstellung gegenüber Gefahren"
(CHAKRABORTY 1991, 1).

Durch die Deutung von Unsicherheiten als Risiko erscheint die Zukunft also nicht mehr als vorgegeben und unbeeinflussbar, sondern als abhängige Variante eigenen Handelns und eigener Entscheidungen. Vice versa öffnet diese Einbindung von Unsicherheit in einen neuen Bewusstseinshorizont des Menschen auch die Türen für neuartige Denk- und Handlungsweisen: Der moderne Mensch kann sich in seiner Lebensweise nun nicht mehr auf die Dominanz alter Traditionen verlassen, denn er muss, will er die Welt selbst erfahren, dabei ein Risiko eingehen. Bei HEGEL heißt es hierzu:

„Das Individuum welches das Leben nicht gewagt hat, kann wobl als Person anerkannt werden, aber es hat die Wabrheit dieses Anerkanntseins als eines selbstständigen Bewusstseins nicht erreicht" (HEGEL 1986, 146).

Wie aber hat sich diese Entwicklung „von der Gefahr zum Risiko“ (EWALD \& NOWOTNY 1987, 25) vollzogen? Die Frage nach den ursächlichen Zusammenhängen dieses Paradigmenwechsel rücken die historischen Arbeiten NERLICHs, KÖHLERs und natürlich BONßs in den Blickpunkt, die sich in verschiedenen Veröffentlichungen der Frage nach der kulturellen Bedeutung von Risiko und Unsicherheit gewidmet haben. Diese Ausführungen sind insofern von Bedeutung für die Arbeit, da hier die grundlegenden Wahrnehmungsmuster von Unsicherheit in einen kulturhistorischen Bezug gesetzt werden und sich ,Risiko' über seine Begrifflichkeit hinaus als Konzept in ganz unterschiedlichen Dimensionen entfalten lässt. 
Die Entwicklung von einem begrenzten, statischen, religiösen und transzendenten Weltbildes zu einem atomistischen, dynamischen und säkularen Weltbild lässt sich nur in Zusammenhang mit einem Übergang von einer allgemeinen ,Gnadenwirksamkeit Gottes', vom ,Ausgeliefertsein' hin zu einem ,Anspruch der Vernunft' verstehen und einer damit in Verbindung stehenden Veränderung im Verhältnis zur Zeit (vgl. BANSE 1996, 26). Was hier zunächst kompliziert klingt, lässt sich mit BONß am Beispiel des vormodernen ,Fortuna'-Konzeptes verständlich nachvollziehen ${ }^{47}$. ,Fortuna' war die bei den Römern mit Glücksrad, Füllhorn, Steuerrad und Flügeln dargestellte Schicksals- und Glücksgöttin, die als Symbol für die Willkür und Wechselhaftigkeit des Lebens stand. Bis weit in das 18. Jahrhundert hinein repräsentiert diese ,Fortuna' das wohl wichtigste vormoderne Unsicherheitskonzept (vgl. BONß 1995, 111). Unsicherheit in der Gestalt ,Fortunas' stand dabei nicht für den reinen Zufall, sondern für den Willen Gottes, der allein die Geschicke jedes Einzelnen bestimmt. Das Schicksal galt damit als von außen bestimmt und unbeeinflussbar - individuelle Anstrengungen konnten den Lauf des Glücksrades, durch das ,Fortuna' bildlich dargestellt wurde, nicht beeinflussen.

\section{„Überwiegend vertraute man jedoch einer Praxis der Divination, die zwar keine verlässliche Gewissheit schaffen konnte, aber jedenfalls garantierte, dass die eigene Entscheidung nicht den Zorn der Götter oder anderer numinoser Mächte erregte, sondern im Kontakt mit geheimnisvollen Schicksalsbestim- mungen abgesichert war" (LUHMANN 1991, 16).}

Vormodern bleibt das Bild der ,Fortuna' insofern, „[...] als es keinerlei Hinweise darauf gibt, wie und wodurch das Rad in Bewegung gehalten wird“" (BONB 1995, 113) ${ }^{48}$. BONB, der seine Thesen mit historischen Literaturbeispielen untermauert, verweist dabei auf die allgemeine gesellschaftliche Bedeutung der ,Fortuna', die in der sich wiederholenden Unbeständigkeit zugleich eine spezifische Gleichheit der Menschen postuliert (vgl. ebd.).

Erst bei den Vordenkern der Renaissance wird das ,Fortuna'-Konzept als Unsicherheitswahrnehmung neu gedeutet ${ }^{49}$ : Im Gegensatz zur Vormoderne wird der ,Fortuna' und damit der bestehenden Unsicherheit nun Tugend und Tapferkeit entgegengestellt. So vertraten verschiedene Autoren - unter ihnen auch Macbiavelli - die Auffassung einer der ,Fortuna' entgegenzustellenden Kraft um das Schicksal ,[...] in die Schranken zu verweisen und einmalige Gestaltungschancen zu nutzen, um sich so die Welt untertan zu machen" (ebd., 114). ,Fortuna' stellt sich somit nicht länger als göttliche Vorsehung dar, sondern wird durch eigenes Handeln beeinflussbar. Unsicherheit im eigenen Handlungs- und Erfahrungshorizont wird nun partiell nicht mehr nur ausschließlich als Bedrohung, sondern darüber hinaus auch als Herausforderung betrachtet. Diese für die Moderne ${ }^{50}$ bedeutende Umstellung im Bezug zur Unsicherheit manifestiert sich auch in der Auseinandersetzung mit der Natur. Während ,Fortuna' als Indiz für eine noch unbeherrschbare Natur und Geschichte zu verstehen ist, steht die der Unsicherheit entgegenzustellende Tapferkeit und Tugend letztlich auch für die aktive 
Ausweitung instrumenteller Naturbeherrschung (vgl. ebd.). Obwohl diese emanzipatorische Entwicklung, die sich in der breiten Gesellschaftsmasse kaum durchgesetzt haben wird, zu den wichtigen Veränderungen der Renaissance mit beigetragen haben wird, lässt ,Fortuna' keine weiterführende Verbindung zum modernen Verständnis des Risikos zu. Bis heute steht ,Fortuna' vielmehr für das Unbeeinflussbare und Schicksalhafte und spiegelt die heutige Vorstellung dessen wieder, was gemeinhin unter Gefahr verstanden wird.

Weit fruchtbarer erscheint hingegen eine parallele Entwicklung, die sich im Wortfeld des heutigen ,Abenteuers' vollzog. Seit dem 12. Jahrhundert lässt sich mit dem Konzept der ,aventiure ${ }^{51}$ ' ein zweiter zentraler Ansatz zur Wahrnehmung von Unsicherheit ausmachen (vgl. NERLICH 1998, 83). Während ,Fortuna' auf die Unsicherheiten und Wechselfälle des Lebens verweist, die als subjektunabhängig und unbeeinflussbar gelten, fußt das Konzept der ,Aventiure' auf der entgegengesetzte Hoffnung, die „,...] an sich autonome ,Fortuna' durch aktives Handeln eben doch beeinflussen zu können“ (ebd., 117). ,Aventiure' steht diesbezüglich in einem engen Zusammenhang zum ,Fortuna'-Verständnis Machiavellis, im Sinne einer individuell beeinflussbaren Herausforderung und Individualisierung von Unsicherheit. Dabei schließen sich ,Fortuna'- und ,Aventuire' nicht grundsätzlich aus. BONß beschreibt sie als „,...] einander ergänzende Thematisierungsformen von Ungewissheit“ (ebd.), die lange Zeit als Parallelkonzepte gehandhabt wurden.

Im Vergleich zum ,Fortuna'-Konzept steht ,Aventiure' als Unsicherheitstopos in einer zentralen Entwicklungslinie zum heutigen Abenteuerverständnis und damit auch zum Risikosport ${ }^{52}$. Zugegeben: auch der erkenntnistheoretische Weg, der zu dieser Feststellung führt, ist zwar bisweilen etwas ,steinig', die fruchtbaren Anschlussmöglichkeiten entschädigen aber für das abstrakte Vorgehen. Zunächst aber zu den Ursprüngen: Als zentrale Kategorie findet ,Aventiure' ihren Ursprung in der höfische Ritterideologie im 12. Jahrhundert (vgl. NERLICH 1977 / I, 23ff). In Verbindung mit der Auflösung von Schlachten in Einzelkämpfen und damit der Erosion einer ihrer wichtigsten Aufgaben, kündigte sich vor allem im westlichen Europa ein Bewusstseinswechsel auf der Ebene des Kleinadels an. Da in den sich herausbildenden feudalistischen Großräumen des Mittelalters nur noch bedingt Platz und Funktionen für Ritter des Kleinadels waren, sahen sich diese ,Ehrenmänner' gezwungen - im Sinne eines Statuserhaltes - ein neues Sozialprestige aufzubauen (vgl. CARTER 2000). Das Bestehen von gefahrvollen Handlungen, z.B. in der Begegnung mit wilden Tieren oder auch menschlichen Kämpfen, wurde hierdurch zu einer Art ritterlichen Abenteuerideologie stilisiert und im Kreise der höfische Ritterkultur als hohe ethische Leistung zelebriert (vgl. NERLICH 1998, 84). Diese Form historischer Bewegungshandlungen setzt sich in einer langen Tradition von Ritterturnieren und Vergleichwettkämpfen fort, die - allein durch das bestehende Verletzungsrisiko - als frühe Form eines sportlichen Risikohandelns gedeutet werden kann. KURRAS (1992), vor allem aber FENSKE (1986) haben diesbe- 
züglich nachgewiesen, dass die verschiedenen Ritterturniere durchaus auch als eine Form von bewusst aufgesuchter Unsicherheit wahrgenommen wurde 5354 .

Maßgeblichen Anteil am Erfolg dieser frühen Abenteuer-Ideologie besitzen zweifelsfrei zahlreiche Schreibkundige, die von den Rittern mit abenteuerlichen Geschichten versorgt wurden. Die bisweilen ironischen Geschichten des Kristian von Troyes, der mit seinen Romanen über ,König Artus und die Ritter der Tafelrunde' (1160 - 1190) zu einer massiven Verbreitung des Abenteuerdogmas beitrug, fanden über ganz Westeuropa hinweg Verbreitung und Nachahmung (vgl. ebd.). Als Beispiele seien hier auch die Abenteuersatiren im ,Don Quijote' oder auch Dumas', Die drei Muskeltiere' genannt. Es ist diesbezüglich auch nicht erstaunlich, dass sich .diese frühe Form des Risikohandelns im späten Mittelalter und bis in die Moderne hinein z.B. in Erziehungsinstitutionen durchsetzte. So wurde in den verschiedenen Ritterakademien auch die bedeutenden Fertigkeiten der Ritterturniere vermittelt (vgl. MAHLER 1921; VON DEM BUSCHE 1989).

„Die Ritterakademien hatten den Zweck, ibre Zöglinge mit allen den Fertigkeiten auszurüsten, die sie befähigten, ein Staats- oder Hofamt zu bekleiden, so dass zu den wissenschaftlichen Lehrfächern als wesentlicher Bestandteil noch Unterricht in gewissen Leibesübungen, den so genannten adeligen Exercitien, binzukam" (MAHLER 1921, 3)5.

Für NERLICH, der für seinen Untersuchungen zeitgenössische Literatur sowie verfügbare Originalquellen heranzog, vollzieht sich mit dieser ritterlichen Suche nach Abenteuern - der, quête de l'aventure' - ein neuartiges Kriterium des Menschseins. Denn die höfische Abenteuersuche verweist den Einzelritter im Sinne einer persönlichen Herausforderung auf sich selbst zurück, die - ohne es zu intendieren - den Strukturen des Mittelalters diametral entgegensteht.

„[...] Diese Definition des Menschseins dürfte mehr als jede andere dazu beigetragen haben, die Welt irreversibel von der Vormoderne in die Moderne zu verwandeln. Zum ersten Mal wird eine bejabte Experimentalbaltung zur Welt als böchstes Menschbeitsideal ausgezeichnet: der klassenindifferente Auszug ins Unbekannte, das Neue, das Risiko [....]" (NERLICH 1999, 1.).

Gegenüber Unsicherheiten der ,Fortuna' wird ,Aventiure' folglich nicht aufgezwungen oder befohlen, sondern gänzlich freiwillig eingegangen. KÖHLER verweist darüber hinaus auch auf den Situationsbezug im ,Aventiure'-Konzept. Er kommt in seiner Analyse historischer Literatur zu dem Schluss, dass ,Aventuire' auch als Ausbruch auf Zeit zu deuten sei: Die ,Aventuire' überfällt einen nicht, sondern sie wird bewusst gesucht. Sie verweist auf ein ,Sicherheitsproben ohne Auftrag, ohne Amt, ohne konkreten geschichtlich-politischen Hintergrund" (KÖHLER 1970, 71).

Im ,Aventuire' wird also ein maßgeblicher Wechsel in der Wahrnehmung von Unsicherheit vollzogen, der auch für die Entwicklung einer historischen 
Perspektive des Risikosports von Bedeutung ist. Denn die Idee eines aktiven und freiwilligen Eingehens von Abenteuern, wie es das ,Aventiure'-Konzept nahe legt, steht in einem direkten Bezug zum heutigen Verständnis von Abenteuer, Wagnis und Risiko in nicht-ökonomischen Zusammenhängen. Wenngleich Handlungsstruktur, Organisation und Motivation des heutigen Risikosports andere Erklärungszusammenhänge nahe legen, zeigen sich doch erstaunliche Parallelen zum gegenwärtigen Verständnis von Abenteuersuche und Risikobereitschaft im Kontext des Sports auf. Es ist diesbezüglich also wenig verwunderlich, dass die von NERLICH forcierte systematischen Explikation der Abenteurer-Ideologie des ausgehenden 12. Jahrhunderts, viele Ähnlichkeiten zu modernen Beschreibungen risikosportlicher Protagonisten aufweist (vgl. Tabelle 3.1).

Der Mensch muß, um wirklich Mensch zu sein:

1. seinen gegenwärtigen materiellen und/oder geistigen Status als unzureichend und vorläufig empfinden;

2. den Wunsch und Willen haben, diesen Status zu überwinden;

3. die dazu notwendigen Veränderungen, Arbeit, Gefahr auf sich nehmen;

4. aus diesen Gründen vom bisherigen vertrauten Ort aufbrechen

5. in einem unbekannten Raum eindringen (den Horizont zur Ferne bzw. zum Unbekannten überschreiten)

6. dort nach etwas suchen, was er nicht genau kennt und

7. was selten, gefährlich, auf jeden Fall aber mehr oder weniger neu sein und materiell und/oder geistig profitabel sein muss;

8. den Zufall (etwas zu finden oder nichts zu finden) akzeptieren

9. Risiken (wie Verwundung; Gefangenschaft, Tod, Verlust, Ergebnislosigkeit der Suche) in Kauf nehmen

10. Findsysteme entwickeln bzw. Kooperationen mit anderen betreiben

11. Risiken (über Planung und Berechnung bzw. Kampfstrategie und Waffentechnik) minimieren oder eliminieren;

12. das bis dahin partiell oder ganz Unbekannte-Neue a priori akzeptieren, suchen, erkennen und geistig verändern;

13. damit - durch das per Zufall und System gefundene Neue - seinen Status materiell und geistig verändern;

14. die durch Auszug und Rückkehr verursachten materiellen und geistigen Veränderungen der sozialen Beziehungen sowie

15. die Vorläufigkeit und Unabschließbarkeit des Vorgangs sowie die Notwendigkeit seiner Wiederholung akzeptieren

Tabelle 3.1: Abenteuersuche als Definition des wahren Menschseins im ausgehenden 12. Jahrhundert (zit. nach NERLICH 1998, 85).

Es ist deutlich hervorzuheben, dass z.B. in den Werken Kristians die zunächst als Privilegien des ritterlichen Standes beanspruchte, quête de l'aventure' für Menschen aller Stände und sozialer Gruppierungen an Bedeutung gewann. Turniere und Bewegungshandlungen wurden bis in die Renaissance als Kriegs-Vorbereitung genutzt, wenngleich in quantitativ gerin- 
gerer Form (vgl. MEYER 1986). Allerdings weist NERLICH in diesem Zusammenhang darauf hin, dass die Ritter weder direkt noch alleine die Ideologie des ,Aventiure' hervorgebracht haben, , [...] ja sich genau gesehen über von ihnen bezahlte oder ihnen nahe stehende Ideologieproduzenten parasitär Denkstrukturen anderer sozialer Gruppierungen bedient haben [...]“" (ebd. 1990, 25). Auch wenn die Durchsetzung dieses Bewusstseinswandels nicht alle Gesellschaftsbereiche postwendend erfasste, so ist doch NERLICH folgend - für das, Aventiure'-Konzept von einem für die Entfaltung der Moderne weitreichenden Paradigmenwechsel zu sprechen:

\section{„Seit dieser Zeit ist dem (west)europäischen Menschen alles Verharren im Repetitiv-Bekannten unerträglich, wird das Bedürfnis nach Neuem zu einer gesellschaftlichen Selbstverständlichkeit bzw. zur unverzichtbaren Seinsquali- tät" (ebd. 1998, 86).}

Die Durchsetzung des ,Aventiure'-Kozeptes als spezifische Wahrnehmung von Unsicherheit im Verständnis des heutigen rationalen Risikos vollzieht sich aber aus einer anderen Entwicklungslinie. So findet sich eine zweite Variante von ,Aventiure' als wesentlich stärker juristisch und ökonomisch geprägter Abenteuerbegriff am Ende des 13. Jahrhunderts, der vor allem den Stadtbürgern zu zuordnen ist und im Rahmen ökonomischer Unternehmungen beschrieben wird (vgl. BONß 1995, 120; auch NERLICH 1977 / I, 24f). Die Städter entwickelten hierbei ein eigenes Konzept von Abenteuer und Abenteurern, welches zwar in Anlehnung an die Begrifflichkeit der ritterlichen Abenteuerideologie zu verstehen ist, inhaltlich aber durchaus Unterschiede aufweist (vgl. ebd.). So wird ,Aventiure' im Rahmen der aufkommenden Handelskultur von den Handelsbürgern für die Unsicherheit ihrer Geschäftpraktiken benutzt, wobei sie mit dem Begriff sowohl die Ware selbst, die Unsicherheit der Handelspraktiken als auch den daraus resultierenden Profit benennen (vgl. NERLICH 1998, 26). Verfolgt man die weitere Historie dieses ,Aventuire'-Konzeptes und damit die endgültige Durchsetzung einer modernen Risiko-Logik, so ist ihre Anwendung und Entwicklung in der Ökonomie besonders hervorzuheben (vgl. BONB 1995, 123f). Der Seehandel, der mit mannigfaltigen Unsicherheiten verbunden war, wurde diesbezüglich zum Inbegriff des ,Aventiure' unter bürgerlichen Standesmitgliedern. Zahlreiche Unternehmungen und Handelsreisen in unbekannte Länder sowie neuartige Handelsbeziehungen lassen sich in der Entwicklung nachvollziehen. Unter Unsicherheitsgesichtspunkte gingen insbesondere die westeuropäischen Händler und Kaufleute neue Wege, so dass SOMBART in der seit dem 15. Jahrhundert zunehmenden Zahl an ökonomisch motivierten Abenteurern in der Gestalt von Kaufleuten und Freibeutern einen entscheidenden Schritt auf dem Weg zum Kapitalismus sieht (vgl. ebd., 125f).

Auch wenn die ritterliche, Aventiure' über die massenwirksame Literatur in verschiedene Teilbereiche späterer Gesellschaftsformen eingedrungen is ${ }^{56}$, darf die diametrale Verschiedenheit von früher Ritterideologie ${ }^{57}$ und ökonimischen Handels-, Aventiure' nicht vergessen werden, zumal die gesellschaftliche Rivalität zwischen dem höfischen Kleinadel und den bürgerli- 
chen Händlern dazu führte, dass die Händler in späteren Zeiten zunehmend auf die Abenteuerterminologie verzichteten (vgl. NERLICH 1998, 89). Mit Ende des 15. Jahrhunderts kommt es in der Handelssprache sogar zu einer sukzessiven Verdrängung des Abenteuerbegriffs zu Gunsten des Risikobegriffs. Dieser Übergang ist verbunden mit einer Verlagerung der Unsicherheiten aus dem individuellen Geschäftsbereich in die gesamtgesellschaftliche Verantwortung, die in der Herausbildung von Handelswegen durch die Hansen und Gilden sowie staatspolitische Maßnahmen wie das Versicherungswesen bestand (vgl. ebd. 1990, 27). In der zentralen Begrifflichkeit des Risikos und seiner Bedeutung im ökonomischen Denken jener Zeit wird auch die historische Bedeutung des ,Aventiure'-Konzeptes als Basis einer neuen Risikoideologie deutlich: Das Risiko als Erfassung und Rationalisierung von lebensweltlich bedeutsamen Zukunftsentscheidungen. Die moderne Akzentsetzung des Risikos findet sich hier in der Ambivalenz aus Chance und Bedrohung, in einer Dialektik aus Furcht vor Niederlage und Hoffnung auf Gewinn, den man nur erreichen kann, wenn man sich den Unsicherheiten der Fremde und des Neuen aussetzt (vgl. ebd.) ${ }^{58}$. Die von BERNSTEIN im Titel seiner Arbeit benutzte Phrase „Wider die Götter ${ }^{59 “}$ (BERNSTEIN 1997) kennzeichnet damit die wesentliche Unsicherheits-Qualität der Moderne, die in der individuellen Zurechenbarkeit von Unsicherheit auf das eigene Handeln besteht und mit ihrem Zukunftsbezug die Rationalität und Reflexionsfähigkeit des Menschen voraussetzt. Zu diesem Schluss kommt auch DOUGLAS, die Risiko als ,,[... key idea for modern times“ deklariert (DOUGLAS 1990, 3).

\section{„Zum ersten Mal in der Menschbeitsgeschichte wird systematisch die Preisgabe des Vetrauten, das Kennenlernen des Fremden, die Überschreitung des (kul- turellen) Horizonts, das Vordringen ins Unbekannte, die Akzeptierung des Zufällig-Risikohaften, die Konstituierung des sozialen Individuums in diesen Koordinaten zur wesentlichen Tätigkeit des Menschen erboben" (NERLICH 1990, 26).}

Auch wenn Vorformen von rationalen Risikohandlungen bereits in der Vormoderne existierten, ist abschließen darauf hinzuweisen, dass das bürgerliche ,Aventiure'-Konzept als Wahrnehmung und Behandlung von Unsicherheit weit über die Renaissance hinaus nur für gesellschaftliche Teilsysteme eine Rolle spielte und sich nur sukzessiv in die Gesellschaftsbreite durchsetzte. Parallel hierzu existierten lange Zeit weiterhin parallele irrationale Konzepte, wie sie im Fortuna-Konzept deutlich geworden sind.

Der kurze Blick zurück auf die Ursprünge des Risikos als spezifische Form des Unsicherheitshandling verdeutlicht, dass Unsicherheit auch in der Vormoderne existentielle Bedeutung für das Handeln der Menschen besaß wenn auch unter anderen Vorzeichen. Dies an sich führt noch zu keinem Erkenntnisgewinn. Aber: Risiko als spezifische Form der Unsicherheitswahrnehmung wird damit zu einer sozialen Konstruktion, die in ihrer geschichtlichen Tradition bis heute nachzuvollziehen ist. Zwar ist der etymologische Pfad - und damit auch seine inhaltliche Konzeption - vom Aventi- 
ure-Konzepte zur heutigen Risikothematik und darüber hinaus zum Sport als zweckfreie Tätigkeit kein stringent nachzuvollziehender Entwicklungsstrang. Trotzdem lassen sich Entwicklungslinien zum heutigen Risikosport rekonstruieren: Versucht man die von NERLICH angeführten Varianten abenteuerlicher Frühformen im Bezug auf risikosportliche Handlungen zu interpretieren, so finden sich in beiden Varianten Ursprünge risikoorientierter Tätigkeiten. Sowohl die Abenteuer der höfischen Ritterideologie als auch die Reiselust der Kaufleute am Ausgang des 15. Jahrhunderts zeigt erstaunliche Parallelen zu heutigen Aktivitäten, die in die Nähe aktueller Risikoaktivitäten zu bringen sind ${ }^{60}$. Trotzdem ist eine eindeutige Differenzierung im Bezug auf das heutige Verständnis von Risiko anzubringen: So besteht bei der frühen Form des ,Aventiure' - der höfischen Ritterideologie - ein direkter Handlungsbezug zur Erschließung neuer sozialer Wirklichkeiten. Letztlich ging es bei der ,Aventiure’ der Rittersöhne um ,[...] eine gute Partie, die reiche Heirat" (NERLICH 1977 / I, 28) und damit um Statuserhalt (vgl. auch CARTER 2000). In dieser Variante erscheint die Suche nach Abenteuer als distinktives Klassenmerkmal - als spezifische Form der Abgrenzung einer Statusgruppe (vgl. ebb., 27).

Für BONB ist diese Form des Abenteuerverhaltens eine ethische Leistung, die zwar kaum mit einem wahren Paradigmenwechsel in der Unsicherheitswahrnehmung einhergeht und in den Bezügen zur heutigen Risikowahrnehmung nur wenige Parallelen aufweist, für die Entwicklung des Risikosports aber doch maßgeblich gewesen sein dürfte. Die Auffassung BONßs, wonach insbesondere die rationale Risikoideologie der Handelsleute zu einem Paradigmenwechsel in der Unsicherheitswahrnehmung geführt hat, ist im Bezug auf die öknomisch-ökologischen Risiken der Neuzeit sicherlich zu zustimmen, sie verkennt aber eine wichtige Entwicklungslinie, die Risiko ohne einen direkten Zweckzusammenhang als Handlungselement begreift. Auch wenn für die gesellschaftliche verbreitete Durchsetzung einer modernen Risikoideologie, der ,Aventiurhandeln' von entscheidender Bedeutung auch im Sinne eines sich durchsetzenden Wirtschaftssystem zu sein scheint, weist die höfische Ritterideologie - will man den empirischen Arbeiten von BONB, NERLICH, WIEDEMANN und LUHMANN folgen doch erstaunliche Parallelen zum Risikosport von heute auf. Sie verweist dabei auf zwei unterschiedliche Entwicklungsstränge, die sich bis heute rekonstruieren lassen: Unsicherheit im Verständnis des ritterlichen Abenteuers findet sich hierbei als Selbstzweck, quasi als Medium zur Selbstverwirklichung. Das Risiko wird hier um des Risikos willen eingegangen und steht damit dem zweckrationalen Risikobegehren des wirtschaftlichen Systems entgegen. Dieses Risiko als Herausforderung findet sich in ihrer heutigen Auffassung als Gefahrenrisiko oder Wagnis im Risikosport wieder. Auch im Risikosport wird - wie im weiteren Verlauf der Arbeit expliziert wird - das Risiko um seiner Selbst willen aufgesucht, ohne dass zweckrationale Begründungen, die ein sichtbares Ergebnis lieferten, daraus resultieren müssen. 


\subsubsection{Risiko und Sicherheit}

Wie im Verlauf der Arbeit zu zeigen sein wird, stehen Risiko und Sicherheit im Risikosport in einem engen konzeptionellen Zusammenhang. Verfolgt man historisch den Weg des Risikos als spezifisches Wahrnehmungskonzept von Unsicherheit, so wird eine entscheidende Paralellentwicklung deutlich, die bis heute weite Teile der Gesellschaft bestimmt: die Minimierung dieser nun kalkulierbaren Unsicherheit und der Aufbau von Sicherungssystemen. Obwohl bis ins 18.Jahrhundert eine verstärkte Hinwendung zu Risiken in Form von Glücksspielen und Wetten zu beobachten ist, kann spätestens mit Ausgang des 18. Jahrhunderts von einer regelrechten Sicherheits-Obsessions in westlichen Gesellschaften gesprochen werden (vgl. BONß 1995, 147f). Mit der Erkenntnis, dass sich die Unsicherheit im eigenen Handeln durch rationales und reflektives Wissen beeinflussen lässt, erwächst auch das Bedürfnis diese Unsicherheit - weil generell beeinflussbar - so weit es geht zu beseitigen. Bereits 1625 formuliert BACON hierzu:

„Wer nur dem sichtbaren Gewinn nachgeht, wird schwerlich zu großem Reichtum gelangen, und wer alles auf Wagnisse setzt, scheitert oft und verarmt. Deswegen ist es gut in Wagnisse Sicherbeiten einzuschalten, um Verluste vorzubengen" (BACON 1625, 122; zit. nach BON $\beta$ ebd.).

Prinzipiell lassen sich dabei zwei unterschiedliche Prinzipien differenzieren, die bis heute zur Risikominimierung eingesetzt werden. Zum einen wurden kognitive Kompetenzen entwickelt, die darin bestehen durch Informationsbeschaffung und rationale Abwägung bestehende Unsicherheitsfaktoren $\mathrm{zu}$ isolieren und schließlich im Handeln zu eliminieren. BONß diskutiert diese Thematik in Anlehnung an WEBER unter dem Stichwort rationale Kalkulation. Auf der anderen Seite hat sich die kollektive Verteilung des Risikos - zunächst in kleinen Soldiargemeinschaften - etabliert. Hinter dieser Versicherung als Prinzip und Institution verbirgt sich der Versuch auf spezifische Weise die Verlustoption, die sich aus dem Risikohandeln ergibt, für den Einzelnen zu minimieren (vgl. BONB 1995, 148).

Rationale Kalkulation beruht in seinem Entwicklungsgang auf einem Verwissenschaftlichungsprozess, der aus der grundlegenden Idee resultiert, durch Wissen und Information Unsicherheiten zu reduzieren. Diese bis heute nachwirkende Geisteshaltung findet sich in den Ideen des Scientismus wieder, die darauf basiert, dass Wissenschaft als rationelle Anstrengung des Geistes kraft ihrer methodischen und kognitiven Möglichkeiten alle Probleme lösen und damit theoretische Gewissheit liefern sowie praktische Sicherheit bieten könne ${ }^{61}$. Der Glaube an die Wissenschaft und ihre vermeintlichen Gesetzmäßigkeiten beherrscht damit auch das Bestreben der Risikominimierung. Für WEBER zeigt sich in dieser aufkommenden okzidentalen Rationalisierung sogar die Idee einer allgemeinen Weltbeherrschung, nämlich der Glaube, ,[...] dass man, wenn man nur wollte, es jederzeit erfahren könnte, dass es prinzipiell keine geheimnisvollen, unsichtbaren Mächte gebe [...] dass man vielmehr alle Dinge - im Prinzip - durch Berechnung beherrschen könne“" (WEBER 1919, 317). 
Dass sich die mit der Verwissenschaftlichung verbundenen Ansprüche im Hinblick auf die Minimierung - wenn nicht sogar Eliminierung - der Unsicherheit, nicht vollständig einzulösen ließen, zeigten durch alle Epochen hindurch zahlreiche Kontingenzerfahren ${ }^{62}$ sowohl im Handelswesen als auch im individuellen Erfahrungshorizont der Menschen. Auch der planvolle Abwägungsprozess, der auf wissenschaftlich gesicherte Informationen zurückgreift, ist nicht in der Lage die Restunsicherheit einer ,riskanten' Unternehmung zu beseitigen. Um sich gegen derartige Restrisiken zu schützen, reichte und reicht auch heute die rationale Kalkulation allein nicht aus. Erforderlich waren Absicherungskonzept, die nicht auf die Verhinderung möglicher Schäden abzielten sondern auf deren Kompensation.

Die Versicherung als Prinzip und Institution ist ein auf den frühen Kontingenzerfahrungen der Händler und Manufakturisten aufbauendes Sicherungsprinzip. Zwar existiert über den Versicherungsbegriff in den wissenschaftlichen Beiträgen derzeit kein definitives Einvernehmen ${ }^{63}$, die inhaltliche Diskussion lässt sich aber unter der Minimalformel ,Übernahme von Verlustrisiken gegen vorherige Prämienzahlung' auf einen brauchbaren Konsens bringen (ebd., 156). Das traditionelle Leistungsbündel der Versicherungsträger stellt damit eine statische Komponente der Stabilisierung dar. Bestände aber auch Prozesse werden dahingehend geschützt, um trotz möglicher Schadensfälle eine bestimmte Lage zu garantieren.

Auf die Arbeits- und damit Funktionsprinzipien des Versicherungswesens kann hier nicht eingegangen werden. Interessanter erscheint in diesem Zusammenhang die historische Entwicklung des Versicherungswesens, die Aufschlüsse über das Zusammenspiel von Unsicherheitswahrnehmung und Sicherheitserwartungen geben kann.

Für BONß stehen Risiko und Versicherung in einem engen Entwicklungsverhältnis, ,[...] deren Schicksal konstitutiv miteinander verknüpft ist. Der Aufstieg der Versicherung begann in dem Maße, wie, uncertainties' Unsicherheiten, Ungewissheiten (Anm.d. Verf.)] als Risiken konstituiert wurden und damit die Voraussetzungen für ein neues Unsicherheitsbewußtsein und eigenständige Sicherheitsdiskurse gegeben waren“ (ebd., 178). Anhand der Entwicklung des Versicherungswesen zeigt sich für BONß auch die zeitliche Entwicklung des Unsicherheitsdiskurses, die er in 4 Phasen differenziert:

In der Vorlauf- und Experimentierphase zwischen 1300 und 1700 finden sich Elemente der Versicherung z.B. bei einfachen Solidargemeinschaften und dem institutionellen Zusammenschluss zu Gilden oder in Einzelverträgen. Ein eigenständiges System der Sicherheitsvermarktung existiert noch nicht. Ganz im Gegenteil: die ersten formalen Versicherungen waren eher in die Nähe des Glücksspiels und der Wetten zu rücken als in die Absicherung riskanter Unternehmungen.

In der Gründungsphase von ungefähr 1700 bis 1850 entsteht die Versicherung als ein auf zahlreichen Unsicherheiten anwendbares System der Sicherheitsgenerierung. In Kombination einer weiten Verbreitung der Wahrscheinlichkeitsrechnung und institutioneller Standards erhält die Versicherung vor allem im Handelssektor eine starke Verbreitung. Neben der Seeversicherung 
entstand schon früh die allgemeine Transportversicherung. Aber auch im Bereich der Haus- und Hofversicherungen wurden erste Fälle bekannt, wie die Brand- und Feuerversicherung. Die massive Verwendung von Versicherung deuten auf das Abklingen irrationaler Unsicherheitswahrnehmung hin, wie sie sich im ,Fortuna'-Konzept finden.

In der weiterführenden Umsetzungs- und Ausbauphase ab 1850 setzt sich erstmals die Versicherung im Rahmen großer Gesellschaften und als politisches System gleichermaßen durch. Sie wird über das ökonomische System hinaus zum standardisierten Umgang mit Unsicherheit und konzentriert über die die individuellen Risiken hinaus auch auf die Handhabung sozialer Risiken.

Im Gegensatz zu anderen Autoren weist BORSCHEID (1997) in einer empirischen Arbeit darauf hin, dass sich in der zweiten Hälfte des zwanzigsten Jahrhunderts schließlich eine vierte Etappe in der Entwicklung der Versicherung etabliert hat, die BONß als Be- und Entgrenzungsphase benennt (vgl. ebd., 180). Zwar wächst auch in dieser Phase die Bedeutung der Versicherungen als Basisinstitution, jedoch existieren darüber hinaus immer mehr Versicherungstatbestände, bei denen die potentiellen Schäden nicht von der Gemeinschaft der Versicherer übernommen werden sondern vielmehr vom Steuerzahler (vgl. BONB 1995, 180f). Diese Phase ist auch geprägt von Hoch- und Großtechnologien, die sich durch eine neue Globalität, Komplexität und Irreversibilität kennzeichnen. Die Handhabung derartiger Technologien im Bezug auf mögliche Risiken ist dabei fast unmöglich, da eine Begrenzung der Folgen in Raum und Zeit nahezu unmöglich ist und ,exaktes' Wissen über Eintrittswahrscheinlichkeiten und Schadensausmaße kaum ermittelbar ist (vgl. auch BANSE 1996, 33).

EWALD sieht in der historischen Entwicklung der Institution Versicherung das entscheidende Mittel, mit dessen Hilfe es den modernen Gesellschaften als System gelungen ist, Gefahren im sozialen und wirtschaftlichen Bereich kalkulierbar zu machen und in Risiken zu verwandeln. Anhand der französischen Entwicklung im 19. Jahrhundert entfaltet der Autor dabei die Entstehung des ,Vorsorgestaates' und der,Versicherungsgesellschaft', die in der allgemeinen Verantwortungsübernahme durch Dritte - in der Regel Versicherungen - bestehen. Die Versicherung als Prinzip bildet dabei eine entscheidende Chiffre für die Vergesellschaftung der Moderne (EWALD 1989). Wie noch zu zeigen sein wird, steht allein dieser Prozess in einem engen Entwicklungszusammenhang des Risikosports.

Aber der Blick auf die historische Entwicklung der Versicherung als Prinzip und Institution offenbart über diese Zusammenhänge hinaus, dass nicht nur das Risiko im Sinne der Unsicherheitswahrnehmung als ein evolutionär später Sonderfall zu betrachten ist. Die Entwicklungslinie der Versicherung verweist auf die nachhaltig wirkende Idee einer kausalanalytisch orientierten Gefahrenbeseitigung als soziale Strategie im Umgang mit Unsicherheit hin und damit auf Sicherheit als eine gesellschaftliche Wertidee (vgl. KAUFMANN 1973, 10). Es ist diesbezüglich „,[...] auch kein Zufall, dass seit dem 17. Jahrhundert Sicherheitsthematiken und Risikothematiken aneinan- 
der reifen" (LUHMANN 1991, 29). Angesichts der geschichtlichen Darstellung ist diese Aussage zwar dahingehend zu relativieren, als dass sich der Sicherheits- im Vergleich zum Risikodiskurs mit einer gewissen Zeitverschiebung entwickelt. Dies ändert aber nichts an der systematischen Verwandtschaft beider Diskurse.

Risiko und Sicherheit müssen also - aus ihrer historischen Entwicklung heraus - zusammenfassend als zwei Seiten einer Medaille verstanden werden müssen, die sich in ihrer Evolution bedingen und aufeinander verweisen ${ }^{64}$. Beide sind dabei in ihrer Wahrnehmung und ihrer Handlungsbedeutung im Sinne einer sozialen Konstruktion zu verstehen. Dabei vertritt LÜBBE die These, dass - auch durch das Prinzip der Versicherung im Zivilisationsprozess - die Risikoakzeptanz dramatisch ab- und das Sicherheitsverlangen in dem selben Maße zugenommen hat. Der Autor konstatiert: „Nie zuvor in der Kulturgeschichte sahen wir uns alltäglich so viel Sicherheitsaufwand umgeben“ (LÜBBE 1993, 38). Für das Erkenntnisinteresse dieser Arbeit ist diese historische Perspektive - wie gezeigt werden wird - ein wichtiger Ansatzpunkt für eine soziologisch-psychologische Einordnung des Risikosports.

Was aber bleibt nun nach diesem etwas abstrakten Ausflug in die Geschichte von Risiko und Sicherheit als Erkenntnisgewinn festzuhalten? $\mathrm{Zu}$ nächst ist zu konstatieren, dass sowohl Risiko als auch die auf Gefahrenbeseitigung und Risikominimierung aufbauende Sicherheit ein soziales Konstrukt der Moderne darstellen. Wenngleich die Ausführungen a priori keinen direkten Zusammenhang zu den risikoreichen Sportaktivitäten von heute aufweisen, sind sie für die folgenden Darstellungen doch gleichsam als theoretische Grundvoraussetzungen von entscheidender Bedeutung. Das Erfassen von Sicherheit und Risiko als aufeinander bezogene Handlungselemente, stellt für das Verständnis des heutigen Risikosports wesentliche Voraussetzungen dar. Dabei verweist ,Risiko' als spezifisches Unsicherheitshandling nicht nur auf Sicherheit, sondern - und dies ist fast ebenso wichtig - auch auf Gefahr. Im Risikokonzept wird Unsicherheit zu einem selbstreflexiven Element, welches eine subjektive Zukunftsoption offen hält und damit mögliche Unsicherheitsfolgen auf die eigene Person bezieht. Gefahr hingegen bleibt außerhalb der Person oder im Sinne des Fortuna-Konzeptes weiterhin eine ,schicksalhafte Bedrohung'.

Die folgenden Abschnitte werden - diese Perspektive aufnehmend - die Fokussierung nun auf die direkten Entstehungs- und Entwicklungszusammenhänge des Risikosports lenken. Mit dem Blick auf den Sport als Handlungsfeld verbindet sich aber auch ein allgemeiner Perspektivwechsel: Nicht mehr Begriffe und ihre phänomenologisch-historische Deutungsmuster stehen im Zentrum der folgenden Ausführungen sondern die sozialkulturelle bewegungsorientierte Operationalisierung von Unsicherheit in der neueren Geschichte. 


\subsection{Das Risiko im Sport - Kulturhistorische Entwick- lung und Zusammenhänge}

Die Frage nach den Wurzeln des Risikosports ist nicht nur auf Grund seiner historischen Verortung von entscheidender Bedeutung für die Diskursanalyse dieser Arbeit. Erweitert um den Aspekt soziokultureller Rahmenbedingungen wird dieses Kapitel zu einer kulturhistorischen Analyse des Risikos im Sport. Denn sportgeschichtliche Analyse sind letztlich ,[...] nur dann von historischem Wert, wenn sie Aufschluss über grundlegende Aspekte des $\mathrm{Zu}$ sammenlebens liefern" (KRÜGER 2004, 22) und damit auf die Entstehungs- und Wirkungszusammenhänge des untersuchten Gegenstandes verweisen. Im Mittelpunkt der historischen Untersuchung steht die Frage inwieweit und in welcher Form gesellschaftliche und kulturelle Entwicklungen zur Entfaltung eines Risikos im Sport und darauf aufbauend des heutigen Risikosports beigetragen haben und welche Bedeutung risikosportliche Aktivitäten für die Protagonisten im historischen Kontext besitzen. Gefragt ist dabei nicht die chronologische Entstehungs- und Entwicklungsgeschichte von heutigen Risikosportarten, wie sie sich in verschiedenen sportartspezifischen Beiträgen findet ${ }^{65}$, sondern die Untersuchung der gesellschaftlichen und soziokulturellen Hintergründe von risikoorientierten Aktivitäten im Umfeld des Sports. Im Blickpunkt des folgenden Abschnitts steht daher das freiwillige Aufsuchen und bewegungsorientierte Bewältigen von sportlichen Risikosituationen, die über alltägliche Risikohandlungen wie das Autofahren hinausweisen. Gegenstand der historischen Analysen sind damit weitestgehend zweckentlastete, spannungsinduzierte und zeitlich begrenzte Handlungskontexte, die im Rahmen körperlicher Bewegung als sportliche Risikosituationen bezeichnet werden können ${ }^{66}$.

Die allgemeine Historie des modernen Sports lässt sich im wesentlichen auf drei unterschiedliche Entwicklungsstränge zurückverfolgen (vgl. KRÜGER 1996, 94). Während die schwedische Gymnastik mit dem Wunsch nach Gleichheit zwischen Belastung und Entlastung argumentierte und damit für die Entwicklung des heutigen Gesundheitssports verantwortlich ist, steht das deutsche Turnen in einer direkten Entwicklungslinie zum heutigen pädagogischen Verständnis des Sports und zu traditionellen Formen des Wehrsports. Für den modernen Wettkampfsport, der sich aus dem ,englischen Modell des Sports' ableitet, findet sich dessen Ursprung in der Adelsgesellschaft des 18. Jahrhunderts (vgl. ebd.). Für KRÜGER hat sich die englische Spiel- und Wettkampfbewegung dabei als dominante Entwicklungslinie durchgesetzt, wobei der Autor zeigt, dass sich die bei GUTTMANN deduzierten Prinzipien des modernen Sports auch im Gesundheitssport identifizieren lassen ${ }^{67}$. Im Bezug auf risikoreiche Aktivitäten, die eine Verwandtschaft zum heutigen Risikosport aufweisen, finden sich in den klassischen sporthistorischen Zugänge nur wenig Anschlussofferten. Ein Überblick über den Forschungsstand offenbart sogar ein deutliches Desiderat sporthistorischer Forschung. So sind nicht nur Darstellungen über 
die historischen Dimensionen des Risikosports Mangelware, es fehlen auch allgemeine historische Ansätze zum Risiko im Sport.

Während es im deutschen Turnen nach Jahn und Spie $\beta^{68}$ vor allem um die Körperdisziplinierung und „Vergeistigung des Leibes“ (KRÜGER 1993, 173) ging, steht der Vergleichswettkampf und das Spiel im Mittelpunkt der englischen Spiel- und Wettkampfbewegung. Im populären Modell der englischen Sportbewegung mit seinen Verweisen auf das Leistungsmotiv und die Rekordjagd lassen sich zwar Hinweise allgemeiner Natur auch für den Risikosport ableiten, eine Konkretisierung im Bezug auf Risiko als Handlungselement erfolgt aber nicht. ELIAS \& DUNNING haben in ihren Beiträgen zum Sport im Zivilisationsprozess zudem dargestellt, dass der Sport allgemein, aber auch der Wettkampfsport im speziellen einen massiven Prozess der ,Zivilisierung' und ,Disziplinierung' durchlaufen hat. Die Formalisierung von Gewalt und Eliminierung von Gefahren im sportlichen Handlungsverlauf verweist in diesem Prozess auf die dominante Entwicklungslinie aller sportlichen Bewegungsprogrammen und Formen von Leibesübungen, die sich gleichsam als Paradigma des modernen Sportverständnisses in der sporthistorischen Forschung darstellt (vgl. auch GRUPE \& KRÜGER 1998, 479). Offerten für eine Historie des Risikosports finden sich auch hier nur auf Umwegen.

$\mathrm{Zu}$ dieser Forschungslücke hat sicherlich auch die Einbettung des Risikosports in den Kontext des Trendsports beigetragen, der in der Sportwissenschaft gerne als ein postmodernes Phänomen ${ }^{69}$ verstanden wird und sich aus den gesellschaftlichen Entwicklungen der letzten zwanzig Jahre heraus erklärt. Zwar weisen LAMPRECHT \& STAMM (2003) erstmals auf die traditionellen Bezüge des Trendsports hin, allgemein wird das Phänomen Trendsport und damit bisweilen auch der Risikosport in der wissenschaftlichen Theoriebildung aber als ein postmodernes Phänomen dargestellt, dessen Wurzeln auf das letzte Drittel des 20. Jahrhunderts beschränkt sind (vgl. z.B. SCHWIER 2000; SIELAND 2003; STUMM 2004).

Einzelbeiträge über historische Vorformen risikosportlicher Aktivitäten finden sich zum einen über das Wellenreiten in den Darstellungen von FINNEY \& HONSTON (1996) und über frühe Jagdformen, zusammenfassend bei KRÜGER ${ }^{70}$ (2004). Auch die Entwicklung verschiedener Kampfsportarten, wie sie beispielsweise bei DIEM (1923) beschrieben werden, können im weiteren Sinne als entfernte Vorformen betrachtet werden. Diese frühen Aktivitäten geben zwar nur bedingt Aufschluss über die soziokulturellen Bedingungen heutiger bekannter Risikosportarten und damit wenig Hinweise auf die Einbettung des Risikosports in den kulturhistorischen Horizont, gleichsam sind sie für eine Entwicklungslinie des Risikos im Sport dennoch von Bedeutung. In einem Exkurs in Abschnitt 3.2.2 werden daher insbesondere die Aktivitäten der frühen Großwildjagd auf ihre Risikoaffinität untersucht.

Im folgenden wird der Fokus aber zunächst auf diejenigen sportliche Aktivitäten gelenkt, für die sich ein direkter Entwicklungsstrang zu heutigen Risikosportarten nachvollziehen lässt. Im Mittelpunkt der folgenden Be- 
trachtung steht der Alpinismus, der als risikoorientiertes Bewegungsfeld recht gut in seinen historischen Zusammenhängen evaluiert wurde. Der erste Abschnitt wird das alpine Bergsteigen in seinen sozial- und kulturhistorischen Bezügen darstellen und Annahmen über das gesellschaftliche und individuelle Verhältnis zum Risiko generieren (3.2.1). Der bereits erwähnte Exkurs über Risiko als bewusstes Handlungselement in der frühen Großwildjagd wird in 3.2.2 vorgestellt. Darüber hinaus lassen sich auch in der Erlebnispädagogik nach HAHN Elemente eines sportlichen Risikos bzw. Wagnisses extrahieren. Im Abschnitt zwei wird daher nach den ideellen Hintergründen gefragt, die für die Entwicklung der Erlebnispädagogik und das sozialpädagogische Verständnis des Risikosports relevant sind (3.2.3). Risiko als konstitutives Element des Sports lässt sich darüber hinaus auch im Wehrsport finden, in dem die Vorbereitung auf kriegerische Handlungen unternommen wurden. In der Form eines Exkurses (3.2.4) wird schließlich diesem Ansatz nachgegangen, wonach sich Risikosport als männliches Abhärtungsrefugium darstellen lässt.

\subsubsection{Das sportliche Risiko als Herausforderung - die Geschichte des Alpinismus}

Der Alpinismus in seiner engeren Bedeutung umfasst das Bergsteigen in all seinen Formen und Spielarten wie Bergwandern, Felsklettern, Eisklettern, Bergbesteigungen, alpine Skitouren u.v.m. in alpinen Landschaftszusammenhängen. Hierbei lassen sich durchaus geruhsame und risikoarme Tätigkeiten wie das Wandern als auch risikoreiche Aktivitäten wie das Klettern oder Bergsteigen identifizieren. Der Alpinismus als sportwissenschaftlicher Forschungsgegenstand - dies offenbart der Überblick über den Forschungsstand - wurde maßgeblich von AMSTÄDTER (1996) beeinflusst, der wie ZIAK (1965) eine Monographie zur Geschichte des Alpinismus vorgelegt hat. ZIAK (1965) lieferte dabei die erste chronologische Darstellung der Alpinismusgeschichte ohne jedoch auf sozial- oder kulturgeschichtliche Prozesse einzugehen Ähnlich verfährt auch GREITBAUER (1977), der zwar psychologische Interpretationen des Alpinismus vornimmt, soziale Dimensionen des Alpinismus aber kaum berührt. Auch AUFMUTH (1988) wählt eine psychologische Perspektive ohne jedoch sozial-kulturelle Aspekte zu behandeln. Dem steht HANSEN (1991) gegenüber, der den britischen Alpinismus in seinen Anfängen sozialhistorisch erforscht. In dieser Perspektive ist auch die Arbeit von LEJEUNE (1988) zu nennen, der den französischen Alpinismus in der Zeit von 1875-1919 untersucht. Im Vergleich zu vielen anderen Arbeiten zum Alpinismus behandelt der Autor dabei insbesondere sozialhistorische, kulturelle sowie politische Aspekte. Ähnlich geht auch AMSTÄDTER (1996) vor, der in seiner Monographie über die Geschichte des Alpinismus insbesondere die Organisationsform und Arbeit der alpinen Vereinen darstellt, wobei der Schwerpunkt seiner Analysen die Vereinsbildung im Zeitalter von Liberalismus und Nationalismus im 19. Jahrhundert sowie die Einbettung des Alpinismus in die nationalsozialistische Ideologie 
ist. Auch ZEBHAUSER (1998; 1999) untersucht die ideologischinstitutionelle Verknüpfung zwischen Nationalsozialismus und Alpinismus, jedoch findet keine übergeordnete Einordnung der alpinen Bewegung in den sozialhistorischen Kontext statt. SLUSARCZIK (2000) explorierte die polnische Geschichte des Bergtourismus auch unter Berücksichtigung sozialer Dimensionen. Ohne konkrete wissenschaftliche Perspektivierung gehen HEICHEL (2003), der die Erschließung des Karakorums aufgearbeitet hat und BROGIATO (2003) vor, der die Geschichte des Süd-Amerikanischen Bergsports untersucht. Zu nennen sind auch die kulturwissenschaftliche Arbeit von MANZENREITER (2000), die die soziale Konstruktion des japanischen Alpinismus behandelt und die Arbeit von SCOTT (2000), der sich mit der kanadischen Geschichte des Bergsports auseinandersetzt.

Dabei kann sich eine Untersuchungen im deutschsprachigen Wissenschaftsraum auf zahlreiche alpinistische Quellen stützen, die eine historische Forschungsarbeit durchaus erkenntnisreich ermöglicht. Neben den zahlreichen Publikationen der alpinen Vereine Deutschlands und Österreichs hier sind insbesondere die Mitteilungen des Deutschen Alpenvereins, die Zeitschrift des Deutschen Alpenvereins sowie die Zeitschrift des Deutschen und Österreichischen Alpenvereins ${ }^{71} \mathrm{zu}$ nennen - existieren von verschiedenen Alpinisten des 19. und 20. Jahrhunderts eigene Erfahrungsberichte (z.B. LAMMER 1905; MEYER 1907), die eine vertiefte Auseinandersetzung mit Primärquellen ermöglichen.

Die Geschichte des Alpinismus als zweckfreie und körperorientierte Auseinandersetzung mit der Bergwelt beginnt mit dem Ende des 18. Jahrhunderts und setzt sich bis in die Gegenwart fort, wobei sich bereits im so genannten ,Präalpinismus' eine wichtige Phase der alpinen Auseinandersetzung z.B. in der Literatur nachvollziehen lässt. Schwerpunkt der folgenden Analyse ist die Identifizierung von Handlungskausalitäten und Entstehungszusammenhängen, die über die Geschichte des Alpinismus hinaus auf soziale und kulturelle Hintergründe des Risikosports verweisen. Selbstverständlich lässt sich auch in der Geschichte des Alpinismus kein einfacher kausaler Zusammenhang von auslösendem Faktor und alpinem Ereignis herstellen. Vielmehr ist es notwendig, aus einem größeren kultur- und sozialhistorischen Kontext heraus auf Faktoren zu verweisen, die die Entwicklung des Alpinismus und damit des Risikosports maßgeblich beeinflusst haben ${ }^{72}$. Trotz grundsätzlicher Veränderungen im Bereich der Sicherheitstechniken erscheint die Handlungsstruktur des alpinen Bergsteigens dabei bis heute weitgehend unverändert, weshalb eine Zuordnung als Risikosportart auch im historischen Kontext zweifelsfrei herzustellen ist. Das Risikomoment im Bergsteigen besteht sowohl heute als auch in früheren Jahrhunderten in den Gefahren der Höhe, dem Wetter, den außerordentlichen Anstrengungen oder den Schwierigkeiten der Orientierung.

"The dangers that snowy mountains provide are mainly of two kinds: there is the danger of things falling on the traveller, and that of the traveller himself falling. [...] Lastly there are dangers arising from weather. [...] In describing 
them (the dangers; Anm. d. Verf,), the nature of the craft itself has been desdribed" (CONWAY 1900, 28ff).

\subsubsection{Der Präalpinismus}

Obwohl im Präalpinismus ${ }^{73}$ noch nicht von einer sportlichen - im Sinne einer zweckfreien - Auseinandersetzungen mit der Bergwelt gesprochen werden kann, stellt die intellektuelle und vor allem ästhetische Aneignung der Alpen im 16. und 17. Jahrhundert eine wesentliche Stufe in der Entwicklung des Alpinismus dar. Es verwundert insofern nicht, dass die Geschichte der ästhetischen Naturwahrnehmung mit der Entdeckung der Alpen als Naturraum in ein und denselben Zeitraum fällt. Bis ins Mittelalter galten die Alpen als unbequeme Reiseroute und waren durchaus Gegenstand irrationaler Angstvorstellungen ${ }^{74}$. Die Ergebnisse alpinhistorischer Forschung geben Aufschluss darüber, dass zwar auch schon im Präalpinismus - z.B. im Mittelalter - ,kleinere' Berge erstiegen wurden, diese Unternehmungen waren aber auf Einzelfälle begrenzt. Als Beispiel kann Petrarca (1304-1374) gelten, der 1336 als erstes den Gipfel des Mont Ventoux bestieg. Obwohl bei einigen Autoren ${ }^{75}$ angezweifelt wird, ob Petrarca die Besteigung wirklich unternommen hat, stellt die aus der vermeintlichen Aktivität erstellte Abhandlung den Beginn einer Natur- und Landschaftswahrnehmung aus ästhetischen Gründen dar. Seine für die damalige Zeit ungewöhnliche Aktivität rechtfertigt der Dichter mit dem Bestreben, ,sich Gott im Anblick der Natur zu vergegenwärtigen" (vgl. AMSTÄDTER 1996, 23). Die Darstellung dieser alpinen Unternehmung erzählt dabei nicht die erste zweckfreie Besteigung eine Berges sondern ist eine philosophisch-theologische Reflexion über Raum und Zeit und deren Bezug zum wahren Leben (vgl. BEIERWALTER 1996). Für RITTER beginnt mit den Abhandlungen Petrarcas die ästhetische Betrachtung der Natur als Entdeckung der Landschaft, denn „Natur und Landschaft ist Frucht und Erzeugnis des theoretischen Geistes" (RITTER 1963, 146). Für den Alpinismus als bewusste Auseinandersetzung mit der alpinen Natur stellt sie eine bedeutende Vorstufe dar (vgl. auch SLUSARICZIK 2000, 64f).

Die in den folgenden Jahrhunderten des Präalpinismus stattfindende Auseinandersetzung mit den Alpen als Naturraum und Landschaft vollzieht sich vor allem in einem kleinen Kreis bildender Künstler, die zu verschiedenen Reisen in die Alpenregion aufbrechen. Dabei repräsentiert die künstlerische Darstellung der Alpen im geschichtlichen Ablauf gleichsam einen wichtigen Wechsel in der Bergwahrnehmung. In Albrecht von Hallers Lehrgedicht ,die Alpen' aus dem Jahre 1729 geraten die Berge dabei erstmals zum Projektionsraum für eine neu entstehende Natursehnsucht (vgl. VON HALLER 1729 / 1959, 14). Die Entdeckung der Alpenlandschaft als ästhetischer Gegenstand wird darüber hinaus maßgeblich von den Reisenden der so genannten ,Grand Tour ${ }^{76^{\prime}}$ multipliziert, die im 18. Jahrhundert auf ihren Reisen nach Italien auch die Alpen überqueren und in ihren Reiseberichten über die Erschwernisse aber auch die Erhabenheit der Alpen berichten. Gerade in den Reisen der, Grand Tour' sowie den romantischen Bildungsreisen 
des 18. Jahrhundert wird die veränderte Natur- und Landschaftswahrnehmung deutlich. WAGNER konstatiert hierzu:

„Galten die Alpen jabrbundertelang als binderliche Barriere auf der Reise ins gelobte Land Italien, veranlasste die vegetationslose Einöde nackter Felsen noch Herder, die Fenster seiner Reisekutsche zu verbängen, so entwickelten sich die Schweizer Hochalpen seit dem ausgehenden 18. Jabrbundert zu einem der interessantesten und besuchtesten Teile von Europa" (W AGNER 1981, 67).

Vor allem die Aufklärung - basierend auf dem naturwissenschaftlichen Weltbild des 17. und 18. Jahrhundert - als stärkste geistige Bewegung der europäischen Neuzeit, beeinflusst die weitere Auseinandersetzung mit der Bergwelt. Da die Alpen bis ins 18. Jahrhundert noch wenig zusammenhängend erforscht sind, wächst in der zweiten Hälfte dieses Jahrhunderts das Interesse der Naturwissenschaften an den Alpen als Lebensraum und Landschaft. Hervorzuheben sind hierbei der Naturforscher Benedict de Saussure in den Westalpen sowie der Arzt Belsazar Hacquet in den Ostalpen. 1787 gelingt de Saussure die Besteigung des Mont-Blanc, nachdem allerdings 1786 die Erstbesteigung des höchsten Berges der Alpen bereits vollzogen ist (vgl. BONNINGTON 2000, 26f).

Hierbei ist von Bedeutung, dass sich diese erste Phase des Präalpinismus nicht nur - wie es die Ausführungen nahe legen - in der deutschsprachigen Literatur vollzieht. Auch die französische Entdeckung vollzieht sich zunächst in einer Phase der romantischen Annäherung und literarischen Auseinandersetzung (vgl. JANTZEN 198877). Für den polnischen Bergtourismus identifiziert SLUSARCZIK $(2000,65)$ eine ähnliche Strömung, die vom Musiker Daniel Stera um 1683 ausging und den Bergtourismus in den Sudenten und Karpaten eröffnete.

\subsubsection{Der Alpinismus als zweckfreie Handlung und Sport}

Während das Bergsteigen vor allem in den Ost- und Westalpen lange über Saussure und Hacquet hinaus noch aus wissenschaftlichen Gründen betrieben wurde, wandeln sich bis zur Mitte des 19. Jahrhunderts die Motivationen des Alpinismus vom naturwissenschaftlichen Interesse hin zum Bergsteigen ,um seiner selbst willen'. Mit dem aufkommenden Interesse der Engländer an den Alpen wird das Bergsteigen Anfang des 19. Jahrhunderts erstmalig zu einem rein sportlichen Unterfangen - maßgeblich beeinflusst von britischen Adligen und dem Modell des modernen Sport ${ }^{78}$. Durch den Ausbau des britischen Kolonialreiches und der frühen industriellen Revolution in England gelangten insbesondere der Landadel in den Besitz von großen Reichtümern und bildeten im 19. Jahrhundert eine neuen Gegenpol zum wohlhabenden Hochadel. Gleichzeitig aber sehen sich insbesondere die Landadligen mit dem Aufkommen des industriellen Bürgertums in England mit dem Verlust weitreichender gesellschaftlicher und politischer Funktionen konfrontiert ${ }^{79}$. Das Bergsteigen wird von vielen von ihnen als neue Freizeitbeschäftigung 
erschlossen, als Herausforderung, die sich von den gesellschaftlichen Vergnügungen und sportlichen Betätigungen des Hochadels, etwa der Jagd, unterscheiden sollte:

„To those gentlemen who could claim their status by birth were added the men of new money, intellect, or professional credentials whose claims to genteel status rested in their wealth, their performance in competitive market or examinations, or their membership in some corporate body such as a professional organization. The new gentleman was usally educated at the reform public schools and universities, where he learned a sense of honor and a commitment to public service" (HANSEN 1991, 88).

Stellvertretend für diesen neue Bergsteiger-Motivation bezeichnet der Engländer Sir Leslie Stephan die Alpen als ,playground of Europe' und gibt damit den Trend des sportlichen Charakters des alpinen Bergsteigens vor (vgl. AMSTÄDTER 1996, 34). Das englische Bergsteigen stellt damit erstmals nicht die visuelle und kognitive Naturaneignung in den Mittelpunkt, sondern die bewegungsvermittelte Überwindung von alpinen Landschaftsarrangements. Ziel dieser neuen Bewegungsaktivität - so die Interpretation von BAHNERS \& HANSEN - ist die Suche nach Herausforderungen, die über die Naturwahrnehmung hinaus dazu dient, den eigenen Status zu manifestieren:

„Die Jagd, der Sport der Aristokratie, wurde als barbarisch verdammt, als blutig und monoton [...] Der Bergsteiger wäblte sich einen edlen Gegner, dem er nichts zu leide tat; er musst Körper und Geist in ständiger Anspannung halten wie der Kapitalist, der mit dem schlimmsten rechnete. Dem Adel, die alte Kriegerkaste, wollte man als funktionslos erweisen, weshalb warf man ibm weibische Verweichlichung vor. [...] Als Mann galt nur, wer jederzeit abstürzen konnte und nicht auf die Kissen eines angeborenen Status fiel" (BAHNERS \&HANSEN 1996, 71).

Die ab der zweiten Hälfte des 19. Jahrhunderts in England einsetzende ,Versportung' der Leibesübungen erfasst dabei auch das alpine Bergsteigen. Im Zentrum steht die Erstbesteigung als Rekord aber auch der Wettkampf um die schnellste Besteigung (vgl. auch LEJEUNE 1988). Ab Mitte des 19. Jahrhunderts nimmt die alpintouristische und bergsteigerische Bewegung dabei solche Ausmaße an, dass sich erstmals Gruppen, Vereine und daraus resultierend auch Fachbücher und Zeitschriften ausbilden. Die Gründung des ersten Alpenvereins in London (1857) drückt dabei die sportlichen Ambitionen der neuen englischen Mittelklasse aus und manifestiert die Vernetzung zur englischen Sportbewegung. Vor allem auf das Betreiben von E.S. Kennedy, der durch zahlreiche Publikationen bekannt geworden war (z.B. ,Where there is a will, there is a way "), konnte nur derjenige Mitglied im ,Alpine Club' werden, der einen Berg von mindestens 4000 Meter Höhe bestiegen hatte. In der, goldenen Zeit' des britischen Sport-Alpinismus von 1859 1865 werden 68 Hauptgipfel der Alpen von Mitgliedern des ,Alpine Club’ 
erobert. Als Höhepunkt wird schließlich 1865 das Matterhorn unter der Führung von Edward Whymper erstbestiegen (vgl. BONNINGTON 2000)

Bergsteigen als Distinktion eines sich neu definierenden Landadels und des neureichen Bürgertums beherrscht zu dieser Zeit die alpine Bewegung. In der Argumentation ZIAK kommt in diesem Zusammenhang zu dem Schluss, dass sich das englische Bergsteigen als kulturelle Unterscheidungspraxis auch als imperialistische Demonstration von Macht interpretieren lässt ${ }^{80}$ :

„Denn darin, wie die Briten, vor allem von 1859 bis 1865, um die Gipfel der Alpen kämpften, vielfach im Wettlauf mit anderen Nationen - man denke nur an den Kampf um das Matterborn -, dürfen wir wobl etwas von dem britischen Imperialismus erblicken, der sich eben Indien unterworfen batte und auch in den Alpen gleichsam Kolonialland - wenn auch nur ideelles sab" (ZIAK 1965, 97).

HANSEN, der die bedeutendste Monographie über das britische Bergsteigen veröffentlicht hat, verdeutlicht die implizite Nähe des Bergsteigens zum Imperialismus auch anhand der semantischen Zusammenhänge:

„Moutnaineers defined their climbing in terms of adventure and conquest, exploration and empire. Their rhetoric mattered; the language they used was not neutral. By comparing climbers in the Alps with exlporers in Africa or the Artic, mountaineers were not simply engaging in the pursuit of desinterested science. The $>$ conquest $<$ of the mountain summit became the moment for symbolic reaffirmation of British imperial strength. [...] By climbing mountains, professional men could participate in the expansive dimension of the Britain's national identity" (HANSEN 1991, 443).

Zur Verbreitung der neuen englischen Bergsteiger-Ideologie trägt in den folgenden Jahren zudem eine bis dahin unbekannte mediale Vermarktung der Erlebnisse bei. Neben zahlreichen wissenschaftlichen Werken und Reiseführern wird eine Vielzahl von literarischen Werken veröffentlicht, die die Erhabenheit der Alpen beschreiben und damit einem größeren Publikum nahe bringen. Die beeindruckenste mediale Inszenierung des Bergsteigens geht dabei auf Albert Smith zurück, der 1852 den Mont-Blanc bestiegen hatte und dieses Erlebnis in der Ägyptischen Halle in London in einer perfekt inszenierten Show präsentiert ${ }^{81}$. Zwar war der Berg zuvor schon von fast 50 anderen Seilschaften bestiegen worden aber „Smith hatte verstanden, dass der Ruhm eines Sportlers nicht nur von der Leistung sondern auch von der Vermarktung abhängt" (BAHNERS \& HANSEN 1996, 71). Die Show, die über 200000 Besucher zählte, wurde von der Times 1853 zu den Hauptsehenswürdigkeiten der Stadt London erhoben.

„1854 gab Smith eine Privatvorstellung für die königliche Familie. Alle Sinne wurden angesprochen, man börte den Donner der Lawinen, roch Felle und Blumen und durfte Rucksäcke anfassen. Die Zuschauer wurden eingeladen, selbst durch den Schnee zu stapfen, die Schluchten zu überwinden und den Gipfel zu erklimmen. In der Pause offerierten Bernhardiner Schokolade. Die 


\section{Illusionstechnike erreichte ibren Gipfel im Blick von der Bergspitze. Oben an- gekommen, erhob man das Champagnerglas zu Ehren von Königin Vikto- ria"(ebd.).}

Das Bergerlebnis wird hierbei erstmalig zur Inszenierung: Das Risikomoment und die damit verbundenen Erlebnisdimensionen werden dem Zuschauer nahezu lebendig vermittelt und lassen die Erlebnisse des heldenhaften Bergsteigers Wirklichkeit werden. Hervorzuheben sind in diesem Zusammenhang auch die Erlebnisberichte von Edward Whymper (1840 - 1911), dem Erstbesteiger des Matterhorns, die ebenfalls zu einer massenwirksamen Verbreitung des Bergsteigens beitragen. Anders als in früheren Reisebeschreibungen steht bei Whymper die Besteigung der Berge mit all seinen Widrigkeiten im Mittelpunkt - also das Risiko als Handlungselement - und weniger der romantische Blick auf die Landschaft. In seinem 1872 veröffentlichten Buch „Berg- und Gletscherfahrten“ werden zahlreiche Erstbesteigungen und Besteigungsversuche der schwierigsten Berg der Alpen beschrieben (vgl. WHYMPER 1872 / 1990). Diese erste massenwirksame Vermarktung risikoreicher Erlebnisse, die sich bis heute in den zahlreichen Veröffentlichungen von Bergsteigern, Kajakfahrern, Fallschirmspringern und Abenteurern fortsetzt, verweist in diesem Zusammenhang auf einen tiefgreifenden Wahrnehmungs- und Motivationswechsel im sportlichen Alpinismus. Hinter der auf den ersten Blick formalen Veränderung in der alpinen Literatur steckt die Mitte des 19. Jahrhunderts einsetzende Tendenz zum sportlichen und damit auch vergleichsweise versachlichten Bergsteigen.

Parallel zu dieser literarischen Verarbeitung des Bergsteigens als Sport vollzieht sich eine neue Qualität der Denk- und Redeweisen über das alpine Geschehen: Die Alpen verlassen sukzessiv den Dunstkreis der romantischen Bildungsreisen und eignen sich - als kulturelle Praxis - neue Stile an ${ }^{82}$. In der Verbreitung der Erlebnisberichte findet das Statusbegehren des Bürgertums einen Nährboden, der zur sozialen Verortung der neuen Mittelklasse beiträgt.

Besonders deutlich tritt bei Whymper erstmals auch ein Fluchtmotiv zur Erklärung des alpinen Bergsteigens hervor. Diese Gegenüberstellung von alpiner Natur und städtischer Zivilisation zählt nach 1860 quasi zu den Konstitutiva der alpinen Theoretiker und gibt wichtige Hinweise auf die sozialhistorischen Hintergründe des Risikosports ${ }^{83}$. Sie lässt sich als eine den Alpinismus bedingende Entwicklungslinie verfolgen, die sich über die Naturbetrachtung zu einer Naturbewältigung entwickelt. Die alpine Bewegung steht diesbezüglich in einem engen Zusammenhang zu Prozessen der Industrialisierung und Modernisierung mit ihren Potentialen für das Individuum $^{84}$ und bezieht sich gleichsam auf die Natur als Gegenwelt (vgl. GROßKLAUS \& OLDEMEYER 1983). Das alpine Erlebnis wird in den Beschreibungen des ausgehenden 19. Jahrhundert als Kontrastfolie zur Zivilisation und Künstlichkeit entworfen. Bereits 1873 betont der Präsident des Österreichischen Touristenclubs Schiestl die Bedeutung des Alpinismus als Fluchtpunkt ,[...] vor dem engen Leben da drunten in die wahre innere Freiheit" (SCHIESTL 1873, 328). Die Berge werden damit zu einer Bühne 
der , condition humaine', die Natur zu einem erlebnisbezogenen kulturellen Handlungsfeld. Neben der Entfremdung von der Natur tritt durch die zunehmende Arbeitsteilung auch die Entfremdung von der Arbeit als Fluchtmotiv hinzu:

„Der moderne Arbeitsmensch aber hat weder Kreuziüge noch Entdeckerfahr-
ten und ist bei der fortgeschrittenen Teilung der Arbeit in seinem Berufe noch
beengter als der Europäer von anno dazumal, der auch in seinem Erwerbs-
schaffen noch manche Abwechselung fand. Besonders übel daran sind die
menschlichen Schreibmaschinen, die Sklaven des Comptoirs und der Studier-
stube, denen die Arbeit nicht einmal körperliche Bewegung bringt. Für diese
Männer ist die Entdeckung des Hochgebirges eine Erlösung, und die blaue
Blume der Abentenerromantike duftet ibnen in ibr Arbeitszimmer binein"
(Ohne Autor in der Österreichische Alpenzeitung 1902, 181).

Beeinflusst vom wilhelminischen Leistungsgedanken rücken neben dem Fluchtmotiv auch die Leistungsvergleiche und Wettkampfgedanken in den Mittelpunkt der alpinen Bewegung, über die sich die Verknüpfung zur englischen Spiel- und Wettkampfsportbewegung vollziehen lässt ${ }^{85}$.

\subsubsection{Die Gründung der alpinen Vereine}

Während sich die Engländer in den folgenden Jahren sukzessiv aus den Alpen zurückziehen und sich bereits 1868 dem Kaukasus zuwendeten, führen Deutsche und Österreicher die systematische Erschließung vor allem der Ostalpen fort. Im Jahr 1863 wird zunächst der, Österreichische Alpenverein' (OeA.V.) und kurz darauf 1869 der ,Deutsche Alpenverein' (DAV) gegründet. 1874 konstituiert sich schließlich der Club Alpin Francais (C.A.F.). Obwohl gewisse Gemeinsamkeiten in der ideologischen Ausrichtung, im Männlichkeitskult und in der politischen Orientierung zu konstatieren sind, zeigen insbesondere die Vereine doch wichtige Unterschiede in der Mitgliedsstruktur auf. Dominiert bei der Gründing des OeA.V. die Zahl akademischer Naturwissenschaftler, so weist die Mitgliedsstatistik des DAV auf eine Dominanz des Bildungsbürgertum hin. Auch im französischen C.A.F. dominiert das Bildungsbürgertum (vgl. LEJEUNE 1988, 54ff). Demgemäß finden sich auch Unterschiede in den Vereinszielen: für den OeA.V. steht die weitere naturwissenschaftliche Erforschung des Alpenraums im Vordergrund, während der DAV und der C.A.F. die Bereisung der Alpen erleichtern und damit zu einer Verbreitung der alpinen Bewegung beitragen will (vgl. AMSTÄDER 1996, 55ff). 1873 schließen sich die beiden deutschsprachigen Vereine zusammen und beenden damit die über Jahre dauernde Diskussion um die politische Ausrichtung zwischen Liberalismus, Nationalismus und unpolitischen Vereinsbürgertum. Seit dem Zusammenschluss entwickelt sich der Deutsche und Österreichische Alpenverein zu einem Massenphänomen, die Mitgliederzahl steigt von 1882 von 10000 auf über 200000 im Jahr 1930 an (PRAHL \& STEINECKE 1989, 50f). 
Wenngleich der Alpinismus des ausgehenden 19. Jahrhundert bisweilen als Massenphänomen ausgelegt wird (vgl. ebd., 48), so ist doch darauf hinzuweisen, dass nicht alle gesellschaftlichen Gruppen daran teilnehmen konnten. Das alpine Bergsteigen war weiterhin ein Privileg der besitzenden Schichten, welches in der Mitgliedsstatistik der alpinen Vereinen deutlich zum Ausdruck kommt. 1895 kommt es in Wien schließlich zur Gründung des Arbeitervereins ,Die Naturfreunde', der entscheidend zur sozialtouristischen Verbreitung des Bergsteigens beiträgt (vgl. PILS 1994; GÜNTHER 2003). Die Gründung dieses proletarischen Touristenvereins im Jahr 1895 versucht das bürgerliche Privileg des alpinen Erlebnisses für alle gesellschaftlichen Gruppen zu öffnen und insbesondere der Arbeiterklasse die Reise in die Alpen zu ermöglichen (vgl. ZIMMER 1995, 42).

\subsubsection{Der Schwierigkeits- und Gefahrenalpinismus}

Nach der Besteigung der Hauptgipfel im goldenen Zeitalter des englischen Alpinismus konzentriert sich der zentraleuropäische Alpinismus auf noch unerstiegenen Nebengipfeln oder auf Graten und Wänden schon bestiegener Berge. Dabei setzt sich mehr und mehr die Idee eines Schwierigkeitsalpinismus als führerloses Bergsteigen durch (vgl. JANTZEN 1988, 61ff; LEJEUNE 1988, 131f; AMSTÄDTER 1996, 93). Mit der Bergbesteigung auf immer schwierigerem Wegen erfolgte eine rasante Weiterentwicklung technischer Elemente vor allem im Bereich des Felskletterns. Die Gipfelbesteigung als Hauptziel gerät zunehmend in den Hintergrund, wichtiger wird der Weg, dessen Schwierigkeiten in Routenbeschreibungen und technischen Ausdrücken festgelegt wird. Die frühen bergsteigerischen Antriebe, die sich ästhetischen Genuss der Naturschönheit finden, treten verstärkt in den Hintergrund. Zwar vertreten zahlreiche einflussreiche Bergsteiger wie z.B. Zsigmondy (1861-1885) am Ende des 19. Jahrhunderts noch den ethischen Genuss der Alpennatur, sie befinden sich aber im Gegensatz zu einer Vielzahl so genannter Gefahrenalpinisten, die in der Überwindung und Bekämpfung der Naturgewalten ihren Hauptantrieb betrachten (vgl. ebd., 95). Eugen Lammer (1863-1945), einer der einflussreichsten Vertreter dieses Gefahrenalpinismus um die Jahrhundertwende, preist die Gefahr als das höchste Glück des Bergsteigens an:

„Wenn sich dieser bewußt in Gefahr begibt, die bebende Angst vor der Vernicbtung kennenlernt und mit dem neu geschenkten Leben wieder aus den Bergen geht. Die Begier nach Kampf und Rettung aus Todesgefahr, die Wonne und das Grauen begleiten ibn auf allen seinen Touren als seine besten Freunde" (LAMMER 1893; rit. nach PICHL 1927, 22)86.

Obwohl Lammer, Gefahr' zum zentralen Begriff seiner Überlegungen macht, sind seine Ausführungen im Bezug zum heutigen Risikosport von entscheidender Bedeutung. Bei Lammer und den von ihm beeinflussten Bergsteigern wird erstmals das Risiko um seiner selbst willen thematisiert und die erlebnisinduzierenden Prozesse in den Mittelpunkt der alpinistischer 
Ideologie gerückt. Er steht damit beispielhaft für eine bedeutende Entwicklungsstufe im Bezug auf die Entdeckung der Alpen als Wirklichkeit und inneres Erlebnis.

„Wir gehen nicht in die Berge um der Berge willen, sondern um unseretwillen. Der Mensch in den Bergen ist es, der uns interessiert. [...] Die besonderen Erlebnisse und Handlungen oder das Individuelle muß mehr in den Vordergrund treten" (LAMMER 1893; zit. nach PICHL 1927, 23).

Das Bergsteigen Lammers ist gekennzeichnet durch die Auseinandersetzung mit dem Berg als Kampf und als Überwindung den damit einhergehenden emotionalen Vorgänge :

„Furcht zu empfinden ist keine Schande; nur ibr zu erliegen schändet. Je kräftiger und gesünder ein Lebewesen, desto stärker wogen in ibm alle Affekte und Leidenschaften empor, also auch Lebensliebe und Todesangst" (LAMMER o.J.; zit. nach GREITBAUER 1973, 22).

Die kampfbetonte Konfrontation mit der alpinen Natur wird um die Jahrhundertwende - durch Lammer beeinflusst - zu einem zentralen Leitmotiv des Alpinismus. Auf der Basis der wilhelminischen Leistungsmoral führt der bürgerliche Mensch dabei einen individualistischen Kampf gegen die alpinen Gewalten. Hierdurch wird das bürgerliche Fluchtmotiv um das spannungsgeladene Risikoerlebnis erweitert: Das Bergsteigen beinhaltet Unsicherheiten und Unberechbarkeiten, die im gewöhnlichen bürgerlichen Leben vermieden werden oder die schlichtweg durch Zivilisierungsprozesse eliminiert sind (vgl. JANTZEN 1988, 83). Die Spannung der alpinen Handlung wird gegen die Monotonie des steifen und zwanghaften Alltags gesetzt, in dem sich das bürgerliche Individuum nur selten ,äußeren' Gefahren ausgesetzt ist ${ }^{87}$. Besonders deutlich tritt diese Zivilisationskritik bei Ludwig Purtscheller (1849 - 1900) hervor, der zahlreiche Gefahrenbesteigungen in den Westalpen durchführte. Obwohl in seinen Ausführungen eine deutliche Distanz zur Gefahr als zentrales Handlungsmotiv des Bergsteigens zu finden ist, betont Purtscheller die entwicklungsgeschichtliche Bedeutung des Alpinismus in der Hochindustrialisierung:

„Des Hochgebirges kunstvoller Bau, seine Schwierigkeiten und Gefabren fordern zu einem Wettstreit mit ihm auf. Es gilt zu zeigen, was das Übermaß an Zivilisation, was städtische Verkehrtheit, Unverstand und konventionelle Sitte noch Tüchtiges und Brauchbares an uns gelassen hat, was körperliche und geistige Energie aufzubieten vermögen" (zit. n. ZIAK 1965, 152).

Bei Oskar Erich Meyer (1883-1940), der als der ,alpine DichterPhilosoph“ (AMSTÄDTER 1996, 97) beschrieben wird, heißt es hierzu:

„Es ist der moderne Mensch, der in die Berge binaufsteigt mit allen seinen Konflikten; aus dessen reichen Worten es oft aufflackert wie ein Blitglicht von Friedrich Nietzsche oder Richard Debmel; der moderne Mensch, der in einem unserer bis aufs äußerste spezialisierten Berufe gleichsam nur die linke Hand 
gebrauchen kann, während die rechte verkümmert, dessen gequältes Individuum aufschreit unter dem Drucke des Dranges nach Betätigung aller Kräfte. Darum geht er auch allein in die Berge" (MEYER 1907, 171).

Diese steigende Anerkennung und Handlungsmotivation des Gefahrenmomentes führt in den 1880er Jahren zu einer drastisch ansteigenden Zahl von Bergunfällen, insbesondere in der Wiener Kletterszene. Als es nach zahlreichen tödlichen Unfällen 1887 zu einem Erlaß der Polizeidirektion Wien über die Möglichkeiten der Unfallreduktion kommt, wehren sich die alpinen Vereinen mit einem gemeinsamen Memoradum gegen eine Beeinflussung des alpinen Geschehens. Die Handlungsfreiheit des Alpinisten wird zu einem entscheidenden Streitwert in der Auseinandersetzung um die Legitimation des Gefahrenalpinimus und rückt die ethische Dimension des Risikos in den Mittelpunkt der alpinen Bewegung (vgl. AMSTÄDTER 1996, 96). Dabei tritt auch das vormals ästhetisch interpretierte Freiheitsideal des Alpinismus nun in deutlich ausgeprägter Form erneut hervor:

\section{„Verkümmern wir und doch nicht an der Freude an der Natur durch eng- berzige Regeln und knöchernde Gesetze und bewahren und vor allem das, was wir im Hochgebirge als beglückendes Ziel sich und finden wollen: die herrliche Freibeit" (Heß 1888; zit. n. PICHL 1927, 35).}

Mit dem Übergang zum Schwierigkeits- und Gefahrenalpinismus in den Vorstellungen Lammer's vollzieht sich die Entwicklung des modernen Felskletterns, welches sich an der Besteigung von schwierigen Teilabschnitten und herausfordernden Felsformationen orientiert. Die Entwicklung technischer Fertigkeiten, die eine gefahrvolle und schwierige Route ermöglicht, wird damit um die Jahrhundertwende des 20. Jahrhunderts zu einem bedeutenden Wendepunkt in der sportlichen Ausführungen des alpinen Bergsteigens. Die Konzentration auf einzelne Wände und Abschnitte gewinnt nun nochmals an Bedeutung und führt zu der Entwicklung des Sportkletterns in seiner heutigen Form (vgl. hierzu ausführlich SCOTT 2000).

\subsubsection{Der Alpinismus als Massenphänomen}

Obwohl sich der Gefahrenalpinismus zunächst nicht als Massenphänomen durchsetzt, widmet sich mit Ende des 19. Jahrhunderts erstmals eine breite Bevölkerung dem Alpinimus. Dies wird insbesondere durch den Ausbau der Infrastruktur - insbesondere der Eisenbahnanbindung - ermöglicht ${ }^{88}$. Schon 1850 verzeichnet die Schweiz ca. 40000 Besucher mit deutlich steigender Tendenz (vgl. ebd.). Durch den Ausbau der Bahnstrecken erweiterte sich zum Anfang des 20. Jahrhunderts auch der Bewegungsradius der arbeitenden Bevölkerung. Waren am Anfang der alpinistischen Entwicklung kaum Handwerker und Gewerbetreibende oder sogar Industriearbeiter zu finden, so drängen im Zuge der Arbeitskampfbewegung um geregelte Arbeitszeiten mehr und mehr Arbeiter in die Alpen.

Obwohl die Aufnahmekriterien der alpinistischen Vereine (OeA.V. und ,Alpine Club') zunächst noch verhinderten, dass das Bergsteigen als Sport 
über die neu gebildeten Eliten hinaus Verbreitung findet, ist der Alpinismus als Sport spätestens Ende des 19. Jahrhunderts nicht mehr nur eine distinktive Praxis gesellschaftlicher Aufsteiger, sondern ein klassenübergreifendes Phänomen. Mit der Durchsetzung der Naturfreunde als alpiner Verein erhält der Alpentourismus als „Schlachtfeld sozialer Distinktionen“ (HENNING 1997, 18) Anfang des 20. Jahrhunderts weitere Dimensionen. Neben den unterschiedlichen bürgerlichen Alpinisten reisen nun auch Städter mit zum Teil konträren Naturauffassungen in die Alpen. Gerade im Bereich des Gefahrenalpinismus und damit der risikosportlichen Variante des Alpinismus setzt sich die Arbeiterklasse aber kaum durch. Die Aktivitäten bleiben zunächst auf Erholung und Landschaftsgenuss beschränkt, wenngleich es zu einzelnen bergsteigerischen Aktivitäten kommt.

In der weiteren Entwicklung im 20. Jahrhundert vollzieht sich in den Alpen eine breite touristische Nutzung der Alpen, die durch die Etablierung des alpinen Skilaufs nicht nur auf die Sommermonate beschränkt bleibt, sondern sich insbesondere im Winter zu einem Massenanlaufpunkt entwickelt. Das alpine Gipfelbergsteigen verlagert sich in der ersten Hälfte des 20. Jahrhunderts in andere Teile der Welt, vor allem in den Himalaya, in dem bis weit in die zweite Hälfte hinein zahlreiche Erstbesteigungen durchgeführt werden. In den Alpen selbst setzt sich sowohl das erholsame Wandern und Spazierengehen als auch der Gefahren- und Schwierigkeitsalpinismus in der Form des Felskletterns und Bergsteigens als Massenbewegungen durch (vgl. auch PRAHL \& STEINECKE 1989). Ziel war nun die schon angedeutete Besteigung ohne Hilfsmittel, in schnellerer Zeit oder aber allein, die sich mit den Elementen der englischen Wettkampfbewegung nun endgültig zu ,Sport' entwickelte.

„Die Generation nach ibnen [den Eroberern, Anm. des Verf.] aber fand die Berge erschlossen. Wo den Vätern die Fülle der Siege geblïbt hatte, dort mussten die Söhne Probleme suchen, mit deren Lösung Lob einzuheimsen blieb. War jene Tat allein genug gewesen, so musste es für diese schon eine einrigartige Tat sein [...]. Das gute alte Bergsteigen, das romantisch und abenteuerlich gewesen war, wurde ein Sport wie das Wettrennen, und das Klettern, einst Hilfsmittel dort, wo der Fuß allein nicht mehr genügte, wurde eine Kunst, die nicht selten zur Akrobatik ausartete" (ZIAK 1965, 157).

Die Entwicklung zu einer breitenwirksamen Bewegung lässt sich auch an der Mitgliederstatistik der alpinen Vereinen belegen. So weist allein der Deutsche Alpenverein heute über 700000 Mitglieder auf (vgl. www.alpenverein.de [Zugriff am 12.02.2005]).

$\mathrm{Zu}$ erwähnen ist diesbezüglich natürlich auch die massive Ideologisierung nationalsozialistischer Prägung, die im dritten Reich zu einer Vernetzung und Gleichschaltung des Alpenvereins mit den Interessen der kriegsvorbereitenden NS-Politik besteht (vgl. AMBROSI 2004). Der HeldenMythos des Bergsteigens wird dabei zum ideologischen Mittel des Nationalsozialismus, indem das Bergsteigen als heroische Lebensform zelebriert wird ${ }^{89}$. 


\subsubsection{Konklusion: Der Alpinismus als historische Wurzel des Risikosports}

Aus der historischen Perspektive des Risikosports stellt der organisierte Alpinismus des beginnenden 20. Jahrhunderts die Grundlage für eine Vielzahl heutiger Risikosportarten dar. Der Tourenskilauf etablierte beispielsweise sich aus der Sektion Bayerland des Deutschen und Österreichischen Alpenvereins heraus ${ }^{90}$ und auch das Kanu Wildwasserfahren findet seinen Ursprung im Alpinismus. Zwar kam der Kanusport zunächst in Frankreich Ende des 19. Jahrhunderts als Freizeitaktivität auf, die ersten Wildwasserbefahrungen wurden in den 30er Jahren aber von bekennenden Alpinisten betrieben und vernetzen auch das Wildwasserfahren mit dem Alpinimus (vgl. DIEM 1960, 664ff).

Als Handlungsraum sind die Alpen auch für die Mehrheit der ,postmodernen' Risikosportarten, wie das alpine Skifahren, das Gleitschirm- und Drachenfliegen, das Canyoning oder das Mountainbiken von zentraler Bedeutung, die sich seit den 60er Jahren des 20. Jahrhunderts teilweise zu Massenphänomenen entwickeln. Durch den allgemeinen wirtschaftlichen Aufschwung, einhergehend mit Materialverbesserungen erfahren die Alpen eine regelrechte sportliche Invasion. Ende des letzten Jahrhunderts zählen die Alpenländer Österreich und die Schweiz z.B. mehr als 100 Millionen Übernachtungen ${ }^{91}$ (vgl. PRAHL \& STEINECKE 1989, 65).

Zusammenfassend erlaubt es die Quellen- und Literaturlage zur historischen Entwicklung des Alpinismus nunmehr die Frage nach den kulturhistorischen Hintergründen des Alpinismus als Quelle des Risikosports mit vier beeinflussenden Strömungen zu beantworten. Ein erstes wichtiges Element kann im Distinktionspotential des alpinen Bergsteigens identifiziert werden, wie es HANSEN am Beispiel der englischen Identitätsbildung verdeutlicht hat. Durch die einschneidenden Veränderungen des Lebensstils eines sich neu entwickelnden Bürgertums, wird der Alpinismus zur kulturellen Praxis im Sinne einer sozialen Abgrenzung zu allen Seiten. Im Mittelpunkt steht hierbei die neue Mittelklasse aus aufstrebendem Bürgertum und Landadel, die im Rahmen der Kolonialisierung und Industrialisierung zu neuen ökonomischen Reichtümern gekommen sind.

Die mediale Vermarktung der alpinen Erlebnisse, als zweiter bedeutender Faktor für das steigende Interesse an den Alpen, steht diesbezüglich in einem engen Zusammenhang. Durch die frühe öffentliche Darstellung und Inszenierung der alpinen Erlebnisse diffundiert der Alpinismus in weite Teile der Gesellschaft hinein und verlässt seinen ursprünglichen Handlungsraum. Das alpine Bergsteigen wird hierdurch erstmals weiten gesellschaftlichen Gruppen zugänglich.

Als dritter entscheidender Faktor für das zunehmende Interesse an den Alpen kann neben den ökonomischen Veränderungen der Ausbau der Infrastruktur gelten, ohne den die Entwicklung des Alpinismus zur sportlichen Bewegung undenkbar gewesen wäre. Durch die Vernetzung von Naturräumen wie den Alpen mit urbanen Lebensräumen wird die Nutzung als Freizeitraum erst erschwinglich und bewältigbar. Diese Entwicklung verstärkt sich erstmalig mit dem Ausbau des Eisenbahnnetzes und ein zweites Mal 
mit der Durchsetzung des Autoverkehrs seit den 50er Jahren des 20. Jahrhunderts.

Als zentrales Element für die Entwicklung des Alpinismus und damit auch des Risikosports muss aber das beschriebene Fluchtmotiv angesehen werden. Im Mittelpunkt steht hierbei zunächst die Natur als ästhetische Landschaftsidylle und schließlich die Naturgewalten als Herausforderung. Das am Berg erlebte Risiko wird zum Versuch, der zunehmende Zivilisierung, Rationalisierung und Industrialisierung der Moderne zu entkommen Natur wird damit zum expliziten Handlungsraum des Risikosports. Offenbar werden die im „Prozess der Zivilisation“ (ELIAS) erreichten Sicherheiten und Beschränkungen im alpinen Risikohandeln ,überwunden'. Den Alpinismus in eine Reihe von Fluchtbewegungen zu stellen ist dabei keine Empfehlung der Kompensationstheorie gegenwärtiger Prägung, sondern evidente und begleitende Deutung alpinistischer Chronik und Reflexion (vgl. auch TSCHOFEN 1999).

Zivilisationsflucht, Bürgerliches Distinktionsbegehren, mediale Vermarktung und der Ausbau der Verkehrswege stehen diesbezüglich in einer Beziehung und geben als zusammenhängende Faktoren Aufschluss über die Entwicklung des modernen Risikosports. Die Entstehung des Risikosports ist damit in komplexe gesellschaftliche und kulturelle Verhältnisse eingebettet, die auf eine wechselseitige Beziehung von Risikosport und Moderne verweisen.

Entgegen den Annahmen eines ,postmodernen' Phänomens, weist der Risikosport also eine klare Traditionslinie auf, die sich aus den gesellschaftlichen Entwicklungen der jungen Moderne entfaltet. Für den Risikosport lässt sich auf diese Weise eine eigene Traditionslinie darstellen, die parallel und zum Teil konträr zu den in der Sportwissenschaft diskutierten historischen Wurzeln des modernen Sports steht. Die Überlegenheit der britischen Alpinisten Mitte des 19. Jahrhunderts erklärt letztlich die Vernetzung des Alpinismus mit der englischen Sportbewegung und damit ihre Einbettung in den Direktive des Sports. Der Risikosport steht trotz dieser dominanten Einflüsse jedoch in einer nicht-linearen Verbindung zur Historie des modernen Sports. Vielmehr zeichnet sich eine eigenständige Entwicklungslinie für risikosportliche Aktivitäten ab. Die weiteren historischen Studien werden prüfen, inwieweit diese bisherigen Überlegungen und Erklärungsversuche belastbar sind. Zunächst wird die Bedeutung von Risiko und Abenteuer bei der Ausübung der Großwildjagd als sportliche Herausforderung des ausgehenden 19. Jahrhunderts evaluiert. Darüber hinaus werden in den folgenden Abschnitten die ideologischen Hintergründe risikosportlicher Aktivitäten evaluiert. Das sportliche Risiko als Erlebnis wird insbesondere bei den ideologischen Vordenkern der heutigen Erlebnispädagogik diskutiert, weshalb eine explizites Quellenstudium dieser Ansätze mit dem Ziel eines erhofften Erkenntnisgewinns durchgeführt wird. 


\subsubsection{EXKURS: Risiko und Abenteuer bei der sportlichen Großwild-} jagd des 19. Jahrhunderts

Wenngleich HANSEN (1991) anführt, dass sich Alpinismus und Großwildjagd in einem diametralen Verhältnis befinden, weil sich die Protagonisten über ihre entsprechenden Aktivitäten voneinander abgrenzen wollten, so erscheint die Großwildjagd, die als dominante Sportbewegung der Aristokratie des ausgehenden 19. Jahrhunderts zu bezeichnen ist, dennoch als risikoreich und abenteuerlich ${ }^{92}$. Ziel dieses Exkurses ist die exemplarische Aufarbeitung von grundlegenden Zusammenhängen der sportlichen Großwildjagd im Bezug auf das bereits diskutierte Risiko im Sport und seine Konzentrierung im Risikosport. Dabei wird neben einigen Primärquellen insbesondere auf die sozialwissenschaftlichen Arbeiten von LEMKE (2000), STEINHART (2001) und RIEDEL (2004) verwiesen, die die Geschichte der Großwildjagd unter soziologisch-psychologischer Perspektivisierung untersuchten.

Zweifelsfrei findet sich für die Jagd als Freizeitbeschäftigung bereits in der geschichtswissenschaftlichen Literatur zum Mittelalter einer reichhaltiger Fundus (vgl. z.B. FLECKENSTEIN 1986). Wenngleich auch für diese Formen mitteleuropäischer Jagdprägungen ein intensives und zugleich nicht ungefährliches Bewegungshandeln zur Zielerreichung erforderlich war, wird ein genuin sportliches Handlungsfeld bisweilen nur in der Großwildjagd des 19. und 20. Jahrhunderts konstatiert. Insbesondere in den kolonialisierten Gebieten Afrikas aber auch in Nordamerika wurde die Jagd auf Großwild zu einer anerkannten - zumeist aristokratischen - Form des Sports (vgl. RIEDEL 2004). Es ist insofern wenig verwunderlich, dass die 1900 vom EARL OF SUFFOLK AND BERKSHIRE publizierte „Encyclopaedia of Sport" in der Mehrzahl Beschreibungen von zu jagenden Großwildarten beinhaltet (vgl. auch FRY 1905). Die ausgeprägteste sportliche Herausforderung wohnte dabei offensichtlich der Büffeljagd inne, wie sie von SAYERS (1930) aber auch ROOSEVELT (1886a; 1886b) beschrieben wird.

„The buffalo is generally regarded as the most dangerous of African game, and to the real sportsman, who has also an accurate, quick and confident shot, buffalo bunting in difficult country is nowhere to be surpassed" (SAYERS 1930, 400)

Zweifelsfrei war die Großwildjagd in Afrika aber auch in den Gebieten Nordamerikas mit vielen Risiken verbunden. Aber welche Rolle spielten Risiko und Abenteuer für die Protagonisten bei dieser sportlichen Herausforderung? RIEDEL hat in einer ausführlichen Quellenstudie der Großwildjagd in Deutsch-Ostafrika zwischen 1891-1916 diesbezüglich festgestellt, dass „[...] sich das Motiv der Abenteuerlust und der Risikosuche in den Quellen zur Großwildjagd häufiger wieder findet" (RIEDEL 2004, 163). Die bewusste Thematisierung von Unsicherheit, die sich ja bereits im Gefahrenalpinismus des 19. Jahrhunderts konstatieren ließ, war damit offensichtlich auch für die Großwildjagd motivationsbildend: 
„Wer in Afrika jagt, hat trotz der überlegenden Schusswaffen immer noch etwas zu wagen. Es ist das Große an dieser Jagd, daß sie eben noch einen Einsatz fordert. Ob Gewehrjagd oder Kamerajagd - immer ist der Mensch dem Zufall anheimgegeben" (PEINER 1937, 33).

Dabei wurde nicht nur die Gefährlichkeit der Großwildarten als riskante Herausforderung erlebt, sondern desgleichen die Auseinandersetzung mit den Naturgewalten wie Hitze, Regenzeiten und Unwettern (vgl. ebd.). Auch vollzieht sich bei der sportlichen Großwildjagd eine dem Alpinismus ähnelnde ,Risiko-Kommunikation': Auch hier werden die durchlebten Abenteuer in diversen Erzählungen literarisch verarbeitet und damit für die unbeteiligte Öffentlichkeit aufgearbeitet. Unterstützt wurde diese Abenteuerliteratur durch die Illustration zahlreicher Bilder, die zunehmend auch durch Photographien ersetzt wurden. Ähnlichkeiten zum Alpinismus offenbaren sich darüber hinaus in der Mythologisierung der Natur, die in der Thematisierung von Freiheit, Einsamkeit und Unberührtheit zum Ausdruck kommt (vgl. RIEDEL 2004, 164).

Bei der Analyse der Primärquellen, die insbesondere auch LEMKE (2000) vorgenommen hat, wird deutlich, dass auch eine gewisse Zivilisationsmüdigkeit zu den zentralen Motivelemeneten der Großwildjagd zu zählen ist. Neben den für den Alpinismus bereits analysierten Gesellschaftsfluchtpunkt einer zunehmenden Rationalisierung und Zivilisierung, bot die Großwildjagd zudem auch das Erleben von Macht und Herrschaft:

„Die ritualisierte Großwildjagd in den Kolonien in Afrika bot nicht nur den Sport, den die Kolonisateure suchten, sondern gleichreitig auch die Möglichkeit die eigene Dominanz und Macht gegenüber den Kolonisierten aufrechtzuerhalten" (LEMKE 2000, 1).

Obwohl eine direkte Verbindung zwischen sportlicher Großwildjagd und einzelnen Sportbereichen des heutigen Risikosports nicht aufgezeigt werden kann, so ist für die Großwildjagd doch eine dem Alpinismus konvergente Entwicklungslinie des Risikos im Sport erkennbar: Das erlebte und kommunizierte Abenteuer dient abermals als Distinktionsgewinn und Kontrapunkt einer sich modernisierenden Gesellschaft. Die Exklusivität der Handlungsräume, die für die Großwildjagd des 19. Jahrhunderts ebenso charakteristisch war wie für den Alpinismus des 20. Jahrhunderts mit seinen Expeditionen nach Asien und Suüdamerika ${ }^{93}$, kennzeichnet darüber hinaus ein weiteres historisch charakteristisches Merkmal des Risiko- und Abenteuersports: Die Flucht aus den sich modernisierenden Gesellschaften Europas wird zugleich zu einer Reise in die Ferne, die von KÖCK (1990) als das zentrale Element des Abenteuers beschrieben wird. Noch heute findet diese Spezies des sportlichen Abenteuers in Form verschiedener Expeditionen eine Traditionslinie ${ }^{94}$. Die im vorstehenden Abschnitt aufgezeigten historischen sowie sozialkulturellen Vernetzungen des Risikosports verdichten sich damit für ein weiteres Handlungsfeld, wenngleich die Protagonisten - will man den Ausführungen HANSENS' $(1991,88)$ glauben - unterschiedlichen sozialen Milieus angehörten. 


\subsubsection{Das sportliche Risiko als Erlebnis - Die ideologischen Hinter- gründe des Risikosports}

Risikosport wird heute nicht nur als Freizeitsport betrieben sondern findet neben dem Tourismus auch einen Einsatz in der Sport- und Erlebnispädagogik. Der Rückgriff auf Risikosportarten wie dem Bergsteigen, Klettern, Kajakfahren, Mountainbiken oder Segeln in der Erlebnispädagogik als pädagogisches Handlungsfeld erfolgt dabei nicht intuitiv sondern theoriegeleitet und reflexiv (vgl. SCHULZE 1995; FISCHER \& ZIEGENSPECK 2000a; 2000b; HECKMAIR \& MICHL 2002). Risiko, Wagnis und Abenteuer sind in der Erlebnispädagogik somit konstitutive Elemente des pädagogischen Konzeptes, welches zur Persönlichkeitsentwicklung oder Teambildung eingesetzt wird (vgl. hierzu SCHÖT'TLER 1994; THIERSCH 1995; SEIFERT 2005). Obwohl die Rezeption und die theoretische Reflexion über Erlebnispädagogik im deutschen Sprachraum erst in den 80er Jahren des 20. Jahrhunderts eine systematische Vorgehensweise erkennen lässt, blickt die europäische Erlebnispädagogik auf eine lange Tradition zurück. Ziel der folgenden Ausführungen ist die Darstellung der historischen Grundlagen, auf denen sich die Erlebnispädagogik aufbaut und die Elemente eines sportlichen Risikoerlebnisses als pädagogisches Kategorien in ihre Konzeption einbaut. Hervorzuheben ist hierbei insbesondere die frühe theoretische Auseinandersetzung mit Erziehungsidealen und Gesellschaftswirklichkeiten, die Aufschluss über das ideologische Verhältnis von Gesellschaft und Risiko, sowie über die kulturkritische Implikation des Risikosport geben können.

Die theoretische Reflexion erfolgt dabei zunächst entlang der grundlegenden Theorien von Rousseau (1712 - 1778), Thoreau (1817 - 1862) und Dilthey (1833-1911) die als die Wegbereiter ${ }^{95}$ der Erlebnispädagogik betrachtet werden. Obwohl Simmel (1858-1918) nicht zu den klassischen Theoretikern der Erlebnispädagogik gehört, sind seine Ausführungen über das Abenteuer von grundlegender Bedeutung. Die Inhalte seiner philosophischen Abhandlung über das Abenteuer wird deshalb ebenso expliziert wie die von Russel (1872-1970). Schließlich wird die Erlebnistherapie nach Habn (1886 - 1974) ausgeführt, da sie für die Wirklichkeit heutiger erlebnispädagogischer Programme besonders relevant ist und die kulturkritische Perspektive für risikosportliche Aktivitäten verdichtet.

\subsubsection{Die natürliche Erziehung bei Rousseau}

Jean-Jacques Rousseau (1712 - 1778) - geboren in Genf - gilt als einer der bedeutendsten Philosophen und Pädagogen der sich konstituierenden Moderne. Rousseau kam als Sohn eines Uhrmachers aus einfachen Verhältnissen und absolvierte eine Lehre als Kupferstecher. Mit 16 Jahren verließ er seine Heimatstadt Genf, um sich in den Metropolen der damaligen Zeit autodidaktisch fortzubilden.

Sein Werk entsteht im Umfeld der französischen Aufklärung, die Vernunft als Quelle jeder Erkenntnis und als Leitfaden menschlichen Handelns ansahen. Zur Aufklärung gehörte eine eindeutig rationalistische Tendenz, 
die aus der Vernunft des Menschen heraus auf die Mündigkeit und Freiheitsideale des neu geborenen Individuums argumentierte. Als politische Argumentation und Geisteshaltung breitete die Aufklärung sich im 18. Jahrhundert von Frankreich aus flächendeckend aus. Ihre erkenntnistheoretischen Höhepunkte erlangte sie in den philosophischen Systemen des deutschen Idealismus von Kant (1724-1804) und Hegel (1770-1831).

Die zentrale pädagogische Forderung sowie philosophische Aussage Rousseaus besteht in einer bewusst proklamierten Abkehr von den Zwängen der feudalen Gesellschaft, die gemeinhin im Appell des ,zurück zur Natur ${ }^{96}$ ' zusammengefasst wird (vgl. FISCHER \& ZIEGENSPECK 2000, 101).

„Alles ist gut, wie es aus den Händen des Schöpfers kommt, alles entartet unter den Händen des Menschen" (ROUSSEAU 1975, 9).

Dieser erste Satz in Rousseau's Abhandlung ,Emile' von 1762 kennzeichnet seine diskrepante Auffassung gegen die empiristische und sensualistische Erkenntnistheorie, wie sie sich beispielsweise bei Locke (1632-1704) findet und nimmt gleichsam die romantische Bewertung der Natur vorweg, die für sein Werk von zentraler Bedeutung ist. In der Aufklärung stand der Naturzustand als Begriff für den ursprünglichen Zustand des Menschen, bevor es organisierte Gesellschaftsformen gab. Der Forderung der Aufklärungstheoretiker, diesen Zustand zu verlassen, um nicht mehr Sklave der Natur zu sein, sondern sie als ihr Gesetzgeber und Subjekt für sich zum Objekt zu machen, widersprach Rousseau. Nur wer sich rückwärts wendet und in der Natur die Einfachheit sucht, nach dem Ursprung schaut und die Sprache der Natur verstehen lernt, nähert sich - seiner Theorie zufolge dem Guten (vgl. HECKMAIR \& MICHL 2002, 5). In pädagogischer Perspektive plädiert er für eine natürliche Erziehung ohne gesellschaftlichen Einfluss ${ }^{97}$, die sich in der Reformpädagogik des 19.Jahrhunderts als ,Erziehung vom Kinde aus' wieder findet. Mit dieser erkenntnistheoretischen Vorstellung von Erziehung vertritt Rousseau die Ansicht, dass sich das Kind zunächst selbst finden muss, bevor es in die gesellschaftlichen Zwänge eingebunden wird. Das Kind kann, soll und darf demnach seine natürlichen Bedürfnisse kennen lernen.

Erlebnis, Erfahrung und Abenteuer tragen als wichtigste Säulen die Erziehungsutopie Rousseaus. Durch das unmittelbare sinnliche Erleben folgt das Kind seinen wahren Bedürfnissen, die von der Natur vorgegeben sind. Auch Risiko und Gefahr wird von Rousseau als Erlebnisdimension thematisiert. Dabei nimmt er Ansätzen und Theorien des heutigen Risikosports vorweg, die sich beispielsweise in den Ausführungen SEMMLERs (1994) wieder finden:

„Man muss sich mir der Gefahr selbst vertraut machen, um zu lernen, sie nicht mehr zu fürchten" (ROUSSEAU 1975, 119).

Das Handeln in der freien Natur wird bei Rousseau nicht nur zur tragenden Säulen des pädagogischen Konzeptes sondern steht auch im Zentrum seiner philosophisch-gesellschaftskritischen Ausführungen ${ }^{98}$. Schon in 
seiner Schrift „Discours sur les sciences et les arts“ von 1750 klagt Rousseau Kultur und Gesellschaft dahingehend an, dass sie der Entfremdung der menschlichen Zusammenhänge von ihren natur- und lebensnahen Urzuständen zu sehr den Weg bereitet und damit vor allem auch der jungen Generation den Zugang zur Freiheit versperrt hätte (vgl. FISCHER \& ZIEGENSPECK 2000, 122). Nur im Naturzustand kann der Mensch demnach ein ursprüngliches und glückliches Leben führen. Während der Mensch in der Gesellschaft fremd bestimmt sei, finde er in der Natur zur Freiheit von dieser Fremdbestimmtheit zurück. Dabei steht das unmittelbare Naturerlebnis - als Gegenwelt zu den Entartungen der Zivilisation - im Zentrum seiner zeitgeschichtlichen Kulturkritik. Gerade die Unbestimmtheit der Natur mache sie zu einem Ort der Freiheit von der Gesellschaft (vgl. AMSTÄDTER 1996, 27). Natur wird bei Rousseau nicht zur ästhetischen Betrachtung sondern zum Ort erlebbaren Abenteuers:

„Ich verlange Gießbäche, Felsen, Tannen, dunkle Wälder, Berge, raube aufund abfübrende Pfade und recht fürchterliche Abgründe neben mir" (ROUSSEAU rit.n. FRIEDELL 1982, 727).

Die von Decartes als zentrale These des rationalen Humanismus geprägte Aussage ,Ich denke also bin ich’ wendet Rousseau in „Ich erlebe, also bin

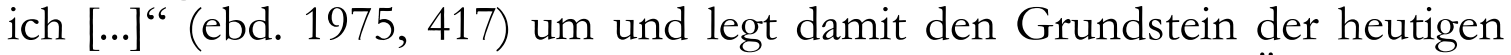
Erlebnispädagogik und des Outdoor-Trainings (vgl. auch DÜPPE 2004, 48f).

Insbesondere mit seinen 1761 erschienenen Romanen „Julie oder die neue Heloise“ und „Geschichte zweier Liebenden am Fuße der Alpen“ beeinflusste Rousseau die zeitgeschichtliche Naturwahrnehmung. Der Naturprogrammatik Rousseau folgend, sucht der beginnende bürgerliche Alpentourismus der ,Künstlichkeit' des zivilisierten Alltags zu entfliehen ${ }^{99}$. Zweifellos steht die Kritik, die sich bei Rousseau mit der Sehnsucht nach dem natürlichen Erleben reflektiert, im sozio-kulturellen Kontext der feudalabsolutistisch-bürgerlichen Übergangsgesellschaft. Als Vordenker der Romantik entwirft Rousseau aber das Naturerlebnis als Kontrast zur Zivilisation, zu Künstlichkeit, kommunikativer Zwanghaftigkeit und wissenschaftlicher Rationalität, welches sich ein Jahrhundert später in den Dokumenten des Alpinismus als Legitimation des Bergsteigens wieder findet. Die Bedeutung Rousseaus für die Entwicklung des aktiven Alpinismus ist damit besonders aufschlussreich (vgl. AMSTÄDTER 1996, 25ff). Für die Rekonstruktion der Historie des Risikosports erhärtet sie die Annahme der kulturkritische Bedeutung der aktiven Naturüberwindung als zivilisatorischer Gegenweltentwurf 100 .

\subsubsection{Das Naturerlebnis als Selbsterfahrung bei Thorean}

Auch David Henry Thoreau (1817 - 1862) begründet seine Erziehungstheorien und philosophischen Abhandlungen moralisch und argumentiert damit ähnlich wie Rousseau kultur- und zivilisationskritisch. Allerdings liegen zwi- 
schen Rousseau und Thoreau ein ganzes Jahrhundert und ein ganzer Ozean, so dass die sozio-kulturellen Ausgangsbedingungen nicht unbedingt vergleichbar sind. Zwar ist die Rezeption von Thoreau im europäischen Raum wesentlich geringer, für die amerikanische Philosophiegeschichte sowie die Entwicklung der Erlebnispädagogik gilt aber Thoreau gleichsam als wichtiger Vordenker. Entgegen dem Theoretiker Rousseau, der seine eigenen Kinder in ein Findelhaus gab, um seine Erziehungsutopie zu entwickeln, setzt Thoreau seine philosophischen Ansätze in praktisches Handeln um und gibt seinen theoretischen Abhandlungen damit einen pragmatischen Rückhalt.

Thoreau studiert an der Harvard University und gründet 1838 in Concord / Mass. eine Privatschule, an der er auch selber unterrichtete. Beeinflusst von der Philosophie Emmersons, der als Hauptvertreter des amerikanischen Transzendentalismus gilt, zieht sich Thoreau 1845 zu einem Experiment in eine einsame Blockhütte am, Walden-See' zurück. Diese Erfahrung des Ausstiegs beschreibt er später als ,Walden-Experiment' auf der er sein philosophisches Denkgebäude aufbaut. Im Zentrum seiner Überlegungen steht die ursprüngliche und unmittelbare Erfahrung, die insbesondere in der Natur zu einem erkenntnisreichen Prozess werden kann. Kulturkritisch beklagt auch er - ähnlich wie Rousseau - den Verlust des unmittelbaren Erlebens durch den herrschenden Zeitgeist, durch Luxus, Bequemlichkeit, Zivilisation und Technik (vgl. HECKMAIR \& MICHL 2002, $11 \mathrm{ff}$ ).

\section{„Das meiste von dem, was man unter dem Namen Luxus zusammenfasst, und viele der sogenannten Bequemlichkeiten des Lebens sind nicht nur zu ent- beheren, sondern geradezu Hindernisse für den Aufstieg des Menschenge- schlechts"(THOREAU 1971, 26).}

Was Thoreau von anderen zeitgenössischen Kulturkritikern unterscheidet, ist seine wiederholte Betonung der Unmittelbarkeit des erlebten Augenblicks und das Erlebnis als authentische Selbsterfahrung. Diese Erfahrungsmöglichkeiten stehen demnach in der gesellschaftlichen Wirklichkeit nur bedingt zur Verfügung, in städtischen Ballungszentren werden sogar Erfahrungsdefizite sichtbar. Gerade die Natur ermögliche derartige reale Situationen, die auch die Erfahrung von Risikobewältigung einschließt. Die aktive Naturbegegnung wird bei Thoreau zu einer Reise nach innen im Sinne eines natürlichen Läuterungsprozesses, der den ,neuen' Menschen hervorbringt (vgl. HECKMAIR \& MICHELS 2002, 16). Dieses Idealbild des ,richtigen' Menschen findet sich auch bei Rousseau wieder, der in seiner ,Emile' einen entsprechenden Idealtypus entwirft. Da Natur allen Menschen als Lern- und Erfahrungsfeld zur Verfügung steht, ist nach Thoreau nun aber jeder selbstverantwortlich in der Lage diese Auseinandersetzung zu suchen ${ }^{101}$ (vgl. DÜPPE 2004, 51ff).

Obwohl Thoreau Sport als spezifische Form der Naturerfahrung nicht anführt, findet sich in seiner Argumentation ein impliziter Legitimationsund Erklärungsversuch für natursportlicher Aktivitäten. Analog zu Rousseau stehen derartige Aktivitäten dabei auch in seinen Überlegungen in einem 
zivilisationskritischen Zusammenhang, der auf den Gegenweltcharakter der Natur als Handlungsraum verweist. Nur die unmittelbare bewegungsvermittelte Naturaneignung - so der Philosoph - kann die Defizite der neuzeitlichen Gesellschaft ausgleichen. Kurzum: auch hier manifestiert sich die Überlegung eines Gegenweltkonzepts für risikosportliche Aktivitäten gegenwärtiger Prägung.

\subsubsection{Das Erlebnis als Voraussetzung des Erkenntnisprozesses (Dilthey)}

Weiterhin sind auch die philosophisch-psychologischen Überlegungen von Wilhelm Dilthey (1833-1911) hervorzuheben, der mit seinen Abhandlungen zum Erleben die Erlebnispsychologie und -soziologie maßgeblich beeinflusst hat (vgl. DILTHEY 1971). Erleben meint bei Dilthey keine bloße Innerlichkeit im Sinne reiner Gefühlszustände, sondern den ganzheitlichen Lebensbezug als eine unhintergehbare Form der Welterfahrung (vgl. SCHWIER 1998b, 129). Der von ihm konzipierte Dreischritt „Erlebnis, Ausdruck und Verstehen“" ist der geisteswissenschaftlich-hermeneutisch orientierten Pädagogik zuzuordnen:

„Wir verstehen einen Menschen nur, indem wir mit ihm füblen, seine Regungen in uns nacbleben" (DILTHEY 1971, 54).

Nach seiner Auffassung ist das Erleben das subjektive Innewerden von Vorgängen, die als bedeutsam empfunden werden und die über die Erfahrung als das Wissen, welches aus den eigenen Erlebnissen hervorgeht, zu Erkenntnissen führt.

"Jeder von uns weiß, dass er Erkenntnis [...] nur aus der Erfahrung ableiten kann"(ebd., 199).

Auch wenn für DILTHEY der natursportliche Bereich wenig Relevanz besitzt und seine pragmatische Pädagogik eher auf musischen oder künstlerischen Erlebnissen aufbaut, besitzen DILTHEY's Ansätze für die sportliche Erlebnispädagogik eine große Bedeutung. Die Hervorhebung des Erlebnisses als Ausgangspunkt eines Erkenntnisprozesses wird schließlich zur Leitmaxime der modernen Erlebnispädagogik, wenngleich insbesondere an den Theorien DILTHEY's deutlich Kritik geübt wird. So kritisiert insbesondere OELKERS, dass das Erlebnis an sich noch keinen pädagogischen Wert besitzt und darüber hinaus gar nicht immer wünschenswert sind (vgl. OELKERS 1992, 105ff).

Diese Verschmelzung von Objekt und Subjekt, von Außen- und Innenwelt wird unter psychologischen Gesichtspunkten bei CSIKSZENTMIHALYI (1993) zum zentraler Bezugspunkt der sog. FlowTheorie und besitzt damit Einfluss auf die Erklärung risikosportlichen Verhaltens.

Zentrale Gemeinsamkeit der philosophisch-pädagogischen Überlegungen bei Rousseau, Thoreau und Dilthey zeigen sich zum einen in der Zentrierung auf die unmittelbare Erfahrung im Sinne eines authentischen Erleb- 
nisses als Bezugpunkt pädagogischen Handelns und zum anderen auf die Natur als gegenweltlicher Handlungs- und Erlebnisraum. Dabei ist die kulturkritische Argumentation, die sich in der Reformpädagogik fortsetzt, keine Polemisierung gegen die zeitgeschichtliche Entwicklung. Die technischen und naturwissenschaftlichen Errungenschaften der jeweiligen Epochen wurden - von Rousseau bisweilen abgesehen - durchaus anerkannt und für sinnvoll erklärt. Kritisiert wurde vielmehr die zweckrationalistischen Domestizierungen, die die zunehmende Industrialisierung und die damit verknüpften Werthaltungen mit sich brachten und eine Negation von Empfindungen und unmittelbarer Erfahrungsvielfalt zur Folge hatten.

\subsubsection{Die Ambivalenz des Abenteuers bei Simmel}

Obwohl Georg Simmel (1858-1918) kein typischer Wegbereiter der Erlebnispädagogik ist und vielmehr als Soziologe und Philosoph in die Wissenschaftsgeschichte eingeht, sind seine lebensphilosophischen Ausführungen für das ideologische Fundament der Erlebnispädagogik durchaus bedeutend (vgl. HECKMAIR \& MICHL 2002; THIERSCH 1996). Für Simmel strebt der Mensch nach einer aktiven Auseinandersetzung mit seiner Umwelt, um sich zu bestätigen, $\mathrm{zu}$ reproduzieren, $\mathrm{zu}$ steigern und sich $\mathrm{zu}$ überwinden. Zentrum seiner philosophischen Auseinandersetzung im Kontext des Risikosports stellt das Abenteuer dar. Für Simmel ist das Abenteuer eine sinnhafte Form des Erlebens, welche - unabhängig vom Inhalt des Abenteuers zu einem lustvollen Spannungserleben führen kann:

„Seine Atmosphäre [des Abenteuers; Anm. d. Verf.] ist [...] unbedingte Gegenwärtigkeit, das Aufschnellen des Lebensprozesses zu einem Punkt, der weder Vergangenheit noch Zukunft hat und deshalb das Leben mit einer Intensität in sich sammelt, der gegenüber der Stoff des Vorganges oft relativ gleichgültig wird" (SIMMEL 1923., 23).

„Das Entscheidende für diese Tatsache ist, dass das Abenteuer seinem spezifischen Wesen und Reize nach eine Form des Erlebens ist. Der Inbalt, der vor sich geht, macht das Abenteuer noch nicht: daß eine Lebensgefahr bestanden [...] wird, [...] das braucht noch nicht Abenteuer zu sein, sondern wird erst durch eine gewisse Gespanntheit des Lebensgefïhls, mit dem solche Inhalte sich verwirklichen [...]" (ebd., 22).

Die Differenzierung zwischen Form und Inhalt stellt die Grundlage späterer psychologischer Ansätze zur Erklärung des Risikosports dar. Der Verweis auf die für das Abenteuer notwendige subjektive Erwartungshaltung, die sich in Form einer Gelingensspannung ausdrückt, gilt darüber hinaus sowohl für die Erlebnispädagogik als auch für den Erklärungszusammenhang des Risikosports als eine wichtiges Element.

Herauszuheben ist bei SIMMEL aber insbesondere die Ambivalenz des Abenteuers. Das Abenteuer als herausgehobenes Ereignis ist charakterisiert durch den inneren Widerspruch, das Ergreifen einer Chance und das sichÜberlassen an Gewalten und Chancen in der realen Welt. Gerade diese Am- 
bivalenz - so wird vor allem im Rahmen der weiteren interdisziplinären Betrachtung deutlich - wird zum zentralen Erkenntnisgewinn. Dabei wird das Abenteuer bei Simmel eine Art Grundfiguration von Leben überhaupt, wie es sich in der Lebensstruktur zwischen Leben und Tod im Wandel der Verhältnisse und der Geschichte zeigt, denn die Konfrontation mit Gefahr und ihrer Begegnung als Risiko stellt eine besonders kräftige Erfahrung von leidenschaftlichem und authentischem Erleben dar.

„[...] gerade auf die schwebende Chance, auf das Schicksal und das Ungefähr hin setzen wir alles ein, brechen die Brücken binter uns ab, treten in den $N e$ bel, als müsste der Weg uns unter allen Umständen tragen" (ebd., 19).

Die bei Rousseau und Thoreau angeführten Gegensätze von Zivilisation und Natur, von Monotonie und Erlebnis, von Mittelbarkeit und Unmittelbarkeit löst Simmel über die Ambiguität des Abenteuers auf. Entgegen der grundsätzlichen Gegensätzlichkeit der Elemenete bei Rousseau beschreibt Simmel das Abenteuer als vom gesellschaftlichen Alltag abhängige Komponente eines ganzheitlichen Handelns. Bei Simmel gibt es damit kein entweder oder, kein gut oder schlecht, sondern nur die Verbindung von gesellschaftlicher Wirklichkeit einerseits und erlebten Abenteuer andererseits. Nur durch die Gegensätzlichkeit wird das Abenteuer überhaupt als ein solches erlebt. Das Abenteuer als bildendes Erlebnis greift letztlich mit den einzelnen Inhalten des Alltags ineinander und bildet damit einen einheitlichen Lebensprozess.

„Indem das Abenteuer aus dem Zusammenhange des Lebens herausfällt, fällt es gleichsam eben mit dieser Bewegung wieder in ibn binein, ein Fremdkörper in unserer Existen₹, der dennoch mir dem Zentrum irgendwie verbunden ist. Das Außerhalb ist, wenn auch auf einem großen und ungewohnten Umweg, eine Form des Innerbalb" (ebd., 13).

Die Betrachtungen Simmels zum Abenteuer als Erlebnisform sind insofern bedeutsam, als dass sie die Ambiguität des Abenteuers hervorhebt und damit die Verknüpfung des bisher Gegensätzlichen herausstellt. Darüber hinaus betont Simmel die besondere Intensität des aktiven Risikoerlebnisses, die sich in der Konfrontation und Bewältigung von Gefahr vollzieht.

\subsubsection{Erziehung zur Verantwortung (Hahn)}

Während Simmel eher als Randfigur für die Entwicklung der Erlebnispädagogik betrachtet wird, gilt Kurt Hahn (1886-1974) als die zentrale Persönlichkeit für die Entwicklung der Erlebnispädagogik ${ }^{102}$. Hahn wird in der Literatur sowohl als engagierte, vitale Persönlichkeit, als Pädagoge und Schulgründer gewürdigt, als auch als Sammel- und Kristallisationspunkt philosophischer und pädagogischer Traditionen der Erlebnispädagogik angeführt. Hahn gilt diesbezüglich nicht als expliziter Theoretiker sondern als pragmatischer Pädagoge und Politiker, der die Zusammenführung unterschiedlicher philosophischer und pädagogischer Strömungen wohl eher unbewusst als 
beabsichtigt vollzog (vgl. HECKMAIR \& MICHELS 2002, 23). So ist die ,Erlebnistherapie' bei Hahn trotz aller Entwicklungslinien auch keine explizite Theorie, die wissenschaftlichen Ansprüchen genügen kann, sondern eine Zusammenfassung fragmentarischer Bausteine einer praktischen Pädagogik.

In seinem Hauptwerk von 1958 „Erziehung zur Verantwortlichkeit“, welches eine Sammlung von Reden und Aufsätzen darstellt, verknüpft Hahn sinnreiche Erfahrungen als auch philosophisch-pädagogische Ansätze zu einem ersten operationalisierbaren Konzept der ,Erlebnistherapie’. Als die Arbeit von Hahn beeinflussende Autoren gelten neben den oben angeführten Persönlichkeiten vor allem Lietz. (1868-1919); Nobl (1879-1960) aber auch Pestalozzi (1746-1827) und Goethe (1749-1832). Dabei setzt Hahn an den von der Reformpädagogik formulierten Ideen der Einfachheit und Echtheit, der Natur und des Erlebens, der Unmittelbarkeit und des Augenblicks an, wobei er diese Prinzipien in einer neuen Weise zueinander in Beziehung setzt und Gewichtungen vornimmt ${ }^{103}$.

Als Ausgangspunkt der ,Erlebnistherapie’ bei Hahn steht - wie schon bei Rousseau und Thoreau - eine kritische Gesellschaftsdiagnose, in der er allgemeine Verfallserscheinungen der modernen Gesellschaft diagnostiziert. Hahn's kritischer Blick fokussiert insbesondere die technische großstädtische Zivilisation, die zu „sozialen Seuchen“ führen können und einen allgemeinen Verfall der menschlichen Anteilnahme provoziert (vgl. VON HENTIG 1966, 45). Er selbst versteht Pädagogik vor diesem Hintergrund als Therapie, weil seine zeitdiagnostischen Zerfallserscheinungen der Gesellschaft im Sinne von Krankheiten damit geheilt werden sollten. In den Mittelpunkt seiner ,Therapie' steht das Erlebnis als ursprüngliche Form der Selbst- und Sozialerfahrung.

\section{„Es ist Vergewaltigung, Kinder in Meinungen bineinaurwingen, aber es ist Verwabrlosung, ibnen nicht zu Erlebnissen zu verhelfen, durch die sie ibrer verborgenen Kräfte gewahr werden können" (HAHN 1958, 83).}

Aus diesen Grundüberlegungen entwickelt Hahn schließlich ein „elastisches System an Erziehungsmitteln" (RÖHRS 1966), welches er an verschiedenen Schulen in England und Deutschland implementiert. Unter dem Grundsatz: "The destiny of character is shaped out of the classroom" fordert Hahn Betätigungen „,...] die den Charakter enthüllen, erproben und entwickeln“ (HAHN zit. n. BAUER 1983, 25) und plädiert damit maßgeblich für Natur als pädagogisches Handlungsfeld. Als Hauptelemente seiner Erlebnistherapie entwickelt Hahn hierbei vier Elemente, die er den diagnostizierten ,Zivilisationskrankheiten' entgegensetzt:

- Das körperliche Training in Form von leichathletischen Übungen und Natursportarten wie Bergsteigen, Segeln und Kanufahren.

- Die Expedition als mehrtägige Tour, meist in herausfordernden Naturlandschaften, verbunden mit Planungs- und Vorbereitungsphasen sowie der Einbettung alltagspraktischer Tätigkeiten.

- Das Projekt als handwerklich-technische oder künstlerische Herausforderung, als zeitlich und thematisch in sich geschlossener Prozess. 
- Der Dienst als soziales Element der Erlebnistherapie. Je nach Standort werden erste Hilfe, Berg- oder Seerettung bzw. andere gemeinnützige Dienste vollzogen.

- Die eigentliche charakterbildende Wirkung der Erlebnistherapie wird bei Hahn erst in der gegenseitigen Verzahnung und im tatsächlichen Vollzug des Zusammenspiels ihrer Elemente unter dem Motiv des Erlebnisses voll sichtbar (vgl. SCHWARZ 1968, 38f).

Für Hahn besitzen insbesondere die Bewährungssituationen besonderen erzieherischen Wert. Dabei zieht Hahn verschiedene Bezugsgrößen für Bewährungssituationen heran: Neben natursportlichen Wagnissen, werden auch Landschaftseindrücke, ungewohnte Tätigkeiten und soziale Herausforderungen als Bewährungsaufgaben herangezogen.

Trotz der Vielfalt erzieherischer Elemente in der Pädagogik Hahn's sind Risiko, Wagnis und Abenteuer dennoch zentrale Elemente der Erlebnistherapie, die sich insbesondere in der pragmatischen Umsetzung in den Hahn'schen Kurzschulen durchgesetzt haben. Die besondere Bedeutung des Risikoerlebnisses wird dabei persönlichkeitsbildenden Prozessen eingeräumt. Hahn's Thesen, die das Risikoerlebnis als ein der Jugend wesensmäBiges Erziehungsmittel fordern, erfahren in der Folge zunehmende Unterstützung aus Wissenschaft und praktischer Pädagogik. HERBART folgert mit Bezug auf Hahn, ,[...] dass Knaben und Jünglinge gewagt werden müssen, um Männer zu werden“ (HERBART 1965, 45). In den Hahn'schen Kurzschulen wird das vernünftig abgewogene Risiko ein unentbehrliches Mittel zur Persönlichkeitsentwicklung und risikosportliche Aktivitäten zu Instrumenten dieser Konzeption. Heute umfasst das Natursportliche Programm innerhalb der Kurzschulen unterschiedliche Sportarten wie Bergsteigen, Klettern, Kajak und Rafting, Skilaufen, Segeln und Rudern (vgl. NEUMANN 1999, 20).

Das Erleben wird nach Hahn in der Gemeinschaft realisiert, indem unmittelbare und ernsthafte Herausforderungen gemeinsam bewältigt werden. Erlebnisse werden im Rahmen dieses Konzeptes zu Kraftquellen, die ihre pädagogische Wirkung insbesondere aus dem Intensitätsgrad des Erlebnisses ziehen und damit Konsequenzen von Verhalten in konkreten Handlungen erfahrbar werden. Elemente des sportlichen Risikos werden bei Hahn bewusst als erlebnisreiche Therapieform eingesetzt, insbesondere als Sport in der Natur. Entgegen der Tradition der Bewegung der Landerziehungsheime, die ihre Schüler gänzlich fern von der menschlichen Zivilisation halten wollen, plädiert Hahn nicht für einen reinen Naturalismus, sondern für ein Nebeneinander von Natur und Zivilisation.

Die Bedeutung Kurt Hahns in der Historie des Risikosport liegt zweifelsfrei in der Durchsetzung von abenteuerlichen Erlebnissituationen in konkrete pädagogische Programme. Auf der Basis der Erlebnistherapie finden Risikosportarten bis heute eine weite Verbreitung in der Erlebnispädagogik, z.B. in den von Hahn gegründeten Outward Bound-Schulen. 


\subsubsection{Konklusion: Das Risiko als Sinnfindung}

Zusammenfassend lassen sich die historischen Ansätze zum Risiko als Erlebnis in die kulturkritische Gesellschaftsdiagnose einordnen, die schon im Zusammenhang des Alpinismus entworfen wurde. Die Grundannahme kann damit erhärtet werden: Die Erlebnisdimensionen risikosportlichen Handelns sind intensive Formen der Selbsterfahrung und lassen sich deshalb pädagogisch durchaus operationalisieren. Ausgangspunkt der vorgestellten Ansätze sind gesellschaftskritische Überlegungen, die von einer Domestizierung des Menschen durch Modernisierungsprozesse ausgehen. Das sportliche Risiko dient demnach der Kompensation gesellschaftlicher Defizite, die sich aus Prozessen der Industrialisierung, Rationalisierung und Zivilisierung für die Individuen ergeben. Durch das Erlebnis von Risiko- und Wagnissowie die Bewältigung von Bewährungssituationen eröffnet sich insbesondere dem jugendliche Individuum eine persönlichkeitsbildende Perspektive. Während bei ROUSSEAU und THOREAU noch für totalitäre Gegenweltentwürfe - im Sinne einer Abkehr von der Gesellschaft - plädiert wird, relativieren sich diese Vorstellungen bei SIMMEL und schließlich bei HAHN im Sinne eines temporalen, Ausstiegs' auf Zeit. Hervorzuheben ist insbesondere die Ambiguität des erlebten Risiko im Verständnis SIMMELs, der damit auf die Doppelwertigkeit risikosportlicher Aktivitäten hinweist.

Sowohl bei ROUSSEAU als auch bei THOREAU wird das Risiko als kompensatorisches Erlebnis nicht als pädagogische Kategorie expliziert. Erst bei SIMMEL und DILTHEY wird das sportliche Risiko als bewusste Inszenierung deduziert, welche sich bei HAHN im Konzept der Erlebnistherapie schließlich als Element wieder findet. Risikosport erhält damit eine pädagogische Konkretisierung als bewusst gestaltete Bewegungshandlung, in der gesellschaftliche Defizite kompensiert werden können.

Natur als Handlungs- und Erlebnisraum spielt in den ideologischen Ansätzen eine bedeutende Rolle, vor allem weil die dynamischen Elemente der Gesellschaftstransformation zu einer Ausgrenzung von Natur im städtischen Lebensraum geführt hat. Natur als risikosportlicher Handlungsraum gewinnt damit über die Annahmen zum Alpinismus hinaus an Schärfe.

\subsubsection{EXKURS: Das sportliche Risiko als Abhärtung - historische Gedanken zum Risikosport als Kriegsvorbereitung und Kriegs- ersatz}

Wertet man die zur Verfügung stehenden Literatur- und Quellenlage zur historischen Verortung des Risikosports aus, so wird eine weitere Dimension des historischen Risikos im Sport deutlich: das sportliche Risiko als Kriegsvorbereitung und Kriegsersatz. Insbesondere im Alpinismus des ausklingenden 19.Jahrhunderts werden die Verbindung des sportlichen Risikomoments mit kriegsvorbereitenden Elementen deutlich ${ }^{104}$. Aber auch in den historischen Vorformen des Abenteuersports (z.B. bei HAHN) finden sich Elemente des Wehrsports wieder. Mit der Form eines Exkurses wird her- 
vorgehoben, dass es sich bei dem folgenden Ausführungen eher um nahe liegende Überlegungen als um direkt zum Erkenntnisprozess gehörige Ausführungen handelt. Für das Verständnis der Arbeit stellt dieser Abschnitt nur eine untergeordnete Rolle, so dass dem eiligen Leser ein Überspringen gestattet ist.

\subsubsection{Risikosportliche Aktivitäten als Kriegsvorbereitung}

Der Einsatz sportlicher Bewegung als kriegsvorbereitendes Medium stellt keine Einzelheit dar, sondern verweist auf die Turn- und Gymnastikbewegung des 19. Jahrhunderts als Form der nationalen Wehrertüchtigung. Johann Christoph Friedrich Gutsmuths (1759-1839) Turnbuch, welches 1817 veröffentlicht wurde, besteht in diesem Sinn aus einer Vielzahl kriegsvorbereitender Übungen, dessen letzter Zweck die Verteidigung des Vaterlandes darstellt (vgl. KRÜGER 1993, 36). Auch Friedrich Ludwig Jahn (1778-1852), der als der Vater der deutschen Turnbewegung gilt, konzipierte seine Turnübungen als eine Form der Wehrertüchtigung, sowie als Programm zur Erhaltung der Volksgesundheit ${ }^{105}$. Die Turnbewegung wurde diesbezüglich zum Sammelbecken des nationalpolitischen Aufbruchswillen der Bevölkerung in Deutschland und steht für den romantischen und nationalen Aufbruch in die Neuzeit. Auch die schwedische Gymnastik, die durch Hugo Rothstein (1810-1865) in die preußische Leibesertüchtigung Einzug erhielt, fand in den Militärschulen des 19. Jahrhunderts ihre Anwendung (vgl. ebd.).

Ähnliche Verbindungen lassen sich auch aus den Dokumenten der Alpenvereine extrahieren ${ }^{106}$ und für die Verwandtschaft zwischen Alpinismus und Militär darstellen, auch wenn sich diese Verbindung erst mit Kriegsausbrauch manifestiert.

Die Einbindung alpiner Strukturen als militärische Vorbereitung und Kriegsunterstützung wird dabei erstmals im ersten Weltkrieg deutlich. In Folge der Kriegserklärung Italiens an Deutschland und Österreich wird der Deutsch-Österreichische Alpenverein erstmals zu einer wichtigen Säule des Kriegsgeschehens. Der Verein stellt finanzielle Reserven zur Verfügung, organisiert Sammlungen und Spendenaktionen und zeigt sich für die Ausbildung ,alpiner Referenten' für die Hochgebirgskompanien verantwortlich (vgl. AMSTÄDTER 1996, 203). Diese sollten nicht nur die Gebirgstruppen im Kampf gegen Italien ausbilden sondern auch aus den eigenen Reihen erfahrene Alpinisten rekrutieren. Als sich im Verlauf des Krieges herausstellt, dass insbesondere das Skifahren von besonderem Nutzen für den Kriegsverlauf sein kann, beginnt der Alpenverein mit dem Aufbau einer systematischen Wintersportstruktur. Zwar gab es schon im auslaufenden Jahrhundert militärische Übungen auf Skiern, diese wurden aber überwiegend im Flachland absolviert und fanden in den alpinen Verbänden nur wenig Anklang (vgl. GÖTZ 1996). Erst mit der Verwendung des Skilaufs für den kriegerischen Einsatz, entwickelt der DÖAV eine eigene Wintersportstruktur und stellt seine Infrastruktur dem alpinen Skilauf zur Verfügung (vgl. AMSTÄDTER 1996, 204.). Insbesondere im Gebirgskampf um die Berggipfel Tirols und Kärntens, bei denen es zu heftigen Kämpfen um einzelne Ge- 
birgsketten mit Italienischen Truppen kommt, finden zunehmend Alpinisten ihren militärischen Einsatz (vgl. ebd., 206).

Was hier zunächst wie eine Instrumentalisierung einer Verbandsstruktur aussieht, offenbart bei näherem Hinsehen eine ideologische Verwandtschaft des Handelns und Denkens zwischen Alpinismus und Militär. Die Ideen des Gefahrenalpinismus im ausgehenden 19. Jahrhundert, die sich u.a. in den Schriften von Eugen Lammer finden, propagieren nicht zuletzt die Besteigung schwierigster Routen und Gipfel auch angesichts möglicher Todesgefahren und rücken das Gefahrenmoment in den Handlungsfokus (vgl. auch Abschnitt 3.2.1). Die Ablehnung von Hilfs- und Sicherungsmaterialien, die sich in den Diskussionen um die Sicherheit am Berg in den alpinen Zeitschriften der Jahrhundertwende findet, untermauert die Vorstellung eines autonomen und kühnen Alpinisten, der auch im ,Ernstfall seinen Mann steht' (vgl. ebd., 199). Die Symbolik des ,hakenlosen Bergsteigens' fördert mit der Idee des wagemutigen Helden die Basis der ideologischen Vorstellungen der Gefahrenalpinisten. Dieses Bild entspricht auch den Beschreibungen von Eduard Pichl (1872-1955) und Heinrich Pfannl (1870-1929), die in der Bergbesteigung ,[...] mit eigener Kraft und mit eigenem Können, auf eigene Gefahr und um den Preis eigensten Glücks" (PFANNL 1928, 218) das eigentliche Ziel des Alpinismus sehen.

Die Inanspruchnahme dieser alpinistischen ,Mannestugenden' für die Kriegsführung und Kriegvorbereitung findet sich in der allgemeinen Begeisterung für den ersten Weltkrieg wieder:

„Doch in prüfenden Gedanken finden wir, dass der Alpinismus, wie wir ihn verstehen, in diesem Krieg seine Erfüllung findet, nicht seine Verneinung. Jetzt, wo wir die Sache selbst erleben, den Kampf um Sein oder Nichtsein im Sinne unserer auf Selbstbestimmung gestellten Kultur, den Kampf um das Einzige also, was das Leben lebenswert macht: um die Möglichkeit der $\mathrm{Ge}$ samtheit frei, das ist nach den Gesetzen des eigenen Wesens, und nicht nach fremden Befeblen zu dienen - jetzt wollen wir uns nicht ablenken durch einen Kult, der uns nur Ersatz und Sinnbild dieses Kampfes war in den Zeiten scheinbaren Alltags, [...] der Krieg für uns die Wirklichkeit dessen ist wovon Ihr [die Berge; Anm.d.Verf.] uns nur Bild wart, die Tat zum Symbol" (PFANNL 1914, 250).

Das alpine Bergsteigen wird im Rahmen dieser Interpretation zu einer besonderen Form der Kriegsvorbereitung, der sich im Kampf gegen die Kräfte der Natur assimiliert. Die Überwindung von Naturgewalten, die in Friedenszeiten noch gänzlich zweckfrei erscheint, wird in der ideologischen Bedeutung des Krieges zu einer besonderen Form der Persönlichkeitsbildung und weist auf die Grundstruktur wehrfreudigen Handelns und Denkens im Alpinismus hin:

„Alpinismus und Hochtouristik waren die Schrittmacher für die Verwendung von Truppen im Hochgebirge; sie lehrten den Kampf mit den elementaren Gewalten des Hochgebirges, der bestanden werden muss vor und zugleich mit den militärischen Kampfhandlungen, die schufen die alpine Möglichkeiten und die 
Vorbedingungen für die Art des Krieges [...]" (STEINITZER 1917, 102).

Eine weitere Vernetzung des Risikosports zu Elementen des Wehrsports ${ }^{107}$ als Kriegsvorbereitung findet sich zudem in der Verwandtschaft zwischen erlebnispädagogischen Programmen in der Tradition HAHNs und dem Wehrsport der 30er Jahre. Offensichtlich wird in diesem Kontext zum einen die Ähnlichkeit der Übungsformen, die z.B. in der Überwindung von Hindernissen oder langen Wanderungen bzw. Märschen festzustellen ist, zum anderen - und dies betont KRÜGER (2000) mit Nachdruck - auch in der personalen Vernetzung zwischen Wehrsport und Abenteuerpädagogik. Wenngleich eine wissenschaftlich differenzierte Analyse weiter aussteht, betont KRÜGER, dass

„[...] die moderne Abentenerpädagogik, wie sie in Deutschland mit einem modernen emanzipatorischen Anspruch betrieben wird, dieselbe Wurzel bat, wie die nationalsozialistische Wehrerieiehung"(KRÜGER 2000, 22).

Aber nicht nur in den Übungsformen finden sich laut KRÜGER Ähnlichkeiten, vielmehr stehen auch die Initiatoren von Wehrsport und Erlebnispädagogik in einer inhaltlichen Verbindung.

„Die Göttinger connection vom Pädagogen NOHL zum Leibeservieher ZIMMERMANN, der dann aufgrund seiner Ehe als ,jüdisch versippt" 1937 aus dem Amt scheiden mußte und sich in Schottland Kurt HAHN anschließen konnte, macht die deutsche Wurzel der Outward Bound Bewegung deutlich. [...] Mit deinen Geländespielen als Wehrerziehung lag Zimmermann auf derselben Linie wie der in der NSDAP und später im REM verantwortliche Minister Dr. KRÜMMEL, der erst zu den Förderern Zimmermanns gehörte, ehe er ibn persönlich aus dem Amt entließ. Krümmel [...] legte Wert auf Fitneß, auf Geländelauf und Geländespiel. Hierin war er Zimmermann [...] ähnlich, der Soldatenspielerei verabscheute, mit seinen Geländespielen aber auch der Webrerziebung Vorschub leistete" (ebd.).

Wenngleich der Forschungsstand zur Vernetzung von Risikosport und kriegsvorbereitendem Wehrsport weitere Untersuchungen erfordert, um die hier aufgezeigten Vernetzungen zu festigen, kann ein Zusammenhang zwischen Risikosport und Wehrsport an dieser Stelle doch zumindest hypothesenartig formuliert werden.

\subsubsection{Männlichkeitsideale im kriegsvorbereitenden Risikosport}

Die ideologische Vernetzung von risikosportlichen Aktivitäten wie dem Alpinismus und kriegsvorbereitenden Aktivitäten wird auch in den gemeinsamen semantischen Verwendungen von Begriffen wie Freiheit, Kampf, Mut und Angstlosigkeit deutlich. Im Bild des kämpfenden Alpinisten offenbart sich zudem ein männliches Heldentum, welches sich an den Motiven Eroberung und Bewährung orientiert. Es verwundert insofern nicht, dass es im Rahmen revanchistischer Mobilmachung auch in Vorbereitung zum zweiten 
Weltkrieg zu einer militärischen Instrumentalisierung des männlichen Heldenepos kommt. Insbesondere im Faschismus ist dieses abenteuererprobte Männlichkeitsbild aufgegriffen worden und mit der Propagierung von Härte, Durchsetzungsfähigkeit und Risikobereitschaft instrumentalisiert worden (vgl. ebd.) ${ }^{108}$.

\section{„Das deutsche Volk braucht Männer, die ihre Kraft erproben im Ringen mit den Naturgewalten, braucht eine Jugend, die gestäblt ist in der barten Schule des Lebens" (GALLLAN 1937, 24)}

Die männliche Besetzung des alpinen Erlebnisses wird dabei schon in früheren Beschreibungen deutlich. Die wagemutige Eroberung des Berges wird vielfach sogar zum Symbol für Männlichkeit, die Bewältigung des Risikos zur heldenhaften Tat:

„Seine körperliche und geistige Kraft ganz einzusetzen, um einem grausen Abgrund zu trotzen oder um eine mit Eis gefüllte Keble zu überwinden, deren finstere Schatten seit Anfang der Welt nur den Nebeln und Lawinen zugänglich waren, ist eine Arbeit, die eines Mannes würdig ist. Dem Tod ins Angesicht sehen entwickelt Charakterstärke" (MUMMERY 1895; zit.n. ZIAK 1965, 140).

Die sich hierin wieder spiegelnde Symbolik des Mannes ist mit altverwurzelten Konzepten und archaischen Verhaltensweisen von Männlichkeit verwandt, wie sie sich in überlieferten Mythen, Sagen und Geschichten z.B. in der Ritterideologie der Aventiure - wieder findet: der männliche Held zieht ins gefahrvolle Unbekannte hinaus, um Herausforderungen zu suchen und sich in seinem Können selbst zu erfahren (vgl. GROPPE 1987). Frauen haben hierbei komplementäre Rollen: sie werden erobert oder befreit und sind höchstens als Begleiterinnen aktiv.
„Dem Weibe sind bestimmte Grenzen gezogen, die es vergeblich zu überschrei- ten suchen wird. Der Mutterberuf, seine Gefolgschaft und Störungen bilden die Scheidewand zwischen den Erfolgen des Mannes und denen des Weibes, so- wobl in jeder Kunstbetätigung, als auch im Sport" (ALTKIRCH 1905, 218).

Der Alpinismus bleibt damit auch durch seine ideologische Verbindung zum Militär stark mit Männlichkeitssymbolen behaftet, die sich bis heute nachvollziehen lassen. So wird auch in der aktuellen Öffentlichkeitsarbeit der Bundeswehr an derartige Männlichkeitsidealen angeknüpft, um damit die Attraktivität militärischer Handlungen zu erhöhen (vgl. KÖCK 1990).

Der risikosportliche Alpinismus wird heute noch von Männern dominiert und ist in der Symbolik für Frauen durchaus befremdlich (vgl. KÖHLER 1995; NEDBAL 1999). Die für die Besteigung von Bergen und den Einsatz in kriegerischen Schlachten notwendigen physischen und psychischen Fähigkeiten orientieren sich an Männlichkeitsattributen wie Wagemut, Härte und Angstlosigkeit. Die Frage nach der Verwandtschaft von Al- 
pinismus und Militär wird damit zu einer Frage von Geschlechtersymbolik und Heldenideologie ${ }^{109}$.

Eine formale Ausgrenzung der Frau aus dem ersten risikosportlichen Handlungsfeld - dem Alpinismus - findet auch durch den Ausschluß von Frauen aus den verschiedenen Verbänden statt. Sowohl der englische Alpine Club als auch der Schweizer Alpenklub nehmen bis weit ins 20. Jahrhundert keine Frauen in ihre Vereine auf. Im Deutsch-Österreichischen Alpenverein werden Frauen zwar geduldet, die Zahl aktiver weiblicher Mitglieder bleibt aber verschwindend gering. So weist die Sektion Austria 1899 beispielsweise nur 97 weibliche Mitglieder (5,2\%) in ihren Reihen aus. Lediglich der Verein „die Naturfreunde“ nehmen zu diesem, in jetziger Zeit recht eigentümlichen Standpunkt" Frauen auf (vgl. AMSTÄDTER 1996, 129). Zwar gibt es einige weibliche Tourenberichte, bis heute gelangten Frauen aber nicht in führende Funktionärspositionen und nehmen auch in den Besteigungschroniken allgemein eine untergeordnete Stellung ein (vgl. MADLENERSIGRIST 1996). Wenn Frauen in historischen Quellen Erwähnung finden, dann insbesondere als vereinzelte Begleiterinnen in der Rolle der Gattin oder Schwester.

Risikosport verweist damit in seiner Historie auf ein geschlechtsspezifisches Phänomen, welches in der symbolischen Dominanz von Männlichkeit und der damit einhergehenden Ausgrenzung von Frauen beschrieben werden kann.

\subsubsection{Risikosportliche Aktivitäten als Kriegsersatz}

Die Frage nach risikosportlichen Aktivitäten als Form des Kriegsersatzes erschließt sich über die theoretischen Abhandlungen von Bertrand Russell (1872-1970) und William James (1842 - 1910), die im Rahmen ihrer theoretischen Überlegungen nach einem moralischen Äquivalent des Krieges ${ }^{110}$ forschten und risikosportliche Aktivitäten als präventive Möglichkeiten aufgreifen.

JAMES, der eigentlich als Psychologe tätig war, erklärt den Krieg als in seiner Urform als Resultat eines dem Menschen angeborenen Kampftriebes, welcher ursprünglich wertneutral als Bewegungs- und vor allem Spannungsbedürfnis zu verstehen ist. Der Autor geht davon aus, dass insbesondere Jugendliche in ihren Lebenswelten ständig Möglichkeiten vorfinden müssen, um ihre inneren Spannungen abzubauen (vgl. JAMES 1911). Das Fehlen bzw. die Unterdrückung dieser Bedürfnisse führt letztlich zu Ersatzhandlungen, zu denen JAMES auch die Begeisterung für den Krieg zählt. Auch RUSSELL wendet sich gegen die Vermutung, dass sich Kriege allein aus den Machenschaften von Politikern heraus erklären lassen. Er geht von einem unterdrückten Bewegungs- und Bewährungsbedürfnis junger Männer aus, welche in der modernen Gesellschaft nicht mehr einlösbar erscheinen. Die Ursache von Kriegen sieht der Autor in der Industrialisierung und der damit einhergehenden Unterdrückung von menschlichen Bewegungs- und Spannungsbedürfnissen (vgl. RUSSELL 1930). Die Maschine als Symbol der Industriegesellschaft zwingt die Menschen demnach zu einem pausenlosen 
Leben, wodurch ursprüngliche Bedürfnisse nicht mehr befriedigt werden können und es zu Kompensationsversuchen kommt.

„In modernen Industriebevölkerungen gibt es viele Männer, Frauen und Kinder, für deren bare körperliche Bedürfnisse nicht entsprechend gesorgt ist" (ebd., 100).

„Inzwischen berauben uns die Maschinen zweier Dinge, die sicher wichtige Bestandteile des menschlichen Glücks bilden, nämlich der Handlungsfreibeit und der Mannigfaltigkeit" (ebd., 102).

Als Ausweg zur Vermeidung der mit den Maschinen verknüpften Gefahren sieht Russel die Möglichkeit durch Abenteuer und Wagniserlebnisse „,...] Breschen in die Monotonie zu schlagen“ (ebd.). RUSSELL plädiert dafür, Abenteuer und Wagnis in alle erdenklichen Pausen zu integrieren, um so durch eine Entladung vitaler Kräfte die Kriegslust im Menschen zum Erliegen zu bringen.

„Der einzige Weg zur Vermeidung der mit dem Maschinentum verknüptten Mißstände besteht darin [...] den Sinn während dieser Pausen auf alle Weise aufs hohe Abenteuer zu stimmen. In vielen erlösche die Kriegslust, wenn sie Gelegenheit bätten, ibr Leben beim Alpensport dranzusetzen[...]" (ebd., 104f).

„Wenn jeder Arbeitsmann einen Monat im Jahr zur Verfügung bätte, in dem er nach $W$ abl den Bau eines Flugzeuges erlernen könnte, oder zur Jagd nach Saphiren in der Sahara angespornt würde, oder sonstwie dazugebracht würde, sich in irgendein gefährliches und aufregendes Unternehmen einzulassen, dass rasche persönliche Initiative verlangte, dann gäbe es volkstümlichen Kriegsdurst nur noch bei Weibern und Krüppeln. Diese letzteren Menschenklassen friedliebend zu machen, kenne ich offengestanden keine Methode" (ebd., 105).

Das Risiko als vitales Erlebnis wird bei JAMES und - unter Fortführung dieser Gedanken - bei RUSSELL letztlich zu einer Kompensation gesellschaftlicher Domestizierungen in Folge der Industrialisierung. Natürlich ist bezüglich derartiger theoretischer Ansätze Vorsicht vor direkten inhaltlichen Ableitungen geboten. Die Annahmen eines kausalen Zusammenhangs von militärischen und risikosportlichen Handlungen unterstreicht aber einmal mehr eine mögliche Verwandtschaft beider Systeme. Wie bei SIMMEL wird auch hierbei nicht auf eine konkrete Gegenwelt verwiesen, sondern auf temporale Ausbrüche und den Rückkehr in den Alltag. Der Verweis auf ein quasi ursprüngliches Risikobedürfnis, welches in der Industriegesellschaft unterdrückt wird, erweitert die bisherigen kompensatorischen Ansätze schließlich um eine konkrete gesellschaftliche Funktion des Risikosports als kriegsverhindernde Maßnahme. 


\subsection{Zusammenfassung und Einordnung der theoreti- schen Befunde}

Es bleibt festzuhalten: Trotz aller Schwierigkeiten, geschichtswissenschaftliche Ansätze im Bezug auf eine historische Verortung des Risikosports zu verwenden, erweist sich der historische Forschungszugang für das übergeordnete Erkenntnisinteresse dieser Arbeit als durchaus fruchtbar. Für die Exploration relevanter Erklärungszusammenhänge des Risikosports wurden in diesem Kapitel zwei aufeinander bezogene Zugänge gewählt:

(1) Durch die Darstellung von Unsicherheitswahrnehmungen im historischen Rückblick konnte zunächst die begrifflich-phänomenologische Differenzierung von Unsicherheitskonzepten vorangetrieben werden. Dies ist insofern relevant, als dass sich unterschiedliche Unsicherheitskonzepte unter dem Begriff ,Risiko' historisch zurrückverfolgen lassen: Neben dem zweckorientierten ökonomischen Risiko, lässt sich auch ein eher distinktives Risikobegehren als ethische Leistung identifizieren. Als individualisierte Form von Unsicherheit konnte das Risikokonzept darüber hinaus als relativ späte sozial Errungenschaft demaskiert werden. Risiko verweist mit seiner Verantwortungszuweisung für das Individuum damit auf die Moderne und die mit ihr verbundenen Säkularisierungsprozesse, gleichzeitig aber auch auf Sicherheit und die mit ihr verbundenen Sicherungsprozesse.

(2) Der Rückgriff auf Erkenntnisse über die kulturhistorische Einordnung des Alpinismus und die ideologisch-historischen Beiträge zum Risiko als Erlebnis konnte diesen Zusammenhang bestätigen und des Weiteren die Bedeutung gesellschaftlicher Rahmenbedingungen für den Entstehungszusammenhang des Risikosports verdeutlichen. Risikosportliche Aktivitäten binden sich damit an konkrete Errungenschaften der Moderne.

Was aber ist der zentrale Erkenntnisgewinn dieses historischen Forschungszugangs für das Phänomen Risikosport? Risikosport - so kann hier abschließend formuliert werden - stellt in seiner Grundfigur des Abenteuers ein prägnantes, spezifisches und kompensierendes Produkt der zunehmenden Zivilisation unserer Gesellschaft dar. Das vitale Risiko wird zu einem historischen Kompensationspunkt für eine mit Industrialisierung und Zivilisierung verbundene Moderne. Anscheinend - so legen es die Befunde der historischer Ansätze nahe - steckt im erlebten Risiko eine Erfahrung von besonders kräftigem, leidenschaftlichem und authentischen Erleben, die einer zunehmenden Domestizierung des Alltags entgegensteht. Der Risikosport weist damit aus seiner historischen Entwicklung heraus auf eine eigene Kulturkritik, wie sie sich ansatzweise bei ADORNO (1955) und PLESSNER (1956) für den modernen Sport findet. Zwar lassen sich die bei GUTMANN für den modernen Sport formulierten Elemente auch auf die Entwicklung des Risikosports übertragen, gleichzeitig stehen sie hierzu - im Rahmen ihres Gegenweltcharakters - aber in einem ambivalenten Verhältnis. Der Risikosport argumentiert diesbezüglich aus der Moderne heraus und in die Moderne hinein: Es setzt eine Lebensweise voraus, die bereits zeitgleich 
mit Attributen wie bürgerlich, städtisch, zivilisiert und industriell versehen wird, richtet sich aber gleichzeitig gegen sie.

Zugegeben: dieser Erkenntnisprozess auf der Basis historischer Forschungszugänge vollzieht sich auf einer abstrakten und phänomenologischen Ebene und bietet nur wenig konkrete Hinweise auf der Ebene der handelnden Akteure, dennoch: die Ergebnisse dieses historischen Zugangs stellen ersten Erklärungsansatz für das Verständnis risikosportlicher Aktivitäten dar. Es wird Aufgabe der folgenden Kapitel sein, diese Annahmen unter psychologischen Gesichtspunkten (Kap. 4) sowie soziologischen Perspektiven (Kap. 5) zu überprüfen und durch weitere Aspekte zu ergänzen. 


\section{Was motiviert Menschen zum Risikosport? Anthropologische Überlegungen und Psycholo- gische Erklärungsansätze}

Die Gründe, von denen die Leute glauben, daß sie uns zum Bergsport anreizen, sind ja erwiesenermaßen unrichtig: Wegen der Aussicht würde ich nicht auf die Trettach gehen, die kann ich auch anderswo schöner haben; wegen des guten Einflusses auf die Gesundheit auch nicht, denn der ist bei solchen Anstrengungen zum mindesten zweifelhaft; wegen des Ansehens bei den Menschen würde ich nicht Geld, Gesundheit und Lebens aufs Spiel setze, um ein Unternehmen auszuführen, das doch von den meisten als Beweis von Geistesstörung aufgefasst wird. W as ist es also, das uns aus den sonnigen

Tälern, aus dem Leben der Freude und des Genusses ins wilde, raube Hochgebirge, in ein Leben der härtesten Anstrengungen und

Entbehrungen fübrt?

(Hans Magnus Enzensberger (*1930), dt. Schriftsteller)

In der vorausgegangenen inhaltlichen Charakterisierung des sportlichen $\mathrm{Ri}$ sikos als ein spezifisches Gefahrenrisiko wurde dargelegt, dass ein Scheitern in sportlichen Risikosituationen mit einer Beeinträchtigung der körperlichen Unversehrtheit verbunden sein kann. Nicht nur deshalb fällt es schwer für derartige Aktivitäten vernünftige Sinnzuschreibungen - im Sinne klassischer Motivationen - vorzunehmen. Unter einer zweckrationaler Perspektive ist zunächst kaum nachvollziehbar, warum ein Bergsteiger den beschwerlichen Weg zum Gipfel über gefährliche Grate oder Rinnen sucht, wo ihm doch auch die Gondelbahn zum Gipfel bringen würde. 
Im Mittelpunkt des folgenden Kapitels steht daher die Frage, welche Faszination diese vermeintlich gefährlichen Aktivitäten ausüben und welche Motivation die Aktiven im Bezug auf risikosportliche Aktivitäten entwickeln - oder zweckrationalistisch formuliert ${ }^{111}$ : Für welchen erhofften Gewinn nehmen Sportler diese scheinbar unnötigen Risiken auf sich? Kann bei wirtschaftlichen Risiken dieser Gewinn in Form materieller Verbesserungen demaskiert werden, so erscheint diese Fragestellung im Sport ungleich komplizierter, denn hier wird Geld, Zeit und Energie investiert, ohne dass die Tätigkeit ein Ergebnis herbeiführt, bei dessen Erreichen materieller Profit entstünde. Anscheinend verfolgen Risikosportler andere Ziele mit der Ausübung ihrer Sportart, die es im folgenden zu veranschaulichen gilt.

Mit der Fokussierung auf das handelnde Subjekt im Risikosport wird in diesem Kapitel nach Erklärungsansätze über die kulturhistorischen Annahmen des Risikosports als gegenweltliches Erlebnisfeld hinaus gesucht. Hierbei werden insbesondere psychologische Forschungsansätze expliziert, die durch anthropologische Forschungsperspektiven ergänzt werden. Bei der Beantwortung der Ausgangsfrage dieses Kapitels findet sich dabei eine Vielzahl unterschiedlichster wissenschaftlicher Perspektive. Zunächst erfolgt deshalb eine Übersicht über die verschiedenen Forschungsperspektiven der Motivationspsychologie sowie eine Definition motivationspsychologischer Grundbegriffe (4.1). Einen ersten Hinweis zur Bearbeitung der Fragestellung dieses Kaptitels bieten schließlich anthropologische Annabmen zum allgemeinen Wesen des Menschen und seiner Handlungen (4.2). Darüber hinaus werden verschiedene theoretische Ansätze und empirische Ergebnisse zur Erklärung risikosuchenden Verbaltens im Sport aus dem wissenschaftlichen Diskurs der Psychologie und der Verhaltenstheorie (4.3) geliefert und erläuternd dargestellt.

Ziel der Ausführung ist keine empirische Motivations- und Anreizstudie, wie sie z.B. BEIER (2001) für den Natursport vorlegt, sondern eine möglichst umfassende Übersicht über mögliche Motivationsstrukturen und Anreizelemente im Risikosport. Da bis dato kein expliziter Ansatz zur Erklärung der Motivation im Risikosport vorliegt, werden einzelne empirische Beiträge und deskriptive Ansätze auf ihre Relevanz für den Kontext des Risikosports analysiert und entsprechend ihrer Bedeutung deduziert. In der folgenden Darstellung werden dabei einander ergänzende aber zum Teil auch sich widersprechende Forschungsansätze vorgestellt. Diese methodologische Schwäche einer multidisziplinären Motivationspsychologie wird in Kauf genommen, um die Gesamtheit aller Beiträge zu erfassen und im Bezug auf risikosportliche Aktivitäten systematisch zu entfalten. Vor dem Hintergrund des bestehenden Forschungsdesiderats in der Sportwissenschaft ist eine solche umfassende Darstellung einer theoretisch integrierenden Vorstellung vorzuziehen. Für das übergeordnete Erkenntnisinteresse dieser Arbeit liefert sie zudem verschiedene Erklärungsansätze, um das Risikosport als Handlungsfeld genauer zu verstehen. 


\subsection{Motivationspsychologische Ansätze als theoretischer Bezugsrahmen}

Die Frage nach der Motivationen für risikosportliche Aktivitäten setzt zunächst allgemeine Erläuterungen zu den grundlegenden Konzepten und Vorstellungen der Motivationspsychologie voraus. Diese Einführung in die grundlegenden Forschungsansätze der Motivationspsychologie dient primär als inhaltliche und begriffliche Ausgangsbasis und soll einen theoretischen Rahmen für die darauf folgende differenzierte Anreiz- und Motivationsanalyse darstellen. Angesichts des interdisziplinären Forschungsrahmens dieser Arbeit, wird die Vielfalt der diskutierten Modelle dabei auf die wichtigsten Konzepte reduziert ${ }^{112}$.

Zunächst werden die Paradigmen der modernen Motivationspsychologie sowie die grundlegenden Begrifflichkeiten erläutert. Anschließend wird eine Übersicht über klassische Ansätze der Motivationspsychologie expliziert, bevor schließlich einige Annahmen zur Analyse komplexer Motivstrukturen entwickelt werden.

\subsubsection{Motiv - Anreiz - Motivation: Motivationspsychologische Grundbegriffe}

In der Psychologie hat die Motivationsthematik als die Frage nach dem Warum und Wozu des Verhaltens eine lange Tradition (vgl. SCHÖNPFLUG 2004). Während in frühen Beiträgen noch von Instinkten als Handlungsbasis ausgegangen wurde, befasst sich die neuere Motivationspsychologie neben den Beweggründen zielorientierten Handelns auch mit interpersonalen Differenzen, sowie Kontinuität und Wechsel im Verhaltenslängsschnitt. Dabei ist der motivationspsychologische Zugriff dadurch charakterisiert, dass intendierte Zielzustände und dass, was sie attraktiv macht, die erklärenden Größen sind (vgl. RHEINBERG 2002). Um einen Einblick in motivationspsychologische Ansätze zu bekommen, ist zunächst die Klärung der wissenschaftliche Grundbegriffe erforderlich. Bei den für die Motivationspsychologie relevanten Begriffen Motiv, Anreiz und Motivation handelt es sich um so genannte ,hypothetische Konstrukte', da keine unmittelbare Messung oder Beobachtung möglich ist und sie sich erst durch ihren Erklärungswert bei der Verhaltensinterpretation rechtfertigen. Die hier aufgegriffene Begriffbestimmung orientiert sich an den kognitionspsychologischen Ansätzen, die u.a. von HECKHAUSEN, RHEINBERG oder SCHNEIDER vertreten werden.

In der Psychologie hat sich der Begriff des Motivs zur Bezeichnung überdauernder Dispositionen einer Person durchgesetzt. Einige Autoren sprechen an der Stelle von Motiven auch von Bedürfnissen, wobei insbesondere HECKHAUSEN für eine einheitliche Begriffsbestimmung plädiert. Der Autor definiert Motive als "charakterisierende Wertungsdispositionen“ (HECKHAUSEN 1989, 2): 
„Motiv ist kein Begriff der etwas beschreiben soll, sondern einer, der etwas erklären soll [...]"(ebd., 9).

„Für die Abgrenzung der ,Motive' voneinander wird ein nicht unbeträchtliches Abstraktionsniveau bevorzugt. Jedes Motiv unterscheidet sich binsichtlich einer ihm eigenen Inhaltsklasse von Handlungszielen [...]" (ebd.)

Anschaulich werden Motive und ihre Wirkungen vor allem, wenn Individuen in bestimmten Situationen anders handeln, als es die meisten tun oder man es für normal erachtet. Die moderne Motivationspsychologie klammert dabei angeborene Motive nicht mehr aus sondern geht vielmehr von einer dynamischen Motiventwicklungen aus (vgl. SCHNEIDER \& SCHMALT 2000, 15f). Angenommen werden sowohl genetische Determinationen, als auch Sozialisationseinflüsse und ontogenetische Beeinflussungen.

Unabhängig von den Anteilen und der Bedeutung genetischer Einflüsse bedarf es für eine Handlungsausführungen in jedem Fall situativen Handlungsaufforderungen, die Motive anregen und damit die Ausbildung einer Motivation bewirken. Dieser Ansatz findet sich bereits bei LEWIN, der davon ausgeht, dass der Lebensraum einer Person in unterschiedliche Bereiche gegliedert werden kann und jeder Bereich eine Handlungsmöglichkeit bzw. ein Ereignis darstellt. Diese Anreize werden als ,Aufforderungscharakter' für bestimmtes Handeln verstanden, indem sie wahrscheinliche Folgen in Form erstrebenswerter Zuständen oder Ereignissen erwarten lassen. Anreize signalisieren demnach, dass geeignete Bedingungen zum Ablauf zielgerichteter Handlungen vorliegen. Motive und Anreize sind eng miteinander verbunden, sie sind in gewissem Maße komplementär (vgl. ebd., 17) ${ }^{113}$. Ein Motiv - als personenseitige Verhaltensdeterminante - kann nur in dem Maße verhaltenswirksam werden, wie es durch situative Anreize angeregt wird.

Motivation ${ }^{114}$ als zentraler Begriff greift diese Interaktion zwischen Motiven und Anreizen auf und bezeichnet letztlich die situationsspezifische Ausprägung eines Motivs. RHEINBERG definiert Motivation als ,[...] aktivierende Ausrichtung des momentanen Lebensvollzugs auf einen positiv bewerteten Zielzustand.“ (RHEINBERG 2002, 18). Genauer erklärt HECKHAUSEN Motivation als
„[...] die durch eine gegebene (oder auch aufgesuchte) Situation verursachten Prozesse der Antizipation von erwünschten oder befürchteten Anreizen der Folgen eigenen Handelns und der erwarteten $W$ abrscheinlichkeiten, geeignete Ergebnisse durch eigenes Handeln zu erzielen, und die erwarteten Instrumen- talitäten der Handlungsergebnisse für die in Frage stehenden Folgen" (HECKHAUSEN 1989, 11).

Motivation bezeichnet damit den Gesamtprozess des Handelns als Interaktionsprodukt aus personellen und situativen Momenten einer Verhaltenssequenz. RHEINBERG hat diesbezüglich ein Grundmodell der ,klassischen’ Motivationspsychologie entwickelt, in dem er diese Person-SituationsInteraktion grafisch darstellt: 


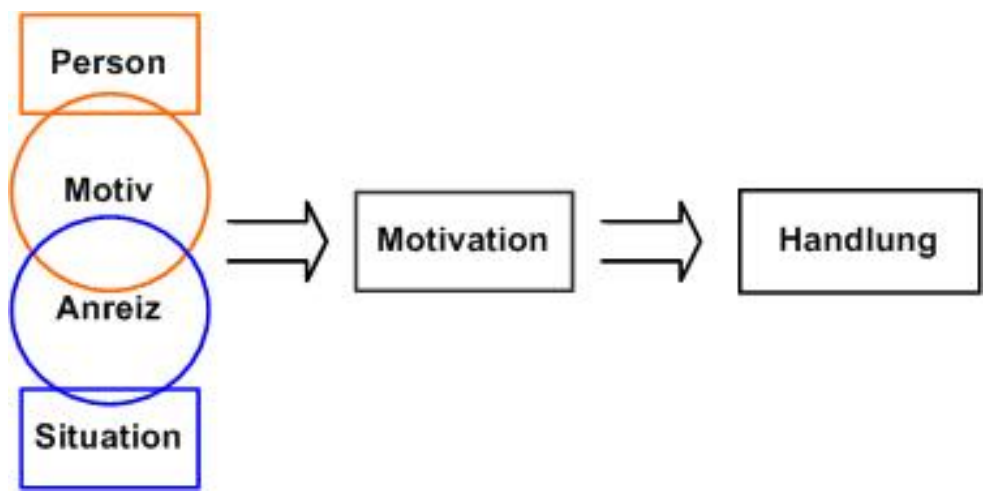

Abbildung 4.1:Grundmodell der klassischen Motivationspsychologie (RHEINBERG 2002, 72).

Motiv, Anreiz und Motivation sind damit als hypothetische Konstrukte in einen Handlungsprozess eingebettet, der - als Prozess verstanden - zu unterschiedlichen Beobachtungsperspektiven einlädt. Während sich die ältere Persönlichkeitspsychologie mit den Verhaltensdifferenzen in gleichen Situationen und zu gleichen Zeitpunkten auseinandersetzt, hat sich insbesondere die Sozial- und Lernpsychologie den situativen Merkmalen angenommen. Die moderne Kognitionspsychologie versucht darüber hinaus im Rahmen des motivationspsychologischen Ansatzes die Wechselwirkungen zwischen personalen und situativen Elementen zu analysieren (vgl. HECKHAUSEN 1989, 5).

\subsubsection{Forschungszugänge der Motivationspsychologie}

Ziel dieses Unterkapitels ist die Darstellung verschiedener Zugänge, die Erklärungsmodelle für das Engagement in risikosportlichen Aktivitäten liefern können. Mit Bezug auf BEIER (2001) ist zu konstatieren, dass bei den folgenden Modellen nicht das tatsächliche Handeln, sondern die Bildung entsprechender Intentionen zur Handlungsausführung die abhängige Größe darstellt.

Im Rahmen motivationspsychologischer Forschung sind eine Reihe von wissenschaftlichen Modellen entwickelt worden, die aus unterschiedlichen Perspektiven und mit divergierenden Methoden der Frage nach den Beweggründen für zielgerichtetes Handeln nachgegangen sind. Auf dem hier zur Verfügung stehenden Raum kann die Vielfalt der publizierten Beiträge kaum vollständig wiedergegeben werden. Vor dem Hintergrund des interdisziplinären Forschungsansatzes dieser Arbeit ist die Darstellung der wichtigsten Forschungsansätze der Motivationspsychologie aber durchaus sinnvoll, um das Verständnis der darauf folgenden Analyse der Motivationsstrukturen des Risikosport zu erleichtern. Die folgende Darstellung wird deshalb auf die bedeutendsten Ansätze reduziert ohne die Vielfalt der Forschungsperspektiven aus dem Blick zu lassen.

Der ethologisch-instinkttheoretische Motivationsansatz, greift die biologische Perspektive der Verhaltenserklärung bei Tieren auf und versucht, human- 
psychologische Phänomene auf der Basis automatisierter Verhaltensmerkmale zu erklären. Angenommen werden hierbei genetisch determinierte Dispositionen, die zu einer angeborenen Handlung führen und durch so genannte Schlüsselreize ausgelöst werden können. Die kognitive Repräsentation sowie kulturelle und soziale Einflüsse der Intentionsbildung werden hierbei weitgehend ausgeklammert. Trotz massiver Kritik an dieser eindimensionalen Betrachtung menschlichen Verhaltens und damit einer Einschränkung des forschungsspezifischen Geltungsbereiches, findet der Ansatz in der Motivationspsychologie durchaus Berücksichtigung. Diese Ansicht gründet auf der Annahme zentralnervöser Strukturen, die im Verlauf der Stammesgeschichte bestimmte Verhaltensweisen kontrolliert haben und auch beim Menschen noch vorhanden sind. Diese geerbten Verhaltensweisen werden in der evolutionären Entwicklung demnach nicht ersetzt, sondern allenfalls überlagert. Im deutschsprachigen Forschungsraum ist eine gewisse Zurückhaltung bei der Integration biologischer-ethologischer Konzepte zu konstatieren (vgl. SCHNEIDER \& SCHMALT 2000, 63f). In der angloamerikanischen Motivationsforschung haben z.B. BUCK (1999) aber auch REEVE (1992) integrative Ansätze vorgelegt.

Evolutionsbiologische Ansätze knüpfen an die Überlegungen DARWINs an, der einem natürlichen Selektionsdruck bei lebenden Organismen beobachtete und daraus ein implizites genzentriertes Fortpflanzungsinteresse ableitet. Diese Perspektive wird für die Motivationspsychologie insofern interessant, als dass das menschliches Verhalten unter dem Blickwinkel seiner Funktionalität für die Gen-Weitergabe zu analysieren ist (vgl. SCHNEIDER \& SCHMALT 2000, 66). Das weitaus wichtigste Forschungsfeld für diesen evolutionsbiologischen Ansatz ist daher auch das Fortpflanzungsverhalten und die damit verbundene Partnerwahl. Der Ausschluss kultureller und sozialer Einflüsse auf die Intentionsbildung wird auch bei diesen Ansätzen gemeinhin kritisiert, wobei gewisse funktionelle Zusammenhänge im allgemeinen nicht geleugnet werden (ebd.).

Als Bezugspunkt der emotionspsychologisch--aktivationstheoretischen Ansätzen dienen zentralenervöse und biochemische Prozesse des Organismus. Es wird davon ausgegangen, dass der Mensch bestrebt ist, das interne Milieu des Organismus - im Sinne einer selbstständigen Regulation aufrecht zu erhalten oder wiederherzustellen. Darüber hinaus ist der Mensch in der Lage als lustvoll empfundene Verhaltensweisen selbst herzustellen und damit seine Reizstimulation selbst zu lenken. Motiviertes Verhalten ist diesem Ansatz folgend eine Reaktion auf einen Ungleichgewichtszustand des Organismus. Durch Verhalten kann der Reizeinstrom und damit auch die hiermit verbundene Emotionsentwicklung beeinflusst werden. SCHNEIDER \& RHEINBERG (1996) haben darauf hingewiesen, dass die Ansätze der Aktivationstheorie, die insbesondere von BERLYNE beeinflusst sind, die Dominanz der neurophysiologischen Prozesse der Motivation akzentuieren. Eine zusammenhängende Erklärung insbesondere von Differenzen im Verhaltenslängsschnitt kann der Ansatz aber nicht erbringen. 
Die tiefenpsychologisch-psychanalytischen Ansätzen der Motivationspsychologie gehen auf die Theoriebildung von FREUD zurück, der von psychischen Prozessen als Ergebnis von inneren Kräften und Konflikten ausgeht ${ }^{115}$. Demnach wird Verhalten durch triebhafte Impulse beeinflusst, deren Herkunft zumeist im Unterbewusstsein verborgen sind. Als Ergänzung der instinkttheoretischen Zugänge geht FREUD dabei nicht nur von Instinkten als Triebquelle aus, sondern schreibt Trieben auch psychologische Qualitäten zu. So entwickeln sich motivationsbildende Triebe z.B. aus pränatalen und frühkindlichen Erfahrungen, die sich im Unterbewusstsein verankern und damit intentionsbildende Funktionen erfüllen (vgl. GABLER 2002, 40). Die wissenschaftliche Errungenschaften dieses Ansatzes für die Motivationspsychologie bestehen zweifelsfrei in der Verbindung von Motivation und Emotionen sowie der Interaktion von Erregungsquelle und Bedürfnisbefriedigung, die FREUD als Grundannahme zur Verhaltenserklärung heranzieht (vgl. SCHNEIDER \& SCHMALT 2000, 70). Gleichwohl sind die kausalanalytischen Zusammenhänge zu hinterfragen und die erkenntnistheoretischen Verknüpfungen zu kritisieren (vgl. KUTTER 2000).

Der Triebbegriff spielt auch in den behavioristische Ansätze eine bedeutende Rolle, wenngleich Verhalten im Behaviorismus als durch Erfahrung gelernt verstanden wird. Lernen erfolgt durch die enge Verknüpfung von beobachtbaren Reiz-Reaktions-Verbindungen, die zu Verstärkungen oder Bekräftigungen bzw. Abschwächungen auf der anderen Seiten führen können. HULL, als führender Vertreter dieser Forschungsrichtung, geht davon aus, dass im Organismus Bedürfniszustände entstehen können, die zu einer erhöhten Triebaktivierung führen können und die ein dieses Bedürfnis befriedigende Verhalten auslöst. Es wird letztlich diejenige Reaktion gelernt und habitualisiert, die in der Lage ist das Bedürfnis erfolgreich zu befriedigen. Motiviertes Verhalten kann aus dieser Sichtweise als Folge von ReizReaktions-Einheiten im Sinne von Gewohnheitsreaktionen verstanden werden (vgl. GABLER 2002, 41f). Auch wenn die empirischen Hinweise aus der behavioristischen Theorie einige interessante Zusammenhänge erklären konnten, so ist die Kritik an diesem Ansatz doch unüberhörbar. Kritisiert wird insbesondere, dass es neben habitualisierten Verhaltenstendenzen auch explorative Verhaltensformen gibt, die keine Gewohnheitsstrukturen besitzen. Gleichzeitig wurde die fehlende kognitive Repräsentation bei der Intentionsbildung kritisiert und in Tierexperimenten nachgewiesen (vgl. SCHNEIDER \& SCHMALT 2000, 76f).

Persönlichkeitstheoretische Ansätze betrachten interindividuelle Differenzen in der Motivationsbildung. Die Persönlichkeit wird im Rahmen der verschiedener Beiträge dieser motivationspsychologischen Richtung als umfassendes Konzept der Individualität des Menschen aufgefasst und die ihr zu zuordnenden Eigenschaften mit Dispositionen beschrieben. Es wird angenommen, dass Personen mit entsprechenden Dispositionen bestrebt sind, diese Motive zielgerichtet zu ,befriedigen'. Die Abgrenzung und Identifikation einzelner Motivklassen wurde im Rahmen dieses inhaltstheoretischen Ansatzes von verschiedenen Autoren untersucht. Personen - so die zentrale 
Annahme dieses Ansatzes - unterscheiden sich danach, wie stark die einzelnen Motive bei den Individuen ausgeprägt sind (vgl. GABLER 2002, 43). Insbesondere MASLOW (1954) hat dabei ein viel diskutiertes System hierarchisch organisierter Motive vorgestellt. MASLOWs Klassifikation ist durch eine Zusammenfassung einzelner Bedürfnisse in übergeordnete Kategorien gekennzeichnet, wobei höhere, Wachstums-Motive' (growth needs) auf den ,niederen Mangel-Motiven' (deficiency needs) aufbauen (vgl. auch HECKHAUSEN 1989, 69ff). Diese Hierarchie allgemeiner Bedürfnisklassen reicht von physiologischen Bedürfnissen über Sicherheitsbedürfnissen bis hin zu Selbstverwirklichungsmotiven. Besonderes die Unterteilung der Motive im Rahmen der Persönlichkeitsentwicklung im Sinne niedriger Bedürfnisse am Beginn und Selbstverwirklichungsmotive am Entwicklungsende haben die Motivationspsychologie maßgeblich erweitert. Die höheren Motive der Selbstverwirklichung und schließlich der Transzendenz sind für MASLOW Ziele emanzipatorischer und mündiger Menschlichkeit, in der das Individuum seine eigenen Wünsche realisiert. Sie sind der Gegenpol der niedrigen Mangelmotive der physiologischen Bedürfnisse und dem Streben nach Sicherheit.

„Even if all these needs are satisfied, we all may still often (if not always) expect that a new discount and restlessness will soon devolop, unless the individual is doing what he, indivually, is fitted for" (MASLOW 1954, 91).

Im Gegensatz zu behavioristischen oder tiefenpsychologischen Forschungsansätzen, die den Menschen eher als reaktives Wesen verstehen und die die auslösenden Bedingungen für ein Verhalten in der Gegenwart als Reaktion auf einen Reiz oder in der Vergangenheit suchen, stehen kognitivhandlungstheoretische Ansätze in der Tradition klassischer anthropologischer Theorien. Hierbei wird der Mensch als ein bewusst planendes und zukunftgerichtetes Wesen verstanden, der sich Ziele setzt und Handlungen wählt, um diese Ziele zu erreichen (vgl. GABLER 2002, 44ff). Grundannahme ist damit ein rational entscheidendes Wesen und die Fähigkeit zur Selbstreflexion. Zentrales Konzept der meisten kognitiven Theorien ist die Erwartung als wahrgenommene Zusammenhänge zwischen eigenem Verhalten und zu antizipierenden Verhaltensfolgen. Dieses Erwartungskonzept findet sich auch in klassischen Erwartungs-Wert-Modellen wieder, die auf der Vorstellung einer Gewichtung von subjektiven Werten mit der Erwartung, dass man diese Werte verwirklichen kann ${ }^{116}$, aufbaut. In den Erwartung $\mathrm{x}$ Wert-Theorien, die u.a. von LEWIN, TOLMAN und VROOM entwickelt wurden, wird die Wahrscheinlichkeit, Stärke oder Geschwindigkeit, mit der ein bestimmtes Verhalten von einer Person ausgeführt wird, als eine Funktion der Stärke der Erwartungen und des Werts, den dieses Ziel für die Person besitzt, verstanden. Der Grundgedanke dieser Modellvorstellungen besagt, dass eine Person sowohl ihre Wertschätzung für bestimmte Handlungsziele als auch die von ihr wahrgenommenen Realisierungschancen bei der Auswahl von Handlungszielen in Rechnung stellt. Die Erwartung x Wert-Theorien gehen damit von einer Interaktion von situativen und perso- 
nalen Komponenten bei der Intentionsbildung aus (vgl. HECKHAUSEN 1989).

LEWIN gilt als der Urvater der Erwartung x Wert-Theorien. Er entwickelte in seiner Feldtheorie ein Umweltmodell, in dem Anreize (= Valenzen) der Ausgangspunkt von Kräften im psychologischen Feld der aktuellen Situation sind, die das Handeln der Person nach Stärke und Richtung bestimmen (vgl. BEIER 2001, 40). ATKINSON (1957) greift diese Vorstellung auf und stellt in seinem Risikowahl-Modell eine klassische Erwartung x Wert Theorie zur Vorhersage der individuell bevorzugten Aufgabenschwierigkeit vor. Hierbei werden neben den Personenfaktoren auch unterschiedliche situative Momente für die Verhaltensdetermination berücksichtigt werden. Das Modell enthält Motiv-, Anreiz- und Erwartungsvariablen, aus deren multiplikativer Interaktion eine resultierende Tendenz entsteht, die Vorhersagen im Bezug auf Leistungs,- Ausdauer-, Aufgabenwahl- und Zielsetzungsverhalten in Abhängigkeit von der Aufgabenschwierigkeit erlaubt ${ }^{117}$ (vgl. RHEINBERG 1989; HECKHAUSEN 1989, BEIER 2001)

Trotze der methodologischen Schwäche, die insbesondere den rationalen Entscheidungstheorien attestiert wird, stellen die entscheidungstheoretischen Ansätze die Grundlage der neueren Motivationsforschung dar, die in ihrem Grundtyp dem Modelltyp der Erwartung x Wert-Modellen entspricht (vgl. HECKHAUSEN 1989).

Entscheidungstheoretische Ansätze lenken den Fokus auf die Frage subjektiven Nutzens und können damit erklären wie ein Individuum bei entsprechenden Präferenzen und Handlungsalternativen entscheiden wird. Sie erklären allerdings nicht warum ein Individuum diese oder jene Handlungsalternative bevorzugt. Für den Kontext risikosportlicher Handlungen geben die Entscheidungstheorien und ihre Erweiterungen zu Erwartung x WertTheorien einen Hinweis auf subjektive Präferenzen, den die Handlung und ihr Ergebnis für die Protagonisten besitzen. Die Frage nach den Ursachen und Beweggründen risikosuchenden Verhaltens im Sport verdichtet sich damit auf die Frage nach dem subjektiven Nutzen, den die Protagonisten mit der Sportausübung verbinden. Motiviertes Handeln ist demnach unter Bedingungen unterschiedlicher Handlungsalternativen als Entscheidungsprozess in Abhängigkeit subjektiver Präferenzen zu beschreiben (vgl. SCHNEIDER \& SCHMALT 2000, 87ff).

Auch wenn die Reichweite eines solchen Ansatzes im Vergleich zu anderen Forschungsansätzen relativ groß ist, so wird seine Begrenztheit doch an habitualisierten oder auch unbewusst ablaufenden Handlungen deutlich. Insbesondere unwillkürliche und Routinetätigkeiten werden von den kognitiv-handlungstheoretischen Ansätzen nicht erfasst.

Die Darstellung der verschiedenen Forschungsansätze der Motivationspsychologie sind als theoretische Minimalbasis für die folgende Motivationsanalyse des Risikosports konzipiert . Mit Bezug auf RHEINBERG (2002, 130ff) ist dabei für den Risikosport von einer komplexen Handlungsmotivation auszugehen. Auch für risikosportliche Aktivitäten gilt, ,,[...] dass nicht nur ein einziger, sondern mehrere Anreize zugleich wirksam werden kön- 
nen“ (ebd.). Die folgende Analyse der motivationspsychologischen Strukturen des Risikosports nimmt den roten Faden einer interdisziplinären Analyse daher nochmals auf, und bezieht verschiedene Forschungsansätze in die Analyse ein. Die Forderung von SCHNEIDER \& SCHMALT $(2000,100)$ nach einer möglichst umfassenden Integration unterschiedlicher Forschungsansätze in die Analyse komplexer Handlungen wird - im Bewusstsein einer methodologischen Schwäche - vollzogen. Dabei wird in Kauf genommen, „[...] dass bei der gegebenen Mannigfaltigkeit der Ursachen von Verhaltensunterschieden auch recht unterschiedliche Erklärungsansätze zustande kommen“ (ebd.). Allerdings - so die Autoren - „,...] dienen diese Konzepte alle dem gleichen Zweck - nämlich die Energetisierung und Zielausrichtung des Verhaltens zu erklären“" (ebd.).

Bevor einzelne Forschungsperspektiven der Motivationspsychologie auf Hinweise für die Motivation im Risikosport untersucht werden, folgen zunächst anthropologische Annahmen, die das menschliche Verhältnis zum Risiko analysieren.

\subsection{Der Mensch und das Risiko - Anthropologische An- nahmen}

Die Anthropologie als die Wissenschaft vom Wesen des Menschen bedient sich in ihrer Theoriebildung der Ergebnisse von Nachbardisziplinen, um der Frage nach dem Kern des Menschlichen im Wandel von Zeit und Raum nachzugehen. Die Methode des Vergleiches steht dabei im Mittelpunkt. Spezifische anthropologische Theorien, die einen direkten Bezug zwischen dem Wesen des Menschen und sportlichen Risikosituationen herstellen, gibt es kaum. Einzig BRUCKHEIMER (1990) definiert einen ,homo adventurus', der durch ein Grundbedürfnis nach Abenteuer und Risiko charakterisiert ist, welches sich aus den Primärbedürfnissen ${ }^{118}$ ableitet (vgl. ebd., 86101). Es handelt sich hierbei jedoch um einen vorläufigen Entwurf, der wesentliche Erweiterungen erfordert. MICHL (1995) versucht die anthropologischen Grundlagen der Erlebnispädagogik zu entschlüsseln, wobei sich seine Darstellungen eher als eine Einführung in die pädagogische Anthropologie lesen. Auf Risiko, Abenteuer und Sicherheit geht der Autor nicht ein. Im folgenden wird daher ein differenzierter anthropologisch-biologischer Argumentationsstrang in Anlehnung an die Überlegungen bei TRIMPOP (1994) und NEUMANN (1999) verfolgt

In einem ersten Abschnitt wird der Mensch als ein riskiertes Wesen dargestellt und das Verhältnis des Menschen zur Sicherheit eingehend erläutert. Dabei wird der zentralen Frage nachgegangen, ob es im Wesen des Menschen an sich - neben einem Sicherheitsbedürfnis - auch eine natürliche Affinität zum Risiko gibt. Auf der Basis der historischen Entwicklung des Risikos als Wahrnehmung von Unsicherheit soll hierbei insbesondere auf die Bedeutung des Risikos aus anthropologischer Sicht eingegangen werden. Diese Bearbeitung erfolgt in Anlehnung an NEUMANN $(1999,57)$ in meh- 
reren Schritten: Zunächst wird das Wesen des Menschen und seine Stellung in der Welt aus anthropologischer Betrachtung charakteristisch dargestellt. Der Argumentation TENBRUCKs (1978) und ZELINKAs (1997) folgend, wird anschließend die Anthropologie des menscblichen Handelns expliziert. In einem weiteren Schritt wird das Nengierverhalten des Menschen im thematischen Zusammenhang dargestellt.

\subsubsection{Der Mensch als ,riskiertes' Wesen}

Ziel anthropologischer Forschung ist es, die Stellung des ,Menschen im Kosmos', die sich von der des Tieres und der Pflanze unterscheidet, darzustellen. Der wohl bekannteste und profilierteste Entwurf stammt dabei von SCHELER (1928), der von GEHLEN (1950) weiterentwickelt wurde und den Menschen als Mängelwesen beschreibt (vgl. ebd., 38f). So skizziert GEHLEN den Menschen als ein Wesen, dass vor allem durch seine Instinktreduktion charakterisiert ist, d.h. dem offensichtlich stammesgeschichtlichen Abbau fast aller speziellen und angeborenen Bewegungsweisen eines Tieres (vgl. ebd.). Während dem Tier eine Vielzahl von Verhaltensweisen als Instinkthandlung, vorgeschrieben' werden, stellt sich der Mensch als weltoffen dar - er muss sich seine Umwelt und seine Handlungen erst selbst erschlieBen (vgl. ZELINKA 1997, 47).

Der Mensch ist damit weitgehend, umweltentlastet'119 und frei von der klaren Orientierung eines Tieres, die durch sein triebhaftes Verhalten gesteuert wird. Er besitzt die mit der Notwendigkeit einer eigenverantwortlichen Weltaneignung verbundene Orientierungsnot, sich seinen Lebensraum selbst zu schaffen (NEUMANN 1999, 58). Da der Mensch im Gegensatz zum Tier ,[...] nicht auf bestimmte Umweltreize hin spezialisiert ist, erscheint ihm [dem Menschen] die Welt als ein ungeordnetes Chaos" (ebd.), der er nicht lebensfähig ausgeliefert ist. Diese Interpretation des menschlichen Wesens als ein ,riskiertes' und gefährdetes' Lebewesen beruht auf eben dieser Mangelhaftigkeit, die dem Menschen mit seiner Geburt auferlegt ist (vgl. GEHLEN 1950, 33).

„Gegenüber der mühelos, still und sicher erscheinenden Existen₹ eines Tieres erscheint der Mensch als unter konstitutioneller Disharmonie und Belastung stehend, d.h. als ein Lebewesen, welches in der Gebrochenheit und Unsicherbeit seiner Existenz böchst gefährdet und riskiert ist" (ZELINKA 1997, 46).

Aus einer ethologisch-biologischen Betrachtung nach LORENZ erscheint der Mensch dagegen als ein Wesen mit ungewöhnlichen Fähigkeiten der Umweltanpassung - als ein „Volltreffer der Evolution“ (ebd., 47), die GEHLENs These des Mängelwesens gegenübersteht.

Gleich welcher Auslegung der Interpretationen man sich anschließt, Ethologie und Anthropologie stimmen überein, dass die Sonderstellung des Menschen im ,[...] Reich lebendiger Wesen [..]“" (ZELINKA 1997, 48) durch die Fähigkeit begründet ist, seine Umwelt zur Welt und seine Natur zur Kul- 
tur umzugestalten. Das menschliche Streben nach Sicherheit kann demzufolge auch als Formierung und Kompensation seiner einzigartigen riskierten Situation im Reich der Natur verstanden werden. Als weltoffenes Wesen, das in der Reflexion auf sich selbst immer wieder mit den Möglichkeiten seiner Existenz konfrontiert wird, bleibt der Mensch daher in ständiger Sorge um seine Geborgenheit und Sicherheit. „Sicherheit ist das elementare Grundbedürfnis eines Lebewesens, das auf seiner Suche nach einem ihm gemäßen Platz zur Lebensgestaltung gezwungen ist" (ebd., 53).

\subsubsection{Die Anthropologie des menschlichen Handelns}

Bestimmt durch seine riskierte Stellung im Reich der Lebewesen, stellt sich der Mensch auch in seinen Handlungen als ,[...] umfassend unsicher dar“ (TENBRUCK 1978, 90). Denn durch das Fehlen zielgerichteter und erfolgssicherer Instinkthandlungen, ist der Mensch gezwungen, eigene Handlungsmuster reflexiv aufzubauen. Die selbsterprobten Handlungsstrukturen müssen dabei einen ständigen Entwicklungsprozess durchlaufen und können daher keine erschöpfende Erfolgssicherheit bieten.

Da der Mensch als weltoffenes Wesen eine große Anzahl von wechselnden und unvorhergesehenen Bedingungen und Zusammenhänge in seine Handlungen integrieren muss, sind die Handlungen einerseits bezüglich der Erfolgschancen, andererseits aber auch hinsichtlich des Handlungszieles selbst unsicher (vgl. ebd., 91). Diese als äußere und innere Unsicherheit bezeichnete Problematik menschlichen Handelns bedingt damit eine ständige Aufmerksamkeit und Kontrolle der Handlungsmuster (vgl. ebd.).

Neben dieser äußeren Unsicherheit über die Erreichung des eigentlichen Handlungszieles, kommt es beim Menschen gleichzeitig zu einer inneren Unsicherheit - einer Art Entscheidungsnot bezüglich der zu erreichenden Zielpräferenzen:

„Auch diese gründet in der Weltoffenheit des Menschen, der zwar eine kaum erschöpfbare Zabl von Zielen und Wünschen haben kann, aber von den wenigen biologisch fundierten Bedürfnissen einmal abgesehen, keine bestimmten haben muss und deshalb immer vor der Frage steht, was seine wahren Bedürfnisse und Ziele sind" (ebd., 95).

Aus dieser Unsicherheit seiner existenziellen Handlungen resultiert das oben beschriebene Sicherheitsstreben, dass sich als Wunsch nach der Reduktion von Unsicherheiten darstellt. Doch neben diesem existenziellen Bedürfnis nach Sicherheit, sieht TENBRUCK im Sicherheitsstreben des Menschen auch einen entscheidenden Lastcharakter. Denn zugleich „,[...] sucht und wünscht sich der Mensch gerade auch Unsicherheit, ja er ist sogar auf Unsicherheit angewiesen $[. .$.$] “ (ebd.).$

Wie erklärt sich nun dieses ambivalente Verhältnis zur Sicherheit? Unsicherheit bedeutet nach den oben angeführten Ausführungen auch Risiko oder Überraschung, die in einem hohen Maße Aufmerksamkeit und Antrieb mobilisieren (s.o.). Wird eine Handlung relativ sicher und habitualisiert, so 
verliert sie an Konzentrationsbedarf und Anreiz. Eine unsichere Handlung hingegen erfordert dagegen die volle Aufmerksamkeit und Kontrolle, die wie die Risikosituation eine zukunftorientierte Gelingensspannung aufrechterhält:

„Unter Berücksichtigung der jeweiligen Ziele reguliert sich die Aufmerksamkeitsspannung also nach der Unsicherheit [...]. Die Ausfübrung entbehrt des Reizes, mehr noch, der Ertrag der Handlung wird subjektiv im Maße der Sicherbeit entwertet. [...] Risiken und Überraschungsmomente haben also in doppelter Hinsicht einen Reiæ, nämlich was die Handlungsfübrung selbst, und was ibren Ertrag angeht" (ebd., 111).

Die ,Prämie' im Sinne einer Handlungsbelohung ist also vom Gehalt an Unsicherheit abhängig. Dies gilt in gleichem Maße für verschiedene Handlungen, als auch für ein und dasselbe Handlungsmuster. Um die Gratifikation einer Handlung zu verstärken, ist also eine gleichzeitige Erböbung der Unsicherheit erforderlich. Der Mensch als Wesen, strebt somit nicht nur nach Sicherheit, er ist damit gleichzeitig auch unsicherheitsbedürftig bzw. in gewissem Umfang risikobedürftig120.

Bei HALLER (1998) drückt sich diese Ambivalenz menschlichen Handels in einer dynamischen, Sicherheitsbilanz'121 aus, in deren Soll Entwicklungs- und Veränderungsgrößen, im Haben Stabilisierungs- und $\mathrm{Zu}$ standsgrößen eingetragen sind (Abb. 4.2). Risiko als positives Merkmal äuBert sich darin, dass Unsicherheit als Chance stets auch den Reiz des Unbekannten und die Möglichkeit neuer Erfahrungen einschließt. Negativ wirkt sich Unsicherheit aus, weil die sich im Risiko eröffnende Chance meist die Möglichkeit von negativen Konsequenzen beinhaltet und damit einen allgemeinen Verlust impliziert. Im Sinne eines Fließgleichgewichts ,„[... zwischen Entwicklung und Stabilisierung“ (ebd., 226) finde ein ständiger Wechsel statt, wobei immer neue Arten der Sicherheit und Unsicherheit empfunden und durchlebt werden.

„Ein allzu langes Verbarren in einem statischen sicheren Zustand fördert das Bedürfnis nach Veränderung; stetige Veränderung und Herausforderungen bewirken den Wunsch nach Ruhe, Geborgenheit und statischer Sicherbeit" (ebd.). 


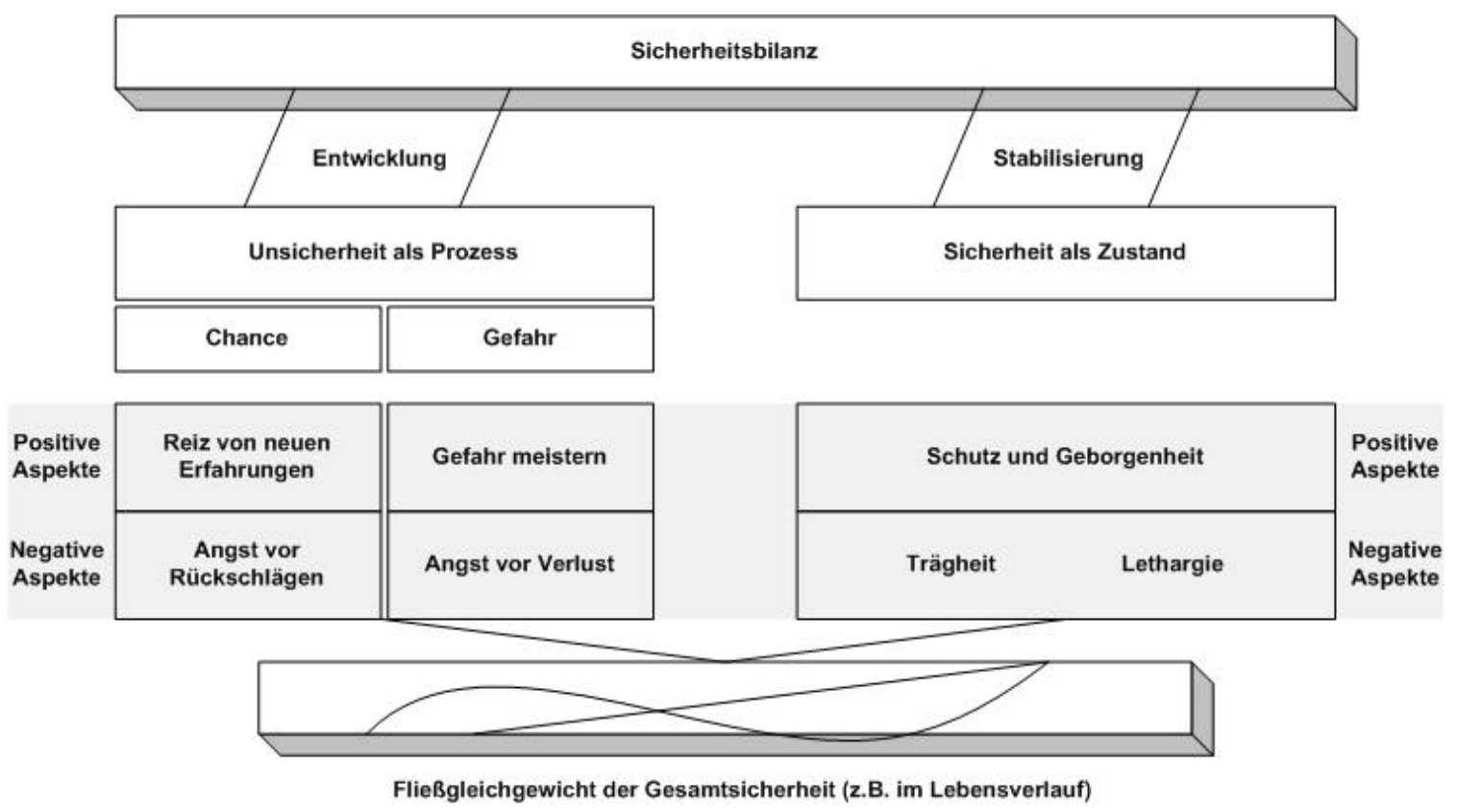

Abbildung 4.2: Die Sicherheitsbilanz: Ausgleich von Entwicklung und Stabilisation (modifiziert nach HALLER 1998, 225).

\subsubsection{Neugierverhalten - ein Sicherheitstrieb}

Die beschriebene anthropologische Weltoffenheit des Menschen, die sich nach TENBRUCK (1978) vor allem in der umfassenden Unsicherheit menschlicher Handlungen wieder spiegelt, gilt auch als Grundannahme für das allgemeine Neugierverhalten. Neugier wird hierbei als ,,[...] Trieb zu erkunden“ (EIBL-EIBESFELD 1986, 716) erklärt bzw. als „,...] Bestreben, das darauf abzielt, Informationen über Sachverhalte, die neu oder unklar usw. sind, zu gewinnen und damit Zustände der subjektiven Unsicherheit oder Ungewissheit zu reduzieren“ (SCHNEIDER 1996, 119). CUBE bezeichnet Neugier als ein Bedürfnis zur Umwandlung von Unsicherheit in Sicherheit und unterstellt Neugierigen somit eine Sehnsucht nach Sicherheit. (vgl. CUBE 1995, 34).

Während für eine von außen angeregte Neugier angenommen wird, dass diese darauf abzielt, subjektive Unsicherheiten abzubauen und damit Wissen und Sicherheit zu erwerben, scheinen „,...] Lebewesen zu anderen Zeiten bestrebt zu sein, solche Zustände selbst zu schaffen, d.h. sie suchen Situationen auf, in denen Unsicherheit durch neuartige oder komplexe Sachverhalte induziert wird" (SCHNEIDER 1996, 130). Vor diesem Hintergrund kann das Aufsuchen von sportlichen Risikosituationen zugleich als eine Form des diversiven Nengierverbaltens verstanden werden, dass auf die Erzeugung subjektiver Unsicherheiten abzielt (vgl. SCHNEIDER 1996, 130).

Ausgelöst wird die diversive Neugier durch den Zustand der Reizhomogenität, also der Langeweile und Monotonie, der durch die allgemeine Reizarmut als belastend wahrgenommen wird (vgl. ebd.). Diversives Neugierverhalten wird folglich als lustvoll und emotional positiv empfunden, wenn in dem Spektrum eines ,Reizkontinuums', zwischen Reizhomogenität und 
Reizüberflutung der Reiz des Neuen sich in einem Mittelfeld bewegt. „Ist ein Reiz zu vertraut, wird er langweilig, ist er zu fremd, flößt er Angst und Unbehagen aus" (OERTER \& MONTADA 1995, 769) und hat eine Vermeidung der Handlung zur Folge.

Die Kennzeichnung des Neugierverbaltens als primärer Sicherbeitstrieb nach CUBE (1995, 34) erscheint - trotz kritischer Betrachtungen (z.B. CLAUSEN 2003) - im Kontext der vorliegenden Arbeit als äußerst bedeutsam. Die Kernaussage dieser Theorie lässt sich auf die Formel „Wer Wagnis sucht, will Sicherheit" zusammenfassen. Das Unbekannte wird zum Bekannten und zum Vertrauten. - Unsicherheit zu Sicherheit. Die lustvolle Suche nach Unsicherheit und ihre Befriedigung durch eigenmächtige Bewältigung stellt sich damit als angeboren und im Wesen des Menschen verankert dar. Auf den Risikosport transferiert stellt CUBE fest:

\section{„Warum ist Klettern so lustvoll? Weil man beim jedem Schritt Unsicherbeit in Sicherheit verwandelt. Das lustvolle beim Klettern, [...] also beim Abenteu- er, besteht in der (lustvollen) Befriedigung des Sicherbeitstriebes" (ebd., 12).}

Auch die evolutionäre Bedeutung der Suche nach Unsicherheit liefert hier einen wichtigen Argumentationspunkt, denn die Risikobereitschaft des Menschen repräsentiert einen bezeichnenden evolutionären Selektionsvorteil: ,[...] Ohne Risikobereitschaft fiele er [der Mensch] der Stagnation anheim" (EIBL-EIBESFELD 1988, 46; vgl. auch KOCH 1994, 23). Die Risikoübernahme im Sinne eines Neugierverhaltens dient damit vor allem im Sozialisationsprozess als ein wichtiger Prozess zur Selbstbestimmung. Gerade bei Kindern ist diese Form der Neugierbefriedigung daher besonders ausgeprägt und kann bei Jugendlichen z.B. bei delinquenten Mutproben auch im Kontext risikoreicher Aktivitäten (z.B. U-Bahn-Surfen, Autorennen) beobachtet werden.

Das vital erlebte Risiko manifestiert sich also - die Thesen der kulturhistorischen Annäherungen in Kapitel 3 - als eine Art anthropologische Grundfigur. Sicherheit und Risiko stehen hierbei in einem scheinbar ambivalenten Verhältnis: Nur im Rahmen einer ausgeglichenen Balance - so die Schlussfolgerung der anthropologischen Überlegungen - kann der Mensch folglich ganz Mensch sein und seine Existenz im Reich der Tiere behaupten. Die erfolgreiche Bewältigung unsicherer sportlicher Risikosituationen wird damit zu einer lustbringende Bedürfnisbefriedigung, die ein gesteigertes Gefühl der Sicherheit mit sich bringt. Die individuelle Risikoübernahme dient hierbei offensichtlich sowohl der Selbsterhaltung als auch der menschlichen Entwicklung. Vor diesem Hintergrund der biologisch-anthropologischen Erkenntnisse stellt sich allerdings die Frage, warum sich nur einige wenige Menschen in derartige unsichere Situationen begeben und warum gerade im Sport Unsicherheit aufgesucht wird, es ließen sich doch eine ganze Reihe von Situationen anführen, in denen sich das Neugierverhalten auch auf anderen Wegen befriedigen ließe. Die Analyse psychologischer Beiträge zur Erklärung risikosuchenden Verhaltens setzt an diesem Punkt an ohne die anthropologischen Ansätze in Frage zu stellen; es folgt eine dezidierte moti- 
vationspsychologische Annäherung aus verschiedenen psychologischen Perspektiven.

\subsection{Risikosport und Motivation: Erklärungsversuche der Psychologie}

Die folgenden psychologisch orientierten Ansätze versuchen das Phänomen Risikosport aus verschiedenen Forschungsperspektiven der Motivationspsychologie zu erklären, deren Grundannahmen im vorstehenden Kapitel vorgestellt wurden. Derzeit existiert in der psychologischen Theoriebildung weder eine einheitliche Theorie noch ein klar definierter Forschungszweig, weshalb im Folgenden eine additive Vorgehensweise verfolgt wird. Eine explizite Theorie zum risikosuchenden Verhalten im Risikosport existiert zudem ebensowenig, so dass die vorgestellten Aussagen lediglich von diversen allgemein-psychologischen Untersuchungen, theoretischen Annahmen und vereinzelten sportpsychologischen Beiträgen übertragen werden können und darüber hinaus ihre Validität lediglich durch Introspektion ${ }^{122}$ erfahren können.

Da auch die Psychologie als wissenschaftlichen Feld von einer Vielzahl unterschiedlicher erkenntnistheoretischer Perspektiven geprägt ist, werden die Beiträge auf ihre Kernaussagen reduziert. Zunächst werden aktivationstheoretische Aspekte zur Erklärung risikosuchenden Verhaltens herangezogen. Daraufhin wird die Risikosuche im Sport unter den Bedingungen des Angsterlebnisses, der Angstüberwindung und der Option vielseitigen Grenzerfahrungen aus verschiedenen motivationspsychologischen Forschungsansätzen (biologischphysiologisch, kognitiv-handlungstheoretisch) betrachtet. In der darauf folgenden analytischen Darstellung der verschiedenen Erlebnisdimensionen von Risikosportarten werden die Motiv- und Anreizelemente isoliert aufgeschlüsselt und wiederum aus unterschiedlichen Forschungsperspektiven analysiert. Abschließend wird eine sozialpsychologische Theorie zur Erklärung des Verhaltens im Risikosport expliziert.

Einige theoretische Annahmen, die zu sportartspezifisch orientiert sind, werden nachfolgend nicht dargestellt, weitere auf bestimmte Teilaspekte reduziert (z.B. AUFMUTH 1989b). Viele Darstellung müssen vor dem Hintergrund ihrer Entstehung deduziert werden, um den Gesamtkontext zu berücksichtigen. Die bei empirischen Arbeiten vorliegenden Ergebnisse werden dabei auf den Gegenstand des Risikosports bezogen und im Hinblick auf ihren Erklärungsgehalt deduziert.

\subsubsection{Aktivationstheoretische und persönlichkeits-psychologische Ansätze: Risikosport als Erregungssuche}

Risikoverhalten ist im Kontext traditioneller persönlichkeits-psychologischer Forschung meist nicht - wie in der vorliegenden Arbeit - als ein freiwilliges Aufsuchen potentiell gefährlicher Situationen aufgefasst worden, sonder als 
Wahl- und Entscheidungsverhalten unter diversen Bedingungen. Bei ATKINSON (1957) und COHEN et al. (1961) wurde Risikoverhalten mit dem Verhalten bei der Anspruchsniveausetzung in Leistungssituationen und mit der Einschätzung der eigenen Erfolgs- und Misserfolgsaussichten unabhängig vom individuellen Anspruchsniveau gleichgesetzt. Untersuchungen von KOGAN et al. (1964) analysierten das Risikoverhalten als Persönlichkeitsmerkmal in Spiel- und Wettsituationen, während es bei PRUITT (1961) als Bedürfnis nach Informationen definiert wurde. All diese Untersuchungen stehen zwar in einem begrifflichen, nicht aber erkenntnistheoretischen $\mathrm{Zu}$ sammenhang mit risikosportlichen Handlungen.

Aber auch bei stärker definitorischer Eingrenzung lassen sich nur wenige Untersuchungen zum Risikoverhalten als Persönlichkeitsmerkmal finden. Lediglich bei TRIMPOP finden sich Untersuchungsergebnisse, die Risikosportaktivität mit unterschiedlichen Persönlichkeitsmerkmalen beschreibt, ,[...] such as being more impulsive and more paratelic oriented“ (TRIMPOP 1994, 185).

Neuere Theorien der Persönlichkeitsforschung beschäftigen sich insbesondere mit Ansätze der Aktivationspsychologie. Aktivationstheoretische Ansätze, die ein Aufsuchen von sportlichen Risikosituationen erklären, lassen sich als Erweiterung der anthropologisch orientierten diversiven Neugiertheorie verstehen. Das als ,[...] Suche nach spezifischen, neugierauslösenden Sachverhalten [...]" (RHEINBERG \& SCHNEIDER 1996, 408) definierte diversive Neugierverhalten dient dabei offensichtlich auch der Einstellung eines optimalen ,Reizeinstroms', der zu einer zentralnervösen sowie peripheren Aktivierung führt (vgl. BERLYNE 1974, 270).

Die zentralen Ansätze hierzu stammen von BERLYNE. Demzufolge strebt der Mensch ein individuelles Aktivierungspotential an, welches durch den Reizeinstrom zu einer spezifischen informationalen Auslastung des Zentralen Nervensystems (ZNS) führt (vgl. RHEINBERG \& SCHNEIDER 1996, 411). Es wird angenommen, dass das subjektiv als angenehm empfundene ,optimale' Aktivierungspotential zwischen Reizarmut und Reizüberflutung liegt (vgl. BERLYNE 1974, 251). Der Autor postuliert für den Menschen daher ein mittleres Erregungspotential als subjektives Ideal ohne allerdings genaue Angaben über Qualitäten des Reizeinstroms anzugeben.

Einen ähnlichen Ansatz vertritt auch HECKHAUSEN (1977), der auf Basis einer neurophysiologischen Aktivierungstheorie einen sogenannten ,Aktivierungszirkel' vorstellt. Demzufolge sucht der Mensch nach einer psychischen Spannung, die sich langsam aufbaut, um schließlich wieder in Entspannung abgebaut zu werden (vgl. ebd., 158). Das optimale Erregungspotential liegt dabei übereinstimmend zu BERLYNEs Theorie der optimalen Erregung in einem mittleren Aktivierungsbereich, der sich zwischen Angst und Langeweile befindet. Sowohl HECKHAUSEN als auch BERLYNE weisen eine Vielfalt an empirischen Untersuchungen zur Untermauerung ihrer Thesen auf, ohne dass sie auf die Frage nach Persönlichkeitspräferenzen eine Antwort geben. 
Die Frage nach individuellen Unterschieden des Aktivierungsoptimums, beantworten insbesondere ZUCKERMAN (1978; 2000) mit persönlichkeitsspezifischen Ungleichheiten ${ }^{123}$. Der Autor erklärt die interpersonellen Unterschiede mit seinem Konzept der ,sensation seeking" - zu übersetzten als „Reiz:" oder „Erregungssuche“ (ALLMER 1998, 66). Das Bedürfnis nach Reiz-Stimulation z.B. in Risikosituationen lässt sich nach ZUCKERMAN dadurch erklären, dass bei Menschen unterschiedlich hohe Erregungsstandards existieren. Verschiedenartige zentralnervöse Erregungsniveaus werden demzufolge individuell unterschiedlich als ,optimal' empfunden (RHEINBERG 1997, 153). Unterstützt werden diese weiterführenden Ansätze von neuro-physiologischen Untersuchungen, die im Bereich des zentralen Nervensystems Differenzen im Bereich der Reizübermittlung und Hormonausschüttung nachweisen konnten. HEBB (1955) wies schon früh mittels EEG-Rhytmen auf einen physiologischen Hintergrund des Risikoverhaltens hin. Diese zentralnervösen Unterschiede lassen sich nach demzufolge auf biochemische Prozesse im limbischen System zurückführen, wobei Dopamine und Monoaminooxidasen im Blut eine wichtige Rolle spielen. FABRY (1990, 10f) hebt in diesem Zusammenhang das Hormon Kortisol besonders hervor, welches eine allgemein aufputschende Wirkung besitzt und den Akteur im Handlungsgeschehen weiterhin handlungsfähig sein lässt. CLONINGER (1987) geht in diesem Zusammenhang von verschiedenen Neurotransmittersystemen aus und ordnet unterschiedlichen Verhaltenssystemen eine unterschiedliche Aktivität der Transmitterstoffe Serotonin, Dopamin und Noradrenalin zu. Insgesamt besteht wenig Zweifel an einer physiologischen Dimension des Risikoverhaltens, auch wenn trotz der Erforschung physiologischer Zusammenhänge noch weitere Fragen offen sind. Weitgehend unklar ist z.B., welche Bedeutung diese Theorie für eine Motivationsanalyse des Sports im Allgemeinen besitzt, so dass im Rahmen dieser Arbeit auf weitere Ausführungen verzichtet wird (vgl. auch RHEINBERG 1997, 155).

Aber auch auf psychologischer Ebene weist das ,sensation seeking' weitere empirische Hinweise auf. ,Sensation Seeking' als Theorie lässt sich demnach verstehen als die ,,...] Suche nach starken Reizen; [...] als ein allgemeines Persönlichkeitsmerkmal, das sich nicht auf einzelne Sinnesorgane beschränkt" (ZUCKERMAN 1978, 16). Menschen mit einem starken Bedürfnis nach Reizsuche haben somit einen entsprechend höheren Level der Stimulation und fühlen sich in reizarmen Situationen eher gelangweilt als Personen mit einem schwachen Bedürfnis nach Reizsuche (ebd.). Das individuelle Erregungsniveau liegt bei diesen Personen entscheidend höher, so dass auch eine entsprechend höhere Reizstimulation erforderlich ist (vgl. HEIL \& HENSCHEN 1996, 232).

Für risikoreiche Gefahrensituationen stellt ZUCKERMAN fest, dass bei gleicher subjektiver Einschätzung des Risikos einer Aktivität ,[...] starke Reizsucher mehr Spaß als Angst damit verbinden, während schwache Reizsucher in der Regel nur Angst empfinden“" (ebd.). ZUCKERMAN beobachtete, dass es neben den Differenzen hinsichtlich des Erregungsniveaus auch 
Unterschiede bezüglich der Reizquelle gibt. Eine Faktorenanalyse, die als „sensation seeking scale“ (SSC) bezeichnet wird, offenbart, dass neben anderen Reizquellen, wie Sexualität oder Drogen, auch das Betreiben von Risikosportarten als spezielles Objekt der Erregungssuche verstanden werden kann. Der Autor konnte sein Konstrukt in vier Komponenten unterteilen, wobei jede Komponente mit zehn Aussagen erfasst wird. Unter anderem verifiziert ZUCKERMANN die SSC mittels persönlichkeitsorientierter Fragebögen auch bei Sportkletterern oder Motorradfahren und weist ihnen die Komponente, Thrill and Adventure Seeking' zu:

(1) Thrill and Adventure Seeking (TAS): Tendenz zu risikoreichen Aktivitäten in Sport und Freizeit mit hohem Erlebniswert (Klettern, Bergsteigen).

(2) Experience Seeking (ES): Tendenz zu neuen Erfahrungen durch Reisen, Kunstgenuß, neue Speisen, interessante Personen, Einnahme von Drogen.

(3) Disinhibition (DIS): Tendenz zur Enthemmung in sozialen Situationen, z.B. auf Partys, in sexuellen Beziehungen, insbesondere Enthemmung im Zusammenhang mit Alkoholkonsum

(4) Bordeom Susceptibility (BS): Tendenz, monotonen, sich wiederholenden Ereignissen und Tätigkeiten sowie langweiligen Personen aus dem Weg zu gehen

Tabelle 4.1: Vier Komponenten des Sensation Seeking nach ZUCKERMANN (1978); leicht modifiziert nach RHEINBERG $(2002,170)$.

Vergleicht man Personen, die in Risikosportarten aktiv sind, mit solchen, die keinen Sport treiben, so haben Risikosportler einen signifikant höheren Gesamtwert im Sensation-Seeking Fragebogen. Bei mäßig riskanten Sportarten wie Rugby oder Ringen fallen die Unterschiede im Vergleich zu NichtSportlern geringer aus, während bei risikogeringen Sportarten wie Laufen oder Tennis keine Unterschiede auftreten (vgl. RHEINBERG 2002, 171). Auch ANDERSEN (2000) extrahiert mittels Faktorenanalyse aus 14 verschiedenen Persönlichkeitsmerkmalen einen eigenen Faktor ,Risk and Competetion-Seeking', der als Disposition für Risikoverhalten verantwortlich sein könnte.

ZUCKERMANs Erklärungsansatz folgend, kann der Risikosport damit als persönlichkeitsabhängige Befriedigung eines individuellen Erregungsbedürfnisses erklärt werden. Eine Differenzierung bezüglich des Risikomoments oder der Handlungsstruktur nimmt der Autor nicht vor, weshalb es unklar bleibt, welche risikosportlichen Aktivitäten genau in das SensationSeeking-Konzept passen und welche Handlungselemente für eine befriedigende Reizsuche relevant sind. Was nahezu unbearbeitet bleibt, ist zudem die theoretische Erläuterung der Beziehung zwischen Disposition und dem beobachtbaren Verhalten. Es fehlt ein interaktionales Modell, welches erklärt, bei welchen Sensation Seeking-Ausprägung welche Situationsfaktoren welche kognitiven und emotionalen Prozesse anregen und deren Interaktion $\mathrm{zu}$ einem sichtbaren Verhalten führt. Leider unterbleibt im ,sensation seeking' Konzept auch eine Rückbindung an soziale Faktoren sowie kulturelle Präferenzen oder externe Motivationsaspekte. Trotzdem besitzt die Konzeption des ,sensation seeking' bis heute weitreichende Bedeutung für die Erklärung individuellen Unterschieden in der Erregungssuche. 
APTER (1994) vertritt in seiner Reversionstheorie eine ähnliche aktivationstheoretische Auffassung, die das Bedürfnis nach Reizen in Erweiterung an HECKHAUSEN (1977) als einen individuell unterschiedlich stark ausgeprägten Wechsel zwischen der Suche nach Erregung (,Nervenkitzel') und deren Vermeidung (,Angst') beschreibt (APTER 1994, 38). Entgegen der Theorie der ,Sensation Seeking' ist APTER der Auffassung, ,[...] dass jeder Mensch ab und zu etwas Nervenkitzel braucht - manche häufiger als andere [...]" (ebd., 22). Für den Autor ist die Suche nach Reizstimulation in Anlehnung an die anthropologischen Annahmen als quasi natürliche Komponente $\mathrm{zu}$ verstehen. Ausgangsthese der Reversionstheorie ist die Feststellung, dass Angst und aufgeregte Freude, und diametral Langeweile und Entspannung auf denselben physiologischen Parametern beruhen (ebd., 29). Aufbauend auf physiologischen Erkenntnissen über die Erregungswahrnehmung und Interpretation geht der Autor nun davon aus, dass Erregung als physiologischer Zustand von verschiedenen Menschen vollkommen unterschiedlich interpretiert werden kann. In Abhängigkeit früherer Erfahrungen und des bestehenden Selbstkonzeptes wird das Individuum auf Erregungszustände ganz unterschiedlich reagieren und sein Verhalten entsprechend der Erregungswahrnehmung gestalten. Obwohl APTERs Ansatz des schützenden Sicherheitsrahmens für eine Analyse des Risikoverhaltens im Risikosport durchaus fruchtbar erscheint ${ }^{124}$, wird in seinen Ausführungen zur Anreizstruktur des Risikosports nicht deutlich, welche Bedeutung gerade der Sport im Rahmen dieser spezifischen Erregungssuche besitzt. Der Autor weist auf das persönliche Befindlichkeitsniveau jedes Menschen hin ohne die Kriterien, die zur allgemeinen Befindlichkeit beitragen, zu explizieren. Es fehlen darüber hinaus klar zu zuordnende Dispositionen oder Anreizelemente, die Hinweise auf einen konkreten Motivationsprozess verdichten.

Zusammenfassend ergibt sich aus den vorgestellten Erläuterungen zur Erregungssuche ein erster Erklärungsansatz für das Aufsuchen sportlicher Risikosituationen: Aus aktivationstheoretischer Perspektive erfüllt das Betreiben von Risikosportarten den Zweck einer optimalen individuellen Erregungsstimulation. Unklar bleiben bei dieser biologisch orientierten Vorgehensweise trotz diverser Forschungsbeiträge aber weiterhin unterschiedliche personale Dispositionen. Zwar können Unterschiede mit unterschiedlichen physiologischen Erregungsniveaus oder deren Interpretation erklärt werden, eine differenzierte Analyse wird lediglich bei ZUCKERMAN vorgenommen. So vermögen es die Ansätze auch kaum zu erklären, warum nur ein bestimmter Teil der Menschen ${ }^{125}$, das Bedürfnis verspüren, risikoreiche Situationen gerade im Sport bewusst und freiwillig aufzusuchen.

Kritisch ist zudem die Geschlossenheit der Ansätze zu begutachten, denn als allumfassendes Erklärungsmodell reichen die aktivationstheoretischen Ansätze zur Sinndeutung des Risikosports zu kurz. Eine auf Reizstimulation ausgelegte Konzeption stellt den umgangssprachlichen ,Nervenkitzel' oder Thrill in den isolierten Mittelpunkt des Anreizinteresses (vgl. RHEINBERG 1997, 157). Thrill-Aktivitäten wie das Bungee-Springen und Risikosportarten wie das Kanu-Wildwasserfahren, das alpine Bergsteigen 
oder das Mountainebiken würden demnach wegen desselben Anreizes aufgesucht, nämlich zum Zweck der Befriedigung eines Erregungsbedürfnisses. Dies erscheint nicht nur aus einer introspektiven Betrachtung sondern auch vor dem Hintergrund der theoretischen Ausführungen im ersten Kapitel als fraglich, so dass das beschriebene aktivitätstheoretische Konzept der Reizsuche zwar als ein grundlegendes Motivelement für die Ausübung von Risikosportarten angenommen werden kann, es aber keinesfalls als einzige oder allgemeingültige Konzeption betrachtet werden darf.

\subsubsection{Risikosport als Angsterlebnis}

Eine Vielzahl von theoretischen Ansätzen zur Erklärung risikosuchenden Verhaltens stellen die Angst und damit einhergehend deren Überwindung in den Mittelpunkt ihrer Argumentation. FINK (1994) zeigt in diesem Zusammenhang am Beispiel des Kanu-Wildwasserfahrens, dass Angst in den unterschiedlichsten Ausprägungen in Risikosportarten präsent ist (vgl. ebd., 66-68) ${ }^{126}$. SCHIEDCK (2003) hat zudem in einer Untersuchung an Fallschirmspringern die Angst-Leistungsbeziehung umfassend untersucht.

Angst als emotionaler Zustand wird mit einer Aktivierung des autonomen Nervensystems beschrieben und kann demnach als eine Form der zentralen Erregung bezeichnet werden. In der Psychologie wird Angst als eine negativ wahrgenommene Emotion etikettierten, die zu einer Vermeidung der Handlung führt oder Fluchtverhalten auslöst ${ }^{127}$ (vgl. DORSCH 1998, 34). Sie ist damit als kognitive, emotionale und körperliche Reaktion auf eine Gefahrensituation bzw. deren Antizipation zu verstehen (vgl. HACKFORT \& SCHWENKMEZGER 1985, 10). Von diesen Grundlagen ausgehend, erscheint es zunächst schwierig anzunehmen, dass Angst auch als Motiv für eine Handlungsausübung wirksam sein kann, und nicht zu deren Vermeidung führt. Unterschieden werden müssen hierbei biologisch ausgerichtete Forschungsbeiträge, die sich als Erweiterung aktivationstheoretischer Ansätze verstehen lassen und kognitiv-handlungsorientierte Theorien.

\subsubsection{Biologisch ausgerichtete Ansätze}

Angst als emotionaler Zustand ist mit einer Reihe physiologischer Vorgänge im Körper verbunden. Ziel ist die plötzliche Aktivierung des Körpers, um der angstauslösenden Gefahrenquellen möglichst schnell zu entfliehen (vgl. HACKFORT \& SCHWENKMEZGER 1985, 24). FABRY (1990) geht von einer Mischung unterschiedlichster hormonaler und nervöser Vorgänge aus, die mit der Ausschüttung des Morphins, Adrenalins und Kortisols einhergehen. Diese aufputschende Wirkung kann demnach auch mit dem Entstehen von Lustgefühlen verbunden werden. Die im Risikosport hervorgerufene Unsicherheitssituation führt demnach zu einer Produktion körpereigener Substanzen mit euphorisierender Wirkung, die zur Beherrschung der Stresssituation erfolgt und mit einem gewissen Lusterleben verbunden ist. Angst und Lust sind somit voneinander abhängige Gefühlsregungen, die nur in Anbetracht einer angstinduzierenden Situation ihr Suchtpotential entfalten 
können. Im englischen Sprachraum hat sich für diesen Zustand der Terminus ,Thrill' durchgesetzt, der übersetzt mit Angstlust den höchsten Moment dieses situativen Erregungszustandes ausdrückt.

In Erweiterung der aktivationstheoretischen Erkenntnisse in Anlehnung an BERLYNE (1974) stellten SPIELBERGER et al. (1981) in einem biologisch ausgerichteten Forschungsbeitrag fest, dass angenehme und unangenehme Gefühle auch simultan erfahren werden können und damit unter Umständen auch als Motivation fungieren (vgl. ebd., 218). Das Konzept des subjektiv optimalen Zustands der Reizstimulation wird von ihnen um den Faktor Angst erweitert, indem sie ein optimales Verhältnis der Erregung dann annehmen, ,[...] wenn Gefühle des Vergnügens oder der Freude mit leichten Gefühlen des Unbehagens verbunden sind. Daraus folgt, dass eine Person das ,optimal level of pleasent feelings' im Kontext starker Gefühle von Neugier und leichten, unangenehmen Gefühlen von Angst erreicht" (ebd.). In diesem Zusammenhang kann Angst vor allem als eine spezifische Erregungsquelle verstanden werden, die das Ziel der optimalen Spannungssituation verstärken kann.

Da die Methodologie in der biologisch orientierten Psychologie vor vielfältige Probleme gestellt ist, stellt sich die ,Angstlust-Theorie' bis dato noch problematisch dar. Leider bleiben im Rahmen der biologisch ausgerichteten Forschungsrichtung zudem einige Fragen offen. So werden personalen Unterschiede im Angsterleben kaum berücksichtigt. Den Vorstellungen dieses Ansatzes folgend müsste sich Angst zudem stets linear in Lust umwandeln. Die Realität beschreibt aber vielfältige gegenseitige Wechselbeziehungen zwischen Angst und Lust, die mit zum Teil schwer nachvollziehbaren Wiederholungen und Rückwandlungen einhergehen ${ }^{128}$. Trotzdem erscheint eine ,Angstlust-Theorie' für den Risikosport vor dem Hintergrund der angstinduzierenden Prozesse, die bei der Handlungsbewältigung auftreten nicht vollkommen abwegig (vgl. auch SCHIDECK 2003). Es bleibt der biologisch orientierten Psychologie überlassen, in weiteren Forschungsansätzen die Bedeutung des Angstempfindens für ein lustvolles Erleben des Risikosports zu verifizieren.

\subsubsection{Kognitiv-bandlungstheoretische Ansätz̨e}

Schon LEWIN erkannte, dass die Begegnung mit tendenziell angstinduzierenden Handlungssituationen, die gleichsam lustvoll besetzt sind, eine Verhaltenskontroverse erzeugt. Während die Angstreaktion ein Fluchtverhalten (,Aversionsverhalten') auslöst, führt die Lustgewinnung zu einem Annäherungsversuch (,Appetenzverhalten'). DOLLARD \& MILLER (1950) sehen in dem Konflikt aus Annäherung und Vermeidung eine sich gegenseitig blockierende Reaktionsmöglichkeit, die durch Ausschaltung des Fluchtreflexes zu massiven Unlustgefühlen führen muss. Die Angstlust-Situation erhält folglich eine sehr negative Bewertung. Die Autoren nehmen schließlich an, dass das lustvolle Erleben derartiger Situationen masochistisch-perverse Züge annimmt und für Risikosituationen nicht als hinreichende Begründung gelten kann. 
LAZARUS et al. (1972) beschreiben Angstlust als Folge der Entscheidungs- und Bewertungsunsicherheit in der konkreten angstinduzierenden Situation. Angelockt von dem Herausforderungscharakter aber abgestoßen von der akuten Bedrohung vermischen sich in der Person demnach Zuwendungsverlangen und Abwehrhaltung zu einer in sich gegensätzlichen Verhaltenstendenz. Folge einer derartigen Situation ist auch hier eine Mischung aus Angst als Folge der Bedrohung und Lust als Folge der persönlichen Herausforderung. Nach LAZARUS kann dieser Vorgang insbesondere im Rahmen fortbestehender Handlungsoptionen demgemäß als durchaus lustvoll empfunden werden.

\subsubsection{Emotionspsychologische Ansätze}

SEMMLER (1994), der als aktiver Fallschirmspringer aus einem Selbstversuch heraus argumentiert, verfolgt bei seinen Analysen einen emotionspsychologischen Ansatz. Er betont, dass nicht nur die Überwindung der Angst, sondern auch ein ,[...] Angsterleben als Moment der Selbsterfahrung“ (SEMMLER 1994, 165) im Blickpunkt des Risikosports steht. SEMMLER unterstellt sportlichen Risikosuchern damit eine allgemeine ,Lust an der Angst' (ebd.). Der Autor geht davon aus, dass in der modernen Gesellschaft eine Vielzahl von angstinduzierenden Gefahrensituationen ${ }^{129}$ existieren, diese dem Menschen aber keine aktive Auseinandersetzung mit ihren Ängsten ermöglichen (vgl. ebd., 85). Die Konfrontation mit gefährlichen Risikosituationen stellt demnach ein Mittel dar ,[...] gegen die Angst vor der Angst. Durch den Erwerb von Fähigkeiten, die ein Kontrollieren der Gefahr ermöglichen, verliert die Angst ihren Schrecken. Als Risikosucher lebt man besser mit ihr, weil man gelernt hat, mit ihr umzugehen“ (ebd., 159).

Die Ausübung von Risikosportarten stellt insofern eine der wenigen Möglichkeiten dar, sich selbst als emotional und angstvoll zu erfahren:

„Sich in seiner Angst zu erleben, bedeutet, sich sehr nabe zu sein, sich zu ,spüren'. Während wir normalerweise in jedem Augenblick damit beschäftigt sind, uns mit den Augen anderer zu sehen, um herauszufinden, welche Erwartungen an uns gerichtet sind, wie wir uns wann zu verbalten haben und welche Rolle gerade erwünscht ist oder erfolgreich sein könnte, ist der Zustand der Angst im Leben vieler Menschen einer der wenigen Momente der völligen Authentizität. Wenn wir massive Angst haben, ist unser äußeres Verhalten identisch mit dem inneren. Angst spielt man nicht, Angst hat man - oder um es noch präziser zu sagen: Angst ist man" (ebd., 166).

Dieser Ansatz findet sich in der französischsprachigen Literatur bereits bei BOUET (1968):

„,...] car, d'une part il recherche le danger, il refuse la securité qui enlèverait tout élément d'incertitude et rendrait le succès assuré : et, d'une autre part, il cherche a mettre toute les chances de son coté et rejette toute démarche qui serait presque certainement exposée a l'échec" (BOUET 1968, 192). 
Dabei vollzieht sich mit der Angstkonfrontation - nach SEMMLERs Auffassung - immer auch ein Stück psychische und geistige Entwicklung des Risikosuchers, die durch alltägliche Angstvermeidung und Verleugnung nicht ermöglicht wird (vgl. ebd., 167). SEMMLER Ausführungen fügen sich damit in das durch die kulturhistorischen Ausführungen entwickelte Bild des Risikosports als Gegenwelt eines als emotionslos empfundenen Alltags ein.

Auch AUFMUTH argumentiert emotionspsychologisch, indem er die Ausübung von Risikoaktivitäten mit einer existentiellen Sinnkrise - mit einer Überwindung der inneren Leere - erklärt. Er stützt seine Aussagen auf die Aussagen von alpinen Bergsteigern, die „den seelischen Mangelerscheinungen der Gesellschaft" (AUFMUTH 1988, 18) durch die Selbsterfahrung im Risikosport begegnen. Angst als emotionales Erlebnis spielt für AUFMUTH dabei eine wichtige Rolle, denn „ohne die Angst wäre die Bergsteigerei für viele uns ganz einfach uninteressant" (ebd., 65).

Leider wird es insbesondere von SEMMLER, aber auch AUFMUTH versäumt, das Angsterleben als positives Erlebnismerkmal von anderen Erlebnisdimensionen und emotionalen Zuständen wie Spannung oder Erregtheit abzugrenzen. Es kommt dadurch teilweise zu einer diffusen Vermischung emotionaler Zustände und ihrer Bewertungen unter der Kategorie Angsterleben. Zudem wird in SEMMLER's Theorienentwicklung die Frage nach den persönlichkeitsspezifischen Differenzen stark verkürzt. Die Frage, wieso einige Menschen das Angsterleben als positiv bewerten und andere negativ wahrnehmen wird bei den Autoren nicht nachvollziehbar.

Zusammenfassend lässt sich die Motivation zum Risikosport mit der Möglichkeit eines authentischen Erlebnisvorgangs im Zustand der Angst erklären. In Anknüpfung an aktivationstheoretische Ansätze wird hierbei von Angst als Erregungsquelle aber auch als Form eines emotionales Selbsterleben ausgegangen. Es bleibt allerdings fraglich, ob es sich beim Angsterleben um eine Motivstruktur im Sinne einer personellen Disposition handelt, bzw. welche situativen Anreizelemente ein positives Angsterlebnis im Risikosport ermöglichen.

\subsubsection{Risikosport als Angstüberwindung}

Neben dem reinen Erlebnis von Angst stellen diverse Beiträge die Überwindung von Angst in den Mittelpunkt ihrer Forschungsbeiträge. Zu differenzieren sind hierbei psychoanalytische Ansätze und wiederum kognitivhandlungstheoretische Theorien, die sich aus verschiedenen Forschungsperspektiven der Thematik nähern.

\subsubsection{Psychoanalytische Ansätz̧e}

In einer Vielzahl verschiedener psychoanalytischer Theorien wird in der Angstüberwindung ein wesentlicher Anreiz zur Ausübung risikoreicher und angstinduzierender Tätigkeiten gesehen. $\mathrm{Zu}$ unterscheiden sind hierbei so genannte kontraphobische Ansätze (FENICHEL; AUFMUTH) und psychoanalytische Neurosetheorien (BALINT ; KOHUT; ARGELANDER). 
Einen der ersten Versuche risikosuchendes Verhalten als eine Form der Angstüberwindung psychoanalytisch $\mathrm{zu}$ deuten, stammt dabei von FENICHEL (1974) Aus der Tatsache, dass bestimmte gefährliche Situationen Angst auslösen, diese aber nicht zu einem Vermeidungsverhalten führen, schloss FENICHEL, dass Angst auch als Motiv wirksam sein musste. Die Neigung angstauslösende und gefährliche Situationen aufzusuchen, anstatt sie zu meiden, beschreibt FENICHEL (1974) mit dem Begriff der ,kontraphobischen Haltung'. Danach steht die Bewältigung von angstinduzierenden gefährlichen Situationen nur stellvertretend für eine ,innere' Überwindung ureigener Ängste. Durch die stellvertretende Bewältigung der Angst in Gefahrensituationen wird die Fähigkeit suggeriert, mit den eigenen ,inneren' Ängsten umgehen zu können, die aus traumatischen Kindheitserlebnissen resultieren (vgl. FENICHEL 1974, 70-71).

Nach FENICHEL kennzeichnet sich der Sport geradezu als ein „kontraphobisches Phänomen“ (vgl. WARWITZ 2001, 178). Überflüssige Ängste, die sich aus der Kindheit angestaut haben, lassen sich besonders im und durch den Sport trainieren und beherrschen.

In diesem Zusammenhang skizziert AUFMUTH (1989a; 1992) für das alpine Bergsteigen die Verknüpfung zwischen dem typischen Risikomoment einer Sportart und den damit verbundenen seelischen Verwundungen. In einer komplexen und scharfsinnigen Analyse kommt AUFMUTH zu der Überzeugung, dass der risikobereite Sportler im wesentlichen aus einer fundamentalen Krise der Ich-Identität heraus handelt. Für das extreme Bergsteigen wird demzufolge eine traumatische Angst vor dem ,Fallen' bzw. dem ,fallen-gelassen-werden' diagnostiziert (vgl. ebd. 1989a, 134). Beim Kanu-Wildwasserfahren ,[...] drohen das Zermalmtwerden durch die Gewalt des tobenden Wassers und das Ersticken" (ebd.), die damit zugleich auf die „[...] seelische Bedrohung verweist [...]“ (ebd.), die es zu bewältigen gilt.

AUFMUTH diagnostiziert ähnlich wie SEMMLER den Verfall emotionaler Grundbedürfnisse durch die zunehmende Zivilisierung und Domestizierung der Gesellschaft. Er registriert eine allgemeine Gefühlsverdrängung, welche zu einer umfassenden Verarmung der Gefühlswelt und damit zu einem Verlust an Lebendigkeit führt (vgl. ebd. 1989b, 112). Im Gegensatz zu den emotionspsychologisch-kognitiven Ansätzen SEMMLERs folgert AUFMTH allerdings, dass der Risikosport ein ,,[...] Vorgang der Realitätsbewältigung“" (ebd., 218) darstellt und damit als Mittel zur Ausschaltung tieferer Selbstbegegnungen dient.

Einen ähnlichen psychoanalytisch orientierten Ansatz entwickelt BALINT (1994), der das Eingehen von Risikosituationen mit dem englischen Begriff ,thrill' (zu übersetzen mit ,Angstlust' (s.o.)) beschreibt. BALINT gilt als profiliertester Vertreter der so genannten Neurosetheorie. Als grundlegende Merkmale der Angstlust bezeichnet BALINT (1994): „Die objektive äußere Gefahr, welche Furcht auslöst, das freiwillige und absichtliche Sich-Ihr-Aussetzen und die zuversichtliche Hoffnung, das schließlich alles gut wird" (ebd., 21). 
BALINT kontrastiert bei seiner Analyse zwei Persönlichkeitsprofile, die in unterschiedlicher Weise mit gefährlichen und risikoreichen Situationen umgehen ${ }^{130}$. Während der Risikosuchende (,Philobat') immer wieder neue Risikosituationen aufsucht, versucht der Risikovermeider (,Oknophile') diesen Situationen kategorisch aus dem Weg zu gehen. Hierbei handelt es sich um einander idealtypische Varianten (,primitive Haltungen“)(ebd., 36), die in Reinform kaum anzutreffen sein werden, jedoch Verhaltenstendenzen im Umgang mir sportlichen Risikosituationen aufzeigen.

Risikosuchendes Verhalten resultiert nach BALINTs (1973), Theorie der Primären Liebe' aus der frühen Trennung der Mutter-Kind-Einheit, durch die das Kind eine Art Geburtstrauma erleidet (vgl. BALINT 1973, 81). Das Aufsuchen von sportlichen Risikosituationen kann demnach als ein Versuch verstanden werden, dieses Trauma zu bewältigen und den ursprünglichen Primärzustand wieder herbeizuführen. Risikosucher akzeptieren die neue Realität der Trennung, indem sie sich wiederholt risikoreichen Situationen aussetzten, um sie zu bewältigen. Durch die Auseinandersetzung mit Gefahren erwirbt der Risikosucher Fertigkeiten (,Skills'), die ihn dazu befähigen, „,...] bis zu einem gewissen Grad die zerstörte Harmonie zwischen ihm und der Welt wieder herzustellen“ (BALINT 1994, 73). Die wiederholte Konfrontation mit Gefahren und Angst, beweist ihm zudem, dass die in der Auseinandersetzung mit Gefahr erworbenen Fähigkeiten ausreichen, um auch mit erneuten traumatischen Erfahrungen fertig $\mathrm{zu}$ werden (vgl. SZCZENY-FRIEDMANN 1982, 31):

„Der Preis, den er dafür zu zablen hat, scheint ein Zwang zu einer nie endenden Wiederbolung des ursprünglichen Traumas zu sein, eine Art traumatische Neurose" (BALINT 1994, 72) ,[...] ein Weg, vielleicht sogar der wichtigste, um sich mit den Folgen eines Traumas auseinanderzusetzen, ist, es aktiv und absichtlich hervorzurufen" (ebd., 97).

Im Bezug auf das Risikoniveau des Risikosuchenden unterscheidet der Autor schließlich exemplarisch drei Stufen:

- den Besucher eines Jahrmarktes und Vergnügungsparks, der sich ganz auf die Sicherheitsvermittelnde Technik verlässt und sich dem Erlebnistaumel eher passiv aussetzt; „ein Kind im Gewande eines Helden [...]“ (ebd., 92).

- den Sportler, der das einzugehende Risiko selber bestimmen und kontrollieren will. Obwohl der Sportler im Handlungsvollzug auf sich selbst verwiesen wird, stehen ihm in Form von Lizenzen, Gerätevorschriften oder Verwendungsvorschriften, wohlwollende Wächter' zur Verfügung.

- den Professional - als Stuntman, Testfahrer oder -Flieger aber auch als Leistungsspitze in den verschiedenen Risikosportarten - der seine Sicherheitsmaßstäbe erst selbst finden und generieren muss und nur schwer kalkulierbaren Risiken ausgesetzt ist (vgl. ebd.).

Der Risikovermeider als idealtypischer Menschentypus weicht risikoreichen Situation demgegenüber aus. Seiner Angst des Verlassen-werdens entgegnet er mit Anhänglichkeit und Nähe. Er klammert sich an Objekte und Men- 
schen, von denen er sich Schutz und Geborgenheit verspricht, um so die traumatische Trennung von Mutter und Kind zu überwinden. Risikosport entspricht somit nicht der Handlungslogik des Risikovermeiders.

So gesehen, verfügt der Risikosuchende über ein größeres Maß an IchStärke und Handlungsfähigkeit als der Risikomeidende. Dieses hohe Maß an Ich-Kompetenzen lässt ihn allerdings weniger beziehungsfähig werden und zwingt ihn ,[... zu einer nie endenden Wiederholung des ursprünglichen Traumas" (ebd., 73). Er muss sich immer wieder riskanten Prüfungen aussetzen, um ,[...] seine Zweifel an der Verläßlichkeit seiner Fertigkeit zu kontrollieren“" (ebd., 98).

Einen weiteren psychoanalytischen Ansatz im Umfeld der Neurosetheorie liefert KOHUT (1981), der Risikoverhalten als Versuch einer Realitätsverleumdung beschreibt (vgl. ebd., 42f). Nach seiner Auffassung entwickelt eine neurotische Persönlichkeit, wer nicht in der Lage ist, seine eigene Begrenztheit anzunehmen oder bereit ist, sich auf das normale Maß herabzustufen ${ }^{131}$. Risikosport stellt demnach den Versuch dar, diese Begrenztheit zu überwinden und im Erleben von Risiko und Gefahr die Realität zu negieren. Der Risikosportler versucht im wiederholten Aufsuchen von Risikosituationen den Begrenzung des Lebens zu entkommen (vgl. ebd.).

Der Psychoanalyse kommt der Verdienst zu, erstmalig ein schlüssiges Gesamtkonzept zu den Beweggründen risikosuchenden Verhaltens entworfen zu haben. Auch die Beschreibung und kontrastierende Darstellung der Persönlichkeitstypen des Risikosuchers und Risikovermeiders sowie die Typologie risikosuchender Persönlichkeiten bei BALINT erscheint gelungen. WARWITZ (2001) bestätigt insbesondere die Evidenz dieser Typisierungen durch zahlreichen qualitativen Befragungen und halboffenen Interviews, wodurch insbesondere die Charakterisierung risikosuchender Persönlichkeiten erhärtet.

Kritisch ist allerdings die Darstellung risikosuchenden Verhaltens im Bereich pathologischer Zustände zu bewerten, denn die Anknüpfung an die Theorie FREUDs zwängen das Risikoverhalten in medizinische Kategorien von ,krank' und ,gesund', die so nur schwer nachvollziehbar ist. Risikosport ist demnach nicht als freudvolles Erleben zu verstehen, sondern als pathologischer Zwang, frühkindliche Verletzungen zu verarbeiten. Hierbei wird übersehen, dass sich Risikosport nicht in ein Schema des Normalen zwängen lässt. Der risikobereite Mensch schafft vielmehr eigene Beurteilungskategorien, die über die Projektion des eigenen Zustandes und Erwartungshorizontes des beschreibenden Forschers aber auch der nicht beteiligten Gesellschaft hinaus verweist. Schwer nachvollziehbar ist auch die tiefenpsychologische Deutung im Sinne eines früh-kindlichen Trennungstraumas, dass die gesamte Entwicklung bestimmt und zur Herausbildung neurotischer Störung führt. Dieses Denkmodell bewegt sich zu sehr in den eigenen theoretischen Vorgaben und verstellt den Blick auf alternative Möglichkeiten. Zudem weisen die psychoanalytischen Modellen auf Grund ihrer mangelnden empirischen Absicherung und geringen Objektivierbarkeit keine direkte Praxisrelevanz auf (vgl. auch WARWITZ 2001, 112). Nur wenige der vorge- 
stellten Autoren verfügen über eigene Risikoerfahrungen, was die Wirklichkeitsnähe beeinträchtigt. Auch kann die Allgemeingültig der Ansätze in Frage gestellt werden und im Bezug auf ihre Evidenz kritisiert werden (z.B. SCHÖNHAMMER 1991, 208).

\subsubsection{Kognitiv-Handlungsorientierte Ansätz̧e}

Neben dem reinen Erleben von Angst finden sich auch für die Angstüberwindung verhaltenstheoretische Ansätze, die zur Erklärung risikosuchenden Verhaltens dienlich sein können. WARWITZ (2001) beschreibt die Risikosuche als Konfrontation mit der Angst, um sich selbst als stark und souverän zu erleben ,,[...] um zu demonstrieren, dass er [der Risikosucher; Anm. des Verf.] trotz Angstbelastung in einer hochgefährlichen Prüfung handlungsfähig und erfolgreich sein kann" (ebd., 189). Risikosport bietet demnach Anreize zur Selbstbestätigung und Selbsterfahrung durch herausfordernde Handlungselemente.

SEMLER (1994), der schon das Moment des Angsterlebens im Risikosport hervorhob, sieht in der Überwindung und Bewältigung der Angst ein weiteres handlungsleitendes Motivelement des Risikosports. SEMLER schlussfolgert, dass „,...] unser Leben durch seine Begrenztheit und der aus ihrem Erkennen resultierenden Grundangst primär angstbestimmt ist" (ebd., 93). Seine Erfahrungen aus dem Fallschirmspringen verallgemeinernd, nimmt er an, dass es bei Risikosuchern darum gehe, die Konsequenzen der Angst - insbesondere die Angst vor der Angst - zu überwinden. Das Erlebnis in angstinduzierenden Situationen - trotz der drohenden Gefährdung handlungsfähig zu sein und die direkten Gefahren kontrollieren zu können führt zu einem erhöhten allgemeinen Selbstbewusstsein und Vertrauen in eigene Fähigkeiten: „Wer mit den gefährlichen Situationen zwischen Himmel und Erde zurechtkommt, wird nicht in banalen Alltagsproblemen scheitern" (SEMLER 1994, 66). Risikosport dient insofern zur stellvertretenden Bewältigung von Grundängsten, die immer dann präsent sind, wenn sich der Mensch seiner Verletztheit bewusst wird.:

„Angst ist immer mit im Spiel, auch bei sebr erfabrenen Risikosuchern. [...] Denn die Angst, mit der sie es zu tun baben, ist nur vordergründig die Angst vor der Höhe, vor der Tiefe des Meeres oder vor der Geschwindigkeit. Tatsächlich verbirgt sich hinter ibr immer unsere Grundangst, also die Angst, den Anforderungen des Lebens nicht gewachsen zu sein, oder vielleicht die Angst vor dessen Folgen - in letzter Konsequenz die Angst vor dem Tod" (ebd., 155).

Das Bestreben sich selbst als angstvoll zu erleben und gleichzeitig die eigene Angst überwinden zu können, führt unweigerlich zu einem Erkenntnisprozess, sich auch mit seinen Ängsten im Alltag zu beschäftigen. Zu differenzieren gilt es hier nochmals im Bezug auf die Dimension der Angstüberwindung und die von BALINT vorgestellte Typologie im Bezug auf die Handlungsstruktur der jeweiligen Aktivität. So ist es sowohl im Risikosport 
als auch bei der Vielzahl an ,Thrill-Aktivitäten' - wie dem Besuch einer Achterbahn - möglich individuell Angst zu erleben und diese zu überwinden. Der sekundäre Gewinn, den Risikosucher aus der freudvollen Selbstbestätigung ziehen können, die angstauslösende Situation mit den eigenen Fähigkeiten kontrollieren zu können, erschließt sich aber nur dem Risikosportler. Auch den von SEMLER angedeuteten Transfer auf alltägliche Lebenssituationen - und damit ihre pädagogische Relevanz als Lernprozess - ist dem Risikosport vorbehalten.

Allerdings weisen die empirischen Arbeiten von EPSTEIN (1977) ${ }^{132}$ darauf hin, dass nicht primär Selbsterfahrungsprozesse und damit die direkte Angstüberwindung gesucht wird, sondern dass Risikosportler über ausgeprägte Angsthemmungssysteme verfügen. In bedrohlichen Situationen seien die Risikosportler demnach besser in der Lage den Erregungsanstieg zu bremsen und eine entsprechende Überreaktion der Angstpotentiale zu verhindern. WIELAND et al. (1993) kommen in ihrer Untersuchung zu dem Schluss, dass die Angstüberwindung nicht gelernt werden könne, sondern als Persönlichkeitsmerkmal zu den Grundvoraussetzungen von Risikosportlern zählen.

Zusammenfassend bietet die Handlungssituation im Risikosport Anreize in der Form einer individuellen Angstüberwindung, die - je nach Forschungsansatz - als identitätsstabilisierende Selbstbestätigung oder als stellvertretende Traumabewältigung verstanden werden kann.

\subsubsection{Risikosport als Existenzvergewisserung}

Ein weiterer Ansatz, risikosuchendes Verhalten im Sport vor dem Hintergrund motivationaler Elemente zu erklären, stammt von LE BRETON (1995; 2001). Mit seinem Denkmodell der Ordaltheorie ${ }^{133}$ knüpft der Autor an die mittelalterlichen Ordalien an, die als Gottesurteil oder Schicksalsmacht verstanden wurden und mit dem Dualismus von Tod und Leben zur Sinnvergewisserung dienten. LE BRETON bezieht seine Theorie dabei auf eine ganze Brandbreite von Handlungsstrukturen, die nur sekundär mit Risikoverhalten in Verbindung stehen (z.B. auch Anorexie oder Computerleidenschaft). Seiner Auffassung folgend, unterziehen sich Risikosportler in der Risikosituation einer Art existenzieller Sinnprüfung, um sich des Lebens zu vergewissern. Dieser Ansatz findet sich in der französischsprachigen Literatur in ähnlicher Form auch bei BAILLETTE (1995) und BROHM (1995) wieder:

„Les sports extremes (tout comme les activités sportives compétitives qui s'extremisent) sont en quelque sorte aussi un entrainement contre la mort. L'aventuirier sportif s'emploie à repousser inexorablement les barrières du vivant, à explorer les chemins de l'avant mort [...]" (BAILLETTE 1995, 322).

Angesichts einer allgemeinen Werteunsicherheit und dem Verlust verbindlicher Sinninstanzen wie Religion oder Politik dient der Risikosport 
demnach als persönliche Sinninstanz. Im Sinne einer exemplarischen Probe versucht der Risikosucher sich seines Dasein im Risikosport durch die existenzielle Konfrontation mit dem Tod zu vergewissern. "Jede Suche nach Grenzen ist ihrem tiefsten Beweggrund ein Versuch, den Tod zu bewegen, die Existenz zu bekräftigen“" (vgl. LE BRETON 1995, 16). Obwohl auch die metaphorische Nähe zum Tod ausreicht, existenzielle Vergewisserungen zu erfahren, wird die Daseinsbekräftigung umso stärker erlebt, je näher die wirkliche Todesnähe spürbar ist.

„Das Risikoverbalten ist ein Liebängeln mit dem Tod: Geschwindigkeitssu-
che, Sprünge in die Tiefe, Besteigungen von Steilhängen [..], in allen Fällen
wird metaphorisch dem Tod getrotzt, geht es darum, mit tödlicher Bedrohung
in Tuchfüblung zu kommen, ibr aber durch geeignete Vorkehrungen zu ent-
gehen. Indem man sich auf diese Weise dem Tod stellt, wird der Tod selbst ge-
stellt und werden die Grenzen seiner Macht aufgereigt: das Identitätsgefühl
wird durch die erfolgreich bestandene Prüfung gestärkt" (ebd.).

In seiner Ordaltheorie nimmt der Autor Bezug auf die soziologische Ansätze zum Erlebnisdefizit des gesellschaftlichen Alltags ohne allerdings konkrete Hinweise auf diese sozialen Wandlungsprozesse zu geben. Im Mittelpunkt des ,ordalen' Erlebnisses steht für LE BRETON der Körper, denn ,[...] nur der Körper kann später den Erfolg zweifelsfrei beglaubigen“" (ebd., 56). Das intensive Leben im Augenblick erschließt sich dabei nicht nur im Sport, der eine zentrale Rolle in seinem theoretischen Konzept einnimmt. Ordalisierungsformen beobachtet

In einem indirekten Zusammenhang ist auch die Arbeit von CLAUSEN (2003) zu verstehen, der in Anlehnung an WUST (1937) eine Theorie über die religiösen Dimensionen des Extrem- und Risikosport entwickelt. Obwohl der Autor kein stringenter Verfechter der LE BRETON'schen Ordaltheorie ist, beantwortet er die Frage nach den Hintergründen extremsportlicher Aktivitäten ebenfalls mit einer existentiellen Suche nach Identität und Sinn:

„W arum begibt sich der Extremsportler bewusst in Situationen, in denen der Ausgang hochgradig unsicher ist? [...] Weil der Extremsportler in sich unsicher ist [...]. Die Unsicherheit hinsichtlich des eigenen Standpunktes lässt sich übersetzen in die mettphysisch-religiöse Frage nach der Identität und dem Sinn (,wer bin ich?' und ,warum bin ich?') “(ebd., 95).

Wenngleich die Ordaltheorie für bestimmte Bereiche risikosuchenden Verhaltens durchaus sinnvoll erscheint, ist doch WARWITZ (2001) zu zustimmen, wenn er konstatiert, dass die Anwendbarkeit des Erklärungsmodells auf den Risikosport auf Ausnahmen begrenzt ist (vgl. ebd., 136). Sicherlich lassen sich insbesondere die extremen Varianten des Risikosports durch die Einbeziehung der Ordaltheorie besser erklären. So weisen auch die Erzählungen einiger Extrembergsteiger eindeutig auf Ordalisierungsformen hin ${ }^{134}$. Diese extremen Ausprägungen des Risikosports sind von der allgemeinen Handlungsstruktur der sportlichen Risikosituation aber weit 
entfernt. Ihre Anwendung auf den allgemeinen Kontext risikosportlicher Handlungen erscheint hier meines Erachtens als fraglich. Zudem verstellt die Verengung der Ordaltheorie auf den Dualismus von Leben und Tod den Blick auf primäre Motivstrukturen, die z.B. das Kompetenzerleben in den Mittelpunkt ihrer Betrachtung stellen ${ }^{135}$

Zusammenfassend lässt sich die Motivation zum Risikosport - insbesondere in seinen extremen Formen - mit Bezug auf die Ordaltheorie als existentielle Selbsterfahrung begreifen, die zur Überwindung von Sinndefiziten und zur Bestätigung der Ich-Identität führt.

Insgesamt gilt für die bislang dargestellten psychologischen Ansätze, dass der Anreiz von Risikosportarten allein in einem Element zu finden ist - sei es in der Überwindung oder dem Erleben von Angst, der Erfahrung von Todesnähe oder der Befriedigung eines individuellen Erregungsbedürfnisses. Intensive Glücksgefühle als Anreiz von Risikosportaktivitäten finden sich dagegen in den Ausführungen nur vereinzelt (z.B. bei SEMLER (1994) im Moment des Angsterlebens) oder als Folge differenzierter Reflexionsvorgänge. Weitgehend ungeklärt bleibt in den explizierten Ansätzen, warum neben vielfältigen anderen Möglichkeiten - gerade der Risikosport zur Befriedigung der beschriebenen Bedürfnisse beiträgt. Darüber hinaus fehlen in den bisher dargestellten Ansätzen konkrete Vorstellungen zu den Abwägungs- und Entscheidungsprozessen, die dem Handeln in Risikosituationen zugrunde liegen. Geht man weiterhin davon aus, dass Risikoverhalten nicht ausschließlich bedürfnisgesteuert ist, sondern aktiv als bewusstes Verhalten organisiert wird, so muss ferner genauer analysiert werden, inwiefern sich die psychische Regulation in Handlungssituationen auch über die spezifische Anreizstruktur beschreiben lässt. Durch eine analytische Trennung der Anreize wird daher im folgenden in einem integrierenden Überblick über den Forschungsstand dargestellt, dass die für die Risikosituation kennzeichnenden Bedingungen auch in Form von Handlungsmotiven bestehen.

\subsubsection{Risikosport als Grenzsuche: Kognitiv-handlungstheoretische Ansätze}

Sich Risikosportaktivitäten zuzuwenden, bedingt, wie wiederholt dargestellt, sich in Situationen zu begeben, die vor allem durch eine umfassende Unsicherheit charakterisiert sind. Weder Zielerreichung (,Schaffe ich das?') noch der Handlungsverlauf (,Was kommt auf mich zu?') können vor der Handlungssituation vollkommen sicher vorhergesehen werden. Durch das Verlassen gegebener Sicherheitszonen begibt sich der Handelnde damit in Situationen, die nicht deutlich von außen, eingegrenzt' sind oder den Akteur selbst in seinem Handlungsraum ,begrenzen'. Das Aufsuchen von Risikosportaktivitäten kann demzufolge als eine vielfältige Suche nach Grenzen verstanden werden (vgl. ALLMER 1998, 73).

Stellt sich das Wesen des Sports allgemein schon als ein Bestreben dar, spannungsinduzierende Grenzerfahrungen zu machen (vgl. FRANKL 1973a, 88), so kann diese Grenzsuche im Handlungs- und Erlebnisfeld des 
Risikosports als Suche nach den persönlichen und menscblichen Grenzen einerseits und als Austesten der persönlichen Risikogrenzen andererseits spezifiziert werden (vgl. ALLMER 1998, 73). Einen weiteren Ansatz zu Grenzerfahrungen in risikosportlichen Aktivitäten lässt sich auch bei MASLOWs (1953; 1981) Theorie der, Peak-Experiences' finden.

\subsubsection{Suche nach den persönlichen und menschlichen Leistungsgrenzen im Risikosport}

Dass es bei Risikosportaktivitäten zu einer Überwindung von ,natürlichen' Grenzen kommt, bedingt allein die Struktur vieler risikosportlicher Handlungssituationen, deren Ausübung in der freien Natur eng mit der menschlichen Unvollkommenheit verbunden ist (vgl. SEMMLER 1994, 93) ${ }^{136}$. Gemeint ist damit die ursprüngliche Begrenzung des Menschen auf einen bestimmten Lebensraum. Diese Überwindung der eigenen Unvollständigkeit zeigt sich vor allem im Wunsch, fremde Wirkungsbereiche aufzusuchen und mit entsprechenden sportlichen Leistungen die frugale Grenze des Menschen zu verschieben. Beim Kanu-Wildwasserfahren wird beispielsweise die ,unnatürliche' Barriere des fließenden Wassers aufgesucht und der persönliche Handlungsraum dadurch entsprechend erweitert - beim Drachenfliegen der uralte Traum vom Fliegen. Dabei steht auch das Gefühl im Mittelpunkt, etwas zu erreichen, dass physisch eigentlich unmöglich ist (vgl. APTER 1994, 126). Risikosportler versuchen demnach die grundlegenden leiblichen Einschränkungen zu überwinden, um sich auch in anderen Lebensräumen zu bestätigen (vgl. ebd.).

ALLMER (1998) sieht neben der Überwindung der menschlichen ,Begrenztheit' auch im daraus resultierenden ,Kampf gegen die Naturgewalten' einen wichtigen Anreiz der Risikosportarten (ebd., 74). Demzufolge sucht der Mensch als weltoffenes Wesen eine ständige Bewährungsprobe mit der Natur, die das Ziel verfolgt, vor den Naturgewalten zu bestehen (vgl. ebd. 74f). In einer von OPASCHOWSKI (2000) durchgeführten Befragung zu den Motiven des Risikosports nannten 38\% der Befragten den „Wettkampf mit den Naturgewalten" als zentralen Anreiz einer Risikosportart (vgl. OPASCHOWSKI 2000, 117).

Ferner kann unter Grenzsuche auch die Bereitschaft verstanden werden, ,innere’ persönliche Grenzen zu entdecken und zu überwinden. Ein „Austesten der Anstrengungsbereitschaft ist die Suche nach den aushaltbaren Entbehrungen und Strapazen, die bereitwillig auf sich genommen werden, um herauszufinden, ob man in der Lage ist, selbst gestellte Herausforderungen zu Wasser, zu Lande und in der Luft gerecht zu werden“" (ALLMER 1998, 76). Damit eng verbunden kann auch der Wunsch verstanden werden, Herausforderungen ,,[...] nur auf sich selbst gestellt zu bewältigen“ (ebd.). In risikoreichen Situationen wird die Bestätigung dadurch gesucht, nur durch eigenes Handeln und in eigener Verantwortung die geforderte Aufgabe bewältigen zu können. Diese Grenzsuche wird von ALLMER (ebd.) als Austesten der Selbstkontrolle beschrieben: „Extreme [...] Situationen aus eigener Kraft meistern zu können, verstärken das Gefühl der Selbstkontrolle (,Ich habe es geschafft') und stellen die grundlegende Intention zum weiteren Austesten der 
persönlichen Leistungsgrenzen dar“ (ebd., 76). Der Einstieg in die Elemente des naturorientierten Risikosports gibt zudem eine gewisse Eigenlogik zum Austesten der Selbstkontrolle vor, die sich aus dem Handlungszwang der Risikosituation ableitet: Bei ,Einstieg' in eine Wildwasserschlucht oder einen alpinen Steilhang gibt es meist kein Zurück mehr, denn die Situation kann häufig nur durch aktives Handeln bewältigt werden. Eine zufällig geglückte Befahrung ist daher recht selten. Um gewissen Handlungssituationen, wie z.B. Walzen oder Rückläufen zu entkommen, ist aktives Paddeln sogar notwendig. Diese Besonderheit von Risikosportarten kann somit das Gefühl der Selbstkontrolle noch verstärken (vgl. KOCH 1994, 29).

Das Empfinden, etwas Besonderes eigenverantwortlich (Selbstverantwortung) durch eigenes Handeln geleistet zu haben (Selbstwirksamkeit), löst zudem ein intensives Gefühl der Befriedigung aus (Selbstbestätigung), dass von AUFMUTH (1983) als eine Form des Machterlebens bezeichnet wird: ,Macht besitzen' heißt

\section{„[...] Macht über sportliche Schwierigkeiten, über die Widrigkeiten der Na- tur, über die Qualen des gemarterten Leibes, über die Angst, über die Regun- gen der Bequemlichkeit und der Lust, über den bautnahen Tod, Macht, gan₹. allein die bärtesten Situationen zu meistern" (AUFMUTH 1983, 253).}

Im Erleben von Macht sieht auch McCLELLAND (1978) ein eigenständiges Motiv. Er kennzeichnet in diesem Kontext ein Machtstadium II, dass er als: „Ich kontrolliere mich selbst, habe Macht über mich“ (ebd., 78) beschreibt. Im Erleben von Macht werden abermals Parallelen zur Angstüberwindung im Konzept von SEMLER deutlich: Angst zu erleben, sie gleichzeitig zu überwinden und in der Aufgabe zu bestehen, kann als Macht über die eigene Emotionalität und das eigene Erleben interpretiert werden.

Das Gefühl der Selbstbestätigung oder ,[...] das Erleben des Ich als Einheit [..]" wie AUFMUTH $(1983,252)$ formuliert, bewirkt somit eine wichtige identitätsbildende Erfahrung, die als extrem positiv empfunden wird. Das Gefühl ,[...] ganz mit sich und der Welt eins zu sein [...]“" (ebd.), kann allerdings auch zu einem Prozess der Aufgabensteigerung führen, der auf immer größere Herausforderungen abzielt:

„Denn der Mensch ist neugierig, wo die Grenzen seiner Möglichkeiten liegen, und siehe da, die Grenzen liegen nirgendwo. Denn so wie der Horizont schiebt der Mensch seine Grenze mit jedem Schritt, den er auf sie zutut, immer weiter vor sich her. Bis an die Grenzen will der Mensch aber gehen. Er geht dadurch über sich binaus [...]"(FRANKL 1973a, 88)

Diese Spirale der Grenzsuche kann dazu führen, dass nicht nur persönliche Grenzen gesucht werden, sondern dass auch der Versuch gemacht wird, für den Menschen unmögliche Grenzen zu überwinden. Diese Tendenz etwas Außergewöhnliches und Nichtalltägliches zu vollbringen, zeigt sich auch im Extrem-Bereich vieler Risikosportarten, wo immer wieder so genannte ,Erstbefahrungen' (Kanu-Wildwassersport) oder ,Erstbesteigung' (Bergstei- 
gen) extrem schwieriger und gefährlicher Handlungsräume gemacht werden (vgl. auch KANUMAGAZIN 5/97: „Erstbefahrung des Rheinfalls“).

\subsubsection{Austesten derpersönlichen Risikogrenze}

Von außen betrachtet erscheint das Aufsuchen einer sportlichen Risikosituation fast absurd, begeben sich doch Menschen darin freiwillig in Gefahr. Daher erscheint es von diesem Standpunkt aus nicht abwegig „,[...] Betreibern riskanter Sportaktivitäten unbewusste Todessehnsüchte zu attestieren [...]“ (RHEINBERG 1997, 157). Dass die Realität des Risikosports ungleich differenzierter zu betrachten ist, wird von RHEINBERG (ebd.) durch folgende Unterscheidung bekräftigt:

„Entscheidend ist danach, inwieweit der Gang der Ereignisse vom Zufall abbängt, oder von mir und meiner Kompeten₹. [...] Betreiber von Risikosportarten baben eine starke Tendenz zu einem fähigkeitsabbängigen [Herv. d. Verf.] Risiko. Der Einfluss von zufallsabhängigen Faktoren wird dabei soweit wie möglich reduriert" (ebd., 157).

Eine Risikosituation wird folglich nicht fahrlässig oder in direkter Aussicht negativer Folgen eingegangen, sondern in Erwartung eines positiven Handlungsausgangs. Trotzdem bleibt eine gewisse Unsicherheit, die keine hundertprozentige Erfolgs- und damit Lebensgarantie verspricht. „In diesem Restrisiko [...] liegt der Anreiz für Risikosportler“ (ALLMER 1998, 79). Handeln in Risikosituationen ist zu erwarten, wenn

„[...] die Überzengung vorliegt, auch überraschend auftretende Gefahrensituationen meistern ₹u können. In diesem Fall ist das Ergebnis des Kalkulationsprozesses zwischen wahrgenommenen Fähigkeiten und Risikoeinschätzung, dass nicht vorhersehbare (nicht kalkulierbare) Risikosituationen als kontrollierbar bewertet werden" (ebd. / vgl. auch 6.2).

Wird das Restrisiko als beherrschbar eingeschätzt, werden somit auch solche Risikosituationen eingegangen, die von Außenstehenden als, unvernünftig' bewertet werden. RHEINBERG \& SCHNEIDER (1996) konstatieren hierzu: „Nicht das vitale Risiko an sich, sondern die Risikokontrolle durch die eigene Tüchtigkeit werden als Anreiz erlebt" (ebd., 422).

Risikosituationen bieten folglich die Möglichkeit, die eigene körperliche Unversehrtheit in Abhängigkeit der persönlichen Fähigkeiten zu erleben. Ähnlich der Leistungstendenz bezüglich der Selbstkontrolle, ergibt sich auch hinsichtlich des einzugehenden Risikos eine Herausforderung im persönlichen Austesten der Risikokontrolle (vgl. ALLMER 1998, 80). Unter Ernstfallbedingungen zu bestehen, wo Erfolg und Misserfolg nicht nur symbolische Konsequenzen haben, kann demzufolge ein weiteres Motiv für die Ausübung von Risikosport sein. Risikosportliche Aktivitäten wie das KanuWildwasserfahren werden als ,[...] Bewährungserlebnis bewusst aufgesucht. Der Akteur bringt sich in Gefahr, bewältigt sie und leitet aus dieser Bewältigung eine Ich-Bestätigung ab“ (BET"TE 1989, 179). 
Bei erfolgreicher Auflösung risikoreicher Situationen ist das Erlebnis der Kontrolle zudem mit hohen Kompetenz- und Glücksgefühlen besetzt (,Ich habe es geschafft'), die bei entsprechender Weiterentwicklung der Fähigkeiten zu einem Austesten der Risikobereitschaft führen. Denn hier gilt das Prinzip des Behaviorismus: „Angenehme Erlebnisse, und insbesondere solche, die eine starke emotionale Beteiligung auslösen, wecken in uns den Wunsch nach Wiederholung“" (SEMMLER 1994, 126). Das Gefühl eine Risikosituation zu kontrollieren und der Wunsch diese Empfindung zu wiederholen, führt dazu, sich immer neuen Herausforderungen zu stellen. SEMMLER (ebd.) sieht in diesem Bestreben sogar einen gewissen Suchtcharakter, der darin besteht, ,[...] sich wie von einem inneren Zwang getrieben immer und immer wieder mit Gefahren auseinander zusetzen" (ebd.).

Zusammenfassend lassen sich eine Vielzahl von verschiedenen Grenzerfahrungen im Risikosport finden, die mit extrem positiven Emotionen besetzt sind. Die sportliche Risikosituation repräsentiert mit ihren spezifischen Anforderungen eine der wenigen Möglichkeiten, persönliche und natürliche Grenzen legal zu erforschen.

\subsubsection{3 ,Peak-Experiences' (MASLOW) und 'Peak-Performance' (PRIVETTE)}

Die bereits älteren Ausführungen von MASLOW (1954; 1981) und PRIVETTE (1997) haben keinen direkten Bezug zum Risikosport, beschreiben aber eine weitere Erfahrungsperspektive, die für den Risikosport Relevanz besitzen könnte. Ausgehend von der bekannten Bedürfnispyramide entwickelt MASLOW ein Modell der Grenzerfahrungen, die er unter ,peak-experiences' resumiert. ,Peak-experiences' als Erlebnisqualität sind demnach im Bereich der Selbstverwirklichung anzusiedeln und beschreiben einen Moment des höchsten Glücks ${ }^{137}$. Diese Grenzerfahrungen bedeuten ein intensives Glückserleben, einen Zustand subjektiver Sicherheit und ein fast schon transzendentes Verschmelzen mit der Welt, welches mit veränderten Zeit- und Raumwahrnehmung verbunden ist (vgl. BEIER 2001, 160ff). Ohne das der Risikosport in seinen Dimensionen direkt als Auslösbedingung für diese Form der Grenzerfahrung von MASLOW identifiziert wird, können bei den situativen Bedingungen durchaus risikosportliche Handlungen in Betracht gezogen werden. So ist den auslösenden Situationen gemein, dass ein Gefühl der subjektiven Perfektion wahrgenommen wird bezogen auf die eigenen Fähigkeiten und die entsprechenden Anforderungen.

Unverkennbar ist die Ähnlichkeit des MASLOW'schen Ansatzen zum Flow-Konzept bei CZIKSENTMIHALYI. Ein weiteres verwandtes Konzept der ,peak-perfomance' bietet PRIVETTE (1997) - zu übersetzen mit Grenzleistungen. Für PRIVETTE sind Grenzleistungen a là ,peakperformance' mit phyisisch und psychisch anspruchsvollen Handlungen am oberen Ende der individuellen Leistungsgrenze verbunden. Grenzleistungen - in diesem Sinne als Erlebnisqualität - sind mit Merkmalen wie einer gesteigerten Zentrierung der Aufmerksamkeit, einem Gefühl gesteigerter Stärke (sowohl psychisch als auch physisch) und einem Freisein von Zwängen und 
Hemmungen verbunden (vgl. PRIVETTE 1997). Auch für PRIVETTE besitzen ,peak-perfomances' erlebnisreiche Glückszustände, die bei unterschiedlichsten Handlungen im subjektiven Grenzbereich erfahren werden. Im Bezug auf die Auslösebedingungen formulieren die Autoren, dass die Person sehr stark in die Situation eingebunden sein muss und für sie einen hohen Stellenwert besitzt (ebd., 200). Zwar wird Sport oder Risikosport in den Ausführungen PRIVETTEs nicht als Auslösebedingung genannt. MEIER (1996) berichtet aber in einer Untersuchung an Sportkletterern $(n=64)$, dass sowohl einfache Grenzerfahrungen, als auch tiefe Gernzerlebnisse für die Befragten motivbildend wirksam sind.

\subsubsection{Risikosport als Erlebnissuche - emotionspsychologische und tätigkeitszentrierte Ansätze}

Risikosportaktivitäten können in der Möglichkeit einer Grenzerfahrung letztlich eine Vielzahl von intensiven Erlebnisse schaffen, die in ihrer Gesamtheit fast ausschließlich als zweckzentriert klassifiziert werden. Das erwünschte Resultat, wie beispielsweise das Kompetenzerleben oder die Selbstbestätigung, setzt schließlich ein bestimmtes (Zwischen-) Ergebnis voraus, als dessen Folge man das beschriebene Erlebnis erfahren kann (vgl. RHEINBERG 1989, 146). Es erscheint jedoch als unzureichend, eine Erlebnissuche ausschließlich auf den antizipierten Erfolg einer risikosportlichen Handlung zu reduzieren. Bei diesen Ansätzen handelt es sich um Erklärungsansätze für das Auftreten und die Qualität von bestimmten Erfahrungen, die sich im Zusammenhang mit Risikosport darstellen lassen.

RHEINBERG (ebd.) schlussfolgert, dass in Risikosportaktivitäten unter anderem auch ,tätigkeitszentrierte' Anreize zu finden sind. Bestimmte Erlebnisqualitäten werden dabei im Vollzug der Handlung gesucht, ohne jegliche zweckzentrierten Erlebnisse zu antizipieren ${ }^{138}$. In einer Untersuchung zur Psychologie des Risikosports ( $\mathrm{n}=100$ ) findet ALLMER (1998) mehrere derartige tätigkeitszentrierte Erlebnisse, die sich als Suche nach intensiven Sinnesreizen einerseits und als Suche nach außergewöbnlichen Emotionszuständen andererseits zusammenfassen lassen (vgl. ebd., 84).

\subsubsection{Suche nach intensiven Sinnesreizen}

Die in diesem Abschnitt beschriebene Suche nach intensiven Sinnesreizen bezieht sich vorwiegend auf sensorische und kinästhetische Empfindungen, die nicht primär einem Bedürfnis nach Reizstimulation gemäß ZUCKERMAN entsprechen. Dabei ist zwischen intensiven Bewegungserlebnissen und verschiedenen Aspekten von Naturerleben zu unterscheiden (vgl. ALLMER 1998, 84).

Bewegungserlebnisse lassen sich mit den in der Sportwissenschaft postulierten Körpererfahrungen gleichsetzen, die bei TREUTLEIN \& FUNKE \& SPERLE (1992) in vielfältiger Weise kennzeichnend für das Wesen des Sports beschrieben werden. Der Anreiz von Bewegungserlebnissen bei Risikosportarten ist hingegen weniger bekannt und erklärt. Für KIPPHARD 
(1995) gehen besondere Bewegungserlebnisse in Risikosportarten allein auf ein intensives Erleben der vestibulären Funktionen zurück (vgl. ebd. 335). Dies lässt sich hinsichtlich der reichhaltigen, sicheren’ Möglichkeiten, die ebenfalls ein intensiveres Gleichgewichtserleben im Sport ermöglichen, jedoch in Frage stellen.

Konkreter lässt sich in diesem Kontext die Argumentation CAILLOIS (1964) verfolgen, der in seiner Klassifikation zweckfreier Tätigkeiten eine Einheit bildet, die er als ,Ilinx' bezeichnet. ,Ilinx' stellt sich dann ein, wenn der Körper in besondere Zustände gebracht wird, wie z.B.: „Sturz oder Schweben im Raum, rapide Rotationen, Gleiten, Geschwindigkeit, Beschleunigung in einer gradlinigen Bewegung oder ihre Kombination mit einer kreisförmigen“ (ebd., 33). Für das Kanu-Wildwasserfahren lassen sich beispielsweise ein gewisser Schwebe- oder Gleitzustände und die „[...] Erfahrung fließender Bewegungen [...]“ (ALLMER 1998, 84) annehmen. Dabei steht ganz besonders die Bewegungseinheit Mensch-Wasser im Vordergrund der Bewegungserfahrung: „Es ist Bewegungserlebnis in und mit dem flieBendem Wasser, im Spüren des gewaltigen Wasserdrucks, im Agieren mit dem Wasser [...]. Nur mit dem Ausnutzen der Kräfte des Wassers, dem spielerischen Umgang mit diesem Elementen kann Wildwasserfahren erfolgreich sein" (BAUER \& SCHULTE 1997, 39). Beim Klettern wird beispielsweise die Erfahrung der Schwerkraftüberwindung zu einem fundamentalen Bewegungserlebnis. Gleiches gilt auch für die unterschiedlichen Flugsportarten mit dem Gefühl der Schwerelosigkeit.

LE BRETON (1995) unterscheidet vier Hauptfiguren des Risikoverhaltens, wobei sich die so genannten ,Vertigo-Erfahrungen' (ebd., 20) als bedeutsame motivbildende Bewegungserfahrung identifizieren lässt. Vertigo lässt sich als das Erleben von Taumel und Schwindel im Sinne einer Hingabe an die Umweltkräfte beschreiben. Der Vertigobedürftige liefert sich den Schwerkräften und Fliehkräften aus und überlässt sich ihnen als eine Art Spielball und erfährt dabei eine Art ,wolllüstiger Panik“ (ebd., 20). Diese Lust am Taumel lässt sich nach LE BRETON beim Geschwindigkeitsrausch, dem Gleiten und Schweben aber auch beim Sprung ins Nichts erleben (vgl. ebd.).

BEIER (2001) stellt in seiner Untersuchung zur Motivation in OutdoorSportarten ( $\mathrm{n}=315)$ fest, dass ,[...] Bewegungserleben einen Anreiz für Outdoor-Aktivitäten darstellt" (ebd., 332). Eine genauere Differenzierung nimmt der Autor nicht vor. Unter den von ihm untersuchten Sportarten wurden insbesondere die risikoreicheren Varianten wie z.B. das Klettern als besonders erlebnisreich im Bezug auf das Bewegungserleben bewertet.

Viele Risikosportarten verkörpern ferner verschiedene für den Menschen neue Arten der Fortbewegung, die durch eine unsichere Gleichgewichtsposition charakterisiert sind (beispielsweise das Kanu-Fahren, das Klettern, das Drachenfliegen etc.). Das fortwährende aktive Aufrechterhalten dieser Lage ermöglicht - vergleichbar mit den ersten Schritten des Kleinkindes - eine intensive Bewegungserfahrung speziell für den Anfänger. 
Für das Naturerleben schlussfolgert MERLET (1990) aus einer Untersuchung zur Motivation des Kanu-Wildwasserfahrens ( $\mathrm{n}=122)$, dass „,...] die Naturliebe und die Faszination von Pflanzen und Tierwelt [...]" (ebd., 68) als eines der Hauptmotive der Sportart betrachtet werden kann. Auch OPASCHOWSKI (2000) charakterisiert Risikosportler insbesondere im naturorientierten Risikosport als sehnsüchtig nach unberührter und ,wilder' Natur (vgl. ebd., 49). Auch BEIER (2001) kommt in seiner Untersuchung zum Outdoor-Sport, bei der auch verschiedene Risikosportarten untersucht worden zu dem Schluss:

„Im Vergleich zu anderen Studien lässt sich detalliert nachvollziehen, dass es neben den verbältnismäßig globalen ästhetischen Naturerfahrungen vor allem taktile Reize, Temperaturreize, natürliche Geräusche sowie visuelle Reize sind, die die Attraktivität der Outdoorsportarten ausmachen" (ebd.,330).

Bedeutungsvoller als die eigentliche Naturverbundenheit, die sich auch auBerhalb des Risikosport erfahren lässt, stellen sich also die über die Sinnesorgane vermittelten unmittelbaren Naturwabrnehmungen dar (vgl. ALLMER 1998, 84). Natur als Handlungsraum wird dabei zu einer unmittelbaren Herausforderungen, die einerseits Kulisse auf der anderen Seite aber auch notwendiges Handlungselement darstellt. Der Wildbach, die Steilwand oder die Thermik beim Gleitschirmfliegen sind fundamentale Handlungsbedingungen ohne die die eigentliche Sportausübung unmöglich erscheint. Es bleibt in diesem Zusammenhang auch fraglich, ob Naturerleben im Risikosport nicht auch Naturbeherrschen bedeutet. Zwar deuten die Kräfte der Natur, die sich beim Kanu-Wildwasserfahren z.B. als ,Gewalt des Wassers' oder als ,Bewegung des Flusses' erleben lassen, auf besonders intensive primäre Sinneserfahrungen hin. Weiterhin ist aber auch ein Motivelement im Kontext unmittelbarer Naturbeherrschung denkbar. Es besteht hierbei eine enge Verbindung zwischen Naturwahrnehmung, Machterleben, ungewohnten Bewegungserfahrungen und den beschriebenen Grenzerlebnissen. Die Auseinandersetzung mit fließendem, wilden' Wasser und den entsprechenden Merkmalen der spezifischen Risikosituation lässt sich daher auf verschiedene Weisen in die Konzepte integrieren.

Es bleibt allerdings fraglich, inwieweit sich Naturerleben aus einer voranschreitenden alltäglichen Naturentfremdung ableiten lässt, wie es z.B. WILKEN (1994) oder TREBELS (1991) formulieren. Der Kausalzusammenhang zwischen fortgeschrittener Naturentfremdung und komplementärer - im Natursportarten befriedigter - Natursehnsucht, erscheint als zweifelhaft. Ausgehend von der Beherrschungsfunktion und der Vielfalt übergeordneter Motivelemente, lässt sich dem Risikosport keine allgemeine Suche nach ,Natürlichkeit' unterstellen (vgl. STERNa 2003, 194). Gesucht wird vielmehr die ,wilde Natur', als Vermittlungsinstanz individueller Herausforderungen. COURNELOUP (1997) bringt dieses Naturverständnis am Beispiel des Bergsteigens und Kletterns auf den Punkt. Demnach werden Risikosportarten durch eine ,[...] nouvelle lecture de la nature“ (ebd., 25) cha- 
rakterisiert, die sich in einer Perspektive auf die Natur ,[...] propre a la modernité toute à la fois scientifique et promoethenne [....]" (ebd.) manifestiert.

\subsubsection{Suche nach außergewöhnlichen Emotionszuständen}

Tätigkeitsanreize können in verschiedenen Formen beschrieben werden. Eine recht umfangreiche und komplexe Darstellung dessen, was Personen an autotelischen (auto = selbst; telos $=$ Ziel) Aktivitäten schätzen, findet sich bei CSIKSZENTMIHALYI (1992; 1993), der hierfür den Begriff ,Flow-Erleben' einführt. Daneben sieht ALLMER (1998) in einer als ,Ups-and-downs-Erleben' bezeichneten Komponente einen weiteren Anreiz der Risikosportarten. Beide Erlebniskomponenten werden im folgenden expliziert.

,Flow-Erleben' nach CSIKSZENTMIHALYI (1993) beschreibt einen Zustand, der durch ein ,„...] Verschmelzen von Handlung und Bewusstsein“ (ebd., 28) und dem gänzlichen Aufgehen im Tun gekennzeichnet ist (vgl. ebd.). Durch die Vereinigung von Außen- und Innenwelt, von Subjekt und Objekt, wird die Aufmerksamkeit auf ein bestimmtes Stimulusfeld gerichtet, die infolgedessen zu einer authentischen, Selbstvergessenheit' führt. Flow bedeutet die Verschmelzung zwischen Subjekt und Objekt - der Dualismus von Sportler und Sportgerät gerät dabei aus dem Bewußtsein. Alltägliche Sorgen oder Selbstzweifel werden im Tätigkeitsvollzug beim Flow-Erleben vergessen und zeitliche Strukturen zum Teil vollkommen anders wahrgenommen: „Oft erscheinen Stunden in Minuten zu vergehen [...]“ (ebd., 29) oder Sekunden werden zu scheinbar endlosen Momenten. In der Gegenwartszentrierung auf den ,[...] schönen und spannenden Augenblick“ (BECKER 1995, 328) entstehen Authentizitätsgefühle, die vom Handelnden als außergewöhnlich intensiv und positiv empfunden werden (vgl. ebd.). „Man erlebt sich selbst nicht mehr als abgehoben von der Tätigkeit, man ist mit ihr verschmolzen" (RHEINBERG 1996, 105).

Notwendige Voraussetzungen für ,Flow-Erlebnisse' sind nach CSIKSZENTMIHALYI (1992) deutliche Ziele und unmittelbare Rückmeldungen im Handlungsvollzug. Beim Risikosport erfolgen derartige Rückmeldung oft sehr direkt, z.B. durch die unmittelbare Kenterung nach einem Fahrfehler beim Kanu-Wildwasserfahren oder einem Trittfehler beim Klettern. Diese Möglichkeit der Handlungsrückmeldung und die möglichen Folgen bedingen damit eine völlige Konzentration und Aufmerksamkeit auf die Ausführung, ohne die ein ,Flow-Erlebnis' nicht möglich erscheint. Gleichfalls spielt das vorausgehend beschriebene Gefühl der Risikokontrolle eine wichtige Rolle (vgl. ebd., 26). Für alle ,Flow-Aktivitäten' besteht ein Zusammenhang zwischen Handlungsanforderungen und individuellen Fähigkeiten. Nur wenn sich diese im Gleichgewicht befinden und ,[...] eine Aufgabe im Bereich der Leistungsfähigkeit des Ausführenden liegt" (ebd. 1993, 62) kann ,Flow' überhaupt erfahren werden. Der individuelle Bereich, in dem ,Flow-Erleben' demnach möglich ist, bewegt sich zwischen Über- und Unterforderung, zwischen Angst und Langeweile und wird von CSIKSZENTMIHALYI als ,Flow-Kanal' bezeichnet (vgl. ebd.). Zur Verdeutlichung dieses Zusammenhangs dient die folgende Abbildung: 


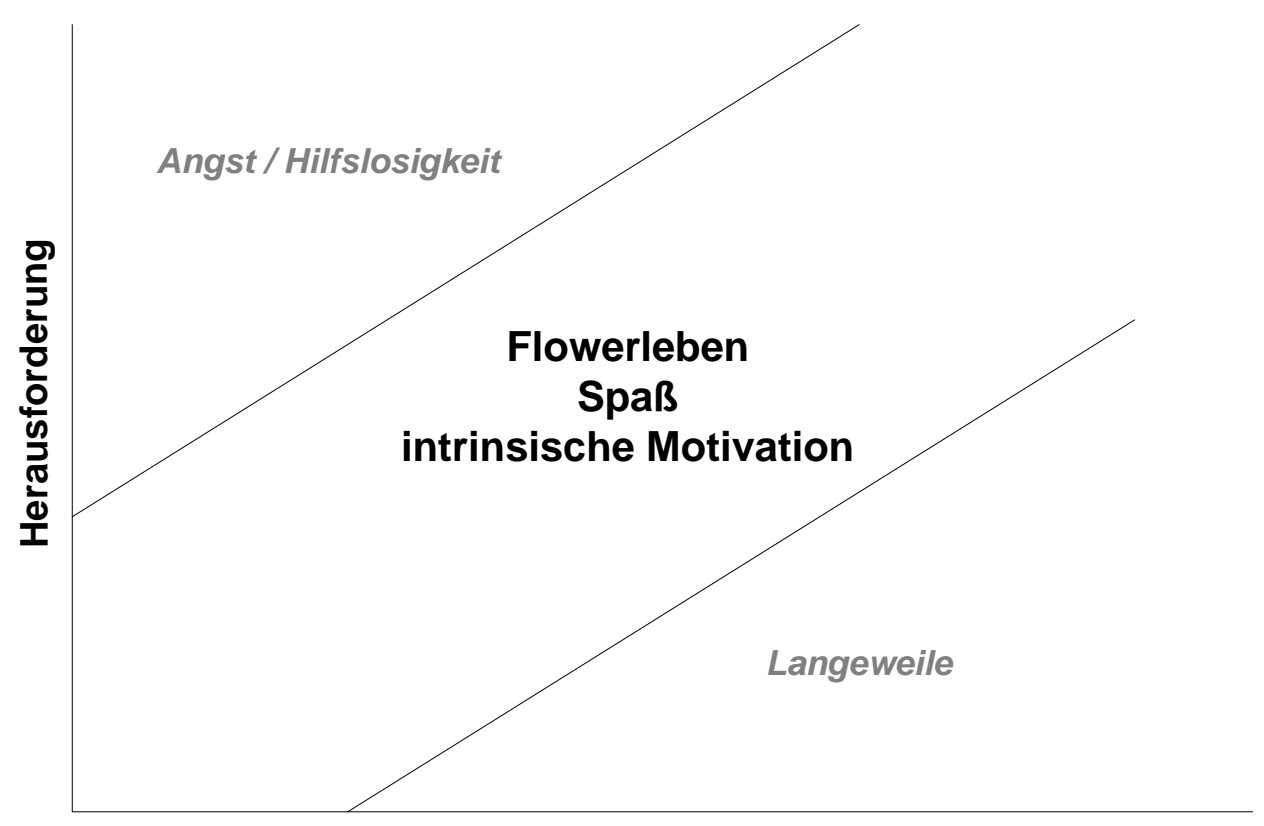

Kompetenz

Abbildung 4.3: Flow-Erleben in Abhängigkeit von Kompetenz und situativen Herausforderungen (modifiziert nach CSIKSZENTMIHALYI \& RATHUNDE 1993, 75)

Introspektiv lassen sich ,Flow-Erlebnisse' in ihrer Stärke und Ausprägung nur schwierig verifizieren, da auch ein entsprechender Vergleich zwischen verschiedenen Tätigkeiten fehlt. CUBE (1995) konstatiert aber, dass ,Flow’ vom Gehalt an Unsicherheit einer Situation abhängig ist und immer dann erlebt werden kann, wenn diese Unsicherheit in Sicherheit abgebaut wird (vgl. CUBE 1995, 49). Für den Risikosport nimmt er daher besonders intensive Formen des ,Flow-Erlebens' an (vgl. ebd., 83). CSIKSZENTMIHALYI (1993) fand gerade bei Felskletterern besonders intensive Flow-Erlebnisse (,deep flow' ${ }^{139}$. Allerdings existieren kaum empirische Untersuchungen zu persönlichkeitsspezifischen Unterschieden, im Sinne verschiedener FlowPräferenzen bei unterschiedlichen Tätigkeiten. Zwar führt WARWITZ (2001, 204ff) einige qualitative Interviewergebnisse aus dem Wagnissport als Nachweis für Flowerlebnisse an, letztlich bleibt es aber weiterhin offen, inwieweit sich das ,Flow-Konzept' für den Einzelnen als Anreiz wirklich präsentiert. Darüber hinaus wird nicht deutlich, warum sich jemand ausgerechnet Risikosportarten als Flow-Aktivitäten aussucht. Schließlich ließen sich Flow-Erlebnisse auch beim Musizieren oder beim Malen erleben (vgl. RHEINBERG 2002, 167).

Neben diesen Erlebnismerkmalen sieht ALLMER (1998) bei Risikosportlern eine weitere Erlebnisqualität, die er als ,Ups-and-downs-Erleben' bezeichnet. Demnach werden nicht nur die isolierten Einzelerlebnisse, wie ,Flow' oder das Erleben von Angst gesucht. Der Reiz verschiedener Risikosportarten liegt vielmehr in der Aufeinanderfolge von spannungsinduzierenden und spannungslösenden emotionalen Erfahrungen (vgl. ebd., 86). Dieses ,,[...] Wechselbad der Gefühle [...]“ (ebd.) - eine oszillierende Mischung aus kontrastreichen Erlebnissen - macht folglich erst den eigentlichen Er- 
lebniswert einer Risikoaktivität aus. ALLMER (1998) nimmt an, dass nicht der einfache Wechsel von angenehmen und unangenehmen Gefühlen als erregend erlebt wird, sondern diese Emotionszustände ,[...] integrale Bestandteile einer Erlebnissequenz sind. Dass heißt, ohne vorherige Anspannung, Aufregung, Angst werden Entspannung, Freude, Glück nicht intensiv erlebt" (ebd., 86).

Zusammenfassend lässt sich das Aufsuchen und Betreiben von Risikosportarten als ein intensives Selbsterleben für die Akteure beschreiben, welches in seiner Gesamtheit als extrem positiv wahrgenommen wird. Dieses „[...] emotionale Selbstmanagement [...]“ (ALLMER 1998, 87) kennzeichnet damit die Erlebnisstruktur der sportlichen Risikoaktivität, denn der Akteur wird zum ,Konstrukteur des Eigen-Erlebens' (vgl. ebd.) und zum ,[...] Manager seiner Innenwelt" (BECKER 1995, 330). Sich selbst solche intensiven Erlebnisse zu verschaffen wird zum eigentlichen Handlungsziel: „Das Subjekt wird selbst zum Objekt, indem es Situationen zu Erlebniszwecken instrumentalisiert" (SCHULZE 1995, 40).

Dabei ist es wie bei der Suche nach Grenzerfahrungen möglich, dass auch die Erlebnissuche im Risikosport suchtähnliche Auswirkungen haben kann:

„Man erlebt sich selbst als Herr über seine Gefühle und weiß, dass man in der Lage ist, positives Erleben zu jeder Zeit selbst herzustellen. Man kann, wenn man es nur will, autark und unabhängig sein. Warum sollte man unter diesen Umständen nicht immer wieder seine Fähigkeiten im Umgang mit extremen Situationen beweisen, wenn man dafür auch noch in Form eines großartigen Erlebnisses belohnt wird?"(SEMMLER 1994, 127).

Auch SCHULZE (1995) unterstellt dieser Suche einen suchtähnlichen Charakter, denn ,[...] Erlebnisse sättigen nicht, sondern stimulieren den Appetit auf weitere Erlebnisse“ (SCHULZE 1995, 450).

\subsubsection{Sozialpsychologische Motivationansätze}

Obwohl viele Risikosportarten individuell ausgeführte Sportarten sind, kann auch das Gruppenerlebnis als ein wichtiger Anreiz für den Risikosport angenommen werden. Nach OPASCHOWSKI $(2000,115)$ ist für $35 \%$ aller Risikosportler das Gruppenerlebnis ein gewichtiges Motiv für die Ausübung von Risikosportarten, wobei keine differenzierten Zahlen für einzelne Aktivtäten vorliegen.

RHEINBERG (1996) argumentiert in der Darstellung eines sozialpsychologischen Anreizes mit der positiven Attribution von Risikosportarten ${ }^{140}$. Wagemut wird in der modernen Gesellschaft als sozial positiv bewertet, wodurch sich Risikosportler als außergewöhnlich und ,heldenhaft' präsentieren können (vgl. ebd., 115). Dieser als ,Gladiator-Komponente' bezeichnete Anreiz drückt sich in der Lebensweise vieler Risikosportler aus, die häufig das Bedürfnis haben, sich nach außen mit ihrem Sport zu identifizieren und zu etikettieren. Dazu gehören eine Vielzahl von Aufklebern auf Autos oder auch 
entsprechende einheitliche Bekleidung. Daneben kommt es oft zu einem inneren Bedürfnis von Risikosportlern ihre sportlichen Höchstleistungen zu dokumentieren und die Produkte einer Öffentlichkeit zu unterbreiten (vgl. ebd.).

Durch die positive Attribution des Risikoverhaltens kann das Betreiben von Risikosportarten auch als soziale Identitätsfindung verstanden werden, die auf eine soziale Anerkennung ausgerichtet ist (vgl. AMESBERGER 1992, 26; vgl. auch HECKER 1989, 329). So weisen GEBAUER et al. (2004) im Rahmen ihrer Studie zu Stilisierungsprozessen im Sport darauf hin, dass informelle Sportarten wie Triathlon oder Inline-Hockey zu so genannten neuen Stilgemeinschaften führen, die neue Formen der Vergemeinschaftung schaffen. Obwohl die Autoren ihre Studie nicht explizit im Umfeld des Risikosports durchgeführt haben, ist anzunehmen, dass auch informelle risikosportliche Engagements zu Wahlgemeinschaften führen. Jenseits des Vereins wird im Risikosport demnach soziale Zugehörigkeit gefunden und Gemeinschaft auf der Basis gemeinsamer Erlebnisse zelebriert (vgl. ebd., 45ff).

DIEHL (2000) hat am Beispiel des Wintersports aufgezeigt, dass Risikosportarten auch im kommerziellen Fernsehen an Bedeutung gewinnen und aus den Strukturen einzelner Sportarten sogenannte ,Lifestyle-Trends' etabliert und vermarktet werden. Es ist diesbezüglich mit Szenebildungen und Life-Style-Strukturen für einzelne Sportarten im Bereich des Risikosports zu rechnen (vgl. SCHWIER 1998). Mut und Abenteuer werden somit zu distinktiven Stilelementen, die eine Abgrenzung gegenüber anderen Sportlern und Nicht-Aktiven ermöglicht. Eine detaillierte Analyse für Risikosportarten steht diesbezüglich noch aus.

\subsection{Zusammenfassung und Einordnung}

Durch dieses Kapitel wird deutlich, dass eine Fülle von Motiv- und Anreizelementen für die Ausübung von Risikosportaktivitäten existieren. Neben den psychoanalytischen Erklärungsansätzen (BALINT 1994; AUFMUTH 1989), die das Aufsuchen von Risikosportarten als eine Art ,pathologisches' Bedürfnis deuten, wurden verschiedene Theorien aus dem Bereich der kognitiv-handlungstheoretischen Motivationspsychologie dargestellt. Dabei kann das Betreiben von risikosportlichen Aktivitäten als Erregungssuche (ZUCKERMAN 1978; BERLYNE 1974), als Erleben und Überwinden von Angst (SEMMLER 1994), als Existen₹vergewisserung (LE BRETON 1995) oder als Suche nach persönlichen Grenzen und individuellen Erlebnissen (ALLMER 1998; CSIKSZENTMIHALYI 1992 ; RHEINBERG 1989) erklärt werden. Die anthropologischen Annabmen (GEHLEN 1950; ZELINKA 1997) und theoretischen Ausführungen über das Neugierverhalten als Sicherheitstrieb (CUBE 1995; SCHNEIDER 1996) verdeutlichen darüber hinaus die Zusammenhänge zwischen Sicherheits- und Risikobedürfnis als komplementäre Teile einer menschlichen Grundfiguration. 
Die analytische Trennung der mit den Risikosportaktivitäten verbundenen Motivationsstrukturen erfolgte aus Gründen der Übersichtlichkeit. In der Praxis des Risikosports ist dagegen mit einer recht komplexen Anreizstruktur der Risikosportarten zu rechnen. Wahrscheinlich werden risikosportliche Aktivitäten durch mehrere zum Teil miteinander verknüpfte Motivdispositionen und situative Anreizen bestimmt (vgl. ALLMER 1998, 89; auch KUHN 2001). Risikosportler verfügen dabei über eine Art individuellen ,Motivationscocktail', der aus den verschiedenen Motiven in unterschiedlicher Gewichtung und Ausprägung und in Interaktion mit Situationsmerkmalen zusammengesetzt ist. Dieser Mix aus verschiedenen Anreizen und Motivelementen kann dabei einerseits zwischen einzelnen Personen divergieren, auf der anderen Seite aber auch interindividuell situativ differieren. Eindimensionale Erklärungsansätze für das Phänomen Risikosport und eine Zuweisung bestimmter Sinnprofile an eine einzelne Sportart stellen sich damit als problematisch dar. Auch wenn die aufgezeigten Anreize und Motivelemente ein Spektrum dessen beschreiben, warum sich Menschen in sportliche Risikosituationen begeben, so kann eine detaillierte und personalisierte Sinnzuschreibung kaum erfolgen. Der Sportler entscheidet letztlich eigenständig und selbstverantwortlich, welche Anreize eine bestimmte Sportart für ihn besitzen (vgl. SCHULZ 1994, 499f).

Zusammenfassend lässt sich festhalten: Risikosportler suchen bewusst Situationen auf, in denen der Handlungsausgang der eingegangenen Risikosituation vom Einsatz der eigenen Fähigkeiten abhängt. Im Mittelpunkt steht dabei, sich in absoluter Selbstverantwortung und Selbstinszenierung Handlungssituationen zu gestalten, die unter den Bedingungen des Risikos die eigene Handlungsfähigkeit unter Beweis stellen. Das Bewusstsein der realen Gefährlichkeit bewirkt - auch unter abgeschwächten Bedingungen im risikosportlichen Breitensport - eine Intensivierung des Erlebens. Die mit der Risikosituation verbundenen Aktivierungen und Angstreize werden dabei aber nur deshalb als positiv empfunden, weil der Risikosportler der Überzeugung ist, die Kontrolle über die signalisierten Risiken zu haben. Entscheidend bleibt die Feststellung, dass das Ziel des Risikosports mit seinen selbstinszenierten Erlebnis- und Erregungsmöglichkeiten eng mit dem Risikocharakter der Situation verbunden ist. Letztlich ist es die vitale, als Herausforderung wahrgenommene Risikosituation, in der die beschriebenen Anreize und Motivelemente etabliert sind.

Damit fügen sich kulturhistorische Annahmen und psychologischanthropologische Theoriebildung nahtlos zusammen und zwar insofern, als dass nun das bis dato leere Risikoerlebnis mit konkreten Inhalten gefüllt und Motivationsstrukturen für den Risikosport identifiziert wurden. Im vitalen Risiko steckt - soviel kann nun nachdrücklich konstatiert werden - eine Erfahrung von besonders kräftigem und authentischem Erleben. Diese ganzheitliche Beanspruchung der unterschiedlichsten Kräfte in höchster Intensität und Leidenschaft widersetzt sich in einer Gegenerfahrung des Authentischen - so legen es die Befunde zumindest nahe - gegen die Moderne und deren zivilisatorische Errungenschaften. In einer erlebnis- und risikoarmen 
Modernen wird der Risikosport scheinbar zu einem erlebnisintensiven Kontrapunkt.

Der aufmerksame Leser wird über derartige kausal anmutende Zusammenhänge sicherlich verwundert die Nase rümpfen. Angesichts von Gesellschaftsdiagnosen wie der ,Risikogesellschaft' oder der ,Erlebnisgesellschaft' sind solche Zweifel auch durchaus berechtigt, denn die scheinbar monotone Industriegesellschaft, die zur Erklärung der Entstehungszusammenhänge des Risikosports in Kapitel 3 herangezogen wurde, erweist sich über ein Jahrhundert später doch maßgeblich verändert. Mit dem Postulat einer wie auch immer gearteten ,Postmoderne' weist die gegenwärtige Gesellschaft auf zahlreichen Veränderungsprozesse hin, die über Prozesse einer, ersten Modernisierung' (BECK) hinaus viele der kulturhistorische Annahmen aus Kapitel 3 diskreditiert. Kapitel 5 wird die ,postmodernen' Veränderungen, die die gegenwärtige Gesellschaft betreffen, aufgreifen und eine Transformation der kulturhistorischen Annahmen aus einer soziologischen Perspektive für die Gegenwart vornehmen. Pädagogische Konsequenzen sowie ethische Überlegungen schließen sich im folgenden Kapitel „Risikosport und Gesellschaft" an. 


\title{
5 Risikosport und Gesellschaft - soziologische, pädagogische und ethische Perspektiven
}

\author{
Sicherheit erreicht man nicht, indem man Zäune errichtet, \\ Sicherheit gewinnt man, indem man Tore öffnet. \\ (Urbo Kekkonen (1900-86), finn. Politiker)
}

In den voranstehenden Kapiteln wurde aufgezeigt, welche Erklärungszusammenhänge aus einer kultur- und soziohistorischen sowie psychologischanthropologischen Perspektive für den Risikosport entwickelt werden können. Durch die kontrollierten Bewältigung von bewegungsvermittelten Bewährungssituationen offeriert der Risikosport demnach eine Erfahrung von besonders kräftigem und authentischem Erleben. Risikosport, so legen es die theoretischen Befunde bis dato nahe, fungiert dabei in seinem historischen Entstehungszusammenhang als Gegenerfahrung einer modernen Zivilisation. KÖCK (1990) betont:

„Erst mit der Disziplinierung von Menschengruppen entsteht das Bedürfnis nach Gegenwelten. Je stärker die Fremd-oder Sachzwänge wirken, um so intensiver äußert sich die Abenteuersehnsucht." (KÖCK 1990, 31).

Und auch HARTMANN (1996) stellt fest, dass es in der Dritten Welt, „[...] die ums Überleben kämpft [...]“ (ebd., 79) kein freiwilliges Aufsuchen von Aktivitäten gibt, die mit offensichtlichen Risiken verbunden sind. Dagegen wird in der,postmodernen' westlichen Gesellschaft ein regelrechten Trend in der Entwicklung von Risikosportarten beobachtet (vgl. LORCH 1995; OPASCHOWSKI 2000). Wie aber ist der Risikosport in einer Gesellschaft zu erklären, die mit Prozessen einer ,zweiten (reflexiven) Moderne' (BECK et al. 1997) eine bedeutende gesellschaftliche Transformation über 
die industriegesellschaftliche ,erste Modernisierung' hinaus vollzogen hat? Wie lässt sich Risikosport als Kontrapunkt einer Gesellschaft verstehen, die kurant auch als ,Risikogesellschaft' etikettiert wird?

Wengleich die kultur- und soziohistorischen Annahmen einen kohärenten Ansatzpunkt für das Verständnis des Risikosports in der Moderne offerieren, reichen die Befunde für die Erklärung und Wirkungsweise risikosportlicher Aktivitäten in der gegenwärtigen Gesellschaft nicht aus. Dabei ist die Frage nach den gesellschaftlichen Bezugspunkten des Risikosports für ein umfassendes Verständnis unabdingbar, denn wie BETTE formuliert:

„Ein Beobachter, der den zeitgenössischen Abenteuer- und Extremsport ver-
stehen und sich selbst und anderen die Frage beantworten möchte, warum
Menschen in einer auf den ersten Blick überflüssig erscheinenden Weise ibr
Leben und ihre Gesundheit auf Spiel setzen, [...] hat nicht nur auf die diver-
sen Erscheinungsformen des Abenteuer-und Risikosports zu schauen und
Akteure zum Sprechen zu bringen, die Extremes tun. Er hat auch die Ge-
sellschaft zeitdiagnostisch zu analysieren, in der das Extreme stattfindet und
eine nicht-lineare Verbindung zwischen beiden Bequgspunkten, Abenteuer
und Gesellschaft, herzustellen"(BETTE 2004, 121).

Zur Beantwortung dieser Fragen werden aktuelle soziologische Theorien herangezogen. Die Soziologie als Wissenschaft zur Erforschung und Erklärung sozialer Wirklichkeiten ist dabei als eine Symbiose von soziologischer Theoriebildung und empirischer Sozialforschung zu verstehen. Dies macht soziologische Forschung aber bisweilen schwierig, weil die Trennung von empirischer Forschung und Theoriebildung nicht - wie beispielsweise in den Naturwissenschaften typisch - zu einer Einheit verschmolzen ist. Im Bezug auf die wissenschaftstheoretische Ausrichtung gibt es in der Soziologie also kein ,Entweder-oder' sondern ein ,sowohl als auch' (WEIß 1999). Auch die als spezielle Soziologie bezeichnete Sportsoziologie greift auf Paradigmen der allgemeinen Soziologie zurück und vermittelt die systematische Erfassung und Deutung sozialer Tatbestände im Sport ebenfalls mittels Theorien sowie soziologischer Forschungsmethoden und -techniken. Da aber kaum empirische Befunde über die sozialen Strukturen und Funktionen des Risikosports aus der Perspektive der Sportsoziologie vorliegen, erfolgt die soziologische Auseinandersetzung dieses Kapitels hauptsächlich theoriegeleitet. An Hand unterschiedlicher soziologische Ansätze wird überprüft, ob sich Wechselbeziehungen zwischen dem freiwilligen Aufsuchen von Risikosportarten und den aktuellen gesellschaftlichen Hintergründen unter dem Topos der reflexiven Modernisierung ${ }^{141}$ ableiten lassen. Dabei werden - in Anlehnung an KÖNIG (1973) sowohl Theorien mittlerer Reichweite als auch Theorien hoher Komplexität zur Erklärung des sozialen Wirklichkeit des Risikosports herangezogen.

Die folgende soziologische Deduktion geht dabei zu den beschriebenen motivationspsychologischen Aspekten des letzten Kapitels auf Distanz ohne diese gleichfalls zu negieren. Im ersten Abschnitt wird zunächst die These der (Post)Modernisierung (5.1) aufgegriffen, die mit als zentraler theoretischer 
Bezugsrahmen für die Entwicklung und gegenwärtige Bedeutung des Risikosports gelten können. In Anlehnung an die in der aktuellen Soziologie erörterten Ansätze werden schließlich Erklärungsansätze für Risikosport in der modernen Gesellschaft unter dem Titel ,Soziologie des Risikosports' (5.2) vorgestellt. Da Sicherheit in einem dialektischen Verhältnis zum Risiko als sozialer Kategorie steht und als zentrales gesellschaftliches Thema dem Aufsuchen von Risikosportarten scheinbar konträr entgegensteht, wird schließlich der gesellschaftliche Umgang mit Sicherbeit und Risiko aus einer soziologischen Perspektive (5.3) erläutert. In Abschnitt 5.4 werden sozialisationstheoretische Ansätze aufgegriffen und die Bedeutung von Risikosport im Lebenslauf evaluiert. Im Rückgriff auf geschlechtssoziologische Theorien wird anschließend der Frage nach ,Risikosport und Geschlecht' (5.5) im Bezug auf risikosportliche Aktivitäten nachgegangen. Um die Bedeutung von Risikosportraten im gesellschaftlichen Zusammenhang zu verdeutlichen, schließen sich eine sportpädagogische und eine ethische Betrachtung (5.6 \& 5.7) des Risikosports an. In 5.8 werden die Ergebnisse zusammengefasst.

\section{1 (Post-)Modernisierung als theoretischer Bezugs- rahmen des Risikosports ${ }^{142}$}

Die gegenwärtigen hoch entwickelten Gesellschaften sind durch besonders tief greifende, radikale und rasante Veränderungen im zeitlichen Vergleich der letzten 50 Jahre geprägt. Vor allem die zweite Hälfte des 20. Jahrhunderts - so legt die gegenwärtige soziologische Diskussion nahe - scheint in westlichen Gesellschaften im Bezug auf das Ausmaß und Geschwindigkeit sozialer Wandlungsprozesse beispiellos zu sein. Als Indikatoren dieser Entwicklung wird die Verbesserung der Wohnraumversorgung sowie die Verbreitung der Massenmedien und -Kommunikation gedeutet (vgl. KLOCKE 1993; 15ff).

Die Soziologie sozialen Wandels zählt zu den stärksten und bedeutsamsten Bereichen der Sozialwissenschaften und kann auf eine umfangreiche Literatur zurückgreifen ${ }^{143}$. Während die Paradigmen der klassischen Theoreme des sozialen Wandels dabei sowohl evolutionistisch-global, als auch analytisch ausgelegt waren, enthalten die jüngeren soziologischen Beiträge gleichzeitig auch einen politisch-normativen Kern: die weltumspannende Angleichung an die industriegesellschaftliche Moderne. Nun treten neben die klassische soziologische Literatur seit einigen Jahrzehnten zunehmend Gesellschaftsdiagnosen, die mit markanten und einschlägigen Begriffen die Wandlungsprozesse unserer Gesellschaft beschreiben ${ }^{144}$. Die Etikette dieser neuen Gesellschaft reichen von der Postmoderne über die Freizeitgesellschaft zur Multioptionsgesellschaft oder Single-Gesellschaft, stimmen in ihrer grundlegende Essenzen aber überein: Zentrale Gemeinsamkeit der neueren Ansätze ist das Moment einer erneuten Modernisierung, welches sich aus nahezu allen Beiträgen in sehr unterschiedlichen Kontexten extrahieren lässt. Traditionelle Strukturen, Muster und Handlungszusammenhänge verlieren dabei 
sukzessiv an Bedeutung und werden durch neue und zum Teil völlig anders geartete Sinnzusammenhänge substituiert. Die Kennzeichen der Moderne waren u.a. die vorangeschrittene Modernisierung, das Aufkommen des Nationalstaates als wichtige politische und gesellschaftliche Einheit aber auch eine zunehmende Zivilisierung des Alltags sowie rationalisierte Arbeitsverhältnisse ${ }^{145}$ (vgl. VAN DER LOO \& VAN REIJEN 1992). Die Postmoderne also soziologischer Topos stellt einen Versuch dar, die über diese erste Modernisierung hinausgehenden Prozesse des sozialen Wandels zu fassen und in einen Kontext zu bringen. Allerdings erfolgt die Theoriebildung hierbei entlang einer Loslösung von der Geschichte: Historische Gesetzmäßigkeiten werden geleugnet und die soziale Beschaffenheit der Gesellschaft in eine erkenntnistheoretische Beliebigkeit geführt. Diese ,[...] postmoderne Intelligenz" (PRAHL 2002, 36) wird hier nicht weiterverfolgt, im Rahmen dieser Arbeit wird vielmehr die Auffassung einer, weitergehenden Modernisierung' (ZAPF 1998, 472) und die damit verbundenen Wandlungsprozesse vertreten.

Nahezu alle Beiträge dieser zweiten Modernisierungdiskussion gehen dabei von einem übersehbaren Zuwachs an materiellen Wohlstand sowie sozialen Sicherheiten in westlichen Gesellschaften aus. Zeigt sich die ältere Theorie des sozialen Wandels noch überwiegend universellen Fragestellungen zugewandt, so stehen bei diesen neueren Modernisierungsbeiträgen auch Fragen der Adaptation und der Steuerbarkeit von Problemen durch die gesellschaftlichen Teilsysteme im forschungsleitenden Interesse (vgl. KLOCKE 1993, 18f).

Sport als Kultursegment einer modernen Gesellschaft (GRUPE 2000) steht seit jeher in einem direkten Bezug zu modernisierungstheoretischen Ansätzen. Die Frage nach den Zusammenhängen zwischen gesellschaftlicher Entwicklung und der Ausbildung eines gesellschaftlichen Teilsystems Sport, zählt letztlich zu den wichtigsten Forschungsfragen der Sportsoziologie (vgl. WEIß 1999, 23f). Insbesondere das gesellschaftliches Phänomen Risikosport wird unter Bezugnahme neuerer soziologischer Konzepte diskutiert (vgl. BETTE 2004; WHEATON 2004; GEBAUER et al. 2004). Des Weiteren impliziert auch der Verweis auf Paradigmen und Theorien der soziologischen Trendsportforschung ${ }^{146}$ - die in ihrer theoretischen Konstruktion explizit auf gegenwärtige Modernisierungsparadigmen zurückgreift - eine Auseinandersetzung mit Theorien und Konzepten der soziologischen Modernisierungsdebatte.

Um zu verdeutlichen, vor welchen Prozessen und sozialen Hintergründen sich Risikosport als gesellschaftliches Phänomen zur Zeit konstituieren könnte, werden im Folgenden zunächst gesamtgesellschaftliche Veränderungen betrachtet, die als Ergänzung und theoretische Basis den eigentlichen Fragestellungen dieses Kapitels vorgeschaltet werden. Auf der Suche nach gesellschaftstheoretischen Ansätzen, die über Begriffe wie die ,Postmoderne' hinausgehend auch die vielfältigen Anzeichen für einen Wandel von Subjektivität, als einen Transformationsprozess zu begreifen versucht, stößt man unausweichlich auf das Konzept der Individualisierung (5.1.1) sowie dem Pos- 
tulat eines $W$ andels der Existen:formen (5.1.2). Angelehnt an BOURDIEU (1982) werden anschließend die Ansätze der modernen Sozialstrukturforschung (5.1.3) expliziert, die einen Brückenschlag zwischen traditionellen Klassenparadigmen und der sich abzeichnenden gesellschaftlichen Veränderungen auf Mikroebene herstellt. Auf BERGER (1996), VESTER (2001) aber auch HRADIL (1992) gehen Theoriebildungen zurück, die unter den Stichworten ,Differeñierung' und ,Pluralisierung' (5.1.4) Modernisierungstendenzen zusammenfassen, die sich durchaus im Handeln und Erleben der Gesellschaftsmitgliedern niederschlagen. Obwohl die Forschung zum Wertewandel (5.1.5) vielseitig und umstritten ist, darf ihre Bedeutung für die Entwicklung im Bereich des Risikosports keinesfalls vernachlässigt werden.

\subsubsection{Individualisierung}

Kaum ein anderes Thema hat in der Soziologie der letzten zwanzig Jahre eine derart steile Karriere erfahren und dabei die Sozialwissenschaften gleichartig umfassend beeinflusst wie das Individualisierungstheorem und die Ausführungen zur Erlebnisgesellschaft nach SCHULZE. Auch wenn die Kerntheorie der Individualisierung bereits über zwanzig Jahre alt ist, besitzt wohl kaum eine andere Gesellschaftsdiagnose weiterhin derartige Aktualität wie das Individualisierungstheorem (vgl. BECK 1997; JUNGE 2002; SCHULZE 2003; BETTE 2004). Als soziologischer Begriff ist Individualisierung der Versuch eine pointierte Zeitdiagnose zu geben, die über die klassischen Kategorien hinaus verweist und als Schlüssel zur Erklärung grundlegendster Veränderungen in der westlichen Gesellschaft dient. Als Gegenwartsbegriff ist Individualisierung aber auch Schlagwort der öffentlichen und privaten Diskussion über eine gesellschaftliche Entwicklung geworden, die für viele unüberschaubar und verwirrend zu sein scheint. Ähnlich verhält es sich auch mit den Kerngedanken der Erlebnisgesellschaft, der zeitdiagnostischen Gesellschaftsanalyse von SCHULZE (1995). Der Autor stellt hierin einen allgemeinen Wandel der Existenzformen dar, der im folgenden Abschnitt ebenfalls erläutert wird. Im Kontext des Risikosports stellen Individualisierung und der Wandel der Existenzformen einen wesentlichen Bezugsrahmen zur gesellschaftstheoretischen Einbettung des Risikosports dar.

Nach BECK (1986), dem wohl exponiertesten Vertreter des Individualsierungstheorems, ist unter Individualisierung zu verstehen, dass ,[...] die Menschen aus den Sozialformen der industriellen Gesellschaft - Klasse, Schicht, Familie, Geschlechtslagen von Männern und Frauen - freigesetzt werden, ähnlich wie sie im Laufe der Reformation aus der weltlichen Herrschaft der Kirche in die Gesellschaft ,entlassen’ wurden“ (BECK 1986, 115). Dabei ist Individualisierung kein grundsätzlich neues Phänomen: Individualisierung als Freisetzung des Einzelnen aus den Abhängigkeiten und Bindungen der ständischen Gesellschaft ist schon bei MARX aber auch bei WEBER als ein Grundprinzip der kapitalistischen Gesellschaft analysiert worden. Prozesse der Individualisierung brachten z.B. den „freien“ Lohnarbeiter wie auch das „autonome“ bürgerliche Individuum hervor - im Sinne 
marktvermittelter Existenzformen - die dennoch in neu entstehenden Klassen und Milieus aufgefangen wurden ${ }^{147}$ (vgl. auch BILDEN 1989, 20f). Dass es sich bei Individualisierung keinesfalls um ein neues Thema handelt, lässt sich auch bei SIMMEL nachvollziehen, der im Rahmen seiner kulturhistorischen Betrachtung Aufschluss über die historischen Zusammenhänge von Individualisierung, persönlicher Autonomie und individueller Freiheit liefert (vgl. SIMMEL 1923) ${ }^{148}$

Die aktuelle Diskussion über Individualisierung als Modernisierungsphänomen wurde maßgeblich von BECK (1983; 1986; 1997), DAHRENDORF 1992 aber auch von KEUPP (1994a; 1994b, 2000) beeinflusst, wobei die Autoren von zum Teil divergierenden Forschungsansätzen ausgehen. Auch die Arbeiten von BERGER (1986), BERGER \& HRADIL (1990), GIDDENS (1989), KOHLI (1989), MOOSER (1984) sind als zentrale Beiträge zum Forschungsstand zu betrachten ${ }^{149}$.

Die durch die Arbeiten von BECK angestoßene Debatte um Prozesse der Individualisierung liegt die Kernthese einer veränderten Wohlstandsgesellschaft zugrunde: Ausgehend von einer zunehmenden sozialen Absicherung, einem relativ hohen Lebensstandard in allen Schichten der Gesellschaft, sowie einer allgemeinen Bildungsexpansion haben sich die Lebensumstände seit den fünfziger Jahren derart verändert, dass die Menschen aus traditionellen Klassenbindungen und Versorgungsbezügen der Familie zunehmend herausgelöst und verstärkt auf sich selbst und ihr individuelles Schicksal verwiesen werden (BECK 1983, 486). Dieser ,Fahrstuleffekt' - im Sinne einer vertikalen Verschiebung der Gesellschaft bei gleich bleibenden sozialen Unterschieden - scheint nun nochmals besonders seit den 80er Jahren in einer bislang ungekannten Dynamik und Reichweite die Lebensverhältnisse der Menschen zu verändern. Strukturelle Wandlungen und massive Einbrüche sind damit in den Rang alltagsweltlicher Erlebnisse und Erfahrungen aufgerückt, die sich in Form neuer Handlungsmöglichkeiten und Entscheidungszwängen unmittelbar im Erleben und Handeln niederschlagen (vgl. BERGER 1996).

\section{„Das historisch Neue besteht darin, dass das, was früher wenigen zugemutet wurde - ein eigenes Leben zu führen - nun mehr und mebr Menschen, im Grenafall allen abverlangt wird" (BECK \& BECK-GERNSHEIM 1994, 21).}

Individualisierung als soziologische Kategorie lässt sich nach BUCHMANN (1989) zusammenfassend in drei Dimensionen untergliedern, die mit dem BECK'schen Ansatz von Individualisierung nur bedingt kongruent sind. Die erste Dimension beschreibt die Differenzierung und Diversifikation sozialer Schichtung und individueller Lebenslaufmuster. BECK (1986) spricht in diesem Zusammenhang von Prozessen der Endtraditionalisierung, die als ,Auflösung' hergebrachter Sozialformen und institutioneller Beschränkungen begriffen werden. Individualisierung meint die Tatsache,

„[...] dass Menschen sich in sozialer Hinsicht von traditionellen gesellschaftlichen Bindungen und Versorgungsarrangements befreien und sich auf kogniti- 


\section{ver Ebene traditionellen Glaubensauffassungen und Sicherbeiten immer weiter entriehen" (VAN DER LOO / VAN REIJEN 1992, 162).}

Individualisierung als soziologische Kategorie lässt sich somit originär als Herauslösung und Befreiung aus bindenden Normen und Lebenszusammenhängen verstehen, die in direkter Anlehnung an SIMMEL als „Freiheit von etwas" verstanden werden können (vgl. EBERS 1995, 344). Individualisierung thematisiert den neu entstandenen gesellschaftlichen Rahmen für individuelle Entwicklung und individuelles Handeln: Menschen werden aus vorbestimmten Lebensläufe und gesellschaftlichen Zwängen sukzessiv herausgelöst und ohne die Einbindung in Klassen- oder Schichtgemeinschaften zur eigenen Biographieführung gezwungen. BECK (1986) bemerkt hierzu: „Der oder die einzelne selbst wird zur lebensweltlichen Reproduktionseinheit des Sozialen“ (ebd., 209). Damit verringert sich nicht nur die selbstverständliche Identifizierung mit Zielen und kulturellen Verhaltensweisen des Herkunftsmilieus; dem Individuum unterliegt damit auch die Ausgestaltung der Gesellschaft und der Formen des Zusammenlebens als Ausdruck.

Hieraus resultierend wird als zweite Dimension die Privatisierung sozialer Beziehung identifiziert. „Der einzelne gilt nicht mehr nur als Adresse in Kommunikationsprozessen. Vielmehr wird das Individuum nun vor allem als Gestalter seiner sozialen Welt gesehen“ (JUNGE 2002, 9). Das Individuum wird zum zentralen Bezugspunkt für sich selbst - die Herstellung sozialer Kontakte eine Leistung eigener Wahl- und Auswahlentscheidung. Der moderne Mensch verliert demnach seine aus einem Guss zusammengehörende Lebenswelt, die in der Einheit aus Familie, sozialer Schicht, Einkommensstruktur, Beruf, Ehepartner und Glauben bestand (vgl. BECK 1986, 206). Während in früheren Gesellschaften beispielsweise soziale Herkunft, der Beruf oder aber die soziale und politische Orientierung für das Individuum vorgegeben zu sein schien und mitunter auch für das Sportengagement von entscheidender Bedeutung sein konnte ${ }^{150}$, zerfällt diese biographische Einheit in der Spätmoderne zusehends in seine Bestandteile (vgl. BRINKHOFF 1998, 252). Dem mit der Durchsetzung der Industriegesellschaft einhergehenden Zugewinn an individuellen Spielräumen steht ein Verlust an traditionellen und zum Teil verbindlichen Werten, Normen und Sinnorientierungen und die zunehmende Abhängigkeit von Institutionen und Organisationen gegenüber. Individualisierung impliziert damit einen Verlust an Sicherheiten und Sinnstrukturen, den die Bindung an traditionelle Gemeinschaften und die Einbindung in primäre soziale Netzwerke einschließt.

Damit ist auch die dritte Dimension des Individualisierungsprozesses bezeichnet: der Zugewinn an persönlicher Autonomie. Vor allem die Arbeiten von KEUPP (1994a; 1994b; 2000; KEUPP et.al. 1997), der die gesellschaftlichen Freisetzungsprozesse in ihren Folgen für die Subjekte untersucht ${ }^{151}$, aber auch die Schriften von KOHLI (1989) erlauben im Kontext dieser Arbeit fruchtbare Anschlussleistungen. Dem Verlust gesellschaftlicher, Ligaturen'152 steht demzufolge eine Zunahme an Optionen - im Sinne gestiegener Handlungsmöglichkeiten gegenüber. 
Versteht man Individualisierung als einen Entstrukturierungsprozess, so bedeutet dies für das Individuum immer weniger auf vorgegebene Muster und Normierungen im Bereich der Lebensführung zurückgreifen zu können und statt dessen auf selbst entwickelte Orientierungen verwiesen zu werden. Das individuelle Leben wird angesichts gestiegener Wahlmöglichkeiten ${ }^{153} \mathrm{im}$ Hinblick auf Lebensführung und Lebenslauf zu einem Projekt - die Normalbiographie wird zur Wahl- (LEY 1984) oder Bastelbiographie (HITZLER \& HONER 1994). Ständische Rollenmuster, die noch in der Zwischenkriegszeit deutlich ausgeprägt waren ${ }^{154}$, sind in den modernen Konsumkulturen des ausgehenden Jahrhunderts nun kaum noch lebensweltlich relevant.

Zusammenfassend zeigt Individualisierung also in hohem Maße ambivalente Effekte: Auf der einen Seite werden die Menschen zunehmend aus ihren gesellschaftlichen Zwängen und Herkunftsschicksalen befreit. Den damit gewonnen Freiheiten, die sich auch als Individualisierungschancen begreifen lassen, stehen aber unausweichlich auch Individualisierungsrisiken gegenüber: Neben dem verstärkten Verweis auf eine selbstbestimmte Biographieführung, die ohne kollektive Sinnquellen auskommen muss, wird das Individuum ferner mit den Zwängen gesellschaftlicher Institutionen und Marktprozessen konfrontiert.

Die Soziologie tut sich zur Zeit noch schwer, das Individualisierungstheorem und seine Folgen wissenschaftlich zu bewerten. Normativ wird die Entwicklung zu einer individualisierten Gesellschaft von einigen Betrachtern durchaus begrüßt und als eine Chance begriffen, von anderen Beobachtern jedoch als Verlust bedauert und im Sinne von Individualisierungsrisiken interpretiert. Gleichzeitig wird insbesondere die generalisierte Argumentation der allumfassenden Wohlstandsentwicklung kritisiert (vgl. BÖLLERT 1995). So verweist beispielsweise eine Untersuchung von KRAUSE \& SCHÄUBLE (1988) zu haushaltsbezogenen Wohlfahrtspositionen darauf, dass im Ergebnis ca. 10-15\% der Haushalte der Bundesrepublik im Armutsbereich bzw. im Grenzbereich zur Armut anzusiedeln sind. Angesichts der jüngeren wirtschaftlichen Entwicklung ist davon auszugehen, dass Individualisierung vor allem eine breite Mittelschicht betreffen wird, wogegen untere Schichten und Arbeitslose auf Grund ihrer sozioökonomischen Haushaltsposition nur in Teilbereichen von Individualisierungsprozessen betroffen sein dürfte ${ }^{155}$.

Empirisch lassen sich sowohl Studien für die Individualisierungsthese anführen als auch dagegen ${ }^{156}$. Dies lässt u.a. damit erklären, dass Individualisierung die Gleichzeitigkeit des Ungleichzeitigen bedeutet (JUNGE 2002, 17):

„Die Gesellschaft befindet sich in einer Transformationsphase, in der gleichzeitig alte und neue soziale Formen nebeneinander existieren. Die Ambivalenz in der Bewertung von Individualisierung spiegelt also die Ambivalenz.der sozialen Entwicklung wieder" (ebd.). 
BECK als einer der zentralen Vertreter der Individualisierungsthese meint mit Individualisierung demgemäß sowohl schon vollzogene als auch gegenwärtige und zukünftige Prozesse der Freisetzung der Individuen. Er versteht Individualisierung als Entwicklung, die sich als eine Art „Trendaussage“ (BECK \& BECK-GERNSHEIM 1994, 16) interpretieren lässt: „In diesem Sinne ist Individualisierung beides - exemplarische Gegenwartsdiagnose und Zukunftsmusik“" (ebd.).

\subsubsection{Der Wandel der Existenzformen}

Die These vom Wandel der Existenzformen geht auf die umfassende Arbeit von SCHULZE (1995) zurück. Wie BECK geht auch SCHULZE (1995) in seiner Gesellschaftsanalyse zur Erlebnisgesellschaft von einer allgemeinen Wohlstandsentwicklung aus, die das existenzielle Grundproblem der Lebenssicherung auf der kollektiven Ebene verdrängt und der Kultur als subjektiven Handlungs- und Bestimmungsraum eine wesentliche Bedeutung für die individuelle Lebensorientierung zuspricht. Mit dem Übergang von der „Knappheitsgesellschaft' zur ,Überflussgesellschaft' haben sich die Bedingungen der Lebensführung fundamental verändert und mit der Knappheitsgesellschaft auch die sozioökonomisch strukturierten Klassenmilieus aufgelöst ${ }^{157}$.

Grundüberlegung SCHULZEs Theorie ist ein fundamentaler Wandel des grundlegenden Verhältnisses von Subjekt und Situation, der Existenzform (ebd., 197f). Nicht mehr Begrenzungen und Zwänge der Situation zeichnen die Existenzform demnach heute aus als vielmehr die Modi des Wählens, der freien Symbolisierung und der gewollten Prägung. Soziale Ordnung resultiert demzufolge heute nicht mehr aus der Festlegung der Subjekte durch die Außenwelt, sondern aus deren Selbstfestlegung in einem fast unbegrenzten Optionsraum. Unter diesen Bedingungen verändert sich die Grundstruktur des Denkens und Handelns: Vormals außenorientiert, d.h. auf die Verbesserung der Umstände durch gezieltes ,Einwirken' gerichtet, ist das Denken und Handeln nunmehr innenorientiert, d.h. die Menschen wählen permanent vielfältige Aspekte der Situation aus, um sich schöne Erlebnisse zu verschaffen. Daraus resultierendes Ziel ist Verwirklichung eines aufregenden Lebens, Lebensgenuß, persönliche Entwicklung und Selbstbestimmung (vgl. VAN DER LOO \& VAN REIJEN 1992, 160).

Die Individuen begreifen im Rahmen dieser innengerichteten Modernisierung ihre Lebenswirklichkeit immer weniger in ökonomischen als immer mehr in subjektbezogenen Kategorien. An die Stelle der außenorientierter Lebensauffassung - die sich um existenzielle Probleme der materiellen Versorgung und Reproduktion konzentriert - tritt die Erlebnisorientierung (ebd., 37f) als innenorientierte Handlungsmaxime. Handlungsleitend in der Erlebnisgesellschaft ist demnach die Produktion angenehmer Erlebnisse, die dem ,Projekt des schönen Lebens' gewidmet sind. Durch die Erlebnisorientierung wird zwar das traditionelle Statusdenken abgelöst, SCHULZE macht aber klar, dass an die Stelle der Statusdistinktion eine neue Form der Ab- 
grenzung tritt. Diese neue sozialstrukturelle Ordnung basiert auf ,Erlebnisroutinen', die dem Individuum Orientierung bietet und über die alltagsästhetischen Schemata zur mikrosozialen Reproduktion sozialer Milieus in der Gesellschaft beiträgt.

Auch SCHULZE sieht hierbei massive Verunsicherungsprobleme auf die Individuen zukommen. Nicht nur die Frage, was will ich eigentlich' erfordert in diesem Rahmen ungekannte Reflexionsleistungen sondern auch die umfassende Wahl- und Handlungsfreiheit führt zu neuen Überforderungen, die systematisch Unsicherheit und Enttäuschung produziert (ebd.; 60ff). Die zentrale Frage der Gesellschaft ist nun nicht mehr, wie erreiche ich X?' sondern ,was ich will ich eigentlich?'.
„Für die Wäblbarkeit von Waren, Lebensläufen und Beqiebungspartnern haben wir mit dem Verlust jener emotionalen Balance zu bezablen, die uns das Vorgegebene selbst dann vermittelt, wenn es uns das Leben schwer macht. An die Stelle von engen und langfristig bestehenden Verwandtschaftsgruppen, Nachbarschaften und ökonomisch restringierten oder privilegierten Milieus ist kein gesellschaftliches Vakuum getreten. Neue psychisch schwierigeren Formen von Gesellschaft keristallisieren sich heraus" (ebd., 77).

\subsubsection{Moderne Sozialstrukturanalyse}

Die Analyse der Sozialstruktur als eine Form der Gesellschaftsanalyse zielt seit jeher auf die Erklärung und Erforschung von sozialen Ungleichheit ab. Sie nimmt als eine der ältesten Disziplinen der Soziologie einen zentralen Stellenwert innerhalb der soziologischen Wissenschaftsdisziplin ein (vgl. SCHÄFERS 1979, 4). Nach dem herkömmlichen Verständnis verweist die Analyse der Sozialstruktur auf ,objektive' Gegebenheiten, bzw. auf den $\mathrm{Zu}-$ sammenhang zwischen vermeintlich, objektiven' Strukturen und soziokulturellen Handlungsweisen.

Strukturelle Individualisierung geht dabei von einer Lockerung bzw. Auflösung des lange Zeit in der Soziologie vorherrschenden Klassen- und Schichtparadigmas als strukturierende Ungleichheitsstruktur aus. An den zentralen Arbeiten von MARX und WEBER ansetzend, gehen die von BECK $(1983 ; 1986 ; 1997)$ und BERGER (1986) initiierten Thesen von einer zunehmenden Entkopplung von sozialer Lage und kultureller Lebensweise aus ${ }^{158}$. Ziel der in den 80er Jahren vor allem auf Grund der Forschungsergebnisse MOOSERs (1984) aufkeimenden Diskussion über die moderne Sozialstruktur ist die Konzeption einer ,neuen sozialen Ungleichheit'. MOOSER (1984) aber auch BECK (1983; 1986) stellten als erstes die mannigfaltigen Umwälzungen der Arbeitergesellschaft an einigen empirischen Beispielen dar, die sich im Kern an der Auflösung bestehender Sozialmilieus, der Auflösung von Schicht- und Klassenstrukturen, einer Verschiebung der Geschlechterrollen und der Erosion bestehender Biographieführung festmachen lassen. Im Mittelpunkt steht die Beobachtung einer vor allem in der Gleichzeitigkeit einander entgegengesetzten Entwicklung: Trotz relativ kon- 
stanter Ungleichheitsrelationen zeigen sich deutliche Auflösungserscheinungen klassengesellschaftlicher Deutungsmuster (vgl. BERGER 1986, 225).

Die industriegesellschaftlichen Lebensformen, die sich an sozialen Klassen und Schichten orientieren und die mit dem Aufkommen der Moderne sukzessiv entschärft wurden, verlieren dabei scheinbar zunehmend an soziokultureller Bedeutung. Die zentrale These besagt, dass die klassischen Indikatoren sozialer Ungleichheit, wie Beruf, Einkommen und Bildung nicht mehr hinreichend Handlungsorientierungen und Verhaltensweisen von Menschen prognostizieren oder gar erklären können (vgl. BÖLLERT 1995, S. 29). BECK geht mit seiner Individualisierungsthese dabei nicht von einer Nivellierung sozialer Ungleichheit wie beispielsweise noch GEIGER (1949) aus, sondern folgert aus seinen Beobachtungen, dass Faktoren sozialer Ungleichheit in früheren Jahren lediglich eine wesentlich stärker strukturierende Wirkung auf die individuelle Biographieführung hatten als heute. Dabei zielt die Wiederbelebung der Individualisierungsthese nicht ausschließlich auf eine Veränderung von Familien oder Geschlechterverhältnissen sondern letztlich auch auf eine einschneidende Veränderung im Verhältnis von Individuum und Gesellschaft, die ,[...] nun ohne die Puffer quasi-ständischer Vergemeinschaftungsformen - wie Familie, Milieu, Schicht oder Klasse - miteinander verkoppelt sind“" (BERGER 1996, 58).

Die wohl kontroverseste Diskussion im Rahmen des Individualisierungstheorems stellt die Art der sozialen Integration dar, die sich in der Lebensstilforschung fortsetzt und zu weitreichenden Debatten geführt hat ${ }^{159}$. Die Frage wie die Individuen sich in die Gesellschaft integrieren und ob auf Individualisierung Atomisierung folgt, ist eines der strittigen Themen in der aktuellen Sozialstrukturforschung ${ }^{160}$ und gleichzeitig im Rahmen subjektorientierter Überlegungen von Relevanz.

Soziale Beziehungen müssen dem Individualisierungtheorem folgend zunehmend - jenseits traditioneller Gemeinschaften - selbst bestimmt, gesucht und gepflegt werden. Zwar verlieren Klassen und Schichten - so die These - ihre sozialstrukturelle Bedeutung. Diese Befreiung bedeutet aber letztlich kein Vakuum, sondern die Einbindung in stärker marktgesteuerte Austauschprozesse und „sekundäre Institutionen“ (vgl. LEISERING 1999). Auch VAN DER LOO / VAN REIJEN (1992) haben in ihren Ausführungen über die Ambivalenzen der Moderne darauf aufmerksam gemacht, dass die Freisetzung des Individuums aus traditionellen Bindungen mit einer zunehmenden Abhängigkeit von Staat, unpersönlichen Organisationen und Institutionen verbunden ist. In ,individualisierte' und ,enttraditionalisierte' Gesellschaften werden die Schicksale nicht mehr an althergebrachten und ständisch eingefärbten Sozialformen geknüpft, sondern ,,[...] an anonyme Arbeitsmarktgesetzmäßigkeiten sowie oftmals undurchsichtige sozialpolitische Regulierungen“ (BERGER 1996, 59).

In der überaus umfangreichen Literatur zur aktuellen Diskussion nach BECK $^{161}$ lassen sich drei zentrale Dimensionen sozialstruktureller Individualisierung besonders hervorheben: Neben der Kontinuitätsthese, die die oben beschriebene Entwicklung einer konstanten Ungleichheitsrelation bei 
insgesamt wesentlich höherem Gesamtniveau beschreibt, vertritt vor allem BECK eine Transformationsthese. Diese geht trotz konstanter Ungleichheitsrelationen von einer veränderteten Analyseperspektive jenseits von „Stand und Klasse" (BECK 1983) aus und beschreibt damit einen Wandel des Privaten, der in seinen Dimensionen auch für das gesellschaftliche Subsystem Sport von großer Bedeutung ist. Die Prognose eines zunehmenden Bedarfs an selbstbestimmter Auswahl, Entscheidung und Begründung des eigenen Handelns und Erlebens lässt sich insbesondere aus der oben beschriebenen Herauslösung des Individuums aus den angegebenen historisch vorgebenden Sozialformen und -Bindungen schließen. „Du darfst und Du kannst, ja Du sollst und musst eine eigenständige Existenz führen " (BECK \& BECKGERNSHEIM 1994, 25). Damit rücken kulturelle Unterscheidungsmuster vermehrt in den Vordergrund der Diskussion um Ungleichheitserfahrungen in westlichen Gesellschaften.

Die Konzentration auf die individuelle Lebensführung betont sowohl in der analytischen Perspektive als auch in der individuellen Selbstbeschreibung Stile, Konsumgewohnheiten und Geschmackspräferenzen. Die entscheidende These in der gegenwärtigen Sozialstrukturforschung stellt allerdings die schon angedeutete Entkopplungsthese dar: Sozialkulturelle Formationen oder soziokulturelle Selbstbeschreibungen seien zunehmend von der sozialstrukturellen Selbstbeschreibung abgelöst (vgl. JUNGE 2002, 59). Auf eine Entkopplung ,objektiver Lebensbedingungen' und ,subjektiver Lebensformen' weist u.a. auch HRADIL (1990) hin und unterstreicht damit die Bedeutsamkeit der These für die sozialwissenschaftliche Diskussion. Allerdings wird insbesondere die Entkopplungsthese in der Auseinandersetzung um Individualisierung und Sozialstruktur oft vernachlässigt, wobei die Vertreter des Theorems den empirischen Nachweis für die Entkopplungsthese schuldig bleiben.

In der aktuellen Diskussion über Individualisierung als sozialstrukturelles Phänomen stehen sich zur Zeit weiterhin Klassentheoretiker (STRASSER \& DEDERICHS 2000; KONIETZKA 1994), Schichttheoretiker (GEISSLER 1994) und Vertreter einer radikalen Individualisierungsthese gegenüber (MÜLLER-SCHNEIDER 2000; MICHAILOW 1996). Diese fordern im Hinblick auf die beschriebenen Transformationsprozesse eine feinkörnigere und multidimensionale Analyse von sozialer Ungleichheit und befürworten damit ein an Lebensführung und Lebensstil angegliedertes Konzept.

Gegner der Individualisierungsthese (GEISSLER 1994; KOHLI 1989) argumentieren mit einer Reihe empirischer Analysen und kommen zu dem Schluss, dass Individualisierung nicht völlige Entstrukturierung und Entschichtung bedeute, sondern das schichtspezifische Lebensverläufe und Lebenschancen durchaus exisitieren, bzw. sozialstrukturelle Vorgaben weiterhin eine starke Wirkung auf den Lebenslauf ausüben. Die aktuelle Sozialstrukturforschung versucht deshalb Verbindungslinien zwischen Individualisierung, Lebensführung und Lebensstilen zu ziehen (vgl. SPELLERBERG 1998). Individualisierung wird damit als Differenzierung sozialer Lagen, Privatisierung von Sozialbeziehungen und persönlicher Autonomie verstanden. 
Einen bedeutenden und viel beachteten Ansatz zur Sozialstrukturforschung, der für weite Teile der Lebensstilforschung von großer Bedeutung ist, liefert zudem BOURDIEU (1982). In seiner soziologischen Theorie der „Feinen Unterschieden“ versucht er die gesellschaftliche Makroebene mit der personellen Mirkoebene zu verbinden. Er beschreibt die Gesellschaft als einen mehrdimensionalen Raum, der sich in das Kapitalvolumen, die Kapitalstruktur und die zeitliche Entwicklung unterteilen lässt (vgl. ebd., 195f). Kapital ist dabei nicht eine rein ökonomische Größe, sondern wird erweitert um das soziale Kapital als Ausdruck sozialer Beziehungen und dem kulturellen Kapital, welches als Bildungsmaßstab gedeutet werden kann. Die Aufteilung des sozialen Raumes erfolgt nun anhand dieser Dimensionen und ihrer Beziehung untereinander. Von Klassen kann man im Sinne BOURDIEUs nur sprechen, wenn die symbolischen Formen berücksichtigt werden, in denen die Individuen soziale Positionen zum Ausdruck bringen und sich voneinander abgrenzen - im sozialen Raum der Lebensstile.

Ohne auf die detaillierte Beschreibung seines Werkes einzugehen, ist BOURDIEU's Einbeziehung symbolischer Ausdruckweisen in die Sozialstrukturanalyse und damit in die Gesellschaftsanalyse von großer Bedeutung für die Entwicklung der neueren Sozialstrukturforschung und gleichsam für das Forschungsinteresse dieser Arbeit. BOURDIEUs Leistung ist es, das Verhalten und die Handlungsweisen eines Menschen als ,symbolisches Kapital' - soll heißen seine Bedeutung für Prestige, Anerkennung und soziale Schließungsprozesse - herausgestellt zu haben. Die Frage nach der konstitutionellen Bedeutung von kulturellen Praktiken wie dem Sport für soziale Schließungsprozess stellt darüber hinaus ein Novum der Sozialstrukturanalyse dar. Stil und symbolhaftes Verhalten im Rahmen sportlicher Betätigung wird damit zu einer Frage sozialer Schließungsprozesse und für sportsoziologische Fragestellungen von großer Bedeutung.

\subsubsection{Pluralisierung und Differenzierung}

Auch die soziologische Debatte um Pluralisierung und Differenzierung ist eng an die Individualisierungsdiskussion gekoppelt.

„Die Tendenz zur Pluralisierung meint, dass mit der Auflockerung oder
Auflösung der historischen, von Klassen und Konfessions:wängen geprägten
sozialmoralischen Großmilieus die Gesellungen, insbesondere die Formen des
Zusammenlebens und des Gemeinschaftshandelns der Menschen vielfältiger
und situationsoffener gestaltet werden (VESTER et al 1992, 38f).

Als Ursache für eine Pluralisierung von Lebensformen wird die oben beschriebene Veränderungen der sozialen Schichtung als auch die Ausdifferenzierung individueller Lebensläufe gedeutet (vgl. GLUCHOWSKI 1988, 9). Aber auch die im zeitlichen Verlauf starke Auffächerung der allgemeinen Lebensbedingungen, die in vielen unterschiedlichen Formen der Erwerbsarbeit, des Zusammenlebens, der Freizeitgestaltung und des Konsums ihren 
Ausdruck finden, wird unter dem Topos der Pluralisierung diskutiert (vgl. ZERGER 2000, 24f; auch HRADIL 1996, 20ff).

Empirisch wurde diese Pluralisierung der Lebensformen u.a. von DELL (1979) angestoßen, der zu Beginn der 1970er Jahre die These vertrat, der soziale Wandel führe die Industriegesellschaft in eine postindustrielle Dienstleistungsgesellschaft. Obwohl diese These in ihrer Absolutheit keine Bestätigung fand, können doch mannigfaltige Pluralisierungstendenzen im Bereich der Arbeitswelt festgestellt werden ${ }^{162}$.

Die Veränderungen in den Haushalts- und Familienformen werden in der Soziologie als die markantesten Umbrüche in der Sozialstruktur angesehen und als Indiz einer großen Variationsbreite an familialen und außerfamilialen Lebensformen charakterisiert (vgl. zusammenfassend bei HRADIL et al. 1990). Waren früher Haushalts- und Familienformen durchaus synonym zu lesen, zeigt der Funktionswandel der Familie heute ein neuartiges Bild der Haushaltsformen z.B. in Form einer massiv gestiegenen Zahl der Einpersonenhaushalte 163

Die Zunahme unvollständiger oder alternativer Lebensformen verweist zudem auf die Pluralisierung der Ehe- und Familienformen. So nahm die Zahl der geschiedenen oder getrennt lebenden Paare in den letzten Jahrzehnten deutlich zu (vgl. LOCKE 1993). Auch die Zahl alleinerziehender Menschen verweist schon seit Anfang der 1990er Jahre auf eine deutliche Pluralisierung der Lebensformen in Deutschland (vgl. SCHÄFER 1990, 135).

Ein weiteres Feld der Pluralisierung der Lebensformen lässt sich im Bereich der kultureller Interessen aber auch im Feld der Freizeitaktivitäten darstellen. So lässt sich nicht nur eine Auffächerung unterschiedlichster Kunstrezeption anhand der Museumszahlen belegen (vgl. LOCKE 1993, 61ff). Auch die Quantität und Qualität von Mediennutzungen, sowie die Mitgliedschaft in Sport- oder Gesangsvereinen weist auf eine Differenzierung der Lebensformen hin (vgl. auch BETTE 2004).

Die Bedeutung der pluralisierten Lebensformen für die individuelle Lebensgestaltung wird in diesem Zusammenhang zwar weiterhin kontrovers diskutiert. Zweifelsfrei kann aber eine durch die Individualisierungsdynamik hervorgebrachte Auflösung familialer Strukturen und damit eine veränderte Identitätslogik konstatiert werden. Nicht die Familie im traditionellen Rahmen führt zur Einbindung und Selbstvergewisserung des Einzelnen, sondern eine ganze Vielfalt von Lebensformen sind für die Individuen in dieser Gesellschaft heute handlungsleitend und damit auch sozialstrukturell relevant (vgl. auch BETTE 1999). Insgesamt muss die beobachtbare Pluralisierung der Ehe- und Familienform sowie der markante Wandel im Bereich der Erwerbsarbeit auf Grund ihrer Bedeutsamkeit für die Lebensform des einzelnen als eine der gravierendsten Veränderungen der Sozialstruktur in den letzten Jahren gedeutet werden. 


\subsubsection{Wertewandel}

Obwohl die soziologischen Beiträge zum Wertewandel in westlichen Gesellschaft durchaus kontrovers diskutiert werden, steht die Thematik des Wertewandels als Themenfeld sowohl für die Sportsoziologie als auch für die soziologische Untersuchung des Risikosports in einem wichtigen inhaltlichen Zusammengang. Wertorientierungen gehören zu den zentralen Kohäsionsinstanzen einer jeden Gesellschaft. Im Vergleich zu gesellschaftlichen Normen und politischen Einstellungen, können Wertorientierungen als ein individuelles Überzeugungssystem verstanden werden, das verhaltensrelevant ist und gleichzeitig eine gewisse Änderungsresistenz besitzt (vgl. LOCKE 1993, 57f; vgl. auch KLEIN 2000). Ein wie auch immer gearteter Wertewandel hat damit erheblich Bedeutung für das soziokulturelle, als auch für das politische System einer Gesellschaft.

Maßgeblich beeinflusst wurde die sozialwissenschaftliche Auseinandersetzung dabei von INGELHART (1995; 1998), der mit bemerkenswerter Konsequenz das Thema des kulturellen, wirtschaftlichen und sozialen Wandels entfaltet hat. Ausgehend von einer hohen Nachkriegsprosperität in den westlichen Gesellschaften geht INGLEHART von einer Ablösung materialistischer Werte $\mathrm{zu}$ Gunsten einer Hinwendung zu postmaterialistischen Werten aus. Sein zentraler Erklärungsansatz geht von einer sogenannten Mangelhypothese aus, der zufolge die Menschen nach Dingen streben, die für sie im allgemeinen knapp sind. Insofern lassen sich an Wertorientierungen auch die sozialökonomische Lage der Befragten wieder spiegeln. Seine Sozialisationshypothese, die davon ausgeht, dass Wertorientierungen hauptsächlich während der Jugendphase begründet werden und dann entsprechend für den Lebenslauf gefestigt sind, werden heute unter dem Vorwurf der Vereinfachung kritisiert ${ }^{164}$. Im Vergleich zu INGLEHART konstatieren die sog. Speyerer Werteforschung rund um KLAGES (1984; 1988; 1999) einen Wandel von Pflicht und Akzeptanzwerten hin zu Selbstentfaltungswerten. Ein wesentlicher Unterschied der beiden Konzepte liegt in der den jeweiligen Annahmen bezüglich einer Ein- oder Mehrdimensionalität des am Wandel beteiligten Werteraums. INGLEHART postuliert einen eindimensionalen Raum der materialistischen und postmaterialistischen Werte, so dass ein Wachstum der einen Werte zwangsläufig zu einer Schrumpfung der anderen Wertegruppe führen muss. KLAGES nimmt dagegen die Möglichkeit verschiedenartiger Kombinationen aus Pflicht - und Akzeptanz- sowie Selbstentfaltungswerten in einem mehrdimensionalen Werteraum an (vgl. KLAGES 1999, 698f).

STENGEL (1997) führt in einer Auflistung Schwerpunkte eines Wertewandels an. Demnach wird durch den Wandel der Werte vor allem die eigene Selbstentfaltung und ein individueller Hedonismus betont. Im diesem Rahmen ist auch eine höher Bewertung der Freizeit und eine gewisse Abwertung der Arbeit zu konstatieren. Aber auch das Streben nach Gesundheit oder die zunehmenden Befreiung der Sexualität von gesellschaftlichen Normen sind als Folgen des Wertewandels festzustellen. 
Auch im Bezug auf die Ursachen dieses - wie auch immer im Detail gearteten - Wertewandels besteht keine völlige Einigung (vgl. GIESEN 1992). Während INGLEHART - in Anlehnung an MASLOWs Bedürfnistheorie den Wertewandel an eine Knappheitsthese verknüpft und davon ausgeht, dass sich Werte nach den jeweils aktuellen Mangelsituationen richtet, vertritt vor allem die Speyerer Werteforschung einen Mehrebenenansatz. Demnach lassen sich sowohl langfristig wirkende Ursachen, als auch lebenszyklische Effekte und Katalysatoren sowie unmittelbare Auslöser ausmachen. KLAGES (1998) stellt vor allem Modernisierungsprozesse in einen kausalen Zusammenhang des Wertewandels. Formen des Wertewandels von Gesellschaften, die sich im Modernisierungsprozess befinden, werden daher als ein ,normaler' Vorgang sozialen Wandels interpretiert.

Im Zusammenhang der forschungsleitenden Fragestellung dieses Kapitels ist vor allem die Frage der Dynamik und den Trägern des Wertewandels relevant. STENGEL (1997) konstatiert hierzu:

„Bei differenzierter Analyse der Daten stellt man fest, dass verschiedene Be-
völkerungsgruppen den Wertewandel in jeweils unterscbiedlichem. Ausmaß
tragen. Besonders ausgeprägt ist der Wandel der Werthaltungen bei jüngeren
Personen mit höherer, insbesondere mit akademischer Bildung"(ebd., 246).

KLAGES und die Speyerer Wertforschung kommt in ihrer Analyse des Wertewandels zu fünf besonders hervorstechenden Wertetypen, welchen ,[...] sich die Gesamtbevölkerung fast vollständig zurechnen lässt" (KLAGES 1999, 706). Neben ,Konventionalisten' (Typ 1) und ,Perspektivlosen Resignierten' (Typ 2) - eine in der Tendenz eher älteren Gruppe sind vor allem die ,aktiven Realisten' (Typ 3), die ,Hedonistischen Materialisten' (Typ 4) und vor allem die ,Nonkonformen Idealisten' (Typ 5) mit eher jüngeren Personen Träger des spontanen Wertewandels in der Bundesrepublik Deutschland (vgl. ebd.).

Die Folgen des Wertewandels beeinflussen unter anderem auch den sozialen Handlungsbereich des Sports, in dem eine merkliche Veränderung des individuellen Engagements stattgefunden hat (vgl. BETTE 1999, 147ff). Der aktive Sport erhält in Folge des Wertewandels einen eigenen Erlebnis- und Bedeutungsgehalt im Leben der Menschen im Sinne individueller Selbstverwirklichungsideale. Insbesondere der Trendsport und mit ihm auch verschiedene risikosportliche Aktivitäten als inszenierte Formen des Freizeitsports weist in ihren Ausprägungen und Handlungsweisen deutliche Bezüge zum multidimensionalen Wertewandel auf.

Für die folgenden Ausführungen zur Soziologie des Risikosports stehen die Ausführungen zum Wertewandel sowie die anderen deduzierten Theorien in einem erkenntnistheoretischen Zusammenhang. Wenngleich zugegeben werden muss, dass nicht immer offensichtlich wird, inwieweit die Prozesse einer erneute Modernisierung einen Erkenntnisgewinn für das Verständnis des Risikosports liefern können, so ist doch zu konstatieren, dass die hier praktizierte theoriegeleitete Auseinandersetzung nur unter Bezugnahme dieser Ansätze zu legitimieren ist. Nur wenn a priori deutlich ge- 
macht werden kann, um was es sich bei Prozessen wie Individualisierung oder Etiketten wie der Erlebnisgesellschaft eigentlich handelt, kann im Rahmen der theoretischen Deduktion darauf zurückgegriffen werden ${ }^{165}$.

\subsection{Soziologie des Risikosports}

Aus soziologischer Perspektive lassen sich eine Reihe von Erklärungs- und Interpretationsmustern von Risikosport deduzieren ${ }^{166}$. Aus den dargestellten gesellschaftsdynamischen Prozessen der Modernisierung und Individualisierung, der modernen Sozialstrukturanalyse und dem Wandel der Existenzformen lassen sich zunächst verschiedene Interdependenzen auf den Risikosport übertragen. Zum einen kann der Risikosport als individuelle Sinnsuche und Selbstvergewisserung gedeutet werden. Gleichzeitig lässt sich Risikosport in Verbindung mit Distinktionsformen und moderner Identitätsarbeit setzen, die als Reaktion auf Individualisierungsprozesse gelten. Ein weiterer Ansatz geht von einem Erleben individueller Risikokompetenz aus. Abschließend werden die in Kapitel 3 aus der Historie dargestellten Ansätze des Risikosports als Gegenwelt einer defizitären Gesellschaft expliziert. Hierbei muss zwischen einer Suche nach authentischer Lebendigkeit als Reaktion auf eine spannungsarme Gesellschaft und dem Wunsch nach Selbstermächtigung und Subjektaufwertung als Folge der funktionaldifferenzierten Gesellschaft unterschieden werden.

\subsubsection{Sinnsuche und Subjektivtätspflege}

Individualisierung als subjektorientierter Prozess impliziert hohe Anforderungen an die Individuen der betroffenen Gesellschaft - quasi als Regisseure ihres Lebens - und an ihr individuelles Gestaltungspotential: Ohne die Fähigkeit zur Selbstreflexivität und selbst entwickelter Handlungsfähigkeit ist das Individuum im Dschungel der Möglichkeiten ${ }^{167}$ nahezu verloren. Denn Individualisierung als ambivalenter Prozess ist auch in seinen Folgen für das einzelne Individuum hochgradig doppelwertig: Individualisierung eröffnet den Individuen einerseits Gestaltungsspielräume, sie zwingt aber auch zur Ausformung und Führung eines eigenen Lebens und damit zur eigenen Sinngebung, ,[...] zur Suche nach den jeweils besten Möglichkeiten und zu immer neuen Entscheidungen" (BILDEN 1989, 23).

„In der individualisierten Gesellschaft muss der einzelne entsprechend bei Strafe seiner permanenten Benachteiligung lernen, sich selbst als Handlungsraum, als Planungsbüro in Bezug auf seinen eigen Lebenslauf, seine Fähigkeiten, Orientierungen, Partnerschaften usw. zu begreifen "(BECK 1986, 217).

Es entsteht auf diese Weise ein zunehmender Zwang zur selbstbestimmten Auswahl, Entscheidung und Begründung des eigenen Handelns (vgl. GIEß-STÜBER 1998, 136). Das Individuum wird in diesem Prozess auf sich selbst zurück verwiesen und zur Selbstreflexion gezwungen. Eine Biographisierung im Sinne traditioneller Vorgaben ist nur noch bedingt möglich 
- vielfach sogar unmöglich ${ }^{168}$ (vgl. BECK \& BECK-GERNSHEIM (1994, 25).

Diese fehlende Orientierung und der Zwang zu einer reflexiven Lebensführung geht mit dem Verlust an kollektiven Bezugspunkten einher und beinhaltet dabei einen Zugewinn an individuellen Gestaltungsspielräumen. Gleichzeitig impliziert dieser Prozess die individuelle Generierung von eigenen Sinnquellen.
„Zu den entscheidenden Merkmalen von Individualisierungsprozessen gehört derart, dass sie eine aktive Eigenleistung der Individuen nicht nur erlauben sondern fordern. [...] Die Individuen müssen, um nicht zu scheitern, langfris- tig planen und den Umständen sich anpassen können, müssen organisieren und improvisieren, Ziele entwerfen und neue Anfänge versuchen "(BECK \& BECK-GERNSHEIM 1994, 14f).

Wie SCHULZE (1995) in seinen Ausführungen über die Erlebnisgesellschaft deutlich macht, sind insbesondere die materialistischen Sinnquellen der späten 70er Jahre wie Reichtum, Erfolg oder Karriere nur noch bedingt handlungsleitend ${ }^{169}$. Man ist geradezu ,[...] besessen von dem Ziel der Selbstverwirklichung“" (BECK 1986, 156) ${ }^{170}$. Die Lebensplanung kann nun mitunter auch auf Ziele ausgerichtet sein, die bedingt durch zunehmenden Wohlstand, Freizeit und Fortschritt abseits traditioneller Lebensmuster ihre Gültigkeit gewinnen. Diese Tendenz individuellen Verhaltens nimmt auch SCHULZE (1995) als Leitidee an. Die Suche nach Sinnerfüllung und Identität bezieht sich demnach auf die eigene Innenwelt und wird zur Maxime der Gesellschaft: ,[...] ,Erlebe Dein Leben' ist der kategorische Imperativ unserer Zeit" (ebd., 59). Die Erlebnisorientierung als Norm bestimmt folglich den Sozialcharakter des modernen Alltags:

„Personen, die sich mit dieser Norm identifizieren, begreifen ibr ganzes Leben als Erlebnisprojekt, für das sie individuell verantwortlich sind. Sie mobilisieren die ibnen zur Verfügung stehenden Ressourcen, um möglichst erlebnisintensiv ₹u leben. [...] Es erscheint ibnen als rational, nach intensiven Erlebnissen zu streben und demzufolge ibr Leben auch danach zu bewerten, wieweit es ibnen gelingt dieses Ziel zu erreichen" (HAUBL 1998, 7).

Mit Bezug auf SCHULZE sieht RITTNER (1998) insbesondere für den modernen Sport vielfältige Korrespondenzen zwischen den Prinzipien der Innenorientierung und den Formen eines veränderten Sporterlebens. Gerade im modernen Sport, zu dem er auch den Risikosport zählt, zeige sich die Steigerung des Selbsterlebens und des Interesses an einer selbstbezüglichen Selbstinterpretation. Den Ausführungen folgend, liefert gerade der Risikosport - z.B. durch die Abschwächung des traditionellen Leistungsprinzips im Sport oder die fehlende Orientierung an universalen Vergleichsmaßstäben prägnante Beispiele für den Abbau von tradierten Prinzipien der Außenorientierung (vgl. ebd., 30f).

Risikosport kann damit im Rahmen der individuellen Orientierungslosigkeit mit seinen vielfältigen Erlebnismöglichkeit (vgl. Kapitel 4) zu einer indi- 
viduellen Sinnquelle erhoben werden. Dabei ist weniger entscheidend wirklichen Sinn im Risikosport zu erkennen, als vielmehr den Risikosport als Subjektivierungsvergewisserung zu missbrauchen. Die im Risikosport bereitgestellte Erlebnisdimensionen der Körperlichkeit, des Risikos und der Emotionalität werden für Prozesse der Sinnfindung und der Vergewisserung von Subjektivität entdeckt und fungieren dabei als Ressourcen von Selbstvergewisserung und als Quelle von Selbstvertrauen. Risikosport verspricht damit einzigartige Erfahrungen und schafft unmittelbar - zumindest bei erfolgreicher Bewältigung - direkt und individuell Orientierung und Sinnhaftigkeit (vgl. auch RUMMELT 2003, 212). Das individuell bewältigte Risiko wird hierbei zur Projektionsfläche der Selbstrepräsentation verwendet und als solches inszeniert.

Genau hier liegen auch die Stärken des Risikosports: In wenig anderen Aktivität wird die Fähigkeit zur selbstproduzierten Erlebnisfähigkeit stärker betont und von den Akteuren wahrgenommen als bei risikosportlichen Aktivitäten. Risikosport wird in diesem Kontext zu einer Art erlebnisorientiertem Leistungsausweis - zu einem individuellem Symbol der Subjektivitätspflege.

Es verwundert in diesem Zusammenhang nicht, dass Risikosport in seinen vielen Varianten eben in den sozialen Milieus besonders attraktiv ist, in denen Individualisierung und damit die individuelle Sinnsuche als besonders relevant angenommen werden. Der Verweis auf die historische Entwicklung des Risikosports (Kap. 3) und erste aktuelle empirischen Befunde belegen, dass die Mehrheit der Risikosportler soziostrukturell zur breiten Mittelschicht gehören ${ }^{171}$ (vgl. z.B. AUFMUTH 1989; ALLMER 1998; OPASCHOWSKI 2000). Für die junge Generation einer wohlhabenden Mittelschicht kann Risikosport demnach im Sinne eines Selbstverwirklichungsmotivs wirksam werden. Die Frage nach den Sinnzusammenhängen erschließt sich letztlich aus der realen Herausforderung der Situation, die im klaren Kontrast zu den vielfältigen Erlebnisfelder des Alltags steht.

\subsubsection{Risikosport als Identitätsarbeit und Distinktion}

Wenn es in der modernen Gesellschaft - wie im Individualisierungstheorem postuliert - zu einer Herauslösung des Individuums aus traditionellen Einbindungen kommt und dies parallel zu einem Zugewinn an persönlicher Autonomie führt, ist Individualisierung auch im Bezug auf die Identitätsbildung relevant. Das Gesellschaftsmitglied erfährt sich - angesichts des angesprochenen Individualisierungsprozesses - als eigenverantwortliche Zurechnungsinstanz und Steuerungszentrale für Lebensplanung und Lebensführung (vgl. MICHAILOW 1996, 74). Die Frage nach dem eigenen Selbstverständnis lässt sich daraus ableitend nicht mit Bezug auf die klassische Einbindung in die Gesellschaft beantworten. Traditionelle Identitätsmuster verlieren in diesem Kontext zunehmend an Bedeutung und bieten kaum noch Anknüpfungspunkte für die eigene Selbstzuschreibung. 
„Der Prozess der Individualisierung nimmt dem Subjekt die Möglichkeit, sein Selbst auf der Grundlage fester, unbinterfragbarer und allgemein gültiger Modelle zu beschreiben. Die Freisetzung von den klassischen Sinngebungsinstanzen beinhaltet einen Bedeutungsverlust vormals sicherbeitsstiftender Wirklichkeitskonstruktionen" (BETTE 2004, 51).

Identität wird damit zu einem narrativen Prozess („Ich erzähle mich

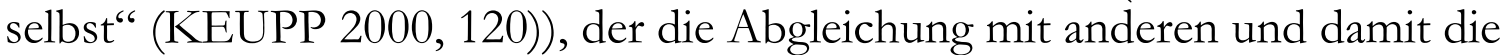
Auseinandersetzung mit Gegenidentitäten impliziert. Unter diesen Konkurrenzbedingungen wird die Stilisierung und Ausformung der eigenen Persönlichkeit zur vordringlichen Aufgabe. Definitionsräume, die wie z.B. der Sport identifikatorische Kraft besitzen, werden im Hinblick auf ein ,erfülltes Leben' mit der Suche nach sich selbst und einem neuartigen Drang nach Selbsterfahrungen gefüllt. Identitätsarbeit wird dabei zum Bemühen, sich von anderen zu unterscheiden und dies performativ darzustellen.

BETTE (1999) formuliert hierzu treffend:

„Der Mensch steht vor der existenziellen Notwendigkeit, die eigene Individualität festzulegen, und er unternimmt dies, indem er sich gegenüber seiner Umwelt als etwas Eigenständiges und Einmaliges abgrenzt. [...] Es bekommt sich selbst nur als Differenz zu sehen, nämlich in Form einer positiven oder negativen Tautologie: ich bin, wie ich bin oder: ich bin wie ich nicht bin, aber sein möchte172 "(ebd., 154)

Dementsprechend verändern sich auch die Bilder, die für ein gelungenes Leben oder eine erfolgreiche Identität herangezogen werden. BAUMANN (1997) spricht in diesem Zusammenhang von „Vagabunden“ oder „Flaneuren“ oder auch „Chamäleon-Identität“. Er bemüht auch die Metapher des „Videobandes“, welches ,[...] leicht zu löschen und wieder verwendbar ist" (ebd., 133). Die subjektiven Anteile von Definitionsleistungen und Relevanzsetzungen zur Strukturierung der Lebenslage werden dabei massiv aufgewertet, wobei auch die Revidierbarkeit einmal getroffener Entscheidungen deutlich zunimmt (z.B. Scheidung, Berufswechsel etc.).

Schon BOURDIEU (1982) hat darauf aufmerksam gemacht, dass der Sport in seinen vielfältigen Ausdrucksformen kein interessenloses und zweckfreies kulturelles Phänomen darstellt, sondern nicht zuletzt eine distinktive Praxis repräsentiert. Gerade der Risikosport bietet sich mit seinen gesellschaftlich-konträren Vorstellungen von Sicherheit und Risiko geradezu an, zu einer Projektionswand für die Selbstinszenierung zu werden. „Der Hinweis auf Betätigungen in Risikosportarten ist oft eine Möglichkeit, im sozialen Umfeld für Ansehen zu sorgen“" (HECKER 1989, 329). EGGEBRECHT formuliert hierzu treffend:

$$
\begin{aligned}
& \text { "Nie wieder Alltag lautet die Parole des Abenteurers, nie mehr von außen } \\
& \text { produzierte vermeintliche Identität in irgendeinem Status" } \\
& \text { (EGGEBRECHT 1985, 58). }
\end{aligned}
$$

Erfolgreicher Risikosport bedeutet aber nicht nur soziale Anerkennung, wie RHEINBERG (1997) es in seiner, Gladiator-Komponente' ausdrückt. 
Risikosportliche Aktivitäten dienen gleichsam zur Erzielung individueller Distinktionsgewinne, die durch verschiedene expressive Darstellungsmöglichkeiten in weite Gesellschaftsbereiche hineinreichen. Da Risiko und Sicherheit gesamtgesellschaftlich relevante Themen darstellen, verfügen die verschiedenen Risikoaktivitäten über ein umfangreiches Repertoire an Ausdrucksmöglichkeiten zur Selbstdarstellung und Distinktion. SIEGRIST (1998) konstatiert, dass gerade Wildnis als Handlungsmotivation in vielen Risikonatursportarten der Zivilisation antithetisch gegenüber steht (vgl. ebd., 249). Wer sich als Risikosportler beschreibt, stellt sich in Abgrenzung zum gesellschaftlichen Konsens und der breiten Masse in seiner Eigenständigkeit dar. Risikosport bietet mit seinem impliziten Herausforderungscharakter faktisch Anknüpfungspunkte mit denen Menschen die Möglichkeit erhalten, auf Distanz zum Alltags-Ich und zur Normalverwendung des Körpers zu gehen.

„Was differenziert in einer durch Routine, Sicherbeitsorientierung und Alltäglichkeit geprägten Gesellschaft mehr als die Selbstdefinition als Extremsportler und Abenteurer" (BETTE 2004, 52).

Auch GEBAUER et al. (2004) konstatieren in ihrer qualitativen Untersuchung, dass durch Sport vermittelter Stil als Identifikationshilfe und Selbstbestimmungsraum im Sport fungiert. In ihrer komparativen Analyse zwischen Trendsportlern und Akteuren traditioneller Sportarten betonen sie die Bedeutung von Vergemeinschaftungsprozessen und Zugehörigkeitsbetrebungen im Trendsport. Auch wenn diese Analyse bis dato nicht für den Bereich Risikosport repliziert wurde, können sie sehr wohl als empirische Indizien herangezogen werden.

Risikosport kann im Rahmen modernisierungs-theoretischer Annahmen also über die individuelle Sinngenerierung hinaus als Identitätsmedium und Distinktionsinstrument fungieren. In einer individualisierten Gesellschaft, in der sich Menschen nicht mehr durch den Anschluss an eine kollektive Identität identifizieren lassen, funktioniert die Selbstidentifikation der Individuen im Risikosport über eine individuelle Sinnzuschreibung und Erlebnisrationalität sowie eine demonstrative Abgrenzung nach außen.

\subsubsection{Risikosport als Gegenwelt einer defizitären Gesellschaft}

Die Einordnung des Risikosports aus modernisierungs-theoretischer Perspektive kann - wie vorstehend dargestellt - als ein interdependenter Vorgang der individuellen Selbstvergewisserung, der Identitätsunterstützung und der Erlangung individueller Risikokompetenz verstanden werden. Im folgenden wird das Aufsuchen von risikosportlichen Aktivitäten als ,[...] Befreiung des Individuums aus dem verregelten und als langweilig empfundenen Alltag“" (NEUMANN 1999, 107) - oder allgemein als Ausbruch aus einer defizitären Gesellschaft gedeutet. In Erweiterung einer eher traditionellen soziologischen Argumentationsfigur werden die Ansätze ELIAS (1991) 
über den Prozess der Zivilisation berücksichtigt und auf den Gegenstand des Risikosports übertragen.

Eine genaue Analyse lässt zwei miteinander verwandte Perspektiven zu. Als Grundlage des ersten Ansatzes gilt die Annahme der zunehmenden Routinisierung und Bürokratisierung der heutigen Gesellschaft, die eine fortschreitende Wirkungslosigkeit des ursprünglichen Menschen bedingt. Gemäß HIRSCHBICHLER (1987) manifestiert sich diese individuelle Entfremdung hauptsächlich in Langeweile (vgl. ebd., 64). Eine als spannungsarm empfundene Gesellschaft löst somit den Drang nach Aufregung und Anspannung aus, der den anthropologischen Ausführungen folgend (vgl. Abschnitt 4.2) mit einer Suche nach Unsicherheiten verbunden ist. FRANKL (1973b) formuliert diesen Gedanken, der konvergent zum ,Sensation Seeking' - Konzept nach ZUCKERMAN (1978) verstanden werden kann, folgendermaßen:

\section{„1. Der Mensch braucht Spannung. 2. Der Mensch sucht auch Spannung. 3. Gegenwärtig findet er aber zunvenig Spannung. 4. Darum verschafft er sich Spannung"(FRANKL 1973b, 28).}

In der von OPASCHOWSKI (2000) durchgeführten repräsentativen Befragung zum Thema Risikosport nennen 63\% aller Risikosportler und Menschen, die gerne eine Risikosportart betreiben würden, die ,Flucht vor der Langeweile als relevantes Motivelement (vgl. OPASCHOWSKI 2000, 17).

Langeweile und Spannungsarmut als gesellschaftliche Alltagserfahrung muss angesichts unzähliger Erlebnisangebote und der Übermacht an medialen und virtuellen Konsummöglichkeiten allerdings relativiert werden. WEIS (1995) beschreibt die Erfahrungs- und Wahrnehmungsdimensionen des Individuums im Bezug auf die gegenwärtigen Kommunikationsveränderungen mit dem Paradigma der ,Entrealisierung' (vgl. ebd., 54). Die Vielzahl der heutigen Erlebnisse werden demnach nicht mehr real sondern hauptsächlich virtuell - im Sinne sekundärer Erlebnisse - wahrgenommen. Dadurch erfährt der Mensch eine zunehmende Zentrierung der Sinneswahrnehmung auf die visuellen Informationswege. Die Komplexität der Realität wird zu einer vermittelten und gefilterten Wirklichkeit, die den Körper und die Gesamtheit der dort installierten Sinnesorgane aus dem Erfahrungsfeld des Menschen ausschließt. Unter dem Topos der Erfahrungsverluste, der sich seit der zweite Hälfte des 19.Jahrhunderts durch die europäische Kultur zieht, werden neben der ,Entkörperlichung' auch eine fortschreitende Entfremdung der Kommunikation und die als Virtualität bezeichnete ,Entrealisierung' des Menschen diskutiert (vgl. WEIS 1995, 52).

Die Reduktion des menschlichen Körpers auf isolierte Teilfunktionen begründet sich nach WEIS (ebd.) zudem durch den Prozess der Zivilisation, der durch die gültige Produktionsteilung einen physisch einseitigen Menschen begünstigt. Der Philosoph VIRILIO (1989; 1992) bezeichnet die moderne Technisierung des Haushaltes, die durch Computer, Internet und Fernsehen unterstützt wird, als eine „[...] künstliche Domestizierung“ 
(VIRILIO 1989, 54), die einen, liegenden’ Menschen verursacht (vgl. VIRILIO 1992, 124).

Eine durch Spannungsarmut und Erfahrungsverlust geprägte defizitäre Gesellschaft bietet folglich günstige Voraussetzungen für die Verbreitung von Risikosportarten, denn ,die Gelegenheiten für ein intensives, emotionsgeladenes und positiv besetztes Spannungserleben sind in hochkomplexen Gesellschaften knapp geworden“ (BETTE 1989, 175). Risikosportler nutzen demnach die Risikosituation und die stimulierende Wirkung der unterschiedlichen Erlebnisdimensionen, um die Gefühle der Langeweile zu vertreiben und das eigene Lebendigkeitsgefühl zu verstärken. „Der moderne Risikosport ist deshalb als eine Kulturtechnik der Lebensbejahung anzusehen" (BETTE 2004, 20). In einer Gesellschaft, in der Sekundär- und Tertiärerfahrungen das Erfahrungsfeld der Menschen domestiziert, bietet der Risikosport demnach durch ein spezifisches Körper- und Bewegungserleben ein probates Medium zur Erschließung von primären Erfahrungen. Im Risikosport stehen nicht Kommunikation im Vordergrund, sondern sinnliche Wahrnehmung und Körperhandeln.

„Augen, Obren und Nase, aber auch Hände und Füße sowie bierüber vermittelte Orientierungs-, Gleichgewichts- und Balancierfähigkeiten erfahren im Extremsport eine interessante Wiederbelebung" (ebd., 77) ${ }^{173}$.

Auch AUFMUTH (1988; 1989; 1996) verweist in seinen Ausführungen über das alpine Bergsteigen auf das mit dem Risiko verbundene Lebendigkeitsgefühl, welches Risikosportler in der Ausübung ihrer Aktivitäten erfahren:

„Aus dem Schmerz und aus der Muskelarbeit, aus der Entbebrungen der Kampfbegierde und der Todesangst holen sie bergsteigend jenes Maß an gespürter Lebendigkeit aus sich heraus, dass die Leere der gelähmten Gefühle vergessen macht" (ebd.1996, 135).

Die Unausweichlichkeit liegt demnach im Wechselspiel der Erlebniskulturen:

„Wir modernen Menschen sind dazu verurteilt, periodisch die Welten zu wechseln, um zu einem annährend vollen Daseinsgefübl zu gelangen" (ebd., 172).

Allerdings verweist der Autor auf die Persönlichkeitsstruktur von passionierten Naturabenteurern, die im Vergleich zu anderen Menschen und ausgelöst durch frühkindliche Versagenserfahrungen kaum Lebendigkeit verspüren. Um dieser „,...] Brüchigkeit ihrer Lebendigkeitsempfindungen“ (ebd.) entgegenzuwirken, suchen diese Menschen fortwährend nach temporären Ausbrüchen aus ihren Alltagswelten.

Aus differenzierungstheoretischer Perspektive lässt sich ein zweiter Ansatz im Kontext der defizitären Gesellschaft entwerfen. COLEMAN (1986) weist in seinen systemtheoretischen Analysen auf die wachsende Asymmetrie zwischen Personen und Organisationen in modernen Gesellschaften hin. 
Der Mensch ist dabei zunehmend eingebunden in übergeordnete Organisationen und Institutionen, die als funktionale Systeme für das Funktionieren der Gesellschaft verantwortlich gemacht werden können. Aus Sicht der korporativen Akteure sind die Menschen in diesen Systemen austauschbar, um die Funktionalität der Organisationen aufrecht zu erhalten. Menschen werden in diesem Zusammenhang in Mitgliedschaftsrollen berücksichtigt, die sich aus der jeweils spezifischen Einbindung in die Organisationen ergeben. Überspitzt ließe sich formulieren: das Individuum wird in funktionalistisch differenzierten Gesellschaft niemals ganzheitlich wahrgenommen und erlebt sich auch selbst nie als Einheit. „Organisationen erzeugen damit in erwartbarer Weise Gefühle der Entfremdung, Nichtigkeit, Machtlosigkeit und des Ausgeliefertseins auf Seiten ihrer Mitglieder" (BETTE 2004, 26).

Des Weiteren lassen sich Nichtigkeitserfahrungen in arbeitsteilig organisierten Gesellschaften auch auf Grund der komplexen und unüberschaubaren Handlungsunterteilung beschreiben. Handlungsvorgänge, in denen der einzelnen den gesamten Handlungskontext betreut, sind selten, Interdependenzen und Verkettungen die Regel. Sowohl im Bezug auf die Handlungsrealisation als auch auf die Handlungswirkungen ist das Individuum in übergeordnete Vorgänge eingebunden:

"Die meisten Menschen sind im Arbeits- und auch im Freizeitbereich zur
weitgehenden, Wirkungslosigkeit' verdammt und erleben Gefüble einer ,vitalen
Inkompetenz'. Sie erfahren sich nicht als Mittelpunkt ibrer Welt, nicht als
Schöpfer ibrer eigenen Handlungen, stehen sich selbst fremd gegenüber"
(DOEHLEMANN 1991, 88; zit. nach NEUMANN 1999, 108)

In welchem Kontext aber steht der Risikosport vor dem Hintergrund von Bedeutungsverlust und Wirkungslosigkeit? Unter dieser zweiten Perspektive des Risikosports als Gegenwelt einer defizitären Gesellschaft können Risikosportaktivitäten aus dem Bestreben verstanden werden, Macht über das eigene Handeln wiederzuerlangen (vgl. auch BETTE 2004, 28). Im Risikosport lässt sich die Handlungsfähigkeit unter Beweis stellen und selbstständig auflösen. Die Bewältigung von Risikosituationen dient damit nicht nur der Vertreibung von Langeweile durch den so genannten Kick, sondern verweist auf die Einheit der Person mit allen ihren Handlungsfähigkeiten und sinnlichen Bezügen. Die Herausforderung als implizites Element der sportlichen Risikosituation vermittelt dem Sportler ein Gefühl der eigenen Stärke und Wirkungsfähigkeit, die ihm im Alltag weitgehend abhanden gekommen ist. Risikosportarten können demnach als physisch und psychisch ganzheitliche Erlebnissynthesen die Defizite der funktional differenzierten Gesellschaft ausgleichen.

„In einer Gesellschaft, in der das Handeln des einzelnen bäufig spurenlos verläuft und Personen Bedeutungsverluste und Gefüble der Obnmacht binzunehmen haben, zeigen Abenteuerbelden mit ibren spektakulären Aktionen in schlagender Weise genau das Gegenteil: dass Subjekte nicht gänzlich tot sind und ein unabbängiges eigenmächtiges Handeln in einer durchorganisierten Gesellschaft nach wie vor möglich ist" (ebd., 39). 
Zusammenfassung: Da empirische Beweise für die aufgezeigten Erklärungsmodelle bis auf wenige Ausnahmen (OPASCHOWSKI 2000) weiterhin fehlen, sind die gemachten Annahmen in ihrem Kontext als zu verifizierende Thesen zu verstehen. Wichtig erscheint aber die Annahme einer allgemeinen Interdependenz zwischen Risikosport und Gesellschaft aus unterschiedlichen Perspektive. Risikosportliche Aktivitäten lassen sich damit als Antwort und Frage auf gesellschaftliche Bedingungen verstehen, die in einem klaren modernisierungs-theoretischen Zusammenhang stehen. Die Soziologie, die hier als Bezugswissenschaft fungiert, weist mittlerweile mit einer Vielzahl von Konzepten und Theorien über die Risikofrage in modernen Gesellschaften auf (zur Übersicht z.B. BANSE \& BECHMANN 1998; BANSE 1996a). Die Darstellung sollte deshalb als explorativer Beantwortungsversuch für die forschungsleitende Fragestellung dieses Kapitels verstanden werden und weniger als empirisch abgesicherter Status Quo.

\subsection{Sicherheit und Risiko aus soziologischer Perspektive}

So unterschiedlich die verschiedenen Risiken der modernen Gesellschaft auch sein mögen, Sicherheit und Risiko sind zu zentralen Themen in der gesellschaftlichen Diskussion der Moderne geworden (vgl. Kapitel 3; auch STIEHR 1992, 125). Nie haben die Menschen ,[...] gewaltigere Anstrengungen unternommen, um sich gegen Krankheit, Not, Unfälle, Diebstahl, Gewalt, Subversion, und kriegerische Aggressionen zu sichern" (STRASSER $1986,29)^{174}$. Soziologisch lässt sich dieses gesteigerte gesellschaftliche Bedürfnis nach Sicherheit auf die gesteigerte Wahrnehmung von Unsicherheit zurückführen. Eine enge Verknüpfung findet sich zudem zum Individualisierungsprozess: das Herauslösen aus gemeinschaftlichen Zusammenhängen, die ihrerseits Geborgenheit und Schutz bieten, wird in der Moderne durch eine ,[...] staatliche Risikoabsicherung durch eigens geschaffene Institutionen kompensiert.“ (STIEHR 1992, 126).

Risikosport konstituiert sich in einem gesellschaftlichen Bezugsrahmen, der nicht nur über die Begriffsverwandtschaft in einer engen Beziehung zum sozialen Verständnis von Risiko und Sicherheit verstanden werden muss. Begreift man den Risikosport unter gesellschaftstheoretischer Perspektive, dann kann - wie a priori ausgeführt - das aufgesuchte Risiko in seinen Erlebnisdimensionen als Kontrapunkt zum gesellschaftlichen Alltag verstanden werden. Betrachtet man dieses Verhalten als eine Risikosuche oder Jagd nach Unsicherheit, dann präsentiert sich aber ein scheinbares Paradoxon: Denn wie kann der Mensch angesichts der Vielzahl bestehender Unsicherheiten und dem im Zusammenhang stehenden Sicherheitsstreben gleichzeitig noch auf eine freizeitsportliche Risikosuche erpicht sein? Es erscheint für eine kritische Analyse des Umgangs mit Risiko und Sicherheit im Risikosport daher erforderlich zu sein, eine Differenzierung zwischen gesellschaftlichen und individuellen Risiken vorzunehmen und das gesellschaftliche Verhältnis zur Sicherheit zu explizieren. 
Es ist in diesem Kontext zu hinterfragen, welche Unterscheidung zwischen Unsicherheiten und Risiken aus gesellschaftstheoretischer Perspektive entwickelt werden können und wie ein Bedürfnis nach Sicherheit im gesellschaftlichen Alltag überhaupt befriedigt werden kann. Daher wird im folgenden nochmals auf die Differenzierung von Risiko, Unsicherheit und Gefahr eingegangen und schließlich die gesellschaftliche Konstruktion von Sicherheit inklusive der entsprechenden gesellschaftlichen Sicherungsstrategien erläutert. Anschließend wird der Risikosport in den Kontext einer vermeintlich , sicheren Gefahrengesellschaft' eingeordnet.

\subsubsection{Risiko, Unsicherheit, Gefahr - eine Annäherung aus soziologi- scher Perspektive}

Wie viele Begriffe in der Soziologie sind Dogmen wie Sicherheit und Risiko nicht selbstexplikativ. Der eigentliche Sinn erschließt sich erst durch den entsprechenden Referenzrahmen - die Außenwelt selbst kennt weder Risiken noch Sicherheit. Wenn - wie LUHMANN (1991; 1993) betont - Gesellschaften in struktureller Hinsicht durch Kontingenz geprägt sind, ist eine Unterscheidung von Sicherheit und Unsicherheit, von Sicherheit und Risiko generell mit Schwierigkeiten behaftet. Unsicherheit als Referenzrahmen ist universell und bezieht sich auf die immer schon vorhandene Zukunftsungewissheit. Aus der Zurechenbarkeit und Handhabung von Ungewissheit lassen sich dennoch fruchtbare Differenzierungen ableiten: Wie in Kaptitel 2 expliziert, sind Risiken im Unterschied zu Gefahren handlungs- und entscheidungsbezogene Formen der Konstitution und Handhabung von Ungewissheit, die sich dadurch auszeichnen, dass die zur Diskussion stehenden Unsicherheiten als zurechenbar und verantwortbar gelten (vgl. BONB 1995, 62) ${ }^{175}$.

Der geschichtliche Abriss zum Verständnis von Risiko in vormodernen Epochen hat verdeutlicht, dass Risiko als soziales Konstrukt in Abhängigkeit der gesamtgesellschaftlichen Bedingungen konstituiert wird. Es lässt sich zudem an verschiedenen Beispielen veranschaulichen, dass dieses Risikokonzept in der gesellschaftlichen Praxis keineswegs so klar und eindeutig anzuwenden ist, wie es in einer ersten Annäherung erscheint. So ist sowohl der Handlungsbezug als auch die Entscheidungsabhängigkeit dessen, was gemeinhin als Risiko bezeichnet wird, anzuzweifeln.

BONß beschreibt drei grundsätzliche Dimensionen, in denen Zurechnungsprobleme und Entkoppelungsphänomene des Risikos auftreten können, explizit in zeitlicher, sachlicher und sozialer Hinsicht. In zeitlicher Perspektive können Entkopplungsprobleme beispielsweise dann auftreten, wenn die Risikofolgen generationsverschoben auftreten, beispielsweise bei der Nutzung von Energieträgern und den damit verbundenen Folgen des Ausstoßes von Kohlendioxiden. Eine Entkopplung in sachlicher Hinsicht liegt vor, wenn sich Folgen einer Risikohandlung in ganz anderen nichtzusammenhängenden Bereichen bemerkbar macht - z.B. wenn die Düngung mit Pestiziden bewirkt, dass Säuglinge keine Muttermilch mehr bekommen 
können. Die dritte Dimension der sozialen Entkopplung ist schließlich dann gegeben, wenn sich die Grenzziehung zwischen Handelnden und Handlungsbetroffenen verschiebt, z.B. wenn keine Entscheidungsträger mehr auszumachen sind oder aber alle global von den Risikofolgen betroffen sind (vgl. ebd., 62f).

Zur Verdeutlichung dieses gesellschaftlichen Risikophänomens führt BONß die Differenzierung zwischen Risikohandlung und Risikosystem ein, die die Entkopplung von Handlungen und Handlungsfolgen sowie die daraus resultierenden Probleme bei der Herstellung von Zurechenbarkeit fokussieren. Unter Risikohandlungen versteht der Autor klar zurechenbare Handlungen unter Unsicherheit, wobei die Folgen der Risikohandlung in zeitlich, sozialer und sachlicher Hinsicht weitgehend eingrenzbar und überschaubar sind. Zudem besitzt die Risikohandlung einen klar definierbaren Anfang und ein eindeutiges Ende (vgl. ebd., 57). Risikosysteme basieren im Gegenzug auf den beschriebenen Entkopplungsprozessen und werden durch eine Vervielfachung, Ausdifferenzierung und strukturelle Verfestigung von Risikohandlungen charakterisiert (vgl. ebd., 63). Bei Risikosystemen geht es nicht um einzelne Entscheidungen, sondern um Handlungsnetze, die in Abhängigkeit voneinander treten und dadurch eine höhere Gesamtkomplexität des Gesamtzusammenhanges erzeugen. Als Beispiele für Risikosysteme führt BONß Hochtechnologien, Gentechnik oder Kernkraftwerke an. Und im Bezug auf die gesellschaftliche Wirklichkeit konstatiert der Autor: „[...] isolierte Risikohandlungen sind eher die idealtypische Ausnahme, während Risikosysteme die realtypische Normalität repräsentieren“ (ebd., 65).

Auch BECK (1986) beschäftigt sich unter dem Etikett der „Risikogesellschaft - auf dem Weg in eine andere Moderne" mit eben diesen gesellschaftlichen Risikophänomene der späten 80er und 90er Jahre des letzten Jahrhunderts. Neben dem bereits angeführten Individualisierungstheorem, setzt sich BECK in seiner Analyse auch mit den gesellschaftlichen Auswirkungen globaler Risiken auseinander. Moderne Gesellschaften besitzen demnach eine besondere Affinität zum Risiko, die im Unterschied zu früheren Gesellschaftsformen von einer ungekannten Universalität und Globalisierung geprägt sind. Jeder Einzelne ist betroffen, ohne jedoch selbst handlungsfähig zu sein, denn weltumspannende Risiken wie Kernkraft oder andere Fortschrittstechnologien erlauben dem einzelnen kaum noch persönliche Handlungsspielräume. „Die Gefahren der chemisch und atomar hoch entwickelten Produktivkräfte heben die Grundlagen und Kategorien auf in denen wir bisher gedacht haben [...]“" (ebd., 29). In der gegenwärtigen Bedrohung durch Terrorismus sieht BECK die Aktualität seiner Risikotheorie bestätigt:

„Viele dieser Strukturmerkmale der Risikogesellschaft lesen sich heute wie Beschreibungen der Welt nach dem 11. September, nach den Terrorattentaten von New York und Washington.[...] Das Attentat vom 11.September hat die Verletzlichkeit der entfalteten Zivilisation ins allgemeine Bewusstsein gerück.t" (BECK 2002, 64). 
Nun lässt sich angesichts dieser soziologischen Gesellschaftsdiagnose feststellen, dass die das gesellschaftliche Leben betreffenden Risiken in der Mehrheit von einer zeitlichen, sachlichen und sozialen Entkopplung geprägt sind. Weder bei den von BECK als gesellschaftlich relevant beschriebenen Hochtechnologien, noch bei Umweltbedrohungen oder wirtschaftlichsozialen Risiken ist eine direkte Zurechenbarkeit und Verantwortlichkeit des Risikos gegeben. Zwar betont auch BECK die allgemeine Betroffenheit spätmoderner Risiken, die im Ausspruch: „Not ist hierarchisch, Smog demokratisch“ (ebd. 1986) ihren Ausdruck findet. Wenn aber das Risiko durch die individuelle Zurechenbarkeit und Verantwortung gekennzeichnet ist und gleichsam als entscheidungsbezogene Form des Umgangs mit Unsicherheit definiert wird, bleibt der Risikobegriff im Zusammenhang der Risikogesellschaft vakant ${ }^{176}$.

Angesichts dieser fundamentalen Kritik und in Erweiterung des BECKschen Risikogedanken, führt BONß (1995) schließlich den Terminus „Gefahren zweiter Ordnung“ (ebd., 80) zur inhaltlichen Differenzierung ein. Von Gefahren zweiter Ordnung ist dann zu sprechen, „,[...] wenn bestimmte Risikohandlungen und -systeme zeitlich, sozial und/oder sachlich versetzte Nebenfolgen haben" (ebd.).

\section{„Gefahren zweiter Ordnung sind transformierte Risiken, die in dem Maße sichtbar werden, wie die Ergebnisse eines Risikosystems nicht nur die Grenzen dieses Systems außer Kraft setzen, sondern zu einer neuen Ausgangslage füh- ren, die nicht mehr als Risikolage beschreibbar ist" (ebd.).}

Gefahren zweiter Ordnung kennzeichnen die von modernen Risikosystemen produzierten Folgen. Anwendbar auf eine breite Palette von Phänomenen - beispielsweise Kernkraft, Autoverkehr, Treibhauseffekt u.v.m. sind Gefahren zweiter Ordnung Ausdruck des gescheiterten Versuchs der Naturbeherrschung in der Moderne (ebd., 84).

Mit diesem Begriffswechsel beschreibt BONß einen gesellschaftlichen Entwicklungsgang vom Risiko zur Gefahr zweiter Ordnung, welcher mit einem allgemeinen Perspektivwechsel einhergeht. Begreift man die Gesellschaft nicht wie BECK als eine ,Risikogesellschaft', sondern als eine ,Gefahren-zweiter-Ordnung-Gesellschaft', dann wird deutlich, dass nicht das entscheidungsabhängige Risiko das Leben der Gesellschaftsmitglieder bestimmt sondern unzerechenbare Gefahren. Obwohl von den Folgen und Auswirkungen der Gefahren zweiter Ordnung massiv betroffen, erlebt der einzelne das Risiko nicht als einen entscheidungsabhängigen Prozess sondern als nicht handlungsabhängige Verunsicherung.

\subsubsection{Die gesellschaftliche Konstruktion von Sicherheit}

Ausgehend von der sozialen Bedeutung, die Risiken und ,Gefahren zweiter Ordnung' in ihrer allgemeinen Betroffenheit für die Gesellschaftsmitglieder besitzen, rückt erneut Sicherheit als gesellschaftliches Phänomen in den wissenschaftlichen Fokus der Arbeit. Denn nimmt man die anthropologischen 
(z.B. GEHLEN 1950), psychologischen (MASLOW 1954) und soziologischen (KAUFMANN 1973) Ansätze in ihrem Postulat eines menschlichen Sicherheitsbedürfnis ernst, dann stellt sich die Frage nach den gesellschaftlichen Bedingungen und Verfahren unter denen der Einzelne Sicherheit produziert.

Sicherheit - definiert als Zustand der Gefahrlosigkeit und der Unbedrohtheit (DORSCH 1998, 790) - verweist mit dieser allgemeinen Begriffsbestimmung auf eine Realfiktion, frei nach dem Motto: wo Sicherheit ist, kann keine Unsicherheit sein (vgl. BONß 1997, 22). Es konnte in Kapitel 3 anschaulich demonstriert werden, dass sowohl Unsicherheit als auch die auf sie bezogene Sicherheit eine soziale Konstruktion darstellt. Auch kultursoziologischen Arbeiten (z.B. DOUGLAS \& WILDAVSKY 1982) haben gezeigt, dass ein Streben nach Sicherheit in nahezu allen Gesellschaften als eine Art ,Umdefinition' von Unsicherheiten in Sicherheiten verstanden werden kann. Dies erscheint auch als überaus sinnvoll, denn wer die diversen Alltagsrisiken vom Haushalts- bis zum Verkehrsunfall oder die Möglichkeit, überfallen und ermordet zu werden als ,,[...] gleich gültige Wirklichkeit betrachtet und unklare Unsicherheiten als eindeutige Gefährdungen begreift, wird sich irgendwann nicht mehr aus dem Bett trauen" (BONB 1995, 86). Die eigene Handlungsfähigkeit lässt sich demnach langfristig nur durch eine selektive Unsicherheitswahrnehmung bzw. durch deren ,Umdefinition' in Sicherheit aufrechterhalten.

Besonders deutlich wird dieser Zwang zur selektiven Wahrnehmung in potentiell erdbebengefährdeten Gebieten, wie die Beispiele in Japan 1995 und Kalifornien 1994 zeigen. Ein normales Leben wäre dort ohne die ,Ausblendung' der real existierenden Gefahren gar nicht möglich, denn in Folge einer bewussten Antizipation der denkbaren Auswirkungen, müsste es zu massiven Abwanderungen kommen. Es existiert in diesen Gegenden eine Sicherheitsfiktion, im Sinne von selektiven Wahrnehmungs- und Verdrängungsprozessen. Eine solche Strategie zur Herstellung von Sicherheitsgefühlen ist aber nicht allein auf potentielle Katastrophengebiete beschränkt. Vielmehr ist eine derartige Sicherheitskonstruktion ein in allen Gesellschaften verbreitetes Phänomen (vgl. BONB 1997, 23). In den meisten Fällen ist eine solche Disposition sogar unausweichlich, um in der Gesellschaft handlungsfähig zu sein. Denn „,viele potentiellen Bedrohungen sind nur dann zu handhaben, wenn sie gerade nicht vollständig realisiert werden" (ebd., 24). Wer z.B. am Straßenverkehr teilnehmen will - ob als Autofahrer oder Fußgänger - muss zwangsläufig die Eventualität, selbst bei größter eigener Vorsicht in einen Unfall verwickelt zu werden, ausblenden.

Sicherheitsgefühle beruhen demnach nur in Ausnahmefällen auf einer tatsächlichen Beseitigung von Unsicherheiten und Gefahren. LUHMANN (1984) expliziert in diesem Zusammenhang den Begriff der Erwartungssicherheit als Substitution des gesellschaftlichen Wortgebrauchs der Sicherheit (vgl. LUHMANN 1984, 417). Erwartungssicherheit meint ,[...] nichts anderes, als dass aus einem Universum denkbarer Möglichkeiten, bestimmte Möglichkeiten als handlungsrelevant, andere hingegen als irrelevant ausge- 
blendet werden, wobei genau dieser Selektionsprozess zu Eindeutigkeit und Sicherheit führt" (BONß 1997, 24). Das Verdrängen und Ausblenden der negativen Eventualitäten aus dem Spektrum vielfältiger Möglichkeiten eines Handlungsausgangs dominiert auf diese Weise auch den durch die Erwartungssicherheit charakterisierten Alltag:

„Die alltäglichen Handlungen laufen bereits über lange Zeit ohne Widerstände ab. Da sie sich bei der Lösung von Problemen weitgehend reibungslos bewährt haben, werden sie zu einer stabilen Sicherbeit gebenden Ressource, von der wir erwarten, dass sie auch in der Gegenwart und darüber binaus funktionieren wird" (BECKER 1994, 4)

Erwartungssicherheiten sind grundlegend für jegliches sozialen Handeln. Denn Handeln ist letztlich nur dann möglich, ,[...] wenn die Welt nicht als auch immer anders möglich, also als kontingent erscheint, sondern erwartbar stabil und in diesem Sinne komplex" (BONB 1995, 90). Letztlich wird Sicherheit im Rahmen dieser Konzeption zu einer sozialen Konstruktion, die in hohem Maße subjekt- und situationsbezogen ist und individuell akzeptiert und verworfen werden kann.

\subsubsection{Moderne Sicherungsstrategien}

Die heutige Gesellschaft verfolgt in der Bedeutung einer Erwartungssicherheit vor allem die Idee einer kausalanalytisch orientierten Gefahrenbeseitigung, die ,[...] sich vor allem dadurch auszeichnet, dass Sicherheit nicht mehr als eine letztlich außerhalb der eigenen Welt liegende Angelegenheit erscheint, sondern zu einem dem eigenen Handlungskontext zurechnenden Phänomen wird“ (BONß 1997, 26). Die Entwicklung zur Risikogesellschaft ist demzufolge auch damit verbunden, Gefahren und Ungewissheiten, die bislang als unbeeinflussbar gedeutet wurden, in Risiken umzudefinieren, die vom Menschen selbst produziert oder gleichfalls von ihm beherrschbar gemacht werden können. Durch diesen Verständniswechsel erfährt Sicherheit in der heutigen Gesellschaft gleichzeitig die Idee eines, vollständigen' Schutzes, und erhebt sich damit zu einer ,[...] gesellschaftlichen Wertidee“ (KAUFMANN 1973, 10). Bestanden traditionelle Sicherungsstrategien vor allem im Glauben an übermenschliche Mächte, so wird heute das Dogma der absoluten Sicherheit vor allem durch das Vertrauen auf Technik und Fortschritt ermöglicht.

Im Glauben, die Gefahren von gestern als Risiken von heute bewältigen zu können, entsteht ein regelrechter Sicherungszwang, der darin besteht, mit Hilfe von Technik, Wissenschaft und Staat immer mehr Risiken zu institutionalisieren:

„Es ist ein Prozess der ständigen Verlagerung von Verantwortung von den Menschen auf die Apparate, der uns zwar einerseits von quälender Unsicherheit, [...] von der Qual der täglichen Sorge und Vorsorge entlastet, der uns aber gleichzeitig immer gründlicher anonymen Mächten ausliefert" (STRASSER 1986, 32). 
ne und Zurechnungsprobleme feststellen. Risikosportliche Aktivitäten bieten demnach ein Feld, in dem eine individualisierte Risikoübernahme zu erleben ist, in der die eingegangene Bedrohung als direkt abhängig von eigenen Entscheidungen und eigenen Fähigkeiten erscheint.

Risikosport - so entwickelt NEUMANN (1999) - dient in diesem Kontext als eine Art zivilisatorische Schlüsselqualifikation, als spezifische Lernsituation im Umgang mit Unsicherheit.

„Ausgestattet mit den Risiko-Erfahrungen ist man für die Risiken der Risiko-Gesellschaft besser gerüstet als obne sie. Wer eine Risiko-Sport-Karriere über- und durchlebt hat, der wird die persönlichen und beruflichen Herausforderungen der Risiko-Gesellschaft besser meistern können" (RUMMELT 2003, 211).

Risikosport ermöglicht in diesem Kontext eigene Entscheidungen, die zwar auch aber nicht ausschließlich Betroffenheit zulässt. In modernisierungs-theoretischer Perspektive lässt sich Risikosport in diesem Zusammenhang als die Aneignung von individueller Risiko-Kompetenz innerhalb einer veränderten gesellschaftlichen Risiko-Kultur verstehen, die eigentlich zu einer Gefahren-Kultur mutiert ist.

In einer zweiten Annäherung lässt sich diese Argumentation auch im Kontext gesellschaftlicher Sicherheitskonstruktion nachvollziehen. Berücksichtigt man das anthropologisch manifestierte Bedürfnis nach Unsicherheit (vgl. Kapitel 4), so kann die übermäßige Sicherheitskonstruktion einen soziopathischen Zustand ${ }^{179}$ bewirken, in dem das überdimensionale Bestreben nach Sicherheit dem natürlichen Verlangen nach Unsicherheit gegenüber steht. Risikosportarten besitzen somit wesentliche kompensierende Funktionen. „[...] In dem Maße, wie Unsicherheitsfaktoren, Risiken und Gefahren aus dem alltäglichen Leben ausgeschlossen werden, nehmen Monotonie und Routinisierung zu“ (SCHLESKE 1977, 49). Wenn in westlichen Konsumgesellschaften ,[...] Risikosportarten wie Pilze aus dem Boden schießen, ist dies ein untrüglicher Beweis dafür, dass Risiko und Sicherheit aus dem Gleichgewicht geraten sind“ (OPASCHOWSKI 2000, 36).

Risikosport als Gegenwelt einer sicheren Gesellschaft bildet somit die Grundlage der aktivationstheoretischen Erkenntnisse, die sich als Suche nach Erregung und Spannung zusammenfassen lassen (vgl. 4.3). Auch SEMMLERs (1994) verhaltenstheoretische Darstellungen des Angsterlebens, lassen sich auf eine ,übersicherte' Gesellschaft im oben beschriebenen Sinn zurückführen. Um angesichts der mächtigen Bedrohung, die von den Gefahren zweiter Ordnung ausgehen, handlungsfähig zu bleiben, wird Erwartungssicherheit zu einem universellen Glauben. Durch die sozialen Konstruktion ,Sicherheit', die dem Individuum alltäglich suggeriert wird, bekommt Angst einen geringeren Stellenwert, der stellvertretend in risikosportlichen Aktivitäten in wachsendem Maße erfahren werden kann. Zudem fehlt es bei den potentiellen, aus den heutigen Bedrohungen resultieren Ängsten, an direkten Bewältigungsmöglichkeiten, die dem Akteur in sportlichen Risikosituationen gegeben sind. Des weiteren kann der Ernstfallcharakter der 
Risikosportarten die Folgenlosigkeit des gesellschaftlichen Alltags ausgleichen, der durch die beschriebene Versicherungs- und Vorsorgepraxis ,abgesichert' erscheint (vgl. SCHLESKE 1977, 49).

Ein anderer Interpretationsansatz, der aus der Differenzierung von Risiko und Gefahr und ihrer Referenz zur Sicherheit resultiert, bezeichnet die Ausübung von risikosportlichen Aktivitäten nicht als Gegensatz eines individuellen Sicherheitsbedürfnisses, im Sinne des Kontrastes von Unsicherheit und Sicherheit, sondern als konkreten Ausdruck eines Sicherheitsbedürfnisses. In Anlehnung an CUBE (1995), der das diversive Neugierverhalten als Sicherheitstrieb bezeichnet (vgl. 4.2), beschreibt SCHALLBERGER (1995) den Zustand der Sicherheit nicht als Abwesenheit von Gefahr - was eine Art primäre Sicherheit darstellen würde - sondern als aktive und erfolgreiche Bewältigung von vorhandener Gefahr im Sinne einer sekundären Sicherheit (vgl. SCHALLBERGER 1995, 81). Risikosuche in den Risikosportarten stellt auf diese Weise keinen Gegensatz zum Sicherheitsbedürfnis dar, sondern kann als ,[...] Suche nach Erlebnissen von sekundärer Sicherheit“ (ebd.) im Sport verstanden werden. Diese ist aber nur zu gewinnen, wenn „[...] man einer gefährlichen Situationen nicht ausweicht und sie sogar wenn sie sich nicht von selber einstellt - aktiv aufsucht.“ (ebd.).

Durch die zunehmende Konstruktion von primären Sicherheitsvorkehrungen und gleichzeitiger Unterdrückung von sekundären Sicherheitsbedürfnissen, die im Rahmen des Umgangs mit gesellschaftlicher Gefahren zweiter Ordnung entstehen, gewinnt der Risikosport somit an Bedeutung. In der Differenzierung von primärer und sekundärer Sicherheit, von Risiken und Gefahren, wird darüber hinaus der entscheidende Unterschied zwischen gesellschaftlicher und risikosportlicher Unsicherheit deutlich: Das menschliche Sicherheitsbedürfnis richtet sich dieser Interpretation zufolge nicht hauptsächlich auf Sicherheit im Sinne eines statischen Fortdauerns eines einmal gegebenen Zustandes, sondern zielt auf eine dynamische Auseinandersetzung mit Gefahr im Sinne der aktiven Umwandlung von Unsicherheit in Sicherheit ab (vgl. ebd.).

Gleich wie das Aufsuchen von Risikosportaktivitäten im Kontext von gesellschaftlicher Sicherheit interpretiert wird - das gemeinsame Element der Ansätzen ist, dass Risikosportler Situationen suchen, die ,[...] nicht über die im zivilisierten Leben vorhandenen Sicherheitsnetze verfügen “ (CSIKSZENTMIHALYI 1992, 25). Auch wenn die Entwicklung des theoretischen Bezugsrahmens im gesellschaftstheoretischen Kontext auf nichtverifizierte Annahmen beruht, so lässt sich angesichts des explorativen Forschungsdesigns dieser Arbeit eine weitere These über den Zusammenhang von Risikosport und Gesellschaft formulieren: Die Gesellschaft schafft mit ihrer Entwicklungslogik vom Risiko zu Gefahren zweiter Ordnung und der hieraus resultierenden gesteigerten Konstruktion von Sicherheit die eigentliche Grundlage für das Aufsuchen von risikosportlichen Aktivitäten. Menschen, die im fähigkeitsabhängigen Risikosport ihr Unsicherheitsbedürfnis befriedigen, sind somit bestrebt, Sicherheit unter Ernstfallbedingungen selbst und aktiv herzustellen. 
Dieser Paradigmenwechsel eröffnet eine neue Sichtweise auf den Risikosport. Sowohl aus sportpädagogischer Perspektive als auch aus Sicht der Sportethik ist nun eine differenzierte Bewertung des Risikosports unter Berücksichtung der vorgestellten Zusammenhänge erforderlich. Diese erfolgt in Abschnitt 5.5 \& 5.6. Zuvor werden die Zusammenhänge von Risikosport im Bezug auf Geschlechterausprägungen (5.5) untersucht. Des Weiteren erfolgt eine Darstellung der Bedeutung des Risikosports im Lebenslauf und damit für Prozesse der Sozialisation.

\subsection{Risikosport im Lebenslauf}

Betrachtet man die Protagonisten risikosportlicher Aktivitäten, so fallen zwei gravierende soziale Merkmale der Aktiven besonders ins Auge. Zum einen handelt es sich vorwiegend um junge Menschen, die in den seltensten Fällen das 40. Lebensjahr überschritten haben und zum anderen - in der Mehrheit um Männer ${ }^{180}$. Nicht nur die Ergebnisse der empirischen Forschungen von OPASCHOWSKI (2000) sondern auch die Forschungsbeiträge der pädagogischen Psychologie (RAITHEL 2001a; 2004) bestätigen diese Beobachtungen. In der Repräsentativbefragung über die Attraktivität risikosportlicher Aktivitäten ${ }^{181}$ sticht insbesondere die Gruppe der 14-17 Jährigen deutlich hervor. Auch bei den 18-29 Jährigen sind Risikosportarten noch stark nachgefragt. In älteren Altersgruppen und insgesamt bei Frauen verlieren sportliche Risikoaktivitäten den Ergebnissen zufolge deutlich an Attraktivität (vgl. OPASCHOWSKI 2000, 139ff). Während die Geschlechtsspezifik im nächsten Kapitel behandelt wird, geht es im folgenden um die Korrelation von Risikoerleben und Lebensalter unter der besonderen Berücksichtigung der Jugendphase.

Hinweise auf die besondere Affinität von Jugendlichen und Risikosport finden sich bereits bei SIMMEL, der vom Abenteuer als einem spezifisch jugendlichem Phänomen spricht. Er argumentiert, dass das Risikoerleben im Abenteuer eine Affinität zum Jugendalter besitzt, weil in dieser Lebensphase die Dynamik, Intensität und Gespanntheit der Lebensgefühle am ausgeprägtesten sind, während das Alter ,[...] ein neues Gebilde forme: der Beschaulichkeit, der sachlichen Abwägung, der Freiheit vor der Unruhe“" (SIMMEL 1923, 23). Risikoerleben im Alter ist für SIMMEL deshalb eine „,...] nicht gemäße Lebensform“ (ebd.).

Eine aktuellere Perspektive für die Frage nach den Altersunterschieden eröffnet die Sozialisationsforschung nach HURRELMANN und die pädagogisch-psychologischen Arbeiten von RAITHEL. Hier wird die Jugendphase in ihrer identitätsbildenden Funktion in der interaktiven Auseinandersetzung mit der Umwelt dargestellt. Im Zentrum der Theorieentwicklung steht dabei die Verschränkung von persönlicher Individuation und sozialer Integration als interdisziplinärer Ansatz (vgl. HURRELMANN 1999). Der Jugendphase wird in diesem Konzept eine besondere Affinität zum Risikoerleben unterstellt, weil diese Phase durch eine differenzierte Orientierungs- 
arbeit gekennzeichnet ist. Neben der Akzeptanz des eigenen Körpers, der Abnabelung von Eltern und Vormunden, findet in der Jugendphase wohl auch die bedeutendste Phase der Identitätsentwicklung statt ${ }^{182}$ (vgl. FERCHHOFF 1999, 76ff). Jugend wird hierbei als erweiterter Begriff verstanden, der den verlängerten sozialen Entwicklungsbedingungen entspricht und Phasen der Postadoleszenz mit der Altersstufe 16-29 einschließt (vgl. ebd. 68). Es ist in diesem Zusammenhang davon auszugehen, dass Jugendliche in ihrer Entwicklung - im Rahmen ihrer notwendigen Identitätsarbeit Risikoerlebnissen nicht abgeneigt sind und diese als ,Übergangsriten' (LE BRETON 2001) oder Distinktionsprozesse (RITTNER 2001) wahrnehmen. HURRELMANN folgend wird dieser Prozess durch die aktuelle gesellschaftliche Transformation noch verstärkt.

\section{„Es fehlt an tragfähigen moralischen, ethischen, religiösen und politischen Ori- entierungen und Werten, die dem eigenen Leben Sinn geben und eine Identität stiften können. Entsprechend verunsichert sind Selbstbild und Selbstwertgefübl dieser jungen Menschen" (HURRELMANN 2000, 11).}

Risikoerleben als besonders intensive Form der Selbsterfahrung kann damit als eine Form der jugendlichen Selbstvergewisserung spezifiziert betrachtet werden. Im Risikosport und mit ihnen verwandte Aktivitäten können die Selbstständigkeit unter ,Ernstfallbedingungen' erlangt und Projektionsfolien für die Individuation bereitgestellt werden. Die besonderen Anforderungen und Erlebnismomente, die bei der Bewältigung sportlicher Herausforderungen eine Rolle spielen, haben deshalb eine besondere Anziehungskraft auf Jugendliche, weil in ihnen zudem eine bewusste Grenzerfahrung provoziert wird, die für Jugendliche in der Entwicklung von besonderer Bedeutung ist (vgl. HURRELMANN 1999).

„Jugend ist eine Zeit, die in besonders kräftiger Weise darauf angewiesen ist zu erfahren, wer sie selbst ist, die sich selbst spüren, mit sich experimentieren will, die dadurch charakterisiert ist, dass sie Grenzen suchen und Grenzen provoziert, um an ibnen zu erfahren wer sie eigentlich ist. [...] Man will, wie die Jugendlichen dies heute vielfältig formulieren, sich im scharfen Erleben als jemand spüren, den es gibt, der gestalten kann und der Zuständigkeiten hat" (THIERSCH 1996, 11) ${ }^{183}$.

In stärker soziologisch orientierten Ansätzen werden zudem die Abgrenzungspotentiale sportlicher Handlungen für das Jugendalter hervorgehoben. SCHWIER betont in diesem Zusammenhang die Bedeutung körperliche Bewegung in subkultureller Gemeinschaften für soziale Identitätsprozesse. Gerade sportive Aktivitäten können ,,[...] scheinbar schwieriger gewordenen Prozess des Erwachsenenwerdens eine unterstützende Funktion einnehmen" (SCHWIER 1998, 29). Das über den Risikosport codierte Zeichensystem (Kleidung, Sprache, Habitus) kann demnach zu einer distinktiven Stilisierung führen, die im Rahmen modischer Elemente wichtige Bedeutung für soziale Identitätsprozesse besitzt (vgl. FERCHHOFF 1999; RAITHEL 2004). Die von SCHULZE angeführte horizontale Strukturierung der „Er- 
lebnisgesellschaft" verdeutlicht zudem, dass Erlebniswelten und damit auch Vergesellschaftungsprozesse maßgeblich durch das Alter bestimmt werden. Auch hier lässt sich ein Indiz für die unterschiedliche Risikosportnachfrage in verschiedenen Lebensabschnitten finden.

KÖCK entwickelt komplementär die Annahme von allgemein kulturell unterschiedlichen Erlebnisebenen in den verschiedenen Lebensabschnitten und kommt schließlich zu einer Pluralisierung von Risikoerlebnissen als kulturelle Praxen:

\section{„Die kulturelle Linie, die den Gegensatz. und die gegenseitige Bedingtheit von Alltagswelt und Abenteuerwelt beschreibt, ziebt sich durch alle Lebensphasen des von bürgerlichen Denkweisen geprägten Menschen. Die Erlebniswelten - so unterscbiedlich sie in den einzelnen Lebensabschnitten sind - haben stets eine kulturkonservierende Funktion" (KÖCK 1990, 39).}

Zusammenfassend lässt sich eine starke Affinität der Jugendphase im Bezug auf risikosportliche Aktivitäten aufzeigen, die sich durch die Notwendigkeit identitätsbildender Prozesse ergibt. Risikoerleben dient Jugendlichen scheinbar sowohl als intensive Selbsterfahrung, die im Individuationsprozess benötigt wird, als auch zur Identitätsbestimmung durch Abgrenzung und Kompetenzerfahrung. Diese Erkenntnis ist insofern von Bedeutung, als dass die pädagogische Implikation des Risikoerlebens im Sport im Bezug auf ihre Wirkungsweisen eine besondere Reflexion bedarf und darüber hinaus auf die Wechselwirkungen von Gesellschaft und individueller Entwicklung verweist.

\subsection{Risikosport und Geschlecht}

Die Frage nach Geschlechtsspezifika im Risikosport wurde schon in der geschichtlichen Betrachtung aufgeworfen, in der ein männliches Heldenepos als historisches Charakteristikum des Alpinismus des beginnenden 20. Jahrhunderts vorgestellt wurde. Frauen schienen diesbezüglich lange Zeit formal aber auch ideologisch von alpinistischen Handlungen ausgeschlossen (vgl. KÖHLER 1995). Diese Ausgrenzung von Frauen aus risikosportlichen Aktivitäten zieht sich bis weit in das 20. Jahrhundert hinein - erst 1974 öffnet sich beispielsweise der englische Alpine Club für das weibliche Geschlecht, wobei Frauen bis heute eine Minderheit im organisierten Alpinismus darstellen (vgl. MADLENER-SIEGRIST 1996). Die Geschichte des Risikosports ist durchgängig eine männliche Geschichte, die sich auch durch ihre implizite Nähe zu militärischen Handlungsfeldern bestimmt. GROPPE (1987) hat in diesem Zusammenhang dargestellt, dass auch die Geschichte der Kolonisation eine Geschichte des männlichen Abenteurertums darstellt, dessen Mythen bis heute männliche Sehnsüchte auffangen und wieder spiegeln.

Auch die mediale Darstellung des Risikosports und mit ihm verwandter Aktivitäten werden in überwältigender Mehrheit männlich inszeniert ${ }^{184}$ (vgl. auch FRÖHLICH 1991). Man denke hier nur an die Zigarettenwerbung, in 
der erfolgreich mit dem Abenteuer-Ideal des Cowboys gearbeitet wird und Männlichkeit in seiner ursprünglichsten Form präsentiert wird.

\section{„Es gibt kein anderes Medium als die Werbung, in dem scheinbar so un- gebrochen und penetrant gleichwohl liebevoll, abenteuernde Männer gefeiert werden" (GROPPE 1987, 58).}

Betrachtet man die empirischen Befunde zur Quantität der Nachfrage sowie zur Qualität des Risikoerlebens in Risikosportarten, so finden sich ähnliche Ergebnisse: Die heutigen Risikosportarten werden wesentlich weniger von Frauen nachgefragt als von Männern und selbst jene Frauen, die am Risikosport partizipieren, erleben dieses Handlungsfeld anders als ihre männlichen Kollegen (vgl. WINKLER1989). Auch STUMM, der in seiner Untersuchung zum Trendsport ${ }^{185}$ eine zufällige Teilnehmerauswahl trifft, konstatiert eine vergleichsweise hohe Sportnachfrage von Männern insbesondere für die Sportarten Mountainbiken, Windsurfen und Klettern (vgl. STUMM 2004, 151). Auch die Untersuchungsergebnisse von HUGGER (1991), OPASCHOWSKI (2000), ROBINSON (2004) und KUSZ (2004) weisen auf unterschiedliche Quantitäten der Nachfrage sowie Qualitäten des Risikoerlebens von Frauen und Männern hin. Insgesamt lässt sich eine allgemeine Affinität zwischen Risikoerleben und Männlichkeit herstellen, die auf eine Geschlechterspezifik des Risikosports verweist (vgl. ROSE 1991) ${ }^{186}$.

In Anlehnung an PFISTER (2002) ist allerdings darauf hinzuweisen, dass die unterschiedliche Attraktivität von Sportarten für Männer und Frauen in unterschiedlichen sozialen Kontexten - allgemein ein Charakteristikum des Sports darstellt. So unterscheiden sich die Geschlechter nicht nur hinsichtlich des Umfangs und der Art der sportlichen Betätigung, sondern auch in ihrer Zeichensetzung und distinktiven Darstellung.

Die Frage nach den Ursachen dieser Geschlechterdifferenzen im Bezug auf den Risikosport eröffnet dabei erneut verschiedene Perspektive. Zunächst wird ein sozialisationstheoretischer Ansatz in Anlehnung an CHODOROW und HAGEMANN-WHITE vorgestellt, der von unterschiedlichen Individuationsprozessen von Mädchen und Jungen ausgeht. RAITHEL verknüpft diesen Ansatz mit der Theoriekonzeption aktueller ,Gender'-Forschungen und geht von einem kulturellen System der Zweigeschlechtlichkeit aus. THIERSCH hingegen entwickelt einen modernisierungstheoretischen Ansatz, der auf die beschriebenen sozialen Wandlungsprozessen aufbaut und das sportliche Risikoerleben von Männern als aktives Ausleben traditioneller Rollenverständnisse ausweist.

Die geschlechtsspezifische Sozialisationsforschung, die insbesondere von CHODOROW (1986) und HAGEMANN-WHITE (1983) beeinflusst wurde, ist eine Verbindung von psychologischen und soziologischen Theorieansätzen ${ }^{187}$. Ansatzpunkt dieses Theorieentwurf stellt die frühe familiäre Kindheitserziehung dar, die als Ausgangspunkt kindlicher Individuationsprozesse definiert wird. Ausgehend von einer tradierten weiblichen Dominanz in der Erziehung kommt es demnach zu unterschiedlichen Individuations- und Differenzierungsprozessen von Mädchen und Jungen ${ }^{188}$. Die 
Gleichheit zwischen Mutter und Tochter, die sich mit anderen weiblichen Betreuungspersonen (Kindergärtnerin; Tagesmütter etc.) durch die gesamte Kindheit hindurch fortsetzt, lässt den weiblichen Individuationsprozess demnach anders verlaufen als den der Jungen (vgl. ROSE 1997).

„Weil sie dasselbe Geschlecht wie ihre Töchter haben und selbst einmal Mädchen waren, neigen Mütter von Töchtern dazu, diese nicht in gleicher Weise als verschieden von sich selbst zu betrachten wie Mütter von Söhnen. [...] Die primäre Identifikation und die Symbiose mit Töchtern ist im allgemeinen stärker" (CHODOROW 1986, 143).

Das Kind wird von der Mutter demnach als ,Verdopplung' erlebt. Die frühe Mutter-Kind-Trennung als Separation im Individuationsprozess wird damit deutlich erschwert. Die im Rahmen dieses Prozesses notwendige Differenzsetzung erfolgt bei Mädchen wesentlich schwächer als bei Jungen. Mädchen bleiben - der Theorie folgend - wesentlich länger in einer starken Mutter-Kind-Beziehung bzw. geben diese nie gänzlich auf. Das Mädchen kann im Vergleich zum Jungen länger und „,...] unbefangener sich ins Einssein mit der Mutter fallen lassen und daraus Kraft ziehen" (HAGEMANNWHITE 1984, 95). Es entwickelt wesentlich durchlässigere „Ich-Grenzen“ und lernt sich selbst mehr in Beziehungen zu anderen zu definieren (vgl. CHODOROW 1986, 123).

Bei Jungen hingegen, die von ihrer Mutter durch das andere Geschlecht eben nicht als mit sich ähnlich wahrgenommen werden, findet sich keine so starke Mutter-Kind-Dyade. Es wird aus der mütterlichen Symbiose verstärkt herausgedrängt und auf sich selbst verwiesen. Der Junge weiß damit sehr früh, dass er anders ist als die Mutter, findet aber nur selten eine entsprechende Identifikationsfolie. Der meist räumlich und emotional abwesende Vater, der dieses Vakuum füllen könnte, bietet keine identifikatorische Stütze, da er durch die traditionelle Rollenzuweisung meist beruflich eingebunden ist. Die Rolle des Vaters, der eine ,[...] zweite Geborgenheit“" (BÖNISCH 1993, 54) geben könnte und ihn im ,[...] Gleich sein spiegeln könnte" (ebd.) fehlt dem Jungen. Da keine gleichgeschlechtlichen Objekte existieren, an denen er sich orientieren könnte, bleiben letztlich nur ,[...] männliche Medien-Idole, weit entfernt von jeglicher Realität“ (ROSE 1995, 74). Die Individuation des Jungen vollzieht sich daher nicht in Anlehnung an den Vater, sondern als negative Abgrenzung gegen die Mutter.

Nach HAGEMANN-WHITE basiert die Geschlechtsidentität des Jungen letztlich sogar auf einer doppelten Negation:

„Frau ist, wer kein Mann sein kann. Eine Frau ist Nicht-Mann. Dem Jungen aber wird seine Männlichkeit zunächst durch Abgrenzung von der Mutter vermittelt; und diese ibm am nächsten stehende Erwachsene ist das, was er nicht sein darf, um ein Mann zu werden. So wird sein Geschlecht als NichtNicht-Mann bestimmt. [...] Der Junge hat ₹.B. praktisch keine Gelegenheit, hervorragende Beispiele von männlichem Mut zu erleben. Ergreift er nun die Stereotype ,Frauen sind ängstlich', so wird ihm Ängstlichkeit qum Beweis, dass jemand kein Mann. Um sich und anderen zu beweisen, wie männlich er 
ist, wird er sich nun als ,nicht-ängstlich' vorstellen." (HAGEMANNWHITE 1983, 92).

Der Junge entwickelt demnach demonstrativ sich-abgrenzende Tugenden der Härte, Unverletzlichkeit, Selbstständigkeit und Leistungsfähigkeit und stellt sie in Mutproben unter Beweis. Die Herstellung von Männlichkeit wird damit zu einem aktiven Prozess, der sich durch Abgrenzung zum typisch weiblichen definiert. Die erfolgreiche Männlichkeit ist ,[...] im Kern Abwehrstruktur“ (ebd., 94).

Die Heraustrennung des Mädchens aus der Mutter-Kind-Symbiose geschieht allmählicher und ohne Betonung einer Differenzsetzung - die Individuation des Mädchens bleibt damit zwar unvollständig. Die Identitätsbildung verläuft beim Mädchen also stärker durch Spiegelung der Ähnlichkeit als durch Abgrenzung im Anderssein und vollzieht sich damit weniger entlang abenteuerlicher Handlungsaktivitäten. Für Mädchen bleibt ,[...] die Errichtung von Ich-konturierenden Grenzen, von Ich-Kompetenzen“ (ROSE 1995, 75) daher ein vernachlässigtes Thema.

In der unterschiedlichen Bedeutung, die die Differenzsetzung und damit das Erproben von Ich-Grenzen in der weiblichen und männlichen Biographie zukommt, wird die geschlechtsspezifische Anziehungskraft des Risikoerlebens deutlich. Diesen Ansatz übertragend, bietet das Risikoerleben auch und gerade im legitimierten Feld des Sports - für das männliche Geschlecht eine Möglichkeit zur Auseinandersetzung mit seinen drängenden Individuationsthemen und damit eine Chance zur männlichen Identitätsbildung.

„Begreifen wir das Abenteuer als eine kulturelle Praxis der Grenzerfahrung,erprobung und-überschreitung, so erklärt sich nun, warum das Mädchen sich bierzu relativ distanziert verhält, während der Junge magisch angezogen wird. [...] Die Sicherheit der vertrauten Welt zu verlassen und sich in Fremde, Gefabr und Abenteuer zu begeben, sind Anforderungen, die um die Herstellung von Unabbängigkeit, Abgrenzung und Omnipotenz kereisen - um Themen also, die im männlichen Individuationsprozess in besonderer Weise virulent sind" (ebd.).

Diese Form der Risikobewältigung als Abwehr regressiver Wünsche findet sich auch in den Ausführungen BALINT's wieder (vgl. Abschnitt 4.3.3). Die vom Autor angeführten ,primitiven Haltungen“ (BALINT 1994, 36), die in den Persönlichkeitsprofilen des Philobaten und Oknophilen repräsentiert werden, weisen diesbezüglich eindeutige Hinweise auf Geschlechtsspezifika auf ${ }^{189}$. Während der, Philobat' als Abenteuersuchender immer wieder versucht, das Trauma der Individuation zu überwinden und damit eher dem männlichen Individuationsmuster entspricht, flüchtet sich der, Oknophile' in die Nähe sicherheitsvermittelnder Personen - letztlich der schutzbietenden Mutter. Dieses Bild entspricht überwiegend dem weiblichen Sozialisationsmuster ${ }^{190}$.

RAITHEL (2004) greift den in der Geschlechterforschung richtungsweisenden Begriff des, doing gender' in Anlehnung an WEST \& 
ZIMMERMANN (1987) auf. In Ergänzung zu den beschriebenen Individuationsansätzen der Sozialisationsforschung geht der Autor von einem kulturellen System der Zweigeschlechtlichkeit aus. Die Unterscheidung der Geschlechter in Mann und Frau bildet demnach ein grundlegendes Phänomen moderner Gesellschaften. Das Geschlecht ist „,[...] Merkmal der sozialen Realität" (RAITHEL 2004, 106) und strukturiert damit wesentliche Teile der Persönlichkeitsentwicklung. Hiermit eng verbunden ist ein symbolisches System der Zweigeschlechtlichkeit, das gesellschaftliche Strukturen, individuelle Handlungsspielräume und Verhaltensweisen bestimmt.

„Das dichotome Symbolsystem der Zweigeschlechtlichkeit ist ein durchgängiges Ordnungsprinzip unserer Kultur, das alle individuellen und gesellschaftlichen Bereiche durchdringt und auf die Annahme der Existenz von nur zwei Geschlechtern als Natursache zurückgeht" (ebd.).

In dieser dichotomen Struktur sind ,Männlichkeit' und ,Weiblichkeit' Symbole eines polar konstituierten Sinnsystems, welches sich über Verhaltensweisen und Ausdrucksformen manifestiert. Härte, Mut, Leistung und Sieg sind in dieser polarisierenden Struktur zentrale Kategorien für die Geschlechtsidentitätsreproduktion des männlichen Geschlechts (vgl. ebd.). Risikosport als sozial legitimierte Form von Risikoverhalten bieten sich diesbezüglich gerade zu an, männliche Integrität und Invulnerabilität zu demonstrieren. Sportliches Risikoverhalten und die Darstellung ,[...] einer körperlich gebundenen männlichen Überlegenheit und Stärke dient hierbei idealtypisch der männlichen Geschlechtsidentitätsreproduktion“" (ebd., 107). Aufgrund seiner Manifestation differenziert der Autor schließlich unterschiedliche Kategorien des geschlechtsspezifischen Risikoverhaltens. Während das interiosierende Risikoverhalten eher eine feminin Konnotation besitzt und Medikamentenkonsum oder restriktives Essverhalten umfasst, beschreibt das maskuline exteriosierende Risikoverhalten z.B. riskantes Verkehrsverhalten oder auch Risikosport (vgl. ebd., 31). Wenngleich diese prototypische Darstellung einer innen- und außengerichteten Perspektive des Risikoverhaltens empirisch unbestätigt bleibt, verweist der Ansatz doch auf geschlechtlich unterschiedliche Risikoverhaltensweisen.

Abenteuer und Risikoerleben dient aus Sicht der Sozialisations- und Individuationsforschung zusammenfassend als männlicher Kristallisationspunkt für Individuation und Identität. Über das Risikoerleben vollzieht sich die männliche Begrenzung zur weiblichen Rolle, die der Mann in der Person der Mutter erfährt und gegen die er sich in seiner männlichen Identität abgrenzen will und muss. Für Mädchen und Frauen stellt sich das Risikoerleben als unrelevant dar, da durch die Symbiose zur Mutter eine nur unvollständige Individuation stattfindet und sich die weibliche Identitätsbildung am Spiegelbild der Mutter vollzieht. Es bleibt in diesem Zusammenhang fraglich, inwieweit die Individualisierung der Frauenrolle, wie sie von BECK-GERNSHEIM (1983) thematisiert wird, zu einer mittelfristigen Veränderungen der geschlechtlichen Individuation führen wird. In den aktuellen Studien von WHEATON (2004) und ROBINSON (2004) wird die Vermu- 
tung neuer Geschlechtsidentitäten im Sport nicht bestätigt, wenngleich sich Hinweise auf eine Pluralisierung von männlichen und weiblichen Identitätsmodellen finden.

Die modernisierungstheoretischen Ideen von THIERSCH über die männliche Affinität zum Risikoerleben, die sich in ähnlicher Form auch bei KÖCK (1990) und GROPPE (1987) finden, setzen an diesem Punkt an und versuchen die Perspektive des sozialen Wandelns zu integrieren. Im Bezug zu den vorgestellten sozialisationstheoretischen Ansätzen stehen diese Ansätze vielmehr in einem komplementären Verhältnis und sind dementsprechend als Reflexionsanstoß zu verstehen.

Von den einleitend beschriebenen Individualisierungs- und Pluralisierungstendenzen in der aktuellen Gesellschaft ausgehend, entwickelt THIERSCH (1995; 1996) die Perspektive des Risikoerlebens als männliche Rückzugsposition. Wenn Individualisierung und Pluralisierung zu einer Freisetzung aus tradierten Normen und Lebensbezügen führt und damit die Einbindung in traditionelle soziale Strukturen wie z.B. die Familie aufweicht, dann kann dies - so THIERSCH - auch für die traditionelle gesellschaftliche Rollenmuster von Männern und Frauen gelten. Als Folge der gesellschaftlichen Veränderungen, die sich auch in einer allgemeinen Emanzipation und Gleichberechtigung wieder spiegelt, öffnen sich auch traditionelle geschlechtliche Handlungsmuster. Risikosport bietet sich demnach als Handlung an

\section{„,...] in der sich traditionelle Männlichkeit gegenüber dem Zeittrend, sich ihm sperrend und aus ibm zurückziehend, behaupten könnte" (THIERSCH 1995, 45).}

Einer Erosion von traditionellen Rollenmutstern wird die im Risikosport vermeintlich inszenierte Härte, Angstlosigkeit und Risikobereitschaft entgegengesetzt, die damit männliche Identität unterstützt.

GROPPE (1987) und AUFMUTH (1988) argumentieren ähnlich, wenngleich sie von einem genetisch überlieferten männlichen Freiheitsdrang ausgehen, der den Mann auch heute noch dazu bringt, sich in seiner ,NomadenIdentität' zu beweisen:

„Die unserer Seßhaftigkeit ist verschwindend kurz. gegenüber den riesigen Zeiträumen, die wir frei und unstet streifend gelebt haben. Und unsere unmittelbaren Vorfahren in der Abstimmungsreibe waren bekanntlich auch eine sehr unrubige Gesellschaft. [...] Wir haben den Nomaden in uns durch unsere Kultur der tausendfältigen Daseinsregulierung angekettet, vielleicht auch geschwächt, ausgelöscht haben wir ibn nicht. Er lebt weiter in unseren Sebnsüchten nach Freibeit, Weite und Regellosigkeit, die manchmal in uns mächtig werden bis zur Schmerz̧haftigkeit" (AUFMUTH 1988, 46).

Einen völlig anderen Ansatz, der von HOBERMAN (2005) diskutiert wird, sieht dagegen hormonelle Ursachen für ein risikosportliches Engagement. Mit seinen thesenhaften Ausführungen über die Bedeutung des Testosterons für den leistungssportlichen Erfolg, verweist der Autor auf die 
Möglichkeit einer biologischen Determination risikosuchenden Verhaltens aufgrund der unterschiedlichen - genetisch bedingten - hormonellen Ausstattung von Männern und Frauen. Wenngleich differenzierte Untersuchungen hierzu nicht vorliegen, so konstatiert auch die Entwicklungsbiologie $\mathrm{Zu}$ sammenhänge zwischen Hormonkonzentration und Verhaltensdispositionen. Die Frage nach der Geschlechtssystematik des Risikosports wird damit - zumindest hypothesenartig - um eine biopsychosoziale Dimension erweitert (vgl. z.B. KLEINHEMPEL 1996), die in einer engen Verbindung zur anthropologischen Theoriebildung steht (vgl. Kapitel 4.2). Da sowohl die empirischen Grundlagen als auch die wissenschaftliche Theoriebildung in diesem Kontext wenig fortgeschritten ist, kann über die Bedeutung dieses Ansätze nur spekuliert werden. Es ist wird die Aufgaben weiterer Forschungsarbeiten sein, diese Hypothesen durch angemessene Forschungsarbeiten zu bearbeiten ${ }^{191}$.

Zusammenfassend kann für die Frage nach den Geschlechtsspezifika des Risikosports aus verschiedenen Wissenschaftsperspektiven eine gewisse Affinität zwischen Risikoerleben und Männlichkeit konstatiert werden. Diese begründet sich - wie hier deduziert - nicht nur aus einer Historie des Risikosports, sondern einerseits aus dem Individuationsbegehren des kindlichen Jungen in Abgrenzung zur Weiblichkeit und andererseits als Rückzugsposition für das Erleben traditioneller Männlichkeit im Sinne tradierter Rollenmuster. Die Möglichkeit genetisch disponierter Verhaltensdispositionen konnte darüber hinaus angedeutet werden. Die Zusammenhänge zwischen Risikosport und Geschlecht sind insbesondere für sportpädagogische $\mathrm{Zu}$ sammenhänge von Bedeutung, weshalb sich ein sportpädagogisches Fazit anschließt, in dem die Ambivalenzen des Risikos im Sport zusammengefasst werden.

\subsection{Das Risiko im Sport - ein sportpädagogisches Fazit}

Folgt man den Beschreibungen der gesellschaftlichen Veränderungen, dann kann im Wissen um die Defizite und die durch Risikosport ermöglichte Kompensationen gleichfalls eine pädagogische Dimension des sportlichen Risikoverhaltens expliziert werden. Diese wurde mit den Ausführungen in Kapitel 3 schon im Rahmen der historischen Zusammenhänge aufgegriffen und wird hier einer abschließende Diskussion unterzogen ${ }^{192}$. Das Risiko im Sport lässt dabei eine ganze Vielzahl von sportpädagogischen Betrachtungen zu, weist in seiner pädagogischen Implikation aber auch zahlreiche Ambivalenzen auf ${ }^{193}$.

Wird das Aufsuchen und Betreiben von risikosportlichen Aktivitäten als Kompensation eines spannungsarmen und domestizierten Alltags verstanden, so kann unter pädagogischen Aspekten mit dem Angebot von Risikosportarten sozial legitimierte und dosierte Spannung erzeugt werden. Die spannungsreichen und erlebnisdominierten Anreize (s. Kap. 4) ermöglichen darüber hinaus auch eine ganzheitliche Selbsterfahrung, die - wie dargestellt 
wurde - identitätsstiftende Erfahrungen bietet. Ein pädagogisches Angebot von Risikosportarten kann vor allem Heranwachsenden dazu dienen, sich ein ,[...] angemessenes Selbstbild aufzubauen und zukünftig selbstbewusst(er) zu handeln" (NEUMANN 1999, 131).

ANDRECS (1987) betrachtet insbesondere die in den Sportarten zugrunde liegenden Grenzerfahrungen als pädagogisch zweckmäßig. Demnach kann die Erfahrung von Grenzen und das Eingehen von Risiken zur Erforschung eigener Kapazitäten dienen und damit die Ausbildung eines Selbstkonzeptes fördern (vgl. ANDRECS 1987, 129). Ferner vermag eine risikoreiche Natursportart durch die Auseinandersetzung mit Naturgewalten auch im Sinne einer Umwelterziehung eingesetzt werden. Mit der Ausübung von Natursportarten können Erfahrungen verbundenen sein, die dem Sportler Einsichten in natürliche Kreislaufprozesse ermöglichen (NEUMANN 1999, 141). Allerdings schränkt WILKEN (1994) die pädagogischen Möglichkeiten von risikoreichen Natursportarten insofern ein, als dass der alleinige Naturkontakt nicht automatisch zu einer Verantwortung gegenüber der Natur erzieht. Hierzu bedarf es weiterer theoretischer Maßnahmen wie Wissensvermittlung oder dem Einblick in menschliches Nutzungsdenken (vgl. WILKEN 1995, 41). THIERSCH $(1996,13)$ weist zudem auf die Erlebnisspirale risikosportlicher Aktivitäten, die zu einer besonderen Form des Umweltverbrauches und der Inszenierung der Natur als Kulisse führen kann.

Entscheidend für die pädagogischen Betrachtung des fähigkeitsabhängigen Risikosport ist an dieser Stelle die Differenzierung und Abgrenzung von kommerziellen Angeboten der so genannten Thrill-Aktivitäten, wie sie die moderne Freizeitindustrie bereitstellt. Zwar verschaffen auch Aktivitäten wie das Bungee-Springen oder hoch technisierte Vergnügungsparks eine Art von Erregung oder ,Nervenkitzel', die als Kompensation eines monotonen Alltags verstanden werden können, doch repräsentieren diese Tätigkeiten kein Bedürfnis nach Kontrolle oder eigener Entscheidung. Im Mittelpunkt des Interesses steht dabei ,[...]das Streben nach dem ultimativen Kick [...]“ (ERDMANN 1997, 82). Die Fähigkeit zur Risikobewältigung und die Verantwortung für die eigene Handlung wird bei diesen Aktivitäten, analog zur Erwartungssicherheit der Gesellschaft, an dritte abgegeben. HAHN (1957) bezeichnet solche Aktivitäten sogar als pädagogisch kontraindiziert:

„Denn Sensationen dieser Art sind ,unverdient, trügerisch und flüchtig': Sie verhelfen zwar zu einem Durchleben von Spannung und Gefabr, zu einem Auskosten der ,Erregung des Gelingens', verlangen aber keine persönlichen Entscheidungen und keine Verantwortung und leisten dadurch der Passivität und einer Verkümmerung der Eigeninitiative Vorschub" (HAHN 1957, 84).

In Anlehnung an die Ausführungen in Kapitel 4 können risikosportliche Aktivitäten aber auch Förderungsaspekte einer sinnlichen und emotionalen Wahrnehmung beinhalten. Die in der Gesellschaft oft auferlegte emotionale Kontrolle kann dadurch durchbrochen und Emotionen in tolerierbarer Weise erlebt werden, ohne befürchten zu müssen an Selbstwertgefühl zu verlie- 
ren. ,Angsthaben' kann in diesem Zusammenhang sogar zu einem emotional positiv besetzten Erlebnis werden, dass mitunter auch Zustimmung von anderen Aktiven hervorruft.

Als einer der wichtigsten pädagogischen Aspekte im Kontext von Risikosuche und Sicherheitsbedürfnis kann aber die sogenannte ,Sicherheitserziehung ${ }^{夭}$ verstanden werden. Der sportpädagogische Grundgedanke verschiedener Auslegungen beinhaltet demzufolge für den Akteur im Umgang mit Unsicherheit die Möglichkeit sein eigenes Sicherheitsbedürfnis kennen zulernen und gefährliche Situationen im Sinn eines sekundären Sicherheitsbedürfnisses zu erfahren (vgl. HECKER 1989; TREBELS 1988; ERDMANN 1990; BAUMANN 1995; GLAS 1995). Damit ist die Ausbildung einer Fähigkeit gemeint, die in der Risikosituation induzierte Unsicherheit nicht als Verunsicherung zu begreifen, sondern als Wahl- und Gestaltungsmöglichkeit zu verstehen. Die Konfrontation mit individuellen Unsicherheiten, den die Gesellschaft mit ihrer Sicherheitskonstruktion behindert, kann in risikosportlichen Aktivitäten somit dosiert erlebt werden: „Denn nur derjenige kann den Umgang mit Unsicherheit und Risiko lernen, der die Möglichkeit hat, Risiken einzugehen“ (NEUMANN 1999, 138). Das Risiko im Sport erhält dadurch nicht nur eine gewisse pädagogische Legitimierung, sondern besitzt darüber hinaus auch wichtige soziale Funktionen. Dabei sind die spezifischen Handlungsintentionen über den individuellen Bezug hinaus auch gesellschaftlich relevant. Es kann damit für fast alle Motivationsprozesse des fähigkeitsabhängigen Risikosports ein gesellschaftlicher und pädagogischer Sinnbezug hergestellt werden.

APTER (1994) fordert in diesem Zusammenhang ein grundsätzliches gesellschaftliches Umdenken im Bezug auf risikoreiche Aktivitäten. Er postuliert, dass Menschen neben dem ,Raum zum Atmen' auch einen vermehrten ,Raum für Risiken' benötigen, in dem die Menschen ihre erforderlichen Risikoerfahrungen machen können: „Wir sollten solche Gefahren zulassen, in denen nur das abenteuerlustige Individuum selbst Risiken eingehen kann" (APTER 1994, 242). Gleichzeitig unterscheidet er zwischen entwicklungsfördernden ,Nervenkitzel-Strategien' und inhaltsleeren ,SackgassenStrategien'. Er schlägt ein theoretisches Modell vor, indem solche Erlebnisse nur dann genussvoll sind, solange man sich im Zustand tätigkeitszentrierter Motivation befindet. In zweckzentrierten Zuständen hingegen sollten Personen Risikoerlebnisse meiden (vgl. ebd., 232). Die tätigkeitszentrierten Motivationen sind dabei eng an CSIKSZENTMIHALYI (1992) und dessen ,Flow-Konzept' angelehnt.

Ähnlich argumentiert auch SEMMLER (1994), der das Sprichwort, Wer sich in Gefahr begibt, kommt darin um' in das Gegenteil umdefiniert: Indem Risikosucher sicherheits-signalisierende Gewohnheiten verlassen, um die eigenen Grenzen auszuloten und Unbekanntes neugierig zu erschließen, eröffnen sich für sie immer wieder neue Entwicklungschancen (vgl. SEMMLER 1994, 179f). Er unterstellt Risikosuchern damit eine gewisse ,Aufbruchsstimmung', im Sinne einer Freude an der eigenen Entwicklung (vgl. ebd., 139). Von Risikosuchern zu lernen heißt demnach 


\begin{abstract}
„[...] die Gewohnheiten, die unsere Entwicklungen blockieren zu durchbrechen und sich dadurch Freiräume zu schaffen. Sich auf Neues einzulassen, die ursprüngliche Neugier - das Interesse an dem was hinter den Dingen stecktwiederzubeleben und weiterzuentwickeln [...]"(ebd., 183).
\end{abstract}

Dabei geht es weniger um das Aufsuchen von Risikosportarten, als um die Bereitschaft, auch im Alltag hin und wieder Unsicherheiten und Risiken zu suchen ${ }^{194}$. Was Risikoverhalten in der Risikosituation kennzeichnet, sollte demnach auch für den Alltag gelten, nämlich

\title{
„[...] an den Anforderungen zu wachsen und eine Entwicklung zu vollaiehen, die die Person als Ganzes mit all ibren Eigenschaften und Fähigkeiten mit einbeziebt" (ebd., 184).
}

Auch wenn eine solche Transferleistung umstritten ist, kann zumindest allgemein für die gesellschaftliche Funktion des Risikosports mit dem Soziologen BECKENBACH (1994) geschlussfolgert werden: „Die moderne Gesellschaft ohne individuelle Wagnisbereitschaft und Explorationsdrang wäre wie ein Fluss ohne Strömung" (BECKENBACH 1994, 162).

Obwohl die Literaturlage zur pädagogischer Implikation des Risikosports durchaus umfangreich ist, finden sich nur wenige kritische Beiträge, die sich mit den Ambivalenzen des Risikos als pädagogisches Handlungsfeld auseinandersetzen. Dies ist insofern verwunderlich, als dass gerade risikosportliches Handeln besonders deutlich von pädagogischen Ambivalenzen gekennzeichnet ist ${ }^{195}$. Zum einen sind risikosportliche Aktivitäten generell nur unter der Prämisse der Freiwilligkeit pädagogisch wertvoll ${ }^{196}$. Die Entscheidung für das Eingehen einer derartigen Situation ist von unterschiedlichen Variablen - wie der situativen Befindlichkeit - abhängig und erfordert einen individuellen Reflexionsprozess. Dies ist angesichts unterschiedlichster pädagogischer Handlungsfelder nur bedingt möglich und bedeutet letztlich ein Zuschneiden der Situation auf ganz individuelle Bedürfnisse. Sozialer Druck oder die Integration in Pflichtangebote (Schulsport, Sozialarbeit) verfälschen damit die Ausgangssituation und letztlich die pädagogische Implikation (vgl. THIERSCH 1995, 49f).

Grundsätzliche Kritik wird auch im Bezug auf die kompensatorischen Leistungen des Risikosports geübt. Erlebnispädagogik setzt auf pragmatisches Ereignis und Handeln als Kompensation zu einer zunehmenden Rationalisierung der Gesellschaft. Die Handlungssituation erfordert damit eine gewisse körperliche Leistungsfähigkeit und Konzentration als kompensatorische Erfahrung. Im kulturkritischen Kontext wird dies unter antimodernen Vorbehalten gegen die Aufgaben einer Verständigungsgesellschaft ausgelegt, die Instrumentalisierungsoptionen beinhalten (vgl. THIERSCH 1996, 12). In Folge dieser Kritik werden zunehmend Reflexionselementen in die pädagogische Praxis integriert, die das Risikoerleben als diskursives Element behandeln und den Selbsterfahrungsprozess kommunikativ begleiten.

NEUMANN (2003) weist zudem auf die pädagogischen Risiken hin, die im Gelingen bzw. Misslingen risikosportlicher Handlungen liegen. So ist sowohl der Erfolg als auch der Misserfolg bei derartigen Handlungen - wie 
oben beschrieben - mit zahlreichen pädagogisch wünschenswerten Folgen verbunden. Gleichzeitig weisen aber sowohl Erfolgs- als auch die Misserfolgssituationen zahlreiche pädagogisch negative Aspekte auf. Kritisch zu betrachten ist hierbei z.B. das Risikoerlebnis unter gemeinschaftlichem Druck, die endliche Steigerungsspirale risikosportlicher Situationen oder die sich aus der Situation ergebende Selbstdarstellung. AUFMUTH (1989) hat im Rahmen seiner Identitätsüberlegungen ebenfalls darauf hingewiesen, dass die gewaltigen Anstrengungen und Entbehrungen im Risikosport auch durchaus identitätszerstörend wirken können.

Desgleichen wenig diskutiert werden in den pädagogischen Beiträgen bis dato die Folgen eines Scheiterns in der Risikosituation, die mit massiven Enttäuschungen, Angstgefühle und Entmutigung einhergehen können. Die sich hieraus ableitende Forderung von OELKERS (1992) lehrreiche aber negative Erlebnisse aus der erlebnispädagogischen Praxis auszuschließen, findet sich auch bei HECKMAIR \& MICHL (2002) wieder (vgl. ebd., 65f). Inwieweit die Anwendung dieser Forderungen sinnvoll und möglich erscheinen, wird dabei angesichts der Bedeutung des Misslingens für die Gelingensspannung und damit die gesamte pädagogische Implikation bezweifelt (vgl. auch NEUMANN 2003).

Bezieht man die historischen und sozialpsychologischen Annahmen zur Geschlechtsspezifik des Risikoerlebens in die pädagogische Diskussion ein, so werden weitere Ambivalenzen risikosportlicher Aktivitäten im Rahmen pädagogischer Handlungsfelder deutlich. Wenn der Risikosport mit einer gewissen Affinität zu Männlichkeitsidealen beschrieben werden kann, stellt sich eine Nutzung im Rahmen erlebnispädagogischer Maßnahmen als überaus schwierig dar. PFISTER weist diesbezüglich auf die Folgen eines pädagogisch instrumentalisierten Risikosports hin:

\section{„Gerade die risikoreichen und aggressiv inszenierten Sportformen tragen er- beblich zur Re-Produktion von Geschlechterdifferenzen bei und sind als In- szenierung und Demonstration von Männlichkeit zu interpretieren" (PFISTER 2002, 206).}

Pädagogische Risikosituationen beinhalten die Gefahr einer Vernachlässigung weiblicher Bedürfnisse und Herangehensweisen an Risikosituationen sowie die Verstärkung von Männlichkeitsattributen. Die Frage nach geschlechtsspezifischen Unterschieden und hieraus erwachsenden sozialen Dispositionen erfordert eine differenzierte Betrachtung und Behandlung von Männern und Frauen in risikopädagogischen Maßnahmen.

Ähnliche Kritik lässt sich auch aus den biografischen Zusammenhängen zwischen Risikoerleben und Lebensalter ableiten. Wenn das aktive Risikoerleben vor allem in der jugendlichen Lebensphase wichtige sozialisatorische Funktionen erfüllt, stellt sich die Frage des pädagogischen Wertes risikosportlicher Aktivitäten allgemein für alle andere Lebensphasen sowie die Erweiterung der Jugendphase auf die Adoleszenz (vgl. THIERSCH 1995). Die unterschiedliche Bedeutung und Verarbeitung von Risikoerlebnissen im 
Lebensverlauf erfordert auch hier eine differenzierte Herangehensweise, die in der Literatur kaum aufgegriffen wird (vgl. ebd.).

Bezüglich der pädagogischen Aspekte von risikosuchendem Verhalten stellt sich nunmehr die Frage nach einer zusammenhängenden Risikobewertung im Sport. Da diesbezüglich schon eine Vielzahl von Perspektiven dargestellt wurde, schließt sich im folgenden eine zusammenfassende ethische Betrachtung des risikosuchenden Verhaltens im Risikosportart an.

\subsection{Ethische Betrachtung des risikosuchenden Verhal- tens im Sport}

Vor dem Hintergrund der vielfältigen Aspekte von individueller Motivation, gesellschaftlichen Zusammenhängen und pädagogischen Möglichkeiten des Risikosports, kann nunmehr der Versuch einer Antwort auf die eingangs gestellte Frage nach dem Sinn und der Notwendigkeit des Risikosports formuliert werden. Die traditionelle Beantwortung, wonach ein gewisses Risiko eingegangen werden darf, ein anderes aber vermieden werden sollte, kann an dieser Stelle in keiner Weise mehr befriedigen oder muss zumindest konkretisiert werden. Der Annahme, dass ein sportliches Risiko keinerlei stichhaltige Notwendigkeit besitzt, kann angesichts der vielfältigen Anreize des Risikosports und deren gesellschaftlichen Sinnbezügen endgültig widersprochen werden. Essentiell für eine ethische Betrachtung ist die eingangs angeführte Differenzierung von Risiko, Gefahr und Sicherheit im Verständnis LUHMANN's. Nur unter Berücksichtigung der Subjektivität der Risikosituation (vgl. Kap. 2.1) und der Differenzierung zwischen Risikohandlung und Risikosystem (vgl. Kap. 5.2) kann eine ethische Betrachtung des Risikosport entwickelt werden.

Wird Risikoverhalten als Kompensation eines spannungsarmen und ,übersicherten' Alltags verstanden, so gewinnt der Sport allgemein, insbesondere aber der Risikosport eine notwendige, dieses Spannungs- und Erlebnisdefizit ausgleichende Funktion. Im Gegensatz zum Risiko als System (vgl. BONB 1995) ermöglicht die Risikohandlung eine individuelle Auseinandersetzung mit Unsicherheit. Damit entfällt zugleich die herkömmliche negative Bewertungsgrundlage, gemäß der ein Risiko im Sport grundsätzlich als ein unnötiges Wagnis betrachtet und konsequenter Weise disqualifiziert wird (vgl. ELSÄSSER 1979, 79). Im Gegenteil: Das Risiko als Handlung - im Unterschied zum Risiko als System - kann als ein Refugium individuell bewältigbarer Unsicherheit verstanden werden.

Die psychologischen Anreize des Risikosports und speziell der Anreiz der Risikokontrolle verdeutlichen, dass es bei dem ,realen' sportlichen Risiko, dass der Einzelne eingeht, niemals um absolute Größen geht. Vielmehr handelt es sich dabei um variable Beziehungs- oder Verhältnisgrößen zwischen Individuum und Situationsanforderungen. Deshalb kann deren jeweiliger Umfang auch nur von Fall zu Fall immer wieder neu ermittelt werden und zwar aus ,[...] der Relation aller objektiven, subjektiven und situativen 
Faktoren eines Tuns“ (ebd.). Letztlich geht jeder Sportler sein eigenes individuelles Risiko ein, dass sich mit dem Risiko eines anderen Sportlers oder mit der gleichen sportlichen Handlung unter anderen Bedingungen nicht vergleichen lässt. Über die sittliche ,Erlaubtheit' oder ,Unerlaubtheit' einer sportlichen Risikoaktivität lässt sich mit den herkömmlichen Maßstäben der Gesellschaft folglich keine Aussagen treffen (vgl. BIRNBACHER 1996, 197). Im Gegenteil, die gesellschaftliche Konstruktion von Sicherheit steht dem Aufsuchen von sportlichen Risikosituationen sogar kontradiktorisch gegenüber. Denn das gesellschaftliche Streben nach einer vermeintlichen Gefahrenreduktion bei analoger Sicherheitsmaximierung lässt sich mit dem freiwilligen Aufsuchen von entsprechenden sportlichen Risikosituationen nicht vereinbaren. Die zum Teil negative Bewertung des Risikosports resultiert folglich nicht aus dessen sinnfreien und unnötigen Charakter, sondern aus dem allgemeinen gesellschaftlichen Streben nach Gefahrenreduktion bei gleichzeitiger Unkenntnis über die Differenzierung zwischen Risikohandlung und Risikosystem. Dass es sich beim sportlichen Risikoverhalten letztlich um eine direkte Folge dieses Umgangs mit Sicherheiten und Gefahren handeln könnte, wird dabei scheinbar nicht wahrgenommen (vgl. ebd.).

Aus den beschriebenen Zusammenhängen zwischen den gesellschaftlichen Defiziten und den pädagogisch-sozialen Möglichkeiten sportlichen Risikoverhaltens darf allerdings keinesfalls die voreilige und unkritische Schlussfolgerung gezogen werden, dass Situationen mit besonders hohem Risiko gleichfalls außerordentlich wünschenswert sind - ganz nach dem Motto: Je höher das Risiko, um so wertvoller die entsprechende Entwicklung für das Individuum. Auch wenn heute ,,[...] die Toleranz gegenüber freiwilligen Risiken wächst" (ebd., 200) und Risikoverhalten zum sozial positiven Attribut modifiziert wird, so muss NIETZSCHEs Parole: „Lebe gefährlich - if you are tired of life, then risk it" (NIETZSCHE 1955, 166) doch weitestgehend differenziert werden. So wird das vermeintliche Risikoerleben im Rahmen vielfältiger kommerzieller Thrill-Aktivitäten zu einem flüchtigen ,Kick-Erleben'.

Aber auch das Aufsuchen von fähigkeitsabhängigen Risiken bleibt mit einer gewissen Verantwortung verbunden. Die fahrlässige oder leichtsinnige Inkaufnahme von Risiken ist im Sport desgleichen nicht als positiv zu bewerten. Eine einseitige Befriedigung des spannungsarmen und übersicherten Alltags durch eine ebenso einseitige Risikotendenz kann daher die bisher gewonnenen Erkenntnisse über das sportliche Risiko nur diskreditieren.

Welcher ,richtige' Maßstab an Verantwortung dem Risikoverhalten zugrunde liegt, kann hiermit zwar nicht beantwortet werden. Allerdings kann jedes Risiko als sittlich gerechtfertigt werden,
„,[...] das der betroffene Sportler unter Berücksichtigung aller Umstände für seine Person und in seiner Situation für verantwortbar, d.h. vor seinem Gewis- sen für angemessen und vertretbar bält - auch wenn ein anderer dies von vornherein anders beurteilt oder der Betreffende selbst im nachbinein feststellen sollte, dass er sich in der Beurteilung der Sachlage geirrt hat" (ELS ÄSSER 1979, 82). 
Unter diesen Vorraussetzungen kann an der positiven Bewertung des sportlichen Risikoverhaltens festgehalten werden und damit die ausgleichende Funktion des Risikosports im Sinne der vielfältigen Erlebnis- und Selbsterfahrungsmöglichkeiten gefestigt werden. „Nicht das Risiko an sich ist schlecht, denn es können nur Personen unangemessen handeln, die es für sich und/oder andere falsch eingeschätzt haben“" (BAUMANN 1995, 8).

\subsection{Zusammenfassung und Einordnung}

Dieses Kapitel zeigt, dass die Bedeutung des Risikosports in enger Beziehung zu den gesellschaftlichen Strukturen der Gegenwart steht. Angesichts erweiterter modernisierungs-theoretischer Ansätze - wie beispielsweise dem Individualisierungstheorem oder dem Wandel der Existenzform - kann das mit den Risikosportarten assoziierte Erlebnis als eine Form der modernen Sinnsuche verstanden und zur individuellen Identitätsvergewisserung herangezogen werden. Parallel werden risikosportliche Aktivitäten wie das KanuWildwasserfahren oder das Freeclimbing weiterhin als Kompensation einer defizitären Gesellschaft erklärt, die durch Langeweile und Bewegungsarmut gekennzeichnet ist. Risikosportarten reflektieren hierbei das Bedürfnis nach authentischer Aufregung und Spannung, die über einen sekundär vermittle Erlebniskonsum auch den Einsatz der ganzen Person erfordert. Im Rahmen theoretischer Annahmen über die funktional-differenzierte Gesellschaft lässt sich Risikosport zudem als eine Form der individuellen Selbstermächtigung und Subjektaufwertung verstehen.

Gleichzeitig gewinnt der Risikosport im Kontext einer vermeintlich ,sicheren Gefahrengesellschaft' die Funktion einer individualisierten Risikoübernahme, die als Wunsch gedeutet wird, aus der zunehmenden gesellschaftlichen Sicherheitskonstruktion auszubrechen und Sicherheit im Verständnis eines sekundären selbst hergestellten Prozesses zu erfahren. Dieser Argumentation folgend, dient Risikosport als spezifische Form der Risikoerfahrung, die durch den klaren Entscheidungsbezug und eine deutliche Zurechenbarkeit der gesellschaftlichen Unsicherheiten in Form Gefahren zweiter Ordnung entgegensteht.

Auf der Grundlage sozialisationstheoretischer Ansätze konnte zudem die Besonderheit identitätsbildender Prozesse für jugendliche Lebensläufe hervorgehoben werden. Darüber hinaus wurde auf der Basis neuerer Theorien der Gender-Forschung eine Affinität zwischen Risikosport und Männlichkeit postuliert.

Aus den Annahmen einer gesellschaftlichen Risiko-Kultur, die eigentlich eine Gefahren-Kultur bedeutet, wurden zudem ergänzende pädagogische Dimensionen eines risikosportlichen Angebots abgeleitet. Als Resultat der verschiedenen sportpädagogischen Ansätze (APTER 1994; SEMMLER 1994; NEUMANN 1999) ergibt sich ein zentrales Anliegen, das vor allem darauf abzielt, den individuellen Umgang mit Risiken im und durch Risikosport zu erlernen. Die Ausführungen wurden mit einer ethischen Betrach- 
tung zum Risikosport abgeschlossen, die die Individualität des Risikos hervorhebt und als positives Erlebnisfeld akzentuiert.

Zusammengefasst: Als Handlungs- und Erfahrungsfeld einer sich ,reflexiv erneuernden Moderne' bieten risikosportliche Aktivitäten einen kompensierenden Kontrapunkt unter - so ist nun zu konstatieren - sehr vielfältigen strukturellen Auflagen: Gegen eine durchrationalisiert-effektive und unüberschaubar organisierte Welt als Erfahrung von Authentizität, als Erfahrung von Gemeinschaft, Zugehörigkeit und Identität gegenüber den Strapazen von Individualisierung, als Erfahrung von Entschiedenheit gegenüber vielfältigen Notwendigkeiten des Aushandelns und der Auseinandersetzung, als Erfahrung von Unsicherheit, die selbstbestimmt und durch eigenes Handeln zu lösen ist, als Erfahrung von Männlichkeit in einem kulturellen System der Zweigeschlechtlichkeit und schließlich als Erfüllung der in Individualisierung angelegten Möglichkeit des individuellen, eigenen und authentischen Erleben um des Erlebens willen. Zugegeben - diese Zusammenfassung ist verkürzend und bisweilen polemisierend und dennoch: Risikosport als gesellschaftliches und individuelles Handlungsfeld hat nun - zumindest auf phänomenologischer Ebene - an Schärfe gewonnen. Der im Rahmen des interdisziplinären Ansatzes gewählte Forschungszugang konnte Erklärungszusammenhänge liefern, die - aufeinander bezogen - ein durchaus prägnantes und schlüssiges Bild von den Funktionsprinzipien, den gültigen Strukturen und den Wirkungsweisen des Risikosports darstellen.

Aber selbst wenn Risikosport als kulturelles Handlungsfeld in seinen individuellen Sinn- und Motivstrukturen evaluiert werden konnte, bleiben doch - insbesondere auf der realen Handlungsebene - vielfältige Fragen offen: Wie ist Handeln im Spannungsfeld von Risikosuche und Sicherheitsbedürfnis überhaupt realisierbar? Wie transformiert sich Unsicherheit als Gefahr in subjektzentrierte Risiken und wie ist Handeln im Risikosport strukturiert. Im nächsten Kapitel wird mit dem Blick auf die konkrete Handlungssituation nach Antworten aus dem Bereich der regulativen Verhaltensforschung und kognitiven Handlungstheorie gesucht. Die Fokussierung auf das interagierende Subjekt des Risikosport stellt dabei keine Wiederholung motivationspsychologischer Ansätze dar, sondern repräsentiert den Versuch Handeln im Risikosport eingehend zu untersuchen 


\section{6 Über den Umgang mit Risiko - Risikosport und Handlungstheorie}

Take time to deliberate, but when the time for action arrives, stop thinking and go in. (Andrew Jackson (1767-1845), Politiker)

Ausgehend von der vorstehenden interdisziplinären Annäherung an das Phänomen Risikosport, wird in diesem Kapitel der Fokus auf die konkrete Handlung im Risikosport gelenkt und die sie konstituierenden Entscheidungs- und Beurteilungsvorgänge evaluiert. Im Zentrum der folgenden Ausführungen steht die Frage nach den regulierenden und strukturierenden Bedingungen sowie beeinflussenden Variablen der Gefahrenkognition, Risikobeurteilung und dem daraus folgenden Verhalten und Handeln in sportlichen Risikosituationen. Dies scheint angesichts der Besonderheit der Handlungssituation im Risikosport von hervorgehobenen Interesse, denn die sportliche Handlung in Risikosituationen ist durch ein spezifisches Risikoverhalten gekennzeichnet, in der

„[...] Zielerreichen-wollen und Beeinträchtigung-verhindern-wollen deshalb untrennbar miteinander gebunden sind, weil in dieser Situation das Handlungsziel nur unter Gefahr erreicht werden kann, während Ausscheidung der Gefahr auch das Zielerreichen unmöglich machen würde" (KLEBELSBERG 1969, 60).

Begreift man den Menschen - wie es u.a. die anthropologischen Annahmen nahe legen - als ein grundsätzlich bipolar strukturiertes Wesen, dass einerseits die Anreize von sportlichen Risikosituationen sucht, andererseits aber auch fundamental sicherheitsbedürftig ist, dann muss auch für das 
sportliche Risikoverhalten angenommen werden, dass es sich gleichfalls in einem Spannungsfeld von Risikosuche und Sicherheitsbedürfnis befindet.

Ausgangspunkt der folgenden Darstellungen sind handlungstheoretische Grundlagen, die davon ausgehen, dass sich Individuen ihre Handlungssituationen im Sport gemäß ihrer Zielsetzungen selbst konstruieren (vgl. NITSCH 1991, 27). In Anlehnung an HOLLAND (1973) kann in diesem Zusammenhang angenommen werden, dass Individuen Situationen so arrangieren, dass sie in ihnen ihre Fähigkeiten anwenden, ihre Wertvorstellungen verwirklichen und ihre Motive befriedigen können, ohne dabei körperliche Schädigungen in Kauf nehmen zu müssen (vgl. auch SZCZESNYFRIEDMANN 1982, 90). Die zentrale Frage dieses Kapitels lautet daher, inwieweit sich dieses Risikoverhalten zwischen Motivbefriedigung einerseits und Sicherheitstendenzen andererseits im Spannungsfeld von Risikosuche und Sicherheitsbedürfnis verwirklichen lässt, welche Entscheidungs-, Regulations- und Beurteilungsvorgänge stattfinden und welche Variablen diese Vorgänge beeinflussen. Gegenüber den klar theoretisch strukturierten Kapiteln 2-5, wird im Folgenden ein jeweils anwendungsorientierte Praxistransfer intendiert. Da grundlegende Ansätze zur Handlungsregulation im Risikosport fehlen, werden allgemeine Annahmen unterschiedlichster Forschungsrichtungen nach dem bekannten Muster deduziert und in einen konkreten Handlungskontext eingeordnet. An einigen Stellen werden - zum besseren Verständnis - beispielhafte Anwendungen der theoretischen Zusammenhänge an ausgewählten Risikosportarten vollzogen.

Die Frage nach den psycho-regulativen Prozessen, die ein derartiges Verhalten lenken, rücken damit in das forschungsleitende Interesse dieses Kapitels. Zunächst werden im Rückgriff auf handlungstheoretische Ansätze, grundlegende Zusammenhänge für den Risikosport deduziert (Abschnitt 6.1). Anschließend werden anhand empirischer Befunde der Risiko- und Sicherheitsforschung die das Risikoverbalten beeinflussenden Variablen auf situativer und personaler Ebene expliziert und auf risikosportliche Handlungssituationen bezogen (6.2). Das Kapitel schließt mit einem Versuch das Handeln im Risikosport modelltheoretisch darzustellen. Hierzu wird ein transaktionales Handlungsmodell des Risikosports (6.3) entworfen und in seinen internen Komponenten erläutert.

\subsection{Risikoverhalten und Handlungsregulation}

Wird Risikoverhalten als dynamische Auseinandersetzung zwischen subjektiven Bewältigungsmöglichkeiten und objektiven Bedingungen verstanden, so muss gleichfalls analysiert werden, wie sich diese Auseinandersetzung psychisch strukturiert und welche emotionalen und kognitiven Prozesse beim Risikoverhalten stattfinden.

Risikoverhalten im Sinne eines Gefahrenrisikos ist auf die gleichzeitige und möglichst weitgehende Befriedigung der ambivalenten Zielstruktur, einer Leistungstendenz auf der einen (Risikosuche) und einer Sicherheitstendenz (Sicherheitsbedürfnis) auf der anderen Seite ausgerichtet. Vor diesem Hin- 
tergrund stellt die Entscheidung in Risikosituationen immer einen Kompromiss zwischen Motivbefriedigung einerseits und Sicherheitsbedürfnis andererseits dar. Das Verhalten in Risikosituationen ist folglich als ein Prozess des Abwägens von objektiven Bedingungen und subjektiven Fähigkeiten unter Berücksichtigung emotionaler Einflüsse zu verstehen. Das bestimmende Charakteristikum liegt somit nicht in der Entscheidung des Risikoverhaltens selbst,

„,...] sondern in der Phase des vorausgehenden Entscheidungsprozesses, dessen Ziel nicht eine Entscheidung überhaupt, sondern eine Entscheidung unter möglichst gleichmäßiger Berücksichtigung beider Bedürfnisse ist" (KLEBELSBERG 1969, 61).

Im folgenden wird der Prozess und die Struktur dieses Wahrnehmungsund Entscheidungsverhaltens dargestellt. Zunächst erfolgt eine Analyse der Handlungsanforderungen beim Kanu-Wildwasserfahren (6.1.1) - als ausgewähltes Beispiel einer Risikosportart. Der darauf folgende Abschnitt Handlungsregulation und Phasenstruktur (6.1.2) ermöglicht schließlich einen Einblick in die zeitliche Struktur des Risikoverhaltens, um anschließend den eigentlichen kognitiven, physiologischen und emotionalen Wabrnebmungs- und Bewertungsprozess (6.1.3) des Risikoverhaltens zu explizieren. Abschließend wird anhand des ,Adventure Experience Paradigm" (6.1.4) und der individuellen Regulierungsmöglichkeiten des Risikoverhaltens (6.1.5) das Handeln in Risikosituationen verdeutlicht.

Die angeführten Beispiele der Sportart Kanu-Wildwasserfahren erfolgen hierbei unter der Zielsetzung einer konkreten Präzisierung. Dabei sind einige Beispiele sicherlich nicht auf alle Risikosituationen einer Sportart und schon gar nicht auf alle Risikosportarten gleichermaßen zu übertragen. Die theoretischen Ausführungen sind aber zunächst allgemeiner Natur und damit prinzipiell auf andere Situationen und Handlungszusammenhänge zu übertragen.

\subsubsection{Individuelle Schwierigkeitsanforderungen am Beispiel des Ka- nu-Wildwasserfahrens}

Wie in Kapitel 2 dargestellt wurde, ist das Kanu-Wildwasserfahren in besonderer Weise dadurch geprägt, mit individuellen Fähigkeiten die Anforderungen zu bewältigen, denen sich der Sportler unter bestimmten Bedingungen stellt (vgl. BAUER 1991, 5). Die Besonderheit der situativen Faktoren, wie Wasserwucht, Wellen, Walzen, Fließgeschwindigkeit usw. besteht darin, dass im Wildwasser praktisch nie unter konstanten Verhältnissen gehandelt werden kann. Die ständig wechselnden Bedingungen verlangen vom Kanusportler daher einen fortwährenden Wahrnehmungsprozess, der auf eine dynamische Auseinandersetzung mit den situativen Bedingungen abzielt. Die eigentliche Schwierigkeitsanforderungen einer Situation sind somit einerseits abhängig ,,...] von den situativen Bedingungen, die der Fluss vorgibt, andererseits von den jeweils individuellen Zielsetzungen und Erlebnis- 
schwerpunkten [...]“" (BAUER \& SCHULTE 1997, 40). Das Anforderungsprofil im Wildwasser kann einerseits bei verschiedenen Flüssen durch einen unterschiedlichen Flussverlauf oder Fließgeschwindigkeiten verschieden sein, anderseits auch bei einem einzelnen Fluss je nach Wasserstand erheblich variieren. Die Abbildung 6.1 stellt eine Übersicht dieser Anforderungskriterien beim Kanu-Wildwasserfahren dar.

\begin{tabular}{|l|l|l|}
\hline $\begin{array}{l}\text { Subjektive Fähigkeiten } \\
\text { (personale Bedingungen) }\end{array}$ & $\begin{array}{l}\text { Objektive Anforderungen } \\
\text { (situative Bedingungen) }\end{array}$ \\
motorische Leistungsfaktoren: & Wasserführung: \\
Beherrschen von Schlag und & Wasserstand \\
Haltetechniken, Schlagkombinationen \\
und Kombinationsschlägen \\
Bewegungskoordination:
\end{tabular}

Abbildung 6.1: Bestimmung von subjektiven Schwierigkeitsanforderungen anhand subjektiver Fähigkeiten und objektiver Faktoren im Kanu-Wildwasser (in Anlehnung an BAUR et al. 1977, 377).

Im Hinblick auf die individuelle Handlungsregulation wird ein Wildwasserkanute die objektiven Bedingungen so wählen und ,konstruieren', dass er mit seinen subjektiven Bewältigungsmöglichkeiten seine Motive in der Wildwassersituation befriedigen kann, ohne körperliche Schädigungen direkt in Kauf nehmen zu müssen. Dafür stehen in der Regel unterschiedliche Flussabschnitte zur Verfügung, die in ihrem Schwierigkeitsprofil vom Deutschen Kanu-Verband (DKV) mit leicht bis unfahrbar beschrieben werden. Dies erscheint insofern wichtig, da diese dynamische Auseinandersetzung einen ,natürlichen' kognitiven Prozess der Selbsteinschätzung verlangt. Nur wenn der Sportler über seine individuellen Fähigkeiten und Motive reflektiert und diese in Beziehung zu den objektiven Bedingungen bringt, ist ein adäquates und individuelles Schwierigkeitsprofil realisierbar. Risikoverhalten beim Kanu-Wildwasserfahren setzt durch die situativ wechselnden Bedingungen somit auch ein hohes $\mathrm{Maß}$ an persönlicher Antizipation voraus (vgl. SCHWIERSCH 1991a, 43). 
Im Sinne der vorangestellten Begriffsdefinition lassen sich bei den situativen Bedingungen zwischen prinzipiell wahrnehmbaren und damit kognitivemotionalen verarbeitbaren Risiken und außerhalb der Wahrnehmung existierenden Gefahren differenzieren. Eine Gerölllawine oder ein Steinschlag in einer Schlucht sind beim Kanu-Fahren aber auch in allen anderen Risikosportarten im Allgemeinen nicht wahrnehmbar und damit als Gefahren einzustufen. Risiken lassen sich demgegenüber durch eigenes Verhalten beeinflussen, hierzu gehören alle situativen Variablen, die in einem direkten Fähigkeitsbezug zur Handlung stehen und die Handlungssituation direkt beeinflussen.

Auch wenn die vorgenommene Beschreibung der Schwierigkeitsanforderungen am Beispiel des Kanu-Wildwasserfahrens keinen direkten Transfer $\mathrm{zu}$ anderen Risikosportarten zulässt, so kann eine derartige Analyse auch auf andere Bewegungsformen im Umfeld des Risikosports transferiert werden. Risikosportarten zeichnen sich letztlich dadurch aus, dass die in ihnen zu findenden Herausforderungen, den Einsatz der ganzen Person erfordern und die Person auf unterschiedliche situative Anforderungen eingestellt sein muss (vgl. Kapitel 4).

\subsubsection{Handlungsregulation und Phasenstruktur}

Die Handlungssituation einer Risikosportart auf den eigentlichen Zeitraum des motorischen Handlungsvollzugs zu reduzieren, wäre im Sinne handlungstheoretischer Grundlagen irreführend und würde zu trügerischen Konsequenzen im Hinblick auf das sportliche Risikoverhalten führen. Sowohl im Vorfeld des Handlungsvollzugs als auch nach der Handlungsrealisation finden die Handlung regulierende Phasen statt. Handlungsregulation meint dabei ergebnisbezogen die Herstellung einer entsprechenden Organisation und prozessbezogen die Herstellung eines geordneten Ablaufs (vgl. auch BEIER 2001). HACKER differenziert diesbezüglich zwischen motivationalen Aspekten der Regulation - der sog. Antriebsregulation - und einem operationalen Aspekten - der sog. Ausführungsregulation (vgl. HACKER 1998, 63f). Die in Kapitel 4 explizierten Motivationsansätze zum Risikosport sind somit für die weiteren Ausführungen zur Handlungsregulation zu berücksichtigen, da sie die Organisation der Handlung bestimmen. Die Grundmodelle der Handlungsregulation lassen sich darüber hinaus in strukturellfunktionaler Perspektive nach Regulationsebenen und in zeitlich-funktionaler Perspektive nach Handlungsphasen differenzieren.

Aus strukturell-funktionaler Perspektive führt NITSCH $(1986,224 f)$ drei Ebenen an, die er in Regulationssystemen zusammenfasst: Das ,automatische Regulationssystem' umfasst demnach reflektorische und homöostatische Mechanismen wie instinktives Verhalten - bestimmte Reize führen demnach zu genetisch angelegten Reaktionen. Emotionen im Sinne von aus Motiven und Befriedigungserfahrungen resultierende Erlebnisse stehen im Mittelpunkt des ,emotionalen Regulationssystems'. Hierbei erhalten bestimmte Verhaltensmuster erfahrungsbedingte Bedeutungszuschreibungen, 
die damit handlungsorganisierend auf der Regulationsebene wirken. Die Zuordnung von handlungsbedingten Reizen und Reaktionen erfolgt erfahrungsabhängig und ist je nach emotionaler Bewertung sehr flexibel. Die kognitive Verarbeitung von Reizen und deren Erfahrungszusammenhang bestimmen schließlich das ,kognitive Regulationsmodell'. Diese Regulationsebene umfasst vor allem kognitive Situationsanalysen und Planungsprozesse, die zum Teil durch Modellbildungen oder erfahrungsabhängige Codierungsprozesse vollzogen werden. Verhaltensmuster werden hierbei nicht im Rahmen eines Reiz-Reaktions-Schemas abgerufen, sondern zielbewusst geplant und konstruiert.

HACKER (1998, 238ff) differenziert in diesem Zusammenhang zwischen einer, intellektuellen Regulationsebene', die Heuristiken und zielgesteuerte Strategien umfasst sowie einer, perzeptiv-begriffliche, wissensbasierten Regulationsebene, die als Verarbeitungssystem bewusstseinsfähige Handlungsschemata beinhaltet. Als dritte Regulationsebene führt der Autor die ,antomatisierte Regulationsebene' an, die in Anlehnung an NITSCH nicht bewusstseinsfähige Stereotypen und Bewegungsentwürfe bezeichnet.

Aus zeitlich-funktionaler Perspektive stellt sich die Handlungsregulation als ein Prozess dar, der sich in einzelne Phase mit inhaltlichen Merkmalen differenzieren lässt. NITSCH (1986) unterscheidet diesbezüglich drei Handlungsphasen (Antizipationsphase, Realisationsphase, Interpretationsphase), die sich in ähnlicher Form auch bei THOMAS (1995) wieder finden. In PÖHLMANNs (1986, 21) Modell der phasenstrukturellen Klassifikation unterteilt sich die Gesamtsituation sportlicher Handlungen in vier Phasen, die sich in Anlehnung an SZCZESNY-FRIEDMANN (1982, 94) in eine Vorbereitungsphase, eine Übergangsphase, eine Aktionsphase und eine PostAktionsphase (Reflektion) gliedern. Im folgenden werden diese Phasen in ihrem zeitlichen Verlauf, ihren zugeschrieben Regulationsprozessen und ihrer inhaltlichen Gestaltung am Beispiel des Kanu-Wildwasserfahrens beschrieben:

Vorbereitungsphase: In der Vorbereitungsphase, die analog zu PÖHLMANNs (1986) Phase der Handlungsantizipation zu verstehen ist, wird die Aktionsphase zunächst in allen ihren Einzelheiten gedanklich vorweggenommen. In intentionaler Hinsicht bedeutet dies die Vorwegnahme der Handlungsergebnisse und ihrer Folgen sowie den Wertgehalt der Handlungsergebnisse. Auf instrumenteller Ebene wird der Handlungsablauf vorweggenommen und in Form eines grob strukturierten Plans antizipiert. NITSCH (1986, 230f) differenziert auf der Regulationsebene zwischen Kalkulationsprozessen, die die Bewertung der Ausgangssituation sowie vorliegender Handlungsalternativen umfassen und Planungsprozessen. Dabei richtet sich die Aufmerksamkeit vor allem auf die möglichen Schwierigkeitsanforderungen der konkreten Handlungssituation. Durch aktive Informationsbeschaffung werden beim Kanu-Wildwasserfahren z.B. gezielt Gebiete und Flüsse gewählt, die zur individuellen Motivbefriedigung führen sollen. Absprachen mit anderen Paddlern dienen in dieser Phase dazu, eine Gruppe von Individuen mit möglichst ähnlichen Motivationsstrukturen zu bilden. 
Gleichzeitig werden zu diesem Zeitpunkt Mittel und Wege gesucht, mit deren Hilfe man den antizipierten Schwierigkeiten in der Realität begegnen könnte. Dazu gehört das Beschaffen notwendiger Ausrüstungsgegenstände oder Hintergrundinformationen (vgl. hierzu SCHWIERSCH 1991a, 39). Insgesamt ist diese Phase der Handlungsantizipation durch ein Wunschdenken im Hinblick auf die Zielerreichung geprägt.

Übergangsphase: Zwischen der Aktionsphase und ihrer Vorbereitung liegt eine zeitlich gesehen zwar unbedeutende, für den Sportler jedoch sehr wesentliche Phase, in der die Entscheidungen zum Eintritt in die Aktionsphase getroffen und vollzogen werden. Sie entspricht der Antriebsphase bei THOMAS $(1995,54 f)$. Hierbei kommt es erneut zu Kalkulations- und Planungsprozessen, die im Rahmen der individuellen Informationsbeschaffung aber mit einem feineren Filtersystem arbeitet. Zentrales Merkmal dieser Phase ist hierbei eine Situationsbewertung aus Handlungskompetenz und Handlungsvalenz - im Sinne der individuellen Motivationsstruktur. Dabei nimmt der Sportler eine Abgleichung der objektiven Bedingungen und subjektiven Bewältigungsmöglichkeiten vor. Es wird versucht Gefahren im Sinne ,unfreiwilliger Risiken' wie z.B. Gewitter oder minderwertige Materialien zu antizipieren und zu vermeiden, während die in der Vorbereitungsphase angestellten Überlegungen zu den fähigkeitsabhängigen Bedingungen für das Eingehen freiwilliger Risiken wie Wasserstand, Gefälle oder Wasserwucht überprüft werden.

Die Übergangsphase ist durch einen starken Annährungs- und Vermeidungskonflikt und einen Kampf gegen die Vermeidungstendenzen gekennzeichnet (vgl. HEITZLHOFER 1979a, 123f). Das Spannungsfeld von Risikosuche und Sicherheitsbedürfnis ist zu diesem Zeitpunkt am ausgeprägtesten, denn einerseits wird die lustbringenden Motivationsbefriedigungen antizipiert, auf der anderen Seite existiert ein Angstgefühl, die Situation nicht bewältigen zu können. Die endgültige Entscheidung für die eigentliche Risikoaktivität wird nun aufgrund der Einschätzung der jeweils gegebenen Umstände und der eigenen subjektiven Parameter erneut getroffen.

Aktionsphase: In der Aktionsphase kommt es schließlich zur eigentlichen Handlungsrealisation und Vollzugsteuerung (vgl. PÖHLMANN 1986, 21). Besondere Kennzeichnung der Aktionsphase ist die empfundene ,Gefährlichkeit', die es zu kontrollieren (,Kontrollbedürfnis') und zu erleben (,Angstlust'/Wahrnehmung außergewöhnlicher Körperzustände) gilt. Die Erregungs- und Spannungszustände werden dabei durch die meist beeindruckende Naturkulisse verstärkt (vgl. auch SZCZESNY-FRIEDMANN 1982, 94).

Im Gegensatz zu anderen Sportarten ist mit Eintritt in die Aktionsphase die Situation beim Kanu-Wildwasserfahren wie auch bei anderen Risikosportarten aber keinesfalls festgelegt, sondern muss durch die ständig wechselnden situativen Bedingungen immer wieder neu bewertet werden. „Da jede Befahrung als Summation der Bewältigung einzelner Stellen spezifischer Schwierigkeiten verstanden werden kann, ist die Flussstelle die kleinste analytische Einheit" (MÜCKE 1983, 17). NITSCH (1986) differenziert in die- 
sem Zusammenhang auf der Regulationsebene zwischen der Basisregulation im Sinne der optimalen Funktionstüchtigkeit und -bereitschaft des psychophysischen Systems und der Prozessregulation, die als zielbezogene Steuerung der psychomotorischen Funktionsabläufe zu verstehen ist. Insofern ist auch die Aktionsphase durch einen ständigen Wechsel von Orientierung, Bewertung und Entscheidung geprägt, wobei sich das individuelle Risikoverhalten immer an der bipolaren Zielerreichung orientiert: Einerseits an der Befriedigung der autonom gesetzten Motive und andererseits an der Vermeidung einer möglichen individuellen Gefährdung (vgl. SZCZESNYFRIEDMANN 1982, 98).

Post-Aktionsphase (Reflektion): Dem Abschluss der Aktionsphase schließt sich eine zeitlich nicht begrenzte Phase der Handlungsinterpretation an, in der das Erlebte in Gedanken, Gesprächen und unter Umständen auch in Träumen verarbeitet wird. Diese Post-Aktionsphase entspricht der Interpretationsphase bei NITSCH (1986) und bezeichnet Regulationsprozesse nach Beendigung der Handlung. Bei Kontrollprozessen der Regulation kommt es zu Soll-Ist-Vergleichen bezüglich der Art und des Grads der Zielerreichung. Als Evaluationsprozesse werden Regulationsprozesse bezeichnet, die eine Bewertung der Abweichungen und ihrer Ursachen beinhaltet. Hierbei kann es oft zu intensiven Gefühlen von Erleichterung, Verunsicherung oder Triumphempfindungen kommen, die den Wunsch nach Wiederholung bewirken oder den Abbruch der Handlung nach sich ziehen.

Obwohl die Strukturierung einzelnen Handlungsphasen für die Regulation von entscheidender Bedeutung ist, muss darauf hingewiesen werden, dass die einzelnen Phasen nur selten bewusst wahrgenommen werden. Es ist davon auszugehen, dass in risikosportlichen Handlungen alle vier Handlungsphasen repräsentiert werden, auch wenn ein Teile oder die gesamte Regulation unbewusst abläuft. Situationsspezifische Schemata sind zwar eher für automatisierte Handlungen bekannt, lassen sich aber auf risikosportliche Handlungssituationen übertragen (vgl. ebd.).

Es ist zudem darauf hinzuweisen, dass die Abfolge der einzelnen Handlungen nicht zwangsläufig - wie hier dargestellt - linear-sequentiell ablaufen, sondern eine zyklisch-iterative Form der Handlungsphasen und ihrer Regulation vorliegen kann (vgl. NITSCH 1986, 231). Handlungsphasen können dementsprechend verschmelzen oder Teile vorweg genommen werden. $\mathrm{Zu}$ Abweichungen von der linear-sequentiellen Handlungsstruktur kommt es auch bei überlappenden Handlungen oder Handlungen, bei denen gleichzeitig mehrere Ziele verfolgt werden (vgl. auch BEIER 2001, 29).

Mit Bezug auf FUHRER (1984) ist für risikosportliche Handlungen zudem die Existenz von Mehrfachhandlungen anzunehmen, d.h. es werden mehrer Ziele gleichzeitig verfolgt und entsprechende Handlungen parallel abgewickelt. Derartige Handlungen gehorchen gleichzeitig mehreren SollIst-Wert-Vergleiche und lassen eine Überschneidung von Regulationsprozessen auf unterschiedlichen Ebenen zu. 


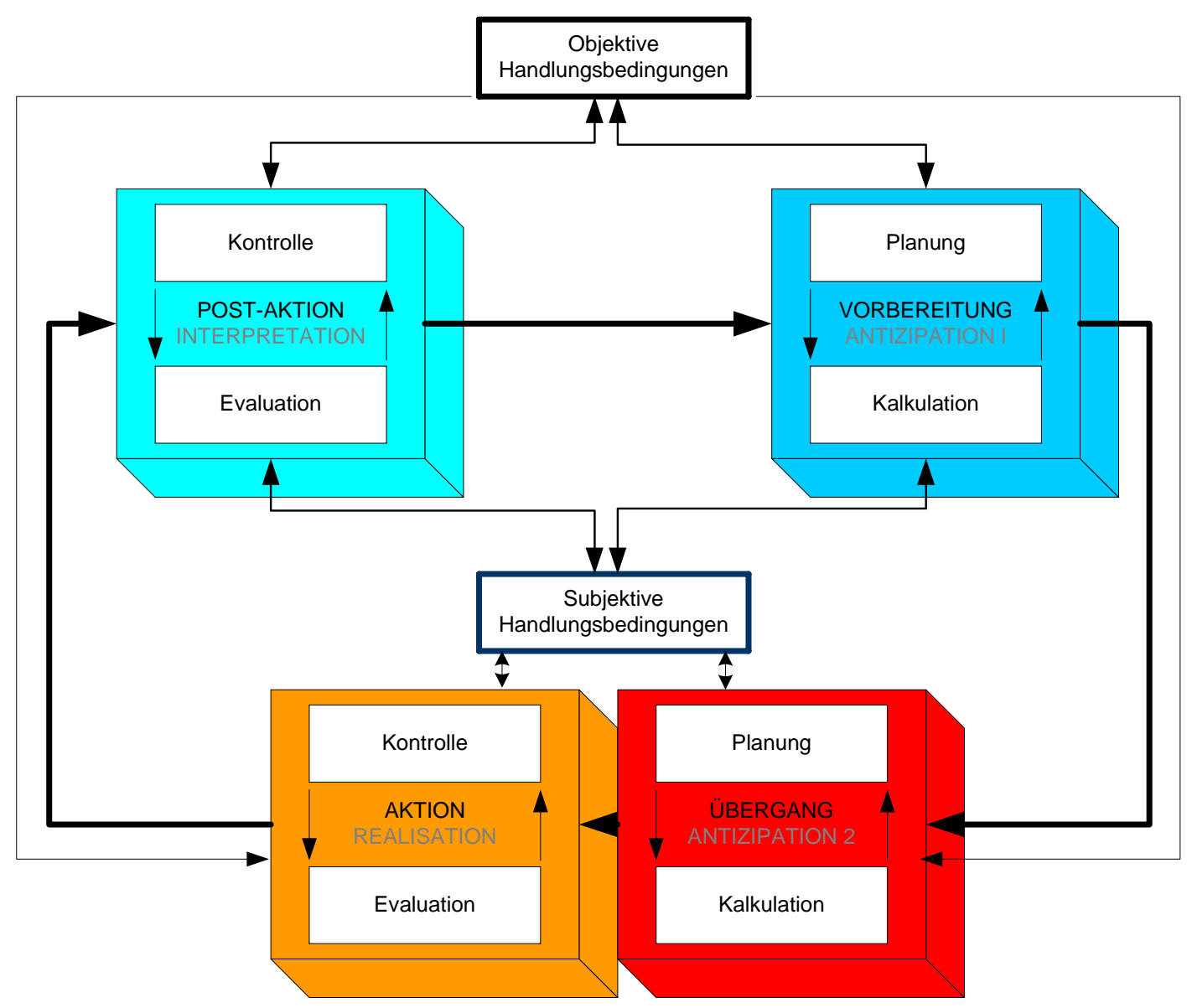

Abbildung 6.2: Phasenstruktur des Handlungsprozesses im Risikosport (erweitert nach NITSCH $(1986,230)$.

\subsubsection{Kognition und Risikoverhalten}

Um die Handlungsrealisation und das Handlungsziel im Risikosport zu verwirklichen, ist es für den Protagonisten unter der Berücksichtigung des Spannungsfeldes zwischen Risikosuche und Sicherheitsbedürfnis unvermeidlich, das Risiko der Situation kognitiv zu erfassen und in Abhängigkeit seiner personalen Faktoren zu bewerten (vgl. BÄNI 1991a, 3). Wahrnehmen, Verhalten, Handeln und Erleben einer Person sind dabei stets untrennbar miteinander verbunden und nur in ihrer Gesamtheit zu verstehen. „Sie sind gewissermaßen Elemente eines zusammenhängenden psychischen Systems, regulieren und beeinflussen sich wechselseitig" (EBERSPÄCHER 1988, 12). Hinzu kommt beim Risikosport, dass sich die komplexen Wahrnehmungs- und Handlungsprozesse in der Handlungssituation vielfach überschneiden, wie BAUR am Beispiel des Kanu-Wildwasserfahrens verdeutlicht:

„schon während er [der Paddler] den entworfenen Handlungsplan für einen bestimmten Streckenabschnitt (₹.B. für die Befahrung einer Blockstrecke) realisiert, muss er die Gewässerbedingungen der Folgestrecke (auf der ₹:B. zwei Walzen zu befahren sind) identifizieren, um daraufbin einen neuen Hand- 


\section{lungsplan für den nächsten Streckenabschnitt zu entwickeln" (BAUR 1982, 65).}

Die folgende Darstellung der kognitiven Prozesse wird daher allein aus Gründen der Übersicht analytisch vorgenommen. Da eine Untersuchung der Wahrnehmungs- und Bewertungsprozesse im Risikosport noch aussteht, stützen sich die folgenden Ausführungen vor allem auf Erkenntnisse der Verkehrs- und Entscheidungspsychologie, die introspektiv für den Risikosport erweitert werden.

Der eigentlichen Risikoanalyse im Wildwasser geht ein Prozess der Gefahrenkognition ${ }^{197}$ voraus, der darauf ausgerichtet ist, mögliche Gefahren zu identifizieren und in Abgleich eines kognitiven Gefahrenmodells zu bewerten. Im Rahmen der Arbeitspsychologie handelt es sich bei der Gefahrenkognition um eine Prozess der Aufnahme und Verarbeitung von Gefahrenindikatoren, die - übertragen auf den Risikosport - gleichfalls als Erfassen adäquater Situationsanreize aufgefasst werden können. Im Spannungsfeld von Risikosuche und Sicherheitsbedürfnis ist diese Gefahrenerfassung für die sich anschließende Risikobeurteilung von besonderer Bedeutung, denn einerseits signalisieren die äußeren Bedingungen (z.B. hohe Wellen oder Abfälle beim Kanu-Wildwasserfahren) starke Anreize, die im Sinne der Motivbefriedigung antizipiert werden. Gleichzeitig enthalten diese gefährlichen Bedingungen aber auch stets beträchtliche Reize, die zu den unbedingten Furchtauslösern zu rechnen sind und ,[...] deshalb überaus geeignet sind, die Realgefahr, in der sich der Risikosportler befindet, zu akzentuieren" "198 (SZCZESNY-FRIEDMANN 1982, 124).

Da nicht alle Gefahren im Wildwasser direkt erkennbar sind, müssen eine Reihe von Gefahrenfaktoren aus bestimmten Indikatoren, wie dem Schätzen von Strömungsverläufen usw. erschlossen werden. In Anlehnung an RUPPERT et al. (1985) und PREUSS (1996) können in diesem Zusammenhang vier Dimensionen im Bezug auf die Wahrnehmung bestimmter Indikatoren differenziert werden:

1. Direkte primäre Risikoindikatoren können unmittelbar wahr genommen werden (z.B. die Atemnot beim Bergsteigen in große Höhen). Sie können auf allen Regulationsebenen verarbeitet werden. Nicht selten führen diese direkten Indikatoren zu automatisierten Reaktionen.

2. Indirekte primäre Risikoindikatoren als Normabweichung oder als Veränderung einer Signalfolge (z.B. Geräuschveränderungen bei Lawinenabgängen, Veränderungen im Wasserdruck beim Kanu-Wildwasserfahren). Die Informationsverarbeitung findet hierbei auf einer perzeptivbegrifflichen oder wissensbasierten Regulationsebene statt (vgl. HACKER 1998, 244).

3. Direkte sekundäre Risikoindikatoren als Interpretationsleistung aus anschaulichen Ereignissen, die nur durch Erfahrungen auf mögliche Gefahren hin erkannt werden können (z.B. die Spiegelung eines Weges als Hinweis auf Rutschgefahr). Hierbei sind insbesondere kognitive Verarbeitungs- und Regulationsprozesse auf der wissensbasierten und intellektuellen Regulationsebene gefordert. 
4. Indirekte sekundäre Risikoindikatoren, die nur auf Grund statistischen Wissens als Gefahren erkannt werden (z.B. die Hangexposition als Indikator für Lawinengefahren). Die Regulationsprozesse finden hierbei insbesondere auf der intellektuellen Regulationsebene statt.

Dabei werden die Risikofaktoren nicht nur wahrgenommen, sondern im Sinne der Gefahrenkognition in den übergeordneten Zusammenhang eingeordnet. Schließlich ist es für die weitere Analyse und Bewertung notwendig, dass bestimmte Strömungsformen nicht nur erkannt, sondern gleichfalls hinsichtlich ihrer, Wirkung' erfasst werden.

RUPPERT et al. (1985) haben im Rahmen mehrerer Studien zum Risikoverhalten in Betrieben diesbezüglich ein Schema von zehn Dimensionen von Risikoindikatoren entwickelt, dass neben der Anschaulichkeit, der $\mathrm{Zu}$ gänglichkeit u.a. auch das Verhalten anderer bei der Risikoindikation beinhaltet. Diese Faktoren beeinflussen demnach die Gefahrenkognition in einem dynamischen Feld. Für ihr Untersuchungsdesign in Arbeitsabläufen stellen sie dabei fest, dass insbesondere die Anschaulichkeit von Risikoindikatoren nur in geringem Maße gegeben ist. Ähnliche Zusammenhänge lassen sich auch für den Risikosport formulieren. Auch hier ist anzunehmen, dass nur ein geringer Prozentsatz der Risikoindikatoren das Kriterium direkter Anschaulichkeit erfüllt und damit die Mehrheit der Gefahrenindikatoren aus sekundären Hinweisen abstrahiert werden muss.

Die anschließende Risikoanalyse oder Risikobeurteilung bezeichnet nach RENN ,[...] die subjektive Aufnahme und Speicherung von negativen bzw. positiven Konsequenzen und der Wahrscheinlichkeit ihres Eintreffens durch Individuen“ (RENN 1984, 96). Hierbei werden die wahrgenommenen Gefahren in Abgleichung der subjektiven Fähigkeiten bewertet und damit in individuelle Risiken transformiert. Die Informationsverarbeitung ist dabei maßgeblich von der individuellen Motivationsstruktur abhängig - z.B. Naturwahrnehmen oder Grenzen erfahren - die als konstituierendes Grundelement die Überlegens, Entscheidungs- und Entschlussmomente reguliert (vgl. PÖHLMANN 1986, 83). Dabei ist das

„.... Identifizieren von möglichen Gefahrenquellen kein Selbstzweck, sondern
setz. Aktionen in Gang, die zur Bewältigung der antizipierten Bedrohung ge-
eignet erscheinen. Für jede potentielle Gefahrenquelle werden Maßnahmen er-
sonnen und erprobt, mit Hilfe derer sich die signalisierten Konsequenzen aller
Voraussicht nach vermeiden lassen; je vollständiger die Erfassung der Gefähr-
dungsmöglichkeiten ist, desto vollständiger ist auch der Schutz vor ibnen"
(SZCZESNY-FRIEDMANN 1982, 100).

Das Ziel der Risikoanalyse besteht folglich darin, den Konflikt zwischen Leistungstendenz und Sicherheitstendenz unter ausgewogener Berücksichtigung beider Komponenten und dem individuellen Zielmotiv zu lösen (vgl. HOYOS 1980, 119). Es werden daher zunächst die Aussichten auf einen möglichen Handlungserfolg - und damit die Verwirklichung der Handlungsmotivation - und die eventuellen Folgen eines möglichen Misserfolges antizipiert. Da hauptsächlich fähigkeitsabbängige Risiken analysiert werden, ist 
die Selbsteinschätzung (der physischen, taktischen und technischen Fähigkeiten) während dieses Prozesses die wesentliche Einflussgröße. Wird eine Flussstelle beim Kanu-Wildwasserfahren in der Risikoanalyse als individuell kontrollierbar eingeschätzt, dann herrscht eine umfassende Kontrollüberzeugung, die auch überraschend eintretende Anforderungen berücksichtigt und diese als kontrollierbar bewertet (vgl. ALLMER 1998, 79).

Obwohl sich das Risiko im Wildwasser nicht mathematisch darstellen lässt, kann der Prozess der Risikoanalyse mit Hilfe der allgemeinen Risikoformel verdeutlicht werden: R (Risiko) = W (Wahrscheinlichkeit) x S (Schadensausmaß) (vgl. BANSE 1996, 35f). Bei geringem Schadensausmaß können dementsprechend eher Risiken mit einem relativ hohen Wahrscheinlichkeitsgrad eingegangen werden, während bei erheblichem Schadensausmaß die Wahrscheinlichkeit des Handlungserfolges entsprechend groß sein muss.

Risikobeurteilungen im Wildwasser stützen sich indessen nicht primär auf objektives Urteilsvermögen, sondern werden in hohem Maße durch subjektive Erfahrungen geprägt. Grundsätzlich führen alle Handlungen zu Erlebnissen, die zu impliziten (nicht-bewussten) oder expliziten (bewussten) Erfahrungen führen. Die subjektive Risikoanalyse stützt sich auf subjektive Verfahrenswege der Urteilsoperation wie z.B. Intuitionen, also gefühlsmäßigen Situationsbeurteilungen sowie auf private Heuristiken ${ }^{199}$ und Plausibilitätsannahmen. Für diese subjektive Risikobeurteilung besteht keine wissenschaftliche Option der Nachvollziehbarkeit ohne dass derartige Beurteilungen und Analysen damit falsch sein müssen (vgl. auch PREUSS 1996). Vor allem im Erkennen und Verstehen der indirekten Gefahrenindikatoren spielt die eigene Erfahrung die entscheidende Rolle. Erfahrungsbedingte Regulationsprozesse beeinflussen sowohl auf der kognitiven, als auch auf der emotionalen Regulationsebene nach NITSCH die Risikobeurteilung.

Ausschlaggebendes Merkmal des Risikoverhaltens im Spannungsfeld von Risikosuche und Sicherheitsbedürfnis ist das Vorhandensein von Handlungsalternativen, die eine Risikoentscheidung erst ermöglichen (vgl. KLEBELSBERG 1982, 46). Besteht zwischen dem Wunsch, die Motivation zu befriedigen und dem Sicherheitsbedürfnis eine zu große Diskrepanz, so wird in der Risikoentscheidung schließlich zwischen den Handlungsalternativen abgewogen.

Neuere Ansätze liefern in diesem Zusammenhang Hinweise auf so genannte Risiko-Entschärfungs-Operatoren (HUBER et al. 2001): die Protagonisten in Risikosituationen suchen demnach nicht nur Handlungsalternativen sondern auch nach Handlungen, die das Risiko einer bestimmten Alternative reduzieren. Dabei stellten die Autoren einen Zusammenhang zwischen der Attraktivität der Handlungsalternative, der Erfolgserwartung und der damit in Zusammenhang stehenden Akzeptanz von RisikoEntschärfungs-Operatoren. Je attraktiver eine Alternative im Sinne der Motivdisposition eingeschätzt wird, desto höher sind die Erfolgserwartungen in der Handlungssituation und die Akzeptanz entsprechender EntschärfungsOperatoren. Bei Risikosportarten wie dem Wildwasserpaddeln werden daher Situationen, die über keine alternativen Handlungsmöglichkeiten verfügen - 
so genannte Zwangspassagen - weitestgehend gemieden. Die Entscheidung, das Risiko einzugehen und die Handlung durchzuführen, hängt damit letztlich von der Attraktivität der Handlungsalternativen und dem persönlichkeitsspezifischen Risikoniveau ab. Dabei handelt es sich ,[...] um den individuellen, relativ situationskonstanten Ausprägungsgrad jenes Risikos, das der Einzelne einzugehen bereit ist" (KLEBELSBERG ebd., 257). Auch bei identischer Risikobeurteilung hängt die Entscheidung somit vom Sportler selbst ab, der in der Form eines individuell akzeptierten Risikos seine Entscheidung je nach den subjektiven Präferenzen trifft ${ }^{200}$.

Die Handlungsrealisation ist im Prozess der Risikobeurteilung das letzte Element und gleichzeitig die entscheidende beeinflussende Größe der situativ nachfolgenden Risikoanalysen. Da eine Handlungsinterpretation die im Anschluss folgenden kognitiven Bewertungsprozesse beeinflusst, stellt die Handlungsrealisation letztlich das verbindende Glied zwischen den kognitiven Wahrnehmungs- und Bewertungsprozessen dar (vgl. BOYES 1991, 16). Ein Misserfolg mit traumatischen Erlebnissen wird schließlich die Situationswahrnehmung der folgenden Befahrung genauso entscheidend beeinflussen, wie die dauerhaft erfolgreiche Bewältigung von Risikosituationen (vgl. hierzu Abschnitt 6.2.3). Letzteres verstärkt die Einschätzung der subjektiven Fähigkeiten positiv und wirkt sich somit modifizierend auf den Bewertungsprozess aus.

Der Erfolg, mit dem Risiko in Risikosportarten richtig umzugehen, hängt nun tatsächlich davon $a b$, ob es gelingt, die Gefahren der Situation richtig zu erkennen und im Bezug auf die eigenen Fähigkeiten und Motivationen zu bewerten (vgl. auch BÄNI 1991a, 3). Die individuelle Motivationsstruktur und das individuell ausgeprägte Sicherheitsbedürfnis können nur befriedigt werden, wenn es dem Kanusportler gelingt, die Anforderungen in der Handlungssituation mit den entsprechenden Risiken wahrzunehmen, mit seinen Fähigkeiten abzugleichen und dadurch eine Kongruenz zwischen situativen Anforderungen und personalen Bewältigungsmöglichkeiten zu erreichen. Entscheidend für den Umgang mit Risiken im Wildwasser ist somit die Ausprägung eines Risikobewusstseins, dass PÖHLMANN (1986) als das $\mathrm{Maß}$ definiert, in dem sich der objektive Schwierigkeitsgrad einer Aufgabe sowie die subjektiven Möglichkeiten zu ihrer Bewältigung im Denken und Fühlen wiederspiegeln und diese bestimmen (vgl. PÖHLMANN 1986, 98). Die Ausbildung eines derartigen Bewusstseins stellt für das Risikoverhalten im Sport die für den Erfolg der Handlung ausschlaggebende Einflussgröße dar.

\section{„Genau diese Überlegung möchten wir auch Risikosuchern unterstellen: daß nämlich seine Verhaltensergebniserwartung in bezug auf den Umgang mit Ge- fabren nur dann positiv ist, wenn es gelingt, das Bewußtsein der Gefahr auf- rechtzuerhalten" (SZCZESNY-FRIEDMANN 1982, 125).}

Allgemein lässt sich das sportliche Risikoverhalten als ein Prozess der Wahrnehmung, Beurteilung, Entscheidung und Interpretation in Risikositua- 
tionen analytisch darstellen. Die folgende Abbildung gibt diesen RisikoProzess zusammenfassend wieder:

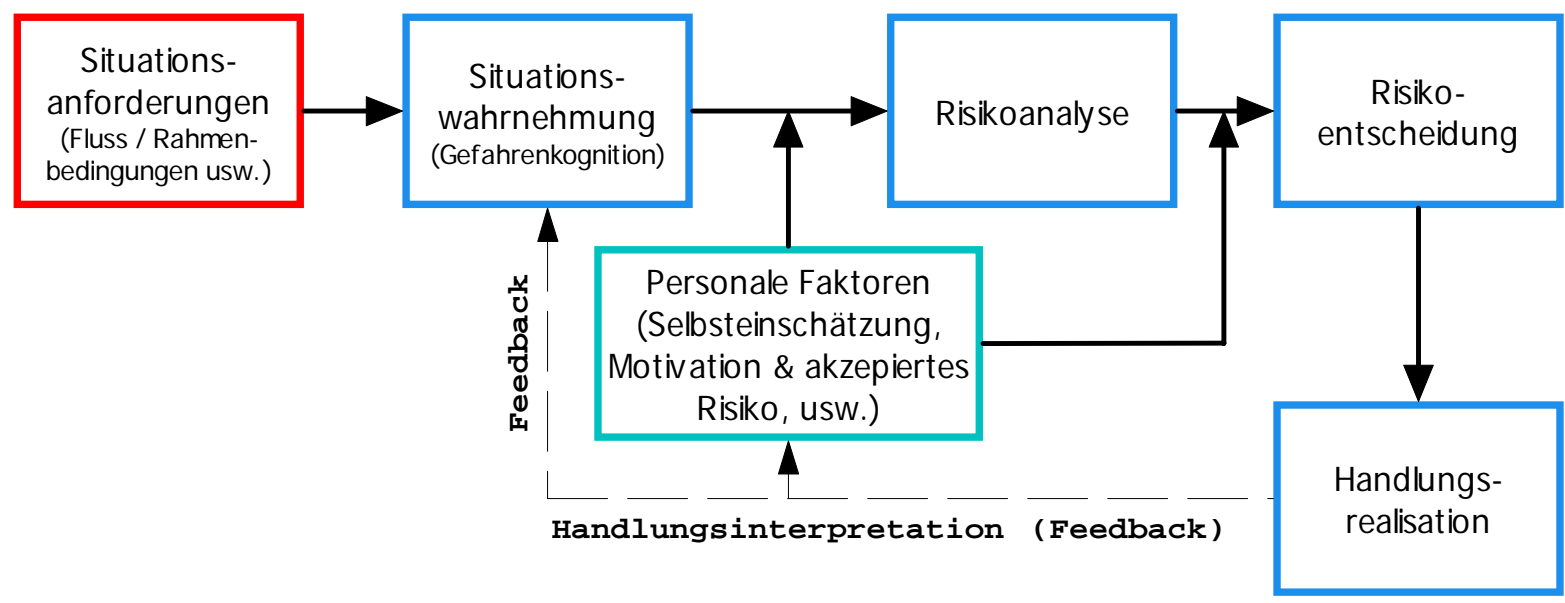

Abbildung 6.3: Risikoverhalten als kognitiver Prozess( mod. n. BOYES 1991, 16).

\subsubsection{Emotion und Risikoverhalten}

Den Wahrnehmungs- und Analyseprozess im Risikosport auf kognitive Prozesse zu reduzieren, erscheint angesichts der emotionalen Erlebnismöglichkeiten im Risikosport als ungeeignet. TRIMPOP (1994) hat in einer Metaanalyse aufgezeigt, dass Emotionen in Risikosituationen einen bedeutenden Platz in der Wahrnehmung und Verarbeitung von handlungsrelevanten Reizen einnehmen.

,[...] the average person engage in behaviors because it ,feels good' and in spite of better knowledge and although a rational decision would have suggested a different course of action. Furthermore, a strong threat of physical injury ca elicit panic reactions, which in turn can prevent a person from following logical cognitive problem solving strategies" (TRIMPOP 1994, 247).

Auch NITSCH (1986) stellt eine Interdependenz von kognitiven Prozessen und emotionalen Erfahrungen fest. Es ist in diesem Zusammenhang abzuleiten, dass

„[...] Emotionen und Kognitionen in direkter Interaktion stehen, insofern als Kognitionen bei der Entstehung von Emotionen beteiligt sind und umgekehrt Art und Ablauf kognitiver Prozesse von emotionalen Vorgängen beeinflusst werden"(SPIES \& HESSE 1986, 75).

Eine der profiliertesten Theorien zur Entstehung von Emotionen in Abhängigkeit kognitiver Prozesse liefert SCHACHTER (1966) in seiner Zweifaktorentheorie der Gefüble. Er konstatiert, dass Emotionen ihren Ursprung in einer undifferenzierten körperlichen Erregung besitzen und erst eine kognitive Interpretation dieses Erregungszustandes schließlich zu dem spezifisch wahrgenommenen Emotionszustand führt (vgl. auch FINK 1994, 30ff). Demnach würden die unbedingten Furchtreize der Risikosituation im Risikosport - z.B. Wellen, Wasserwucht oder hohe Abfälle beim Wildwasserfahren - zunächst zu einem unspezifischen Erregungspotential führen, dass 
dann als Angst interpretiert wird, wenn ,[...] ein Individuum wahrnimmt, dass es einer Herausforderung nicht gerecht wird" (SARASON 1978; zit. nach HACKFORT \& SCHWENKMEZGER 1985, 17). Gleichfalls ist es möglich, dass die Situation als weitestgehend kontrollierbar bewertet wird, so dass die Erregung als eine positive emotionale Reaktion - als eine Art ,Thrill' oder, Angstlust' - gedeutet wird. Die erste sozusagen unwillkürliche Reizinterpretation wird dann von einer zweiten Einschätzung überlagert, „[...] die im wesentlichen den Glauben daran beinhaltet, dass man die Kontrolle über die Gefahren habe. Solange sich keine Hinweise darauf einstellen, dass man die Kontrolle zu verlieren droht, resultiert aus dieser subjektiven Sicherheitsgewissheit ein insgesamt positives Erleben" (SZCZESNYFRIEDMANN 1982, 145) 201.

Ähnliche Zusammenhänge lassen sich auch für das in Kapitel 4 dargestellte Gefühl der Ich-Bestätigung beschreiben. Dieses Triumphgefühl resultiert einerseits aus der Erleichterung darüber, dass die antizipierten Bedrohung nicht Wirklichkeit wurde, andererseits aber aus der Würdigung, dass man es selbst durch die eigene Geschicklichkeit vermochte, den positiven Handlungsausgang herbeizuführen (vgl. ebd. 143).

LAZARUS et al. gehen von einer erweiterten wechselseitigen Beeinflussung von Emotionen und kognitiven Vorgängen aus. Danach ist die eigentliche Bewertung der Risikosituation als ein mehrphasiger Prozess zu verstehen, in dem es zu Interaktionen von Emotionen und kognitiven Vorgängen kommt. Emotionale Reaktionen fungieren im kognitiven Bewertungsprozess als eine Art ,Signalfunktion', die dem Sportler Rückmeldungen über das SollIst-Wert Verhältnis zwischen Anforderungen und Bewältigungsmöglichkeiten liefert. Diese durch eine Emotion - wie z.B. Angst oder Langeweile verdeutlichte Diskrepanz zwingt den Handelnden wiederum zu einer kognitiven Neueinschätzung, die einen Ausgleich dieses Verhältnisses anstrebt (vgl. LAZARUS et al. 1977, 188f). Wird im Wildwasser eine Walze im Hauptstrom z.B. als bedrohlich bewertet, führt die ,induzierte' Angst wahrgenommen über physiologische Parameter - dazu, sich Handlungsalternativen zu suchen. Kann die Walze z.B. weiträumig umfahren werden, so kann diese Neubewertung der Situation die ursprünglich bedrohliche Einschätzung revidieren.

„Eine Neueinschätzung kann eine erfreuliche Einschätzung in eine bedrobliche verwandeln und eine anfänglich bedrobliche Einschätrung in eine erfreuliche" (LAZARUS et al. 1977, 195).

Dementsprechend ergänzen sich emotionale und kognitive Bewertungen im Bezug zur Handlung zu einer ganæheitlichen Situationseinschätz̧ung. Angst als situativ gebundene Emotion ist folglich nicht nur eine unspezifische ,Begleitreaktion', sondern stellt im Zusammenhang mit der kognitiven Bewertung einer Situation ein ausgeprägtes Sicherheitsmoment - in Form eines Warnsignals - dar.

SPIELBERGER (1966) geht unter Berücksichtigung persönlichkeitsspezifischer Merkmale davon aus, dass z.B. die empfundene Angst in einer Situ- 
ation in hohem Maße von einem individuellen, Ängstlichkeitsniveau' abhängt. Demzufolge neigen hochängstliche Personen im Gegensatz zu niedrigängstlichen Personen eher dazu, auf als bedrohlich empfundene Reize mit einem hohen Anstieg von situativer Angst zu reagieren. SCHÖNPFLUG (1989) charakterisiert die ängstliche Person in Anlehung an SPIELBERGER schließlich als einen ,Sicherheitsexperten'. Er nimmt an, dass sie infolge ihres subjektiv erhöhten Ängstlichkeitsniveaus eine überdurchschnittliche Orientierungsaktivität entfaltet, die ihr auch überdurchschnittliche Präventionschancen eröffnen kann (vgl. auch MÜLLER 1991, 59).

DÖRNER et al. (1983) stellen unabhängig davon fest, dass es in Folge intensiver emotionaler Reaktionen - wie z.B. starker Angst - zu recht eingeschränkten kognitiven Prozessen kommen kann. Demgemäß werden im Zustand der Angst die Neben- und Fernwirkungen des Risikoverhaltens nur bedingt berücksichtigt, da die Aufmerksamkeit auf Veränderungen der aktuellen Situation verlagert wird (vgl. SPIES \& HESSE 1986, 80). RADLINGER \& SEILER (1984) konstatieren zudem, dass starke Emotionen auch auf physiologische und motorische Prozesse einen entscheidenden Einfluss ausübt. Während für die positive Angsterregung - als eine Form der Angstlust - eine optimale Mobilisierung des Bewegungsverhaltens angenommen wird, kann es bei starker, negativer' Angst zu Einschränkungen in der situationsspezifischen Bewältigungskompetenz kommen (vgl. RADLINGER \& SEILER 1984, 16f).

„Angst kann demzufolge als Antrieb gesehen werden, der kraft seiner energetisierenden Wirkung einen leistungs- und lernaktivierenden Einfluss hat, der aber andererseits bei zu starker Intensität desorganisierend wirkt und einen leistungs- und lernhemmenden Einfluss bat" (HEITZELHOFER 1979b, 335).

Ähnlich argumentieren UNGERER \& MORGENROTH, die Stresssymptome und ihre Bedeutung für die Risikobeurteilung in Risikosituationen untersucht haben. Die Autoren registrieren u.a. vielfältige psychophysische Auswirkungen von Risikosituationen auf den Organismus. Die von ihnen angeführte Differenzierung von Eustress - als angenehm empfundene Mobilisation psychophysischer Abläufe - und Distress - als negative Bedrohungsempfindung, korreliert mit der angeführten Unterscheidung zwischen Angstlust und Bedrohungsangst (vgl. UNGERER \& MORGENROTH 2001, 87).

Zusammenfassend ist für die erfolgreiche Bewältigung der Risikosituation in Risikosportarten also - zumindest legen dies die Ansätze nahe - neben dem kognitiven Risikobewusstsein auch ein emotionales Befindlichkeitsbewusstsein unerlässlich, dass darauf abzielt, die subjektive Erregung im Handlungskontext wahrzunehmen und zu interpretieren. Insbesondere der situativen Angst kann hierbei eine wichtige regulierende Funktion bei Risikosportarten zugeschrieben werden (vgl. auch MÜCKE 1985, 174). Nicht Angstreduktion, sondern eine individuelle Angstkontrolle - im Sinne eines bewussten Umgangs mit Angst und deren Bewältigung - muss im Risikosport demnach 
erlernt werden. Emotionen wie Angst aber auch Langeweile fungieren dabei im Sinne eines emotionalen Verstärkers, der die kognitive Bewertung der Situation bekräftigt. Eine umfassende Risikobewertung im Risikosport ist folglich überhaupt nur unter Berücksichtigung der Interaktion von Emotionen und Kognitionen möglich.

\subsubsection{Das ,Adventure Experience Paradigm'}

Das von PRIEST (1990) (vgl. auch PRIEST \& GLASS 1997) entwickelte ,Adventure Experience Paradigm' verdeutlicht die bisher dargestellten Regulationsvorgänge beim Risikoverhalten im Risikosport in einer handlungstheoretischen Matrix. Risikoverhalten wird dabei - in Anlehnung an CSIKSZENTMIHALYIs (1993), Flow-Kanal' - als Interaktion der Variablen situative Schwierigkeitsanforderungen und subjektive Bewältigungsmöglichkeiten abgebildet.

„The process of risk involvement is essentially a continual interaction between the individual and the specific stimuli offered by the natural environment" (BOYES 1991, 16).

Wie bereits in den voranstehenden Abschnitten aufgezeigt, besteht das Ziel des Risikosportlers darin, seine Handlungen in einem Bereich zwischen Unter- und Überforderung, zwischen starker Angst und Langeweile, im Bereich des Eustress unter Vermeidung von Disstress, zu realisieren. PRIEST \& GASS (1997) bezeichnen diesen Verhaltensbereich als ,Peak Adventure'. Die Autoren konstruieren hierbei eine zweidimensionale Matrix aus Bewältigungsmöglichkeiten und Schwierigkeitsanforderungen einer Risikosituation, in dem verschiedene Handlungssegmente differenziert werden können. Übertreffen die Bewältigungsmöglichkeiten die Schwierigkeitsanforderungen, so befindet sich der Wildwasserpaddler ,[...] in a condition of Adventure or Experience and experimentation", während der Bereich der Überforderung in ,Misadventure' und bei starker Überforderung in ,Devastation and disaster' differenziert wird (PRIEST \& GASS 1997, 45). Im Spannungsfeld von Motivationsbefriedigung und Sicherheitsbedürfnis charakterisiert der Bereich des Peak Adventure die ,optimale' Herausforderung für den Wildwasserpaddler. Hier lassen sich seine Motivdispositionen in Abgleichung der Anreize optimal realisieren, ohne die Kontrolle über die Situation zu verlieren. Während im Bereich der Unterforderung die Motivation nur bedingt befriedigt werden kann, führt das Risikoverhalten überhöhter Schwierigkeitsanforderungen zu einer Überforderung. Mit dem Bereich des, Peak-Adventures' ist zudem eine optimale Aufmerksamkeit bezüglich der kognitiven Regulationsvorgänge und eine hohe Konzentration bezüglich des Handlungsvollzugs gegeben. Dieser Handlungsraum entspricht damit einem idealisierten Optimalbereich für den Risikosport, ohne allerdings personelle Unterschiede und Einflüsse auf die das Risikoverhalten regulierenden Prozesse zu berücksichtigen. 


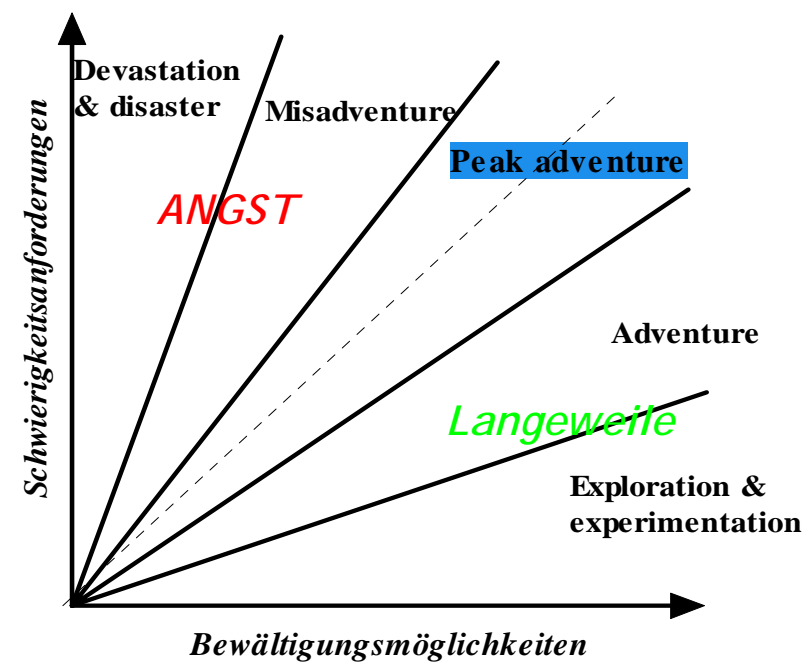

Abbildung 6.4: Das ,Adventure Experience Paradigm' (mod. n. PRIEST \& GASS 1997, 46).

Anhand des ,Adventure Experience Paradigm' lässt sich auch die Bedeutung des kognitiven und emotionalen Wahrnehmungs- und Bewertungsprozesses beim Risikosport anschaulich verdeutlichen. Nur durch die übereinstimmende Wahrnehmung und Bewertung von Anforderungen und Bewältigungsmöglichkeiten und den damit verbundenen Regulationsprozessen, kann eine Handlung als ,Peak Adventure' verwirklicht werden. Bei Fehleinschätzungen der Situation kann es daraufhin zu erheblichen Diskrepanzen zwischen subjektiv wahrgenommenen und objektiv bestehenden Handlungsbedingungen kommen (s. Abb. 6.4).

Die Prozesse der Gefahrenkognition und der Risikoanalyse zielen somit auf eine Annäherung der beiden Parameter ab, um die Handlung im Sinne einer optimalen Herausforderung zu erleben. Die Handlungsregulation im Spannungsfeld von Risikosuche und Sicherheitsbedürfnis ist folglich im Sinne einer natürlichen Regulation des Verhaltens in Risikosituationen zu verstehen, die darauf ausgerichtet ist, einen relativ ausgeglichenen Gleichgewichtszustand herbeizuführen.

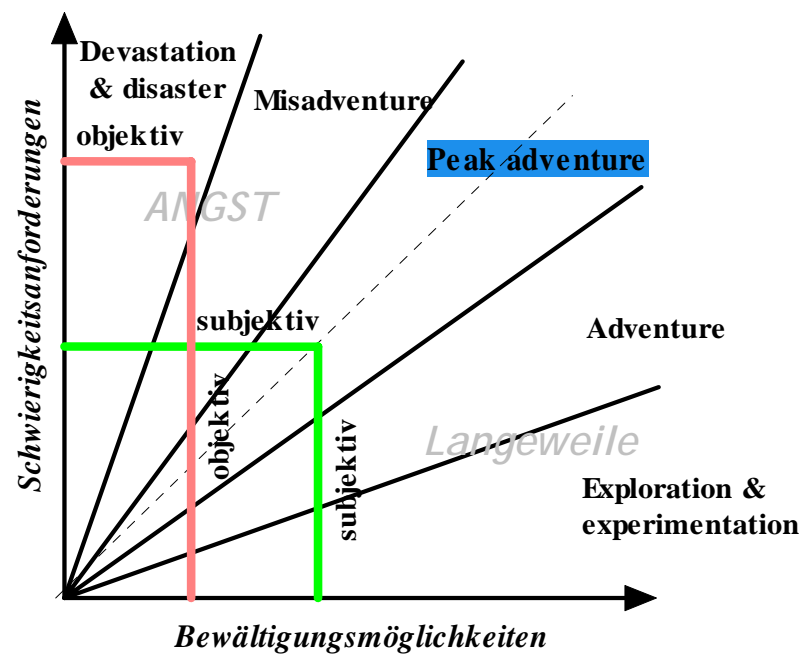




\section{Abbildung 6.5: Diskrepanz zwischen subjektiver Wahrnehmung und objektiven Handlungsbedingungen (mod. n. PRIEST \& GASS 1997, 47).}

RHEINBERG (2002) äußert jedoch Kritik an derartigen Darstellungen und führt die Ergebnisse der Leistungsmotivationsforschung an, die einen pauschalen Rückschluss auf Regulationsvorgänge im Bereich des ,peakadventures' relativieren ${ }^{202}$. So führt der Autor verschiedene Studien der Leistungsmotivationsforschung an, die massive individuelle Unterschiede für den Fall fähigkeitsangepasster Anforderungen konstatieren. Die von PRIEST \& GLASS angenommene Passung von Anforderungen und Fähigkeiten führt demnach nur bei erfolgsmotivierten Personen zu optimalen Herausforderungen während misserfolgsmotivierte Personen unter Passungsbedingungen eher Stress und Angst empfinden ${ }^{203}$ (vgl. RHEINBERG 2002, 158). In diesem Zusammenhang findet SCHALLBERGER \& PFISTER (2001) bei Flow-Untersuchungen im Alltag, dass unter Passungsbedingungen nicht nur positive Aktivierungen unter erhöhter Aufmerksamkeit sondern bei einem Großteil der Probanden auch negative Aktivierungen wie Angst oder Nervosität.

Inwieweit sich derartige Zusammenhänge auf den Risikosport übertragen lassen, ist zu diesem Zeitpunkt noch unklar, eine allgemeine Differenzierung der ,Peak-Adventure'-Matrix erscheint aber angebracht. So ist es sinnvoll von individuellen Herausforderungen als optimalen Raum zu sprechen und die Passung von Schwierigkeitsanforderungen mit ihren individuellen Bewältigungsmöglichkeiten zu relativieren. ,Peak-Adventures' sind demnach auch möglich, wenn eine wahrgenommene Unterforderung vorliegt. Zentraler Bezugspunkt der Matrix sollte daher nicht die Differenzierung zwischen Anforderungen und Bewältigungsmöglichkeiten, sondern zwischen wahrgenommenen Aufgabenschwierigkeiten und Herausforderungen (= subjektive Passung von Anforderungen und Fähigkeiten) sein.

\subsection{6 ,Risiko-Regulierung' im Risikosport}

In Anlehnung an CSIKZSENTMIHALYI (1993) lässt sich der Bereich des ,Peak adventure' auch als verwandtes Konzept zum ,Flow-Erlebnis' - oder als subjektive Herausforderung - beschreiben, bei der die Handlungsmöglichkeiten von einer Person ihren Fähigkeiten entsprechend eingestuft werden (vgl. CSIKZSENTMIHALYI 1993, 24). Es wird auf diese Weise deutlich, dass auch bei minimaler Bewältigungskompetenz, z.B. bei Anfängern eine Handlung im Bereich des ,Peak adventures' möglich ist. Eine Steigerung der Bewältigungskompetenz erfordert aber gleichzeitig erhöhte Anforderungsmöglichkeiten, um die Situation als Herausforderung zu erleben. Neben einer sukzessiven Steigerung der Schwierigkeiten bietet sich in einigen Risikosportarten vor allem ,[...] der Vorstoß der Kreativität“" (CUBE 1995, 52) zur individuellen Handlungsregulation an.

Es erscheint zudem nur bedingt möglich, auch bei bewussten Schwierigkeitssteigerungen immer, optimale' Bedingungen in der Handlungssituation 
anzutreffen. Zwar wird der Wildwasserpaddler als exemplarisches Beispiel eines Risikosportlers - wie oben dargestellt - die Risikosituationen im Vorfeld so konstruieren, dass er darin seine Handlungsziele den Motivationsbedingungen entsprechend verwirklichen kann - selbst in darauf abgestimmten Situationen sind wirklich, ideale Bedingungen' aber nur eingeschränkt über einen längeren Zeitraum zu erwarten. Vor diesem Hintergrund haben sich in der Praxis eine Reihe von Maßnahmen etabliert, die auf eine bewusste Regulierung des Spannungsfeldes von Risikosuche und Sicherheitsbedürfnis ausgerichtet sind.

Bewertet beispielsweise ein Paddler beim Kanu-Wildwasserfahren eine Handlungssituation z.B. als subjektive Überforderung - im Bereich des ,Misadventures' - so beginnt zunächst in Anlehnung an LAZARUS et al. (1977) eine intensive Neubewertung der Risikosituation. Dazu prüft der Sportler die Situation hinsichtlich denkbarer Handlungsalternativen, verlässt wenn möglich das Boot und inspiziert die Risikosituation vom Ufer aus. Erkennt er eine andere Fahrroute, die für ihn ein subjektiv geringeres Risiko darstellt, so setzt er die Befahrung fort. Bestehen keine alternativen Fahrrouten, kommt es zu einer weiteren differenzierten Neuberwertung, die auf eine Minimierung der Risiken abzielt. Das individuelle Risiko der Situation $(\mathrm{R}=\mathrm{W}$ x S) kann so durch eine geringere Bewertung der möglichen Folgen optimiert werden.

Desgleichen lässt sich aber auch beobachten, ,[...] dass das Risiko, so es nicht in ausreichendem Maße gegeben ist, durch geeignete Vorkehrungen in die Situation, eingebaut' wird“" (SZCZESNY-FRIEDMANN 1982, 117). Dabei werden z.B. beim Kanu-Wildwasserfahren schwierigere Fahrrouten gewählt oder kleinere Kehrwässer angefahren. Daneben können im kreativen Spiel mit bestimmten Strömungsformen - dem Surfen in Wellen, Spielen in Walzen usw. - weitere Herausforderungen gesucht werden, die auf eine Annäherung von Anforderungen und Bewältigungsmöglichkeiten abzielen. Dieses von SCHWIER (2003) für den Trendsport als Virtuosität bezeichnete Handlungsmerkmal lässt sich in fast allen Risikosportarten wieder finden. Die dabei erfunden Figuren und Spielformen sind insbesondere für die individuelle Regulation der Handlung von Bedeutung und lassen sich in verschiedenen Schwierigkeitsstufen einbauen.

Eine weitere Möglichkeit, das persönliche Risiko und somit die Anforderungen heraufzusetzen, sieht GOFFMANN (1994) auch im Verzicht auf Hilfsmittel:

„,[...] unter Umständen muss die Ausrïstung, die der Handelnde verwendet, in angemessener Weise eingeschränkt werden. Schließlich könnte ein Stierkampf einen Mann kaum auf die Probe stellen, wenn anstelle der Mantilla und des Degens eine Wetherby 460 verwendet würde [...]" (GOFMANN 1994, 223).

Zwar können auch im Risikosport vereinzelte Handlungen unter bewusst erschwerten Bedingungen beobachtet werden ${ }^{204}$, allgemein wird insbesondere im Breitensport aber auf Hilfsmittel nicht verzichtet. Gleichwohl lässt 
sich in diesem Zusammenhang aber die Modernisierung von Materialien einordnen. So sind z.B. Wildwasser-Boote in den letzten Jahren zunehmend kleiner und geringvolumiger gebaut wurden, wodurch eine Erweiterung der Handlungsoptionen erreicht wurde. Die Möglichkeiten auch auf Bächen im unteren Schwierigkeitsbereich die individuellen Anforderungen heraufzusetzen, lassen sich mit diesen Bootsformen erhöhen. Einerseits besitzen die Boote durch geringere Geschwindigkeit und geringfügigeren Auftrieb weniger ,Sicherheitsreserven' - die Bewältigungsmöglichkeiten sind somit eingeschränkt - andererseits lassen sich die oben beschriebenen kreativen Spielformen damit intensivieren.

Durch geeignete Maßnahmen kann die Risiko-Handlung dem individuellen Risikoniveau im Spannungsfeld von Risikosuche und Sicherheitsbedürfnis also angepasst werden. Risikosportarten stellen dabei eine der herausragenden Aktivitäten dar, die - mit gewissen Einschränkungen - in Risikosituationen die Möglichkeit bietet, sein persönliches Maß an Risiko - im Sinne der Risikohandlung bei BONß (1995) - zu wählen und je nach Befindlichkeit und Anreizstruktur zu variieren. Die sukzessive Leistungssteigerung ist somit nur noch bedingt an eine gefährlich-offensive Schwierigkeitssteigerung gebunden. Durch kreative und bewusste Heraufsetzung des Anforderungsprofils können im Risikosport auch Situationen als Herausforderung erlebt werden, die sonst nur im Unterforderungsbereich wahrgenommen werden. Der Wahrnehmungs- und Bewertungsprozess dient damit auch dem Erkennen und Vorbereiten von kreativen Handlungsmöglichkeiten als Regulationsmöglichkeit zur Optimierung der Risikosituation.

Zusammenfassend kann nun festgehalten werden: Die Handlungsregulation im Risikosport vollzieht sich über einen komplexen Prozess von Wahrnehmung und Bewertung, der sich über rein kognitive Vorgänge hinaus auch über emotionale Regulationsvorgänge manifestiert. Der Risikosportler intendiert dabei - soviel scheint sicher - ein Passungsverhältnis aus Schwierigkeitsanforderungen und Bewältigungsmöglichkeiten, welches sich in der Handlungsrealisation als ,optimale Herausforderung' auflöst. Handeln im Risikosport ist demnach zunächst ein Interaktionsprozess, der über motorische Fertigkeiten hinaus kognitive Kapazitäten erfordert, die im Rahmen der erwähnten Wahrnehmungs- und Bewertungsprozesse zur ,Risikoregulation' eingesetzt werden.

\subsection{Einflüsse auf Risikoverhalten, Gefahrenkognition und Risikobeurteilung - Ansätze und Befunde der Verhaltenspsychologie}

Obwohl ein systematischer Ansatz zum Verhalten in Risikosituationen als Forschungsergebnis der Verhaltenspsychologie noch aussteht, weist die psychologische Risikoforschung eine Vielzahl unsystematischer Forschungsergebnisse über die Beeinflussung des Risikoverhaltens in unterschiedlichen Handlungssituationen, insbesondere über die Einflüsse auf Gefahrenkogni- 
tion und Risikobeurteilung auf. Dabei werden sowohl auf personaler Ebene, als auch auf situativer Ebene akzentuierte Merkmale konstatiert, die die Wahrnehmungs-, Bewertungs- und Entscheidungsvorgänge des Risikoverhaltens maßgeblich beeinflussen. Zunächst werden Zusammenhänge zwischen personalen Verbaltensdispositionen (6.2.1) und dem Risikoverhalten dargestellt. Weiterhin werden Heuristiken im Rahmen situativ akzentuierter Merkmale beschrieben, die den Beurteilungsprozess in Risikosituationen beeinflussen können (6.2.2). Anschließend werden lerntheoretische Befunde wie die Theorie der gelernten Sorglosigkeit aufgegriffen (6.2.3). Auch soziale Einflüsse wirken auf die kognitiven Prozesse in Risikosituationen, wie in der Theorie des ,risky-shift-effekts' formuliert wird. (6.2.4). Schließlich wird - die Annahmen der interdisziplinären Annäherung auf die Handlungssituation übertragend - das Risikoverbalten unter Bedingungen subjektiver Sicherheit analysiert und Schlussfolgerungen für das Verhalten in Risikosituation gezogen (6.2.5).

\subsubsection{Persönlich bedingte Variablen}

Die Frage nach den persönlichen Beeinflussungen des Risikoverhaltens und ihrer Wahrnehmungs-, Bewertungs- und Entscheidungsvorgänge ist von verschiedenen Forschungsdisziplinen im Rahmen der Verkehrspsychologie aufgegriffen worden. Dabei ist vor allem die Bedeutung der jeweiligen persönlichen Verfassung für die Aufnahme und kognitive Verarbeitung konstatiert worden. Einflüsse auf dienkognitiven Bewertungsprozess besitzen vor allem Müdigkeit, Alkohol, Medikamente, psychische Probleme oder auch biologische Rhythmen (vgl. HOYOS 1980).

HAZARD (1993) stellt in Anlehnung an das transaktionale Bewältigungsmodell (LAZARUS \& LAUNIER 1981) zudem eine enge Wechselbeziehung zwischen Bedrohungseinschätzung und den jeweiligen Bewältigungsmöglichkeiten fest. Ein Anstieg in den zur Verfügung stehenden Handlungsressourcen verringert demnach die Risikoeinschätzung. PREUSS (1996) konstatiert diesbezüglich:

\section{„Das Verbältnis zwischen Risikobeurteilung und Gefahrenkognition einer- seits und den zur Verfügung stehenden persönlichen Copingstrategien anderer- seits kann als komplementär bezeichnet werden: Je wirkungsvoller das eigene Handlungspotential eingeschätzt wird, umso niedriger fällt die Bewertung des Gefahrenpotentials aus"(PREUSS 1996, 414).}

HERZBERG \& SCHLAG (2003) untersuchen im Rahmen einer MetaAnalyse die Korrelation zwischen Sensation-Seeking 205 als Verhaltensdisposition und dem Risikoverhalten im Straßenverkehr. Dabei konstatieren sie einen deutlichen Zusammenhang zwischen Sensation Seeking und dem individuellen Fahrstil. Personen mit hohen Sensation-Seeking-Werten - z.B. in der Kategorie ,Thrill und Adventure' - zeigen einen deutlich riskanteren Fahrstil als Personen mit niedrigen Werten (vgl. HERZBERG \& SCHAG 2003, 171ff). Zwar bleibt generell offen, inwieweit diese Zusammenhänge auf das Verhalten in risikosportlichen Situationen zu übertragen sind, ein 
grundlegender Verknüpfung kann hier aber dennoch konstatiert werden. Personen - so muss geschlussfolgert werden - reagieren auf mögliche Gefährdungen mit unterschiedlichem Verhalten.

\subsubsection{Heuristiken und Urteilsfehler}

Schon die theoretischen Modellierung des Risikoverhaltens im Risikosport offenbart, dass es sich bei den Wahrnehmungs-, Bewertungs- und Entscheidungsvorgängen in Risikosituationen nicht um rationale oder gar objektivierbare Prozesse handelt. Die kognitiven Prozesse des Risikoverhaltens sind vielmehr in hohem Maße subjektiv konstituiert und entziehen sich damit einer modelltheoretischen Übertragung in die Praxis.

Die Arbeitsweise der Kognitionen im Risikoverhalten ist Gegenstand der kognitiv-psychologischen Handlungsforschung. Im Bezug auf Handlungen in Risikosportarten und den darin enthaltenden kognitiven Beurteilungsprozessen, weist der Forschungsstand zwar nur wenig explizite Ansätze auf206, die kognitiven Sicherheitspsychologie als Anwendungswissenschaft der Verhaltenspsychologie ist aber mit zahlreichen Forschungsergebnisse weitestgehend etabliert. Die wichtigsten und umfangreichsten Beiträge zu den Prinzipien der „kognitiven Ordnungsstiftung“ (MUHSAHL 1997, 57) stammen hierbei von TVERSKY \& KAHNEMAN sowie FISCHHOFF \& SLOVIC (im Überblick vgl. u.a. ELZER et al. 1990; kritisch auch MUHSAHL 1997).

In ihren Studien entwickeln die Autoren der kognitiven Verhaltenspsychologie ein komplexes System von situativen und personalen Einflüssen auf das Verhalten in Risikosituationen und beschreiben hierbei so genannte heuristische Prinzipien.

„Als Heuristik bezeichnet man eine Methode, mit der die Lösung eines Problems nicht systematisch durchgeführt wird und (selbst bei richtiger Anwendung) nicht unbedingt mit den korrekten Ergebnis abgeschlossen wird, wie dies bei algorithmischen Methoden der Fall ist: vielmehr verläuft die Lösung vereinfachend, daher manchmal auch fehlerhaft" JUNGERMANN \& SLOVIC 1987, 91f).

Im konkreten Bezug zum Risikoverhalten im Risikosport lassen hierbei unterschiedliche heuristische Prinzipien identifizieren:

Die Annahmen einer Repräsentativ- und Ähnlichkeitsheuristik gehen von einer menschlichen Tendenz zur beständigen Suche nach Ähnlichkeiten bei der Bewertung neuer Situationen aus. Die Identifikation eines neuen Elementes erfolgt demnach ,[...] über dessen Ähnlichkeitsbeziehungen zu bekannten Ereignissen“" (MUHSAHL 1997, 59) 207. Zu negativen Urteilsbeeinflussungen führt dieses heuristische Prinzip, den Untersuchungsergebnisse von TVERSKY \& KAHNEMAN (1973) folgend, vor allem bei Entscheidungen, die einen hohen Verwandtschaftsgrad zu bekannten Situationen sowie stereotypische Charakteristika aufweisen. Urteilsprozesse resultieren demnach aus der Erwartung, dass sich ein Verhalten in ähnliche Situationen übertragen lässt und verwandte Entscheidungssituationen angewendet wer- 
den können. Auch eine Überschätzung der Vorhersagemöglichkeit aufgrund ähnlicher Situationsbedingungen resultiert aus der Repräsentativheuristik.

UETZINGER (2004) kennzeichnet im Rahmen einer Untersuchung zur Entscheidungsfindung bei der Lawinenbeurteilung eine eng verwandte $V e r$ trautheitsheuristik. Demnach gehen Menschen in vertrauten Situationen von einer größeren Beherrschbarkeit der situativen Anforderungen aus, als in unbekannten Handlungssituationen. Für risikosportliche Handlungssituationen bedeutet dies, eine Übertragung von Analyseschemata und Bewertungsclustern von verwandten Situationen auf eine aktuell zu bewertende Risikosituation.

Auch die Verfügbarkeitsheuristik steht diesbezüglich in einem engen $\mathrm{Zu}-$ sammenhang und beschreibt die Informationsselektion bei Entscheidungsprozessen. Urteile werden demnach von dem Ausmaß bestimmt, in dem man sich an verwandte Ereignisse erinnert und diese im Rahmen der Informationsverarbeitung verfügbar sind. Dies erscheint auf einer ersten Ebene auch durchaus sinnvoll ${ }^{208}$, gleichzeitig aber in hohem Maße fehleranfällig. So weisen u.a. TVERSKY \& KAHNEMAN (1973) darauf hin, dass Menschen in der Regel eine Nachrichtenselektion in Abhängigkeit vom subjektiven Bedrohungspotential vornehmen. Besonders bedrohliche Ereignisse werden dabei stärker kognitiv berücksichtigt als weniger bedrohliche Ereignisse. Ähnliches gilt für die Kommunikation von Gefahrenindikatoren: So werden häufig kommunizierte Indikatoren stärker kognitiv repräsentiert als weniger kommunizierte Nachrichten - unabhängig von ihrer tatsächlichen Wahrscheinlichkeit (vgl. SCHWIERCH 2004, 16). Bezüglich der Risikobeurteilung werden folglich denjenigen Faktoren eine erhöhte Aufmerksamkeit geschenkt, die in ihrer kognitiven Verfügbarkeit am präsentesten sind - unabhängig von ihrer tatsächlichen Bedeutung für die Risikobeurteilung.

Als weiteres heuristisches Prinzip wird die Anpassungs- und Verankerungsheuristik, beschrieben, die davon ausgeht, dass bei der Beurteilung eines Sachverhaltens das Ergebnis vom Startpunkt der Überlegung abhängt (vgl. MUHSAL 1997, 69). Die Ausgangssituation besitzt demnach bedeutungsvollen Einfluss auf die individuelle Risikobeurteilung. So ändern sich beispielsweise die Beurteilungskriterien einer Risikosituation, wenn dem Protagonisten bestimmte Hintergrundinformationen über die spezifische Situation mitgeteilt werden. Von besonderer Bedeutung für den risikosportlichen Kontext ist in diesem Zusammenhang auch die von UETZINGER gekennzeichnete Festlegungsheuristik. Demnach wird die kognitive Informationsverarbeitung in Risikosituationen von der Festlegung von konkreten Zielen deutlicht beeinflusst. Menschen mit klar festgelegten Handlungszielen - z.B. der Besteigung eines identifizierten Gipfels beim alpinen Bergsteigen - gehen eher von einer Konsistenz einmal gemachter Beurteilungen aus als Menschen ohne klare Handlungsziele (UETZINGER 2004, 54).

Weitere heuristische Prinzipien formuliert darüber hinaus PREUSS ohne allerdings auf die sie strukturierenden Kognitionen zu verweisen. Die Autorin konstatiert, dass auch die Darstellung von Risikoindikatoren die Risikobeurteilung beeinflusst. Komplizierte Formulierungen würde ein Risiko 
demnach vollkommen anders erscheinen lassen als leicht verständliche Beschreibungen (vgl. PREUSS 1996, 413). Eine grundlegende Tendenz zur Verzerrung von Risikobeurteilungen ergibt sich zudem durch das Prinzip des unrealistischen Risikooptimismus. Danach erfolgt eine Unterschätzung des individuellen Risikos durch eine regressive Abwehr („mich trifft es nicht" / „mir kann so etwas nicht passieren“) die mit den Prinzipien der Erwartungssicherheit (vgl. Abschnitt 5.3) übereinstimmt. Allerdings steigt die Bedrohungseinschätzung mit der Identität der Opfer: „Persönliche Kenntnis der Betroffenen führt zu einer Erhöhung der Risikowahrnehmung gegenüber anonymen, statistischen Opfern“ (ebd., 415).

Zusammenfassend bleibt festzuhalten, dass Heuristiken als kognitive Ordnungsstiftungen (MUHSAL 1997) - JUNGERMANN \& SLOVIC (1987) sprechen von ,Daumenregeln des Denkens' - die individuelle Risikobeurteilung auch im Risikosport beeinflussen. Vor allem für die praktische Unfallprophylaxe ist die Berücksichtigung von Heuristiken besonders bedeutend. Ein Interventionsansatz als Wahrnehmungs- und Beurteilungshilfe im Risikosport - wie er in Kapitel 9 vorgestellt wird - muss letztlich relevante Heuristiken berücksichtigen, um Beurteilungsfehler zu verhindern.

\subsubsection{Lerntheoretische Einflüsse}

Lerntheoretische Ansätze zur Erklärung des Verhaltens und der Entscheidungsfindung in Risikosituationen finden sich insbesondere in neueren Forschungsansätzen. Als wichtigster Vertreter gilt MUHSAL (1997), der die Beeinflussung von Entscheidungs- und Beurteilungsprozessen in Abhängigkeit von lerntheoretischen Verstärkungen analysiert. Mit Bezug auf die strukturell- und zeitlich-funktionellen Regulationsebenen und Handlungsphasen geht der Autor davon aus, dass jede Erfahrung in Risikosituationen alle künftigen Entscheidungsprozesse in ähnlichen Situationen beeinflusst ${ }^{209}$.

„Da das registrierte ,Handlungsergebnis' zumeist subjektiv erfolgreich ist, tatsäcbliche Gefährdungen, selten' und potentielle Gefahren und Beinahe-Unfälle nicht erkannt werden, sind - ceteris paribus - ,positive Verstärkungen' (bei denen ein Vorteil resultiert) und ,negative Verstärkungen' (bei denen ein Nachteil, eine angekündigte Gefahr ausbleibt) bäufig" (MUHSAHL 1997, 178).

Die lernabhängigen Verstärkungen fungieren demnach als Operatoren, die den kognitiven Bewertungsprozess beeinflussen. Werden also über einen längeren Zeitraum keine, negativen' Erfahrungen im Rahmen des Handlungsvollzugs gemacht, verlagert sich die Risikoeinschätzung auf ein individuell höheres Niveau (vgl. auch MUHSAL 1996). Führt beim KanuWildwasserfahren beispielsweise die Entscheidung bei einer Flussbefahrungen auf umständliches Sicherheitsmaterial zu verzichten, nicht zu damit korrelierenden negativen Erfahrungen, so wird auch bei zukünftigen Befahrungen vermutlich auf das Material verzichtet. 
Ähnlich wird dieser Zusammenhang im Rahmen der lernpsychologischen Theorie der gelernten Sorglosigkeit (SCHULZ-HARD et al. 1996) beschrieben. Bei diesem Erklärungsansatz zum Verhalten in Risikosituationen wird Sorglosigkeit als „kognitiv-affektive Monopolhypothese“ (ebd., 469) definiert:

,Alles ist gut und wird auch (von selbst) gut bleiben, d.h. potentiell negative Ereignisse werden aus Gründen, die innerbalb oder außerbalb seiner Person liegen, nicht oder in nicht-bedroblichem Ausmaß eintreten, baw. positive Ereignisse aus Gründen, die wiederum innerbalb oder außerbalb seiner selbst liegen, fast zwangsläufig auf. Man meint, sich über die Zukunft keine Sorge machen zu müssen, da in der Vergangenheit dem eigenen Tun [...] stets die erwünschten Konsequenzen gefolgt sind" (ebd.).

Für die Herausbildung von Sorglosigkeit identifizieren die Autoren zwei zentrale Lernerfahrungen:

„Zum einen machen Menschen oft die Erfahrung, sie könnten obne großen eigenen Aufwand enormen Erfolg erzielen [...]. Eine Anstrengung ist nicht notwendig, alles entwickelt sich obnehin zum Guten"(ebd.).

Die zweite Lernerfahrung ist identisch zu der von MUHSAL beschriebenen lerntheoretischen Verstärkungsansatz: Wenn auf ein bestimmtes Verhalten keine direkten negative Konsequenzen folgen, wird die entsprechende Person auch in Zukunft dieses Verhalten fortführen. Unterstützt wird diese individuelle Lernerfahrung zusätzlich durch soziale Lernprozesse, z.B. durch Beobachtung anderer Personen, die mit sorglosem Verhalten Erfolg haben (Modelllernen) oder Sorglosigkeit als sozial wünschenswert darstellen („Vorsicht ist Feigheit" (ebd.)).

Gelernte Sorglosigkeit führt dabei zu einer direkten Beeinflussung der Gefahrenkognition und Risikobeurteilung. SCHULZ-HARDT et al. kennzeichnen diesbezüglich vier Symptome gelernter Sorglosigkeit: Neben der verringerten Fähigkeit zur Gefahrenwahrnehmung besteht auch eine geringere Motivation das eigene Handeln zu hinterfragen und somit auf bedrohliche Risiken aufmerksam zu werden. In der Annahme einer trügerischen Immunität vor Gefahren der Situation wird eine realistische Gefahrenkognition unmöglich. Gleichzeit wird eine unkritisch gehobene Stimmung im Sinne einer Realitätsverzerrung und eine verkürzte Zeitperspektive als Symptome und Konsequenzen gelernter Sorglosigkeit beschrieben (vgl. ebd., 471). Die Projektion der Sorglosigkeit auf zukünftige Handlungssituationen verhindert demnach die Einbeziehung der eigenen Handlung in einem langfristig zusammenhängenden Kontext.

Die Autoren führen darüber hinaus eine Reihe von so genannten Defensivstrategien an, die Menschen im Rahmen der gelernten Sorglosigkeit an Stelle von Verhaltensänderungen annehmen. So werden Gefahreninformationen in bestimmten Zusammenhängen verleugnet oder verdrängt. Mit Überoptimismus bezeichnen die Autoren die Tendenz erkannte Risiken nicht auf sich selbst zu beziehen und sich persönlich für wenig gefährdet zu halten 
(„mir wird so etwas nicht passieren“). Die systematische Überschätzung als Defensivstrategie wird als Kontrollillusion beschrieben: diese ermöglicht es dem einzelnen, sich trotz des Wissens um Gefahren weiterhin riskant zu verhalten. Mit Alibihandlungen und Fatalismus sind Verhaltensweisen gemeint, bei denen eine Risikoreduktion auf höhere Mächte oder Objekte abgeschoben werden (vgl. ebd., 472).

Inwieweit derartige Defensivstrategien im Risikosport zum Ausdruck kommen, ist nicht nachzuweisen. Die Annahmen einer Kontrollillusion sowie die Verleugnung von Informationen und ein unrealistischer Optimismus erscheinen induktiv aber auch in risikosportlichen Zusammenhängen relevant. Die folgende Darstellung der Theorie der gelernten Sorglosigkeit fasst die vorgestellten Zusammenhänge abschließend zusammen:

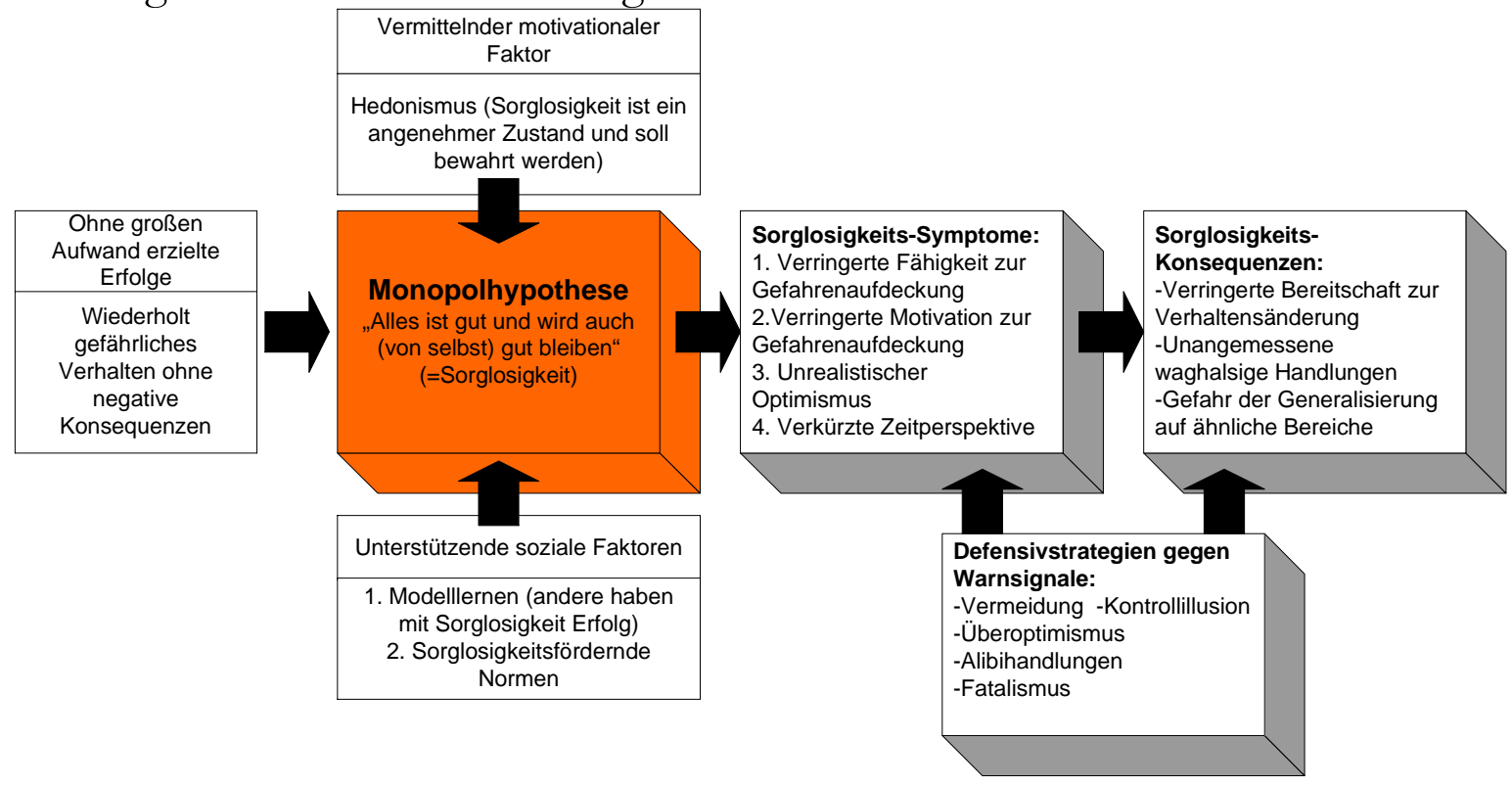

Abbildung 6.6: Schematische Darstellung der Theorie der gelernten Sorglosigkeit (in Anlehnung an SCHULZ-HARDT et al. 1996, 470).

\subsubsection{Sozialpsychologische Annahmen}

Auch sozialpsychologische Forschungsansätze haben sich mit Risikoverhalten und den hierin stattfindenden kognitiv-affektiven Prozessen unter beeinflussenden sozialen Parametern auseinandergesetzt. KOGAN et al. (1964) untersuchten beispielsweise die Risikobereitschaft einzelner Personen bei Gruppenentscheidungen im Vergleich zu isolierten Einzelentscheidung. Sie kommen im Rahmen ihrer qualitativen Untersuchungen zu dem Ergebnis, dass sich in Gruppen einzelne Personen wesentlich risikofreudiger verhalten als in isolierten Entscheidungssituationen. Dieses, risky shift'-Modell des Risikoverhaltens lässt sich auch in risikosportlichen Zusammenhängen nachweisen. NETZER (2001) berichtet beispielsweise von GruppendruckSituationen, in denen sich der Einzelne der Gruppen verpflichtet fühlt und eigene Entscheidungen der Gruppenentscheidung unterwirft. Ähnlich argumentiert auch SCHWIERCH (1991c) und LERCHER (2001). Die Ursa- 
chen hierfür werden in der Abgabe von Verantwortung auf der Ebene der Einzelmitglieder einerseits und durch soziale Erwartung im Bezug auf Rollenzuweisungen andererseits identifiziert.

UETZINGER (2004) beobachtet in Entscheidungssituationen bei SkiTouren-Gehern, dass insbesondere Gruppen mit mehr als 4 Mitgliedern besonders anfällig für gruppenspezifische Fehlentscheidungen sind. Als Ursachen identifiziert der Autor vor allem eine gewisse „Expertenheuristik“ (UETZINGER 2004, 55). Danach wird in vielen Gruppen eine Art informeller Führer ausgemacht, der aufgrund vermeintlicher Erfahrung oder ihm zugewiesene Kompetenzen Entscheidungen für die Gesamtgruppe fällt. Gruppen mit einem klar bestimmten Führer setzen sich den Untersuchungen zufolge höheren Gefahrenstufen aus, als Gruppen ohne Führer oder Gruppen, wo die Führung nicht klar besetzt war (vgl. ebd.).

Des Weiteren lassen sich Einflüsse einer so genannte, Soziale Förderungen' - gemeint ist die Anwesenheit anderer Gruppen bei der Risikobeurteilung - konstatieren. Das Risikoverhalten wird demnach durch die Anwesenheit weiterer Gruppen oder Akteure verstärkt. Allerdings ist dieser Einfluss von der Selbsteinschätzung der zu vollziehenden Handlung abhängig.

\section{„[...] gut beherrschte Fähigkeiten [werden] durch die Anwesenheit anderer verstärkt [...], während schlecht beherrschte Fäbigkeiten in der Anwesenheit anderer noch schlechter werden" (ebd., 56).}

Gut ausgebildete Gruppen setzten sich den Untersuchungsergebnissen folgend in Anwesenheit anderer Personen eher höheren Gefahrensituationen in Form von lawinengefährdeten Hängen aus, während in der Selbstwahrnehmung schlecht ausgebildete Gruppen derartige Gefahrensituationen bei Anwesenheit weiterer Personen eher vermieden (vgl. ebd.).

Einen weiteren sozialen Einflussbereich auf das Risikoverhalten klassifiziert UETZINGER unter der Kategorie Anerkennung. In Anlehnung an die ,Gladiator-Komponente' bei RHEINBERG (1996, 115; s. auch Abschnitt 4.3.7) geht der Autor von der Annahme aus, dass Menschen die Neigung besitzen sich in Aktivitäten zu engagieren, von denen sich die Protagonisten eine Beachtung und Anerkennung in sozialen Interaktionsprozessen erhoffen. Die von UETZINGER zitierte Untersuchung des amerikanischen Lawinenzentrums untersucht unter diesem Aspekt das Verhalten von rein männlichen im Vergleich zu geschlechtsheterogenen Gruppen. Gemischte Gruppen setzten sich den Ergebnissen zufolge wesentlich höheren Gefahren aus, als geschlechtshomogene Gruppen. Männer zeigen demnach in der Anwesenheit von Frauen eine bedeutend größere Risikobereitschaft als bei deren Abwesenheit (vgl. UETZINGER 2004, 55).

\subsubsection{Risikoverhalten unter Bedingungen subjektiver Sicherheit}

In den vorstehenden Abschnitten konnte expliziert werden, dass sich das sportliche Risikoverhalten maßgeblich über die individuelle Motivationsstruktur und dem daraus resultierenden Spannungsfeld von Risikosuche und 
Sicherheitsbedürfnis reguliert. Greift man aber auf die gesellschaftliche Dimension des Risikosports zurück, so wird deutlich, dass das Bedürfnis nach sportlichen Risiken, scheinbar als Kontrapunkt einer übersicherten Gesellschaft provoziert wird. In der Funktion eines kompensatorischen Ausgleichs werden Risikosituationen im Sport anscheinend nur dann aufgesucht, wenn im Alltag keine individuellen Herausforderungen existieren die eine Befriedigung der beschriebenen Motive zulassen (vgl. Kapitel 5). Verkürzt induziert Sicherheit somit den Wunsch nach Risiko, während Risiko das Bedürfnis nach Sicherheit akzentuiert.

Im folgenden wird dieser Zusammenhang auf die Handlungssituation übertragen und anhand APTERs (1994) psychologischen Sicherbeitsrahmens, dem Sicherbeits-Risiko-Gesetz nach CUBE (1995) und der Theorie der Risikokompensation nach WILDE (1978) expliziert. Abschließend wird das Modell der subjektiven und objektiven Sicherbeit in Anlehnung an KLEBELSBERG (1982) vorgestellt.

\title{
6.2.5.1 Derpsychologische Sicherbeitsrabmen
}

APTER (1994) beschreibt das Aufsuchen von sportlichen Risiken in Abhängigkeit eines psychologischen schützenden Rabmens, der das Betreiben risikosportlicher Aktivitäten überhaupt erst ermöglicht. Die Bewertung der Situation hängt demnach nur bedingt mit rationalen Entscheidungen zusammen, sondern wird aufgrund subjektiver Sicherheitsempfindungen getroffen:

\begin{abstract}
„Dieser scbützende Rabmen kann auf der eigenen Zuversicbt, der potentiellen Hilfe anderer Menschen, technischer Unterstützung und äbnlichem beruben. [...] Der schütrende Rahmen bilft dem Betrachter, die Welt auf eine bestimmte Weise wahrzunehmen - wobei wir uns einmal auf die Möglichkeit einer realen Gefabr konzentrieren, das andere mal von einem Rahmen ausgehen, der uns unserer Meinung nach schütz̧t und uns vor möglichen Gefahren bewahren kann"(APTER 1994, 44f).
\end{abstract}

In einer recht ursprünglichen Form lässt sich dieser schützende Rahmen am Beispiel des naiven explorativen Verhaltens beschreiben. Diese frühe Form des Neugierverhaltens lässt sich bei Kleinkindern nur beobachten, wenn die Mutter - oder die entsprechende Bezugsperson - dem Kind ein Gefühl der Sicherheit vermittelt. Das Hinbewegen auf neue Gegenstände, also „,...] das Aufsuchen von Unsicherheit“ (CUBE 1995, 43) hängt dabei unmittelbar vom kindlichen Sicherheitsrahmen ab. Ist die entsprechende Bezugsperson nicht anwesend, so stellt das Kind seine Erkundungen ein und richtet die Aufmerksamkeit auf Zeichen, die das Wiederkommen der Mutter andeuten, folglich auf das Wiedererlangen von Sicherheit ${ }^{210}$ (vgl. SPIELBERGER et al. 1981, 208). Ähnliche Wechselbeziehungen lassen sich aber auch auf die erwachsene Handlungssituation z.B. beim Risikosport übertragen, wo das subjektive Gefühl der Sicherheit oft durch die „ExpertenAura“" (UETZINGER 2004, 55) bestimmter Personen verkörpert wird. 
Das Engagement in Risikoaktivitäten ist somit in hohem Maße von einem subjektiven Gefübl der Sicherheit abhängig, welches sich rationalen Erklärungsversuchen weitestgehend entzieht. Es ist vor allem das Vertrauen in die eigenen Fähigkeiten aber auch der Glaube in die technische Ausrüstung oder die Mitstreiter, der die Ausübung risikosportlicher Aktivitäten ermöglicht. Sicherheit als subjektive Erfahrung wird damit zu einer antezenden Bedinung des Risikosports und zu einer die Handlung regulierenden Komponente.

\subsubsection{Das Sicherheits-Risiko-Gesetz.}

CUBE (1995) erweitert die Überlegungen zur subjektiven Sicherheit aus der Sicht der Verhaltensbiologie zu einem Sicherheits-Risiko-Gesetz, dass die Handlungssituation als direkte Wechselbeziehung von Risikosuche und Sicherheitsbedürfnis beschreibt. Subjektive Sicherheit ist demnach nicht nur antezedente Bedingung des Risikoverhaltens, sondern bestimmt gleichzeitig die ,Intensität' des aufgesuchten Risikos:

„Je sicherer man sich fühlt, desto weiter muss man gehen, um auf den Reiz. der Unsicherbeit zu stoßen. [...] Je größer also das Sicherbeitsgefübl, desto größer muss auch die Schwierigkeit sein - sonst wird sie ja nicht als Reiz empfunden"(CUBE 1990, 42).

Für die risikosportliche Handlungssituation gilt folglich, dass, je größer das Sicherheitsempfinden einer Person und damit das Vertrauen in die Bewältigungschancen in einer Handlungssituation ist, desto größer muss auch die Schwierigkeit gewählt werden, um den Reiz und die Lust am Risiko zu empfinden. CUBE (1995) formuliert das Sicherheits-Risiko-Gesetz folgendermaßen: „Je sicherer man sich fühlt, desto größer ist das objektive Risiko, das man eingeht oder aufsucht" (ebd.) ${ }^{211}$.

Da CUBE (1995) aus der Sicht der Verhaltensbiologie ähnlich wie TENBRUCK (1978) 212 von einem grundlegenden und ursprünglichen Risikobedürfnis ausgeht, lassen sich aus dem Sicherheits-Risiko-Gesetz weitere Ergänzungen ableiten. Da ein Mangel dieser ,Primärbedürfnissen` zu starkem Appetenzverhalten führt, lässt sich für das Spannungsfeld von Risikosuche und Sicherheitsbedürfnis eine Art Abhängigkeit zwischen Intensität des Risikoverhaltens und der Dauer des Sicherheitszustands ableiten. Zusammenfassend formuliert er, dass, ,je länger die Sicherheit währt, desto intensiver wird die anschließende Suche nach Unsicherheit" (ebd.).

Analog zu CUBEs verhaltensbiologisch orientierter Theorie, finden sich auch in der Psychologie theoretische Ansätze, die die Bedeutung der subjektiven Sicherheit für das Risikoverhalten akzentuieren.

\subsubsection{Die Theorie der Risikokompensation}

Die Theorie der Risikokompensation wurde im Rahmen der Verkehrspsychologie von WILDE (1978) und seinen Mitarbeitern entwickelt 213 und konnte in verschiedenen Untersuchungen, insbesondere in verkehrspsychologischen 
Untersuchungen empirisch belegt werden. Demzufolge steuern Individuen ihr Verhalten in Risikosituationen stets so, dass das letztlich eingegangene Risiko (Risikoniveau), als Produkt von Schadenswahrscheinlichkeit und Schadensausmaß, konstant bleibt. Wird das subjektive Sicherheitsgefühl angehoben, dann gleicht sich das Risikoverhalten diesem Sicherheitsempfinden an. Bemühungen um mehr Sicherheit in entsprechenden Risikosituationen werden folglich durch eine erhöhte Risikobereitschaft kompensiert, so dass letztendlich kein wirklicher Sicherheitsgewinn beobachtet werden kann (vgl. WILDE 1978, 138). Ähnlich dem SPIELBERGERschen (1966) Ängstlichkeitsniveau ist dieses Risikoniveau zwar individuell divergierend, tatsächlich bleibt aber das persönliche, akzeptierte Risiko' relativ konstant (vgl. BERGHOLD 1988, 16).

KRÄMER (1999) illustriert diesen Sachverhalt der Risikokompensation am Beispiel des Antiblockiersystems in den Vereinigten Staaten. Kraftfahrzeuge mit dieser ,Sicherheitsausstattung' verursachten dort nicht weniger, sondern sogar deutlich mehr Unfälle als Autos ohne eine entsprechende Ausgestaltung:

„Bewusst oder unbewusst, wir gleichen unser Verbalten an geänderte Risiken nur derart an, dass das Gesamtrisiko sich nur wenig verändert" (KR ÄMER 1998, 23).

RÜMMELE (1988) verdeutlicht die Theorie aber auch in ihrer gegenteilig kompensierenden Wirkung:

„Als aus Umweltschutzgrü̈nden im Winter auf den Straßen der Städte kein Salz mehr gestreut wurde, haben viele Politiker unbedacht ein Ansteigen der Verkehrsunfälle vorhergesagt. Doch das Gegenteil ist eingetreten, obwohl die objektive Gefährdung anstieg" (RÜMMELE 1988, 20).

Beide Beispiel illustrieren das ambivalente Spannungsfeld zwischen Sicherheitsbedürfnis und Risikoverhalten, wie es im Sicherheits-Risiko-Gesetz beschrieben wird: Je sicherer sich der Mensch fühlt, desto größer ist das objektive Risiko, das er in Risikosituationen eingeht (Bsp. Antiblockiersystem). Fühlt er sich aber unsicherer, so wird das objektive Risiko auf das er sich einlässt entsprechend geringer sein (Bsp. Salzstreuung). Pointiert formuliert hierzu auch APTER (1994): „Hier weckt Gefahr das Bedürfnis nach Sicherheit und Sicherheit das Bedürfnis nach Gefahr" (APTER 1994, 245)

Obwohl sich die Verkehrssituation und der Risikosport in ihren Handlungsvoraussetzung und Strukturen deutlich unterscheiden, so lassen sich die Merkmale der Risikokompensation auch auf Risikosportarten übertragen. Die Entwicklung von Sicherheitsausrüstungen wie z.B. Helme oder Schwimmhilfen beim Kanu-Wildwasserfahren führten in der Entwicklung des Wildwassersports schließlich nicht zu einer sicheren Bewältigung bekannter Flüsse, sondern zum Aufsuchen von neuen Situationen mit entsprechend höheren Schwierigkeiten und somit letztlich auch vergleichbaren Risiken (vgl. SCHNOOR 1987, 560). Desgleichen repräsentieren auch die oben dargestellten Möglichkeiten zur individuellen Handlungsregulation den 
Wunsch, das persönliche Risikoniveau relativ konstant zu halten. Bietet ein Fluss trotz der oben beschriebenen Möglichkeiten zur individuellen Handlungsregulation für den Sportler kein Risiko mehr - fühlt er sich also zu sicher - so wird er eine neue oder schwierigere Risikosituation aufsuchen, in der die beschriebenen Anreize wiederholt entdeckt werden. Das subjektive Sicherheitsgefühl - auch im Sinne einer allgemeinen Kontrollüberzeugung reguliert somit in hohem Maße das Verhalten in sportlichen Risikosituationen.

\subsubsection{Das Modell der subjektiven und objektiven Sicherbeit}

Um die Bedeutung des subjektiven Sicherheitsgefühls für die Handlungsregulierung hervorzuheben, differenziert KLEBELSBERG (1982) in Anlehnung an KAUFMANN (1973) in seinem Modell zwischen erlebter Sicherheit einerseits und objektiver Sicherheit andererseits. Als subjektiv erlebte Sicherheit bezeichnet KLEBELSBERG (1982) das situationsabhängige Sicherheitsgefühl des Einzelnen - vergleichbar mit APTERs (1994) Sicherheitsrahmen während die objektive Sicherheit die tatsächlichen Begebenheiten - im Sinne physikalischer Sicherheitsübereinkünfte - charakterisiert (vgl. KLEBELSBERG 1982, 258). KAUFMANN formuliert diesbezüglich in seiner Phänomenologie der Sicherheit:

„Objektiv sicher ist der Mensch, dem keine Gefahr drobt oder der vor möglichen Gefahren geschützt ist. Sicher füblt sich der Mensch, der sich keiner Gefahr bewusst ist, sei es infolge fehlender Gefahr, fehlender Wahrnehmung von Gefahr oder infolge eines Bewusstseins, Gefahren gewachsen zu sein. [...] Dinge und Zustände sind sicher, insofern ibr Bestand nicht gefährdet ist oder sie keine Gefabr für den Menschen darstellen" (KAUFMANN 1973, 151).

Die dem Modell zugrunde liegende Hypothese geht dabei von zwei grundsätzlichen Annahmen aus:

1. Sicherheit im übergeordneten Sinn hängt weder allein von der subjektiven, noch allein von der objektiven Sicherheit ab, sondern kann nur als dynamische Wechselbeziehung zwischen beiden Sicherheitskomponenten und deren gegenseitiger Relativierung verstanden werden.

2. Angepasstes Verhalten in Risikosituationen, im Sinne einer erfolgreichen Bewältigung, ist folglich nur möglich, wenn die objektive Sicherheit mindestens gleich groß oder aber größer als die subjektive Sicherheit ist. Nur dann werden die geltenden objektiven Grenzwerte der entsprechenden Gesetzmäßigkeiten nicht überschritten (vgl. ebd.).

Damit lassen sich die Erkenntnisse der Handlungsregulation und die Überlegungen zur subjektiven Sicherheit in ein Modell integrieren. Dies lässt sich an einem kanusportlichen Beispiel verdeutlichen: Eine Erhöhung des subjektiven Sicherheitsgefühls, z.B. durch eine Schwimmhilfe, kann dazu führen, ein erhöhtes objektives Risiko einzugehen. Diese subjektive Sicherheit kann in bestimmten Situationen durchaus berechtigt sein, denn die verbesserten Auftriebsbedingungen erleichtern das Schwimmen im Wildwasser 
zum Teil bedeutend. Die objektive Sicherheit wird in diesen Situationen also gleichfalls erhöht. In anderen Situationen, z.B. bei der Befahrung von Wehren kann das Empfinden von Sicherheit aufgrund der Benutzung einer Schwimmweste aber gleichfalls trügerisch sein, da die objektive Sicherheit in diesem Fall nicht oder nur geringfügig erhöht ist.

Das Postulat einer ,umfassenden Sicherheit“ im Risikosport (vgl. MÜCKE 1986, 54) erweist sich infolgedessen als Illusion, solange bei Sicherheitsbeurteilungen nicht die Rückwirkung auf das Teilsystem Mensch mit seinem individuellen Sicherheitsempfinden berücksichtigt wird. Eine übermäßige ,Sicherheitskonstruktion' würde nach dem Sicherheits-RisikoGesetz letztlich nicht zu angepasstem Verhalten führen, sondern - im Gegenteil - es würde somit zu einem echten Risiko, oder mit den Worten CUBEs zu einer ,gefährlichen Sicherheit“ (CUBE 1995, 10).

Zusammenfassend lassen sich nun also eine Vielfalt von Einflüssen auf das Risikoverhalten im Risikosport identifizieren. Dabei ist von einer dynamischen Interaktion der beeinflussenden Variablen auszugehen, so dass eine analytische Trennung nur mit der Zielsetzung einer verständlichen Darstellung legitimiert werden kann. Neben persönlichkeitsspezifischen Einflüssen sind vor allem so genannte Heuristiken aber auch lerntheoretische und sozialpsychologische Beeinflussungen des Risikoverhaltens zu konstatieren. Besonders interessant erscheint zudem die handlungsregulative Wechselwirkung von Risikoempfindung und dem Gefühl subjektiver Sicherheit. Wenngleich sich diese Erkenntnisse nur theoretisch auf den Risikosport übertragen lassen, sind sie als Erkenntnisgewinn an dieser Stelle hervorzuheben. Risiko, Sicherheit und Gefahr stehen damit auch in der konkreten Handlungssituationen in einem handlungskonstitutiven Zusammenhang, der entgegen zweckrationalen Annahmen - die Sonderstellung des Risikosports als Handlungsfeld akzentuiert. Die folgende Modellentwicklung versteht sich in diesem Sinn als zusammenfassende Darstellung handlungsregulativer und -strukturierender Prozesse für den Risikosport.

\subsection{Handeln im Risikosport - ein transaktionales Modell}

In den vorausgegangenen Kapiteln wurde der Forschungsstand zum Risikosport aus unterschiedlichen Forschungsdisziplinen expliziert. In diesem Kapitel wurden zudem die eine Handlung strukturierenden und regulierenden Strukturen und Prozesse evaluiert. Ziel der Darstellungen war eine möglichst umfassende Analyse der Handlungsbedingungen und regulativen Strukturen im Risikosport mit dem Endziel einen Rahmen für praktische Anwendungsbezüge zu konstruieren. Bis dato fehlt eine derartige Deduktion für den Risikosport, welche die einzelnen Beiträge der verschiedenen Forschungsrichtungen zusammenführt und modelltheoretisch integriert. Versuche Risikoverhalten modelltheoretisch abzubilden finden sich im deutschsprachigen Forschungsraum aus entscheidungspsychologischer Perspektive bei MÜLLER-GETHMANN (2000), aus motivationspsychologischer Sicht bei 
ATKINSON (1957) und RHEINBERG (2002). Im englischsprachigen Forschungsraum finden sich motivationspsychologische Ansätze bei YATES (1992) aber auch bei TRIMPOP (1994), der ein allgemeinpsychologisches Handlungsmodell in Risikosituationen vorstellt.

Im folgenden wird der Versuch einer integrativen Modellkonstruktion des Handelns im Risikosports vorgenommen. In Anlehnung an die ,Risk Motivation Theory' (RMT) bei TRIMPOP (1994) werden hierzu zunächst die grundlegende Annabmen des Modells (6.3.1) expliziert. Anschließend wird das Handlungsmodell des Risikosports als schematische Abbildung vorgestellt und die einzelnen Modell-Komponenten (6.3.2) näher erläutert.

\subsubsection{Grundlegende Annahmen des Modells}

Risikosport wird im folgenden Modell in Anlehnung an die Ausführungen in Kapitel 2 als bewusstes Eingehen von sportlichen Risikosituationen mit dem Ziel der Bewährung durch einen aktiven Einsatz eigener Fähigkeiten definiert. Kernelement des Risikosports ist damit die Bewältigung von subjektiven ,Bewährungssituationen', die den Einsatz der ganzen Person erfordern und darüber hinaus spezifisch erlernte Fähigkeiten und Fertigkeiten zur erfolgereichen Bewältigung verlangen.

Ausgehend von den bisherigen Sinndeutungen des Menschen als gleichermaßen risikosuchend und sicherheitsbedürftig und unter Berücksichtigung der interdisziplinären Forschungsbeiträge zum Risikosport, muss ein Handlungsmodell für den Risikosport von einer Interaktion von personenabhängigen und situationsabhängigen Variablen ausgehen.

„[...] the personality structure determines the basic target level of risk a person prefers, while the situation variables determine the momentary fluctuations" (TRIMPOP 1994, 240).

Dabei ist Risikosport als ein Handlungsfeld zu betrachten, welches eng mit gesellschaftlichen Prozessen der Modernisierung und der mit ihr entstandenen Kultur verbunden ist und in seinen historischen Bezüge auf Natur als Handlungsraum verweist (vgl. Kap. 3). Grundlegende Annahme des Handlungsmodells ist damit eine wechselseitige Beeinflussung von Individuen und Gesellschaft sowie zwischen Individuen untereinander. Gesellschaft bzw. die von ihr ausgehenden gesellschaftlichen Einflüssen - wie Sinn- und Wertorientierungen, Politik, Sozialstruktur usw. - beeinflussen das Handeln im Risikosport vor allem über das sich in Prozessen direkter Vermittlung zwischen Risikosport und Gesellschaft entstehende Erfahrungsfeld des individuellen Risikoerlebens (vgl. Kap. 5).

Handeln im Risikosport impliziert damit - im Rückgriff auf anthropologische Forschungsbeiträge - ein allgemeines und natürliches ,Risikobedürfnis' im Sinne eines notwendigen physiologischer Reizeinstroms und einem damit in Verbindung stehenden psycho-sozialen Wohlgefühl. Basierend auf biologischen Konzepten, ist davon auszugehen, dass Individuen dabei ganz unterschiedliche reizverarbeitende Systeme besitzen und daher ungleich auf 
,Risikoreize' reagieren. In Anlehnung an WILDEs Risiko-Homöostase wird angenommen, dass Menschen - unabhängig von der jeweiligen Risikohöhe ihr individuelles Risiko-Level weitestgehend konstant halten und Veränderungen im Sicherheitsempfinden nur Adaptationen im Risikoverhalten hervorruft.

Handeln im Risikosport lässt darüber hinaus durch eine ganze Vielfalt von Motivations- und Anreizbedingungen erklären (Kap. 3.2), die in ihrem Zusammenwirken das Handeln in risikosportlichen Situationen beeinflussen. Neben zweckzentrierten Anreizen werden auch tätigkeitszentrierte Anreize im Risikosport wahrgenommen und motivationsbildend verarbeitet. Im Mittelpunkt risikosportlicher Handlungen steht damit die fähigkeitsabhängige Bewältigung von subjektiv wahrgenommenen Herausforderungen, die aus der Handlungssituation antizipiert werden und die bei erfolgreicher Bewältigung zu positiven Empfindungen während und nach dem Handlungsvollzug führt.

Darüber hinaus wird angenommen, dass sich Handeln im Risikosport nur durch einen kognitiv-emotionalen Wahrnehmungs-, Beurteilungs- und Entscheidungsprozess vollziehen lässt. Dieser findet als Abgleichungsprozess von individueller Motivation, Risikoneigung, Situationsanforderungen, Bewältigungsmöglichkeiten und Handlungsalternativen statt und unterliegt personalen sowie situationsabhängigen Einflüssen. Eine direkte Rückwirkung von bewältigten Risikosituation auf aktuelle und folgende Handlungssituationen wird zudem als lerntheoretisches Postulat modelltheoretisch integriert.

Zusammenfassend lässt sich das hier konstruierte Handlungsmodell des Risikosports als ein transaktionales Handlungsmodell ${ }^{214}$ beschreiben, welches Persönlichkeits- und Situationsvariablen vor dem Hintergrund gesellschaftlicher Rahmenbedingungen und unter der Annahme eines kognitivemotionalen Entscheidungsprozesses integriert.

\subsubsection{Die internen Modellkomponenten und ihre Interaktion}

Ziel eines Handlungsmodells ist die modelltheoretische Darstellung von Verhaltensstrukturen und ihren dynamisch-interagierenden Handlungsbezügen. Im Risikosport stellen sich diese Strukturen und Bezüge mannigfaltig in ihrer wechselseitigen Beeinflussung dar, so dass die Berücksichtigung aller interagierender Komponenten nur schwer zu vollziehen ist. Das Handlungsmodell des Risikosports wird daher einen idealtypischen Handlungsvollzug abbilden ohne jedoch die diversen Einflüsse auf das Risikoverhalten (Abschnitt 6.2) zu berücksichtigen. Handeln im Risikosport als Modell abzubilden bedeutet darüber hinaus immer Teilkomponenten auf ihre grundlegenden Funktionen und Strukturen zu reduzieren und dabei eventuelle Interaktionen zu unterschlagen. Das hier explizierte Handlungsmodell versteht sich daher auch eher als ein erster Versuch das Handeln im Risikosport modelltheoretisch darzustellen, ohne daraus einen Anspruch auf Vollständigkeit zu erheben. 
Im folgenden wird das Modell zunächst vorgestellt (Abb. 6.7). AnschlieBend werden die internen Modellkomponenten erläutert und ihre Interaktion untereinander beschrieben.

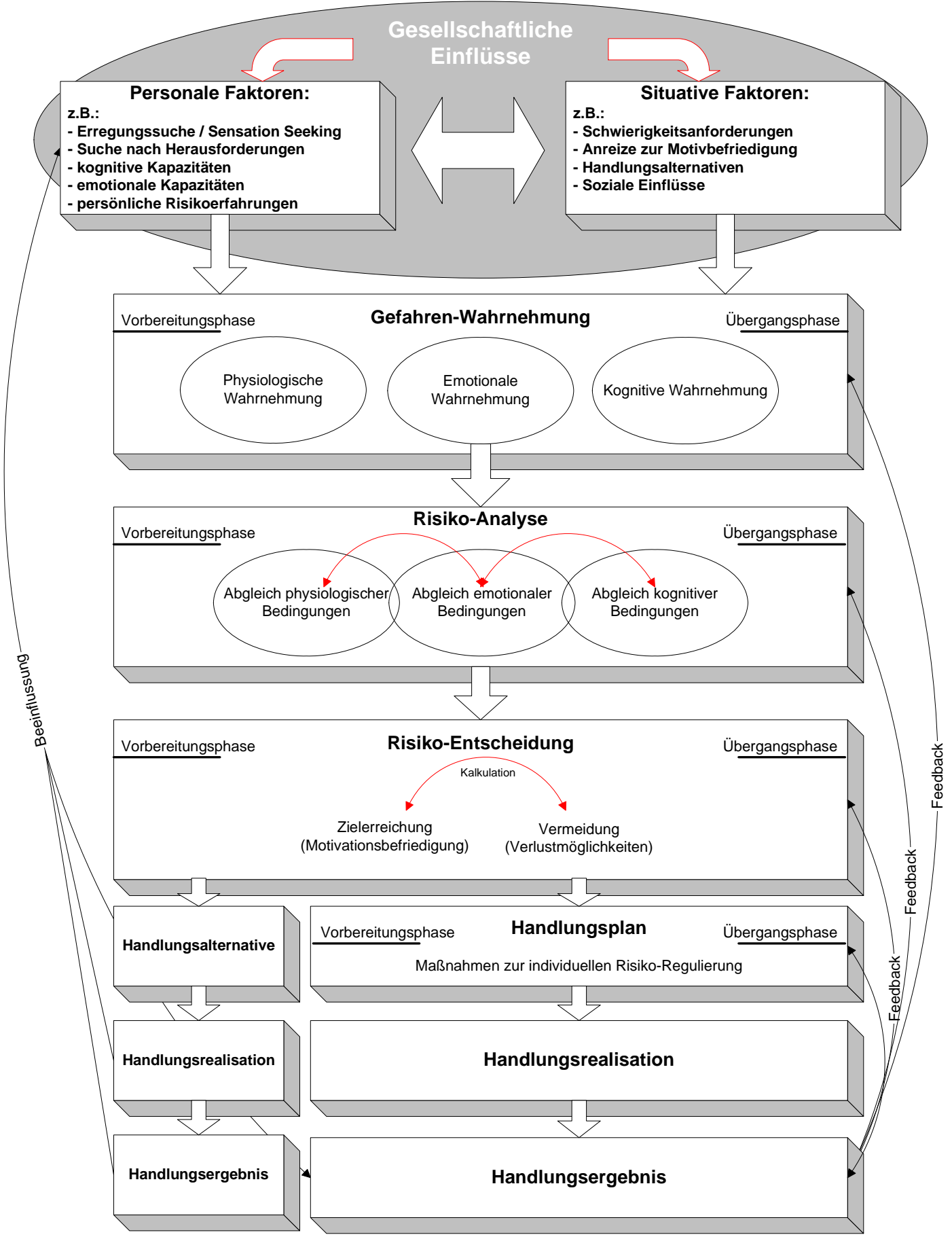

Abbildung 6.7: Handeln im Risikosport - ein integratives Modell (eigene Darstellung)

Personale Faktoren: Um das Handeln in einer risikosportlichen Situationen bestimmen zu können, ist zunächst die Persönlichkeit des Protagonisten mit all seinen Risikoerfahrungen, Motivdispositionen und physiologischpsychologischen Konstitutionen zu berücksichtigen. TRIMPOP spricht von „risk personalities“ (ebd.) und bezeichnet damit nicht nur die Motivation eine Risikosituation bewusst aufzusuchen, um seine Motivdispositionen dar- 
in zu verwirklichen, sondern auch seine durch Filter vorgenommene Fähigkeit zur Risikowahrnehmung. Je nach Persönlichkeit (z.B. Motivdisposition, individuellem Risiko-Level, emotionaler Befindlichkeit etc.) ändert sich auch die Risiko-Wahrnehmung und die sich anschließenden Prozesse. Personen, die sich im Risikosport aktiv engagieren - so wird hier unterstellt - suchen nach Herausforderungen, die eine Aktivität der eigenen Person erfordert und die positive Empfindungen bei erfolgreicher Bewältigung oder während des Handlungsvollzugs auslöst.

Die Risiko-Persönlichkeitsfaktoren (vgl. Kap. 4.3) sind in Anlehnung an TRIMPOP (ebd.) als relativ stabil und überdauernd zu betrachten, wenngleich biographische sowie situative Anpassungen zu vermuten sind.

Situative Faktoren: Die Persönlichkeits-Faktoren stehen in einer direkten Interaktion mit situativen Risiko-Faktoren, die sich aus dem Anforderungsund Aufforderungscharakter der Situation, den darin antizipierten Anreizen aber auch sozialen Einflüssen oder vorhandenen Handlungsalternativen ergeben. Die Risiko-Situationsfaktoren beeinflussen ihrerseits wiederum die Risiko-Wahrnehmung durch äußere Einflüsse wie z.B. das Wetter aber auch durch innere Einflüsse wie Stimmungen, Hoffnungen oder Ängste.

Gesellschaftliche Einflüsse: Handeln im Risikosport ist über die Beeinflussung der personalen sowie situativen Faktoren gesellschaftlichen Einflüssen direkt und indirekt ausgesetzt (vgl. Kapitel 5). Gesellschaft als Vermittlungsinstanz von Wert- und Normvorstellungen und als identitätsstiftende Kultur beeinflusst auf der personalen Ebene die Motiv- und Bedürfnislage der Protagonisten sowie auf situativer Ebene die Bedingungen diese zu verwirklichen.

Gefahrenwahrnehmung: Die Wahrnehmung von Gefahrenindikatoren und vermeintlich gefährdenden Elementen wird in der Risikosituation zum einen durch die Situation selber, zum anderen durch die die Situation wahrnehmende Person bestimmt. Die Wahrnehmung selbst vollzieht sich dabei auf unterschiedlichen intra-individuellen Ebenen, die bei Wahrnehmungsprozessen in Risikosituationen nur analytisch zu trennen sind: Physiologische Faktoren wie Herzschlag, Hormonausschüttung finden quasi automatisch statt und bestimmen die meist unbewusste physiologische Wabrnehmung. Die emotionale Wahrnehmung der Situationen lässt sich auf größtenteils unbewusst stattfindende emotionale Reaktionen, wie Angst oder Euphorie zurückführen, die ihrerseits zu einer Gesamtwahrnehmung der Handlungssituation beitragen. Auf kognitiver Ebene werden mögliche Gefahrenindikatoren mit Hilfe von Schemata oder Vergleichsprozessen zunächst wahrgenommen und den verarbeitenden Prozessen zugeführt.

Die Identifizierung von potentiellen Gefahren ist dabei kein Selbstzweck sondern dient einerseits zur Motivationsbildung und damit der Antizipation möglicher Handlungsergebnisse. Gleichzeitig werden durch die Gefahrenwahrnehmung Aktionen in Gang gesetzt, die zur Bewältigung der antizipierten Situation geeignet erscheinen.

Prozesse der Risikowahrnehmung lassen sich sowohl in der Vorbereitungsphase, also beispielsweise in einem von der Situation zeitlich und 
räumlich entfernten Zusammenhang, als auch in direkter räumlicher und zeitlicher Nähe, der Übergangsphase, nachvollziehen.

Risikoanalyse: In einem Abgleich der wahrgenommenen Gefahrenindikatoren mit den persönlichen Vorstellungen, Wünschen und Bedingungen findet schließlich eine Transformation der Gefahren in individuelle Risiken statt. Dieser Prozess der Gefahrenkognition als Integration von Wahrnehmungsprozesse in ein internes kognitives Gefahrenbild vollzieht sich vermutlich in Form einer kognitiver Repräsentation. Dabei finden auf den drei Ebenen der Wahrnehmungsfelder rückgekoppelte Soll-Ist-Vergleiche statt, wobei Interaktionen der Ebenen zu einer ganzheitlichen Bewertung der Situation führt. Gegenüber anderen Handlungsfeldern ist insbesondere die emotionale Beeinflussung der Risikoanalyse hervorzuheben:

„Without feelings of uncertainty, or a certain level of arousal, a risk will hardly be perceived. Without an emotional experience of risk, however, only an abstract cognitive decision about a bypothetical problem can be made [...].

If no arousal is noticed and no feeling experienced, a stimulus is not likely to be interpreted as 'risky' " (ebd., 245f).

Risiko-Entscheidung: Im Gegensatz zu anderen Handlungsmodellen, die Risikoentscheidungen darstellen (z.B. die ,Prospect-Theory' (WANG \& FISCHBECK 2004)), geht das hier vorgestellte Handlungsmodell für den Risikosport nicht von einem isoliert kognitiven Entscheidungsprozess aus. Die Entscheidung, eine Handlung in risikosportlichen Situationen zu realisieren - so kann als Ableitung der ersten Abschnitte hier angenommen werden - wird im Risikosport auch von emotionalen und zum Teil nicht bewusstseinpflichtigen Prozessen bestimmt. Dabei lässt sich die RisikoEntscheidung als Annährungs-Vermeidungs-Konflikt darstellen: Zielerreichung und damit Motivbefriedigung steht dem Wunsch nach Unversehrtheit und einem situativen Unbehagen gegenüber. Nur wenn die Zielerreichung (positive Empfindung als Motivbefriedigung) positiv eingeschätzt wird und es eine positive Übereinstimmung von Bewältigungsmöglichkeiten und Situationsanforderungen gibt, wird eine Handlungsrealisierung in Betracht gezogen. Überwiegen Vermeidungstendenzen, die aus einer antizipierten Uberforderungssituation erfolgen oder aus starken emotionaler Reaktionen resultieren, so wird die Suche nach Handlungsalternativen forciert. Die Risikoentscheidung ist dabei maßgeblich von personalen Faktoren - insbesondere den bisherigen Risikoerfahrungen - abhängig. Der Risikoentscheidung können sowohl die Handlungsrealisation, als auch ein Abbruch oder eine alternative Handlungsmöglichkeit folgen.

Handlungsplan: Der aus der Risiko-Analyse und der daraus resultierenden Entscheidung folgende Handlungsplan beinhaltet nicht nur eine konkrete Umsetzungsstrategie der Risikohandlung. Ziel des Handlungsplans ist die Optimierung des Handlungsvollzugs im Sinne einer optimalen Motivbefriedigung. Bei der Erstellung eines Handlungsplans wird auf gelernte Handlungsschemata und Bewegungsscripte zurückgegriffen, die im Rahmen der Risikoentscheidung im Abgleich der situativen Bedingungen als erfolgsver- 
sprechend evaluiert werden. Dazu können die in Abschnitt 6.1.6 angeführten Elemente einer ,Risiko-Regulierung' eingesetzt werden, oder Modifikationen des ursprünglichen Handlungsplans durchgeführt werden. Auch die Umsetzung der Handlungsalternative erfordert ein konkretes Handlungsskript.

Trotz der Annahme einer konkreten Plan- oder Schemagenerierung, werden auch Ungewissheiten des Handlungsverlauf als Handlungsplan abgebildet. Natürlich ist bei Handlungssituationen im Risikosport zu berücksichtigen, dass sich nicht alle Handlungsdetails vorwegnehmen lassen und eine gewisse Offenheit bezüglich der Handlungsrealisation besteht und im Sinne der Motivbefriedigung als wünschenswert erachtet wird.

Auch bei der Erstellung des Handlungsplans findet eine Fokussierung der Handlungsdetails von der Vorbereitungsphase zur Übergangsphase statt. Während ein Handlungsplan in der zeitlich entfernten Antizipation noch grobe Vorstellungen der Handlungsrealisation beinhaltet, wird in der zeitlich-räumlichen Übergangsphase ein detailliertes Handlungsschema entworfen.

Handlungsrealisation und Handlungsergebnis: Die Handlungsrealisation vollzieht sich als motorische Bewältigung der antizipierten Handlungssituation als Vollzugshandlung des Handlungsplans. Angesichts bestehender Ungewissheiten sind Abweichungen und damit Neuschematisierungen des Handlungsplans während der Handlungsrealisation keine Seltenheit im Risikosport. Insofern ist auch die Handlungsrealisation durch einen ständigen Wechsel von Orientierung, Bewertung und Entscheidung geprägt, wobei sich das individuelle Risikoverhalten immer an der bipolaren Zielerreichung zwischen Motivbefriedigung und dem Wunsch nach Unversehrtheit orientiert.

Schon während des Handlungsvollzugs finden also Feedback-Schleifen bezüglich der antizipierten Handlungsverlaufes und damit Regulationsprozesse auf allen Ebenen statt. In der Reflexion des Handlungsverlaufs und des Handlungsergebnisses in einer Post-Aktionsphase wirken diese Feedback-Prozesse sogar verstärkt auf die Evaluation der Handlung zurück. Ergebnisse gehen als Erfahrungen in sich anschließende Handlungssituationen ein und beeinflussen über das individuelle Risiko-Niveau die Wahrnehmungs- und Analyseprozesse folgender Risiko-Handlungen im Risikosport.

\subsection{Zusammenfassung und Einordnung}

Das Kapitel verdeutlicht die strukturelle und psychologische Konstitution des Risikoverhaltens im Risikosport. Die Handlungssituation im Risikosport ist dabei vor allem durch die situativ wechselnden Handlungsbedingungen geprägt, die eine dynamische Auseinandersetzung mit den objektiven Anforderungen voraussetzt.

Risikoverhalten im Risikosport ist - so legen es die Erkenntnisse der Handlungstheorie nahe - charakterisiert durch einen intensiven kognitiven, 
emotional-physiologischen Prozess der Wahrnehmung, Bewertung und Entscheidung. Ziel dieses Bewertungsprozesses ist es, die Handlung im Hinblick auf die bipolare Zielstruktur - der individuellen Motivation einerseits und dem Bedürfnis nach Sicherheit und Unversehrtheit andererseits - zu realisieren. Entsprechend des subjektiven ,Anreizcocktails' wird die Handlung somit maßgeblich über das Anreizinteresse reguliert. Der mithin falschen Charakterisierung von Risikosportlern als leichtsinnige Menschen, kann damit endgültig widersprochen werden ${ }^{215}$. Risikosportler versuchen Sicherheit gerade dadurch zu erreichen, dass sie ein Höchstmaß an Kontrolle über die als gefährlich erachteten Reizgegebenheiten zu erlangen suchen.

Anhand des ,Adventure Experience Paradigm' konnte gezeigt werden, dass die bilateralen Motiv- und Sicherheitstendenzen in einem als ,Peak adventure' gekennzeichneten Bereich - ähnlich dem ,Flow-Kanal' - im Sinne einer optimalen Herausforderung gleichermaßen befriedigt werden können. Eine Vielzahl von individuellen Regulierungsmöglichkeiten erlaubt es dem Sportler zudem, seine Handlung immer wieder als individuelle Herausforderung gemäß seinen Anreizstrukturen zu steuern.

Allerdings stehen die kognitiv-emotionalen Regulationsprozesse des Handelns im Risikosport unter verschiedenen Einflüssen. Neben persönlich bedingten Variablen, konnte zudem der Einfluss von individuellen Heuristiken und lerntheoretische Einflüsse aufgezeigt werden. Besondere Berücksichtigung muss auch sozialen Einflüssen wie Gruppenprozessen oder Anerkennungsphänomenen eingeräumt werden.

Nach APTER und CUBEs Sicherheits-Risiko-Gesetz ist das eingegangene objektive Risiko einer Situation, schließlich von einem subjektiven ,Sicherheitsrahmen' abhängig. Eine äußere Erhöhung der Sicherheit wirkt sich demzufolge nur auf die entspreche Risikotendenz aus. Das individuelle akzeptierte Risiko bleibt nach der Theorie der Risikokompensation aber letztlich konstant.

Da es sich beim Risikoverhalten um eine individuelle Reaktion auf eine subjektiv erlebte Risikosituation handelt, ergänzt KLEBESLBERG (1982) das Modell des Risikoverhaltens mit dem Modell der objektiven und subjektiven Sicherheit. Demnach ist die Sicherheit einer gesamten konkreten Gefahrensituation nur aufgrund des Verhältnisses der beiden Komponenten subjektive und objektive Sicherheit zu beurteilen. Angepasstes Verhalten in Risikosituationen setzt voraus, dass die objektive Sicherheit gleich groß oder größer ist, als die subjektive Sicherheit. Grundlegendes Problem des menschlichen Risikoverhaltens ist laut KLEBELSBERG (1982) eine Wechselbeziehung zwischen objektiven Sicherheitsmaßnahmen und subjektivem Empfinden, da bei Erhöhung objektiver ,Sicherheitsstandards' oftmals ein übermäßiger Anstieg des subjektiven Sicherheitsempfindens zu beobachten ist. Das eigentliche Risiko einer Situation wird also bestimmt durch das Verhältnis von objektiver zu subjektiver Sicherheit und damit wesentlich beeinflusst durch die beiden Verhaltenstendenzen.

Abschließend wurde das Handeln im Risikosport schließlich als Modell abgebildet, indem die bisherigen interdisziplinären Kenntnisse und empiri- 
schen Hinweise modelltheoretisch integriert wurden. Dieses Modell versteht sich als Anregung und zusammenfassende Strukturierung von auf den Risikosport übertragenen Forschungsansätzen. Es wird weiteren Forschungsvorhaben überlassen, dieses Modell zu erweitern oder - wenn sich die in diesem Kapitel aufgestellten Annahmen als falsch erweisen sollten - zu verwerfen.

Abschließend bleibt zusammenzufassen: Die Handlungsregulation im Risikosport vollzieht sich als komplexer Prozess von perzeptiv-kognitiven Vorgängen und deren emotionale Beeinflussung. Ziel des Kapitels war es von wissenschaftlichen Erkenntnisse der Handlungstheorie sowie der Verhaltenspsychologie ausgehend - einen Bezugsrahmen für pragmatische Interventionsanliegen im Risikosport zu schaffen. Die Ausführungen dieses Kapitels gelten damit als grundlegende Legitimation für die in Kapitel 8 durchgeführte empirische Studie. Um die bisherigen theoretischen Erkenntnisse zusammenzufassen wird in Kapitel 7 zunächst ein Zwischenfazit als Abschluss des theoretischen Teils vollzogen. 


\section{Zwischenfazit}

\section{Ich bätte viele Dinge begriffen, hätte man sie mir nicht erklärt. (Stanislaw Jersy Lec (1909-66), poln. Schriftsteller)}

Die bisherigen theoretische Vorgehensweise, die über einen interdisziplinären Forschungsansatz das Thema Risikosport in seinen inhaltlichen Bezügen und Zusammenhängen darstellte, erfordert - bedingt durch die Komplexität - an dieser Stelle ein zusammenfassendes Zwischenfazit. Es stellt insofern den Abschluss der theoretischen Deduktion dar.

Mit der inhaltliche Differenzierung von Risiko und Gefahr und ihre Abgrenzung gegenüber dem Begriff Sicherheit in Anlehnung an LUHMANN konnte in Kapitel 2 zunächst der terminologische Bezugsrahmen des Risikosports verdeutlicht werden. Durch die begrifflich-konzeptionelle Abgrenzung des Risikosports von anderen sportwissenschaftlichen Begriffsbestimmungen konnte zudem eine kategoriale Eingrenzung des Forschungsgegenstands vorgenommen werden. In Kapitel 3 wurde unter Berücksichtigung eines geschichtswissenschaftlichen Forschungsansatzes ein sozialhistorischer Bezugrahmen für den Risikosport rekonstruiert. Das vitale Risiko wurde hierbei als ein historischer Kompensationspunkt für eine mit Industrialisierung und Zivilisierung verbundene Moderne gekennzeichnet. In risikosportlichen Aktivitäten - so legten es die Befunde der historischer Ansätze nahe stecken Erfahrungsoptionen von besonders kräftigem, leidenschaftlichem und authentischen Erleben, die einer zunehmenden Versicherung des Alltags entgegenstehen.

Mit der Explikation relevanter Motivationselemente für den Risikosport konnte in Kapitel 4 vor allem die subjektive Seite des Risikosports erhärtet werden. Risikosport bietet - psychologischen Forschungsansätzen folgend - demnach über den ,Erregungs-Thrill' hinaus vielfältige Erfahrungsoptionen, die vor allem im au- 
thentischen Selbsterleben und - als Bewährung-in der Form einer Selbstbestätigung angesichts vermeintlicher Gefahr - seine spezifische Wirkung entfaltet. In Kapitel 5 wurde - bezugnehmend auf die soziologische Theoriebildung - eine Transformation der historischen Annahmen für die gegenwärtige Gesellschaft vollzogen. Als Handlungs- und Erfahrungsfeld einer sich ,reflexiv erneuernden Moderne' bieten risikosportliche Aktivitäten demnach über die historischen Erklärungszusammenhänge hinaus einen kompensierenden Kontrapunkt unter sehr vielfältigen strukturellen Auflagen.

In Kapitel 6 wurde schließlich der Fokus auf die konkrete Handlungsebene des Risikosports gelenkt. Dabei konnte ein komplexes Zusammenwirkung von perzeptiv-kognitiven und emotionalen Prozessen sowie vielfältige Beinflussungen auf der Ebene der kognitiven Handlungsregulation konstatiert werden

Vorherrschendes Erkenntnisinteresse der interdisziplinären Annäherung war, neben der Exploration unterschiedlichster wissenschaftlicher Perspektiven, insbesondere die Evaluation von Erklärungszusammenhängen, die Risikosport als gesellschaftliches Phänomen und individuelles Handlungsfeld zusammenhängend erläutern. Zugegeben: die wenigsten der deduzierten Ansätze sind explizit für den Risikosport empirisch überprüft worden, dennoch kann Risikosport auf der Basis der theoretischen Deduktion nun besser erklärt und vor allem erfasst werden. Das Verständnis des Risikosports dies lässt sich wohl an dieser Stelle nachdrücklich sagen - erschließt sich insbesondere durch ein den gesellschaftlichen Konventionen bisweilen widersprechendes Verständnis von Risiko, Sicherheit und Gefahr. Der Risikosportler sucht in der bewegungsvermittelten Herausforderung eben nicht die Gefahr, was einem Spiel mit dem Schicksal nahe kommen würde, sondern ein auf seine Person zugeschnittenes Risiko und die damit verbundene starke Intensität des Erlebens. Der Mensch - so wird im Risikosport transparent - ist demnach kein monokausales Sicherheitswesen, sondern im Sinne einer erhöhten Handlungsgratifikation auch an Risikosituationen interessiert. Zweifelsfrei ist der Mensch im allgemeinen ein vorsichtiges Wesen ist, welches sich nach Schutz und Geborgenheit sehnt, auf der anderen Seite aber empfindet er offensichtlich auch Lust und Freude an Risiko und Unsicherheit. Dieses Verhältnis zwischen Risiko und Sicherheit ist - so muss konstatiert werden dabei aufeinander bezogen, denn je bedeutungsvoller es für den Menschen ist, sich abzusichern, desto notwendiger erscheint es zugleich, dass er etwas riskiert: , ,...] nur wenn er sein Leben einzusetzen wagt, wird er es gewinnen. Wenn er es dagegen ängstlich festzuhalten versucht, wird er es in Wirklichkeit verlieren“" (ELSÄSSER 1979, 72).

Die wissenschaftliche Auseinandersetzung mit dem Forschungsgegenstand Risikosport verlangt also, von gebräuchlichen Sicherheitskonventionen abzurücken und eine Differenzierung von Risiko, Sicherheit und Gefahr voranzutreiben, die über den wissenschaftlichen Status Quo hinausgeht. Notwendig ist zudem die Erkenntnis, dass es sich bei dem Bedürfnis nach Sicherheit und der Suche nach Risiko nicht um zwei absolute Gegensätze - im Sinne eines ,entweder / oder' - handelt, sondern um ein Spannungsfeld mit 
zwei nahezu gleichwertigen Polen. Der Mensch präsentiert sich in diesem Zusammenhang als bipolar strukturiertes Wesen, welches sich ganzheitlich nur entfalten kann, wenn und solange dieses bipolare Spannungsverbältnis zwischen Sicherheits- und Risikotendenzen lebendig gehalten werden kann bzw. einigermaßen ausgewogen gestaltet ist. Dieses Bedürfnis nach Sicherheit und Planung wird in der Gesellschaft indessen in einem so hohen Maße abgedeckt, dass für die Suche nach Risiken im zivilisierten Leben kaum noch Platz bleibt. Der Sport und insbesondere der Risikosport - dies konnte anschaulich deduziert werden - erfüllt damit die Funktion einer gegen-poligen Kompensation.

Natürlich - auch das muss hier angeführt werden - repräsentieren Risikosportarten nur eine von vielen Möglichkeit, die bipolare Grundstruktur des Menschen zu kompensieren. APTER (1994) und SEMMLER (1994) verweisen in diesem Zusammenhang darauf, dass grundsätzlich alle Menschen Risikosucher seien - ,[...] auch wenn sich dies nur in Form einer Sensations- und Katastrophen-Lüsternheit zeigt" (HUBER 1994, 67). Neben dem tagtäglichen passiven ,„[...] Rausch der Erregung vor dem Bildschirm und Kinoleinwand" (ebd.) lassen sich nach APTER (1994) auch sexuelle Abenteuer, Experimente mit Drogen und Alkohol oder das Verhalten im Straßenverkehr als Risikosuche bezeichnen (vgl. APTER 1994, 13f). Andererseits stellen die beschriebenen Anreize des Risikosports (vgl. Kapitel 4) eine besonders existenzielle Form der Sinnvergewisseerung dar, die neben dem reinen Erregungswechsel auch die beschriebenen Möglichkeiten zur Identitätsbildung und Selbsterfahrung bietet.

Es ist wissenschaftlich ungewiss, welchen Stellenwert dieses bipolare Spannungsfeld für die Entwicklung des Menschen besitzt, APTER (1994) sieht in dieser ambivalenten Veranlagung jedoch den entscheidenden Schlüssel zum Glücklich-Sein. Er vermutet, dass jene Menschen am intensivsten leben, denen es gelingt ,[...] beide Zustände ausgewogen zu erleben und zwischen ihnen im jeweils richtigen Moment zu wechseln" (APTER 1994, 112). Ähnlich argumentiert auch HECKER (1991), der konstatiert: „Wer im Leben glücklich sein will, muss ein bestimmtes Maß an Unsicherheit nicht nur akzeptieren, sondern sogar lieben“ (HECKER 1991, 225). Zugegeben: für den wissenschaftlichen Erkenntnisprozess bleiben diese Annahmen bis dato unbestätigte Spekulationen, wenngleich diesen Aussage introspektiv durchaus zugestimmt werden kann.

Die empirische Studie, die im Rahmen dieser Arbeit durchgeführt wurde, setzt an den grundlegenden Erkenntnissen der interdisziplinären Annährung an, wenngleich mit dem Fokus auf Prozesse der Gefahrenkognition die phänomenologische Ebene von Risiko und Sicherheit verlassen wird. Im folgenden Kapitel 8 wird die empirische Untersuchung in ihren konkreten theoretischen und methodischen Grundlagen vorgestellt. Darüber hinaus werden die Ergebnisse dargestellt und diskutiert. Die sich anschließende Ableitung der empirischen Befunde im Rahmen eines Interventionskonzeptes im Risikosport greift schließlich beide Ebenen des Erkenntnisprozesses wieder 
auf und versucht im Rahmen eines Anwendungsbezugs den theoretischempirischen Schulterschluss. 


\section{Empirische Befunde und Anwendungsbezüge}

In den bisherigen Kapiteln wurden grundlegende Zusammenhänge und inhaltliche Bezüge des Risikosports aufgedeckt. Ziel der interdisziplinären Vorgehensweise war die umfassende Evaluation und Darstellung von individuellen und strukturellen Bedingungen des Risikosports in der modernen Gesellschaft. Im Hinblick auf einen praktischen Anwendungsbezug wurde von den theoretischen Darstellungen ausgehend - ein Handlungsmodell für risikosportliche Aktivitäten in Kapitel 6.3 entworfen. Darauf aufbauend soll in diesem zweiten Teil der Arbeit die Ambiguität des Risikos im Sport in seiner wechselseitigen Beziehung zur Sicherheit am Beispiel des Unfallgeschehens in ausgewählten Risikosportarten untersucht werden. Bezug nehmend auf die handlungstheoretischen Zusammenhänge des voranstehenden Kapitels wird in diesem Abschnitt anhand einer empirischen Studie nachgeprüft, inwieweit sich Gefahrenkenntnis und Gefahrenkognition als Handlungskonstitutiva für ein Modell der Unfallprävention anbieten. Am Beispiel des Kanusports und des alpinen Bergsports wird der Frage nach den Unfallursachen im Risikosport mit Hilfe einer sicherheitspsychologischen Untersuchung nachgegangen. Dabei wird in Anbetracht der zusammenhängenden Erkenntnisse über das Spannungsfeld von Risikosuche und Sicherheitsbedürfnis insbesondere die Gefahrenkognition - also die „,[...] Gesamtheit aller Informationen, Assoziationen und Redundanzen über Gefahren“" (MUSAHL 1997, 97) - in der sportlichen Risikosituation berücksichtigt.

Modelle zur Unfallvermeidung im Risikosport sind zur Zeit noch wenig etabliert, wenngleich es in den letzten Jahren zu einer ersten Auseinandersetzung mit dieser Thematik gekommen ist. Eine sportwissenschaftliche Unfalltheorie ist zudem wenig evaluiert. Im Rahmen der Deduktion theoretischen Grundlagen in Kapitel 8 erfolgt daher zunächst in eine Übersicht über den aktuellen sportwissenschaftlichen Forschungsstand zur Sportunfallforschung (8.1). Eine theoretische Annäherung an die Grundlagen der Unfall- und Sicherbeitsforschung (8.2) schließt sich über die inhaltliche Auseinandersetzung mit dem Unfallbegriff und verschiedenen theoretischen Ansätzen der Unfallforschung in Abschnitt (8.3) an.

In Kapitel 9 wird zunächst die konkrete Aufgabe der Untersuchung (9.1) und der methodologische Ansatz der Studie wird in Abschnitt 9.2 vertieft. Die Ergebnisdarstellung (9.3), die Diskussion der Untersuchungsergebnisse (9.4) und ein Untersuchungsfazit (9.5) schließen das Kapitel ab.

In Kapitel 10 wird auf der Grundlage der theoretischen Erkenntniszusammenhänge und den Ergebnissen der empirischen Studie schließlich ein Interventionskonzept für den Risikosport vorgestellt. 


\section{Theoretische Grundlagen der Unfall- und Sicherheitsforschung}

Die einen, so scheint mir, haben viele Werkzeuge und wenig Ideen; die anderen haben viele Ideen und gar keine Werkzeuge. Das Interesse der Wahrheit würde verlangen, daß die Denkenden sich endlich dazu herbeilassen, sich mit den Schaffenden zu verbünden.. (Denis Diderot (1713-84), frz. Philosoph u. Schriftsteller d. Aufklärung)

Um eine differenzierte Sicherheitsstudie für das Verhalten im Risikosport allgemein - und speziell bei bestimmten Risikosportarten - vorzunehmen, ist zunächst eine vertiefende Auseinandersetzung mit den theoretischen Hintergründen der Unfall- und Sicherheitsforschung erforderlich. Einer solchen theoretischen Auseinandersetzung kommt insofern besondere Bedeutung $\mathrm{zu}$, da theoriegeleitete Untersuchung über das Unfallgeschehen im Sport bis dato selten sind ${ }^{216}$. Einleitend wird hierzu zunächst ein Überblick über den sportwissenschaftlichen Forschungsstand gegeben und der Unfall- 
begriff inhaltlich und konzeptionell diskutiert. Nachfolgend werden die insbesondere von RÜMMELE deduzierten Theorieansätze der Unfallforschung und ihre Erweiterung zu sicherheitspsychologischen Forschungsmodellen in ihren zentralen Paradigmen dargestellt.

\subsection{Unfallforschung in der Sportwissenschaft}

Der Sportunfall als sportwissenschaftlicher Forschungsgegenstand ist spätestens seit der umfassenden Arbeit von RÜMMELE (1988) ein explizites Thema der Sportwissenschaft. Das von dem Autor konstatierte sportwissenschaftliche Forschungsdesiderat ist fast zwei Jahrzehnte später zwar deutlich $\mathrm{zu}$ relativieren ${ }^{217}$, wenngleich eine umfassende Forschungssystematik weiterhin aussteht (vgl. hierzu auch PFITZNER 2001; AMESBERGER 2002).

Neben dem Bemühen das Unfallgeschehen im Sport theoretisch zu fassen $^{218}$, ist die Auseinandersetzung mit Unfällen im Sport vor allem durch zahlreiche empirische Studien geprägt. Bereits seit den 1960er Jahren lassen sich Untersuchungen unter Verwendung unterschiedlicher Methoden für viele Einzel-Sportarten finden. Dabei sind nur wenige dominante Forschungsansätze zu identifizieren. Neben dem epidemiologischen Ansatz in der Sportunfallforschung finden sich Einzelfallstudien und - in neueren Studien im Bereich der Schulsportforschung - auch mehrdimensionale Ansätze. ${ }^{219}$

Die Epidemiologie als Forschungsansatz in der Sportwissenschaft entstammt ursprünglich der Medizin und befasst sich mit der Entstehung und Ausbreitung von Krankheiten. Die Anwendung epidemiologischer Ansätze im Bereich der Sportunfallforschung wurde in unterschiedlichen Facetten und unter Anwendung verschiedener Methoden vor allem im Bereich des Schulsports sowie des organisierten Vereinssports betrieben. Neben deskriptiven epidemiologischen Studien, die die Anzahl und Verteilung von Unfällen beschreiben sind auch wenige analytische epidemiologische Untersuchungen bekannt. Hierbei werden aufgrund der in der deskriptiven Epidemiologie ermittelten Ergebnisse Hypothesen formuliert, die anhand kausaler Faktoren erklärt werden sollen (vgl. HOYOS 1980, 353ff). Mit epidemiologischen Studien wird die Zielsetzung verfolgt, auf Unfallschwerpunkte hinzuweisen und mögliche Gründe für das Unfallgeschehen aufzudecken, wobei „,...] die Aussagekraft der Ergebnisse epidemiologischer Forschungsansätze stark von der Qualität der sekundäranalytisch verwerteten Quellen abhängt" (PFITZNER 2001, 152). In der Mehrzahl greifen epidemiologische Studien Versicherungsdaten auf ${ }^{220}$, vereinzelt finden sich auch sekundäranalytisch verwendete Arztberichte und Krankenhausberichte ${ }^{221}$.

Einzellfallstudien stehen den quantitativ und massenstatistisch orientierten epidemiologischen Studien konträr entgegen. Hierbei wird auf der Basis von qualitativen Interviews mit Verunfallten oder am Unfall beteiligten nach Hintergründen und Ursachen der Unfallentstehung geforscht ${ }^{222}$. Im Mittelpunkt steht das Unfallgeschehen im Einzelfall und ihre Korrelation zu Le- 
bensereignissen, biographischen Zusammenhängen oder auch sozialen Bezügen (vgl. HARTGEN 1997).

Mehrdimensionale Ansätze der Sportunfallforschung verknüpfen epidemiologische Studien mit Einzellfallanalysen, Videoanalysen oder anderen Befragungsinstrumenten. In methodisch sehr aufwendigen Verfahren haben u.a. RÜMMELE (1988) und ALTENBERGER (1994) sowie die Forschungsgruppe um HÜBNER \& HUNDELOH (1997) mehrdimensionale Ansätze verfolgt. Der Unfall wird dabei als ,[...] hochkomplexes Geschehen, das ein ihm adäquates (multivariantes) Forschungsprogramm fordert [...]“ (KLEINER et al. 1990, 42) definiert. Ziel des von den Autoren gewählte Forschungsdesign ist die Berücksichtigung miteinander kombinierter Verfahren, die ,[...] sowohl die phänomenale subjektive Wirklichkeit, [...] als auch die für alle gleich erkennbare physikalische Wirklichkeit“ (ebd., 50) erfassen. Insgesamt weisen die Studien allerdings nur äußert geringe Fallzahlen und einige methodische Kritikpunkte auf, so dass die Aussagekraft der ermittelten Ergebnisse durchaus kritisch zu beurteilen ist. Allein der von der Forschungsstelle „Mehr Sicherheit im Schulsport“ seit 1988 an der Universität Münster entwickelte ,forschungsstrategische Mittelweg' verknüpft verschiedene Verfahren der Unfallforschung auch mit einer repräsentativ hohen Fallzahl (vgl. u.a. HÜBNER \& MIRBACH 1991).

Der Hauptgegenstand der sportwissenschaftlichen Forschungsbeiträge über Unfälle im Sport liegt im Bereich der Schulsportforschung, wobei auch hier epidemiologischen Studien dominieren (vgl. SCHARRFE 1999). Daneben existiert eine Vielzahl allgemeiner vergleichender Studien über das Unfallgeschehen in verschiedenen Sportarten (aktuell z.B. bei BRÜGGER 2004 oder HENKE 2004). Das Ziel der Unfallprävention wird hierbei über die Analyse von Unfallschwerpunkten und Verletzungsstatistiken verfolgt. Im Bereich des Risikosports existieren epidemiologische Studien für den alpinen Bergsport (z.B. SLADEK 2003), für den allgemeinen Bereich Wintersport (BURTSCHER 2003; WÜRTL 2003) sowie einzelne Beiträge der Sportfachverbände (z.B. Deutscher Alpenverein, Deutscher Kanu-Verband, Verband der Hanggleiter; etc.). Mehrdimensionale Ansätze finden sich bei MUNTER (1999) für den alpinen Tourenskilauf und für das Klettern in künstlichen Anlagen bei SCHWIERSCH et al. (2004). Einzelne Beiträge haben sich zudem mit Materialfragen im Zusammenhang mit der Unfallentstehung im Sport auseinandergesetzt (z.B. bei BRÜGGER 2003) oder die kognitive Repräsentation über Unfälle untersucht (zusammenfassend bei RÜMMELE 1993).

Da eine präzise Klärung des Unfallbegriffs als zentraler Gegenstand der sportwissenschaftlichen Unfallforschung für ein weiteres Vorgehen notwendig ist, werden nachfolgend die theoretischen Grundlagen der Unfallforschung deduziert, um die gebrauchten Begrifflichkeiten zu bestimmen und den terminologischen Rahmen der eigenen Untersuchung zu erläutern. 


\subsection{Der Unfall: Terminologisch-konzeptionelle Ausei- nandersetzung mit dem Gegenstand der Unfall- und Sicherheitsforschung}

Unter einem Unfall ist im allgemeinen Sprachgebrauch ein seltenes Ereignis mit unerwünschten Folgen zu verstehen, wobei der Unfall eine plötzliche Begebenheit bezeichnet, wohingegen die Erkrankung - die ebenfalls unerwünschte Folgen mit sich bringt - eher langsam zu Schädigungen führt. Die allgemeinen und gebräuchlichsten Definitionen erfassen den Unfall anhand seiner Folgen ${ }^{223}$. Nach SKIBA (1973)

„[...] ist der Unfall das plötzliche, ungewollte, eine Körperverletzung bewirkende Zusammentreffen eines Menschen mit einem Gegenstand" (SKIBA 1973, 4).

Die Akzentuierung von Gegenständen in der Unfalldefinition wird allerdings bereits bei HOYOS relativiert. Demnach resultieren Unfälle ,,[...] aus einer Wechselbeziehung und Wechselwirkung von Mensch und Gefahr“" (HOYOS 1980, 24), wobei Gefahr als unabhängig vom Handelnden betrachtet wird. Die Gefährdung entsteht schließlich mit dem Zeitpunkt des Wirksamwerdens einer Gefahr in der Interaktion von Subjekt und Objekt ${ }^{224}$ (vgl. KIRCHNER 1996, 401). Der Autor weist in diesem Zusammenhang darauf hin, dass der Unfall selbst terminologisch nur das Ereignis und seinen Ablauf bezeichnet, während die aus dem Unfall resultierenden Schädigungen als Unfallfolgen bezeichnet werden sollten. Wegen dieser Unfallfolgen, die in der Regel unerwünscht sind, interessiert man sich in der Unfallforschung auch für die Unfallursachen und -Bedingungen (vgl. ebd., 399ff).

In Anlehnung an COMPES (1992) sind an den Unfallbegriff unterschiedliche Anforderungen zu stellen. Neben der Funktion eines Terminus muss der Unfall auch konzeptionelle Bedeutung besitzen, denn

„[...] neben der Aufgabe des Begriffes, der mit Unfall etwa zur Beschreibung, Mitteilung oder zur Abgrenzung gegen andere Ereignisse ibn treffend und umfassend zu benennen, kommt die Forderung an einen Begriff hinzu, dass er in das Gedankengebäude einer Methodik der Unfallursachen -Forschung undVerbütung passen, dessen ,Bausteine’ liefern muss"(COMPES 1992, 21).

Terminologisch werden Unfälle bei COMPES - angelehnt an SKIBA und HOYOS - als ein Ereignis mit plötzlichem Eintritt und schädlichen Folgen als systemischer Terminus definiert. Der Unfall als Begriff wird hierbei kasuistisch als einzelnes Ereignis in einem Mensch-Umwelt-System begriffen.

Ähnlich auch bei HOYOS: hier resultiert ein Unfall aus einem ,,[...] Versagen gegenüber der Gefahr, das durch Wahrnehmung, Entscheidung und Handlung, also durch psychische Prozesse des Betroffenen herbeigeführt wird“" (HOYHOS 1980, 24.) ${ }^{225}$. Konzeptionell fassen HOYOS und COMPES den Unfall also als Ereignisfolge, welche aus Ereignissen vor dem 
eigentlichen Unfall, dem Unfall selbst und aus Ereignissen nach dem Unfall mit dem Unfallschaden als resultierende Komponente besteht.

Der Sportunfall als eine spezielle Unfallform wird bei WEHMEYER (1992) als unkontrolliertes und ungesichertes Freiwerden von Energie definiert, detailliert als ,[...] Störung des Normalablaufs durch Kollision, wobei das Fähigkeitsprofil durch die Komplexität und Dynamik der Anforderungsbedingungen überfordert wird“" (WEHMEYER 1992, 464). Nach MIRBACH (1995) kann von einem Sportunfall gesprochen werden,

„[...] wenn durch äußere und/oder innere Störfaktoren eine ungewollte, plötzliche Abweichung vom geplanten Ablauf einer sportlichen Situation hervorgerufen wird und es dabei zur Verletzung einer anwesenden Person kommt" (MIRBACH 1995, 47).

Für risikosportliche Handlungen definiert MÜCKE (1983) für das KanuWildwasserfahren einen Unfall im engeren Sinn ,[...] als ein Ereignis, bei dem ein Kanute ursächlich im Zusammenhang mit der Befahrung eines Wildbaches einen Schaden erleidet, wobei das Schadensereignis zumindest subjektiv erwartungswidrig vom Handlungsziel abweicht" (MÜCKE 1983, 14). Damit wird der Wildwasserunfall deutlich von anderen Unfallereignissen abgegrenzt, wie z.B. dem Ausrutschen am Ufer oder Schnittverletzungen an einer Scherbe und damit auf die Kernhandlung beschränkt. Im Rahmen dieser Arbeit wird diese Terminologie beibehalten und der Unfall im Risikosport im Rückgriff auf SKIBA als ungewolltes, einen Personenschaden bewirkendes Ereignis bezeichnet, welches im direkten Handlungszusammenhang der auszuführenden Sportart steht.

Es ist in Anlehnung an TAYLOR (1976) davon auszugehen, dass dem Menschen eine, axiomatische' Absicht zu postulieren ist, unfallartige Ereignisse generell zu vermeiden (vgl. HOYOS 1980, 27). Wenngleich psychoanalytische Forschungsansätze bisweilen von einer ,Selbstschädigung' (FREUD) ausgehen, so wird im Rahmen dieser Arbeit angenommen, dass ein sicherer Ablauf von Ereignissen und Handlungen zu den ausgesprochenen Zielsetzungen des Menschen gehört, was in der Betonung der Sicherheitstendenz bei der Handlungsregulation in Risikosportarten zum Ausdruck kommt (vgl. Kapitel 6).

Insbesondere für sicherheitspsychologische Untersuchungen bedeutend sind auch sogenannte ,Beinahe-Unfälle', die sich vom Unfall nicht aufgrund verschiedener Gefährdungsbedingungen unterscheiden, sondern lediglich darin, dass die Unfallfolgen ausbleiben (vgl. RÜMMELE 1988, 25). Es ist anzunehmen, dass die Analyse von Unfällen nur einen Ausschnitt von sicherheitswidrigen Verhalten abbildet und Verhaltensfehler sowie Systemstörungen nur über die Integration von Beinahe-Unfällen vollständig erfasst werden können. Dabei bleibt zunächst unklar, ob Beinahe-Unfälle von den Akteuren immer wahrgenommen werden (vgl. auch MUSHAL 1997). 


\subsection{Theoretische Ansätze der Unfallforschung}

Im Bereich des Sport finden sich nur wenige theoriegeleitete Untersuchungen, die ein Unfallgeschehen im Sport konzeptionell auf modelltheoretische Ansätze der Unfallforschung zurückführen. RÜMMELE hat sich diesem Desiderat der sportwissenschaftlichen Unfallanalysen angenommen und aus den Ansätzen einer interdisziplinären Unfallforschung vier Theorien für einen systematischen Forschungsansatz der Unfallforschung klassifiziert. Neben persönlichkeitszentrierten Ansätzen konstatiert der Autor Interaktionstheorien, systemtheoretische Ansätze und Handlungstheorien. Die einzelnen Forschungsansätze stellen dabei den jeweiligen theoretischen Rahmen für eine idealisierte Beurteilung von Sportunfällen und bestimmen darüber hinaus das jeweilige methodische Vorgehen der Untersuchungen (vgl. RÜMMELE 1988, 58). Es ist diesbezüglich zu bemerken, dass es in der Praxis der Unfallforschung zu einer Vermischung und Kombination der theoretischen Ansätze kommt, so dass die folgenden theoretischen Annahmen nur bedingt forschungsleitende Paradigmen repräsentieren.

\subsubsection{Persönlichkeitszentrierte Ansätze}

Ausgehend von der Annahme, dass sich die überwiegende Anzahl von Unfällen auf Verhaltensfehler zurückführen lassen, werden in der psychoanalytisch ausgerichteten Unfallforschung die verunfallte Personen in den Mittelpunkt einer persönlichkeitszentrierten Unfalltheorie gestellt. Der Rückgriff auf psychoanalytische Paradigmen in Anlehnung an FREUD wird dabei mit motivationspsychologischen Ansätzen kombiniert (vgl. HARTGEN 1998, 19f).

Die Existenz von sogenannten ,Unfallpersönlichkeiten' - auch als ,Unfäller' bezeichnet - wird dabei in der Unfallforschung kontrovers diskutiert. Eine Person, die Unfälle erleidet oder verursacht, ist demzufolge mit gewissen Disposition ,ausgestattet', die eine Entstehung von Unfällen fördern (vgl. HOYOS 1980, 149). Vielfach werden bestimmte Persönlichkeitsmerkmale beschrieben, die mit einer individuellen Unfallneigung (,accident proneness') scheinbar korrelieren (vgl. HARTGEN 1997, 15f).

In eher motivationspsychologischen Untersuchungen werden leistungspsychologische Determinanten im Bezug auf eine allgemeine Unfallneigung untersucht (z.B. bei MITTENECKER 1981). Da die vermeintlichen Unfalldispositionen jedoch nur schwer empirisch erfassbar sind, haben sich persönlichkeitszentrierte Studien vor allem für sportliche Kontexte nicht durchgesetzt. Zudem scheint es keinen einheitlichen Persönlichkeitstyp zu geben, von dem gesagt werden kann, dass diese Menschen, ,[...] und zwar alle diese und nur diese eine erhöhte Unfallneigung haben" (HARTGEN 1998, 13).

Trotz dieser allgemeinen Kritik haben sich insbesondere im angelsächsischen Forschungsraum personenzentrierte Ansätze etabliert. Für risikosportliche Kontexte haben PRIEST \& GASS (1997) diesen Ansatz für eine Unfalluntersuchung angewendet und für den Umgang mit sportlichen Risi- 
ken neun verschiedene Persönlichkeitstypen beschrieben, die in unterschiedlicher Weise mit Risiken umgehen und daher unterschiedlich stark unfallgefährdet sind. Demnach überschätzt die als, angstlos und arrogant beschriebene Person die eigenen Fähigkeiten eher, als die ,schüchterne und ängstliche’ Persönlichkeit (vgl. PRIEST \& GASS 1997, 47). Eine empirische Überprüfung dieses Modells steht jedoch noch aus.

In diesem Zusammenhang sind auch die von SPIELBERGER (1966) und SCHÖNPFLUG (1989) konstatierten individuellen Ängstlichkeitsniveaus $\mathrm{zu}$ berücksichtigen, die ebenfalls einen persönlichkeitsspezifischen Umgang mit dem sportlichen Risiko bestimmen und sich als Unfalleigenschaften klassifizieren lassen. Beide Ansätze beschreiben aber lediglich Persönlichkeitsmerkmale, die im Sinne klassischer trait-orientierter Theoriebildung davon ausgehen, dass ,,[...] je nach Abstraktionsniveau nur eine oder mehrere Persönlichkeitsvariablen oder damit kovariierende Konstrukte postuliert und identifiziert werden müssen, um ihnen einen Zusammenhang zu Unfällen zuschreiben zu können“ (RÜMMELE 1988, 66).

Das Verwendungsinteresse persönlichkeitszentrierter Ansätze für eine Unfallanalyse im Risikosport ist im Rahmen dieser Arbeit gering, da hier ein konsistentes Verhalten in verschiedenen Situationsklassen vorausgesetzt wird. Darüber hinaus erscheint die operationalisierbare Erfassung von so genannten Unfalldispositionen als schwierig, da konvergierende Validitätsversuche häufig misslingen (vgl. auch RÜMMELE 1988, 66).

\subsubsection{Interaktionistische Ansätze}

Interaktionistische Ansätze der Unfallforschung treten persönlichkeitszentrierten Ansätzen mit ihrer Dichotomie zwischen Person und Situation entgegen. Interaktionistische Unfallforschung beschreibt die Suche nach situativen Problemen zwischen der Person und seiner Umwelt. Schon bei NEULOH (1957) wird der Unfall als eine „,...] Störung des Normalblaufs“, als „Zusammenwirken innerer und äußerer Ursachenfaktoren technischer, physischer, psychischer und sozialer Natur“ (NEULOH 1957, 48) beschrieben. Auch die von KLEBELSBERG (1969) oder WILDE (1978) formulierten Theorien der subjektiv-objektiven Sicherheit bzw. der Risikokompensationstheorie kennzeichnen frühe interaktionistische Ansätze. Dabei wird die Person-Umwelt-Beziehung in erster Linie als kognitiv-evaluativer Vermittlungsprozess begriffen.

In modernen interaktionisitischen Ansätzen wird diese Perspektive um handlungs- und systemtheoretische Ansätze ergänzt (vgl. RÜMMELE 1988, 71). Nicht nur die Situationswahrnehmung sondern auch die zur Verfügung stehenden Verarbeitungs- und Bewältigungsmöglichkeiten werden in so genannten transaktionalen Analysen berücksichtigt. Im Blickpunkt stehen hierbei die personalen Vorgänge der Situationswahrnehmung und die der Person zur Verfügung stehenden Verarbeitungs- und Bewältigungsstrategien. Bereits in der frühen Unfallforschung bei SHEDLETSKY \& 
ENDLER (1974) wird in der Situationswahrnehmung der entscheidende, das Verhalten bestimmende Faktor gesucht:

\section{„Die Intensität (eines Verbaltens) ist proportional zum Ausmaß der wahrge- nommenen Bedrobung "(SHEDLETSKY \& ENDLER 1974, 513; zit. nach RÜMMELE 1988, 71).}

Unfälle ereignen sich demnach aus einer fehlerhaften Wahrnehmung und Verarbeitungen von situativen Anforderungen im Abgleich mit personalen Bewältigungsmöglichkeiten (vgl. auch HOYOS 1980). Dieser Ansatz entspricht dem in Abschnitt 6.3 entwickelten transaktionalen Handlungsmodell des Risikosports. Das Verwendungsinteresse interaktionaler Ansätze im Rahmen der Unfallanalysen dieser Arbeit ist dementsprechend hoch.

Die Unterscheidung von personenbezogenen und umweltbezogenen Determinanten ist dabei bei verschiedenen Studien unterschiedlich gewichtet und im Bezug auf die empirische Überprüfung mittels verschiedener Variablen operationalisiert worden (vgl. im Überblick RÜMMELE 1988). Insbesondere für Unfallanalysen im Sport sind interaktionistische Ansätze vermehrt herangezogen worden (vgl. BAUMANN 1992). Allerdings ist die vollständige Interaktion multivariabler Handlungssituationen nur bedingt empirisch überprüfbar, so dass allgemeingültige Unfalltheorien bis dato nicht vorliegen und auch für die Zukunft nicht zu erwarten sind.

\subsubsection{Systemtheoretische Ansätze}

Systemtheoretische Ansätze der Unfallforschung vollziehen sich unter der Annahme einer komplexen Geschlossenheit von Unfallereignissen. Ein System ist dann eine geordnete Einheit, wenn es aus Subsystemen oder Elementen besteht, die durch Relationen miteinander in Verbindung stehen und zu einer Struktur verknüpft sowie auf einem bestimmten Niveau von seiner Umwelt abgrenzbar sind. Darüber hinaus wird eine funktionale Verknüpfung der einzelnen Systemelemente postuliert, d.h. ein System weist gewisse Funktionen als Beziehungen zwischen bestimmten Attributen auf (vgl. im Bezug zur Unfallforschung RÜMMELE 1988, 78).

In systemtheoretischen Unfallkonzepten sind die einzelnen Elemente des zu untersuchenden Systems nicht isoliert voneinander zu betrachten. Neben der strukturellen Ebene werden dabei auch funktionelle Abläufe und Prozesse berücksichtigt, die auf einer hierarchischen Ebenen wiederum geordnet werden (vgl. im Überblick auch HOYOS 1980). Die Verwandtschaft zu interaktionistischen Ansätzen ist dabei immanent.

Systemtheoretische Unfallanalysen reduzieren den Unfall nicht auf eine Folge menschlichen Verhaltens, sondern berücksichtigen die Handlung als „eine Anordnung von Komponenten, die miteinander verbunden sind und in bestimmten Wechselbeziehungen stehen, um eine Aufgabe zu erfüllen [...]“ (HOYOS 1987, 585).

Dieses systemtheoretische Modell entspricht dem Gedankenmodell der Unfallgefährdung von SKIBA (1973), der ebenfalls von einer systemorien- 
tierten Gefährungsbereich ausgeht. PRIEST \& GASS (1997) nehmen sogar an, dass, je bedeutender die Wechselbeziehung zwischen den Gefahren der einzelnen Teilsysteme ist, desto größer wird auch die Wahrscheinlichkeit eines Unfalls sein (vgl. ebd., 89) ${ }^{226}$.

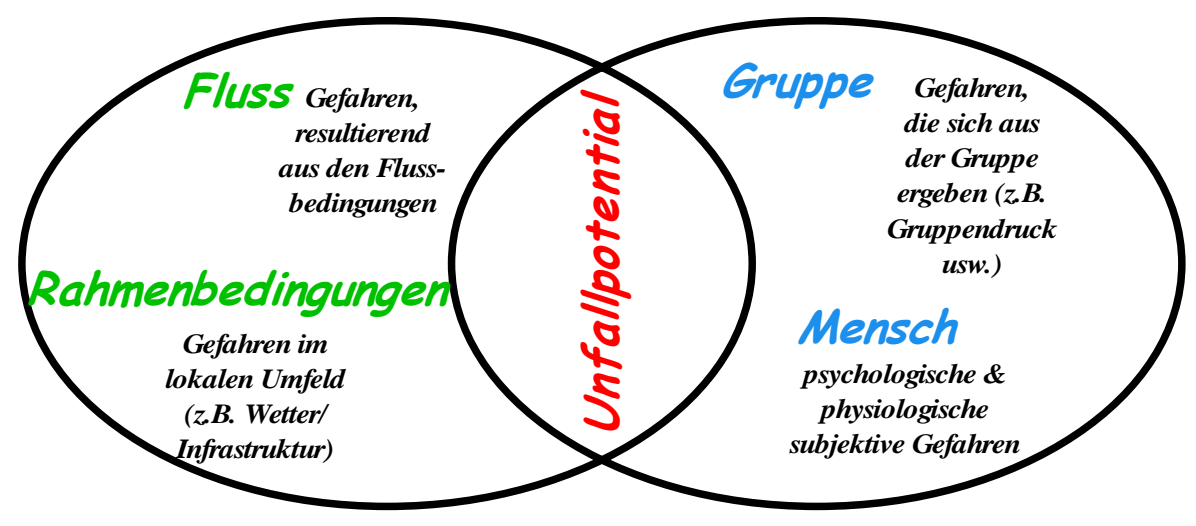

Abbildung 8.1: Risikosport als System am Beispiel des Kanu-Fahrens. In Anlehnung an die 'Accident Theory'; verändert und erweitert nach PRIEST \& GASS 1997, 89.

Es ist der Verdienst systemtheoretischer Unfallstudien, auf die Bedeutung von Systemgrenzen hingewiesen zu haben und monokausale Erklärungsprinzipien zu differenzieren.. Insbesondere diese Abgrenzung von Handlungsbedingungen stehen hierbei in einem wichtigen Verwendungszusammenhang. Sowohl bei zu kleiner, als auch bei zu großer Definition des Handlungssystems, kann es zu fehlerhaften Erklärungen bezüglich der Unfallursachen kommen (vgl. HOYOS 1987, 586). Allerdings dominieren in systemtheoretischen Unfallanalysen die Mensch-Maschine-Ansätze, welche Bedingungsebenen (Verhalten, Organisation, Technik) mechanistisch untersuchen. Für sportwissenschaftliche Forschungsvorhaben stellt sich zudem die Frage nach den konkreten Variablen, über die sich eine systemtheoretische Überprüfung des Unfallgeschehens operationalisieren ließe.

\subsubsection{Handlungstheoretische Ansätze}

Handlungen spielen bei allen Unfällen im sportlichen Zusammenhängen eine bedeutende Rolle. Wie Systemanalysen auch, sind handlungstheoretische Ansätze der Unfallforschung ebenfalls Situationsanalysen, die in Verwandtschaft zu Interaktionstheorien das Beziehungsgeflecht zwischen Person und Umwelt unter dem Gesichtspunkt einer zu bewältigenden Aufgabe aufzudecken versuchen (vgl. RÜMMELE 1988, 97). Im Vergleich zu systemtheoretischen Ansätzen wird unter handlungstheoretischen Perspektiven der Selbststeuerungsprozess der Handlung in den Mittelpunkt gerückt. Im Gegensatz zu Interaktionistischen Ansätzen wird zudem die Strukturierung sowie die sich hierbei abspielenden Prozessanalysen betont (vgl. HÜBNER 1997). Dabei wird das Handlungskontinuum einer sportlichen Handlung in Sequenzen inhaltlich differenzierbarer Einzelhandlungen zerlegt und eine handlungsspezifische Phasenstruktur postuliert ${ }^{227}$ (vgl. Abschnitt 6.1.2). 
Risikosportliche Handlungen sind als problemlösende Handlungen ein Spezialfall des Handlungskontinuums menschlicher Handlungen. Wie in Abschnitt 6.1.3 dargestellt werden hierbei Entscheidungsprozesse vollzogen, die die Ausgangssituation, mögliche Handlungsalternativen, deren Effekte und die sich hieraus erwarteten Konsequenzen berücksichtigen. Handeln wird hierbei als ein ,In-Beziehung-Setzen' von Elementen verstanden (vgl. RÜMMELE 1988, 102). Unfälle sind dann Folge eines fehlerhaften Soll-IstVergleiches, der auf eine Diskrepanz zwischen subjektiven Handlungsvorrausetzungen und -Anforderungen zurückzuführen ist. Unfallforschung unter handlungstheoretischer Perspektive ist dabei vielfach HandlungsfehlerForschung, also die Suche nach Störgrößen im Handlungsprozess (vgl. hierzu MEHL 1996) 228.

Für eine umfassende Unfallanalyse ist es nützlich, den Unfall in allen Ereignisphasen auf mögliche Handlungsfehler hin zu untersuchen. Ein solches Vorgehen, wie es sich z.B. bei HAHN (1983) für einen Wildwasserunfall findet, ist aufgrund mangelnder Informationen allerdings nur selten möglich. Es ist zudem zu bedenken, dass Handlungsfehlerforschung in erster Linie von situationsspezifischen Gegebenheiten abstrahieren und die internen Strukturvorgaben einer Person reflektieren. Sie repräsentieren damit lediglich einen Ausschnitt möglicher Unfallursachen, die sich außerhalb der eigenen Person finden (z.B. Lawinen oder Blitzschlag). Da in der hier angestrebten empirischen Untersuchung nach Möglichkeiten der gezielten Handlungsintervention geforscht werden soll, ist das Verwendungsinteresse eines handlungstheoretischen Ansatzes im Rahmen risikosportlicher Unfallforschung durchaus bedeutend.

\subsubsection{Ansätze einer erweiterten Sicherheitsforschung}

Bei reinen Unfallforschungen erfolgt die Frage nach den Unfallursachen als Analyse eines konkreten Unfalls, je nach Forschungsperspektive mit unterschiedlichen Schwerpunkten. Die Rekonstruktion des Unfallgeschehens impliziert dabei, dass eine Unfallforschung erst möglich wird, weil sich Unfälle ereignen. MUSAHL (1997), HINRICHS et al. (2002) aber auch RINKE (2002) haben in diesem Zusammenhang mehrfach darauf hingewiesen, dass es sich bei dieser retrospektiven Form der Unfallanalyse um einen subjektiv konstruierten Vorgang handelt, bei dem die Ursachenforschung mit den aus dem Unfall resultierenden Folgen vermischt werden. Die subjektive Konstruktion von Wirklichkeiten erfolgt demnach stets aus dem Blickwinkel des Beobachters und repräsentiert damit ein verzerrtes Bild der Realität. Bereits 1982 haben SLOVIC et al. im Rahmen der Unfallforschung diesen Effekt in verschiedenen Untersuchungen nachweisen können ${ }^{229}$.

Sicherheitspsychologische Ansätze als Erweiterung einer kausalanalytischen Unfallforschung verfolgen demgemäß vermehrt das Ziel, den Fokus von der reinen Analyse von Unfälle auf das ,,[...] antizipatorische und kompensatorische Handeln in gefährlichen Situationen“ (MONTEAU et al. 1999, 812) zu erweitern. Der sich vollziehende Paradigmenwechsel von der 
Unfall- zur Sicherheitsforschung impliziert ein übergeordnetes Forschungsinteresse, welches über die eigentliche Unfallsituation hinaus geht und interdisziplinäre Ansätze integriert. Die Analyse von ,folgenlose Unfälle' oder ,kritische Ereignisse' steht hierbei ebenso im Vordergrund wie die Analyse kognitiver Vorgänge in Risikosituationen. Der sich damit ändernde Anspruch einer erweiterten Sicherheitsforschung wird von ZIMOLONG verdeutlicht:

\section{„Sicherheit ist mehr als das Verbüten von Unfällen. [...] Nicht das Ergebnis \\ - Unfall, Beschwerden, Umweltbelastung - steht im Mittelpunkt, sondern der Prozess der Verbesserung" (ZIMOLONG 1996, 24)}

Bedeutende Anregungen hat die Sicherheitsforschung insbesondere aus dem Bereich der Technologie-Untersuchungen bekommen, wo Unfälle durch das enorme Ausmaß möglicher Unfallfolgen gar nicht erst auftreten sollten (z.B. bei Kernkraftwerken) - Präventionsmaßnahmen müssen daher ohne direkte Unfallanalysen entwickelt werden. Im Mittelpunkt der Forschungsansätze einer erweiterten Sicherheitsforschung stehen neben der Zuverlässigkeit technischer Systeme vor allem Informationsverarbeitende Prozesse der beteiligten Protagonisten, wodurch kognitionspsychologische Forschungsmethoden in den Mittelpunkt rücken (vgl. SCHRÖDER 2004). Für die Entwicklung übergeordneter Verhaltens- und Präventionsmaßnahmen werden über die Unfallsituation hinausgehende Kognitions- und Verhaltensprozesse untersucht.

In Anlehnung an THEIS (2002) lassen sich sicherheitspsychologische Ansätze der ersten und zweiten Generation inhaltlich und methodisch voneinander differenzieren. Den Methoden der ersten Generation liegt dabei ein sequenzielle Informationsverarbeitungsmodell zu Grunde ${ }^{230}$ (z.B. BYE 1998). Prozessual wird hierbei zwischen Wahrnehmung, Entscheidung und Handeln differenziert. Darüber hinaus wird davon ausgegangen, dass Handlungen auch durch einen abgekürzten Prozess von Wahrnehmen zu Handeln, d.h. ohne, dass ein Entscheidungsprozess vollzogen wurde, hervorgerufen werden können. Diese sequenzielle kognitive Verarbeitungsmodelle basieren auf einer zwangsweise gekoppelten Reihenfolge seiner Einzelprozesse, bei der das menschliche Handeln als eine direkte Reaktion auf ein Ereignis, auf eine Warnung oder auf ein Signal betrachtet wird. Aus sicherheitspsychologischer Sicht wird der kognitive Verarbeitungsprozess dabei auf ein einfaches Reiz-Reaktionsmodell reduziert (vgl. THEIS 2002, 30).

Diese Verfahren einer ersten sicherheitspsychologischen Generation stoßen insbesondere auf Grund des sequenziellen Informationsverarbeitungsprozesses an ihre Grenzen. Transaktionale Einflüsse wie persönliche Fähigkeiten und motivationsbildende Zielsetzungen beeinflussen - wie in Abschnitt 6.3 zusammenfassend dargestellt - das Verhalten in Risikosituationen. Diese Faktoren werden auf Grund der Kopplung im sequenziellen Informationsverarbeitungsmodell nicht als Einflussgrößen berücksichtigt. Das menschliche (Fehl)Verhalten ist jedoch nur unter Berücksichtigung des fortlaufenden Abgleichs zwischen der äußeren, d.h. auf den Menschen ein- 
wirkenden Faktoren und den internen Modellen des Menschen, d.h. seinen Erwartungen an die Welt zu ergründen (vgl. ebd.).

HOLLNAGEL (1998) aber auch NUREG (1996) begründen sicherheitspsychologische Ansätze der zweiten Generation, bei denen sowohl personale Faktoren als auch über die konkrete Handlungssituation hinausgehende Einflüsse berücksichtigt werden. Gegenüber der sequenziellen Modellbildung der Verfahren der ersten Generation können innerhalb des kognitiven Verarbeitungsprozesses, der den Methoden der zweiten Generation zu Grunde liegt, Rekursionsschleifen integriert werden. Geringfügige Abweichungen der situativen Faktoren oder der persönlichen Erwartungen und Ziele führen jeweils zu einer neuen kognitiven Strategie der Informationsverarbeitung und damit der sich anschließenden Vollzugshandlung (vgl. HOLLNAGEL 1998, $151 \mathrm{ff}$; THEIS 2002, 31f).

Obwohl die Verfahren auch eine systematische methodische Konzeption aufweisen ${ }^{231}$, sind sie eindeutig auf technisch dominierte Handlungsfelder ausgerichtet. Die von den Autoren aufgestellten Analysesysteme bauen auf so genannten Fehlerbäumen auf, für die ein umfangreiches Wissen über die Verhaltensweisen, Zielsetzungen und Einflussfaktoren in der Handlungssituation benötigt wird (vgl. hierzu HAUPTMANNS et al. 2004). Derartig detaillierte Verhaltensangaben über Handlungssituationen liegen im Sport oftmals nicht vor und sind auch aufgrund der Variabilität der Handlungssituation nur bedingt möglich. Die Verwendungsoptionen für sportliche Sicherheitsstudien sind dementsprechend gering.

Weitere sicherheitspsychologische Forschungszugänge zum Verhalten in ,gefährlichen Situationen' finden sich im Rahmen einer Arbeitssicherheitsforschung u.a. bei ZIMOLONG (1978), HALE \& GLENDON (1987), SLOVIC (1987), MUSAHL (1997) sowie HINRICHS et al. (2001). Auch hierbei stehen informationsverarbeitende Prozesse im Blickpunkt der Untersuchungsansätze. Im Gegensatz zu den oben vorgestellten sicherheitspsychologischen Konzepten der Handlungszuverlässigkeit werden bei diesen Ansätzen aber vielmehr Prozesse der Gefahrenwahrnehmung und deren Folgen für die Handlungsregulation untersucht. Zentrale Annahme dieser kognitionspsychologischen Forschungsrichtung ist, dass nur ein frühzeitiges Erkennen von Gefahren zu einer adäquaten Handlungsregulation und damit $\mathrm{zu}$ einer Vermeidung von Unfällen führen kann (vgl. HINRICHS et al. 2001).

In Anlehnung an die theoretische Deduktion der interdisziplinären Beiträge im ersten Teil dieser Arbeit ist das Verwendungsinteresse dieser neuren Ansätze der Sicherheitspsychologie besonders groß. Die Frage nach Unfallund Gefahrensituationen wird hierbei nicht auf die einzelne Unfallsituation reduziert, sondern in einen übergeordneten Prozess der Gefahrenwahrnehmung und -Verarbeitung eingeordnet.

Im folgenden wird das von MUSHAL entwickelte Modell der Gefahrenkognition als Bezugsrahmen für die eigene Studie vorgestellt. Es stellt das etablierteste sicherheitspsychologische Modell im Bereich der Arbeitssicher- 
heit dar und integriert unfalltheoretische Ansätze mit Paradigmen moderner Sicherheitsforschung.

\subsubsection{Das Modell der Gefahrenkognition aus der Arbeitssicherheit}

Im Rahmen der in dieser Arbeit durchgeführten sicherheitspsychologischen Studie wird auf das grundlegende Forschungsparadigma der modernen Sicherheitsheitspsychologie als Erweiterung einer monokausalen und retrospektiven Unfallforschung zurückgegriffen. Über die Analyse einzelner Unfälle hinaus wird in der folgenden Untersuchung nach übergreifenden Verhaltens- und Kognitionszusammenhängen im Risikosport gefragt. Der dabei zugrunde Forschungsansatz leitet sich aus dem Modell der Gefahrenkognition ab, welcher der Arbeitspsychologie entstammt und von MUSAHL (1997) entwickelt wurde. Die diesem Konzept immanenten theoretischen Hintergründe sowie die Ergebnisse der empirischer Untersuchungen und das methodische Vorgehen werden im Folgenden deduziert.

Gefahrenkognition als sicherheitspsychologisches Modell wurde von MUSAHL (1997) in der Arbeitssicherheit am Beispiel des Bergbaus entwickelt und in verschiedenen empirischen Untersuchungen bei unterschiedlichen Arbeitstätigkeiten angewendet (MUSAHL 1992; 1994; 1997; MUSHAL \& ALSLEBEN 1990; HINRICHS et al. 2001). Das Arbeitssicherheitskonzept auf Grundlage der Gefahrenkognition geht davon aus, dass sich Menschen „wissenskonsistent ${ }^{232}$ “ verhalten, sie Handlungen also aufgrund ihrer kognitiven Repräsentation in Form eines subjektiven Bezugsmodells regulieren. Der Mensch handelt demnach so, ,[...] wie es ihm aufgrund seines vorhandenen Wissens richtig und sinnvoll erscheint" (RINKE 2002, 32). Für vermeintlich gefährliche und risikoreiche Handlungsfelder bedeutet dies: In Situationen, die ein Mensch subjektiv für gefährlich hält, wird er sich tendenziell umsichtig und aufmerksam verhalten. In Handlungssituationen, die aufgrund der subjektiven Repräsentation als vermeintlich sicher und habitualisiert gelten, wird er der Handlung dagegen nur wenig Aufmerksamkeit ${ }^{233}$ und Konzentration schenken.

Hierbei wird davon ausgegangen, dass es sich bei der Gefahrenkognition um einen dynamischen und subjektiven Prozess von Wahrnehmung, Beurteilung und Entscheidung unter Risikobedingungen ${ }^{234}$ handelt. Gefahrenkognition bedeutet letztlich die Integration potentieller Gefährdungssituationen unter Zuhilfenahme von Heuristiken in ein internes Gefahrenmodell. Dieses Gefahrenmodell - auch als kognitive Repräsentation der Gesamtheit aller Informationen, Assoziationen und Redundanzen bezeichnet (MUSHAL 1997, 97) - wird je nach Situation und Person durch subjektive Erfahrungen, kognitive Lernprozesse und emotionale Befindlichkeiten beeinflusst. Es besitzt damit subjektiv einen hohen Wahrheitsgehalt und bestimmt über die Wahrnehmung hinaus das Verhalten in potentiellen Risikosituationen. Menschen - so die Argumentation bei MUSHAL - regulieren ihr Verhalten in gefährlichen Situationen also maßgeblich über das situativ wahrgenommene und beurteilte subjektive Risiko, wobei sich Wahrneh- 
mungs- und Beurteilungsprozesse an den internen Vorstellungen über die spezifischen Bedingungen orientieren (vgl. MUSAHL 1997, 220). Die Abkehr von vermeintlich objektivierten Unfalldaten zu einer subjektzentrierten Analyse von Kognitionsprozessen illustriert den mit der Durchsetzung der Sicherheitspsychologie verbundenen Paradigmenwechsel im Rahmen der Unfallforschung. Im Forschungsinteresse bei MUSAHL steht damit nicht der einzelne Unfall sondern die subjektive Repräsentation von Situationen und ihre jeweilige Bewertung.

„Welche Entscheidung binsichtlich der Durchführung bestimmter Handlungen getroffen werden, hängt von der Gefahrenkognition ab, also von dem subjektiven Abbild, welches durch die Wabrnehmung der Situation unter der Berücksichtigung des internen Gefahrenmodells entstanden ist" (RINKE 2002, 31).

$\mathrm{Zu}$ verstärkten Unfällen kommt es dem Modell zufolge dann, wenn das subjektiv repräsentierte Wissen über die Gefährdungssituation bzw. die Einschätzung des subjektives Risikos und die objektiven Gefahren nicht situativ deckungsgleich sind. Den handelnden Akteuren ist in bestimmten Situationen also nicht die Gesamtheit aller situativen Gefährdungspotentiale bekannt oder sie empfinden subjektiv ein Gefühl der Sicherheit in Anlehnung an das Sicherheitsmodell von KLEBELSBERG (vgl. Abschnitt 6.2.5.4). Der kognitiv-emotionale Beurteilungsprozess vollzieht sich dann auf der Grundlage unvollständiger Informationen, so dass eine realistische Beurteilung der Gesamtsituation nicht möglich ist. Zwischen den situativen Anforderungen und den Bewältigungskompetenzen besteht also eine Differenz, die das Unfallpotential drastisch erhöht. Die Zusammenhänge sind in Abbildung 8.2 veranschaulicht dargestellt.

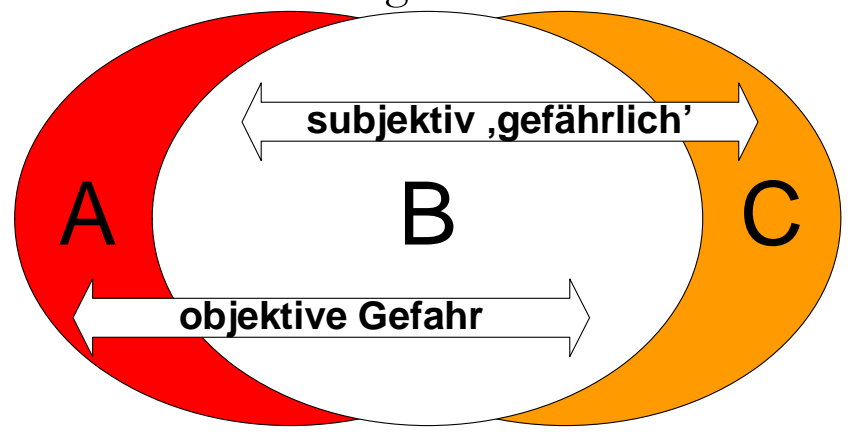

Abbildung 8.2: Veranschaulichung der Beziehung zwischen dem Bereich objektiver Gefahr und dem Bereich, der als subjektiv gefährlich erkannt wird (in Anlehnung an RINKE 2002, 33; mod. nach MUSHAL 1997, 30).

Während der Bereich B die Deckungsgleichheit zwischen wahrgenommener Gefahr und objektiver Gefahr kennzeichnet, stellt C einen Bereich dar, indem der Handelnde von Gefahren ausgeht, die reell nicht vorhanden sind. Der Bereich A kennzeichnet einen Bereich vermeintlicher Sicherheit der Handelnde erwartet hier keine Gefahren und fühlt sich subjektiv sicherer als es sich in der Realität objektiv darstellt. Insbesondere dieser Bereich 
stellt der Konzeption folgend ein großes Unfallrisiko dar, weil hier eine Kontroll-Illusion begünstigt wird, die in der Realität nicht vorhanden ist.

Verschiedene Studien zur Arbeitssicherheit konnten diese Wechselbeziehung bekräftigen. Die Operationalisierung der Gefahrenkognition wird bei MUSAHL über die Einschätzung von Unfallhäufigkeiten und der Schwere möglicher Unfälle bei unterschiedlichen Tätigkeiten vollzogen. So konnte der Autor einen signifikanten Zusammenhang zwischen einer fehlerhaften Gefahrenkognition und tatsächlichen Unfallzahlen im Bergbau nachweisen. Er konnte zeigen, dass sich die Mehrheit der Unfälle auf Tätigkeiten verteilten, die von den Protagonisten tendenziell unterschätzt wurden und mit einem vergleichsweise, sicheren Gefühl' verbunden waren. WORTMUTH et al. (2001) replizierten die Ergebnisse in einer Aluminium-Gießerei und HINRICHS et al. (2001) fand ähnliche Ergebnisse für Tätigkeiten in einem Walzwerk. Die Autoren reanalysierten hierbei jeweils Unfallanzeigen der gesetzlichen Unfallversicherer und bestimmten dabei Tätigkeiten und Handlungssituationen, in denen es zu Unfällen kommt. Über einen Fragebogen wurden schließlich die Arbeitnehmer der jeweiligen Tätigkeitsbereiche gebeten, die Unfallhäufigkeit der einzelnen Arbeitstätigkeiten auf einer sechsstufigen Skala einzuschätzen ${ }^{235}$. Aus dem Vergleich der subjektiven Urteile mit den Unfalldaten konnte in der Folge jede Arbeitstätigkeit entweder als unter, über oder realistisch eingeschätzte Tätigkeit identifiziert werden. Die Ergebnisse der Untersuchungen zeigten, dass sich die Unfallzahlen dabei keineswegs in einem ausgeglichenen Verhältnis zu den Einschätzungen der Arbeitnehmer verteilten. In allen Studien fanden sich die verhältnismäßig meisten Unfälle im Bereich der unterschätzten Tätigkeiten, obwohl diese quantitativ nicht den größten Teil der Tätigkeitsbereiche einnahm (vgl. auch Abbildung 8.3). 


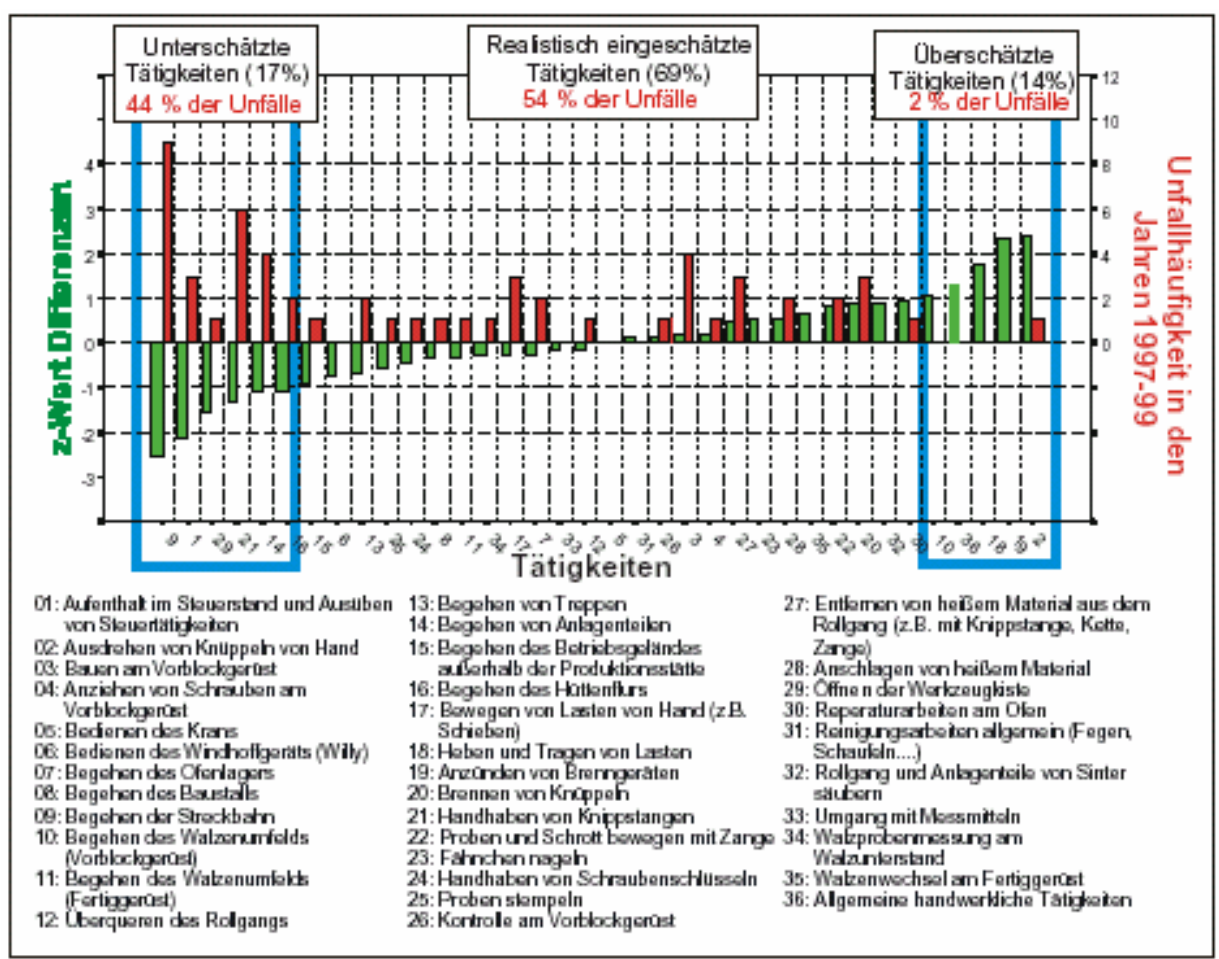

Abbildung 8.3: Gegenüberstellung der Gefahrenkognition und der tatsächlichen Unfälle bei Arbeitstätigkeiten in einem Walzwerk (in RINKE 2002, 35). Insgesamt 17\% der Tätigkeiten wurden in ihrer Gefährlichkeit überschätzt, 69\% der Tätigkeiten wurden realistisch eingeschätzt und $14 \%$ unterschätzt. Gemessen an der subjektiven Einschätzung der Arbeitnehmer ereigneten sich die meisten Unfällen bei unterschätzten Tätigkeiten (44\%). Für diese unterschätzten Tätigkeiten wird angenommen, dass hier Gefährdungspotentiale nicht erkannt wurden (vgl. auch MUSAHL 1997, 269ff).

Eine Anwendung des Modells der Gefahrenkognition im außerbetrieblichen Bereich hat RINKE (2002) ${ }^{236}$ für den Schulsport vorgenommen. In Anlehnung an das Erhebungsverfahren von MUSAHL wurden in dieser Studie Schulsportunfälle in verschiedenen Sportarten und spezielle Turntätigkeiten im Bezug zur Gefahrenkognition von Sportlehrern und Schülern der Sekundarstufe I analysiert. Die eindeutigen Befunde der Sicherheitsstudien in fertigenden Unternehmen über Zusammenhänge zwischen Gefahrenunterschätzung und Unfallhäufigkeit konnten hierbei fast vollständig repliziert werden: Während die Gefahrenkenntnis der Sportlehrer mit den Unfallzahlen bei einzelner Sportarten noch weitestgehend übereinstimmte, konstatierte der Autor bei den befragten Schülern eine eindeutige Korrelation zwischen der Unfallhäufigkeit und der Einschätzung einzelner Sportarten. Auf 25\% der hinsichtlich ihrer Gefährlichkeit unterschätzten - im Schulsport ausgeübte - Sportarten entfielen 70\% aller registrierten Schulsportunfälle (vgl. ebd.). Ähnliche Zusammenhänge konnten auch für einzelne Turntätigkeiten sowohl bei Lehren als auch bei Schülern unterschiedlicher Schulformen konstatiert werden (vgl. Abbildung 8.4). 


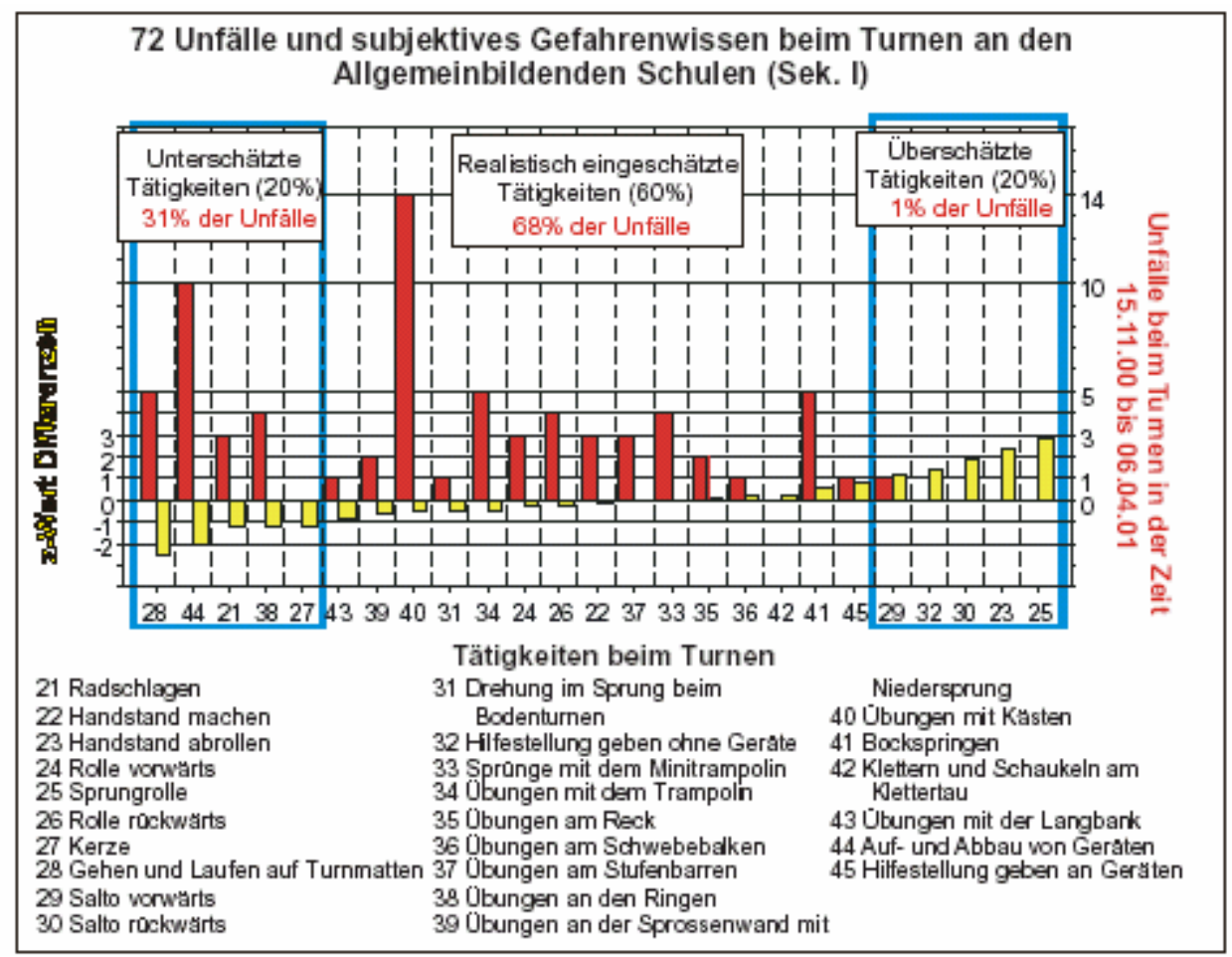

Abbildung 8.4: Unfälle und subjektives Gefahrenwissen bei Turntätigkeit in Allgemeinbildenden Schulen (nach RINKE 2002, 80).

Es muss konstatiert werden, dass die theoretischen Ansätzen der Unfallforschung in der Sportwissenschaft durch die sicherheitspsychologischen Ansätzen einer erweiterten Sicherheitsforschung eine sinnvolle Ergänzung und Erweiterung erfahren. Die Abkehr von retrospektiven Unfallanalysen zu Gunsten eines multidimensionalen Forschungsdesigns erweitert den Blick von reinen Handlungsfehlern eines verunfallten Individuums auf die übergreifenden Handlungskonstitutiva unter Bedingungen von Risiko und Sicherheit im Sport und - im Kontext dieser Arbeit - im Risikosport. Die von RÜMMELE initiierte Diskussion um theoriegeleitete Ansätze einer sportwissenschaftlichen Unfallforschung wird dadurch keineswegs obsolet, sondern um wesentliche Aspekte erweitert. Obwohl sich die Durchsetzung sicherheitspsychologische Erkenntnisse und Forschungsvorhaben in der Sportwissenschaft nur zögernd vollzieht, weisen die neueren Forschungsansätze von RINKE (2002); SCHWIERCH (2003) sowie MERSCH et al. (2005) auf eine , sicherheitspsychologische Wende' in der Sportwissenschaft hin. Die hierbei eingenommene Forschungsperspektive mit dem besonderen Fokus auf perzeptive Wahrnehmungs- und kognitive Beurteilungsprozessen wird in der folgenden empirischen Studie aufgegriffen und anhand eines eigenen Forschungsansatzes angewendet. 


\section{Empirie: Unfälle und Gefahrenkognition im Ri- sikosport - eine sicherheitspsychologische Stu- die}

Das größte Risiko auf Erden laufen die Menschen, die nie das kleinste Risiko eingehen wollen

(Bertrand Russell (1872-1970), brit. Philosoph u. Mathematiker)

Wie sich in den vorhergehenden Kapiteln zeigte, bestand im Hinblick auf risikosportliche Zusammenhänge eine Vielzahl interessanter offener Frage. Der interdisziplinäre Ansatz, der zur Beantwortung einzelner Fragestellungen im Kontext des Risikosports gewählt wurde, konnte dabei aus verschiedenen wissenschaftlichen Perspektiven einen konstitutiven Zusammenhang von Risiko und Sicherheit aber auch von Risiko und Gefahr im Sport offenbaren. Die Ambivalenz risikosportlichen Handelns im Spannungsfeld von Risikosuche und Sicherheitsbedürfnis manifestierte sich hierbei sowohl aus phänomenologisch-soziologischer Perspektive als auch aus dem Blickwinkel der kognitiven Psychologie und rationalen Handlungstheorie. Bislang liegen 
keine empirische Studien vor, die dieses Spannungsfeld umfassend, theoriegeleitet und detailliert untersuchen. Gleichzeitig fehlt im Bereich des Risikosports ein geeignetes Instrument, welches die subjektive Perspektive des handelnden Protagonisten im Bezug auf Wahrnehmungsprozesse in ein multidimensionales Forschungsvorhaben integriert ${ }^{237}$ und für eine pragmatische Unfallforschung deduziert. Im Bezug auf die folgende empirische Studie ergibt sich daher neben den Fragestellungen zu Sicherheit, Risiko und ihren kognitiven Repräsentanz im Bezug auf das Unfallgeschehen in Risikosportarten auch das Ziel ein geeignetes Forschungsdesign zu entwickeln bzw. anzuwenden.

Das Forschungsvorhaben dieser Arbeit hat neben der Evaluation konstitutiver Zusammenhänge von Risiko und Sicherheit im Risikosport die praktische Aufgabe, Hinweise für eine gezielte Intervention im Risikosport mit dem Endziel zu geben, Unfälle in risikosportlichen Handlungskontexten zu reduzieren. Zwar erscheint eine solche Ableitung pragmatischer Hinweise auch auf der Basis theoretischer Annahmen als möglich, wie die Praxis des Risikosports in den letzten Jahren vermehrt zeigt. Der positive Effekt, der sich aus einem veränderten Risikohandling ergibt und der das Vorhandensein ursächlicher Beziehungen nahe legt, führt aber zu keinem wissenschaftlichen Erkenntnisgewinn - provoziert wird lediglich eine entsprechende, theoretisch aber nicht erklärte Praxis (vgl. auch MUSAHL 1997, 238). Selbst wenn angenommen wird, dass Aufklärungen und Verhaltensänderungen zu einer pragmatischen Unfallreduktion im Risikosport führen, so heißt dies nicht, zu verstehen, warum dies so ist. Ein derartiges Wissen provoziert ,[...] unreflektiertes, pragmatisches Handeln, verführt zu beliebigen naiven Theorien - die man zumeist ohnehin vorher schon hatte und nunmehr erneut bestätigt sieht" (ebd.). Die empirische Überprüfung theoretischer Inhalte vollzieht sich damit nicht nur aufgrund eines wissenschaftlichen Erkenntnisinteresses sondern auch vor dem Hintergrund einer theoretisch fundierten und empirisch abgesicherten Ableitung handlungsorientierten Wissens in die Praxis.

Im ersten Abschnitt dieses Kapitels werden zunächst die Grundlagen der empirischen Studie, auf denen die Untersuchung aufbaut, vorgestellt. Hierzu wird das in Abschnitt 8.3.6 beschriebene Modell der Gefahrenkognition als zugrunde liegendes Modell in den Kontext des Risikosports transferiert und die Fragestellung der Untersuchung erläutert. Im zweiten Abschnitt wird das methodische Vorgehen beschrieben und der Versuchsplan dargestellt. Schließlich werden die Ergebnisse der Studie in Abschnitt 9.3 vorgestellt und in Abschnitt 9.4 abschließend diskutiert. Das Kapitel endet mit einem zusammenfassenden Fazit der Untersuchung in Abschnitt 9.5.

\subsection{Grundlagen, Ziele und Fragestellung der Studie}

Mit Bezug auf die in Kapitel 6 entworfenen handlungstheoretischen Zusammenhänge des Risikosports verdichtet sich in der vorliegenden Untersuchung der Fokus auf die kognitive Repräsentation von risikosportlichen Handlungssituationen 
im Bezug auf ihre subjektiven, Gefährlichkeit'. Bezogen auf das phasenstrukturelle Handlungsmodell bei NITSCH (1986) wird hierbei postuliert, dass ein Abgleich von situativen Wahrnehmungsprozessen und dessen kognitiver Repräsentation die Handlungsrealisation in unterschiedlichen Handlungsphasen im Risikosport beeinflusst. Ein Teilziel der Studie ist es, diese Antizipationsprozesse in einen Handlungszusammenhang einzuordnen und mit Unfalldaten abzugleichen. Dieses aus der theoretischen Deduktion generierte Erkenntnisinteresse entspricht im wesentlichen dem Modell der Gefahrenkognition von MUSHAL (1997) aus der Arbeitspsychologie. Die Berechtigung des Modells für sicherheitspsychologische $\mathrm{Zu}$ sammenhänge in der Arbeitswelt wurde bereits in Abschnitt 8.3.6 verdeutlicht. Eine Legitimation für risikosportliche Handlungsfelder steht dabei noch aus. Eine Übertragung des Modells in den Zusammenhang des Risikosports erfordert dabei eine entsprechende kontextbezogene Transformation, welche im folgenden Abschnitt vorgenommen wird.

\subsubsection{Gefahrenkognition als zugrunde liegendes Modell}

Die vorangestellten Darstellungen der theoretischen Hintergründe von Forschungsansätze zur Analyse von Unfällen und ihre Erweiterung zu sicherheitspsychologischen Forschungskonzepten wird in diesem Abschnitt zu einer theoretischen Rahmenkonzeption für die im Rahmen dieser Arbeit durchgeführte sicherheitspsychologische Studie im Risikosport integriert. Dabei wird der konzeptionelle Ansatz der Gefahrenkognition nach MUSAHL (1997) als forschungsleitendes Bezugsmodell für risikosportliche Aktivitäten transformiert.

MUSAHL geht - wie im Abschnitt 8.3.6 deduziert - im Rahmen seiner Modellentwicklung davon aus, dass sich Unfälle im Arbeitsalltag durch eine Diskrepanz von subjektiver Gefährlichkeitseinschätzung und tatsächlicher Gefährlichkeit erklären lassen. Menschen handeln demnach situativ unangemessen, weil sie sich der realen Gefahren nicht bewusst sind und sie ihre Handlungen deshalb nicht aufmerksam und konzentriert vollziehen. Diese Zusammenhänge wurden auf einer abstrakten Ebene schon durch die Anthropologie menschlicher Handlungen postuliert, ohne dass ein konkreter Unfallbezug entwickelt wurde. Wenngleich eine greifbare Operationalisierung von Sicherheit und Risiko als Handlungsdeterminanten auch bei MUSHAL nicht vorgenommen wird, so lassen sich aus seinen Ergebnisse auch konstitutive Handlungsdimensionen von Sicherheit und Risiko ableiten. Die Untersuchungsergebnisse des Autors legen nahe, den Prozessen der Wahrnehmung, Beurteilung und Entscheidung in Risikosituationen und ihre kognitive Repräsentation nicht nur handlungsleitende Bedeutung zu zuweisen, sondern ihnen darüber hinaus auch einen unfallkonstitutiven Einfluss zu zuschreiben. Unfälle im Risikosport ließen sich damit - zumindest teilweise mit einer unzureichenden oder fehlerhaften Gefahrenkognition oder anders ausgedrückt mit einem Gefühl „,...] trügerischer Sicherheit“ (MUSHAL 1997, 221) erklären. Sicherheit wird bei MUSAHL damit zu einem Risikoproblem und Risiko zu einer das Verhalten beeinflussenden Größe. Aus sicherheitspsychologischer Sicht rücken damit die eine Handlung regulierenden Prozesse der Informationsverarbeitung sowie ihre handlungskonstituti- 
ve Bedeutung im Mensch-Umwelt-System in den besonderen Forschungsfokus. Die Operationalisierung der Gefahrenkognition geschieht deshalb in Anlehnung an MUSAHL über die Erfassung von Gefährlichkeitsurteilen einer Aktivität, die über die Einschätzung von Unfallhäufigkeiten und den Unfallfolgen erfolgt.

Wenn aber der kognitiven Repräsentation von Gefahren eine konstitutive Bedeutung für die Unfallentstehung zugestanden wird, muss zunächst ein Unfallmodell entwickelt werden, welches eine Integration der gefahrenkognitiven Prozesse nach MUSAHL erlaubt. Betrachtet man eine Verletzung als die Folge eines Unfalls und einen Handlungsfehler aufgrund einer gestörten Gefahrenkognition als Unfallursache, dann schrumpft der Unfall auf ein punktuelles Ereignis zusammen, dessen kognitive Beurteilungsanteile nur noch schwer auszumachen sind. Einer umfassenden Sicherheitsstudie wäre damit aber nur wenig gedient. Mit KIRCHNER (1996) wird der Unfall im Risikosport daher als Ereignissequenz begriffen, welche aus Ereignissen vor dem eigentlichen Unfall, aus dem Unfall selbst und aus Ereignissen nach dem Unfall besteht ${ }^{238}$. Neben dem Unfall sind damit auch andere Situationen., in denen es zu einer Inkongruenz von Handlungszielen und Handlungsergebnissen kommt, forschungsrelevant. Dies entspricht der Kategorisierung eines Beinahe-Unfalls in Anlehnung an MUSAHL (1997). Natürlich ist für die Beobachtungsperspektive von Unfällen auch entscheidend, ob es dem Handelnden gelingt, nach einem Störfall bzw. Handlungsfehler die Kontrolle über das Geschehen zurück zugewinnen, z.B. durch gezieltes Schwimmen nach einer Kenterung beim KanuWildwasserfahren. Der Störfall ist demgemäß nur eine Vorraussetzung des Unfalls und nicht mit dem Unfall gleichzusetzen. Kommt es in der Handlungssituation zu einer Überforderung durch eine fehlerhafte Gefahrenkognition, dann ist zwar eine potentielle Unfallsituation gegeben, durch eine Anpassung der Situation, z.B. durch eine Neuorientierung, ist aber eine Korrekturhandlung möglich, die eine konkrete Unfallsituation abwendet.

Wenn sich die sicherheitspsychologischen Annahmen der Arbeitssicherheit auf risikosportliche Kontexte transferieren ließen, dann stellen die Prozesse der Gefahrenkognition das Herzstück jeglicher Unfallanalysen dar und die sicherheitspsychologischer Intervention würde einen klaren Bezugspunkt erhalten. Aufbauend auf den Grundlagen des transaktionalen Handlungsmodells im Risikosport (vgl. 6.5) wird in Abbildung 9.1 ein zu überprüfendes sicherheitspsychologisches Modell zur Erklärung des Unfallgeschehens am Beispiel des Kanusports vorgestellt, welches die Ansätze einer interaktionistischen Unfalltheorie ebenso integriert wie die grundlegenden Annahmen des Modells der Gefahrenkognition nach MUSAHL. Dieses integrative Unfallmodell entspricht sowohl den Hauptbefunden der Arbeitssicherheit, wonach eine Divergenz zwischen objektiv und subjektiv empfundener Gefährlichkeit im Sinne eines Ungleichgewichts zugunsten der subjektiven Gefährlichkeit (Gs > Go) zu einem erhöhten Unfallpotentials führt, als auch den Annahmen einer interaktionistischen Unfalltheorie. Unfälle im Risikosport würden - diesem integrativen Modell folgend - also aus einer Unterschätzung der tatsächlichen Gefährlichkeit resultieren. 


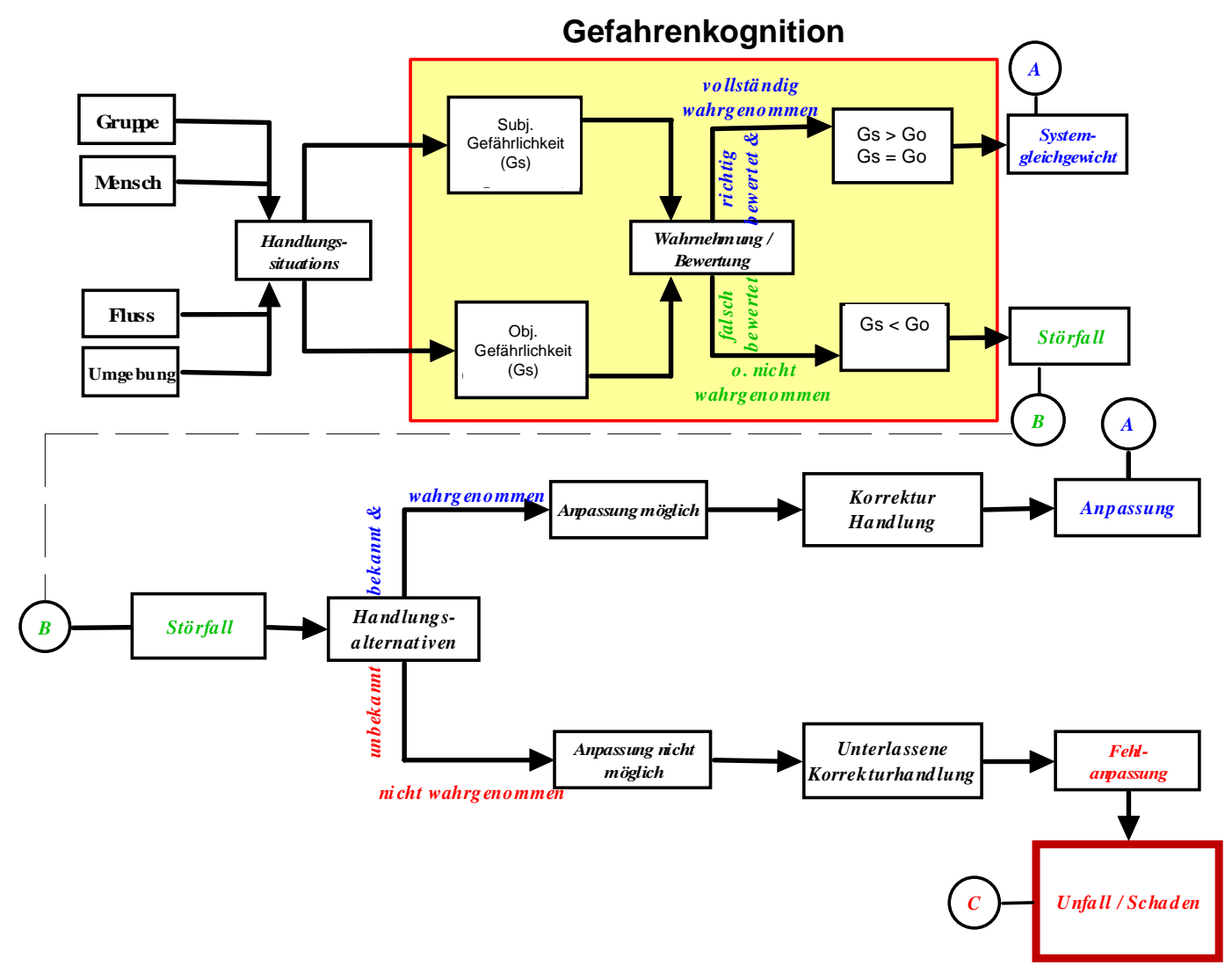

Abbildung 9.1: Sicherheitspsychologisches Modell des Unfallgeschehens im Risikosport am Beispiel des Unfallsystems Kanusport. (eigene Darstellung in Anlehnung an HOYOS 1980, 13).

Es bleibt diesbezüglich aber fraglich, ob sich ein derartiges Modell für den Risikosport adaptieren lässt, denn, Gefährlichkeit' stellt in diesem Sinne gleichfalls ein motivationsbildendes Element dar, welches im Rahmen einer individuellen Herausforderung als subjektives Risiko wahrgenommen wird. Durch eine Replikation der Untersuchungsergebnisse von MUSAHL und RINKE könnte die Gefahrenkognition als Ansatzpunkt eines sicherheitspsychologischen Modells der Unfallentstehung begründet und damit die Gültigkeit des Modell erhärtet werden. Die konkreten Aufgaben, Fragestellungen und Hypothesen der Untersuchung werden im folgenden Abschnitt erläutert.

\subsubsection{Aufgaben, Fragestellungen und Hypothesen}

Das Erkennen und Einschätzen von Gefahren und ihre Transformation in individuelle Risiken durch die Integration in ein internes Gefahrenmodell stellt das Kernelement und gleichzeitig Grundvoraussetzung jeglichen Handelns in gefährlichen Handlungssituationen dar - dies ist der zentrale Befund der sicherheitspsychologischen Studie von MUSAHL (1997). Nur bei einer realistischen Wahrnehmung und Beurteilung der situativen Gefahren und ihrer kognitiver Repräsentation - so die Ergebnisse der Untersuchung - ist ein adäquates und damit befriedi- 
gendes Handeln in potentiell gefährlichen Situationen möglich. Zweifelsfrei stellt die Wahrnehmung und Einschätzung von Reizen aus der Umwelt und ihre kognitive Darstellung im Sinne eines internen Gefahrenmodells ein notwendiges Element jeglichen Handelns dar. In risikosportlichen Handlungszusammenhängen erfährt dieser Prozess aber eine erweiterte Bedeutung: Die Gefahrenkognition im Risikosport dient nicht nur zur Vermeidung möglicher Gefahren sondern gleichsam zur Antizipation adäquater Handlungsanreize (vgl. SCHIEDCK 2003). Eine kausale Risikominimierung - wie sie im Alltag und auch in Arbeitszusammenhängen bisweilen vollzogen wird - würde im Risikosport gleichzeitig die motivationsgenerierenden Anreize reduzieren und damit den subjektiven Sinn der Handlung. Insofern erfährt der bisherige Ansatz der Gefahrenkognition im Risikosport einen neuartigen Kontext, der über die bisherigen inhaltlichen Erklärungen der Arbeitssicherheit weitere Fragen aufwirft.

Wenn durch eine empirische Untersuchung die subjektive Einschätzung des Gefahrenwissens verschiedener risikosportlicher Handlungssituationen erfasst werden würde und mit objektiven Daten dieser Handlungsfelder abgleichen werden könnten und sich damit die Befunde von MUSAHL (1997) und RINKE (2002) in risikosportlichen Kontexten replizieren ließen, so wären konkrete sicherheitspsychologische Interventionsoptionen zur Unfallprävention auch im Risikosport möglich. Die Replikation der Untersuchungsergebnisse von MUSAHL im Risikosport würde darüber hinaus das im voranstehenden Abschnitt aufgestellte sicherheitspsychologische Modell der Unfallentstehung legitimieren und einen wesentlichen Beitrag zur Sicherheitsdiskussion in der Sportwissenschaft beitragen. Als Baustein einer sich entwickelnden sportwissenschaftlichen Risikodiskussion könnte zudem der beschriebene Paradigmenwechsel von retrospektiven Unfallanalysen zu sicherheitspsychologischen Ansätzen in der Sportwissenschaft vorangetrieben werden.

Die zentralen Teilaufgaben dieser Untersuchung, die das Hauptziel einer wissenschaftlich legitimierten Interventionsmöglichkeit im Risikosport ermöglichen können, sind damit vielschichtig:

(1) einerseits die Transformation und Anwendung eines sicherheitspsychologischen Verfahrens als ein erweitertes Unfallanalysekonzept der Sportwissenschaft,

(2) andererseits die Überprüfung der Replikabilität der von MUSAHL und RINKE konstatierten Hauptbefunde für risikosportliche Handlungsfelder und schließlich

(3) die explorative Evaluation von Zusammenhängen zwischen Gefahrenkognition und Unfalldaten im konkreten Kontext des Risikosports.

Diese differenzierte Aufgabenstellung bestimmt damit die konkrete Teilzielsetzungen und Fragestellungen der Untersuchung. Während sich die Transformation des von MUSAHL entwickelten Verfahrens als rein methodologisches Problem vollzieht, sind im Rahmen der geforderten Replikabilität die Überprüfung konkreter Hypothesen notwendig. Die Exploration von möglichen Kovariationen zwischen der subjektiven Gefahrenkognition und den tatsächlichen ,objektiven' Gefahren im konkreten Kontext des Risiko- 
sports muss dagegen ohne konkrete Fragestellungen auskommen und weitestgehend ergebnisoffen erfolgen.

Im Bezug auf die Aufgabenstellung (1), die sich in der Transformation des Forschungskonzeptes der Gefahrenkognition sensu MUSAHL darstellt, wird der Fokus auf die Sachdienlichkeit des Verfahrens für risikosportliche Kontexte gelenkt. Das empirische Verfahren zur Bestimmung der Gefahrenkognition im Abgleich mit objektiven Unfalldaten soll dabei auf seine Anwendbarkeit im Kontext des Risikosports überprüft werden. Die hierzu gehörige Fragestellung lautet: Ist ein im Bereich der Arbeitssicherheit entwickeltes Verfabren für sportwissenschaftliche Anwendungsbereiche außerbalb des Schulsports verwendbar?

Im Bezug auf die Aufgabenstellung (2) bezieht sich die Fragestellung auf die von MUSAHL (1997) hervorgehobenen und von RINKE (2002) replizierten Hauptbefunde. In Anlehnung an MUSHAL wird in der vorliegenden Studie dabei angenommen, dass auch Risikosportler wissenskonsistent handeln. Dies bedeutet, dass das subjektive ,Gefahrenwissen' - zu verstehen als kognitiv-affektive Gesamtheit der situativen Beurteilungskriterien - das Verhalten in Risikosituationen bestimmt. Aktivitäten und Situationen, die die Sportler also subjektiv als unsicher wahrnehmen, werden demnach aufmerksam und konzentriert realisiert, während in als risikolos und sicher wahrgenommenen Situationen, Handlungen mit nur wenig Aufmerksamkeit begleiten werden (vgl. auch Abschnitt 8.3.6). Aus diesem Mangel an Aufmerksamkeit und eine die Handlungsausführung begleitende Unkonzentration - so die Annahme - resultieren Handlungsfehler und damit auch potentielle Unfallsituationen. Subjektive Sicherheit im Sinne einer Unterschätzung des realen Gefahren- und Risikopotential einer Situation würde damit zu einer Funktionslogik von Unfällen und Fehlhandlungen im Risikosport. In Anlehnung an MUSAHL (ebd.) wird dabei angenommen, dass die Mehrzahl der Tätigkeiten und Sportarten durchaus realistisch eingeschätzt werden, dass aber gleichzeitig ein überproportional großer Anteil von Unfälle in risikosportlichen Kontexten zu finden sein wird, der von den Protagonisten der Aktivitäten in ihrer Gefährlichkeit unterschätzt werden (vgl. auch RINKE 2002, 53). Die übergeordnete Fragestellung lautet hierbei: Lassen sich Zusammenhänge zwischen der subjektiven Einschätzung bęüglich der Gefährlichkeit bestimmter Aktivitäten und den objektiven Unfallzablen dieser Aktivitäten im Kontext des Risikosports darstellen? Alle Autoren, die dieses Verfahren bereits angewendet haben, konstatierten aufgrund ihrer Ergebnisse einen eindeutigen Zusammenhang zwischen Gefahrenkognition und Unfallaufkommen. Sie konnten nachweisen, dass für die in ihrer Gefährlichkeit unterschätzten Tätigkeiten im Bergbau aber auch im Schulsport überproportional viele Unfälle zu registrieren sind. Aufgrund der von den Autoren gefundenen Ergebnisse soll die Fragestellung dabei durch folgende Hypothesen überprüft werden:

H1: Auf risikosportliche Aktivitäten, die in ihrer Gefährlichkeit unterschätzt werden, entfallen überproportional viele Unfälle.

H2: Auf Aktivitäten, die in der Schwere der Unfallfolgen unterschätzt werden, entfallen ebenfalls überproportional viele Unfälle 
Im Bezug auf die Aufgabenstellung (3) können keine Hypothesen generiert werden, da keine expliziten Zusammenhänge über die Bedeutung von Gefahrenkognition im Kontext des Risikosports bekannt sind. Hier ist eher ein explorativer Forschungszugang gefragt, der über die Replikation der Ergebnisse von MUSAHL und RINKE hinaus eine offene Exploration der Untersuchungsergebnisse fordert. Diese kontextbezogene Offenheit soll dazu dienen, neue Zusammenhänge und inhaltliche Bezüge zu erkennen und für weitere Untersuchungen nachfolgender Forschungsarbeiten aufzuarbeiten.

Das zur Überprüfung der Hypothesen und damit zur Beantwortung der Fragestellungen gewählte methodische Verfahren wird im folgenden Kapitel vorgestellt.

\subsection{Untersuchungsmethodik}

Im folgenden werden die Verfahrensschritte zur empirischen Bestimmung der Gefahrenkognition für den alpinen Berg- und Kanusport dargestellt. Der hier gewählte Begriff, Methodik' kennzeichnet eine Klasse von Prinzipien, Techniken - also auch Methoden, die zur Untersuchung eines Gegenstandes herangezogen werden. Methodik geht über den Begriff der ,Methode' hinaus, welcher demgegenüber ein bestimmtes regelgeleitetes Vorgehen im Sinne einer Werkzeugfunktion bezeichnet.

Eine für die Seriosität einer Untersuchung bedeutende Voraussetzung ist deren Replikabilität, also die Wiederholbarkeit des zugrunde liegenden empirischen Befundes. Angesichts eines multidimensionalen empirischen Ansatzes und der mit ihr verbundenen Kovariation von Maßen subjektiver Einschätzungen und objektiven Daten, ist die Anwendung eines standardisierten Verfahrens für diese Replikabiliät der Befunde notwendig. Eine qualitative Methodik schließt sich für die hier gewählte Fragestellung daher aus, wenngleich qualitative Methoden im Kontext sicherheitspsychologischer Studien durchaus Anwendung finden (vgl. SCHWIERCH 2003) ${ }^{239}$.

Die Untersuchungsmethodik der empirischen Studie dieser Arbeit erfolgt in Anlehnung an das von MUSAHL (1997) entwickelte Verfahren zur Erhebung der Gefahrenkognition in Arbeitszusammenhängen. Dieses Verfahren wurde u.a. von RINKE (2002) und WORTMUTH et al. (2001) weiterentwickelt und für sicherheitspsychologische Untersuchung in unterschiedlichen Arbeitszusammenhängen legitimiert. Für die forschungsleitende Fragestellung dieser Studie ist das Verfahren dem Untersuchungsgegenstand erweitert und angepasst worden. Methodisch konzeptionell lassen sich hierbei zwei unterschiedliche Untersuchungsstränge differenzieren, die im Rahmen eines Vergleiches einen methodischen Schnittpunkt erfahren. Insgesamt können 6 Verfahrensschritte differenziert werden: Auf der Seite der objektiven Datenerfassung werden zunächst Einzeltätigkeiten und Disziplinen innerhalb eines risikosportlichen Handlungsfeldes aus der Aufgliederung des Unfallgeschehens definiert. Darüber hinaus wird die tatsächliche Unfallzahl dieser Tätigkeiten durch die Auswertung von Unfallbögen und statistischen Unfallmaterialen bestimmt. Diese Daten werden schließlich mit 
den subjektiven Einschätzung der Unfallhäufigkeit und der Unfallschwere für die definierten Tätigkeiten von beteiligten Sportlern verglichen. Aus dem Grad der Übereinstimmung zwischen objektiven Unfalldaten und der subjektiven Einschätzungen des Gefahrenpotentials der Protagonisten lässt sich schließlich auf die Gefahrenkognition der Sportler schließen. Die Untersuchung beruht damit auf einem Ex-post-facto Versuchplan, bei dem die tatsächlichen Unfälle einzelner Aktivitäten mit den Urteilen der Aktiven verglichen werden. Es handelt sich auf diese Weise um einen korrelativdeskriptiven Untersuchungsansatz (vgl. hierzu auch RINKE 2002, 62).

Die einzelnen Verfahrensschritte sind in Tabelle 9.1 zusammengefasst:

\begin{tabular}{||l|l|l||}
\hline Schritt & Aufgabenstellung & Ziel \\
\hline 1 & $\begin{array}{l}\text { Definition aller einzelner Tätigkeiten } \\
\text { eines übergeordneten Sportberei- } \\
\text { ches (Kanusport \& alpiner } \\
\text { Bergsport). }\end{array}$ & $\begin{array}{l}\text { Aufgliederung des Unfallgeschehens auf } \\
\text { die Gesamtheit definierbarer Tätigkeiten } \\
\text { eines übergeordneten Sportbereichs. }\end{array}$ \\
\hline 2 & $\begin{array}{l}\text { Reanalyse der Unfallstatistik und } \\
\text { Unfallbögen }\end{array}$ & $\begin{array}{l}\text { Ermittlung der objektiven Unfalldaten für } \\
\text { die einzelnen Aktivitäten }\left(G_{0}\right) .\end{array}$ \\
\hline 3 & $\begin{array}{l}\text { Einschätzung der Gefährdung der } \\
\text { einzelnen Tätigkeiten }\end{array}$ & $\begin{array}{l}\text { Ermittlung der subjektiven Einschätzun- } \\
\text { gen für die Gefährlichkeit der einzelnen } \\
\text { Aktivitäten }\left(G_{s}\right) .\end{array}$ \\
\hline 4 & $\begin{array}{l}\text { Datentransformation der subjektiven } \\
\text { Einschätzungen und objektiven Un- } \\
\text { falldaten }\end{array}$ & $\begin{array}{l}\text { Vergleich der beiden Datensätze anhand } \\
\text { von } G_{0} \text { und } G_{s} \text { für jede einzelne Aktivität. }\end{array}$ \\
\hline 5 & $\begin{array}{l}\text { Differenzberechnung } G_{s}-G_{0}=\lambda G \\
\text { nach Transformation }\end{array}$ & $\begin{array}{l}\text { Klassifikation der Aktivitäten nach ihrer } \\
\text { subjektiven Gefährlichkeit und Risikoopti- } \\
\text { on }\end{array}$ \\
\hline 6 & $\begin{array}{l}\text { Bestimmung der unterschätzten Tä- } \\
\text { tigkeiten }\end{array}$ & $\begin{array}{l}\text { Definition und Analyse der sicherheits- } \\
\text { psychologisch kritischen Aktivitäten. }\end{array}$ \\
\hline
\end{tabular}

Tabelle 9.1: Verfahrensschritte zur empirischen Bestimmung der Gefahrenkognition anhand der relativen Übereinstimmung zwischen subjektiven Urteilen über die Gefährlichkeit vertrauter Aktivitäten und deren objektiven Entsprechung in Form von Unfalldaten (in Anlehnung an MUSHAL $(1997,234)$.

Als zu untersuchende Handlungsfelder des Risikosports wurden der alpine Bergsport und der Kanusport ausgewählt. Diese Auswahl wurde einerseits aufgrund des induktiven Zugangs des Autors, andererseits aber auch aus Gründen einer größtmöglichen Pragmatik gewählt. Sowohl im alpinen Bergsport als auch im Kanusport liegen ausführliche statistische Datensammlungen und Unfallberichte vor. Die Reanalyse von Unfalldaten, die für die Bestimmung der ,objektiven' Gefahr notwendig ist, kann damit auf der Basis einer größtmöglichen Vollständigkeit erfolgen.

Im folgenden Abschnitt wird die Erhebung des objektiven Datenmaterials, welches sich in der Reanalyse von Unfallberichten und -Auswertungen manifestiert, vorgestellt. Anschließend wird das Erhebungsverfahren des subjektiven Datenmaterials erläutert und die Durchführung der hierbei stattgefundenen Befragung dargestellt. Da die Vergleichbarkeit der objektiven und subjektiven Datensätze nur mittels Datentransformation möglich ist, 
wird abschließend das hierzu angewendete statistische Verfahren und die sich anschließende Differenzberechnung erklärt.

\subsubsection{Objektives Datenmaterial}

Unter dem objektiven Datenmaterial wird die tatsächliche Unfallanzahl, voneinander aufgrund der Handlungsstruktur abgrenzbarer Aktivitäten, im Kanusport und im alpinen Bergsport verstanden ${ }^{240}$.

Die Untersuchung von Unfällen und Unfallzusammenhängen richtet sich dabei nach dem vorhandenen Informationsmaterial, welches über die Unfallsituationen vorliegt ${ }^{241}$. Unfälle sind in einer Analyse zunächst nichts als statistische ,Daten' - was geschah, wer verunglückte, warum geschah es. Die Verwendung von Unfalldaten als Kriterium für weitreichende Maßnahmen der sicherheitspsychologischen Intervention setzt hohe Ansprüche an die Datenerhebung voraus. Anders als in betrieblichen Organisationen ist die Elevation von Unfalldaten im Risikosport jedoch ungleich schwieriger. Zwar liegen Unfallanzeigen und -berichte sowohl im Kanusport als auch im alpinen Bergsport vor, die Aussagekraft der Unfallbeschreibung ist allerdings sehr begrenzt. Zudem sind die einzelnen Unfallberichte auch für wissenschaftliche Erkenntnisprozesse aufgrund von Datenschutzbestimmungen nur begrenzt zugänglich. Die Analyse des objektiven Datenmaterials erfolgte deshalb anhand der von den zuständigen Verbänden herausgegebenen Publikationen und statistischen Datensammlungen zum Unfallgeschehen in den einzelnen Handlungsfeldern sowie einzelnen Unfallanzeigen im Bereich des Kanusports.

\subsubsection{Abgren₹ung und Definition der Aktivitäten}

Für den Vergleich objektiver Unfallzahlen mit subjektiven Einschätzungen bezüglich der Gefährlichkeit ist die Abgrenzung und Definition einzelner Tätigkeiten und Aktivitäten notwendig. Diese sollten allgemein bekannt und durch die Handlungsstruktur und evt. den Handlungsraum deutlich voneinander getrennt sein. Die Arbeiten von ZIMOLONG (1984) und POTEMPA (1980) haben in diesem Zusammenhang gezeigt, dass subjektive Urteile umso sicherer und inhaltlich klarer interpretierbar werden, je differenzierter sie eine Aktivität erfassen. Während im alpinen Bergsport eine solche Differenzierung durch das Österreichische Kuratorium für alpine Sicherheit (SLADEK 2003; 2004) und den DAV (RANDELZHOFER et al. 2004) bereits vorgenommen werden, wurde die Definition und Abgrenzung einzelner Aktivitäten im Bereich des Kanusports durch die Auswertung von zusammengefassten Unfallanzeigen durchgeführt. Die Differenzierung einzelner Kanusport-Disziplinen lehnt sich dabei weitestgehend der Kategorisierung des Deutschen Kanuverbandes an.

Die Reanalyse von kanusportlichen Unfallanzeigen wurde mit Unterstützung des Deutschen Kanu Verbands (DKV) realisiert. Der DKV versucht über einen standardisierten Unfallmeldebogen möglichst umfangreich, Daten über Kanu-Unfälle zu sammeln. Diese Erfassung erfolgt weitestgehend freiwillig und genügt damit nur bedingt wissenschaftlichen Ansprüchen ${ }^{242}$. 
Um möglichst umfangreiche Informationen zu analysieren, wurden die Daten des DKV mit Unfallberichten des Alpinen-Kajak-Clubs (AKC) ergänz, der seinerseits eine eigene Unfalldatensammlung vollzieht. Insgesamt liegt aus den Jahren 1998 - 2003 somit eine glaubwürdige Auswahl an Informationen über Kanu-Unfälle im deutschsprachigen Raum oder mit deutschsprachiger Beteiligung vor, die allerdings nur wenig detaillierte Informationen über den Unfallhergang enthalten und gleichsam keine Repräsentativität beanspruchen kann. Berücksichtigt werden konnten bei der Auswertung der Kanu-Unfälle nur Unfälle mit eindeutiger Verletzungs- oder Todesfolge, da nur hierüber verlässliche Informationen existieren ${ }^{243}$.

Aus den vorliegenden Statistiken der alpinen Bergsportverbände und aus den Unfalldaten des Kanusports wurden schließlich 6 unterschiedliche Aktivitäten für den Kanusport und 5 Aktivitäten für den alpinen Bergsport differenziert, denen sich eindeutige Unfallsituationen und -Zahlen zuweisen las$\operatorname{sen}^{244}$. Die Unfallkategorie Pisten- und Variantenskilauf wurde schließlich für die anschließende Fragebogenuntersuchung allerdings nochmals unterteilt, um Beurteilungsdifferenzen zu erfassen. Eine weitere Differenzierung der Aktivitäten in einzelne spezielle Tätigkeiten, bei denen sich Unfälle ereignen, ließ die Datenbasis nicht $\mathrm{zu}^{245}$ (vgl. zu dieser Problematik auch RINKE 2002, 58). Tabelle 9.2 stellt den Aktivitätskatalog für den alpinen Bergsport und den Kanusport zusammenfassend dar. Die im Fragebogen verwendeten Erläuterungen der einzelnen Aktivitäten wurden in Anlehnung an die verbandsinternen Begriffsbestimmungen des DKV bzw. des DAV erstellt ${ }^{246}$.

\begin{tabular}{|c|c|c|}
\hline \multicolumn{3}{|c|}{ A) Unfallbelastete Aktivitäten im Bereich des alpinen Bergsports: } \\
\hline NR. & Bezeichnung der Aktivität & Erläuterung \\
\hline 1 & Bergwandern & $\begin{array}{l}\text { Wandern und Gehen auf markierten Wegen } \\
\text { (auch im unwegsamen Gelände). }\end{array}$ \\
\hline 2 & Hochtourenbergsteigen & $\begin{array}{l}\text { Alle Besteigungen und Wanderungen im hoch- } \\
\text { alpinen Gelände (auch Gletscher). }\end{array}$ \\
\hline 3 & Felsklettern & \begin{tabular}{|l|} 
Besteigungen von Felsrouten ab Schwierig- \\
keitsgrad III - eingeschlossen ist das Klettern an \\
Eisrouten ab $50^{\circ}$ Neigungswinkel im Blankeis \\
sowie Aktivitäten in Kletterstiegen. \\
\end{tabular} \\
\hline \multirow[t]{2}{*}{4} & Pistenskifahren / -snowboarden & $\begin{array}{l}\text { Alle Wintersportarten, die auf markierten Pisten } \\
\text { in alpinen Skigebieten ausgeübt werden. }\end{array}$ \\
\hline & \& Variantenskifahren & \begin{tabular}{|l} 
Tätigkeiten im alpinen Schneesport, die sich \\
abseits markierter Pisten abspielen. Ausge- \\
nommen ist hier explizit das Tourenskilaufen
\end{tabular} \\
\hline $\mid 5$ & Tourenskifahren & Gehen einer Hochtour mit Wintersportgeräten. \\
\hline \multicolumn{3}{|c|}{ B) Unfalllbelastete Aktivitäten im Bereich des Kanusports } \\
\hline NR. & Bezeichnung der Aktivität & Erläuterung \\
\hline 1 & $\begin{array}{l}\text { Kanu-Wandern auf stehenden } \\
\text { Gewässern }\end{array}$ & $\begin{array}{l}\text { Befahren von Seen mit unterschiedlichen } \\
\text { Bootstypen (Kajak, Kanadier, Drachenboot) }\end{array}$ \\
\hline 2 & $\begin{array}{l}\text { Kanu-Wandern auf leicht flie- } \\
\text { Benden Gewässern }\end{array}$ & $\begin{array}{l}\text { Befahrungen auf langsam fließenden Flüssen } \\
\text { ohne größere Hindernisse mit unterschiedlichen } \\
\text { Bootstypen (Kajak, Kanadier). }\end{array}$ \\
\hline 3 & Seekajak & Kanu-Fahren auf dem offenen Meer - auch in \\
\hline
\end{tabular}




\begin{tabular}{||l|l|l||}
\hline & & direkter Küstennähe \\
\hline 4 & $\begin{array}{l}\text { Kanu-Fahren auf leichtem und } \\
\text { mäßig schwerem Wildwasser }\end{array}$ & $\begin{array}{l}\text { Flüsse im Schwierigkeitsgrad 1-2 der Wildwas- } \\
\text { serskala des DKV mit langsam fließender Strö- } \\
\text { mung und wenigen Hindernissen }\end{array}$ \\
\hline 5 & $\begin{array}{l}\text { Kanu-Fahren auf schwierigem } \\
\text { Wildwasser }\end{array}$ & $\begin{array}{l}\text { Flüsse im Schwierigkeitsgrad 3-4 der offiziellen } \\
\text { Wildwasserskala mit schnell fließender Strö- } \\
\text { mung und zahlreichen Hindernissen. }\end{array}$ \\
\hline 6 & $\begin{array}{l}\text { Kanu-Fahren auf extrem schwie- } \\
\text { rigem Wildwasser }\end{array}$ & $\begin{array}{l}\text { Flüsse im Schwierigkeitsgrad 5-6 der offiziellen } \\
\text { Wildwasserskala des DKV mit rasant fließender } \\
\text { Strömung und extremen Hindernissen }\end{array}$ \\
\hline \hline
\end{tabular}

Tabelle 9.2: Zusammenstellung der abgrenzbaren Aktivitäten für den Bereich des alpinen Bergsports und des Kanusports.

Sowohl im alpinen Bergsport als auch im Kanusport werden damit auch wenig offensichtliche Risikosport-Aktivitäten wie das Bergwandern bzw. das Kanu-Wandern in die Analyse einbezogen. Obwohl es sich hierbei nicht um klar definierte Risikosportarten handelt, werden sie in die folgende Analyse mit dem Ziel integriert, einen möglichst umfassenden Überblick über die Unfallsituationen und deren subjektive Einschätzung der einzelnen risikosportlichen Bereiche zu erhalten. In Anlehnung an die Konzeption risikosportlicher Aktivitäten bei PARLEBAS (1986) und den Verweis auf die phänomenologische Ambivalenz des Risikos, werden die weniger risikoreichen Varianten von typischen Risikosportarten in der Erwartung einer möglichst umfassenden Differenzierung in die Untersuchung einbezogen. Sportausübungen in künstlichen Anlagen (z.B. Kletterhallen, Schwimmhallen) wurden in der Analyse nicht berücksichtigt.

\subsubsection{Reanalyse der Unfalldaten}

Das Basismaterial der „[...] tatsächlichen Gefahr““ (MUSHAL 1997, 250) also der ,objektiven' Daten - waren alle verfügbaren Unfallinformationen im Kanusport für die Jahre 1998 - 2003 sowie im alpinen Bergsport für die Jahre 2000 - 2003. Die Auswahl der auszuwertenden Unfalldaten wurde dabei maßgeblich durch die Verfügbarkeit des Datenmaterials bestimmt. Während die Unfalldaten im Kanusport seit 1998 systematisch in ihren Einzelheiten dargestellt sind und darüber hinaus einzelne Unfallbögen und weiterführendes Material zur Verfügung stand, konnte im Bereich des alpinen Bergsports nur bereits ausgewertetes Datenmaterial reanalysiert werden ${ }^{247}$.

Für die Untersuchung im Teilbereich alpiner Bergsport ist damit keine weitere Reanalyse des Datenmaterials erforderlich, weil die vom Verband veröffentlichte Datenauswertung bereits detaillierte Statistiken enthält. Notwendig ist die Aufbereitung des Datenmaterials allerdings für den Teilbereich Kanusport. Die in diesem Handlungsfeld vorgenommene Unfallanalyse wurde anhand von Basisinformationen in der Klassifizierung in Anlehnung an WALLNER $(1977,13)$ über die Schwerpunkte Unfallbedingungen, Unfallhergang und Unfallfolgen vorgenommen. Die im Bereich des alpinen Bergsports herangezogene Unfallstatistik des Deutschen Alpenvereins 
(DAV) wurde der Datensammlung des Österreichischen Kuratoriums für alpine Sicherheit vorgezogen. Diese von SLADEK (2003; 2004) für das Österreichische Kuratorium ausgewerteten Unfalldaten betreffen zwar alle alpinen Bergsport-Aktivitäten im österreichischen Alpenraum und sind zudem nach Aktivitätsprofilen geordnet, eine detaillierte Aufschlüsselung der Unfalldaten erfolgt aber nicht. Die sehr detaillierte Unfalldatensammlung des DAV wurde daher aus Gründen der Vollständigkeit den Datensammlungen des Kuratoriums für alpine Sicherheit vorgezogen, denn die von RANDELZHOFER (2002; 2004) im Auftrag des DAV erhobene Unfallstatistik erfasst alle Unfälle der eigenen Verbandsmitglieder und gibt darüber hinaus Auskunft über die Schwere der Unfallfolgen. Da davon ausgegangen werden muss, dass auch im alpinen Bergsport einige informeller Sportengagements zu finden sind, stellt die Datensammlung das DAV zwar eine Unfallvollerhebung des Alpenvereinsmitglieder dar, letztlich ist sie aber keine repräsentativen Unfallstatistik. Da eine solche aber nicht existiert, wurde die DAV-Auswertung als die vollständigste und gleichsam detaillierteste Unfalldatensammlung ausgewählt248. Die ursprüngliche Überlegung, beide Statistiken als ergänzend zu kombinieren, wurde vor dem Hintergrund möglicher Überschneidungen verworfen.

Die vorliegenden Unfalldaten wurden schließlich anhand des aufgestellten Tätigkeitskatalogs neu kodiert. Dadurch wurde jeder Unfall einer Aktivität im alpinen Bergsport bzw. im Kanusport zugewiesen. Ließ sich ein Unfall aufgrund unzureichender Informationen nicht eindeutig zugeordnet werden oder konnte eine zweite Zuordnungskategorie nicht ausgeschlossen werden, wurde der entsprechende Datensatz nicht in die Analyse einbegriffen. Insgesamt wurden 2118 Unfälle reanalysiert, wobei im Kanusport 83 und im alpinen 2035 unfallbezogene Datensätze in die Analyse einbezogen wurden.

Eine Klassifizierung der Unfälle nach ihren Unfallfolgen konnte aufgrund der Datenlagen nur im alpinen Bergsport verwirklicht werden. Die Unfallanzeigen im Kanusport beinhalten hauptsächlich Unfälle mit schweren Verletzungen oder tödlichem Ausgang - eine Differenzierung bezüglich der Unfallfolgen erfolgt nicht. Anhand der Schadenspyramide nach HOWLAND, die BÄNI (1991a) für den Kanusport modifiziert, lassen sich gleichwohl die quantitativen Zusammenhänge zwischen Unfällen mit Todesfolgen, schweren Verletzungen und Beinahe-Unfällen verdeutlichen (vgl. Abb. 9.2) auch MUSAHL $(1997,380)$ ). Die statistische Varianz, die sich im Bereich der einzelnen Aktivitäten im Bezug auf die Schwere der Unfallfolgen ergibt, kann damit eingeschränkt werden ${ }^{249}$. Gleichwohl lassen sich für die vergleichende Transformation der Unfalldaten im Kanusport keine Aussagen für die relative Schwere der Unfallzahlen darstellen. Bei der Ergebnisdiskussion wird dieser Punkt erneut aufgegriffen und kritisch erörtert.

Die Klassifizierung der Unfallfolgen bezüglich ihrer Schwere wurde an das Kodierungssystem von RANDELZHÖFER (2003; 2004) angelehnt. Hierbei wird zwischen tödlichen Unfällen (einbezogen sind hier auch vermisste Personen), schwer verletzten Unfallfolgen (bei lebensbedrohlichen 
Unfällen), leichten Verletzungen als Folgen der Unfälle und Unfällen ohne Verletzungsfolgen differenziert ${ }^{250}$

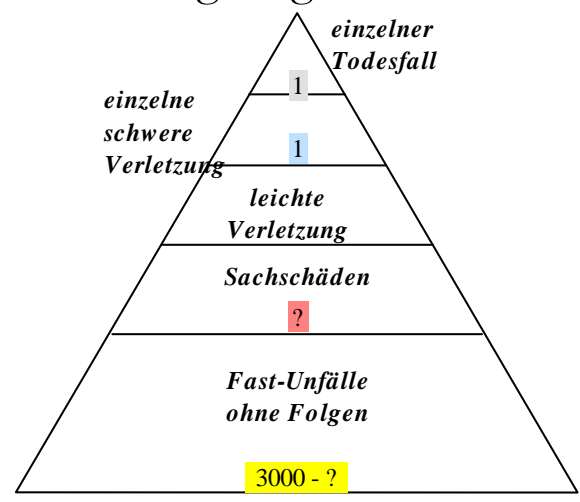

Abbildung 9.2: Schadenspyramide nach HOWLAND (mod. n. BÄNI 1991a, 3).

Dass die Reanalyse des objektiven Datenmaterials damit nicht vollständig durchführbar ist, soll hier kritisch angemerkt werden. Die ,objektiven' Daten beruhen letztlich auf Unfallanzeigen und -Statistiken, die mit deutlichen Mängeln der Zuverlässigkeit und der Gültigkeit behaftet sind. Da bei der vergleichende Analyse zwischen objektiven Daten und subjektive Einschätzungen keine absoluten Zahlen sondern relative Maße verwendet werden, geben die verwendeten Unfalldaten aber den größtmöglichen repräsentativen Wert der Gefährlichkeit der einzelnen Aktivitäten wieder. Mit der vollständigen Erfassung tödlicher und verletzungsschwerer Unfälle im Kanusport und aller vom DAV registrierten Unfälle im alpinen Bergsport wird letztlich der brauchbarste Referenzrahmen mit überprüfbarer Gültigkeit zur Bestimmung der Gefahrenkognition in den fokussierten Sportbereichen verwendet.

\subsubsection{Subjektives Datenmaterial}

Die Bestimmung des subjektiven Datenmaterials meint die Ermittlung der subjektiven Gefährlichkeitseinschätzung von aktiven Berg- und Kanusportlern. Um den objektiven Daten in der Form der tatsächlichen Unfallzahlen, die Einschätzungen der Sportler gegenüber zu stellen, wurde ein spezieller Fragebogen entwickelt. Die Konzeption des Fragebogen wurde auf der Grundlage der unfallbelasteten Sportaktivitäten in Anlehnung an die grundlegende Fragebogenstruktur bei MUSAHL erstellt. Da sowohl für den Kanusport als auch für den alpinen Bergsport institutionalisierte Strukturen fehlen, die eine Befragung der Untersuchungsteilnehmer erleichtern, wurde eine internetbasierte Erhebungsform ausgewählt.

\subsubsection{Teilnehmer der Befragung}

Eine kleine Probandenstichprobe, deren Urteile gemittelt werden, stellt nur wenig gesicherte Maßzahlen bezüglich der Gefährlichkeitseinschätzung dar. Deshalb war die Befragung einer relativ großen Probandengruppe im Rahmen dieser Untersuchung notwendig. Dieser Sachverhalt, der für die Mehr- 
heit quantitativer Studien gilt, verschärft sich im Rahmen der vorliegenden Untersuchung durch die Transformation der subjektiven Befragungswerte, bei denen die Differenzberechnung bei einer kleinen Probandengruppe durch Extremurteile verzerrt werden würde. Um ein möglichst umfassendes und differenziertes Meinungsbild bezüglich der Gefährlichkeitseinschätzung von alpinen Berg- und Kanusportler zu bekommen, ist die Befragung zudem mit möglichst unterschiedlichen Zielgruppen durchzuführen, um Einschätzungsdifferenzen unterschiedlicher Personengruppen möglichst exakt abzubilden. KÖHNE (2003) skizziert für das Medium Internet diesbezüglich verschiedene Möglichkeiten unterschiedliche Probandengruppen anzusprechen und zu einer Thematik zu befragen ${ }^{251}$. Die Befragung wurde vom 10.06.2005 bis 12.07.2005 durchgeführt. Insgesamt haben 907 Personen an der Befragung teilgenommen.

Die Befragung der Gefährlichkeitseinschätzung alpiner Bergsportdisziplinen wurde dabei über ein bergsport-spezialisiertes Internetforum des DAV und eine in Zusammenarbeit mit dem DAV durchgeführte Mailingaktion realisiert. Durch die Auswahl unterschiedlicher Befragungskanäle wurde ganz gezielt versucht, sowohl durchschnittliche Bergsport-Aktivisten als auch so genannte Spezialisten, also Fachübungsleiter der einzelnen Bergsportaktivitäten zu befragen. An der Befragung zur Gefährlichkeitseinschätzung im alpinen Bergsport nahmen insgesamt 724 Personen teil.

Auch die Befragung der Kanusportler hinsichtlich ihrer subjektiven Einschätzung der Gefährlichkeit der verschiedenen Kanusportdisziplinen wurde über verschiedene Internetforen und eine Mailingaktion in Zusammenarbeit mit dem DKV verwirklicht. Die Resonanz auf die Befragung viel im Bereich des Kanusports allerdings etwas geringer aus, nichtsdestotrotz gelang es hier ebenfalls neben gewöhnlichen Kanusportlern auch Fachübungsleiter zu interviewen. Insgesamt nahmen an der Befragung zur Einschätzung der Gefährlichkeit einzelner Kanusportaktivitäten 183 Personen teil.

Da eine internetbasierte Befragung nicht zum Standardrepertoire der Sportwissenschaft zählt, wird nachstehend ein kurzer Exkurs über die Methode, internetbasierte Befragung' erfolgen. Anschließend wird das Untersuchungsmaterial des subjektiven Datenmaterials, der Fragebogen zur subjektiven Gefährlichkeit, vorgestellt.

\subsubsection{Exkurs: Internetbasierte Befragungen - eine kritische Diskussion}

Internetbasierte Befragungen - oder Online-Befragungen (AMD et al. 2001) - repräsentieren eine neue Form der Fragebogenübertragung, wobei es sich nicht um eine gänzlich neue Methode der Sozialforschung handelt, sondern lediglich um eine neuartige Befragungsinteraktion. Dabei impliziert OnlineForschung keinesfalls einen Verzicht auf klassische Methoden und Verfahren empirischer Befragungsmethoden, internetbasierte Befragungen sind lediglich durch einige Besonderheiten gekennzeichnet.

Laut den „Standards zur Qualitätssicherung für Online-Befragungen“ (ebd.) schließt der Begriff Online-Befragung inhaltlich jene Befragungen ein, bei denen Befragungsteilnehmer a) den Fragebogen von einem Server her- 
unter laden und per E-Mail zurücksenden, b) den Fragebogen per E-Mail zugeschickt bekommen und zurücksenden und c) den auf einem Server abgelegten Fragebogen im Internet online ausfüllen (vgl. ebd., 5). In der vorliegenden Untersuchung wurde die letzte Variante gewählt. Diese verspricht zum einen ein Höchstmaß an Anonymität und gilt - wenn keine technische Hindernisse zum Ausfüllen des Fragebogens vorliegen - als weitestgehend effizienteste Befragungsform (vgl. auch KÖHNE 2003, 362ff). Im Vergleich $\mathrm{zu}$ traditionellen Befragungen, z.B. telefonischen Interviews oder schriftlichen Umfragen, ist die Rücklaufquote als auch die geographische Reichweite bei Online-Befragungen vergleichsweise hoch (vgl. Tab. 9.3):

\begin{tabular}{||l|l|l|l|l||}
\hline \hline & $\begin{array}{l}\text { Online- } \\
\text { befragung }\end{array}$ & $\begin{array}{l}\text { mundliche be- } \\
\text { fragung }\end{array}$ & $\begin{array}{l}\text { telefonische } \\
\text { befragung }\end{array}$ & $\begin{array}{l}\text { schriftliche be- } \\
\text { fragung }\end{array}$ \\
\hline Kosten & Sehr gering & Sehr hoch & mittel & Gering \\
\hline Rücklaufgeschwindigkeit & schnell & unmittelbar & unmittelbar & Langsam \\
\hline Rücklaufquote & hoch & sehr hoch & mittel & Gering \\
\hline Erreichbarkeit & wenige & alle & viele & nahezu alle \\
\hline Geograph. Reichweite & sehr hoch & sehr niedrig & mittel & hoch \\
\hline Zugänglichkeit & gering & unterschiedlich & mittel & sehr hoch \\
\hline Sendegeschwindigkeit & schnell & langsam & mittel & langsam \\
\hline \hline
\end{tabular}

Tabelle 9.3: Eigenschaftsvergleich von internetbasierten, mündlichen, telefonischen und schriftlichen Befragungen (aus THEOBALD 2000, 25).

Kritisch zu betrachten ist bei internetbasierten Befragungen allerdings die Tatsache der geringen Zugänglichkeit, denn an Online-Umfragen kann im Allgemeinen nur teilnehmen, wer über einen entsprechenden Anschluss und die damit verbundenen technischen Grundkenntnisse verfügt. Dieser Aspekt beinhaltet Effekte bezüglich der Auswahl und Nichtteilnahme von bestimmten Personengruppen und damit auf die Qualität der Daten (vgl. KÖHNE 2003, 460ff). Zwar prognostizieren EIMEREN et al. (2002, 346) für das Jahr 2005 für die Zahl der Internetanwender einen Anteil von 55\% der Gesamtbevölkerung, gerade untere Bildungsschichten und ältere Personen nutzen das Internet aber weiterhin nur wenig. Allerdings konstatieren EIMEREN \& GERHARD \& FREES (2001) in diesem Zusammenhang:

„Wurde noch 1997 die Klientel des Internets nabezu umfassend beschrieben durch die Attribute männlich, bochgebildet, 20bis 39 Jahre alt, findet das Internet beute in Schichten Anwendung, die noch vor wenigen Jabren eine Minderbeit unter den Nutzern dieses Mediums stellten. So stieg die Zabl weiblicher Internet-Nutzer im Vergleich 1997 bis 2001 um den Faktor 10. Ein Anstieg um den Faktor 13 ist bei den ab 50-Jährigen festzustellen. Die formal niedriger Ausgebildeten zeichnen sich durch Zuwachsraten in äbnlicher Größenordnung aus" (EIMEREN \& GERHARD \& FREES 2001, 383).

Diese Entwicklungen werden auch durch die jüngsten Studien zur Internet-Nutzung bestätigt ${ }^{252}$. Der Ausschluss wesentlicher Bevölkerungsgruppe schränkt sich damit auf wenige soziale Gruppen ein. Da auch bei einer ande- 
ren Befragungsform der Ausschluss potentieller Zielgruppen nicht vollständig verhindert werden kann, wurde das Internet, trotz dieser methodischen Schwäche, als Befragungsmedium ausgewählt.

Um die durchgeführte internetbasierte Befragung gegenüber anderen Befragungsinstrumenten zu legitimieren, findet im Folgenden eine methodenkritische Auseinandersetzung mit den gängigen Gütekriterien zur Bestimmung der Datenqualität empirischen Forschung statt. In Anlehnung an KROMREY (1998) sind als gängige Gütekriterien empirischer Sozialforschung die Objektivität, Reliabilität, Validität und Repräsentativität zu bezeichnen.

Als Objektivität wird das Ausmaß charakterisiert, in dem ein Untersuchungsergebnis in Durchführung, Auswertung und Interpretation vom Untersuchungsleiter nicht beeinflusst werden kann (vgl. LIENERT \& RAATZ 1994, 7). Eine Beeinflussung des Antwortverhaltens ist beispielsweise durch die Anwesenheit eines Interviewers in verschiedenen Fällen nachgewiesen worden (vgl. KÖHNE 2003, 370). Diese Problematik wird z.B. bei schriftlichen Befragungen durch selbst auszufüllende Fragebögen reduziert. Auch bei internetbasierten Befragungen ist mit einer geringen sozialen Beeinflussung zu rechnen, da die Beantwortung der Fragen anonym und weitestgehend allein durchgeführt wird. THEOBALD schlussfolgert schließlich: „Auf der Grundlage dieser Erkenntnisse [...] ist auch für die Umfragemethode im World Wide Web davon auszugehen, dass diese sich gegenüber persönlich vermittelten Befragungen durch eine bessere Durchführungsobjektivität auszeichnet"“ (THEOBALD 2000, 109f).

Die Reliabilität einer Messmethode gibt ihren spezifischen Grad der Zuverlässigkeit an. Eine Untersuchung wird dann als reliabel bezeichnet, wenn es bei einer Wiederholung der Messung unter denselben Bedingungen und an denselben Gegenständen zu demselben Ergebnis kommt (vgl. auch ALTESLANDER 1995, 342). Sie lässt sich u.a. durch eine Untersuchungswiederholung (Retest-Methode) oder einen andere, gleichwertige Untersuchung ermitteln (Paralleltest-Methode). Das Maß ist der Reliabilitätskoeffizient und definiert sich aus der Korrelation der beiden Untersuchungen. Da sich die Reliabiltät primär nach der Standardisierung der Meßmethode richtet, ist eine Online-Befragung bei standardisierten Items einer traditionellen Befragung vergleichbar. Die allgemein auftretenden Probleme der Varianz einer empirischen Untersuchung sind demnach nicht am Medium Internet festzumachen, sondern beziehen sich auf den Einfluss zufälliger Faktoren, wie Reihenfolgeeffekte der Fragen oder Ermüdung der Teilnehmer (vgl. THEOBALD 2000, 112).

Validitäts- oder Gültigkeitsmaße geben den Grad der Genauigkeit an, mit der ein Verfahren misst, was es zu messen vorgibt. Bei der Validitätsmessung wird zwischen verschiedenen Validitätskonzepten unterschieden (Begriffs-, konvergente, logische, Kriteriums-, Inhalts- oder Ökologische Validität), wobei sich drei übergeordnete Validitätskategorien klassifizieren lassen: Inhalts-, Kriteriums- und Konstruktvalidität (vgl. KÖHNE 2003, 371) ${ }^{253}$. BATINIC (2001) hat auf verschiedene Validitätseffekte empirischer Sozial- 
forschung hingewiesen und zur allgemeinen Steigerung der Gültigkeit folgende Empfehlungen ausgesprochen: a) Vermeidung interviewspezifischer Einflussquellen, b) Vermeidung ungeplanter Aktionen, c) Vermeidung der Entstehung von Sympathie-Relationen, d) die Trennung von Datenerhebung und -auswertung und schließlich e) statistische Urteilsbildung. Aufgrund seiner eigenen vergleichenden Analyse kommt der Autor zu dem Schluss, dass ,[...] WWW-Befragungen eine mit Papier-Bleistift-Befragung vergleichbare Validität und Realibilität erzielen“" (BATINIC 2001, 122).

Die Repräsentativität einer Befragung bezeichnet die Auswahl einer Stichprobe, für die alle in einer Grundgesamtheit typischen und charakteristischen Merkmale und Merkmalskombinationen gemäß ihrer relativen Häufigkeit vertreten sind.

„Um von einer repräsentativen Untersuchung sprechen zu können, muss sich die Auswabl der Teilnehmer auf eine sachlich, regional und zeitlich klar definierte Grundgesamtheit berieben und die auszuwäblenden Personen müssen unter Bezug auf die Auswablkriterien auf der Grundlage eines eindeutig definierten Auswablverfahrens individuell angesprochen werden" (AMD et al. 2001, 3).

Hier offenbart sich eine deutliche Schwäche von internetbasierten Befragungen. Aufgrund des derzeitigen Verbreitungsgrades wird eine Interneterhebung für die Gesamtbevölkerung nur selten repräsentativ sein, und auch die Repräsentativität für die Grundgesamtheit der Internetnutzer ist a priori nicht erreichbar, da die Tests innerhalb eines inhaltlichen Kontextes liegen, der eine gewisse Vorselektion trifft. Zwar weist THEOBALD darauf hin, dass ,[...] sich die Verteilung der genannten Variablen unter den WWWNutzern [...] denen in der Gesamtbevölkerung angleicht" (THEOBALD 2001, 88), eine repräsentative Untersuchung wird dennoch erst in ferner $\mathrm{Zu}$ kunft möglich sein ${ }^{254}$.

$\mathrm{Da}$ die interessierenden Parameter der Grundgesamtheit in der vorliegenden Untersuchung nicht bekannt sind, geht man davon aus, dass die Grundgesamtheit dann gut abgebildet wird, wenn die Stichprobe zufällig gezogen wird und keine systematischen Ausfallprozesse vorliegen. Annähernde Repräsentativität ist dann vorhanden, wenn die Stichprobenparameter (Mittelwert, Varianz) als gute Schätzungen der Parameter der Grundgesamtheit gesehen werden können. Die diesbezügliche Schwäche einer internetbasierten Befragung offenbart sich hierbei wiederum als Stärke, da über einen regionalen Kontext hinaus Probandengruppen angesprochen werden könne, die sonst kaum erreichbar gewesen wären. Nichtsdestotrotz gilt die Beachtung der Repräsentativität als wichtiges Kriterium wissenschaftlicher Güte, denn ,,...] so lange nicht ein Mindestmaß an Repräsentativität gewährleistet werden kann, bzw. die entsprechenden Kriterien dazu nicht erfüllt werden, erlauben auf solche Art erhobene Daten auch nur Aussagen über die jeweilige Stichprobe“ (THEOBALD 2001, 118). Allerdings ist mit COOMBER (1997) anzumerken, dass auch nicht repräsentative Daten für den wissenschaftlichen Erkenntnisprozess von Bedeutung sind. 
Insgesamt erscheint die Datengüte von internetbasierten Befragungen als mit traditionellen Befragungsinstrumenten vergleichbar, wenngleich insbesondere die Problematik einer nicht erreichbaren Repräsentativität zu berücksichtigen ist. Obwohl BATINIC (2001) die Datengüte von OnlineBefragungen als sehr gut einschätzt, weisen PÖTSCHKE \& SIMONSON darauf hin, dass ,[...] eine explizite Auseinandersetzung mit dem Medium Internet und seinen Möglichkeiten als Befragungsinstrument für die empirische Sozialforschung noch aussteht" (PÖTSCHKE \& SIMONSON 2001, 7).

Für das forschungsleitende Ziel der vorliegenden Untersuchung hat sich die internetbasierte Befragung vor allem aufgrund der großen Zahl erreichter Probanden als überaus sinnvoll dargestellt. Da eine Repräsentativität der Grundgesamtheit aller Berg- und Kanusportler auch bei anderen Erhebungsinstrumenten nicht möglich gewesen wäre, zeigt sich die Verwendung des Internets als nützliches Befragungsmedium, welches insbesondere im Bezug auf die Datengüte nur geringe Nachteile besitzt ${ }^{255}$.

\subsubsection{Untersuchungsmaterial: der Fragebogen zur subjektiven Gefäbrlichkeit im alpi- nen Berg- und Kanusport.}

Für die Einschätzung der subjektiven Gefährlichkeit wurden ein internetfähiger Fragebogen auf der Basis der Standards zur Qualitätssicherung für Online-Befragungen (AMD et al. 2001) entwickelt. Neben allgemeinen Instruktionen zur Studie wurden dabei Urteile über die Unfallhäufigkeit, die Unfallschwere und die individuelle Ausübungshäufigkeit der zuvor als unfallbelastet definierten Disziplinen und Aktivitäten im alpinen Bergsport und im Kanusport erhoben. Um die entsprechenden Aktivitäten inhaltlich abzugrenzen, wurden die oben angeführten Beschreibungen den einzelnen Fragekomplexen vorweg geschaltet (vgl. Tab. 9.2). Die Probanden sollten dabei in Anlehnung an das Verfahren bei MUSAHL (1997, 255f) die Tätigkeiten unter drei Gesichtspunkten beurteilen:

a) wie häufig sie die einzelnen Aktivitäten ausüben,

b) wie viele Unfälle bei diesen Tätigkeiten passieren und

c) wie schwerwiegend' die möglichen Folgen bei einem Unfall bei dieser Aktivität wären.

Die hierzu formulierten Fragen ließen sich mit Hilfe einer sechsstufigen Skala beantworten. Die Abstufungen der Skalen wurden - als Erweiterung des Verfahrens bei RINKE $(2002,60)$ durch entsprechende Hinweise erläutert, so dass die Probanden in der Lage waren, den Skalenwerten auch entsprechende Inhalte zu zuweisen.

Je Aktivität wurden drei Fragen (Ausübungshäufigkeit, Unfallhäufigkeit und Unfallschwere) gestellt, die jeweils gesondert erläutert wurden. Bei der Formulierung der Fragen wurde die Handhabbarkeit und Einfachheit einer augenscheinlichen Genauigkeit der Einzelangaben vorgezogen („Wie viele Unfälle ereignen sich Ihrer Einschätzung nach...?“ oder „Wie schlimm schätzen Sie die Folgen eines Unfalls...?). Die in einem Pretest geäußerte Kritik einer zu geringen Genauigkeit der Fragestellungen, konnte durch eine 
anschaulichere Erläuterung des Forschungsvorhaben entkräftet werden ${ }^{256}$. Insgesamt enthielt der Fragebogen zur Gefährlichkeitseinschätzung im alpinen Bergsport sowie der Fragebogen zur Einschätzung der Gefährlichkeit im Kanusport 18 Items.

Zusätzlich enthielt der Fragebogen einige sozialdemographische Fragen zur Person sowie Fragen zur Qualifikation und Erfahrung. Die Beantwortung der Fragen konnte innerhalb von 5 Minuten vollzogen werden ${ }^{257}$. Abbildung 9.3 gibt Struktur des Fragebogens beispielhaft für die Aktivität Bergwandern wieder.

\section{Zunächst einige Fragen zum Bergwandern (mit Bergwandern wird das Gehen auf markierten Wegen im alpinen Gelände bezeichnet, wobei auch unwegsames Gelände inbegriffen ist). \\ $\nabla$ Bitte kreuzen Sie an: \\ 1. Wie häufig üben Sie Aktivitäten aus, die Sie zum Bergwandern zählen würden?

\begin{tabular}{|c|c|c|c|c|c|}
\hline 1 & 2 & 3 & 4 & 5 & 6 \\
\hline nie & Fast nie & selten & gelegentlich & häufig & sehr häufig \\
\hline
\end{tabular}

2. Was denken Sie, wieviele Unfälle ereignen sich beim Bergwandern? Hierbei geht es nicht um Ihre persönlichen Erfahrungen, sondern um Ihre Einschätzung für das Bergwandern im Allgemeinen.

\begin{tabular}{|c|c|c|c|c|c|}
\hline 1 & 2 & 3 & 4 & 5 & 6 \\
\hline keine & geringe & erträgliche & ernste & schwere & sehr schwere \\
\hline
\end{tabular}

3. Wenn Sie der Meinung sind, es kommt beim Bergwandern zu Unfällen, wie schwer sind dann Ihrer Einschätzung nach die allgemeinen Unfallfolgen?

\begin{tabular}{|c|c|c|c|c|c|}
\hline 1 & 2 & 3 & 4 & 5 & 6 \\
\hline gar keine & wenige & einige & mehrere & viele & sehr viele \\
\hline
\end{tabular}

Abbildung 9.3: Exemplarische Darstellung der Items am Beispiel des Bergwanderns. Durch Ankreuzen einer Antwortvorgabe sollten 6 Bergsportdisziplinen und 6 Kanusportdisziplinen anhand der jeweils sechsstufigen Skalen zur Ausübungshäufigkeit, Unfallhäufigkeit und Unfallschwere beurteilt werden.

\subsubsection{Z-Wert-Transformation als statistisches Verfahren zur Konver- sion der objektiven und subjektiven Datensätze}

Entsprechend der Modellierung der Gefahrenkognition in Anlehnung an MUSAHL (1997) kann die Art der Gefahrenkognition (realistische Einschätzung, Unterschätzung oder Überschätzung) nach der relativen Übereinstimmung zwischen der subjektiven Gefährlichkeit $\left(\mathrm{G}_{\mathrm{s}}\right)$ als Ergebnis der Gefährlichkeitseinschätzung durch die Befragung und der objektiven Gefährlichkeit $\left(G_{o}\right)$ als Ergebnis der Unfallanalyse klassifiziert werden. Die Gefahrenkognition ergibt sich damit operational aus Richtung und Größe der Differenz beider Begriffe (vgl. ebd., 235). Zur Bildung dieser Differenz muss allerdings zunächst eine gemeinsame Berechnungsgrundlage mit Hilfe einer geeigneten Skalentransformation geschaffen werden. Standardnormen, so genannte z-Werte stellen diesbezüglich ein geeignetes Maß zum Vergleich von objektiver und subjektiven Daten dar. Nach BORTZ $(1999,45)$ werden durch z-Werte einzelne Werte im Bezug zum Kollektiv der Werte dargestellt (vgl. auch AMELANG \& BARTUSSEK 2001, 73; WILLIMCZIK 1997, 62). Z-Werte sind also auf die jeweilige Standardabweichung normierte Ab- 
weichungen der Einzelwerte vom jeweiligen Mittelwert, der daher stets „0“ ist; die Entfernung des einzelnen Wertes vom Mittelwert wird in Einheiten der Standardabweichung angegeben (vgl. MUSAHL 1997, 211).

Nach BORTZ $(1999,46)$ lautet die Formel zur Berechnung von z-Werte:

$$
\begin{aligned}
\bar{z} & =\frac{\sum_{i=1}^{n} z_{i}}{n}=\frac{\sum_{i=1}^{n}\left(x_{i}-\bar{x}\right)}{n \cdot s_{x}}=\frac{\sum_{i=1}^{n} x_{i}-n \cdot \bar{x}}{n \cdot s_{x}}=0 \\
& \left(\text { wegen } n \cdot \bar{x}=\frac{n \cdot \sum_{i=1}^{n} x_{i}}{n}=\sum_{i=1}^{n} x_{i}\right) \\
s_{z}^{2} & =\frac{\sum_{i=1}^{n}\left(z_{i}-\bar{z}\right)^{2}}{n} \\
& =\frac{\sum_{i=1}^{n} z_{i}^{2}}{n}(\text { wegen } \bar{z}=0) \\
& =\frac{\sum_{i=1}^{n}\left(x_{i}-\bar{x}\right)^{2}}{s_{x}^{2} \cdot n}=1 \\
& \left(\text { wegen } \sum_{i=1}^{n}\left(x_{i}-\bar{x}\right)^{2} / n=s_{x}^{2}\right)
\end{aligned}
$$

Hierbei sind die einzelnen Variablen in Anlehnung an das Erhebungsverfahren der Gefahrenkognition bei MUSAHL $(1997,211)$ folgendermaßen definiert:

$z_{i}=\frac{x_{i}-\bar{x}}{s}$

$\checkmark Z_{i}$ der $z$-Wert des Probanden (i) oder der Berg- oder Kanusportdisziplin (i)

$\checkmark \quad X_{i}$ der einzelne Messwert für den Probanden (i) bzw. die mittlere Unfallzahl, Unfallschwere oder deren jeweilige Schätzung der Tätigkeit (i)

$\checkmark$ x der Mittelwert der entsprechenden Urteil bzw. der Mittelwert aller Unfalldaten oder deren Schätzung, z.B. für eine bestimmte Aktivität, und $\checkmark$ s die entsprechende Streuung der Messwerte oder Urteile $\mathrm{X}_{\mathrm{i}}$ ist.

Nach SIXTL (1967) können intervallskalierte Daten unabhängig von ihrer Verteilungsform durch eine Transformation in z-Werte umgewandelt werden. Dass es sich bei den Gefährlichkeitseinschätzungen der befragten Probanden um intervallskalierte Daten handelt, steht außer Frage. Auch Unfallzahlen sind weitestgehend intervallskaliert, allerdings sind Unfälle statistisch gesehen immer seltene Ereignisse und somit in ihrer Verteilung schief, also poisson-verteilt (vgl. hierzu BORTZ 1999, 70f). Zwar stellt das arithmetische Mittel auch bei schiefen Verteilungen den Durchschnitt aller betrachteter Werte dar, der errechnete Mittelwert wird hierbei aber von wenigen extremen Werten deutlich verzerrt. Diese Verzerrung würde sich auch auf die z-Wert-Transformation auswirken und zu einer Verzerrung der zu vergleichenden $z$-Werte führen. Durch eine Neudefinition der Intervallgrenzen durch eine geeignete Datentransformation werden die Unfallzahlen deshalb 
zunächst ,normalisiert'. Eine solche Neudefinition der Intervallgrenzen stellt die Quadratwurzeltransformation dar. Durch diese ,Normalisierung' der Unfallzahlen wird die Verteilung der Unfälle insgesamt näher zusammen geschoben - Aktivitäten mit einer großen Unfallzahl werden dadurch proportional zu solchen Aktivitäten mit einer kleinen Unfallzahl stärker verkleinert. Die schließlich resultierenden $z$-Werte werden dadurch insgesamt kleiner. Diese Vorgehensweise wird von MUSAHL $(1997,277)$ und RINKE (2002, 68) als konservatives Klassifikationskriterium bezeichnet, denn es entstehen bei der Differenzbildung der $z$-Werte letztlich geringe Abstände, da mit vergleichsweise kleineren objektiven z-Werten gearbeitet wird. Zu beachten ist, dass sich diese Transformation hypothesenkonservativ verhält und mit ihr gegen die formulierte Hypothese $1 \& 2$ und für die entsprechende Nullhypothese gearbeitet wird.

Die Arbeitsschritte der Quadratwurzeltransformation und anschließender z-Wert-Transformation führen mit je einem z-Wert für die, objektiven' und ,subjektiven' Gefährlichkeitsmaße für jede Aktivität zur Angabe ihrer relativen Lage zum Gesamtmittelwert - in Einheiten der jeweiligen Standardabweichungen. Wenn sowohl die ,objektiven' Unfalldaten als auch deren ,mittlere subjektive' Einschätzung für die jeweilige Aktivität dem Mittelwert der jeweiligen Verteilung entsprechen, so ergibt sich ein z-Wert von 0. Ein z-Wert von 1 resultiert, wenn die geschätzte Gefährlichkeit oder die ,objektive' Unfallzahl um genau eine Standardabweichung oberhalb des Mittelwertes der Verteilung liegt (vgl. MUSAHL 1997, 236).

\subsubsection{Differenzberechnung}

Durch die z-Wert Transformation der objektiven und subjektiven Datensätze konnte eine geeignete Berechnungsgrundlage zur Differenzberechnung zwischen den tatsächlichen Unfallzahlen und den Gefährlichkeitseinschätzungen der einzelnen Aktivitäten im alpinen Berg- und Kanusport geschaffen werden. Die relative Übereinstimmung zwischen objektiver und subjektiver Gefährlichkeit findet damit einen sehr einfachen und anschaulichen Ausdruck in der Differenz zwischen den beiden Maßzahlen der einzelnen Aktivitäten. Die Differenz zwischen $G_{\mathrm{s}}-\mathrm{G}_{\mathrm{o}}=\in \mathrm{G}$ kann mit Hilfe der ztransformierten Werte in skalentheoretisch und mathematisch-statistisch zuverlässiger Weise berechnet werden (vgl. ebd.). Da es sich bei z-Werten wie beschrieben - um auf die jeweilige Standardabweichung normierte Abweichungen der Einzelwerte vom jeweiligen Mittelwert handelt, ist eine gültige Ergebnisberechnung von der Anzahl der Probandenanzahl abhängig. Bei kleinen Probandenstichproben bestimmen Extremwerte die Interpretation der Daten und damit die Differenzberechnung. Da an der vorliegenden Untersuchung mit 724 Personen für den alpinen Bergsport und 183 Personen für den Kanusport eine vergleichsweise große Probandenstichprobe rekrutiert wurde, ist die Gefahr eines ,zufälligen Ausreißers', der im Wiederholungsfall zu Mitte regrediert, relativ gering.

Bei einer positiven Differenz $\left(\mathrm{G}_{\mathrm{s}}>\mathrm{G}_{\mathrm{o}}\right)$ resultiert eine Überschätzung der tatsächlichen Gefährdung der jeweiligen Aktivität, bei negativer Diffe- 
renz $\left(G_{s}<G_{o}\right)$ eine Unterschätzung. In Anlehnung an MUSAHL (ebd.) ist der Geltungsbereich für realistische Einschätzungen qua conventionem festzulegen. Das von ZIMOLONG (1978) vorgenommene Kriterium, gemäß dem die statistische Grenzen für ein realistisches Urteil $-1.0<Z_{\in G}<1.0$ gilt, soll auch im Rahmen dieser Untersuchung verwendet werden. Im Bezug auf die Urteilsgüte ist dieses Kriterium ein relativ großzügiges Maß, denn erst wenn ein Urteil außerhalb des nach der Standardverteilung gültigen Bereichs von 34,13\% beiderseits des Idealwerts von $Z_{\in G}=0$ liegt, wird es als unterschätzt bzw. überschätzt eingeordnet ${ }^{258}$. Aussagen über die Gefahrenkognition und ihre Koviariation mit den tatsächlichen Unfallzahlen können statistisch gesehen aber auch innerhalb des für die Entscheidungstheorie signifikanten Bereichs getroffen werden (vgl. auch RINKE 2002, 75).

Die Differenzberechnung führt letztlich zu einer Aussage über die Gefahrenkognition des Probandengruppe im Sinne der relativen Beurteilungsgüte der Gefährlichkeit der untersuchten Aktivitäten. Anhand der Differenz $Z_{\in G}$ kann schließlich eine Aussage zur Gefährlichkeitseinschätzung vorgenommen werden, so dass realistisch eingeschätzte Disziplinen von unterschätzten und überschätzten Aktivitäten differenziert werden können.

\subsection{Ergebnisse}

Nachfolgend werden die Ergebnisse der Studie zu Unfällen und Gefahrenkognition im Risikosport vorgestellt, erläutert und veranschaulicht. Am Beispiel des alpinen Bergsports sowie des Kanusports wurde hierbei ein mehrdimensionales Forschungsdesign realisiert, bei dem sowohl objektive Daten in der Form einer Reanalyse von Unfällen als auch subjektive Daten als Ergebnisse einer internetbasierten Befragung zur Überprüfung der Hypothesen herangezogen wurden. Anhand eines Vergleiches zwischen tatsächlichen Unfallzahlen von verschiedenen Aktivitäten im alpinen Berg- und Kanusport, die durch eine Reanalyse von Unfallstatistiken und Unfallkurzberichten bestimmt wurden, und den Einschätzungen der Unfallhäufigkeit und Unfallfolgen dieser Aktivitäten durch aktive Sportler, konnten Differenzen und Übereinstimmungen zwischen beiden Datensätzen erfasst werden.

Zunächst werden in Abschnitt 9.3.1 die Untersuchungsergebnisse für den alpinen Bergsport dargestellt. Anschließend erfolgt die Ergebnisdarstellung für die kanusportlichen Aktivitäten in Abschnitt 9.3.2. Die der sich anschließende Diskussion (Abschnitt 9.4) werden die Untersuchungsergebnisse mit Bezug auf die Hypothesenüberprüfung interpretiert.

\subsubsection{Unfälle und Gefahrenkognition im alpinen Bergsport}

Die Untersuchungsergebnisse über eine Kovariation zwischen Unfällen und Gefahrenkognition im alpinen Bergsport beziehen sich auf die beschriebenen objektiven Daten der Unfallanzeigen und die im Rahmen einer internetbasierten Befragung realisierten Daten subjektiver Urteile von alpinen Bergsportlern. Zunächst werden die Ergebnisse der Unfallanalyse dargestellt 
(Abschnitt 9.3.1.1). Anschließend erfolgt die Ergebnisdarstellung der subjektiven Daten - also der Befragung aktiver Bergsportler (Abschnitt 9.3.1.2). Der Vergleich zwischen objektiven und subjektiven Daten in Abschnitt 9.1.3.3 stellt das Kernstück dieser ersten Teiluntersuchung dar. Geschlechts-, Alters- und Erfahrungsdifferenzen im Bezug auf die Gefahrenkognition werden abschließend in Abschnitt 9.1.3.4 vorgestellt.

\subsubsection{Die objektive Datenbasis: die Ergebnisse der Unfallanalyse}

In die Analyse der Unfälle alpiner Bergsportarten aus den Jahren 2000 2004 wurden insgesamt 2035 Unfälle einbezogen. Diese vom DAV erfassten Unfalldaten der Mitglieder verteilen sich dabei auf die zuvor differenzierten Aktivitäten (vgl. Tab. 9.2).. Während die Unfallzahlen für die Jahre 2000 2002 auf einem vergleichbaren Niveau zu verzeichnen sind, ist für das Jahr 2003 ein Anstieg an Unfällen zu konstatieren. Im Vergleich zu den anderen Jahren zeichnet sich das Jahr 2003 durch einen extremen Sommer mit langen Hitzeperioden sowie einem schneereichen Winter aus (vgl. RANDELZHOFER 2004, 4). Die auf die Jahre 2000 - 2003 verteilten Unfalldaten zeigt Abb. 9.5: 


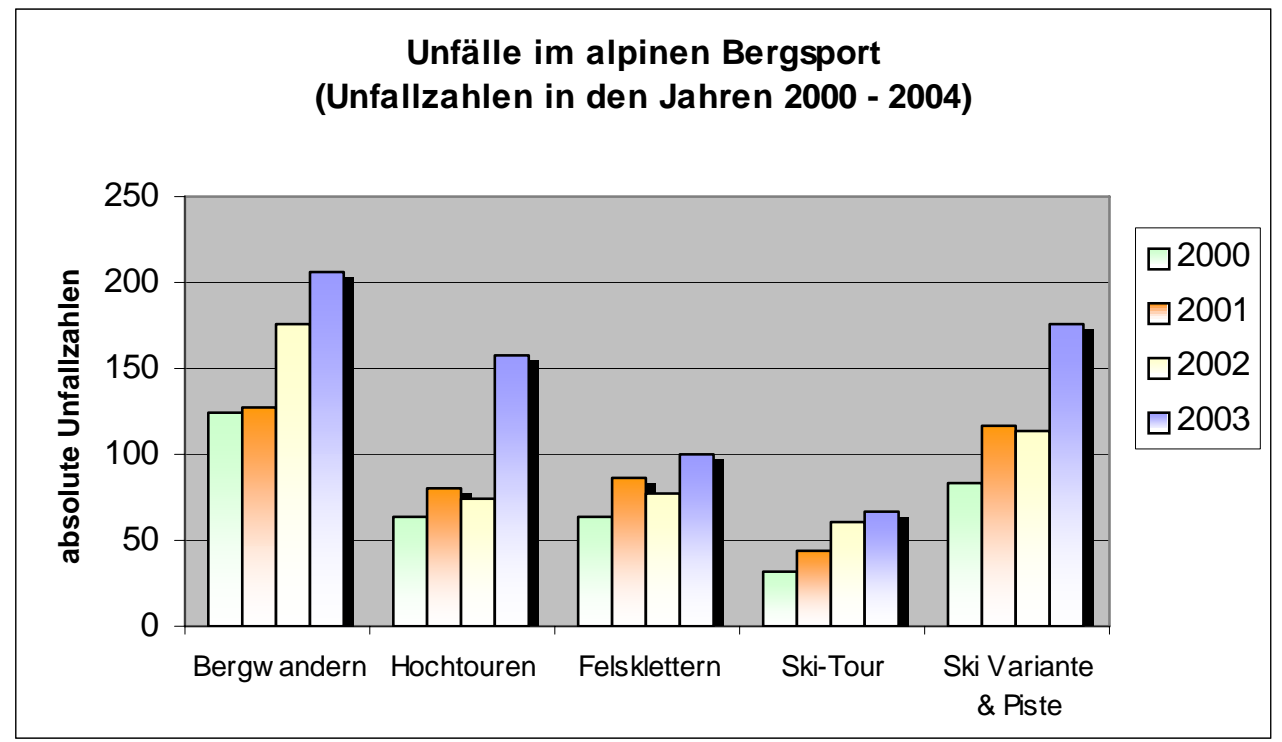

Abbildung 9.4: Unfälle im alpinen Bergsport für die Aktivitäten Bergwandern, Hochtouren, Felsklettern, Ski-Touren und Ski-Pisten \& -Variante für die Jahre 2000 2003. Die Datenbasis stellen die von RANDELZHOFER (2002; 2004) ausgewerteten Unfallanzeigen der DAV-Mitglieder dar.

Abbildung 9.4 zeigt das Gesamtunfallaufkommen der Jahre 2000 - 2003 und die Verteilung auf die einzelnen Aktivitäten. Das Bergwandern mit 625 Unfällen und Skifahren auf Pisten und Varianten mit 491 Unfällen weist dabei die mit Abstand größten Unfallzahlen auf, während das Skitourengehen mit 203 registrierten Unfallanzeigen das geringste Unfallaufkommen aufweist. Eine ähnliche Verteilung zeigt auch die Unfalldatensammlung des Österreichischen Kuratoriums für alpine Sicherheit, so dass von einer repräsentativen Unfallverteilung auszugehen ist (vgl. SLADEK 2004). 


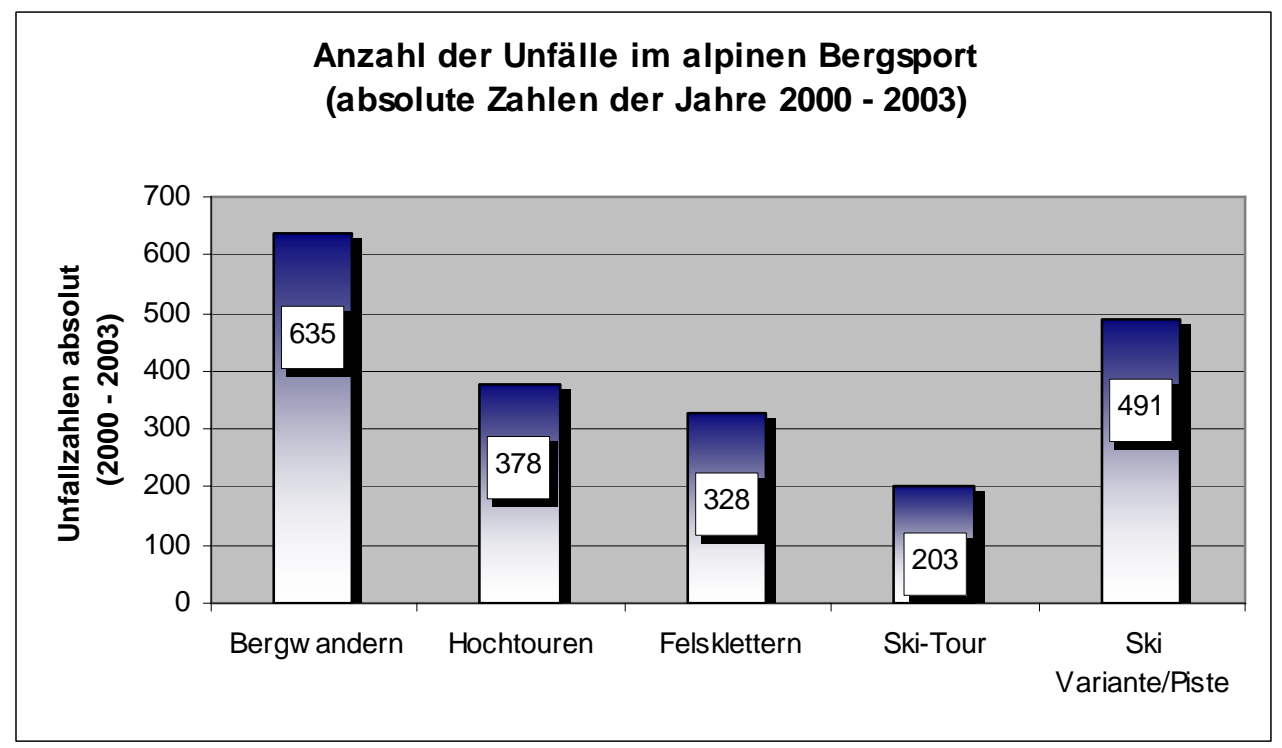

Abbildung 9.5: Absolute Unfallzahlen der einzelnen Aktivitäten für die Jahre 2000 2003. Einbezogen sind sowohl Unfälle ohne eindeutige Verletzung als auch Unfälle mit leichten und schweren sowie tödlichen Verletzungen.

Im Bezug auf die Verletzungsfolgen gibt Abbildung 9.5 Aufschluss über die spezifische Verteilung bei den einzelnen Aktivitäten. Die hierbei festzustellende Dominanz schwerer und tödlicher Anzeigen ergibt sich aus der Qualität der Datensammlung: Erfasst werden beim DAV alle Unfälle, die von Mitgliedern an den Alpinen Sicherheitsservice (ASS) gemeldet werden, um Bergungskosten etc. erstattet zu bekommen. Unfälle, die weder mit einer aufwändigen Bergung oder nur mit geringen Kosten für die Krankheitsbehandlung verbunden sind, werden in der Regel nicht gemeldet. Dies gilt für alle Handlungsbereich in gleicher Weise, so dass von einer in der Relation ausgeglichenen Datensammlung ausgegangen werden kann. Wenngleich für das Bergwandern, Hochtourenbersteigen, Felsklettern und Ski-Tourengehen von einer den absoluten Unfallzahlen entsprechenden Datenlage auszugehen ist, so stellen die Unfallmeldungen des alpinen Skilaufs zweifelsfrei nur einen Ausschnitt aus dem Gesamtunfallaufkommen dar. Da bei der statistischen Behandlung der Daten eine Expositionsbereinigung eine auf die Relativierung der Unfälle in Bezug auf die Schwere der Unfallfolgen vorgenommen wird, kann auch mit den Datensätzen des alpinen Skilaufs weitergearbeitet werden. 


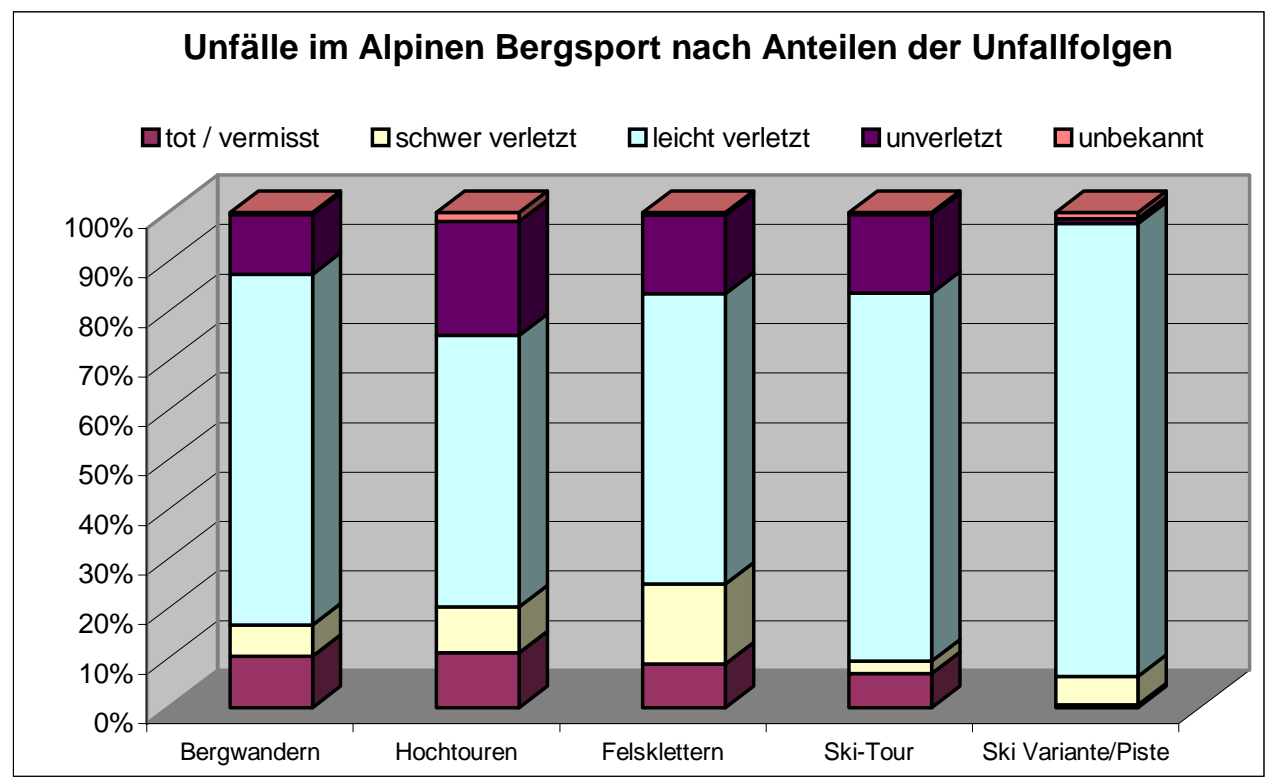

Abbildung 9.6: Unfälle im alpinen Bergsport nach Anteilen der Unfallfolgen (Angaben in Prozent).

Es zeigt sich hierbei deutlich, dass der Anteil von Unfällen mit schwerem Verletzungsausgang (tot / schwer verletzt) beim Hochtouren-Bergsteigen (77) und Felsklettern (82) gemessen am Gesamtunfallaufkommen am größten ist. Während das alpine Skifahren nur 31 Unfälle mit lebensbedrohlichen oder tödlichen Folgen aufweist, sind im Bergwandern 106 Unfällen mit entsprechend schweren Unfallfolgen zu verzeichnen. Beim Ski-Tourengehen sind 19 Unfälle mit lebensbedrohlichen oder tödlichen Unfallfolgen zu verzeichnen.

Da die Auftretenswahrscheinlichkeit von Unfällen bei einer sehr häufig ausgeführten Aktivität wesentlich größer sein wird, als bei einer nur wenig ausgeübten Tätigkeit, muss für eine Vergleich der objektiven Unfalldaten mit den subjektiven Einschätzungen eine Expositionsbereinigung der Unfalldaten erfolgen. Diese Expositionszeit, also die Zeit, in der Sportler sich in den Wirkungsbereich von Gefahren begeben, variiert unter den Sportaktivitäten. In dem Fragebogen zur Einschätzung der Gefährlichkeit wurden die Probanden gebeten, die Ausübungshäufigkeit der einzelnen Aktivitäten anzugeben. Mit diesen für jede Aktivität gemittelten Werten (siehe Anhang) wurden die tatsächlichen Unfallzahlen schließlich expositionsbereinigt: Die tatsächliche Unfallhäufigkeit einer Aktivität wurde durch die gemittelte Ausübungshäufigkeit der Aktivität dividiert und mit dem Mittelwert der Ausübungshäufigkeit aller Aktivitäten gemittelt.

$$
\text { UHex. }=\frac{\text { tatsächlicheUnfallhäufigkeit }}{\text { eingeschätzteAusübungshäufigkeiten }} * \text { Mittelwert (Ausübungshäufigkeit) }
$$

Durch diese Expositionsbereinigung wurde eine Unabhängigkeit der Unfallhäufigkeit von der Ausübungshäufigkeit geschaffen. Mathematisch ist die Ausübungshäufigkeit jeder Aktivität nun gleich, da proportional zu den Un- 
terschieden der mittleren Ausübungshäufigkeit die tatsächliche Unfallhäufigkeit verkleinert oder in Relation vergrößert wurde (vgl. auch RINKE $2002,78)^{259}$. Für jede Aktivität ergibt sich demnach eine neue expositionsbereinigte Unfallzahl (vgl. Abbildung 9.7).

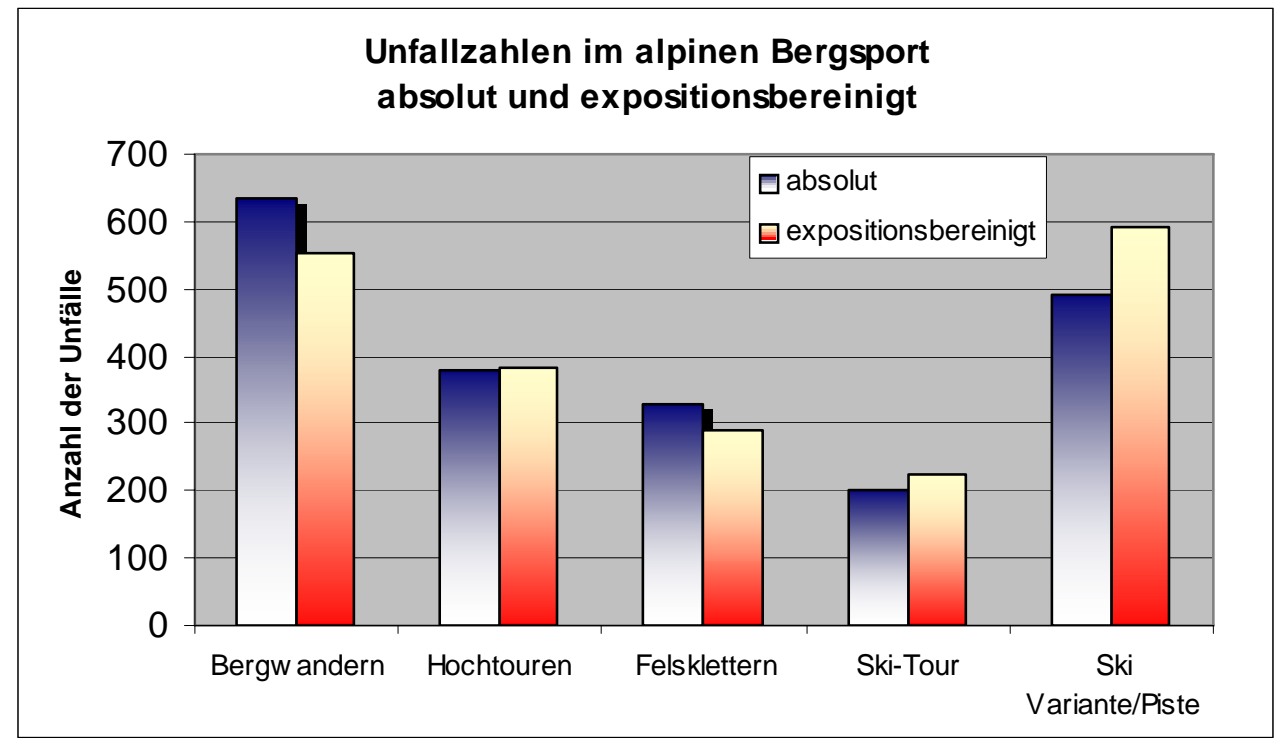

Abbildung 9.7: Unfallzahlen im alpinen Bergsport (absolut und expositionsbereinigt auf der Basis der Einschätzung der Ausübungshäufigkeiten der Probanden).

Durch die große Ausübungshäufigkeit ${ }^{260}$ des Bergwandern $(M W=4,33)$ und des Felskletterns (MW $=4,23)$ haben sich die Unfallzahlen dieser Tätigkeiten nach der Expositionsbereinigung verkleinert, die Zahlen für das Hochtouren-Bergsteigen ( $\mathrm{MW}=3,7)$, das Ski-Tourengehen $(\mathrm{MW}=3,42)$ und das Skifahren auf Pisten und Varianten $(\mathrm{MW}=3,12)$ entsprechend vergrößert.

Um die hier beschriebenen objektiven Unfalldaten einem Vergleich mit subjektiven Einschätzungen unterziehen zu können, wurden die Daten der absoluten Unfallzahlen und der expositionsbereinigten Unfalldaten - gemäß dem beschriebenen methodischen Vorgehen - zunächst quadratwurzeltransformiert und schließlich in z-Werte umgewandelt. Für jede Aktivität ergibt sich folglich ein gemittelter z-Wert, der die, objektiven' Unfalldaten im alpinen Bergsport repräsentiert (vgl. Abb. 9.9). 


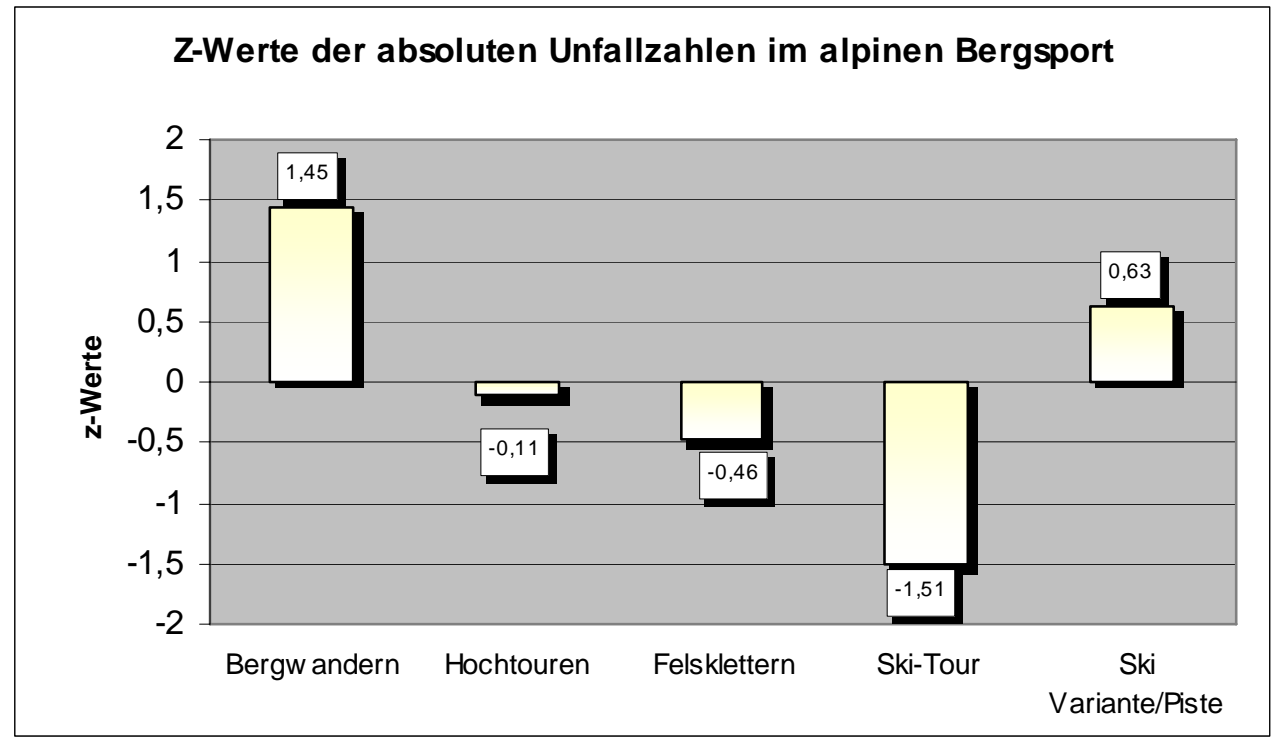

Abbildung 9.8: z-Werte der absoluten Unfallzahlen im alpinen Bergsport auf der Basis der analysierten Unfalldaten des DAV (für die Jahre 2000 - 2003).

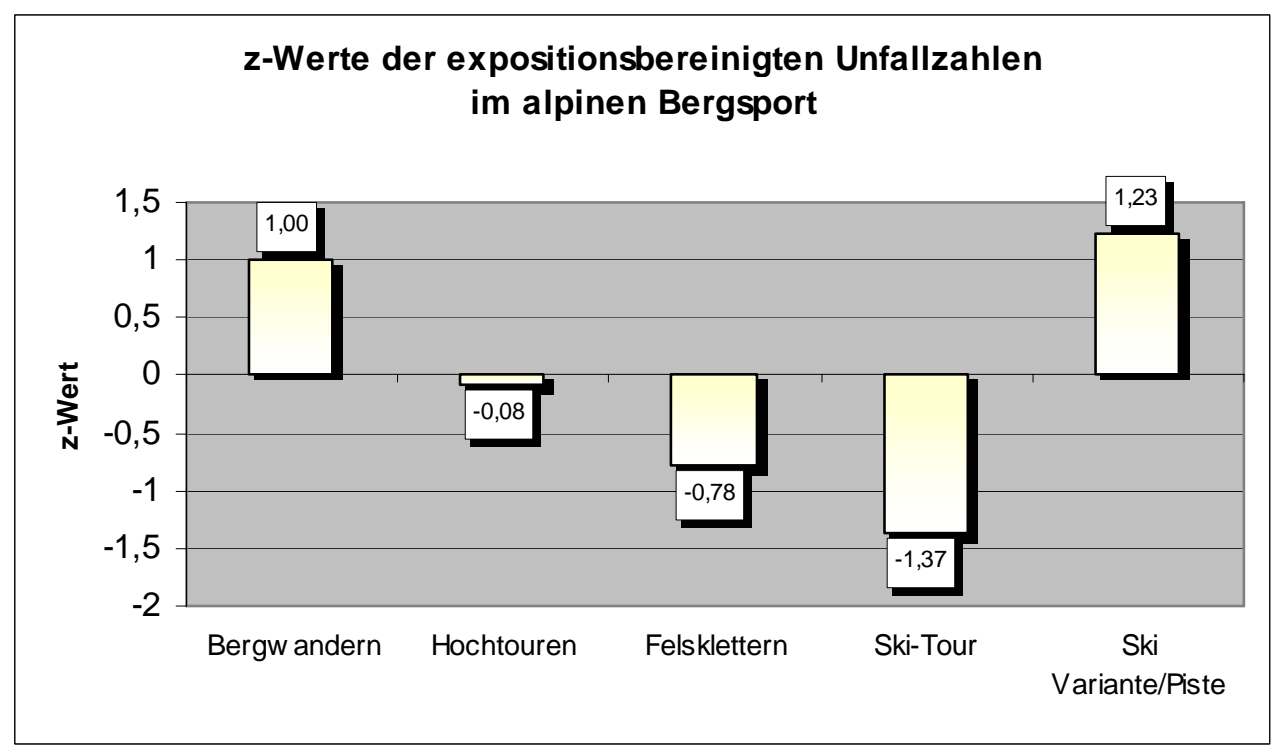

Abbildung 9.9: z-Werte der expositionsbereinigten Unfallzahlen im alpinen Bergsport auf der Basis der analysierten Unfalldaten des DAV (für die Jahre 2000 - 2003).

Da nicht nur die absoluten und expositionsbereinigten Unfallzahlen mit den Einschätzungen der Probanden im Bezug auf die Unfallhäufigkeit verglichen werden sollen, sondern auch ein Vergleich der Einschätzung bezüglich der Unfallfolgen erfolgt, muss eine Transformation der Unfallzahlen auch im Bezug auf die Schwere der Unfallzahlen erfolgen. Um die Unterschiede zwischen Unfallhäufigkeit und Unfallschwere bei der operationalen Definition der objektiven Gefahr zu berücksichtigen, musste ein entsprechendes Gewichtungsverfahren gewählt werden. In Anlehnung an MUSAHL (1997, 261ff) wurde ein Verfahren zur Ermittlung von auf ihre relative Schwere bezogenen Unfallzahlen entwickelt. Hierzu wurde ein Pro- 
portionalitätskoeffizient anhand der Relation zwischen dem Anteil von Unfälle mit sehr schweren oder tödlichen Verletzungsfolgen und dem expositionsbereinigten Unfallhäufigkeitsanteil (in \%) bestimmt. Die Anzahl der Unfälle mit sehr schweren und tödlichen Verletzungsfolgen gibt Abb. 9.10 wieder.

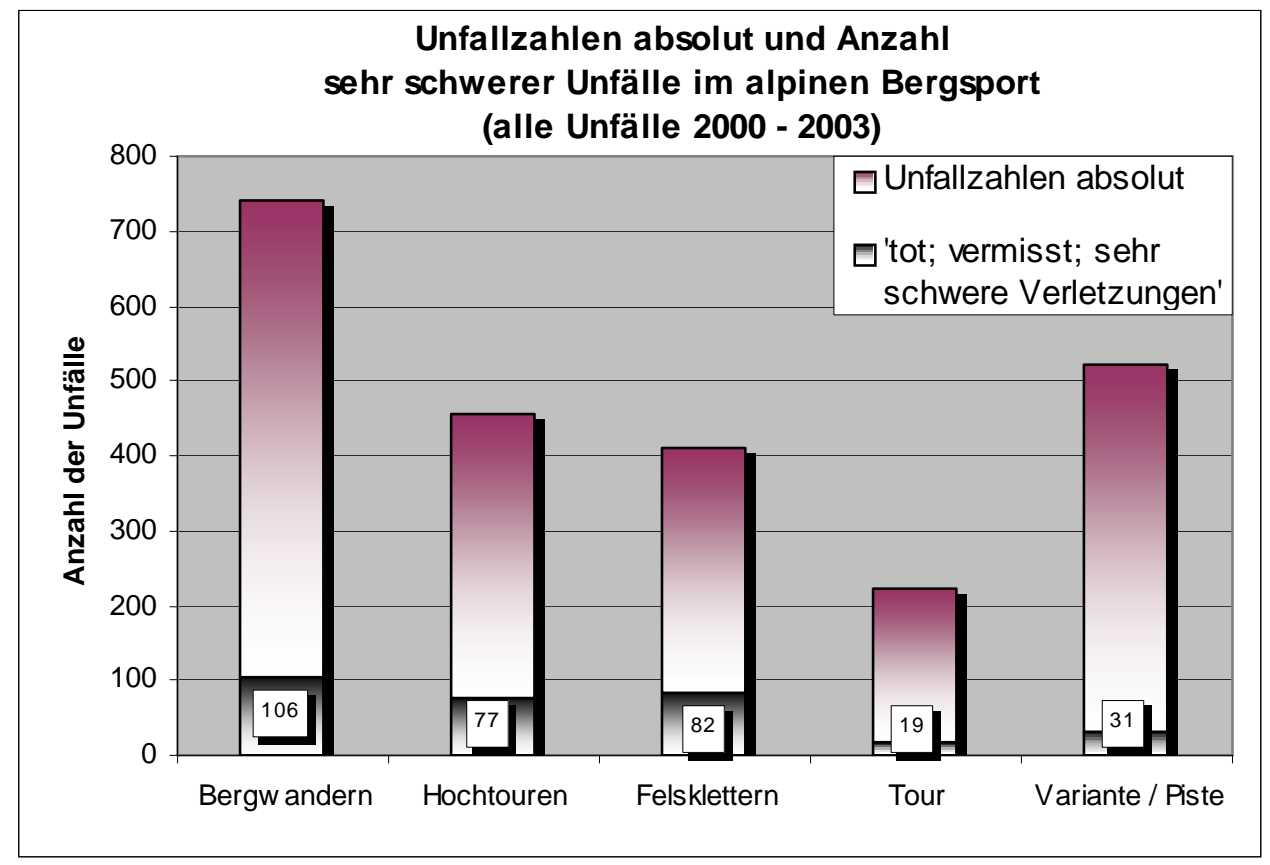

Abbildung 9.10: Unfallzahlen absolut und Anzahl der Unfälle mit tödlichen oder sehr schweren Verletzungsfolgen im alpinen Bergsport. Sehr schwere Verletzungsfolgen werden hierbei als lebensbedrohlich und nachhaltig die Gesundheit beeinträchtigend definiert (vgl. auch RANDELZHOFER 2003).

Da die Anzahl schwerer Unfälle ein im Bezug zur absoluten Unfallzahl geeignetes Maß zur Bestimmung der relativen Schwere der Unfallzahlen darstellt (vgl. auch RINKE 2002), werden diese auf ihre Schwere relativierten Unfallzahlen für den Vergleich mit den subjektiven Einschätzungen bezüglichen der Unfallschwere einzelner Aktivitäten herangezogen. Werden die expositionsbereinigten Unfallzahlen schließlich mit dem errechneten Proportionalitätskoeffizienten multipliziert, ergibt sich schließlich eine relativierte Unfallzahl mit gleicher neutralisierter Schwere. Die ursprünglichen Unfallzahlen für das Bergwandern, das Hochtourenbergsteigen und das Felsklettern werden durch den hohen relativen Anteil schwerer Unfallfolgen aufgewertet, die Unfallzahlen für das Ski-Tourengehen und das Skifahren auf Pisten und Varianten entsprechend abgewertet. Durch diese gewichtete Unfallzahlen entsteht also eine normierte Unfallzahl, die faktisch alle Unfälle so behandelt, als seien sie gleich schwer und beruhten auf gleich häufig ausgeübten Aktivitäten. Damit relativieren sich auch entsprechende statistischmathematischen Fehleinschätzungen: die Unfallzahlen sind nunmehr nicht mehr nach leichten oder schweren Unfallfolgen oder dem statistischen Effekt der Häufigkeit der einzelnen Tätigkeiten angreifbar. Die neu errechneten, auf ihre nach der Schwere relativierten, Unfallzahlen wurden schließlich 
ebenfalls durch Quadratwurzeltransformation neutralisiert und in z-Werte umgerechnet. Abbildung 9.11 stellt diese auf ihre Schwere relativierten und z-Wert transformierten Unfallzahlen dar. Die z-Werte der relativierten Unfallzahlen unterscheiden sich insbesondere für die Aktivitäten Ski Piste / Variante sowie im Bereich Hochtouren und Felsklettern massiv von den zWerten der tatsächlichen und expositionsbereinigten Unfallzahlen. Die Bedeutung dieses Verfahrens für einen anzustellenden Vergleich im Bezug auf die Unfallfolgen wird damit deutlich untermauert.

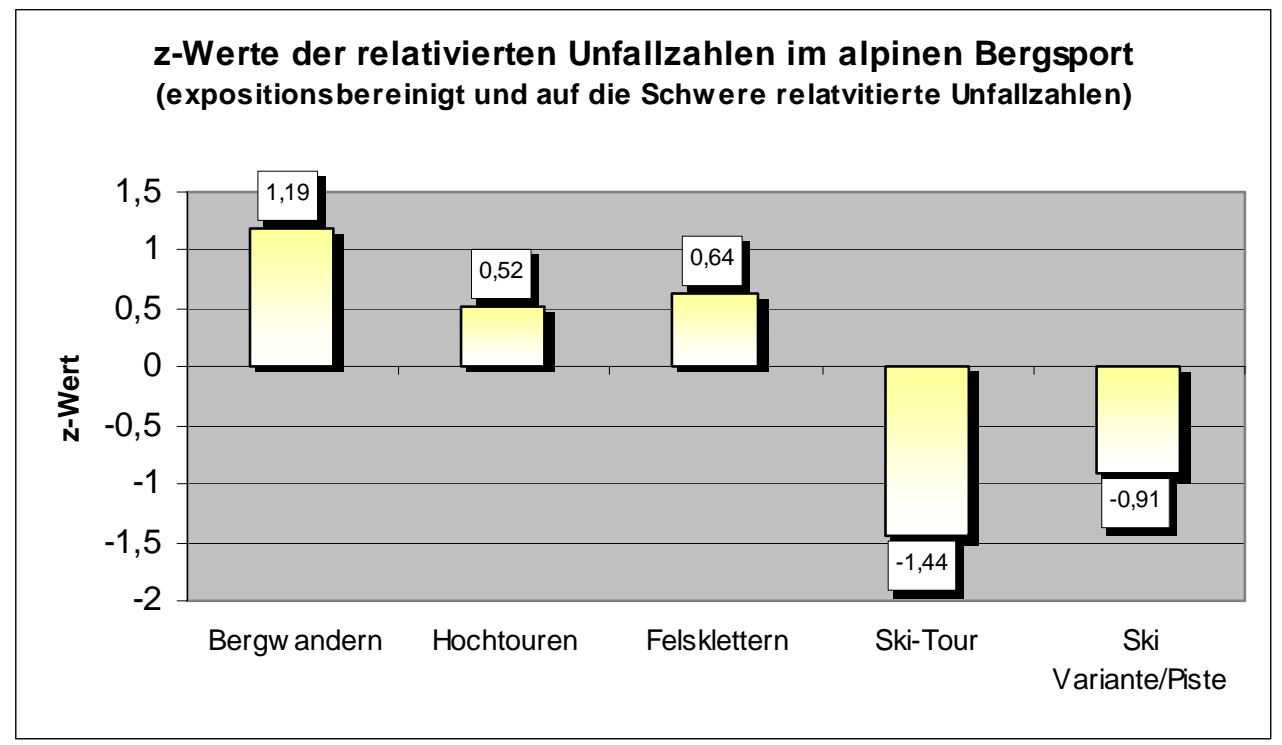

Abbildung 9.11: z-Werte der auf ihre Schwere relativierten Unfallzahlen im alpinen Bergsport. Datenbasis sind alle Unfälle im Zeitraum 2000 - 2003 und der hierin zu konstatierende Anteil schwerer Unfallfolgen mit tödlichen oder lebensbedrohlichen Verletzungen.

Mit den auf die absoluten und expositionsbereinigten sowie auf die in ihrer Schwere relativierten Unfallzahlen bezogenen $z$-Werten steht nun ein Vergleichmaß zur Bestimmung der Gefahrenkognition zur Verfügung. Diese vergleichende Analyse erfolgt im folgenden Abschnitt. Vorab werden die Ergebnisse der internetbasierten Befragung als operative Basis der subjektiven Datensätze dargestellt.

\subsubsection{Die subjektive Datenbasis: die Ergebnisse der internetbasierten Befragung}

Die über die beschriebene internetbasierte Befragung realisierte Umfrage wurde mit Hilfe des Programm Grafstat $(\mathrm{C}$ ausgezählt und schließlich der weiteren statistischen Auswertung unterzogen. An der Befragung, die zwischen dem 10.06. und 12.07.2005 durchgeführt wurde, nahmen insgesamt 724 Personen mit gültigen Antworten teil. 7 Fragebögen mussten aufgrund von Unvollständigkeit verworfen werden. Der Altersdurchschnitt betrug 36,4 Jahre. Neben 587 Männern nahmen 137 Frauen an der Untersuchung teil. Unter den Probanden befanden sich zahlreiche Übungsleiter aus den Bereich Alpinklettern ( $n=69)$, Bergsteigen $(n=124)$, Hochtouren $(n=134)$, 
sowie Ski ( $\mathrm{n}=60)$, deren Urteile im Rahmen der Ergänzungsuntersuchung gesondert ausgewertet wurden. Darüber hinaus wurde in der Befragung nach der individuellen Erfahrung - unabhängig von der Übungsleiterlizenz gefragt. Die hier ermittelten Urteile bezüglich der Unfallhäufigkeit und Unfallschwere von alpinen Bergsportdisziplinen wird ebenfalls im Rahmen der Ergänzungsuntersuchung vorgestellt (vgl. Anhang A).

Die Einschätzungen der Probanden im Bezug auf die Unfallhäufigkeit und die Unfallschwere wurden für jede Tätigkeit gemittelt und in entsprechende z-Werte transformiert (vgl. auch Anhang A). Für die einzelnen Tätigkeiten ergeben sich somit $z$-Werte für die Einschätzung der Unfallhäufigkeit der einzelnen Aktivitäten und z-Werte für die entsprechenden Unfallfolgen (Abb. $9.12 \&$ Abb. 9.13).

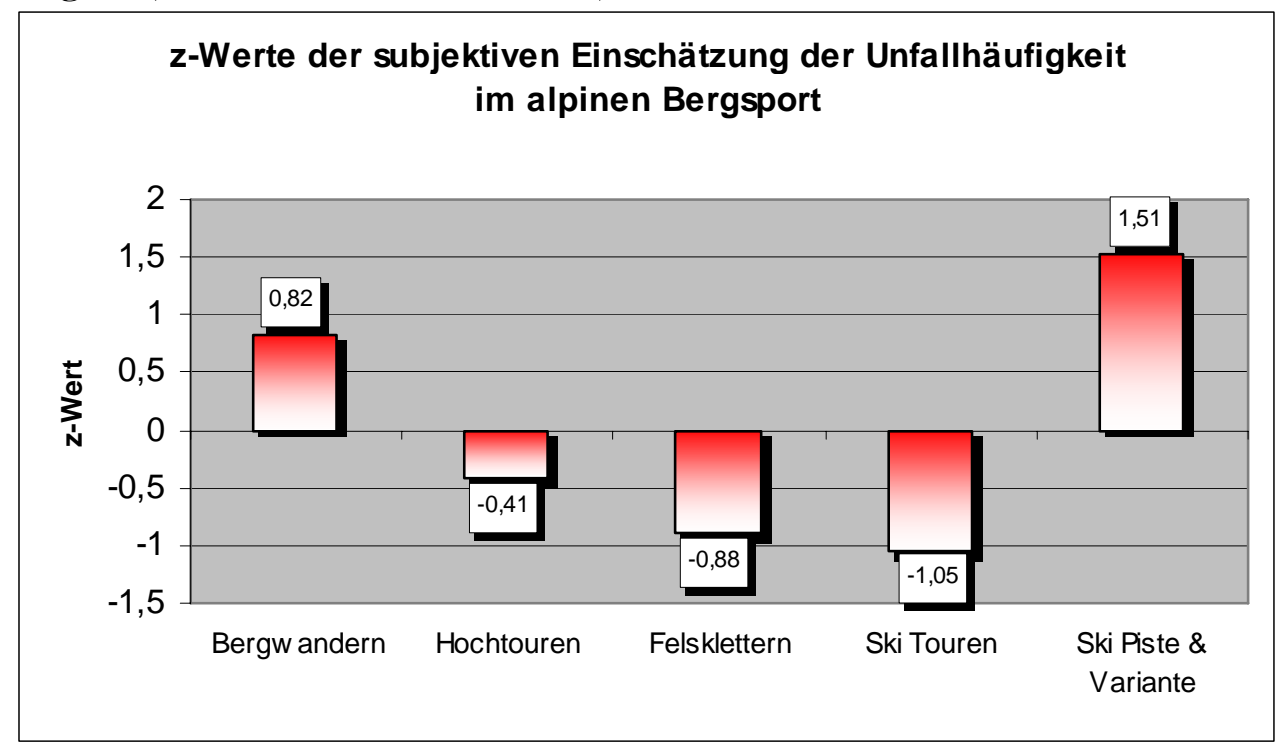

Abbildung 9.12: Einschätzungen der Unfallhäufigkeit bei den vorgegebenen Aktivitäten des alpinen Bergsports. Die Rohdaten wurden gemittelt und in z-Werte umgerechnet. 


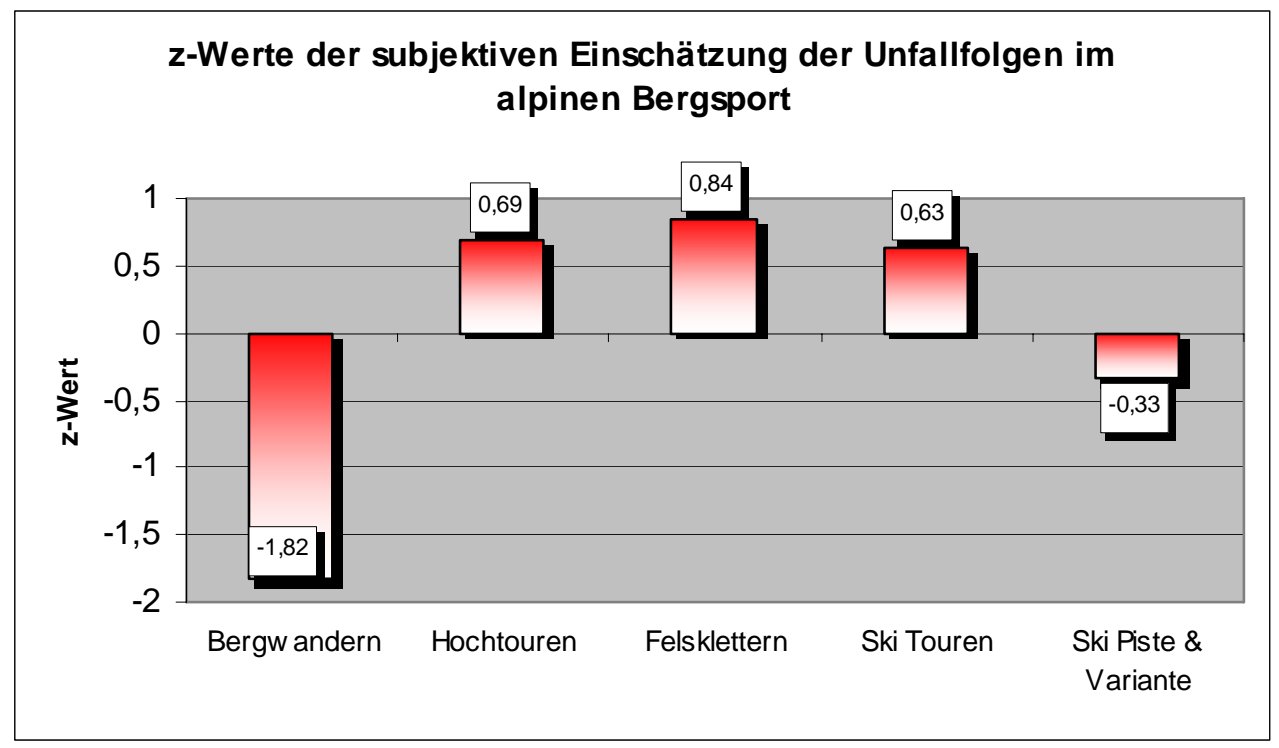

Abbildung 9.13: Einschätzungen der Unfallfolgen bei den vorgegebenen Aktivitäten im alpinen Bergsport. Die Rohdaten wurden gemittelt und in z-Werte transformiert.

Die beiden Diagramme zeigen dabei deutliche Abstände im Beurteilungsverhalten für die einzelnen Aktivitäten. Je größer die z-Werte im positiven Bereich sind, desto überdurchschnittlich unfallbelastet wird die entsprechende Aktivität eingeschätzt. Je kleiner die z-Werte ausfallen, desto unterdurchschnittlich - bezogen auf die Einschätzung aller Aktivitäten - werden diese Sportarten beurteilt. Im Bezug auf die Unfallhäufigkeit werden also sowohl das Bergwandern als auch das alpine Skifahren auf Pisten und Varianten im Vergleich zu den anderen Aktivitäten überdurchschnittlich unfallbelastet eingestuft, während das Felsklettern, das Ski-Tourengehen und das Hochtourengehen unterdurchschnittlich eingestuft werden. Bei der Beurteilung der Unfallfolgen werden diese letztgenannten Sportaktivitäten dagegen überdurchschnittlich beurteilt.

Entgegen den Annahmen MUSAHL's und RINKE's, wonach ,[...] bei einer Folgeuntersuchung auf die Erhebung der Unfallschwere verzichtet werden kann, da die Angaben über die Verletzungsschwere den Einschätzungen der Unfallhäufigkeit entnommen werden können“" (RINKE 2002, 101), zeigt sich in der vorliegenden Untersuchung ein sehr differenziertes Beurteilungsverhalten. Die Probanden im alpinen Bergsport taxieren die Unfallhäufigkeit und die Unfallfolgen durchaus differenziert und können zwischen der Einschätzung von Unfallfolgen und der Unfallhäufigkeit klar unterscheiden. Besonders deutlich zeigt sich dies bei der Kategorie ,Felsklettern': Während für die Einschätzung der Unfallhäufigkeit ein unterdurchschnittlicher Mittelwert von 3,09 resultierte, wurde die Frage nach den Unfallfolgen im Mittel mit 4,47 überdurchschnittlich eingestuft (vgl. auch Anhang A). Eine Vernachlässigung der Ergebnisse zugunsten einer eindimensionalen Betrachtung der Einschätzung der Unfallhäufigkeit würde die Gefahreneinschätzung der Probanden nur unzureichend abbilden. Dies zeigt sich auch in der differenzierten Einschätzung des alpinen Skilaufs. Hierbei wurde 
die Probanden gebeten, das Skifahren auf Pisten und das Skifahren auf Varianten gesonderten zu beurteilen. Tabelle 9.4 stellt die Mittewerte der Einschätzungen dar.

\begin{tabular}{||l|l|l||}
\hline \hline & $\begin{array}{l}\text { Alpines Skifahren } \\
\text { auf Pisten }\end{array}$ & $\begin{array}{l}\text { ALpines Skifahren auf } \\
\text { Varianten }\end{array}$ \\
\hline $\begin{array}{l}\text { Mittelwert Ausübun- } \\
\text { gshäufigkeit } \\
\text { =nie }-6 \text { sehrnüurig }\end{array}$ & 3,49 & 2,75 \\
\hline $\begin{array}{l}\text { Mittelwert Unfallhäu- } \\
\text { figkeit } \\
1=\text { gar keine - } \\
6=\text { sehr viele }\end{array}$ & 4,43 & 3,44 \\
\hline $\begin{array}{l}\text { Mittelwert Unfallfol- } \\
\text { gen } \\
1=\text { keine - } \\
6=\text { sehr schwere }\end{array}$ & 3,72 & 4,47 \\
\hline
\end{tabular}

Tabelle 9.4: Differenzen im Einschätzungsverhalten am Beispiel des alpinen Skifahrens auf Pisten und Varianten $(n=724)$.

Im Folgenden werden die Einschätzungen bezüglich der Unfallhäufigkeit und Unfallschwere mit den objektiven Unfalldaten verglichen, um ein genaues Bild der Gefahrenkognition im Risikosport zu bekommen.

\subsubsection{Differen₹bildung: objektive und subjektive Daten im Vergleich}

Der Vergleich zwischen objektiven Unfalldaten und den für diese Aktivitäten abgegebenen Urteilen über die Unfallhäufigkeit und die Unfallschwere wird über die Differenzbildung der entsprechenden z-Werte in Anlehnung an ZIMOLONG (1978) und MUSAHL (1997) sowie RINKE (2002) vollzogen (vgl. Abschnitt 9.2.3). Qua conventionem wurde festgelegt, dass bei einer Differenz mit den statistischen Grenzen $-1,0<Z_{\in G}<1,0$ eine realistische Gefahrenkognition resultiert. Die subjektive Einschätzung stimmt in diesem Bereich der z-Wert-Differenzen also mit den tatsächlichen Unfalldaten relativ gesehen überein. Bei einer $z$-Wert-Differenz $a b Z_{\in G}>1,0$ wird eine Überschätzung der tatsächlichen Gefahr der Situation angenommen die subjektive Einschätzung der Gefährlichkeit liegt demnach deutlich über den tatsächlichen Unfalldaten. Wenn die subjektive Gefährlichkeitseinschätzung geringer ausfällt als die tatsächliche - durch die Unfalldaten vermittelte - Gefährdung, dann liegt eine Unterschätzung der Gefährlichkeit vor, also $Z_{\in G}<-1,0$.

Die Ergebnisse zeigen, dass bezogen auf die Unfallhäufigkeit eine weitestgehend realistische Gefahrenkognition im alpinen Bergsport vorherrscht. Dies gilt sowohl für die Differenzbildung zwischen subjektiver Einschätzung und absoluten Unfallzahlen (Abb. 9.14) als auch für den Vergleich zwischen subjektiver Einschätzung und expositionsbereinigten Unfallzahlen (Abb. 9.15). 


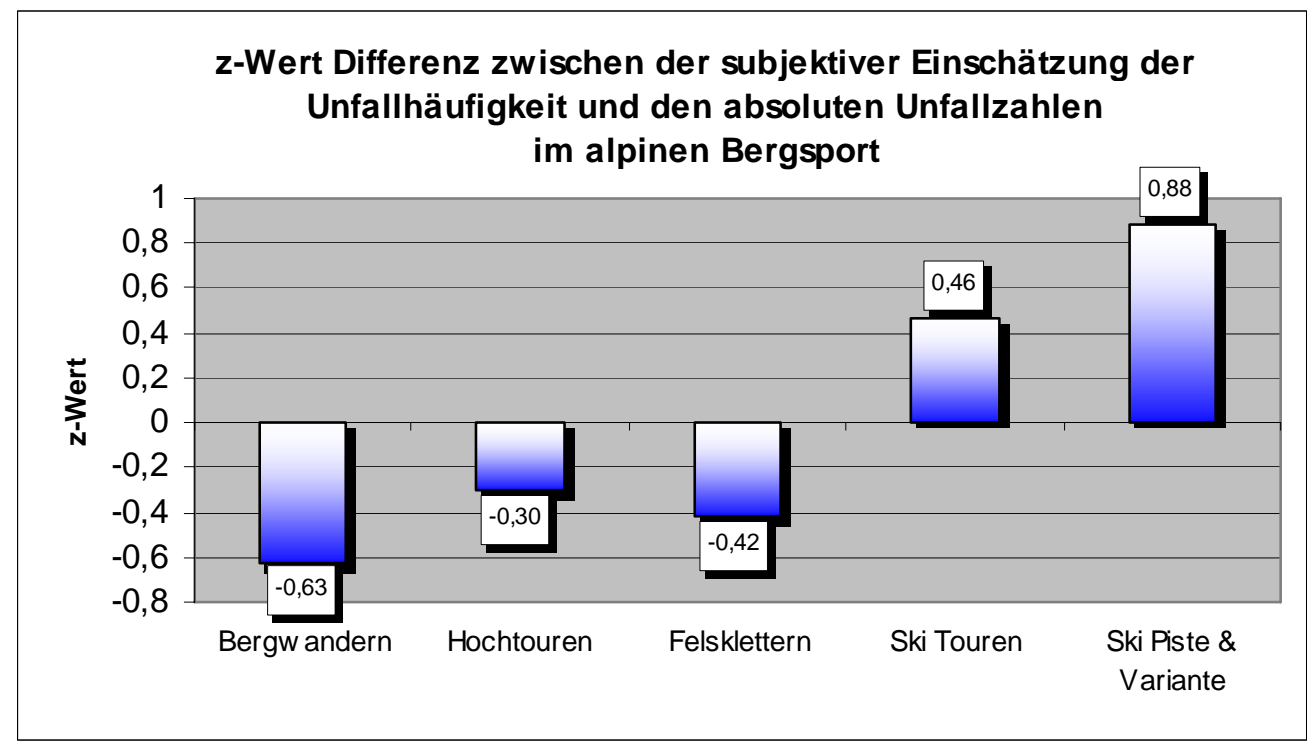

Abbildung 9.14: z-Wert Differenz zwischen den subjektiven Einschätzungen über die Unfallhäufigkeit und den tatsächlichen absoluten Unfallzahlen im alpinen Bergsport ( $\mathrm{n}$ (subjektiv) $=724 ; \mathrm{n}$ (objektiv) $=2035$ ).

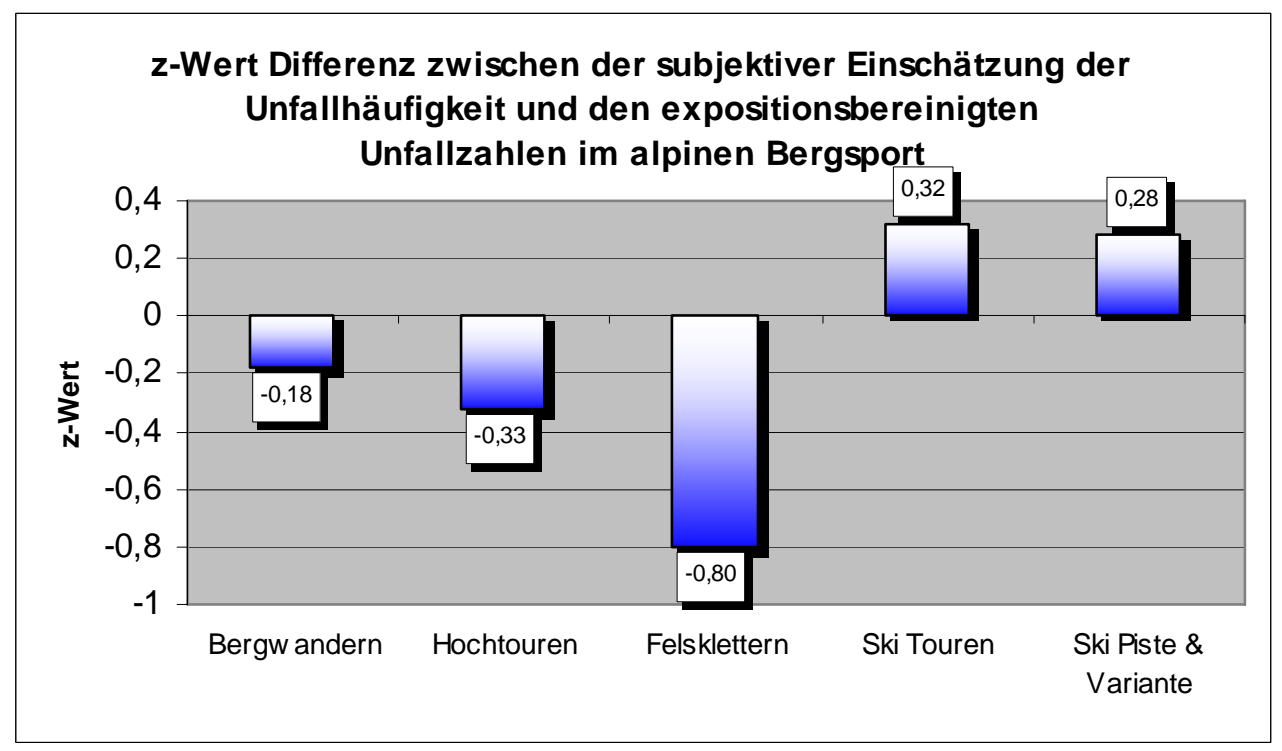

Abbildung 9.15: z-Wert Differenz zwischen den subjektiven Einschätzungen über die Unfallhäufigkeit und den expositionsbereinigten Unfallzahlen im alpinen Bergsport ( $\mathrm{n}$ (subjektiv) $=$ 724; $\mathrm{n}$ (objektiv) $=2035$ ).

Hierbei wird deutlich, dass sowohl das Bergwandern, als auch das Hochtourengehen und Felsklettern in ihrer Gefährlichkeit leicht unterschätzt werden. Angesichts des eher konservativen Klassifikationskriteriums von $Z_{\in G}<-1,0$ fällt aber keine Aktivität in den Bereich einer signifikanten Unter- oder Überschätzung. Das Ski-Tourengehen und das alpine Skifahren auf Pisten und Varianten werden in ihrer Unfallhäufigkeit leicht überschätzt, allerdings zeigen sich auch hier keine signifikanten Zusammenhänge ${ }^{261}$. Den 
Probanden ist die Gefährlichkeit der Sportausübung im alpinen Bergsport im Bezug auf die Unfallhäufigkeit durchaus bewusst.

Der Vergleich zwischen den Einschätzungen der Unfallschwere und den auf ihre Schwere relativierten Unfallzahlen relativieren dieses Bild jedoch offensichtlich (Abb. 9.16).

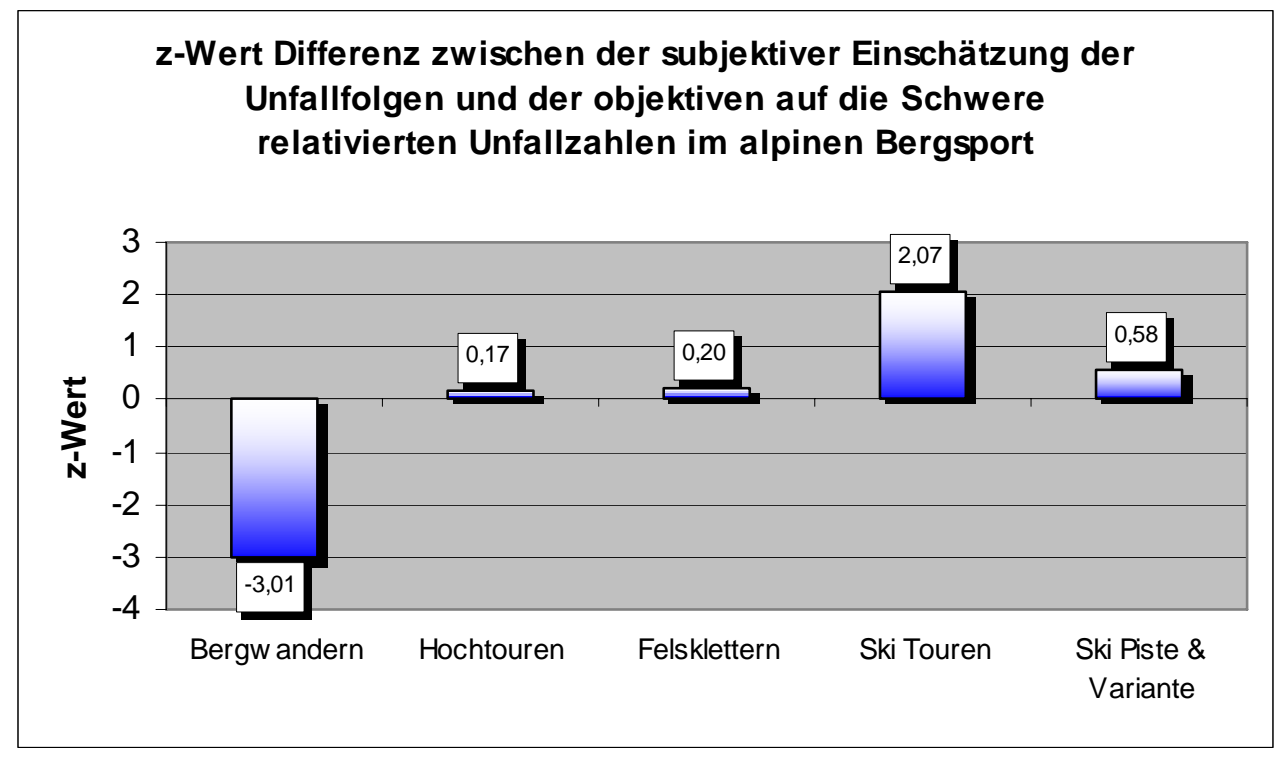

Abbildung 9.16: z-Wert Differenzen zwischen der subjektiven Einschätzung der Unfallfolgen und der objektiven auf die Schwere relativierten Unfallzahlen im alpinen Bergsport ( $($ subjektiv) $=724 ; \mathrm{n}$ (objektiv) $=2035$ ).

Das Bergwandern wird mit einer z-Wert-Differenz von -3,01 nun hinsichtlich der möglichen Unfallfolgen deutlich unterschätzt: Die Probanden beurteilen das Bergwandern im Bezug auf die Unfallhäufigkeit als weitestgehend unfallanfällig, gleichzeitig schätzen sie die Folgen möglicher Unfälle aber als vergleichsweise leicht ein. Eine Einschätzung, die offensichtlich nicht der Realität entspricht. Entsprechend konträr verhält es sich mit dem Tourenskilaufen. Hier wird mit einer z-Wert Differenz von 2,07 die Schwere der Unfallfolgen deutlich überschätzt, während die Unfallhäufigkeit abermals realistisch eingeschätzt wurde. Hier wird also angenommen, dass die Folgen eines Unfalls beim Touren-Skigehen vergleichsweise schwerwiegend sind. Auch dies entspricht nicht vollkommen der ,objektiven' Realität. Bemerkenswert ist auch die nahezu realistische Einschätzung der Unfallfolgen beim Hochtourenbergsteigen und Felsklettern. Hier zeigen sich mit 0,17 und 0,20 nur geringe $z$-Wert-Differenzen und damit eine verhältnismäßig gute Gefahrenkognition.

Die Richtung des Unterschieds zwischen dem subjektiven Urteil und der Realität ist, wie oben dargestellt wurde (vgl. Abschnitt 9.2.4), von hervorgehobener Bedeutung: Während im Falle einer Überschätzung von einer erhöhten Aufmerksamkeit und psychophysiologischer Aktiviertheit ausgegangen wird, stellt die Unterschätzung vermutlich eine zusätzliche Gefährdung für die Ausübung der Aktivitäten dar: Der Akteur fühlt sich in diesem Sinne wenig gefährdet und unternimmt dementsprechend auch nur wenig sicher- 
heitsrelevante Handlungen, um die Gefährdung und das auf den Handelnden bezogene Risiko anzupassen.

Zusammenfassend bleibt für die Gefahrenkognition im alpinen Bergsport ein uneinheitliches Bild festzustellen: Bezüglich der Unfallhäufigkeit werden alle Aktivitäten weitestgehend realistisch eingeschätzt, während bei der Einschätzung der Unfallfolgen vor allem das Bergwandern massiv unterschätzt und das Ski-Tourengehen deutlich überschätzt wird. Und dennoch bleibt festzuhalten: gemessen am konservativen Klassifikationskriterium wird nur das Bergwandern und auch dieses nur im Bezug auf die Einschätzung der Unfallfolgen signifikant unterschätzt. Letztlich sind die Ergebnisse aber durch die eindeutige Differenzierung zwischen den Einschätzungen der Unfallhäufigkeit und den Unfallfolgen nur schwierig zu interpretieren. Inwieweit diese Ergebnisse hinsichtlich sicherheitspsychologischer Intervention ,belastbar' sind, wird anhand der Ergänzungsuntersuchungen zu Geschlechts-, Alters- und Erfahrungsunterschieden sowie Differenzen zwischen Fachübungsleitern und normalen Bergsportlern überprüft.

\subsubsection{Ergebnisse der Ergänzungsuntersuchung: Unterschiede im Bezug auf Geschlecht, Alter, Erfahrung und Übungsleiterlizenzen.}

Im Rahmen der internetbasierten Befragung wurde neben den Einschätzungen bezüglich der Unfallhäufigkeiten und Unfallschwere sowie der Ausübungshäufigkeiten auch nach zusätzlichen soziodemographischen Daten (Alter \& Geschlecht) und spezifischen Erfahrungen im Bergsport gefragt. Darüber hinaus wurden gezielt Fachübungsleiter des alpinen Bergsports zu ihren spezifischen Einschätzungen gefragt. Im Rahmen der Hauptuntersuchung wurden diese Merkmale im Rahmen einer Ergänzungsstudie gesondert betrachtet, um mögliche Differenzen im Beurteilungsvermögen aufzudecken.

\subsection{Geschlechtsspezifische Unterschiede}

Um zu prüfen, ob es geschlechtsspezifische Unterschiede in der Gefahrenkognition gibt, wurden die Rohdaten der Unfallhäufigkeitseinschätzungen und der Unfallfolgeneinschätzungen getrennt nach Männern und Frauen untersucht. Die Ergebnisse der geschlechtsspezifischen Differenzierung zeigen deutliche Unterschiede in der spezifischen Einschätzung der Unfallhäufigkeit. So urteilen die befragten Frauen $(n=137)$ in nahezu allen Bereichen ausgeprägter als die befragten Männer (vgl. Abb. $9.17 \&$ 9.18). Männer zeigen damit ein deutlich realistischeres Urteilsvermögen im Bezug auf die Unfallhäufigkeit. Insbesondere das Bergwandern wird von Frauen ( $\mathrm{z}$-Wert = $1,3)$ deutlich ungefährlicher eingeschätzt als von Männern $(z$-Wert $=-0,49)$. Gleichzeitig wird aber sowohl das Ski-Tourengehen und das Skifahren auf Pisten und Varianten von Frauen überschätzt, während es von Männern weitestgehend realistisch eingeschätzt wird. 


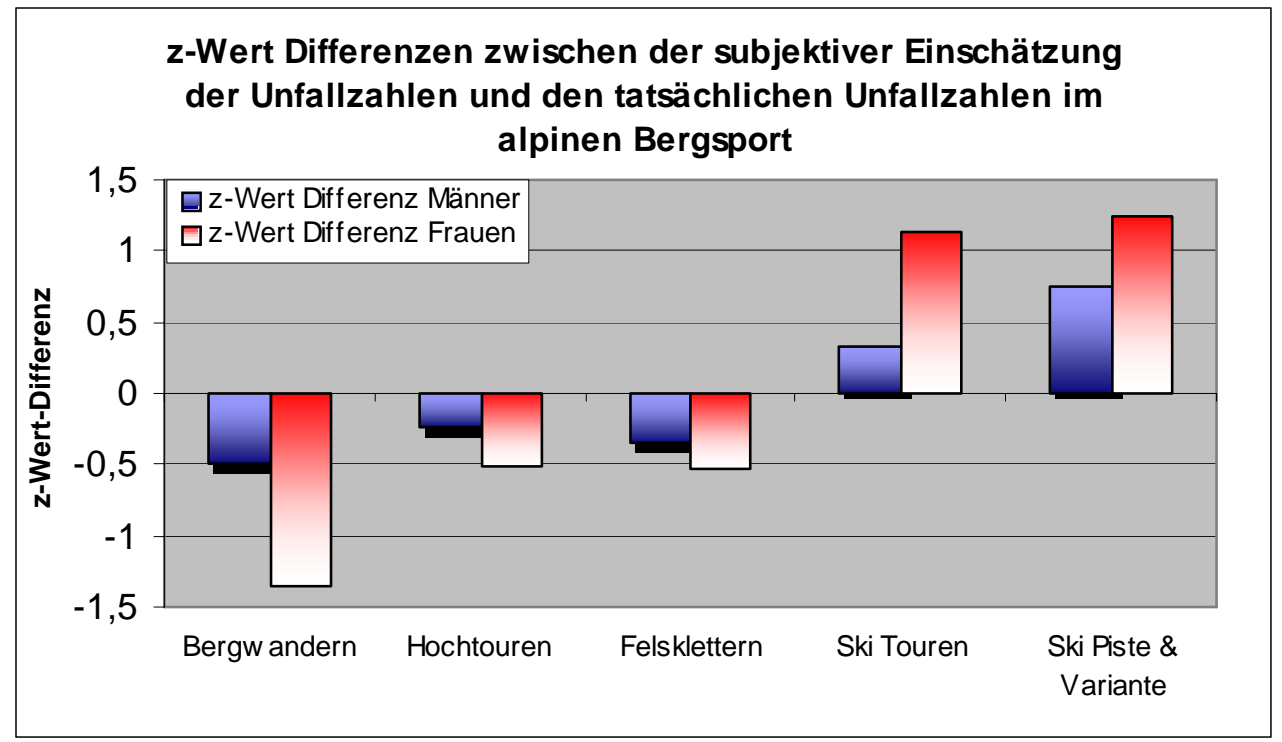

Abbildung 9.17: z-Wert Differenz zwischen Männern und Frauen bezogen auf die subjektive Einschätzung der Unfallhäufigkeit und den tatsächlichen Unfallzahlen im Bergsport ( $($ Männer)= 587; n (Frauen)= 137).

Angesichts der großen Anzahl an Probanden (N (Männer) = 587; N $($ Frauen $)=137)$ sind diese Unterschiede von entsprechender Bedeutung. Natürlich muss hier methodenkritisch eingewendet werden, dass die Anzahl der befragten Frauen von der Anzahl männlicher Probanden deutlicht abweicht. Dennoch die Anzahl weiblicher Untersuchungsteilnehmer stellt mit 137 Personen eine ausreichend große Probandengruppe dar. Die Option durch extremer Beurteilungen eine verzerrte gemittelte Gesamteinschätzung $\mathrm{zu}$ erhalten, ist dementsprechend auch bei der weiblichen Probandengruppe eingeschränkt. Frauen zeigen damit eine Tendenz zu extremeren Einschätzungen als Männer.

In der Einschätzung der Unfallfolgen offenbart sich zwar eine kongruente Übereinstimmung zwischen Männern und Frauen, so dass sich die empirischen Befunde gleichsam relativieren. Die für ein Urteil der geschlechtsspezifischen Gefahrenkognition heranzuziehende Datenbasis muss aber beide Ergebnisse berücksichtigen, so dass die diskrepanten Unterschiede zwischen Männern und Frauen damit keinesfalls neutralisiert sind. Inwieweit sich diese Befunde im Rahmen der Teiluntersuchung im Kanusport erhärten lässt, bleibt abzuwarten. 


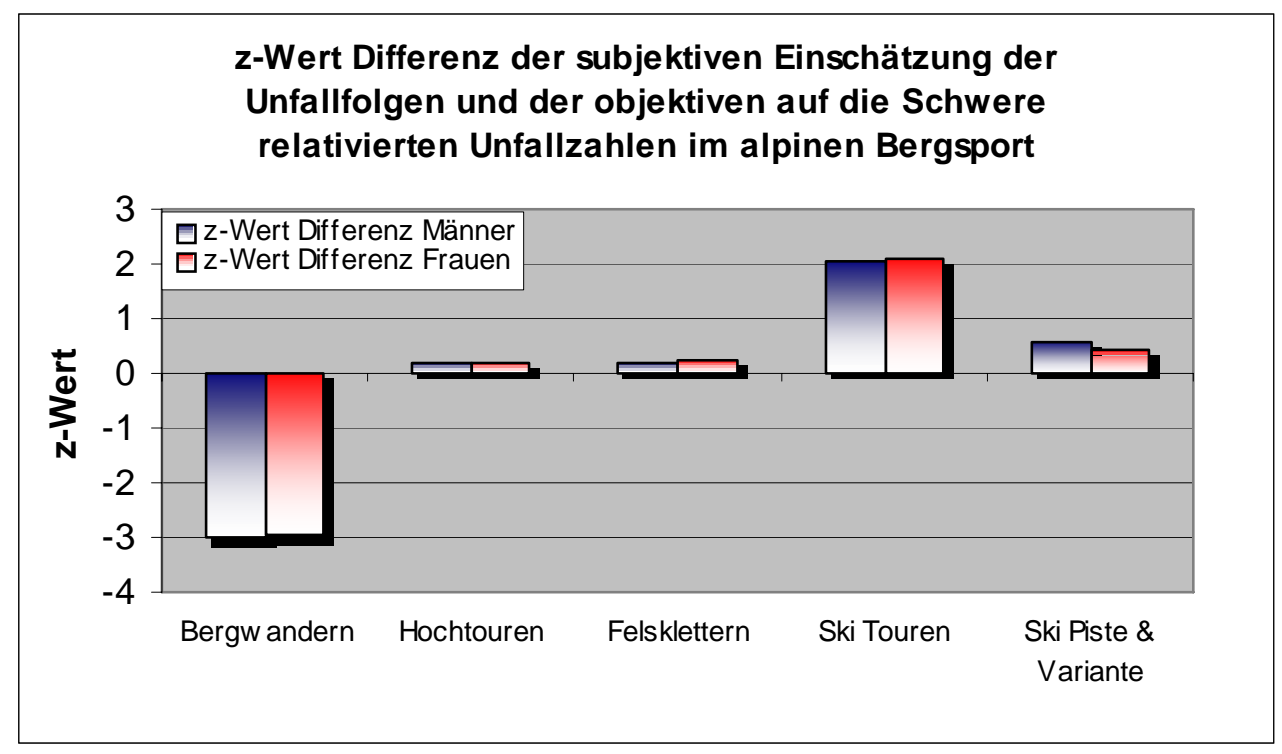

Abbildung 9.18: z-Wert Differenz zwischen Männern und Frauen bezogen auf die subjektive Einschätzung der Unfallfolgen und den auf die Schwere relativierten Unfallzahlen im Bergsport (n (Männer)= 587; n (Frauen)= 137).

\subsection{Altersspezifische Unterschiede}

Dem Vorgehen bei der Analyse geschlechtsspezifischer Unterschiede entsprechend, wurden die Rohdaten auch im Bezug auf Alterskohorten getrennt. Die hierbei vollzogene Trennung der Altersgruppen in a) $<20$ Jahre, b) 21-30 Jahre, c) 31-40 Jahre, d) 41-50 Jahre, e) 51-60 Jahre und f) > 61 Jahre wurde willkürlich vollzogen, sie entspricht aber weitestgehend sozialisationstheoretischen Annahmen (vgl. HURRELMANN 1989).

Die Ergebnisse der altersspezifischen Einschätzung offenbart fernerhin ein sehr differenziertes Bild. Lediglich die Gruppe der über 61 Jährigen zeigt deutliche Unterschiede zu den anderen Altersgruppen auf. Die Einschätzungen bezüglich der Unfallhäufigkeiten sind in der ältesten Gruppe für alle Aktivitäten am realistischsten. Auch bei der Einschätzung der Unfallfolgen zeigt diese Gruppe eine vergleichsweise realistische Gefahrenkognition. Allerdings muss auch hier berücksichtigt werden, dass sich mit $n=29$ auch die wenigsten Probanden in dieser Gruppe befanden. Da nur 8 der Befragungsteilnehmer unter 20 Jahren alt waren, wurde diese Altersstufe gar nicht in die Analyse einbezogen. Die Ergebnisse der altersspezifischen Unterschiede zeigt Abb. 9.19 und Abb. 9.20. 


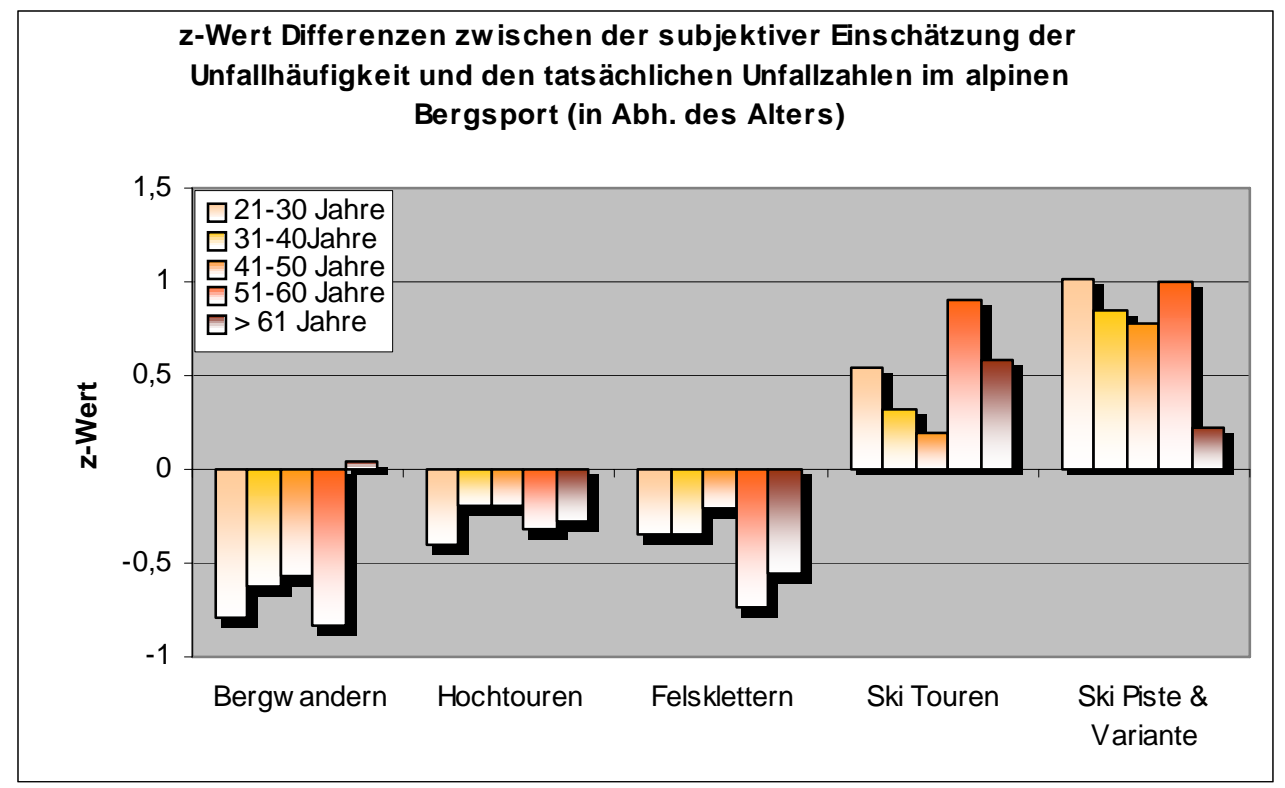

Abbildung 9.19: z-Wert Differenz zwischen verschiedenen Altersgruppen bezogen auf die subjektive Einschätzung der Unfallhäufigkeit und den tatsächlichen Unfallzahlen im Bergsport (n (21-30 Jahre)= 169; $n$ (31-40 Jahre)= 265; $n$ (41-50 Jahre)= $191 ; \mathrm{n}(51-60$ Jahre $)=71 ; \mathrm{n}(>61$ Jahre $)=29)$.

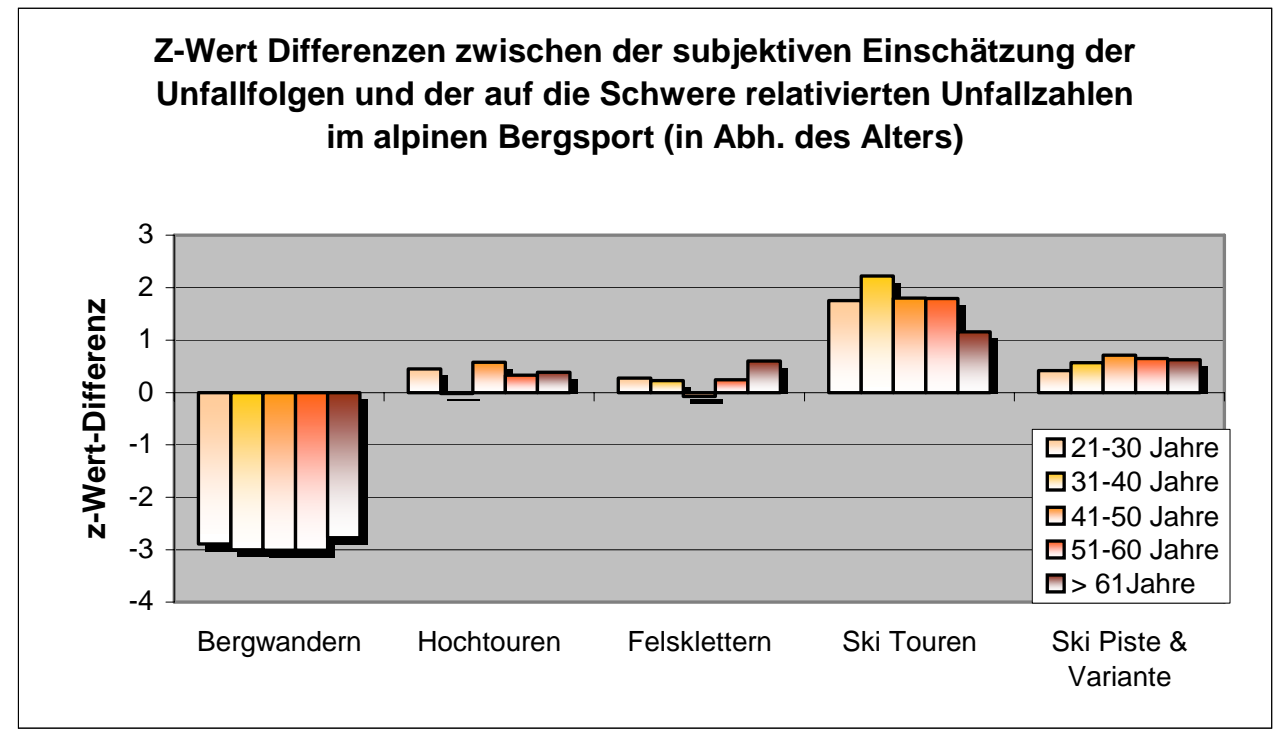

Abbildung 9.20: z-Wert Differenz zwischen verschiedenen Altersgruppen bezogen auf die subjektive Einschätzung der Unfallfolgen und den auf die Schwere relativierten Unfallzahlen im Bergsport ( $\mathrm{n}$ (21-30 Jahre)= 169; $n$ (31-40 Jahre)= 265; $n$ (41-50 Jahre $)=191 ; n(51-60$ Jahre $)=71 ; n$ (> 61 Jahre $)=29)$.

Insgesamt lassen sich im Bezug auf alterspezifische Abhängigkeiten der Gefahrenkognition keine signifikanten Zusammenhänge erkennen. Inwieweit die weitgehend realistische Gefahrenkognition der über 61 Jähre ein replizierbares Ergebnis darstellt, bleibt abzuwarten. 


\subsection{Gefahrenkognition und alpine Erfahrung:}

Erfahrung - so die theoretische Annahme - spielt für Prozesse der Gefahrenkognition eine in doppelter Hinsicht bedeutende Rolle. Zum einen argumentieren verschiedene Autoren (vgl. Abschnitt 6.2) mit der besondere Bedeutung der Erfahrung für eine realistische Gefahreneinschätzung im Risikosport, andererseits zieht MUSAHL aus lerntheoretische Annahmen den Schluss, dass Erfahrung auch zu einer negativen Beeinflussung der Gefahrenkognition führen kann. Um zu erfahren, inwieweit die Probanden über spezifische Erfahrungen im alpinen Bergsport verfügen, wurde auch nach der quantitativen Erfahrungsdauer im alpinen Bergsport gefragt. Die Rohdaten wurden entsprechend den Angaben zu den Erfahrungswerten sortiert und einer gesonderten Analyse unterzogen.

Signifikante Zusammenhänge lassen die Ergebnisse auch hier nicht erkennen. Zwar zeigen die Probanden mit mehr als 28 Jahren Erfahrung im alpinen Bergsport tendenziell realistischere Gefahrenkognitionen als Sportler mit weniger Erfahrung. Dass es sich hierbei aber um kennzeichnende Korrelationen handelt, kann nicht eindeutig festgestellt werden. Weder ein positiver noch einen negativer Einfluss auf die Ausbildung einer Gefahrenkognition kann damit nachgewiesen werden. Abbildungen 9.21 und 9.22 stellen die Ergebnisse dar. Obwohl die Probandengruppen für eine derartige Analyse durchaus angemessen sind, können statistische Artefakte nicht ausgeschlossen werden. Eine eindeutige Interpretation der Ergebnisse ist insofern nicht möglich. 


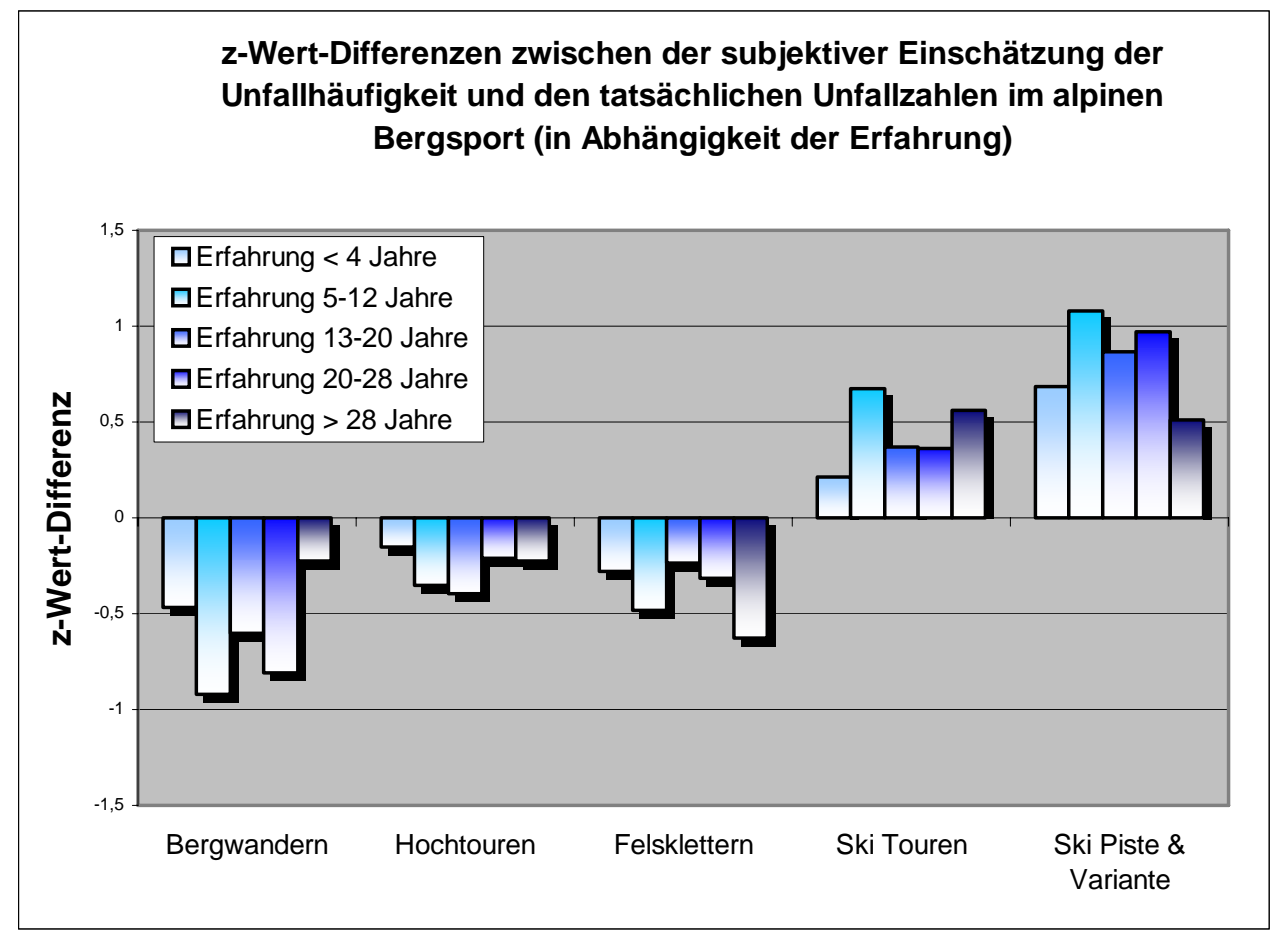

Abbildung 9.21: z-Wert-Differenzen zwischen der subjektiven Einschätzung der Unfallhäufigkeit und den tatsächlichen Unfallzahlen im alpinen Bergsport in Abhängigkeit der alpinen Erfahrung ( $\mathrm{n}(4<$ Jahre $)=24 ; \mathrm{n}$ (5-12 Jahre) $=196 ; \mathrm{n}$ (13-20 Jahre) $=$ 190; $n$ (Erfahrung 20-28 Jahre) = 180; $n$ (> 28 Jahre $)=147$ ).

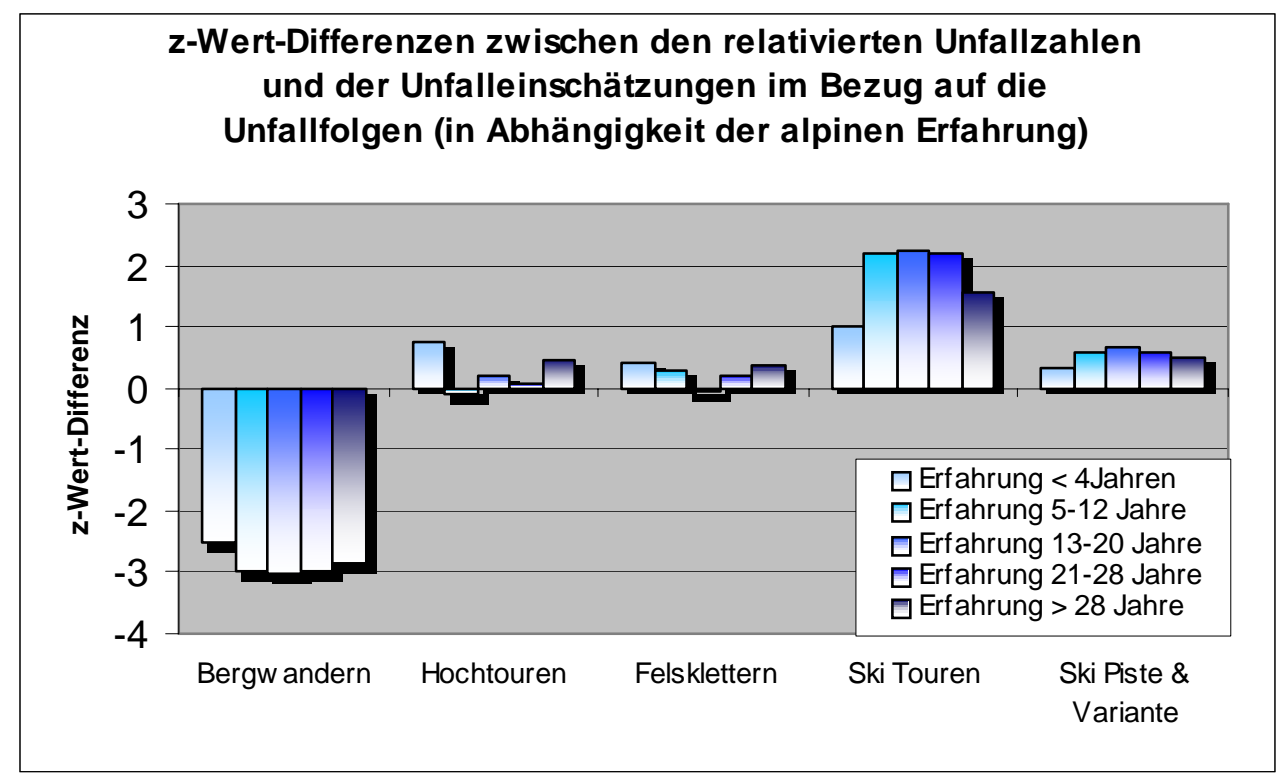

Abbildung 9.22: z-Wert-Differenzen zwischen der subjektiven Einschätzung der Unfallfolgen und den auf die Schwere relativierten Unfallzahlen im alpinen Bergsport in Abhängigkeit der alpinen Erfahrung ( $\mathrm{n}(4<$ Jahre $)=24$; $\mathrm{n}$ (5-12 Jahre) $=196$; $\mathrm{n}$ (1320 Jahre $=190 ; n$ (Erfahrung 20-28 Jahre $)=180 ; n$ (> 28 Jahre $)=147)$. 


\subsection{Gefahrenkognition bei Übungsleitern}

Fachübungsleiter stellen im Bereich alpiner Sportarten so genannte Spezialisten dar, die durch intensive Ausbildungsprozesse und ein hohes motorisches Niveau über ein spezialisiertes Wissen und ein gleichsam ausdifferenziertes Gefahrenmodell verfügen sollten. Im Rahmen der Befragung wurden deshalb gezielt Übungsleiter gebeten, eine Einschätzung der Unfallhäufigkeiten und Unfallfolgen abzugeben. Für die vom DAV ausgegebenen Fachübungslizenzen ,Bergsteigen', ,Alpinklettern', ,Hochtouren', ,Klettersport' und ,Ski' konnten Probanden in statistisch ausreichender Form rekrutiert werden. Nach dem bisherigen Verfahren wurden auch diese Rohdaten getrennt und mit den Einschätzungen von Personen ohne spezifische Übungsleiterlizenz verglichen (Abb. 9.23 \& 9.24).

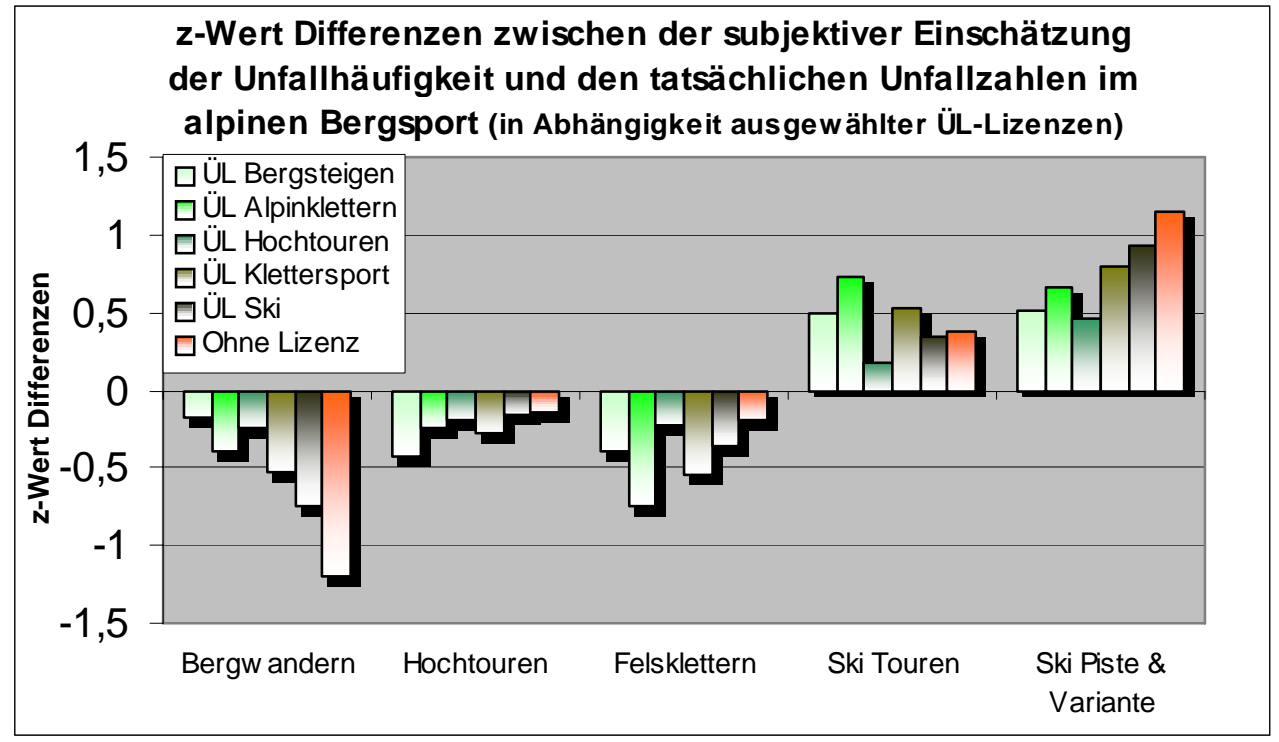

Abbildung 9.23: z-Wert-Differenzen zwischen der subjektiven Einschätzung der Unfallhäufigkeit und den tatsächlichen Unfallzahlen im alpinen Bergsport im Vergleich zwischen ausgewählten Übungsleiter-Lizenzen und Probanden ohne Lizenz ( $\mathrm{n}$ (ÜL Bergsteigen) = 126; $n$ (ÜL Alpinklettern) = 69, n (ÜL Hochtouren) =134; $n$ (ÜL Klettersport) = 119; $n$ (ÜL Ski) = 60); n (Ohne Lizenz) = 207).

Wenngleich nicht signifikant, so zeigen sich doch deutliche Unterschiede in der Einschätzung der Unfallhäufigkeit zwischen Übungsleitern allgemein und Personen ohne Lizenz. Unterschiede zwischen einzelnen Übungsleitergruppen finden sich nicht. Es kann konstatiert werden, dass die Übungsleiter die Einschätzung der einzelnen Unfallhäufigkeiten durchaus realistisch vornehmen und damit das Unfallgeschehen auch sehr differenziert beurteilen können. Die Gruppe der ÜL Bergsteigen zeigt hier die besten Werte bei allen Aktivitäten lassen sich nur sehr geringe Differenzen zwischen den subjektiven und den objektiven Datensätzen darstellen.

Bei der Beurteilung der Unfallfolgen zeigt sich allerdings ein anderes Bild. Zwar sind auch die Übungsleiter allgemein eher in der Lage die Unfallfolgen realistisch einzuschätzen, insgesamt werden aber vor allem die Unfallfolgen beim alpinen Bergwandern von allen Stichprobengruppen unter- 
schätzt (Abb. 9.24). Das differenzierte Wissen der Übungsleiter beinhaltet demnach nur eine realistische Abschätzung der Unfallhäufigkeiten und nur eingeschränkt die Einschätzung der Unfallfolgen. Allerdings bezieht sich diese Aussage vor allem auf das alpine Bergwandern, denn Tätigkeiten wie das Hochtourenbergsteigen oder Felsklettern werden mit einer erstaunlichen Genauigkeit beurteilt. Hier liegen die Differenzen in kaum registrierbarer Reichweite des Nullpunkts.

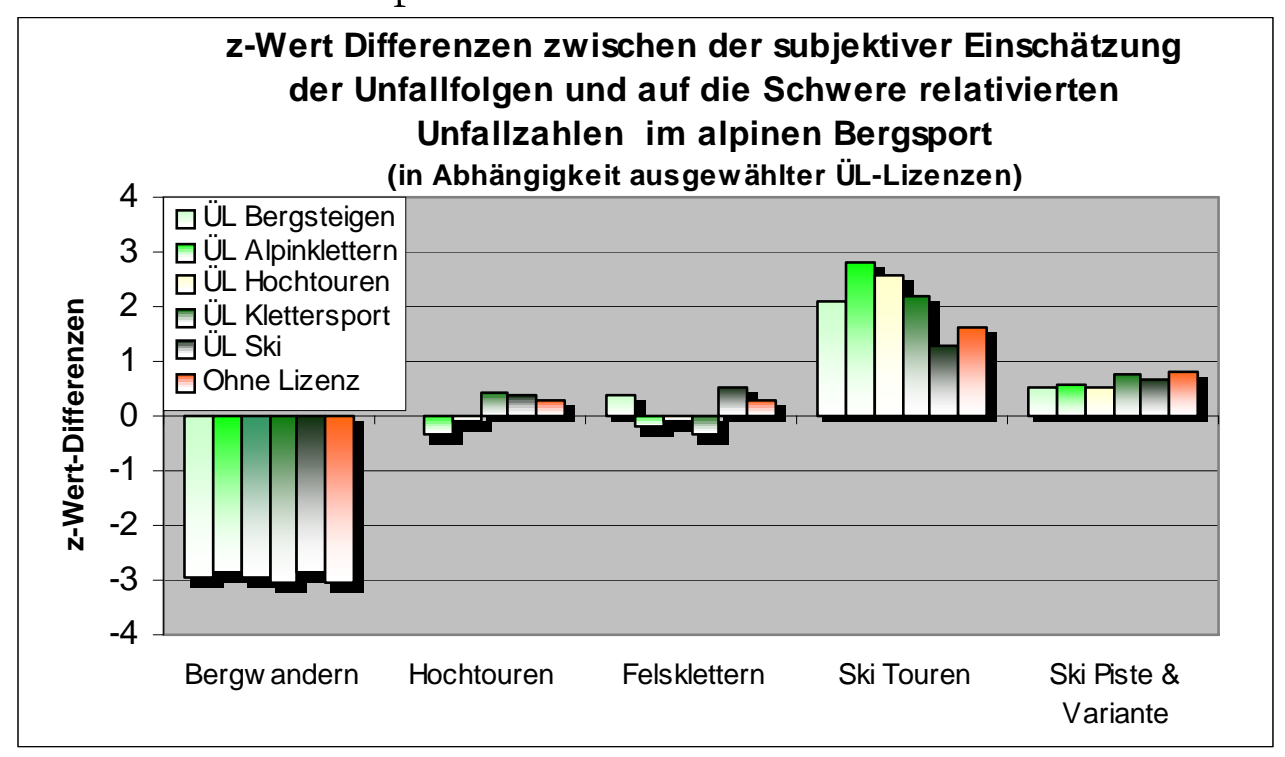

Abbildung 9.24: z-Wert-Differenzen zwischen der subjektiven Einschätzung der Unfallhfolgen und den auf ihre Schwere relativierten Unfallzahlen im alpinen Bergsport im Vergleich zwischen ausgewählten Übungsleiter-Lizenzen und Probanden ohne Lizenz (n (ÜL Bergsteigen) = 126; n (ÜL Alpinklettern) = 69, n (ÜL Hochtouren) =134; n (ÜL Klettersport) = 119; n (ÜL Ski) = 60); n (Ohne Lizenz) = 207).

Es bleibt also festzustellen: Signifikante Zusammenhänge zwischen der Gefahrenkognition und Geschlechts-, Alters- und Erfahrungsmerkmale konnten für den alpinen Bergsport nicht nachgewiesen werden Zwar zeigen sich für den Vergleich zwischen Übungsleitern und Nicht-Übungsleitern, zwischen Männern und Frauen und zwischen über 61 Jährigen und jüngeren Personen greifbare Unterschiede. Diese beziehen sich allerdings nur auf die Einschätzung der Unfallhäufigkeiten und in keinem Fall auf die Einschätzung der Unfallfolgen. Hier wird weitestgehend einheitlich geurteilt, wenngleich ein durchaus differenziertes Beurteilungsvermögen für die einzelnen Aktivitäten zu konstatieren ist. Bis auf das Bergwandern und das SkiTourengehen werden alle Tätigkeiten dabei von weitgehend allen differenzierten Gruppen realistisch eingeschätzt.

In Abschnitt 9.4 werden die Ergebnisse einer abschließende Diskussion unterzogen und eine Interpretation der Daten vorgenommen. Vorab werden aber zunächst die Ergebnisse der Untersuchung im Kanusport vorgestellt. 


\subsubsection{Unfälle und Gefahrenkognition im Kanusport}

Analog zur Vorgehensweise im alpinen Bergsport wurde auch im Kanusport das Verfahren zur Bestimmung der Gefahrenkognition angewendet und der Vergleich zwischen objektiven Unfalldaten und subjektiven Einschätzungen der Unfallhäufigkeiten und Unfallschwere mittels z-Wert-Transformation vollzogen. Im Folgenden werden zunächst die Unfalldaten entsprechend der Vorgehensweise für den alpinen Bergsport vorgestellt. Da im Kanusport nur Unfälle mit schweren Verletzungs- und Todesfolgen vorlagen, ist eine Gewichtung der Unfalldaten im Bezug auf die Verletzungsfolgen im Kanusport nicht möglich. Die Ergebnisse der internetbasierten Befragung werden daher ausschließlich auf die tatsächlichen sowie auf die expositionsbereinigten Unfallzahlen bezogen.

\subsubsection{Die objektive Datenbasis: Die Ergebnisse der Unfallanalyse}

Im Vergleich zum alpinen Bergsport wurden im Kanusport wesentlich weniger Unfälle im Untersuchungszeitraum registriert ${ }^{262}$. Ingesamt wurden 83 Unfälle, die sich in den Jahren 1998 - 2003 ereigneten, in die Analyse einbezogen $^{263}$. Der Altersdurchschnitt der Verunfallten lag dabei bei 36,2 Jahren. In 15,3\% aller Unfälle waren Frauen in die Unfälle verwickelt. Bezogen auf die Jahresdurchschnitte zeigt sich ein sehr differenziertes Bild. Im Vergleich zu den Jahren 1998 und 1999 wurde im Jahr 2003 nur knapp die Hälfte an Unfällen im Kanusport registriert. Inwieweit dies an den tatsächlichen Unfallzahlen oder an einem Erhebungsdefizit der Fachverbände liegt, kann hier nicht eindeutig bestimmt werden. Sicherlich sind jahresabhängige Schwankungen aufgrund von Pegelständen und meteorologischen Besonderheiten möglich, eine derart große Varianz kann aber damit sicherlich nicht bestimmt werden. Abbildung 9.25 illustriert die Unfallzahlen im Jahrgangsverlauf von $1998-2003$.

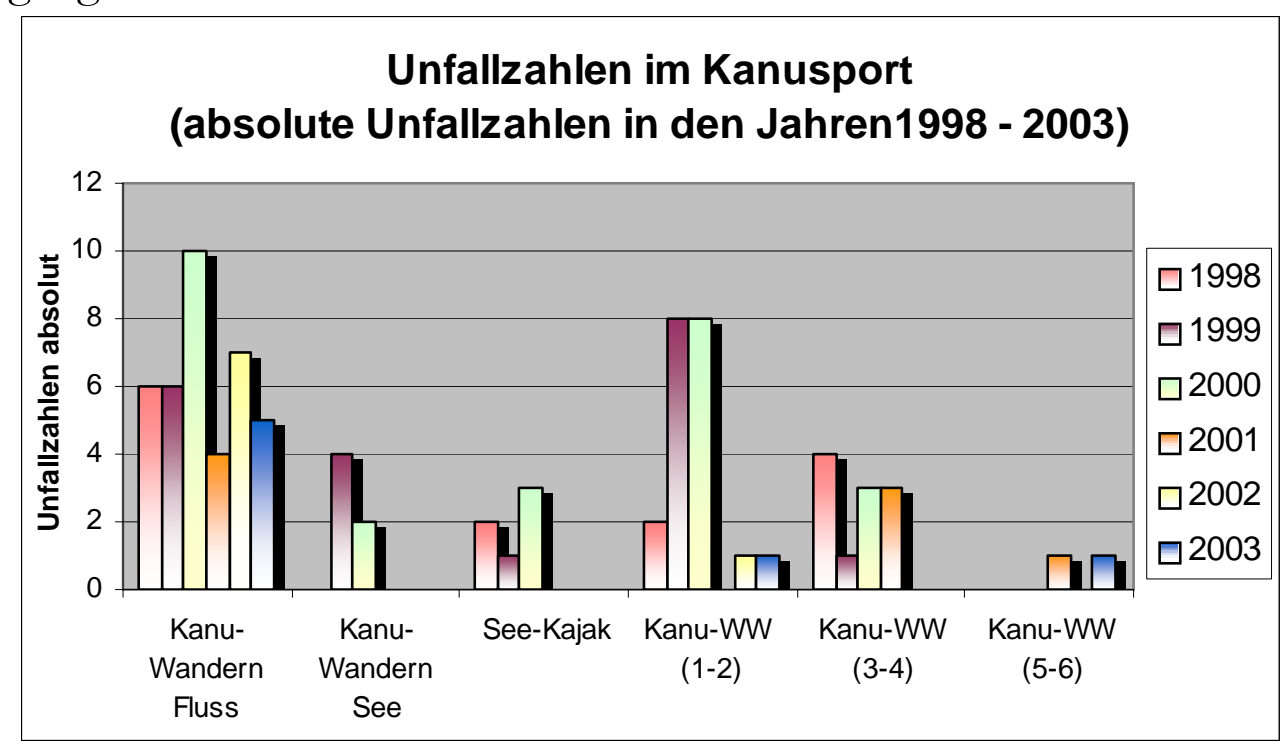

Abbildung 9.25: Absolute Unfallzahlen im Kanusport für die einzelnen kanusportliche Disziplinen mit Unfallaufkommen für die Jahre 1998- 2003. Datenbasis der Darstel- 
lung sind alle verfügbaren Unfallanzeigen (insbesondere des DKV in Ergänzung des AKC).

Betrachtet man die Unfallzahlen für die einzelnen kanusportlichen Disziplinen in ihrem absoluten Unfallaufkommen für die Jahre 1998 - 2003, so wird der große Anteil an Unfällen im Kanu-Wanderbereich deutlich. Insbesondere das Kanu-Wandern auf langsam fließenden Gewässern dominiert diese Unfallstatistik mit knapp der Hälfte aller im Kanusport registrierten Unfälle (Abb. 9.26.).

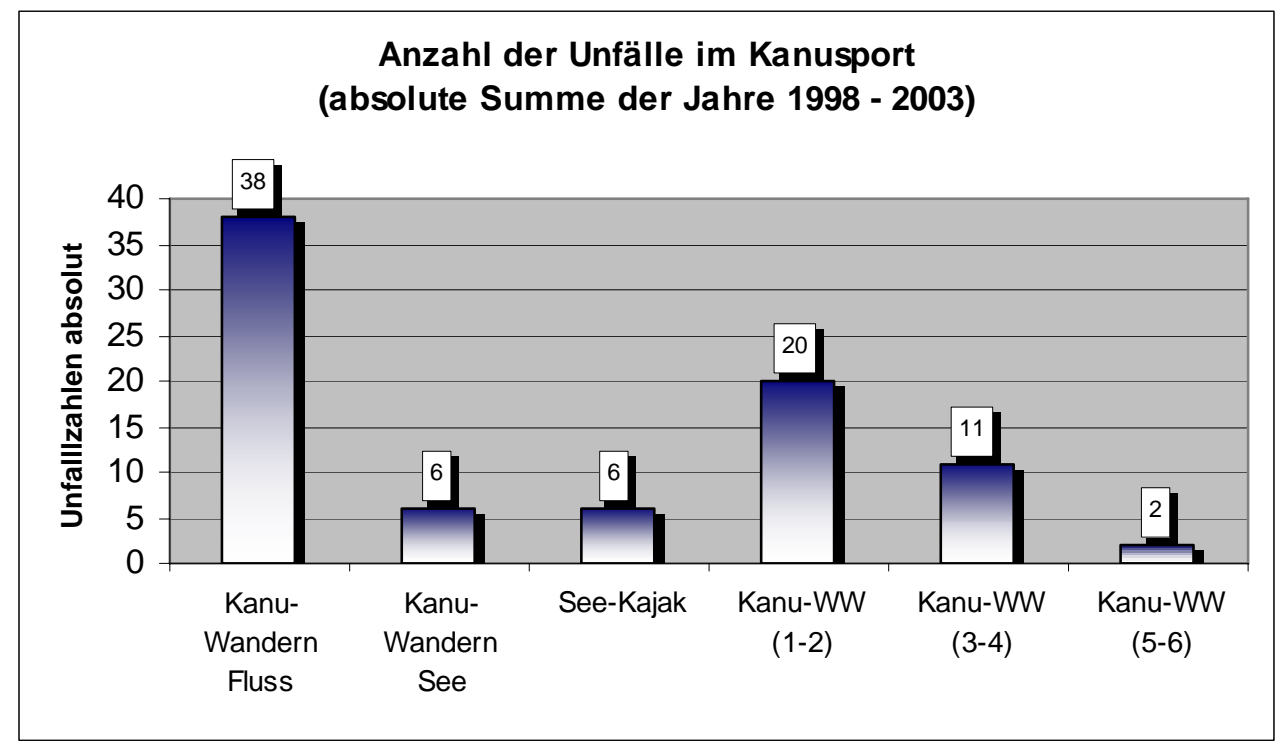

Abbildung 9.26: Summe der Unfälle im Kanusport für die Jahre 1998 - 2003 bezogen auf die einzelnen kanusportlichen Disziplinen. Datenbasis der Darstellung sind alle verfügbaren Unfallanzeigen (insbesondere des DKV in Ergänzung des AKC).

Dies ist zunächst deshalb bemerkenswert, weil alle registrierten Unfälle mit sehr schweren, also lebensbedrohlichen oder sogar tödlichen Unfallfolgen verbunden sind. Die Anzahl tödlicher und lebensbedrohlicher Unfallfolgen ist dementsprechend beim Kanu-Wandern insgesamt deutlich höher als beim Kanu-Wildwasserfahren.

Besonders wenig Unfälle ereigneten sich im sehr schweren bis extremen Kanu-Wildwasser, vor allem im Schwierigkeitsbereich 5-6 der offiziellen Schwierigkeitskategorisierung. Mit zwei registrierten Unfällen fällt die Unfallrate hier sehr klein aus, während im leichten Wildwasser (Schwierigkeitsstufe 1-2) mit 20 Unfällen vergleichsweise viele Unfälle registriert wurden.

Um ein auf die Ausübungshäufigkeit bezogenes Bild der Unfalldaten zu bekommen, wurden die Rohdaten der bekannten Expositionsbereinigung unterzogen. Hierfür wurden die absoluten Unfallzahlen - analog der Vorgehensweise im alpinen Bergsport - durch die gemittelte Ausübungshäufigkeit der Aktivität dividiert und mit dem Mittelwert der Ausübungshäufigkeit aller Aktivitäten gemittelt. Diese auf die Ausübungshäufigkeit bezogenen Unfallzahlen gibt Abbildung 9.27 wieder. 


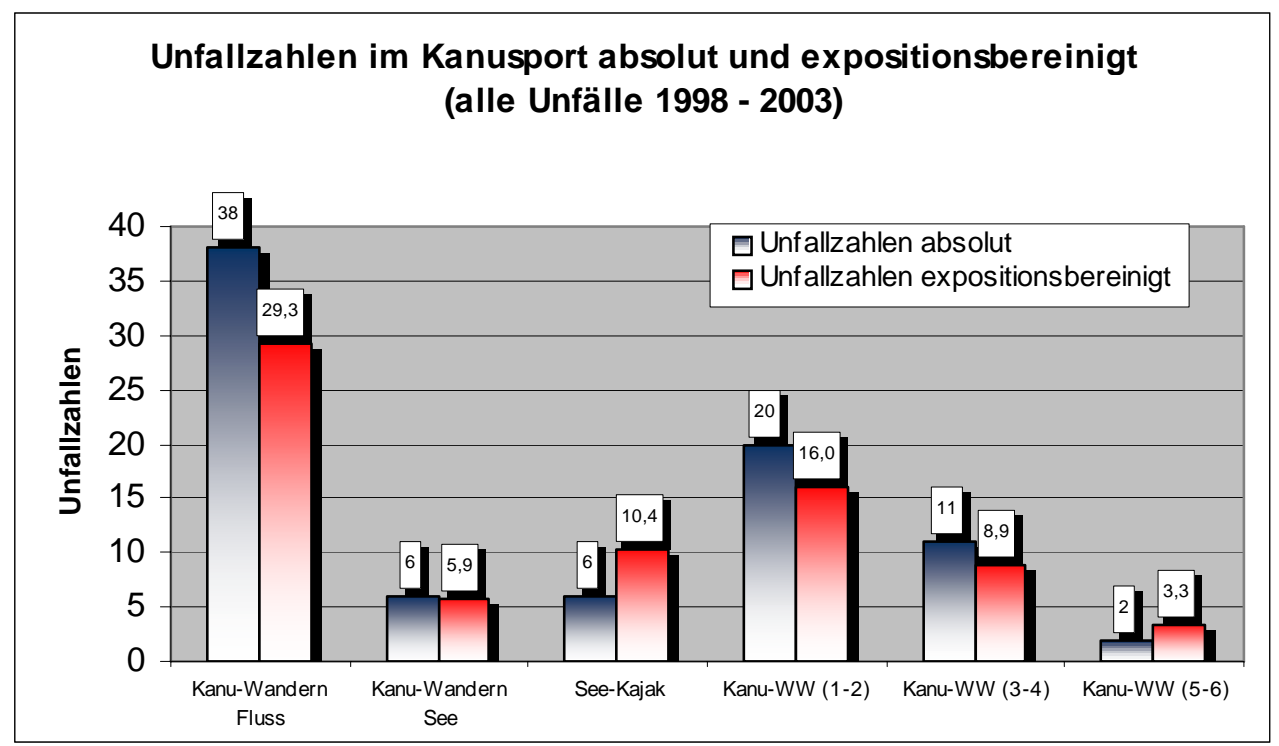

Abbildung 9.27: Absolute und expositionsbereinigte Unfallzahlen im Kanusport als Summe der Jahre 1998 - 2003. Datenbasis stellen alle für die Jahre registrierten Unfallzahlen sowie die im Fragebogen angegebenen gemittelten Ausübungshäufigkeiten $(n=184)$ dar.

Mit einem Mittelwert von 3,97 wurde das Kanu-Wandern auf Flüssen als häufigste Aktivität registriert, während das Seekajak-Fahren und Wildwasserpaddeln auf Wildwasser der Schwierigkeitsstufe 5-6 mit 1,77 und 1,88 am wenigsten ausgeübt werden. Während die Unfallzahlen damit beim KanuWandern auf Flüssen sowie beim Kanu-Wildwasserfahren auf leichten Gewässern (WW 1-2) und auf schwierigen Gewässern (WW 3-4) durch die Expositionsbereinigung verringert wurden, sind die Unfallzahlen des SeekajakFahrens und des Wildwasserfahrens auf extrem schwierigen Gewässern (WW 5-6) durch die geringe Ausübungshäufigkeit nun größer als ihr tatsächlicher Wert. Es ist diesbezüglich zu bedenken, dass sowohl das SeekajakFahren als auch das extreme Wildwasserpaddeln nur von wenigen befragten Personen ausgeübt wird. Ein Mittelwert von 1,77 für das Seekajak-Fahren sowie 1,88 für das Wildwasserpaddeln auf Gewässern der Schwierigkeitsstufe 5-6 repräsentieren eine sehr geringe Ausübungshäufigkeit. Nur 2 Personen gaben an ,häufig' Seekajak zu fahren und nur 8 Personen sind entsprechend häufig auf Wildwasser 5-6 unterwegs. Für die vorliegende Untersuchung bedeutet dies, dass die Einschätzungen bezüglich der Unfallhäufigkeit und der Unfallschwere bei diesen Aktivitäten nur bedingt aus der eigenen Erfahrung resultieren sondern vielmehr abstrakte Vorstellungen über die genannten Aktivitätsbereiche wiederspiegelt.

Für die weitere vergleichende Analyse wurden die Unfallzahlen im Kanusport zunächst quadratwurzeltransformiert und anschließend nach dem beschriebenen statistischen Verfahren in $z$-Werte transformiert. Abbildung 9.28 und Abbildung 9.29 gibt die z-Werte für die absoluten sowie für die expositionsbereinigten Unfallzahlen wieder. 


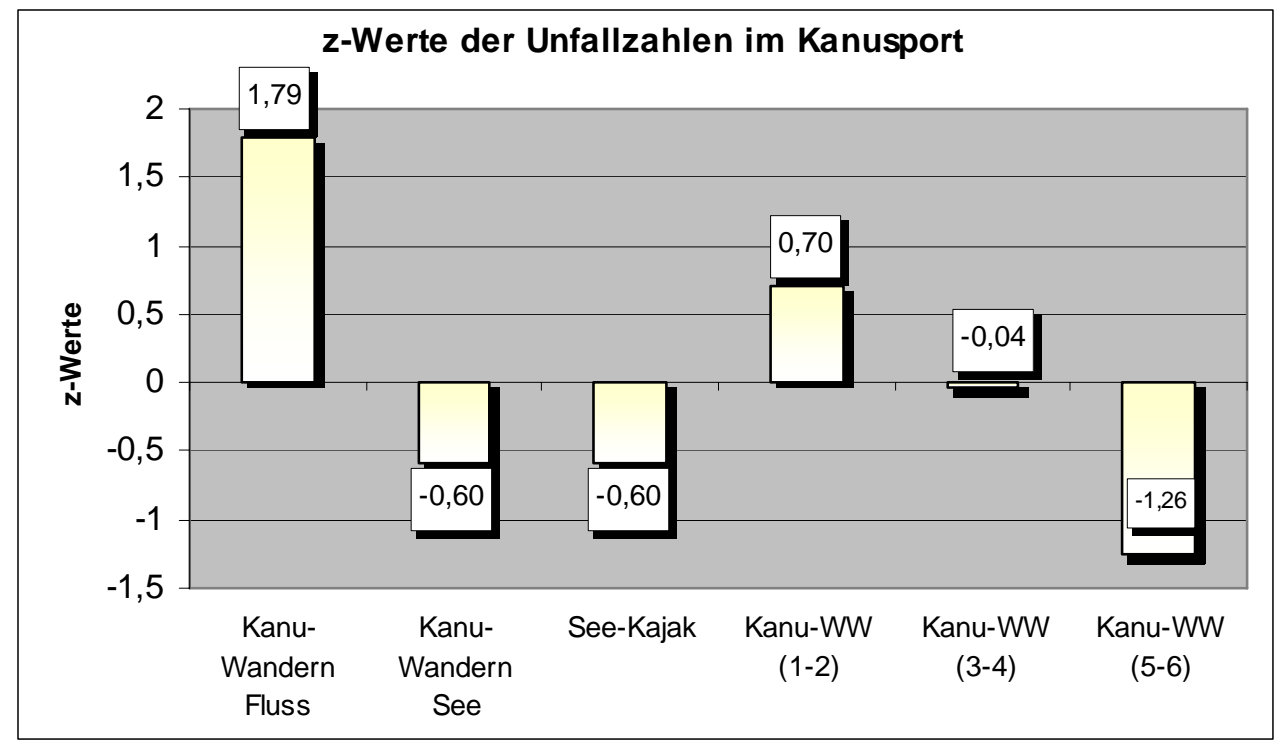

Abbildung 9.28: z-Werte der tatsächlichen Unfallzahlen im Kanusport. Die absoluten Unfallzahlen für die Jahre 1998 - 2003 wurden quadratwurzeltransformiert und schließlich in z-Werte umgerechnet (Datenbasis waren alle 83 registrierten Unfälle für den Bereich Kanusport).

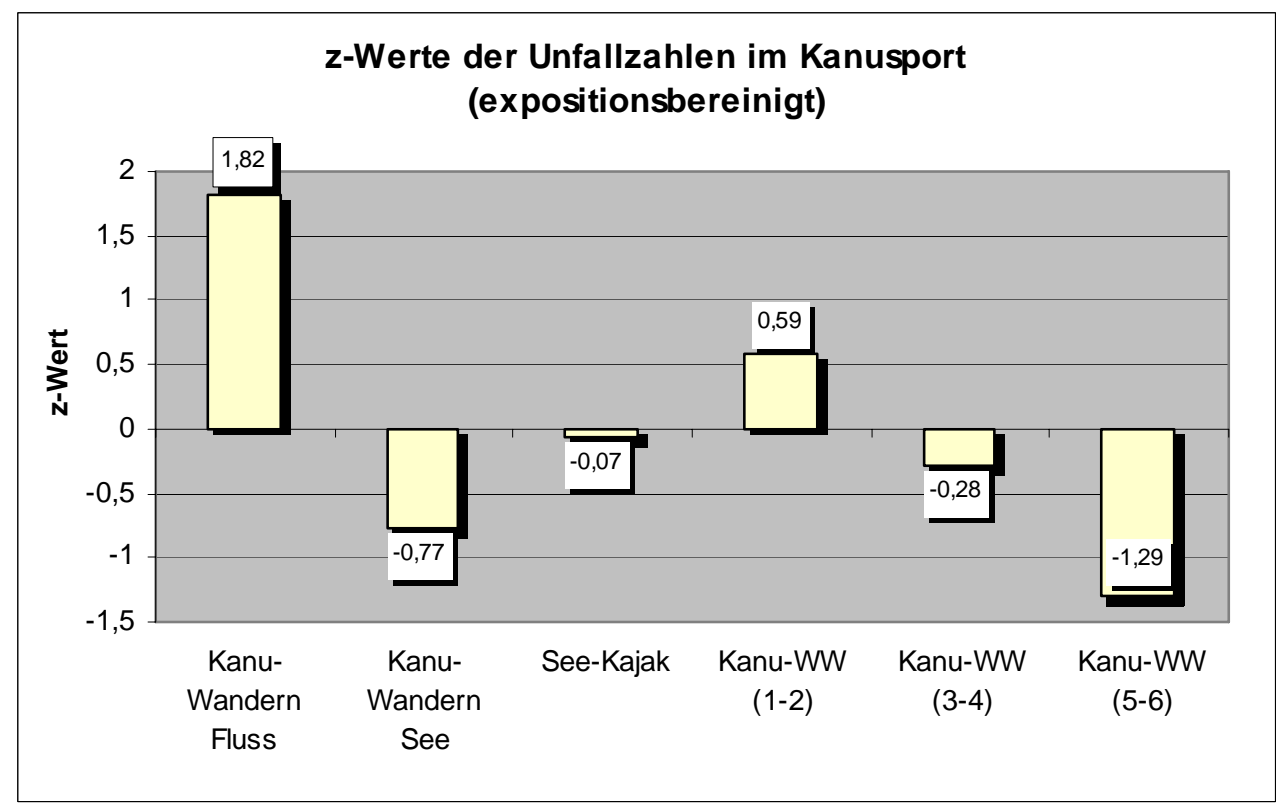

Abbildung 9.29: z-Werte der expositionsbereinigten Unfallzahlen im Kanusport. Die absoluten Unfallzahlen für die Jahre 1998 - 2003 wurden nach der Expositionsbereinigung quadratwurzeltransformiert und schließlich in z-Werte umgerechnet (Datenbasis waren alle 83 registrierten Unfälle für den Bereich Kanusport).

Differenzen in den z-Werten der tatsächlichen Unfallzahlen und den expositionsbereinigten Unfalldaten zeigen sich vor allem für das KanuWandern auf Seen und das Seekajak-Fahren. Beim Kanu-Wandern auf Flüssen sind demnach überdurchschnittlich viele, beim Kanu-Wildwasserfahren 
auf extrem schwierigen Gewässern entsprechend wenig Unfälle zu registrieren.

Eine Gewichtung der Unfallzahlen im Bezug auf die Schwere der Unfallfolgen kann im Kanusport nicht erfolgen, weil hier ausschließlich Unfälle mit schweren und tödlichen Verletzungsfolgen analysiert wurden. Da im Kanusport kein detailliertes Unfall-Erfassungssystem existiert, werden fast ausschließlich Unfälle mit den erwähnten schweren Unfällen registriert. Eine Relationsbildung von Unfällen mit schweren und tödlichen Folgen zu Unfällen mit eher leichten Unfallfolgen ist auf der Basis der bestehenden Rohdaten nicht möglich. Es ist allerdings zu vermuten, dass die durch eine auf die Schwere der Unfallfolgen relativierten Unfalldaten die existierenden Tendenzen eher noch verstärken ${ }^{264}$.

\subsubsection{Die subjektive Datenbasis: die Ergebnisse der internetbasierten Befragung}

Im Zeitraum zwischen dem 10.06. und 12.07.2005 wurden mittels eines Online-Fragebogens und unter Mithilfe des DKV eine Befragung von aktiven Kanusportlern zur subjektiven Einschätzung der Unfallhäufigkeit und der Unfallschwere sowie der Ausübungshäufigkeit durchgeführt. An der Befragung nahmen mit 183 gültigen Fragebögen deutlich weniger Probanden teil, als bei der Befragung der alpinen Bergsportler. Mit 151 männlichen und 32 weiblichen Probanden war auch diese Untersuchung deutlich männlich dominiert. Analog zur internetbasierten Befragung der alpinen Bergsportler wurden auch bei dieser Teiluntersuchung gezielt Übungsleiter für die Befragung rekrutiert. Die Auswertung der Alters-, Erfahrungs- und Geschlechtsmerkmale im Bezug auf das Beurteilungsverhalten wird in der abschließenden Ergänzungsuntersuchung dargestellt. Zunächst erfolgt die Illustration der Befragungsergebnisse.

Die Rohdaten, die sich aus den Einschätzungen der Unfallhäufigkeiten sowie die Beurteilung der Unfallfolgen ermitteln ließen, wurden zunächst mathematisch ausgezählt und schließlich dem beschriebenen statistischen Verfahren unterzogen. Die Mittelwert der Einschätzungen wurde also in entsprechende z-Werte transformiert, wobei erneut auf das Berechnungsverfahren normativer z-Werte zurückgegriffen wurde. Abbildung 9.30 stellt diese aus den Einschätzungen resultierenden $\mathrm{z}$-Werte dar. 


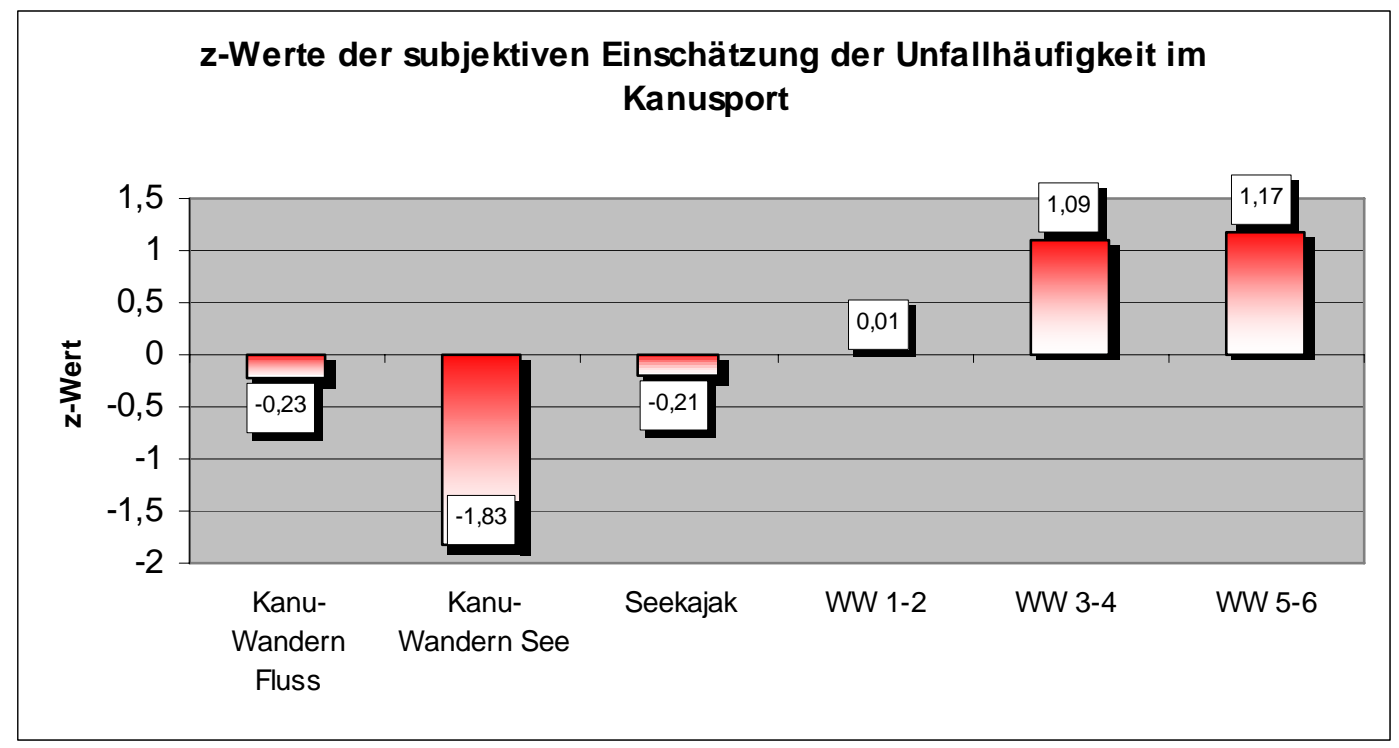

Abbildung 9.30: z-Werte der subjektiven Einschätzung der Unfallhäufigkeit im Kanusport. Datenbasis stellen alle ausgewerteten Fragebögen der internetbasierten Befragung dar $(n=183)$.

Im Mittel wird also das Kanu-Wandern auf Seen (MW 2,45) als ungefährlichste Sportart im Bezug auf die Unfallhäufigkeit beurteilt, während für das Kanu-Wildwasserfahren im Schwierigkeitsbereich 5-6 (MW = 3,56) die meisten Unfälle vermutet werden. Das Wildwasserfahren auf schweren Gewässern im Bereich der Schwierigkeitsskala 3-4 wird mit einem Mittelwert von 3,53 ebenfalls relativ stark unfallgefährdet eingeschätzt.

Obwohl kein objektives Vergleichsmaß für die Beurteilung der Schwere der Unfallfolgen vorliegt, wurden die Einschätzungen über mögliche Unfallfolgen ebenfalls nach dem bekannten Verfahren ausgewertet. Hierbei zeigt sich erneut ein sehr differenziertes Beurteilungsvermögen im Vergleich zu den Einschätzungen der Unfallhäufigkeiten. Mit einen Mittelwert von 4,97 wurden die Folgen eines Unfalls auf Wildwasser der Stufe 5-6 als am schwerwiegendsten beurteilt. Aber auch das Befahren von Wildwasserflüssen im Schwierigkeitsbereich 3-4 (MW = 3,97) und das Seekajak-Fahren $(\mathrm{MW}=4,11)$ wurden als vergleichsweise gefährlich eingestuft. Die Unfallfolgen beim Kanu-Wandern auf Seen $(M W=3,2)$ und Flüssen $(M W=3,34)$ aber auch das Kanu-Wildwasserfahren im Schwierigkeitsbereich 1-2 (MW = $3,14)$ wurden in der Relation aller Einschätzung relativ niedrig bewertet. Abbildung 9.31 zeigt die aus diesen Mittelwerten resultierenden z-Werte. 


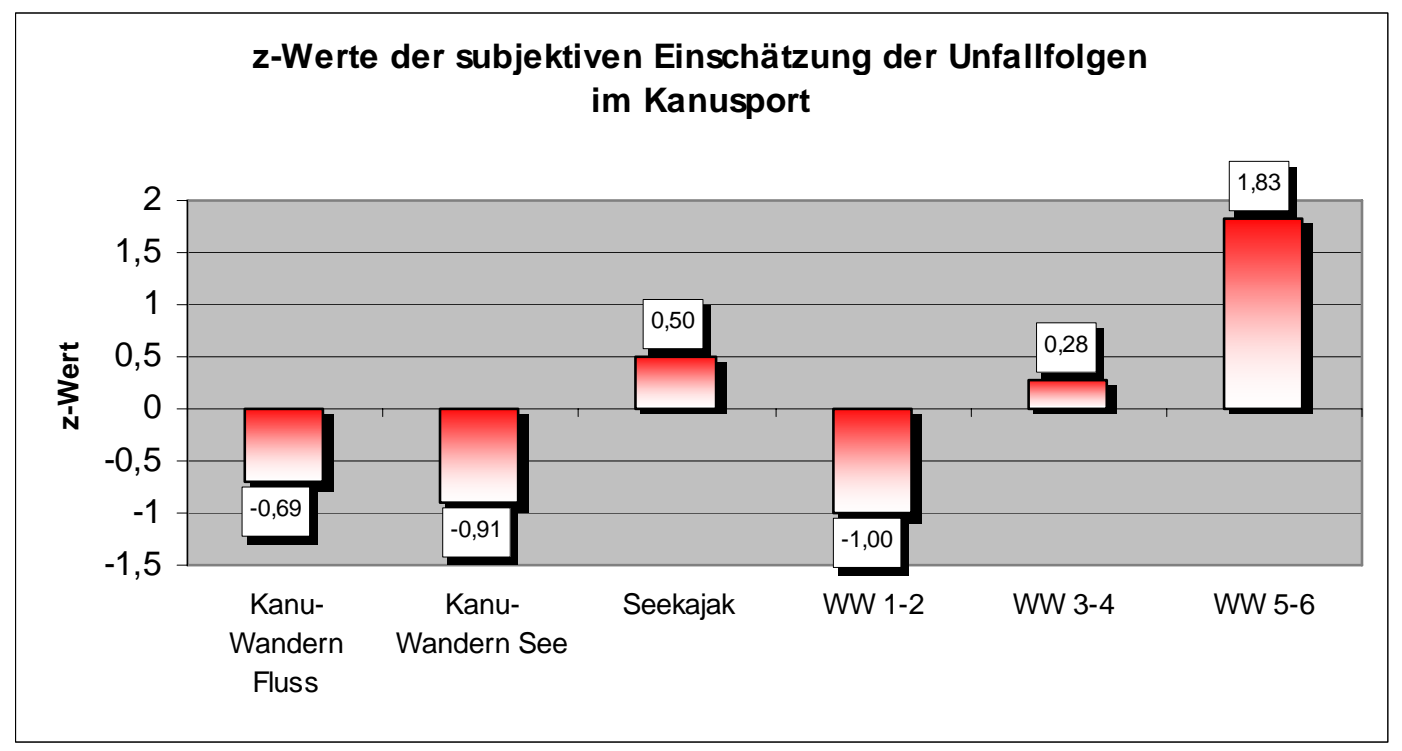

Abbildung 9.31: z-Werte der subjektiven Einschätzung der Unfallfolgen im Kanusport. Datenbasis stellen alle ausgewerteten Fragebögen der internetbasierten Befragung dar $(n=183)$.

Die beiden Diagramme illustrieren erneut, dass die Probanden ein sehr differenziertes Urteil über Unfallhäufigkeiten und Unfallfolgen besitzen. Die Probanden sind also abermals - vergleichbar den Befragungen im alpinen Bergsport - in der Lage ein sehr feinkörniges Urteil über die möglichen Folgen eines Unfalls abzugeben. Die von RINKE und MUSAHL geäußerte Kritik am Befragungsinstrument im Bezug auf die Unfallfolgeneinschätzung kann somit für risikosportliche Kontexte nicht erneuert werden.

\subsubsection{Differen₹bildung: objektive und subjektive Daten im Vergleich}

Die Ergebnisse der Differenzbildung zwischen den objektiven Daten in Form der z-Werttransformierten Unfallzahlen und den subjektiven Einschätzungen werden im Folgenden vorgestellt. Das hierbei gewählte Verfahren ist kongruent zu dem differenzbildenden Verfahren, welches im alpinen Bergsport angewendet und in Abschnitt 9.2 erläutert wurde. Bei der Differenz $Z_{\in G}$ handelt es sich also weiterhin um die Differenz zwischen den ermittelten $z$-Werten der objektiven und subjektiven Daten. Am konservativen Klassifikationskriterium, wonach eine Überschätzung der tatsächlichen Gefährdung erst $\mathrm{ab}$ einem Bereich von $Z_{\in G}>1,0$ und eine Unterschätzung ab einer Differenz von $Z_{\in G}<-1,0$ gelten soll, wird in dieser Teiluntersuchung festgehalten.

Abbildung 9.32 stellt die Differenzen zwischen den z-Werten der subjektiven Einschätzung der Unfallhäufigkeit und den z-Werten der tatsächlichen Unfallzahlen dar. Es wird deutlich, dass im Kanusport sowohl das KanuWandern auf Flüssen als auch das Kanu-Wandern auf stehenden Gewässern deutlich unterschätzt werden. Mit $Z_{\in G}=-2,02$ wird insbesondere das KanuWandern auf Flüssen geradezu massiv unrealistisch im Bezug auf die Un- 
fallhäufigkeit eingeschätzt. Gleichzeitig wird das Kanu-Wildwasserfahren auf Flüssen der Schwierigkeitsstufe 3-4 $\left(Z_{\in G}=1,13\right)$, insbesondere aber der Schwierigkeitsstufe 5-6 $\left(Z_{\in G}=2,43\right)$ deutlich in seinem realen Unfallaufkommen überschätzt.

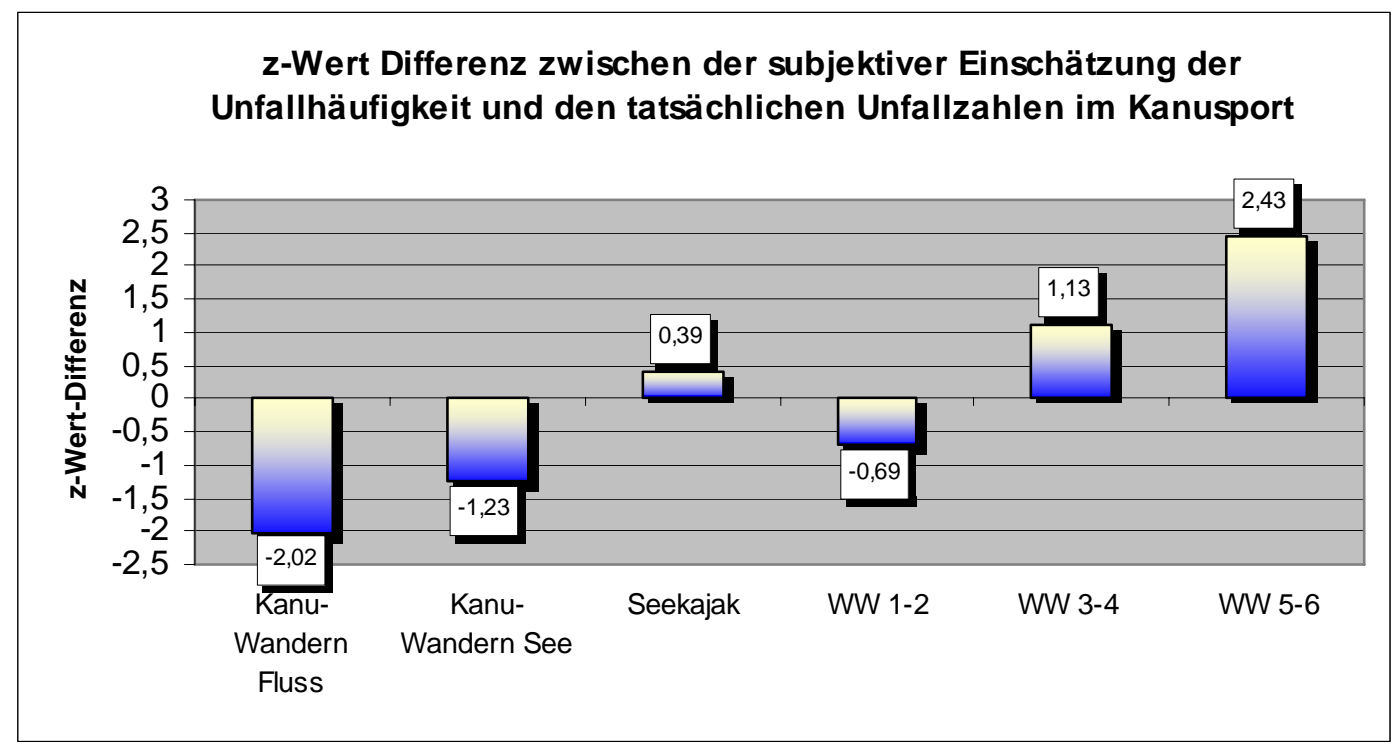

Abbildung 9.32: z-Wert Differenz zwischen der subjektiven Einschätzung der Unfallhäufigkeit und den tatsächlichen Unfallzahlen im Kanusport (n (Unfallzahlen) = 83; $n$ (Probanden) $=183$ ).

Die Ergebnisse werden auch durch die Differenzbildung zwischen den z-Werten der mittleren subjektiven Einschätzungen und den expositionsbereinigten Unfallzahlen nicht entschärft. Auch hier zeigt sich für die genannten Bereiche eine deutliche Unter- (Kanu-Wandern Fluss / See) bzw. Überschätzung (WW 3-4 / WW 5-6). Das Seekajak-Fahren und das Wildwasserfahren auf leichten Flussabschnitten wird dagegen bei beiden Differenzberechnungen als realistisch eingeschätzt (vgl. Abb. 9.33). 


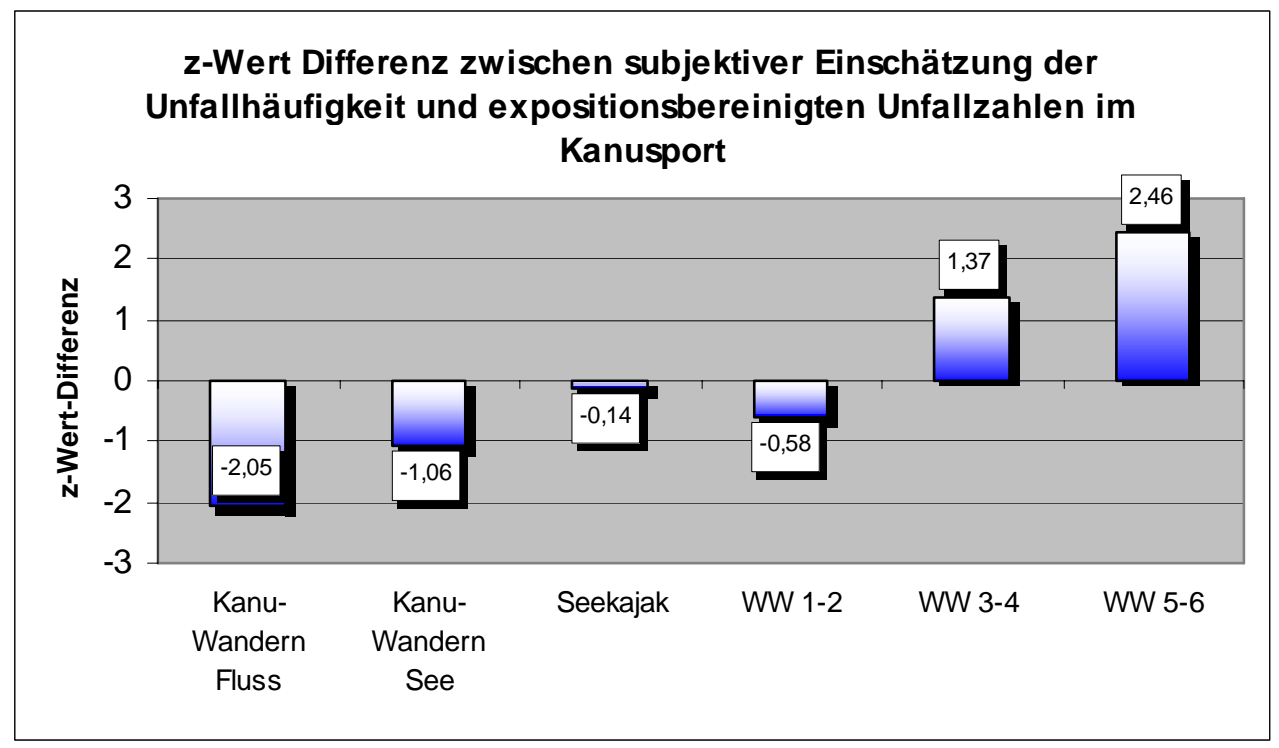

Abbildung 9.33: z-Wert Differenz zwischen der subjektiven Einschätzung der Unfallhäufigkeit und den expositionsbereinigten Unfallzahlen im Kanusport (n (Unfallzahlen) $=83 ; \mathrm{n}$ (Probanden) = 183).

Im Bezug auf die tatsächlichen Unfallzahlen bedeutet dies, dass auf die unterschätzten Aktivitäten im Kanusport über $50 \%$ aller Unfälle fallen, während sich in den überschätzten kanusportlichen Disziplinen nur knapp 16\% der Kanu-Unfälle ereigneten. Oder anders ausgedrückt: obwohl sich über $50 \%$ aller Kanu-Unfälle beim Kanu-Wandern ereignen, werden diese Aktivitäten von den Protagonisten eher als harmlos eingestuft. Dies ist umso erstaunlicher, weil das Kanu-Wandern als kanusportliche Disziplin, welche sich eigentlich nur schwer als Risikosportart einordnen lässt, trotz Expositionsbereinigung damit mehr Unfälle aufweist als die typische Risikosportart Kanu-Wildwasserfahren ${ }^{265}$. Diese Befunde werden in der Diskussion erneut aufgegriffen und einer vertiefenden Interpretation unterzogen. Abschließend werden die Ergebnisse der Ergänzungsuntersuchung vorgestellt, bei der die Probanden nach Alters-, Geschlechts- und Erfahrungsmerkmalen getrennt wurden, um Differenzierungen bezüglich der subjektiven Gefahrenkognition abzubilden.

\subsubsection{Ergebnisse der Ergänzungsuntersuchung: Unterschiede im Bequg auf Geschlecht, Alter, Erfahrung und Übungsleiterlizenzen}

Die im Rahmen der Online-Befragung erhobenen Daten erlauben auch für die Ergänzungsuntersuchung im Kanusport eine Auswertung der Rohdaten im Bezug auf Alters- und Geschlechts- sowie Erfahrungsmerkmale. Darüber hinaus konnten ausreichend Übungsleiter der DKV-Lizenzstufen ,Kanu-Freizeitsport' mit dem Schwerpunkt Kanu-Wandern $(\mathrm{n}=27)$ und mit dem Schwerpunkt Wildwasser $(n=44)$ als so genannte Expertenurteile generiert werden. Die Rohdaten wurden jeweils getrennt und die unterschiedli- 
chen Merkmalsgruppierung gesondert nach dem beschriebenen Verfahren transformiert und ausgewertet.

\subsection{Gefahrenkognition und Geschlecht}

Geschlechtsspezifische Unterschiede im Bezug auf die Einschätzung der Unfallhäufigkeiten, die sich z.B. bei der Teiluntersuchung im alpinen Bergsport darstellen ließen, konnten im Rahmen der kanusportlichen Teiluntersuchung nicht festgestellt werden. Zwar zeigen sich auch hier Differenzen im Bezug auf die Einschätzung der Unfallhäufigkeiten, diese sind aber wenig signifikant. Abbildung 9.34 zeigt die geschlechtsspezifischen Unterschiede in der Einschätzung der Unfallhäufigkeiten anhand der entsprechenden $z$-Werte.

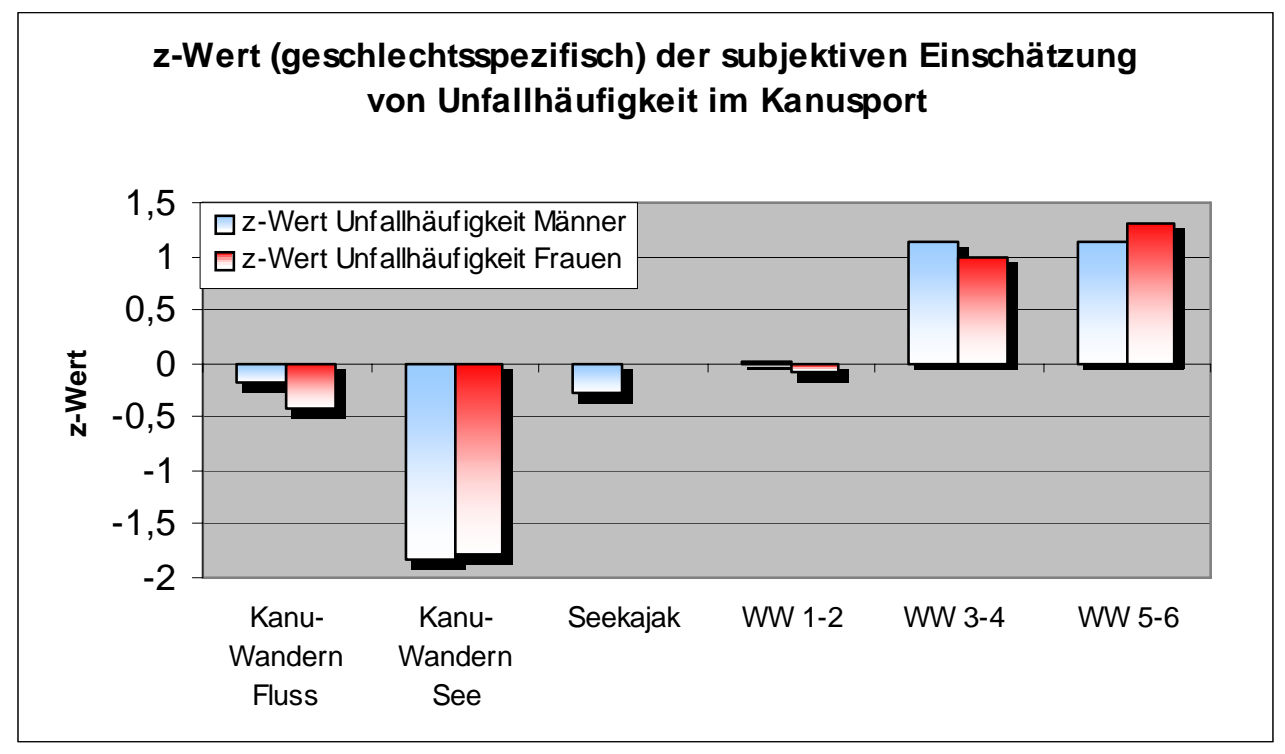

Abbildung 9.34: z-Werte der geschlechtsspezifischen Einschätzung der Unfallhäufigkeit im Kanusport (n (Männer) = 151; n (Frauen) = 32).

Durch die relative Übereinstimmung der geschlechtsspezifischen ZWerte, fällt auch die Differenzberechnung weitestgehend, geschlechtsneutral' aus. Abbildung 9.35 illustriert, dass Männer zwar in der Tendenz insbesondere das Kanu-Wandern auf Flüssen und das Kanu-Wildwasserfahren auf sehr schwierigen Gewässern (WW 5-6) realistischer einschätzen als Frauen. Angesichts der Uneinheitlichkeit dieser Einschätzungen für die übrigen Aktivitäten kann dieser Zusammenhang allgemein aber nicht erhärtet werden. 


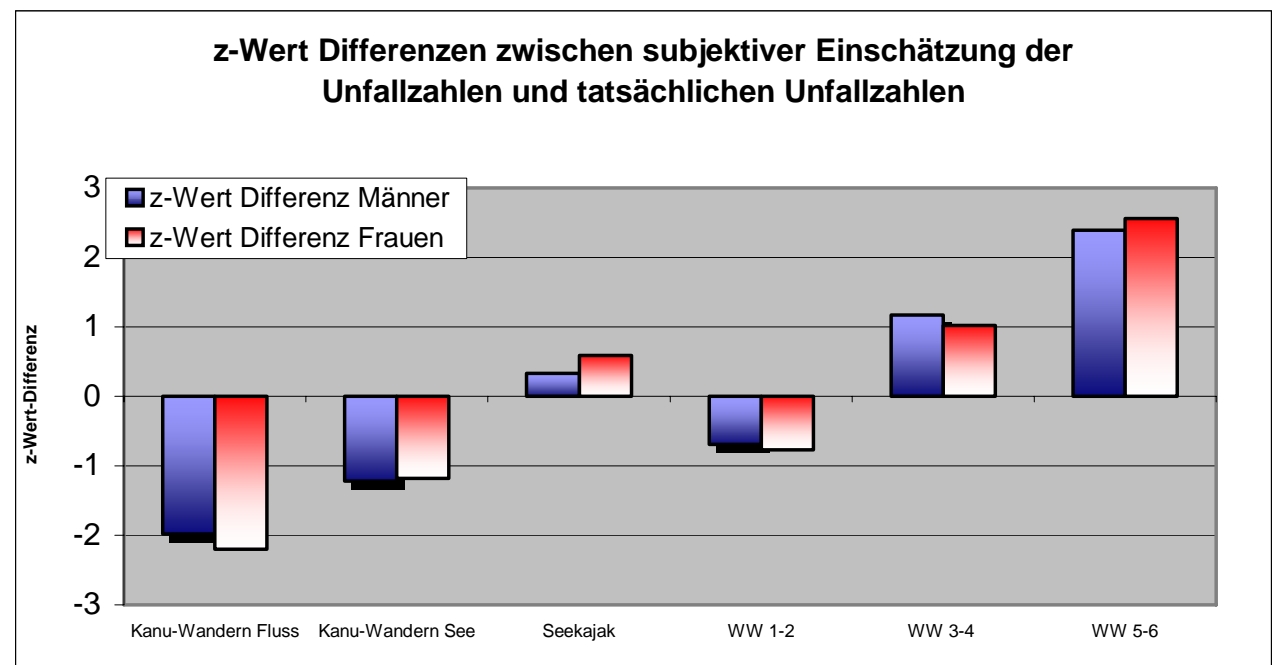

Abbildung 9.35: Geschlechtsspezifische z-Wert Differenzen zwischen der subjektiven Einschätzung der Unfallzahlen und den tatsächlichen Unfallzahlen im Kanusport. Datenbasis sind 83 Unfälle in den Jahren 1998 - 2003 sowie die Einschätzungen von befragten Frauen ( $n=32)$ und Männern $(n=151)$.

\subsection{Gefahrenkognition und Alter}

Die Ergebnisse der altersspezifischen Datenauswertung lassen ebenfalls keine eindeutige Interpretation zu. Keine der Altersgruppen weist Besonderheiten im Einschätzungsvermögen bezüglich der Unfallhäufigkeit auf. Zwar finden sich durchaus Unterschiede im Beurteilungsverhalten der einzelnen Altersstufen, diese sind aber zu heterogen, um signifikante Zusammenhänge darzustellen. Die Gruppe der über 50jährigen konnte gar nicht berücksichtigt werden, da mit $n=9$ (51-60 Jährige) und $n=6$ ( $>61$ Jahre) keine statistisch auswertbaren Stichproben vorlagen. Abbildung 9.36 zeigt die Zusammenhänge als graphische Darstellung. 


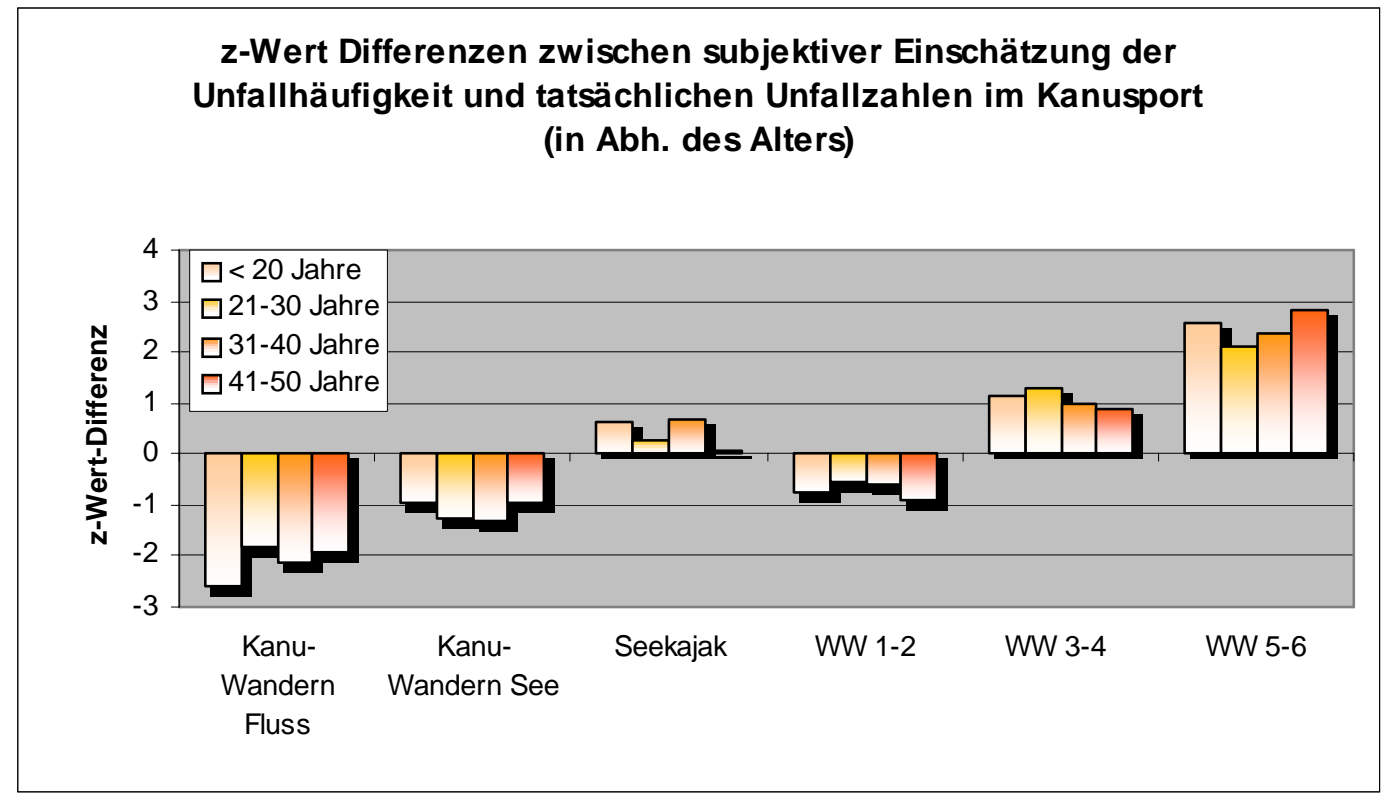

Abbildung 9.36: Altersspezifische z-Wert-Differenzen zwischen der subjektiven Einschätzung der Unfallhäufigkeit und den tatsächlichen Unfallzahlen im Kanusport $N$ (<20 Jahren $)=14 ; \mathrm{N}(21-30$ Jahre $)=67 ; \mathrm{N}(31-40$ Jahre $)=49 ; \mathrm{N}$ (41-50 Jahre)=37)

\subsection{Gefahrenkognition und Erfahrung}

Für die Differenzanalyse zwischen der subjektiven Einschätzungen der Unfallhäufigkeit und den tatsächlichen Unfallzahlen im Bezug auf kanusportliche Erfahrungswerte, wurden die Gruppen nach den Angaben zur Erfahrungsdauer im Kanusport getrennt. Die Ergebnisse der z-WertTransformation zeigen deutliche Unterschiede im Einschätzungsverhalten der einzelnen Probandengruppen. So offenbaren sich deutliche Unterschiede in der Einschätzung der Unfallhäufigkeit für das Kanu-Wandern auf flieBenden Gewässern sowie für das Kanu-Wildwasserfahren auf sehr schweren Gewässern (WW 5-6). Verdeutlicht werden diese Differenzen auch durch die z-Wert-Differenzen. Während die Gruppe mit den längsten Erfahrungswerten ( $>$ 28Jahre Erfahrung; $n=25)$ die realistischsten Gefahrenkognitionen aufweisen, schätzen die erfahrungsjüngsten Probanden ( $<$ 4Jahre Erfahrung; $n=32$ ) die Unfallhäufigkeit in der Tendenz am unrealistischsten ein. Auch zwischen den Erfahrungsbereichen 5-12 Jahre, 13-20 Jahre und 21-18 Jahre sind nuancierte Differenzierungen festzustellen. Allerdings sind auch diese Unterschiede statistisch nicht signifikant. Inwieweit zudem Verzerrungen aufgrund der geringen Probandenzahl zu berücksichtigen sind, kann nicht eindeutig abgeschätzt werden. Abbildung 9.37 und 9.38 verdeutlichen die genannten Ergebnisse. 


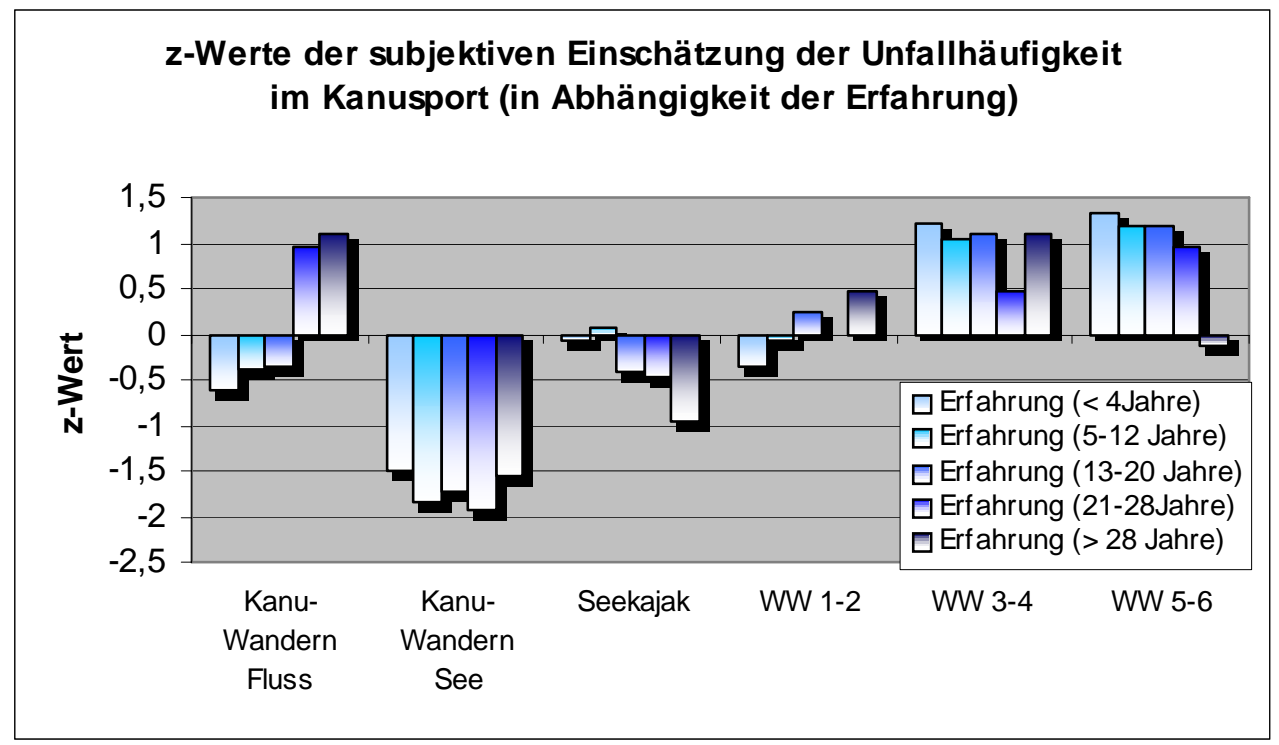

Abbildung 9.37: z-Werte der subjektiven Einschätzung der Unfallhäufigkeit in Abhängigkeit der Erfahrung ( $\mathrm{n}(<4$ Jahre $)=32$; $n$ (5-12 Jahre) $=69 ; n$ (13-20 Jahre) $=$ 41; n (Erfahrung 20-28 Jahre) $=15 ; n$ (> 28 Jahre $)=25$ ).

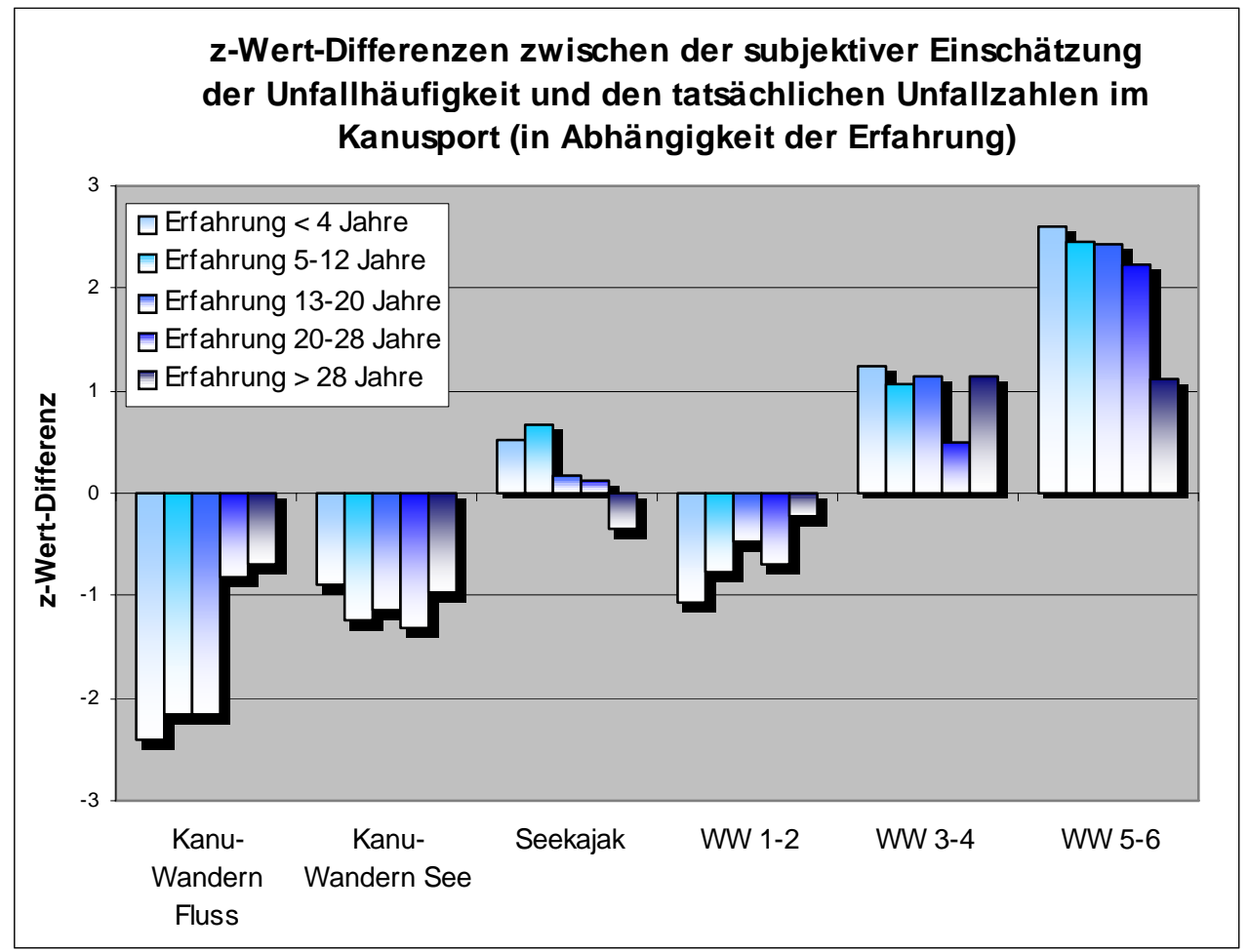

Abbildung 9.38: Erfahrungsspezifische z-Wert-Differenzen zwischen der subjektiven Einschätzung der Unfallhäufigkeit und den tatsächlichen Unfallzahlen ( $n$ ( $<4$ Jahre)= 32; $\mathrm{n}$ (5-12 Jahre $)=69 ; \mathrm{n}$ (13-20 Jahre) $=41 ; \mathrm{n}$ (Erfahrung 20-28 Jahre $)=15 ; \mathrm{n}$ (> 28 Jahre) $=25)$. 


\subsection{Gefahrenkognition von Übungsleitern}

Mit der Befragung kanuspezifischer Fachübungsleiter konnte auch für den Kanusport so genannte Expertenmeinungen eingeholt werden. Insgesamt wurden 71 Fachübungsleiter mit der DKV-Lizenz ,Freizeit und KanuWandersport' zu ihrer Einschätzung der Unfallhäufigkeit und Unfallschwere befragt. Da diese Übungsleiterlizenz sowohl eine Kanuschulung im Bereich Kanu-Wandern als auch Kanu-Wildwasser erlaubt, wurde zusätzlich nach dem Tätigkeitsschwerpunkt der Übungsleiter gefragt. 44 Personen gaben hier einen Tätigkeitsschwerpunkt Wildwasser an, 27 einen Schwerpunkt Wanderpaddeln. Andere Übungsleiterlizenzen (Trainer B; Trainer C) konnten in statistisch nicht ausreichender Form rekrutiert werden. Die Ergebnisdarstellung erfolgt insofern anhand der Tätigkeitsschwerpunkte und wird mit Personen ohne Übungsleiterlizenz verglichen (Abb. 9.39).

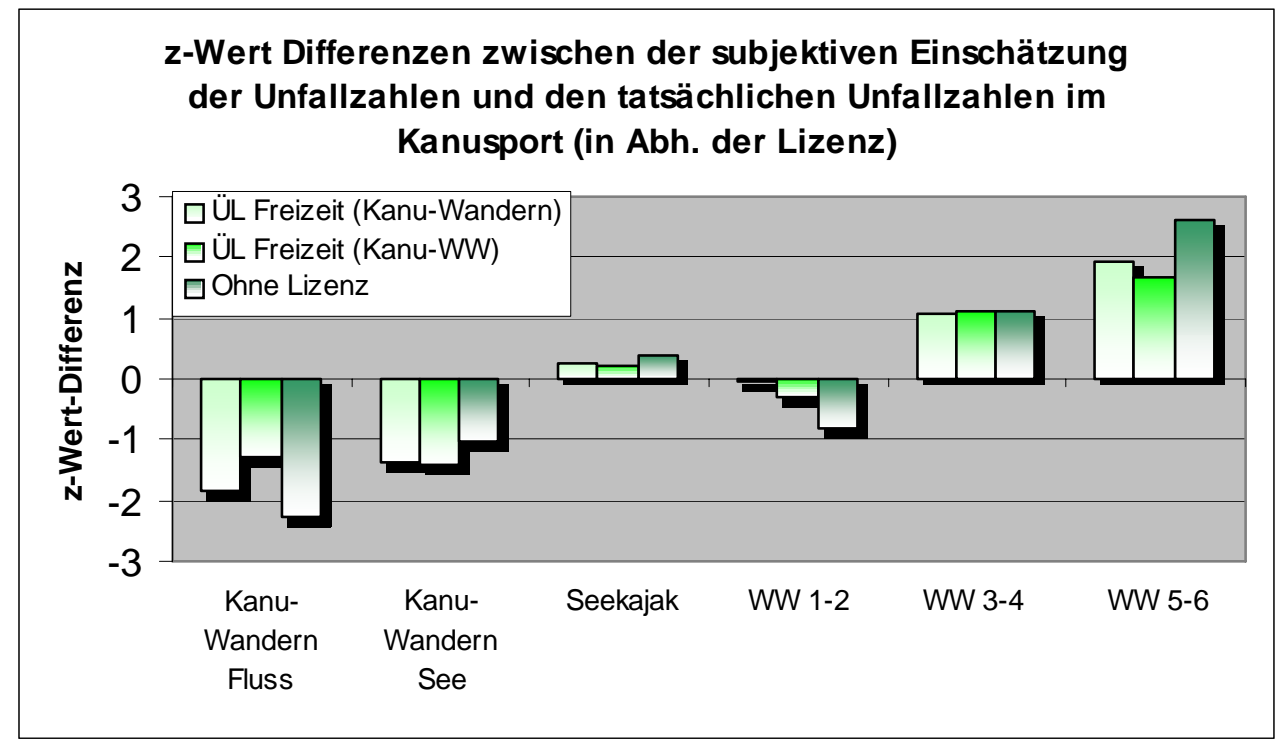

Abbildung 9.39: z-Wert Differenzen zwischen der subjektiven Einschätzung der Unfallzahlen und den tatsächlichen Unfallzahlen bei Fachübungsleitern mit dem Schwerpunkt Wildwasser $(n=44)$, dem Schwerpunkt Kanu-Wandern $(n=27)$ und Personen ohne Lizenzen $(n=112)$.

Die z-Wert-Differenzen verdeutlichen, dass die Übungsleiter die Unfallhäufigkeiten im Kanusport insgesamt wesentlich realistischer einschätzen als Kanuten ohne entsprechende Lizenz. Die statistisch realistischsten Werte zeigt dabei die Gruppe der Fachübungsleiter mit dem Schwerpunkt Wildwasser. Vor allem die beiden, sicherheitspsychologischen Problemfelder' das Kanu-Wandern auf Flüssen und das Kanu-Wildwasserfahren auf Gewässern der Schwierigkeitsstufe 5-6 - werden von dieser Gruppen deutlich realistischer eingeschätzt als von den beiden Vergleichsgruppen.

Zusammenfassend zeigt die Ergänzungsuntersuchung nur für den Vergleich von Übungsleitern mit Personen ohne fachspezifische Lizenz einen deutlichen Zusammenhang im Bezug auf die Gefahrenkognition. Für die Merkmale Geschlecht, Alter und Erfahrung lassen sich keine eindeutigen Zusammenhänge feststellen. Die Ergebnisse der Teiluntersuchung für das 
alpine Bergsteigen konnte damit in Teilen repliziert werden. In der folgenden Diskussion werden auch diese Befunde erneut aufgegriffen und kontextbezogen interpretiert. Das sich an die Diskussion anschließende Fazit der Untersuchung fasst das Untersuchungsverfahren abschließend zusammen und bewertet die Ergebnisse im Kontext der formulierten Hypothesen.

\subsection{Diskussion der Untersuchungsergebnisse}

In der Diskussion der Untersuchungsergebnisse werden die Hauptbefunde der Untersuchung zusammenfassend dargestellt. Dabei werden die Ergebnisse aus sicherheitspsychologischer Perspektive interpretiert und im Bezug auf die Aufgaben- und Fragestellungen der Untersuchungen reflektiert. Es folgt eine theoretische Einordnung der Befunde und ein Ausblick auf offene Forschungsfragen. Ein Fazit der Untersuchung schließt diesen Teilabschnitt ab, bevor das Kapitel schließlich komprimiert zusammengefasst wird. Zunächst wird die Aufgabenstellung der Untersuchung nochmals aufgegriffen, um dem Leser die Ausgangssituation der Untersuchung zu verdeutlichen.

\subsubsection{Aufgabenstellung der Untersuchung}

Praktischer Ausgangspunkt der Untersuchung war eine anwendungsbezogene Aufgabenstellung: Interventionsmaßnahmen im Risikosport sollten mit dem Ziel einer Unfallprävention wissenschaftlich legitimiert und inhaltliche Bezüge aufgedeckt werden. Wie in den Untersuchungen von MUSAHL (1997) und RINKE (2002) gezeigt werden konnte, ließen sich Unfälle in verschiedenen Arbeitssituationen und im Schulsport durch eine fehlerhafte Gefahrenkognition erklären. Handlungssituationen wurden im Bezug auf ihre objektive Gefährlichkeit unterschätzt, so dass eine korrelative Verbindung zwischen Gefahrenkognition und Unfallentstehung aufgezeigt werden konnte. Da weiterführende Konzepte im Risikosport zur Zeit nicht vorliegen, wurde das Hauptziel der Untersuchung mit einer Replikation der Untersuchungsergebnisse von MUSAHL (1997) und RINKE (2002) verbunden. Diese Verknüpfung sollte über zwei Teilaufgaben der Untersuchung realisiert werden, nämlich

(1) über die Überprüfung des von MUSAHL entwickelten Verfahrens für ihre Anwendbarkeit und Validität im Risikosport und

(2) über die Replikabilität der Untersuchungsergebnisse, die einen eindeutigen Zusammenhang zwischen Gefahrenkognition und Unfallentstehung nahe legten. Als Ausgangspunkt der Untersuchung wurden die Hypothesen (1) \& (2) formuliert, wonach auf wenige unterschätzte Aktivitäten überproportional viele Unfälle im Risikosport entfallen.

(3) Darüber hinaus sollten weiterführende Zusammenhängen zwischen Gefahrenkognition und Unfalldaten im konkreten Kontext des Risikosports im Rahmen einer explorativen Herangehensweise aufgezeigt werden.

Es ist folgerichtig, dass die Teilaufgaben (1) und (2) eng miteinander verknüpft sind, weil sich eine Replikabilität der arbeitspsychologischen Unter- 
suchungsergebnisse nur über das von MUSAHL entwickelte Verfahren realisieren lässt. Im Folgenden werden die Hauptbefunde dargestellt und im Bezug zur Aufgabenstellungen interpretiert.

\subsubsection{Hauptbefunde}

Um das Modell der Gefahrenkognition für den Kontext des Risikosports zu überprüfen, wurde das hierzu entwickelte Verfahren für risikosportliche Handlungszusammenhänge transformiert. In Kooperation mit dem Deutschen Alpenverein (DAV) und dem Deutschen Kanu Verband (DKV) wurden Unfallanzeigen und statistische Materialien zum Unfallgeschehen im alpinen Bergsport und im Kanusport analysiert. Für jede unfallbelastete Aktivität wurden die tatsächlichen Unfallzahlen ermittelt und in so genannte Standardwerte transformiert. Diese $\mathrm{z}$-Werte wurden mit Einschätzungen von aktiven Berg- und Kanusportlern im Bezug auf die mittlere Unfallhäufigkeit und die Unfallschwere der jeweiligen Aktivität verglichen. Die subjektiven Daten wurden mittels einer internetbasierten Befragung erhoben, die in Kooperation mit den genannten Fachverbänden durchgeführt wurde. Als Ergänzungsuntersuchung wurde statistische Merkmalsgruppen (Geschlecht, Alter, Erfahrung, Übungsleiterlizenz) getrennt überprüft, um Zusammenhänge und Differenzen genauer abzubilden.

Die folgende Zusammenfassung der sicherheitspsychologisch wichtigsten Einzelergebnisse der beiden Teiluntersuchungen erfolgt entlang den Oben formulierten wissenschaftlichen Zielsetzungen. Zunächst wird die Anwendbarkeit des gewählten Verfahrens diskutiert. Anschließend wird erörtert, inwiefern die Hauptbefunde von MUSAHL (1997) und RINKE (2002) im Kontext des Risikosports repliziert werden konnten und abschließend erfolgt die Darstellungen und Diskussion der wichtigsten weiterführenden Erkenntnisse, die im Rahmen einer Exploration der Untersuchungsergebnisse durchgeführt wurde.

\subsubsection{Anwendbarkeit des gewählten Verfahrens}

Das im Rahmen der Untersuchung gewählte Verfahren wurde in Anlehnung an die von MUSAHL entwickelte Methodik für risikosportliche Kontexte transformiert. Dabei wurden zwei unterschiedliche Untersuchungsstränge angewendet, die in einer Differenzbildung einen gemeinsamen Schnittpunkt erfahren: einerseits die Erhebung objektiver Datensätze in der Form einer Reanalyse für die in den einzelnen Sportbereichen registrierten Unfälle. Andererseits die Generierung subjektiver Daten in Form einer Befragung von aktiven Berg- und Kanusportlern durch eine internetbasierte Befragung.

Insgesamt wurden bei beiden Teiluntersuchungen quantitativ mit unterschiedlich großen Datensätzen gearbeitet. Während im alpinen Bergsport mit 2035 registrierten Unfällen und 724 Untersuchungsteilnehmern relativ große Datenmengen auswertet werden konnten, stand im Kanusport mit 83 registrierten Unfällen und 183 Befragungsteilnehmern ein vergleichsweise geringer Datenpool zur Verfügung. Insofern sich Differenzen im Bezug auf 
Tätigkeiten und Einschätzungen darstellen ließen und es unfallbelastete Aktivitäten gibt, die nicht unterschätzt werden, ist die Forschungsmethode nicht als trivial zu bezeichnen. Die Anwendbarkeit des gewählten Verfahrens stellte sich aber vor allem im Bezug auf die Erhebung der objektiven Datensätze als prekär dar. Während in den sicherheitspsychologischen Studien der Arbeitssicherheit aber auch in der von RINKE (2002) durchgeführten Untersuchung im Schulsport auf institutionalisierte Unfallanzeigen für ein geschlossenes System zurückgegriffen werden konnte, musste im Rahmen dieser Arbeit auf statistische Ausschnitte von Unfalldaten zurückgegriffen werden. Zwar stellt auch die Datensammlung des DAV, die im Rahmen der Teilerhebung im alpinen Bergsport reanalysiert wurde, eine nahezu vollständige Unfalldatensammlung bezogen auf die Gesamtaktivitäten der DAV-Mitglieder dar. Allerdings beschränkt sich der Ausschnitt eben auf eine Teilmenge aktiver Bergsportler, nämlich DAV-Mitglieder, die durch ihre Unfallanzeige Kostenansprüche geltend machen können. Problematisch erscheint dies deshalb, weil diese Gruppe - im Vergleich zu informellen Bergsportlern - über ein differenziertes Wissen im Bezug auf Gefährdungspotentiale und Sicherheitsprobleme verfügen könnte. Wenngleich eine Korrelation zwischen den Unfallzahlen des DAV und anderen alpinen Unfallsammlungen hergestellt werden kann, so ist eine Verzerrung der Daten im Bezug auf die Gesamtheit der alpinen Bergsportler nicht auszuschließen. Beispielsweise ist es denkbar, dass die vom DAV erfassten Unfälle in einer falschen Relation zum Gesamtunfallaufkommen stehen. Ähnliches ergibt sich auch für die Unfalldaten im Kanusport, die zwar für die Unfälle mit tödlichen und schweren Verletzungsfolgen annährend vollständig sein sollten, darüber hinaus aber keine weiterführende Unfalldaten erfassen. Nur wenn auch Unfälle mit leichten Verletzungen oder zumindest die statistisch relevanten Notfälle repräsentativ erfasst werden könnten, wäre die Datenqualität auch in ihrer Validität unangreifbar.

Eine besondere Schwäche, die sich in der Anwendung des Verfahrens durchaus negativ niederschlägt, offenbart sich auch in der Qualität der Unfallanzeigen in risikosportlichen Kontexten. Die Beschreibugen sind insgesamt nur wenig differenziert und uneinheitlich. Eine detaillierte Aufschlüsselung der Aktivitäten in kleinere Tätigkeitseinheiten ist aufgrund des Informationsdefizit der Unfallanzeigen daher nicht möglich. Dies ist in risikosportlichen Aktivitäten auch deshalb durchaus schwierig, weil die einzelnen Handlungssituationen nicht wie in Arbeitsprozessen üblich, standardisiert werden können. Die Handlungsstruktur der gewählten Handlungsbereiche zeigt letztlich eine überdimensionale Varianz und Offenheit, die dazu führt, dass voneinander getrennte Einheiten selbst bei detaillierten Unfallbeschreibungen nicht identifiziert werden können.

Selbst wenn eine fehlende Vollständigkeit der Unfallstatistiken mit dem Hinweis auf die zumindest repräsentative Relation der Unfallanteile der einzelnen Aktivitäten - denn nur diese wird bei der Transformation in z-Werte berücksichtigt - entkräftet werden kann, bleiben bei einer Reanalyse von Unfalldaten doch erhebliche Gültigkeits- und Zuverlässigkeitsmängel. Im 
Bezug auf die Validität der Daten ist insbesondere die Filterung des realen Ereignisses durch die Unfallanzeige und schließlich durch die weitere statistische Bearbeitung anzuführen. Der einzelne Unfall wird durch eine retrospektive Übersetzung in einer Weise gespiegelt, dass Konsequenzen für die Gültigkeit der Daten nicht ausbleibt. Denn fraglich ist auch, ob die Verunfallten überhaupt ein Interesse haben, das Unfallgeschehen vollständig und detailliert weiterzugeben. Um zum Beispiel rechtliche Angriffspunkte zu entschärfen oder die Bedeutung des eigenen fahrlässigen Verhaltens herunterzuspielen, ist eine Entkopplung der Unfallanzeigen vom eigentlichen Unfallgeschehen möglich. Denkbar ist also, dass Unfallanzeigen in der Mehrheit Scheinwahrheiten im Bezug auf die Unfalldetails wiedergeben. Darüber hinaus sind auch Zuverlässigkeitsmängel der Unfalldaten vorstellbar. Die Zuverlässigkeit einer Aussage beschreibt die Genauigkeit und Wiederholbarkeit eines untersuchten Ereignisse - der formale Begriff des Verhältnisses von wahrer Varianz zur empirischen Varianz entspricht diesem Grundgedanken (vgl. auch MUSAHL 1997, 231). Für die Unfallanzeigen würde dies bedeuten, dass unabhängig vom Bearbeiter der Unfallanzeigen eine Übereinstimmung in der ,Übersetzung' der Unfallzusammenhänge für den einzelnen Unfall aber auch für das gesamte Unfallgeschehen erfolgen müsste. Angesichts der geringen Strukturierung der Unfallanzeigen, insbesondere im Kanusport ist eine unabhängige Kodierung der Unfallanzeigen aber kaum möglich. Insbesondere ist eine Trennung von Beschreibung und Erklärung nur schwer einzuhalten. Eine Unfallstatistik - so ist zu vermuten - wird in risikosportlichen Kontexten also tendenziell die ,Handschrift' des individuellen Sachbearbeiters tragen. Unfalldaten repräsentieren damit nur bedingt zuverlässige Datensätze, insbesondere wenn eine Detailuntersuchung der Unfallursachen vorgenommen wird.

Ingesamt kann aber konstatiert werden, dass der hier verwendete Unfalldatensatz im Bezug auf die einzelnen unfallbelasteten Aktivitäten insgesamt wenig verzerrt sein dürfte, denn ein grobmaschiges Kodierungssystem entlang der Sportdisziplinen garantiert ein Mindestmaß an Objektivität, Validität und Reliabilität. Die ursprünglich anvisierte vertiefte Analyse einzelner Unfälle aufgrund von Unfallanzeigen erschien in der vorliegenden Untersuchung aber als prekär.

Anders stellt sich das gewählte Verfahren zur Erhebung der subjektiven Datensätze dar. Das verwendete Befragungsverfahren mittels eines onlinebasierten Fragebogens gestattete es, Urteile über verschiedene Aktivitäten in den beiden risikosportlichen Handlungsfeldern zu erheben. Das angewendete Prinzip der Skalierung einer 6-stufigen Rating-Skala war dabei offenbar für die Mehrheit der Probanden verständlich. Durch die schriftliche, anonyme und rechnergestützte Durchführung sowie die statistischmathematische Transformation der Daten konnten Verletzungen der Auswertungs- und Interpretationsobjektivität zudem weitgehend ausgeschlossen werden. Zwar können Reliabilitätskriterien nur indirekt erschlossen werden, da die klassischen Verfahren zur Zuverlässigkeit der Daten (Retest- oder Paralelltest-Methode) aus organisatorischen Gründen nicht durchgeführt wer- 
den konnten, es ist allerdings anzunehmen, dass vergleichenden Versuchdurchführungen bei ähnlichen Probandengruppen zu übereinstimmenden Ergebnissen kommen. Ein durchgeführter Split-Half-Reliabilitäts-Test - also der Vergleich von frühen und späten Antworten während des Befragungszeitraums - können diese Annahmen zur Reliabilität des Verfahrens untermauern. Sowohl im Bezug auf die Objektivität, als auch auf die Realiabilität und Validität der subjektiven Datensätze ist demnach von einer ausreichenden Güte auszugehen. Dass bei dem gewählten Verfahren das Gütekriterium der Repräsentativität nicht ausreichend erfüllt werden konnte, wurde bereits in Abschnitt 9.2.2.2 ausgeführt.

Zusammenfassend ist die Anwendbarkeit des von MUSAHL entwickelten Verfahrens für risikosportliche Untersuchungskontexte aufgrund der mangelnden Güte der objektiven Daten nur eingeschränkt zu empfehlen. Mit der hier verwendeten Minimaldifferenzierung entlang der Grobkategorisierung der einzelnen Sportdisziplinen konnte das Verfahren zwar in befriedigender Weise angewendet werden, für weitere und vor allem vertiefende Untersuchungen einzelnen Tätigkeitsmodule sind dem Verfahren im Risikosport allerdings Grenzen gesetzt. Inwieweit sich für eine vertiefte Reanalyse der Unfallanzeigen neue repräsentative und vor allem valide Datenquellen auftun können, muss hier bezweifelt werden.

\subsubsection{Replikabilität der Befunde}

Natürlich hat die Schwierigkeit, die sich aus der Anwendung des Verfahrens in risikosportlichen Kontexten ergab, auch Auswirkungen auf die Untersuchungsergebnisse und damit auf die Replikabilität der Hauptbefunde von MUSAHL (1997) und RINKE (2002). Die wichtigsten Befunde, die es zu replizieren galt, waren (a) die allgemein korrelativen Zusammenhänge zwischen subjektiven und objektiven Datensätzen, (b) das dramatische Unfallaufkommen bei signifikant im Bezug auf ihre Unfallhäufigkeit unterschätzten Aktivitäten und (c) das dramatische Unfallaufkommen bei signifikant unterschätzten Aktivitäten im Bezug auf die Schwere der Unfallfolgen.

Ein korrelativer Zusammenhang zwischen subjektiven und objektiven Daten konnte in beiden Teiluntersuchungen zumindest angedeutet werden. Sowohl im Alpinen Bergsport als auch im Kanusport wurden die Aktivitäten durchaus differenziert im Bezug auf ihre Unfallhäufigkeit und die zu vermutenden Unfallfolgen beurteilt. Die hierbei zu konstatierenden Einschätzungen orientierten sich in der Mehrheit der Aktivitäten an der tatsächlichen Unfallhäufigkeit sowie an der Schwere der Unfallfolgen. Die meisten Aktivitäten wurden letztlich - gemessen am konservativen Klassifikationskriterium - realistisch eingeschätzt. Die Gefährlichkeit der Aktivitäten ist den Sportlern in der Mehrheit der Fälle also ebenso bewusst wie eine Differenzierung zwischen Unfallhäufigkeiten und Unfallfolgen. Tabelle 9.5 stellt die Ergebnisse der beiden Teiluntersuchungen nochmals komprimiert dar: 


\begin{tabular}{||l|l|l|l||}
\hline \hline & $\begin{array}{l}\text { Unterschätzte Aktivi- } \\
\text { täten }\end{array}$ & $\begin{array}{l}\text { realistisch eingschä- } \\
\text { tzte Aktivitäten }\end{array}$ & $\begin{array}{l}\text { überschätzte Aktivitä- } \\
\text { ten }\end{array}$ \\
\hline $\begin{array}{l}\text { ALPINER } \\
\begin{array}{l}\text { BERGSPORT } \\
\text { UNFALLHÄUFIGKEIT }\end{array}\end{array} 0 \%$ & $100 \%$ & $0 \%$ \\
\hline $\begin{array}{l}\text { ALPINER } \\
\text { BERGSPORT } \\
\text { UNFALLFOLGEN }\end{array}$ & $20 \%$ & $60 \%$ & $20 \%$ \\
\hline $\begin{array}{l}\text { KANUSPORT } \\
\text { UNFALLHÄUFIGKEIT }\end{array}$ & $33,3 \%$ & $33,3 \%$ & $33,3 \%$ \\
\hline
\end{tabular}

Tabelle 9.5: Zusammenfassung der Schätzungsdifferenzen zwischen subjektiver Einschätzung der Unfallhäufigkeit und expositionsbereinigten Unfallzahlen unterteilt nach unterschätzten Aktivitäten $\left(Z_{\in G}<-1,0\right)$, realistisch eingeschätzten Aktivitäten $\left(-1,0<Z_{\in G}>1,0\right)$ und überschätzten Aktivitäten $\left(Z_{\in G}>1,0\right)$.

Während im alpinen Bergsport alle Aktivitäten im Bezug auf die Unfallhäufigkeit realistisch eingeschätzt wurden, verteilten sich im Kanusport jeweils ein Drittel der Aktivitäten auf die Bereiche ,Unterschätzung, realistische Einschätzung' und ,Überschätzung'. Die Ergebnisse zeigen damit, dass sich die Haupthypothesen nicht in den beiden Teiluntersuchungen vollständigen bestätigen lassen. Die Hypothese (1) „auf risikosportliche Aktivitäten, die in ibrer Gefährlichkeit unterschätst werden entfallen überproportional viele Unfälle "lässt sich nur für den untersuchten Teilbereich des Kanusports eindeutig belegen. Hier fallen mit den unterschätzten Sportdisziplinen ,Kanu-Wandern auf Flüssen' und ,Kanu-Wandern auf Seen' über 50\% aller Unfälle auf Aktivitäten, die in ihrer Gefährlichkeit unterschätzt werden. Im alpinen Bergsport des DAV wird keine Aktivität hinsichtlich der Unfallhäufigkeit signifikant unterschätzt. Zwar ließen sich im Sinne des eher konservativen Klassifikationskriteriums andere Grenzen für die Klassifizierung definieren, das Ziel signifikante Ergebnisse zu produzieren kann aber damit nicht erfüllt werden.

Hinsichtlich der Hypothese (2) „Auf Aktivitäten, die in der Schwere der Unfallfolgen unterschätzt werden, entfallen ebenfalls überproportional viele Unfälle" kann eine Bestätigung nur für die Teiluntersuchung im alpinen Bergsport bestätigt werden. Auf das Bergwandern, welches mit einer Differenz von $Z_{\in G}=-3,01$ massiv hinsichtlich der Unfallfolgen unterschätzt wird, entfallen immerhin knapp 32\% aller Unfälle im alpinen Bergsport. Eine Überprüfung der Hypothese im Kanusport konnte aufgrund der Verfahrensprobleme im Hinblick auf die fehlenden Informationen zur Schwere der Unfallfolgen nicht durchgeführt werden. Insofern ist auch die Hypothese (2) nur teilweise zu betätigen.

Die Replikation der Befunde aus der Arbeitssicherheit und dem Schulsport kann im Rahmen dieser Untersuchung somit nicht vollständig erfolgen. Zwar erhärtet sich die Hypothese (1) für die Teiluntersuchung im Kanusport und die Hypothese (2) für die Teiluntersuchung im alpinen Bergsport, eine eindeutige Aussage kann aufgrund der unvollständigen Datensätze aber nicht erfolgen. Eine Differenzierung zu den Untersuchungser- 
gebnisse der vergangenen Studien muss zudem aufgrund der Unterschiede zwischen der Einschätzung der Unfallhäufigkeit und der Unfallschwere in der vorliegenden Untersuchung vorgenommen werden. MUSHAL (1997, $268)$ und RINKE $(2002,101)$ stellen in ihren Untersuchungen keine signifikanten Unterschiede in der Einschätzung von Unfallhäufigkeit und Unfallschwere fest. Die Bestimmung sicherheitspsychologisch relevanter Tätigkeiten erfolgt hierbei folglich allein aus der Einschätzung der Unfallhäufigkeit, die den Einschätzungen der Unfallfolgen kongruent sind. In der vorliegenden Untersuchung zeigt sich aber ein sehr differenziertes Beurteilungsverhalten, so dass eine Interpretation der Befunde nur über eine Kovariation der Ergebnisse möglich ist. Die Gefahrenkognition im Risikosport und damit auch die Bestätigung der Grundannahmen, wonach sich Unfälle aus einer fehlerhaften Gefahrenkognition - bzw. aus einem trügerischen Gefühl der Sicherheit - erklären könnten, wurden durch die korrelativen Zusammenhänge zwischen der Einschätzung der Unfallhäufigkeiten und den Unfallfolgen erhärtet. Zwar kann eine kognitive Gewichtung auch hier nicht aufgezeigt werden, der Hinweis auf ein differenziertes Beurteilungsvermögen verdeutlicht aber die Bedeutung des kombinierten Verfahrens für eine Replikation der Ausgangsthese. Kombiniert man die Schätzungsdifferenzen der Unfallhäufigkeiten und der Schwere der Unfälle für eine Tätigkeit, dann ergibt sich sowohl für das Bergwandern als auch für das Kanu-Wandern auf Flüssen und Seen eine Unterschätzung. Damit ließen sich die zentralen Befunde der genannten Autoren für risikosportliche Kontexte zumindest replikativ erhärten, ohne das die Hypothesen explizit bestätigt werden konnten. Eine vollständige Replikation kann für risikosportliche Kontexte dennoch nicht vollzogen werden.

\subsubsection{Weiterführende Zusammenhänge zwischen Gefahrenkognition und Unfalldaten im Risikosport}

Neben der Replikation der Befunde aus der Arbeitssicherheit und der Überprüfung des Untersuchungsverfahrens war eine weitere Aufgabe der Studie weiterführende Zusammenhänge zwischen Gefahrenkognition und Unfalldaten im Risikosport zu explorieren. Hierbei sollten keine expliziten Hypothesen überprüft werden. Die Ergebnisse sollten vielmehr im Sinne einer offenen Interpretation nach unbeachteten Zusammenhängen zwischen Gefahrenkognition und Unfalldaten im Risikosport durchleuchtet werden, um über die für arbeitspsychologische Forschungsfelder relevanten Korrelationen hinaus, inhaltliche Beziehungen zu eruieren.

Zunächst fällt auf, dass es sich bei den unterschätzten Aktivitäten, die gleichsam eine erhebliche Unfallhäufigkeit aufweisen, nicht um Risikosportarten im engeren Sinn handelt. Risiko als Erlebniskomponente und Herausforderung spielt bei diesen Sportarten vermutlich eine Nebenrolle. Dies ist insofern umso erstaunlicher, als das gemeinhin davon ausgegangen wurde, dass das vermeintlich objektive Risiko einen Unfall zu erleiden in Risikosportarten wesentlich größer sein würde als in anderen nichtrisikokonnotativen Sportarten. Unterschätzt werden damit ,augenscheinlich 
harmlose' Aktivitäten, für die eine unmittelbare Gefährdung nicht leicht assoziativ ableitbar, erkennbar oder verfügbar ist und an die sich eine subjektiv vermeintliche Kontroll-Illusion bindet. Im Gegenzug werden Aktivitäten, die in ihrer Anschaulichkeit und unmittelbaren Erfahrbarkeit auf eine offensichtliche Gefährdung hinweisen, zum Teil deutlich überschätzt. Dies zeigt sich besonders drastisch am Beispiel des Kanu-Wildwasserfahrens im Schwierigkeitsbereich 5-6. Hier wird eine massive Unfallgefährdung angenommen, die auch in einem sprachlich-konnotativen Wert der Schwierigkeitseinstufung ,extremes Wildwasser' transportiert wird. Vermutlich ist das Kanu- Wildwasserfahren deshalb relativ unfallfrei, weil die Protagonisten wissen, wie ,gefährlich' diese Aktivität ist. Aber auch das Touren-Skigehen wird von den Protagonisten eher überschätzt. Hier spielt sicherlich auch die Kommunikationsintensität eine entscheidende Rolle. Lawinen, die das zentrale Gefährdungselement beim Touren-Skigehen darstellen, werden in ihrer Entstehung und Wirkung auf nahezu allen Ebene des alpinen Bergsports intensiv diskutiert, so dass die kognitive Repräsentation der Gefährdung hier entsprechend präsenter zu sein scheint als z.B. beim Hochtourengehen.

Die Ergebnisse deuten zudem auf einen wichtigen Zusammenhang zwischen der Intensität der kognitiven und motorischen Auseinandersetzung mit dem Handlungsfeld und der sich hieraus konstituierenden Gefahrenkognition hin. Zwar lassen sich diese Zusammenhänge nicht signifikant belegen, die Ergebnisse erhärten aber den Verdacht, dass Übungsleiter gegenüber den Nicht-Übungsleiter eher in der Lage sind das Gefährdungspotential der Aktivitäten realistisch einzuschätzen. Bei beiden Teiluntersuchungen beurteilten die Übungsleiter als so genannte Experten in weitgehend allen Bereichen wirklichkeitsnäher als Personen ohne Lizenz. Da sich ein $\mathrm{Zu}-$ sammenhang zwischen der sportspezifischen Erfahrung und der Qualität der Gefahrenkognition nicht darstellen ließ, ist anzunehmen, dass vor allem die rationale Aufklärung über Gefährdungspotentiale in der verbandsinternen Übungsleiterausbildung zu einer verbesserten Gefahrenkognition führt. Darüber hinaus kann in diesem Zusammenhang aber auch postuliert werden, dass die Vermittlungshandlung einer Sportart bedeutenden Einfluss auf die Ausbildung einer realistischen Gefahrenkognition besitzt. Die Verantwortung, die mit der Ausübung einer Übungsleiterfunktion verbunden ist, zwingt vermutlich zu einer erhöhten Aufmerksamkeit und damit zu einer verbesserten kognitiven Repräsentation der Gesamtgefährdung.

Die für den alpinen Bergsport zu konstatierenden Ergebnisse, wonach Männer eher in der Lage sind, die Gefährdung einer Aktivität angemessen einzuschätzen, ließen sich im Bereich des Kanusports nicht replizieren. Es ist diesbezüglich fraglich, ob geschlechtsspezifische Differenzen im Bezug auf gefahrenkognitive Prozesse vermutet werden können.

Die hier im Rahmen der Exploration aufgestellten korrelativen Zusammenhänge müssen in weiterführenden Forschungsvorhaben durch sachdienliche Hypothesen überprüft werden. Bis dato können sie lediglich als hypothetische Annahmen formuliert und für Interventionsmaßnahmen eingebunden werden. Für die theoretische Einordnung der Befunde stellen diese 
Zusammenhänge aber zweckmäßige Erklärungsversuche für die Handlungsregulation im Risikosport dar, so dass ein vertiefter Blick in Form weiterführender Untersuchung hilfreich wäre.

\subsubsection{Theoretische Einordnung der Befunde}

Durch die Teil-Replikation der sicherheitspsychologischen Befunde von MUSAHL in risikosportlichen Handlungskontexten lassen sich die von verschiedenen Autoren (u.a. auch WORTHMUT (2002); RINKE (2002)) postulierten Zusammenhänge von Gefahrenkognition und Unfallentstehung zumindest teilweise auf den Risikosport transformieren. Die Annahme, dass eine fehlerhafte Wahrnehmung und die kognitive Repräsentation einer Aktivität im Rahmen einer Unterschätzung zu einem erhöhten Unfallaufkommen führt, kann damit für risikosportliche Handlungszusammenhänge bestätigt werden. Das Unterschätzen von gefährlichen Situationen, welches einem situativen Gefühl subjektiver Sicherheit nahe kommt, wird damit zu einem korrelativen Ursachenprinzip für Unfälle auch im Kontext des Risikosports. MUSAHL konstatiert folgerichtig: „Das Fehlen subjektiver Unsicherheit gehört demnach zur Funktions-Logik von Fehlhandlungen, Mißlingen und Unfällen“ (MUSAHL 1997, 220). Wenngleich eine kausale Beziehung zwischen der subjektiven Gefährlichkeit und den tatsächlichen Unfällen im Risikosport eher als Hypothese zu formulieren ist, so kann durch die empirischen Ergebnisse dieser Studie die Annahme eines korrelativen $\mathrm{Zu}$ sammenhangs zwischen einer unzureichenden Gefahrenkognition und der Entstehung von Unfällen zumindest erhärtet werden. Das Fehlen von Unsicherheit könnte demnach eine unzureichende Aufmerksamkeit provozieren, um auf kritische Warnhinweise oder Veränderungen entsprechend zu reagieren. Eine kognitive Klassifikation physikalischer Reize erfolgt unter subjektiven Bedingungen demnach nur unvollständig, denn Wahrnehmung ist ein Prozess der perzeptiv-kognitiven Informationsintegration, der von heuristischen Prinzipien massiv beeinflusst wird (vgl. Abschnitt 6.2). Die Ausprägung der Gefahrenkognition könnte damit auch die situative Verhaltensausprägung bestimmen, die ein unfallträchtiges Verhalten begünstigen oder vermeiden kann (vgl. ebd., 351). Die Untersuchungsergebnisse legen nahe, dass die Unsicherheit im Sport in der Form eines subjektiv wahrgenommenen Risikos eine ,genuin natürlichen' Handlungsregulation hervorrufen könnte, die es Menschen ermöglicht auch gefährliche Situationen ohne gröBeres Unfallaufkommen zu bewältigen

„Die Einordnung eines Ereignisses nach seiner relativen ,Gefährlichkeit' und die daraus resultierende Verbaltensweise berubt danach nicht auf einer Risikobeurteilung nach den Theoremen des entscheidungstheoretischen KostenNutzen-Kalküls, nicht auf einer intraindividuellen stabilen ,Risikobereitschaft' des Unfällers oder anderen Motiv-Dispositionen, sondern sie wird in einem komplexen individuellen Lermprozess entwickelt und verändert"(ebd.)).

Auf der theoretischen Ebene finden die Untersuchungsergebnisse auch in den phänomenologischen Ansätze CUBEs (1995) und den theoretischen 
Annahmen sensu KLEBELSBERG (1982) sowie den anthoplogischmotivationspsychologischen Theorien TENBRUCKs (1978) eine Entsprechung. Bedingungen subjektiver Sicherheit bei vorhandenen Gefährdungspotentialen werden damit zu einem ,Risikoproblem' und zwar insofern, als dass die der Handlung zugrunde liegende Kontrollüberzeugung ein Aufmerksamkeitsdefizit bewirken könnte und Fehlhandlungen und Verhaltensfehler forciert würden. Während bei einer Gefährdungsannahme ohne tatsächlich vorhandenes Risiko - also einer Überschätzung der tatsächlichen Gefahren - ein erhöhtes Unfallpotential aufgrund von Stress- und Angstsymptomen vermutet wird, kann für den Bereich der Unterschätzung reeller Gefahren (gefährliche Sicherheit') von einem erhöhten Unfallpotential durch Überforderung und Unaufmerksamkeit ausgegangen werden (vgl. auch MUSAHL 1997, 221ff). Für Unfälle bei realistisch eingeschätztem Gefährdungspotential wird angenommen, dass die Ursachen in einem Bereich der motorischen Handlungsregulation bzw. der Handlungsorganisation zu suchen sind und daher außerhalb des hier fokussierten Prozesses der Gefahrenkognition $\mathrm{zu}$ finden sind. Unsicherheit als strukturelles Handlungselement - dies war die zentrale Aussage der Anthropologie - erhöht somit nicht nur die Gratifikation der Handlung, sondern führt gleichsam dazu, dass die Handlungsdurchführung mit einer entsprechenden Aufmerksamkeit versehen wird.

Risiko wird damit zu einer zentralen Handlungskategorie: Indem bewusst eine Unsicherheit mit dem Ziel aufgesucht wird, diese zu bewältigen und dadurch sekundär Sicherheit zu gewinnen, wird auch die Handlungsausführung, risikooptimiert'; die bewusste Suche nach zu bewältigen Risiken wird damit zu einer ,Sicherheitsreserve'., wohingegen das Verharren in einem trügerischen Gefühl der Sicherheit zu einer Gefahr werden kann (vgl. Abschnitt $6.2 \&$ 8.3.6).

\subsubsection{Offene Forschungsfragen}

Die hier vorgestellten und diskutierten Hauptbefunde verdichten bestimmte theoretische Annahmen und erhärten die überprüften Annahmen zur Korrelation von Gefahrenkognition und Unfallentstehung. Allerdings reichen die produzierten Ergebnisse nicht aus, um die zu verifizierenden Annahmen nachhaltig zu bestätigen. Nachfolgende Forschungsansätze müssten die Ergebnisse in anderen Risikosportarten zunächst replizieren und die korrelativen Zusammenhänge auf weitere Kategorien überprüfen. Dass hierfür eine Modifikation des Erhebungsverfahrens notwendig sein wird, wurde detailliert ausgeführt. Darüber hinaus lassen sich aus den Untersuchungsergebnissen aber weiterführende Fragestellungen ableiten, die zu einem verbesserten Verständnis der Gesamtsituation führen.

Zum einen muss die ungeklärte Dimensionalität des GefährlichkeitsUrteils hinterfragt werden. Dabei steht nicht die Frage nach der Gewichtung möglicher Urteile über Unfallhäufigkeiten und Unfallfolgen aus, sondern auch die damit verbundene Dimensionalität der Gefahrenkognition. Interventionsmaßnahmen sind letztlich darauf angewiesen, auf detaillierte Infor- 
mationen zurückgreifen zu können. Reichen rein rational vermitteltes Wissen - eine so genannte Aufklärung - aus, um ein Urteil über die Gefährlichkeit zu beeinflussen oder ist die kognitive Repräsentation der Gefährlichkeit ein eher emotional beeinflusster Prozess, der insbesondere durch Erfahrung und die Reflexion bestehender Ereignisse geprägt wird. Hier ist ein experimenteller Zugang gefragt, durch den die Ausprägung und Qualität der Gefahrenkognition an entsprechende Dimensionen rückgebunden werden kann. Zu diesem Forschungsansatz komplementär ist auch die Frage nach der Dimensionalität erlebter Sicherheit zu stellen. Wann und wie empfinden Menschen eine subjektives Gefühl der Sicherheit und durch welche Prozesse kann es beeinflusst werden. Hier ist ein möglicher Einfluss heuristischer Prinzipien ebenso zu berücksichtigen, wie eine kognitive Sicherheitsrepräsentation.

Weiterhin offen sind zudem persönlichkeitsspezifische Unterschiede der Gefahrenkognition z.B. in Verbindung mit Ängstlichkeitsniveaus oder verschiedenen Ebenen der Leistungsmotivation. Ließen sich diesbezüglich $\mathrm{Zu}-$ sammenhänge auf der Basis experimenteller und motivationsdiagnostischer Verfahren herleiten, so könnte eine unfallpräventive Intervention auf der Basis individueller Ansprachen noch erfolgreicher und effizienter durchgeführt werden. Allerdings ist hierbei zunächst ein exploratives Verfahren sinnvoll, um zunächst allgemeine Zusammenhänge zwischen Persönlichkeitsmerkmalen und Gefahrenkognition zu

\subsection{Fazit der Untersuchung}

Ziel der Untersuchung war es, über die Erhebung von subjektiven Gefährlichkeitseinschätzungen und objektiver Unfalldaten die Gefahrenkognition von Risikosportlern zu erfassen. Dabei sollten die Hauptbefunde früherer Forschungsarbeiten repliziert werden und ein möglicher Zusammenhang zwischen Gefahrenkognition und Unfalldaten hergestellt werden. Diese Aufgabe konnte nur teilweise erfüllt werden, da sich vor allem das Erhebungsverfahren nur unzureichend auf risikosportliche Kontexte transferieren ließ. Die Gültigkeit des Gefahrenkognitionsmodells auf den Bereich des Risikosports kann zumindest für den alpinen Bergsport sowie den Kanusport erhärtet werden.

Die wichtigsten Ergebnisse der Studie werden wie folgt zusammengefasst:

(1) Die Einschätzungen der Protagonisten fallen im Bezug auf die Beurteilung der Unfallhäufigkeit und der Unfallschwere sehr differenziert aus. Die Sportler sind dementsprechend in der Lage die Gefährlichkeit von Aktivitäten im Kontext des Risikosport zu beurteilen.

(2) Eine Diskrepanz zwischen dem Handlungswissen der Probanden und der objektiven Gefährlichkeit der Aktivitäten lässt sich angesichts des konservativen Klassifikationskriteriums zumindest für 6 Aktivitäten darstellen: Während das Bergwandern hinsichtlich der Schwere der Unfallfolgen unterschätzt wird, wird das Tourenski-Gehen diesbezüglich drastisch überschätzt. 
Im Kanusport sind es das Kanu-Wandern auf Flüssen und Seen die hinsichtlich der Unfallhäufigkeit unterschätzt werden, während das KanuWildwasserfahren in den Schwierigkeitsbereichen 3-4 und 5-6 überschätzt wird.

(3) Aktivitäten, die in ihrer Anschaulichkeit und Erfahrbarkeit nicht direkt auf eine Gefährdung hinweisen, werden allgemein unterschätzt, während Sportarten, die aufgrund der Handlungsstruktur und/oder des sprachlich-konnotativen Wertes auf ein mögliches Gefahrenpotential hinweisen, eher überschätzt werden.

(4) Übungsleiter weisen in beiden Teiluntersuchungen eine tendenziell realistischere Einschätzung der Gefährlichkeit der Aktivitäten auf.

Insgesamt stellt sich - trotz der methodischen Schwächen - das Untersuchungsverfahren als hilfreich und sinnvoll zur Untersuchung zentraler Ansatzpunkte für eine Intervention im Risikosport dar. Dies ist insofern hervorzuheben, als dass die Untersuchungsergebnisse die spezifischen theoretischern Annahmen zum Spannungsfeld von Risiko und Sicherheit im Risikosport erhärten können.

\subsection{Zusammenfassung und Einordnung}

Dieses Kapitel fokussiert die operative Handlungsebene im Risikosport anhand einer Untersuchung zu Unfällen und Gefahrenkognition im Risikosport. Dabei wurde anhand der theoretischen Aufarbeitung der zentralen unfalltheoretischen Forschungsansätze sowie deren Erweiterung zu sicherheitspsychologischen Konzepten ein grundlegender theoretischer Rahmen für die empirische Analyse geschaffen. Als forschungsleitendes Bezugsmodell wurde das Modell der Gefahrenkognition aus der Arbeitssicherheit für risikosportliche Kontexte transformiert. Die zentrale Annahme des Modells, wonach eine Korrelation zwischen Gefahrenwahrnehmung bzw. dessen Integration in ein kognitives Gefahrenmodell und übergeordneten Zusammenhängen der Unfallentstehung existiert, wurde als zu replizierende Aufgabe für die eigene empirische Studie übernommen.

Die empirische Untersuchung erfolgte für die Handlungsfelder alpiner Bergsport und Kanusport in Anlehnung an das Verfahren von MUSAHL, wonach zunächst Unfalldaten reanalysiert und schließlich subjektive Einschätzungen bezüglich der Gefährlichkeit von zuvor definierten Aktivitäten durch eine online-basierte Befragung erhoben wurden. Probleme wurden hierbei in der Reanalyse von Unfalldaten offensichtlich, die aus einer mangelnden Reliabilität und Validität der Unfallanzeigen resultieren. Die Ergebnisse konnten zwar eine Korrelation zwischen Gefährlichkeitseinschätzung und registrierten Unfalldaten darstellen, die Hauptbefunde früherer Untersuchungen im Bereich der Arbeitssicherheit konnte aber nur teilweise repliziert werden. Die Ergebnisse erhärten dennoch - über das Modell der Gefahrenkognition hinausgehende - theoretische Annahmen über das Verhältnis von Risiko und Sicherheit im Risikosport und ermöglichen damit weiterführenden Forschungsvorhaben auf erste gesicherte Daten im Risikosport 
zurückgreifen zu können. Mit der Integration der Untersuchungsergebnisse in den theoretischen Kontext des Risikosports ist ein konkreter Bezugsrahmen geschaffen worden, der eine anwendungsbezogene Intervention im Risikosport ermöglicht. 


\section{Risikomanagement als anwendungsbezogene Intervention im Risikosport}

Ich habe lange genug gelebt, um mir gerade die Dinge sorgfältig ein zweites Mal anzusehen,

bei denen ich mir auf den ersten Blick ganz sicher bin.

(Josh Billings (1818-85), eigtl. Henry Wheeler Shaw, amerik. Humorist)

Die Anwendung wissenschaftlichen Erkenntnisse in einem sportwissenschaftlichen Wirkungsbereich impliziert die Übertragung theoretischer Befunde in ein $\mathrm{zu}$ definierendes Praxisfeld. Diese Theorie-PraxisTransformation stellt dabei kein konzeptionelles Gerüst dar, in welchem dezidierte Handlungsschritte aufgeführt werden. Vielmehr soll der praktischen Erkenntnisnutzen einer, angewandten' Wissenschaft ausgeführt werden, indem pragmatische Hinweise und Hilfestellungen aus der konsequenten Ableitung der theoretischen Erkenntnisse und der empirischen Ergebnisse für risikosportlichen Handlungsfelder vollzogen werden. Diese Intervention soll dabei primär als Anregungen zu einer veränderten Unfallprophylaxe im (Risiko-)Sport und sekundär als pädagogische Leitlinie im Umgang mit sportlichen Risiken deduziert werden. Eine wirksame Intervention in ein System ist nur dann möglich, wenn man eine zumindest grobe Vorstellung vom $\mathrm{Zu}$ - 
stand des Systems, deren inhaltlichen Zusammenhängen und der Wirkung bestimmter Eingriffe hat. Die interdisziplinäre Deduktion der Kapitel 2-7 sowie die Ergebnisse der empirischen Untersuchung stehen als Basiswissen über die inhaltlichen Bezüge des Risikosports daher in einem indirekten anwendungsrelevanten Zusammenhang.

Zunächst werden in Abschnitt 10.1 einige kurze Gedanken zum Interventionsziel ausgeführt. Mit Risikomanagement als Begriff aber auch als Konzept wird ein Paradigmenwechsel vollzogen, der in Abschnitt 10.2 verdeutlicht wird. Anschließend werden die grundlegenden Eckpfeiler eines Risikomanagement-Konzeptes als Ableitung der theoretischen Erkenntnisse dieser Arbeit vorgestellt (10.3). Da der sportliche Vermittlungsprozess eine zentrale Rolle im Rahmen eines Interventionsprogramms spielen muss, erfolgt schließlich in Abschnitt 10.4 eine Reflexion über Anwendungsbezüge des Risikomanagements in sportlichen Vermittlungsprozessen. Die in der Praxis einiger weniger Risikosportarten bereits etablierten Risikomanagement-Anwendungen werden in Kapitel 10.5 abschließend einer kritischen Begutachtung unterzogen, bevor das Kapitel mit einer kritischen Schlussbetrachtung (10.6) beendet wird.

\subsection{Gedanken zum Interventionsziel}

Ein anwendungsrelevanter Schluss wissenschaftlicher Erkenntnisse erfolgt in der Regel im Glauben an eine Wissenschaft als übergeordnete Instanz. Der zum Teil enge Schulterschluss zwischen Theorie und Praxis vollzieht sich dabei auf der Annahme, dass sich ein mit empirischen Verfahren abgesichertes Wissen auf ein Handlungsfeld übertragen lässt und dessen Praxis nachhaltig verändern kann. DÖRNER (1992) hat in diesem Zusammenhang allerdings eindrucksvoll darauf hingewiesen, dass die Veränderung komplexer Systeme - wenn sie überhaupt erreichbar ist - nur über eine multidimensionales Interventionsverfahren ermöglicht werden kann. Wenngleich in dieser Arbeit mit der interdisziplinären Herangehensweise vielfältige Bezüge und inhaltliche Zusammenhänge eines komplexen Handlungssystems aufgedeckt werden konnten, so ist diese Multidimensionalität des Systems Risikosport doch nur ansatzweise dargestellt worden. Die Ableitung der in dieser Arbeit deduzierten theoretischen Erkenntnisse, die durch die empirischen Befunde erweitert werden konnten, können damit letztlich nur Hinweise und Anregungen für eine systematische Intervention für das System Risikosport geben.

Ansatzpunkt dieser Vermittlung ist die Systemkomponente Mensch. Da sich auch Interventionsmaßnahmen auf der Organisationsebene oder der gesellschaftlichen Ebene ableiten lassen, stellt das hier auszuführende Risikomanagement nur einen Teilausschnitt möglicher Einwirkungsmöglichkeiten dar. Das in dieser Arbeit aufgegriffene Menschenbild, welches für eine sicherheitspsychologische Intervention von Bedeutung ist, orientiert sich dabei an der Vorstellung eines selbstbestimmten und an Selbstverwirkli- 
chung interessierten Wesens. Ausgangspunkt der folgenden Überlegungen zum Risikomanagement ist damit eine lernfähige Persönlichkeit, die mit dem Willen ausgestattet ist, die Welt um sich herum zu verstehen und das, was sie unmittelbar betrifft, möglichst gut zu kontrollieren. Zugegeben: ein solches Menschenbild ist idealisiert und bisweilen auch theoretisch schwierig, denn es geht von einem Interventionssubjekt aus, welches zu Skepsis und realistischer Stellungnahme gegenüber sich selbst, seiner sozialen Umwelt und den organisatorischen Bedingungen befähigt ist. Dass ein solches emanzipiertes Wesen bisweilen nur bedingt anzutreffen ist, sei hier selbstkritisch zu konstatieren (vgl. MUSAHL 1997, 427ff).

Risikomanagement als Interventionsvorhaben greift dabei allgemein gültige Wertvorstellungen von Sicherheit, Risiko und Gefahr an und entfaltet damit eine über das Ziel einer Unfallprävention hinausgehende gesellschaftliche Wirkung. Genau das macht ein solchen Vorhaben allerdings auch anfechtbar, denn eine durch Veränderung grundlegender gesellschaftlicher Perspektiven zu realisierende Intervention geht über den Ansatzpunkt rationaler Aufklärung hinaus. Angreifbar wird eine RisikomanagementIntervention auch insofern, als dass die ,sportwissenschaftliche Communitiy' bis dato eine andere Herangehensweise an das Thema Sicherheit und Risiko praktiziert. Ein sicherheitspsychologisches Programm mit dem Inhalt Risikomanagement wird auch deshalb Kritik ernten, weil es keine systematische,Rund-Um-Lösung' bietet, sondern die Verantwortung ganz bewusst auf die handelnden Akteure überträgt.

Ziel dieses Interventionsvorhabens ist damit auch die Diskussion um Risiko, Sicherheit und Gefahr in der Sportwissenschaft neu zu entfachen und durch Anregungen eine Weiterentwicklung sicherheitspsychologischer Forschung voranzutreiben.

\subsection{Risikomanagement als Paradigmenwechsel}

Die Wahl des Begriffs ,Risikomanagement' als Kennzeichnung eines sicherheitspsychologischen Interventionsprogramms wurde vor dem Hintergrund unsicherheitsrelevanter Termini in der Differenzierung zwischen Risiko, Gefahr und Sicherheit in Anlehnung an LUHMANN (1988) ausgewählt. Vergleichbare Ansätze finden sich in einer klassischen sportwissenschaftlichen Risiko-Diskussion bei NICKEL (1994) und WANG (1994), die für die Ausbildung einer allgemeinen ,Risikobewältigungsfähigkeit' plädieren. Im alpinen Bergsport sind Konzepten und Anregungen unter dem Terminus ,Risikomanagement' seit längerem diskutiert, so z.B. bei JOSI (1991), SCHWIERSCH (1991a, 1991b) oder FAULHAMMER (1994). Zwar ist der Begriff ,Risikomanagement eindeutig wirtschafttheoretisch geprägt ${ }^{266}$, angesichts der bestehenden terminologischen Sinndeutung erscheint eine Übertragung in den Kontext des Risikosports aber durchaus sinnvoll. Nach BANSE (1996) haben: 
„Risikowabrnebmung und Identifizierung, Risikoanalyse und -abschätzung sowie Risikobewertung und -entscheidung letatlich nur ein Ziel, das im Risikomanagement, im ,Handling' von Unbestimmtheit besteht." (BANSE 1996, 59).

Risikomanagement als Interventionsanregung stellt infolgedessen den Versuch dar, die verhaltenspsychologischen, handlungstheoretischen und soziologisch-historischen Erkenntnisse dieser Arbeit zu berücksichtigen und in Verbindung mit den Befunden der empirischen Untersuchungen in ein anwendungsbezogenes Gesamtkonzept für einen bewussten Umgang mit individuellen Risiken zu integrieren.

Ein solches integratives Interventionskonzept zur allgemeinen Unfallprophylaxe und zum bewussten Umgang mit Risiken im (Risiko-) Sport kann nur dann erfolgreich sein, wenn die bipolare Grundstruktur des Menschen in Form des Spannungsfeldes von Risikosuche und Sicherheitsbedürfnis nicht behindert, sondern bipolar verstärkt wird. Im Mittelpunkt eines Risikomanagements im Risikosport steht somit die Förderung und Erweiterung des genuin-natürlichen Handlungsstruktur zwischen Motivbefriedigung (Risikotenden $\approx$ ) einerseits und Sicherheitsbedürfnis (Sicherbeitstenden $\approx$ ) andererseits. Letztlich kann und darf es auch im Rahmen der Unfallprophylaxe nicht darum gehen, auf die lustvollen Erfahrungen, die Risikosportarten im Rahmen des immanenten Risikoerlebnisses bieten, im Bestreben um mehr Sicherheit $\mathrm{zu}$ verzichten, sondern nur darum, diese Erfahrungen möglichst uneingeschränkt zu verwirklichen und zu optimieren. Die Befunde der empirischen Untersuchung hat diesbezüglich aufgezeigt, dass Unsicherheit als subjektiv kalkuliertes Risiko durchaus unfallprophylaktische Wirkung entfalten kann.

Existenzielle Bedeutung in der Interventionskonzeption des sportlichen Risikomanagements besitzt somit das Bewusstsein für Sicherheit, Gefahren und Risiken sowie deren Differenzierung, weshalb für eine Abkehr des Sicherbeitsdenkens zugunsten eines Risikobewusstseins plädiert wird. Dieses ,Risiko statt Sicherheit' bedeutet indes ein Umdenken von gesellschaftlich relevanten Wertvorstellungen und ihrer Integration in wissenschaftliche Theoriebildungen. Sicherheit als kollektiver Wert findet seinen berechtigten Bezugpunkt eben nicht im individuellen Risikohandeln sondern in den vom Einzelnen unbeeinflussbaren Unsicherheiten, die BONB (1995) treffend als „Gefahren zweiter Ordnung“" klassifizierte (vgl. Abschnitt 5.2). Dort wo Risiken als System vom Individuum unbeeinflussbar eine Bedrohung darstellen, ist Sicherheit als gesellschaftliche Wertidee berechtigt. Im Risikosport aber, wo der Einzelne das Risiko ganz bewusst aufsucht, um es als individuelle Herausforderung zu bewältigen, diskreditiert eine kollektive Sicherheitsvorstellung den Wert des Risikos und damit den Sinn der Handlung.

In den voranstehenden umfangreichen theoretischen Ausführungen konnte eingehend erläutert werden, dass das Denken in Sicherheitsgarantien - welches sich auch in der klassischen sportwissenschaftlichen Literatur wiederfindet (vgl. MÜCKE 1986, 54; AKC 1987, 11; HÜBNER \& HUNDELOH 1997) - für ein Interventionskonzept im Risikosport nur bedingt geeignet ist. In Anlehnung an die Untersuchungsergebnisse der durch- 
geführten empirischen Studie kann Sicherheit als subjektives Gefühl des ,ungefährdet-seins' mitunter sogar kontradiktorisch auf das Verhalten in Risikosituationen wirken. Auch die phänomenologischen Ansätze von CUBE (1995) können in dieser Weise interpretiert werden: Konstruierte Sicherheit verhindert letztlich keine Unfälle, sondern verlockt dazu, neue Risiken einzugehen.

Begreift man den Menschen - wie vielfach dargestellt - als ein bipolar disponiertes Wesen, der ein gewisses Maß an selbstbestimmten Risiko und Unsicherheit sucht, dann disqualifiziert sich jedes Denken in Sicherheitsdimensionen im Risikosport von selbst. Die grundlegende Maxime des Risikomanagements lautet daher: Individuelles Risikobewusstsein statt kollektiver Sicherheitsgarantie und Gefährdungsvermeidung durch Risikooptimierung:

„Bekennen wir uns dazu, das Risiko zu optimieren. Wer maximales Risiko sucht, ist dumm und bald tot. Wer maximale Sicherheit sucht, ist scheintot. Denn Risiko gebört zum Leben. Leben ist Risiko" (MUNTER 1999, 179).

Nur über das Bewusstsein für Risiken und Gefahren und ihre inhaltliche Differenzierung kann letztlich die hinreichende und ,natürliche' Handlungsregulation des Menschen in gefährlichen Situationen forciert werden. Auf den 'Terminus ,Sicherheit' wird daher im Rahmen des Risikomanagements weitestgehend verzichtet, da sich die assoziierte Bedeutung dieses Begriffs im Sinne von risikolos - in diesem Zusammenhang als problematisch erweist. Eine Abwendung vom „Phantom Sicherheit“ (MUNTER 1999, 173) hin zu einem bewussten Umgang mit Risiko und Gefahr bedingt allerdings die angesprochene Mentalitätsänderung für das System Risikosport. Aufgrund der mangelnde Differenzierung zwischen Risiko, Gefahr und Sicherheit einer in der Gesellschaft stattfindenden kollektiven ,Sicherheitssozialisation' wird sich ein solcher Paradigmenwechsel allerdings nur schwierig und langsam vollziehen. Selbstbestimmte Unsicherheit als wesentlichen Bestandteil unseres gesellschaftlichen Lebens anzuerkennen, fällt heute schwieriger denn je. Im Sport, insbesondere aber im Risikosport, ist eine derartige Bewusstseinsänderung jedoch dringend erforderlich, will man den veränderten Bedürfnissen einer bestimmten Anzahl von Gesellschaftsmitglieder gerecht werden (vgl. Kapitel 5). Ein verantwortungsvoller Umgang mit Risiken im Sport ist langfristig aber nur zu erreichen, wenn es gelingt, das mögliche Potential und die grundlegende Bedeutung des Risikos für den Menschen anzuerkennen. Dies bedeutet nicht auf Sicherungs- und Sicherheitsmaßnahmen im Risikosport zu verzichten. Im Gegenteil, diese sollen und müssen gezielt im Sinne einer Risikooptimierung eingesetzt werden, jedoch sollte der Grundsatz, weniger ist oft mehr' zunehmend berücksichtigt werden. Die allgemein verbreitete Sicherheitsphilosophie der 70er und 80er, aber auch der 90er Jahre muss langfristig zugunsten eines geschärften Risikobewusstseins und eines aktiven Risikomanagements aufgegeben werden (vgl. MUNTER 1999, 12). 


\subsection{Eckpfeiler eines Risikomanagement-Konzeptes im Risikosport}

Die Untersuchungsergebnisse der im Rahmen dieser Arbeit durchgeführten Studie, die eine Transformation des Gefahrenkognitionsmodells von MUSAHL für risikosportliche Handlungskontexte nahe legen, dienen als Basis der folgenden Ausführungen. Fehlende Aufmerksamkeit im Bezug auf Risikobedingungen und Gefährdungspotentiale durch die Aufforderung, besser aufzupassen, zu forcieren, ist trivial, weil sie ,[...] immer zugleich richtig und falsch" (MUSAHL 1997, 350) ist. Die anwendungsrelevante Schlussfolgerung, die sich aus der Übertragung des Gefahrenkognitionsmodells in den Risikosport ergibt, muss demnach die Fokussierung auf Prozesse der Gefahrenwahrnehmung und deren kognitive Transformation in Risiken betreffen. Ein gefahren-bewusstes und damit gleichsam risiko-bewusstes Verhalten ist Ziel jeglicher Intervention im Risikoport. Effektiv und dauerhaft kann derartigen Unfällen nur durch eine Angleichung von subjektiven Risiko und objektiver Gefährdung entgegengewirkt werden. Risikomanagement bedeutet somit eine Annäherung des Verhaltens an gegebene Anforderungen, anstatt einer Anforderungsangleichung an gegebenes Verhalten.

Wie aber kann man es fertig bringen, Verhalten zu verstärken, bei dem das Spannungsfeld von Risikosuche und Sicherheitsbedürfnis nicht einseitig behindert wird? Am ehesten wird dies dort gelingen, wo die Verhaltensdispositionen der Motivbefriedigung (Risikotendenz) und des Sicherheitsbedürfnisses (Sicherheitstendens) gleichermaßen intensiviert werden. Dies kann nur dann gelingen, wenn situative Unsicherheiten transparent gemacht und die unter dem Begriff Gefahrenkognition zusammengefassten WahrnehmungsBewertungs- und Entscheidungsprozesse allgemein gefördert und verstärkt werden.

In Anlehnung an MUSAHL ist

$$
\begin{aligned}
& \text { "[...] die Erböbung der Erkennbarkeit von Gefahren das wichtigste Instru- } \\
& \text { ment antizipatorischer Gefahrenkontrolle. [‥] Gefahrensignale und - } \\
& \text { indikatoren müssen richtig und rechtzeitig erfasst und verarbeitet werden, da- } \\
& \text { mit die Situation beurteilt, angemessene Entscheidungen getroffen und entspre- } \\
& \text { chend gehandelt werden kann" (MUSAHL 1997, 102f). }
\end{aligned}
$$

Dabei ist ergänzend hinzuzufügen, dass nicht nur eine Erfassung der Gefahrensignale und -Indikatoren für angemessenes Handeln im Risikosport notwendig ist, sondern vor allem deren Transformation in individuelle Risiken. Risikomanagement meint letztlich nichts anderes als die kognitive Aufbereitung situativ relevanter Unsicherheiten und ist damit eng an die Berücksichtigung und Integration kognitionspsychologische Evidenzen, also kognitiver Heuristiken geknüpft (vgl. Abschnitt 6.2). Nur unter Berücksichtigung dieser ,kognitiver Ordnungsprinzipien' kann das Interventionsziel nachhaltig realisiert werden, wenngleich weiterführende Studien für den Bereich Risikosport ausstehen. 
Auf dieser Grundlage bilden eine handlungsorientierte Informationsvermittlung, offene Kommunikation und verbaltensorientierte Reflexion die Eckpfeiler des Risikomanagements im Risikosport (s. Abb. 10.1). Im folgenden werden diese Grundelemente erläutert.

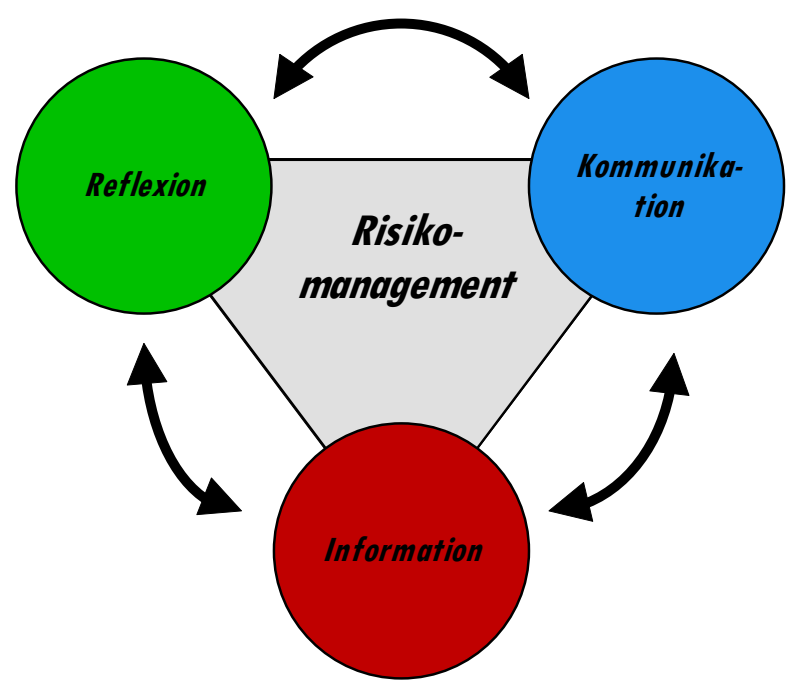

Abbildung 10.1: Die Eckpfeiler des sportlichen Risikomanagements

10.3.1 Information: Erkenntnis objektiver Gefahren und alternativer Handlungsstrategien

Es konnte vielfach dargestellt werden, dass man Unsicherheiten, die man vermeiden oder als Risiken bewältigen will, zunächst einmal erkennen muss (vgl. 6.1). Umfassende Informationen über die objektiven Gefahren der Situation bilden somit die Grundlage einer unfallprophylaktischen Intervention im Risikoport. Allerdings weisen die Erkenntnisse der Sicherheitssychologie darauf hin, dass das Wissen um objektive Gefahren allein nicht ausreicht, um diese Gefährdungen zu erfassen und adäquat als Risiken zu bewerten. CUBE (1995) unterscheidet daher zwischen einfachen Kenntnissen und handlungsorientierten Erkenntnis, indem er betont:

„Der Unterschied zwischen Kenntnis und Erkenntnis liegt darin, dass es sich bei Kenntnissen um Fakten handelt, um einzelne Daten oder Ereignisse. Eine Erkenntnis berieht sich immer auf einen Zusammenhang"(CUBE 1995, 68).

Entscheidend für angepasstes Verhalten in Risikosituationen ist folglich die handlungsorientierte Erkenntnis der objektiven Gefabren und deren kognitive Repräsentation. Die Bemühungen um eine möglichst umfassende Aufklärung über die objektiven und subjektiven Gefährdungen im Risikosport, wie sie sich z.B. ausführlich bei REITHMEIER (1993) für den Kanusport wieder finden, sind damit grundlegende Voraussetzung eines integrativen Interventionskonzeptes. Die Anwendung und Abgleichung von verschiedenen Informationen muss im Rahmen des Risikomanagements aber gleichzeitig permanent und handlungsorientiert erfolgen, so dass sich die Einschätzung der 
Situation auf mehrere verschiedene Parameter stützt. Hier spielt neben rationaler Aufklärung also auch ,erfahrbare Theorie' eine Rolle. Dies bedeutet nicht, Gefährdungssituationen zu provozieren, sondern verantwortungsbewusste Lernerfahrungen zu ermöglichen. Wenn sich - wie MUSAHL postuliert - die kognitive Repräsentation von Gefährdungssituationen über Prozesse so genannter negativer Verstärkungen vollzieht, dann ist ein vorsätzliches Scheitern in Risikosituationen und dessen bewusste Reflexion für die kognitive Festigung gefährdungsrelevanter Informationen unabdingbar. Diese setzt eine gewisse Fehlerfreundlichkeit voraus, denn nur wer Fehler als lerntheoretische Hilfe akzeptiert, kann nachhaltig Informationen verarbeiten (vgl. Abschnitt 6.2).

Neben dem Bewusstsein objektiver Gefahren und subjektiver Risiken, ist auch die Erkenntnis alternativer Handlungsstrategien eine grundlegende Bedingung im Sinne einer erfolgreichen Unfallprophylaxe. Einerseits werden die lustvollen Erfahrungen und Erlebnisse nur deshalb als angenehm empfunden, weil man die Risikobewältigung freiwillig sucht. Andererseits bedeutet ein wie auch immer zustande kommender Handlungszwang einen existenziellen Eingriff in das natürliche Spannungsfeld von Risikosuche und Sicherheitsbedürfnis, woraus sich gravierende Unfälle entwickeln können. Die Erkenntnis alternativer Handlungsstrategien bezieht sich dabei auf alle Phasen der Gesamtsituation (vgl. Abschnitt 6.1.2). Dazu gehören übergeordnete alternative Betätigungsmöglichkeiten, die eine Entscheidung, nicht auch bei widrigen Bedingungen risikosportlich zu handeln, erleichtern. Insbesondere in der Aktionsphase ist ein Wissen über alternative Handlungsstrategien gleichwohl unerlässlich, denn nur unter der Berücksichtigung erreichbarer Handlungsalternativen ist eine Entscheidung in Risikosituationen überhaupt erdenklich und die Wiederherstellung einer Systemhomöostase möglich (vgl. Abschnitt 8.3.3).

Dennoch gilt, dass die handlungsorientierte Erkenntnis objektiver Gefahren und das Wissen um alternative Handlungsstrategien allein kein angepasstes Verhalten in Risikosituationen ermöglichen. Umfassende Informationen sind folglich notwendige, aber keine hinreichende Bedingung für ein Interventionsprogramm im Risikosport.

\subsubsection{Reflexion auf eigenes Verhalten}

Der zweite Eckpfeiler des Risikomanagements besteht in der Reflexion des eigenen Risikoverhaltens, dessen Ursachen und dessen Folgen. Für das Ziel einer allgemeinen Unfallprophylaxe im Risikosport ist es zweckdienlich, das eigene Verhalten in Risikosituationen zunächst hinsichtlich der persönlichen Anreize und Motive aber auch bezüglich emotionaler Aspekte zu reflektieren. Selbstreflexion bedeutet hierbei die Bestandsaufnahme eigener Stärken und Schwächen sowie die Formulierung persönlicher Zielsetzungen. Reflexion eigenen Verhaltens bezieht sich aber auch auf die Überprüfung subjektiver Sicherheitsempfindungen, individueller Angstvermeidungsstrategien, die eigene Überheblichkeit oder empfundene Langeweile. Nur wenn sich der 
einzelne Sportler durch diese ,Kontrollinstanz’ über seine individuellen Ziele und Bedürfnisse bewusst wird, kann eine adäquate und willentliche Handlungsrealisation - im Sinne einer individuellen Risikosituation - verwirklicht werden. Eine gezielte und gelenkte Reflexion kann zudem den Einfluss heuristischer Prinzipien reduzieren und die kognitive Repräsentation von Gefährdungspotentialen fördern.

Eine Verhaltensreflexion schließt weitreichende Überlegung über Gruppenstrukturen und soziales Verhalten in der Gruppe mit ein. Konkurrenzdenken und „Balzverhalten“ (CUBE 1995, 68) lassen sich effektiv nur über die individuelle Definition und Abgrenzung der persönlichen Motivstruktur verhindern. Die gemeinsame Fixierung einer Gruppe auf ein Ziel ist somit allgemein in Frage zu stellen. In Anlehnung an DÖRNER (1992) müssen durch eine Reflexion zudem die Folgen des eigenen Verhaltens für die Gruppe antizipiert werden. Z.B. sollten bessere Sportler ihr Verhalten in Risikosituationen dahingehend überprüfen, ob sich daraus Konkurrenzgedanken und Aufforderungen an andere Gruppenmitglieder ableiten lassen.

\subsubsection{Offene Kommunikation}

„Wer wagt, will sich in der Regel mitteilen“ (NEUMANN 1999, 149). Die Bereitschaft zur Selbstreflexion muss im Rahmen eines aktiven Risikomanagements immer von kommunikativen Prozessen begleitet werden. Offenheit gegenüber den Partnern kann wesentliche unfallfördernde Strukturen wie Heuristiken entgegenwirken. Zudem erscheint es zweckmäßig, die in der Gruppe teilweise unbewusst verteilten sozialen Rollen - z.B. die intuitive Zuweisung bestimmter funktionaler Positionen an gewisse Personen - gemeinschaftlich und kommunikativ zu analysieren und das eigene Verhalten darauf abzustimmen. Kommunikation setzt allerdings die Evaluation persönlicher Verhaltensweisen voraus und ist damit eine ergänzende Maßnahmen reflektiver Prozesse.

Tatsächlich bedeuten offene Kommunikation, umfassende Informationen und die Reflexion des eigenen Handelns keine gravierende Veränderung im Risikoverhalten des Risikosportlers - es handelt sich vielmehr um eine Intensivierung und bewusste, Anwendung' bestehender Verhaltensstrukturen unter geänderten Beobachtungsperspektiven. Übergeordnetes Ziel ist es, das situative Erlebnis zu verstärken und damit einhergehend sicherheitsfördernde Tendenzen durch eine gezielte Risikooptimierung zu festigen.

\subsection{Risikomanagement als Vermittlungsprinzip: Päda- gogische Schlussfolgerung}

Risikomanagement als Vermittlungsprinzip im Risikosport bedeutet zunächst eine generelle Abwendung des häufig benutzten Terminus der ,Sicherheitserziehung', denn 
„zur Sicherheit kann ebenso wenig erzogen werden, wie zur Gesundheit oder zum Frieden. [...] Hinter dem Begriff Eriziehung verbirgt sich immer noch die Theorie, dass durch Belehrungen über vorgegebene Werte und Ziele beim Individuum gewünschte Verbaltensänderungen bewirkt werden" (BAUMANN 1995, 8).

Das Risikomanagement im Unterricht des Risikosport verfolgt demnach die wesentliche Leitidee der Reformpädagogik, die versucht das Individuum zu fördern und in seiner psychischen Strukturierung zu verstärken (vgl. ebd.). Die folgende Ausführungen erfolgen am Beispiel des Vermittlungsprozesses im Kanu-Wildwassersport, wobei die grundlegenden Annahmen und Prinzipien auf weitere Handlungsfelder zu übertragen sind.

Angesichts der zentralen Bedeutung, die die kognitiven und emotionalen Prozesse für die erfolgreiche Bewältigung von Risikosituationen besitzen, sollte insbesondere den Wahrnehmungs-, Bewertungs- und Entscheidungsprozessen ein selbstverständlicher Platz in der Vermittlung von Risikosportarten eingeräumt werden. Die Ausbildung von motorischen und kognitiven Fähigkeiten sowie technischen und taktischen Fertigkeiten bleibt zwar die grundlegende Voraussetzung der Bewältigung einer risikosportlichen Handlungssituation, langfristig müssen kognitive, affektive und motorische Lernziele vor dem Hintergrund der Unfallprophylaxe im Lehrbetrieb aber parallel verwirklicht werden.

Dazu sollte möglichst früh mit dem Einstieg ins Handlungselement begonnen werden. Im Sinne einer gezielten Unfallvermeidung beinhaltet die Ausbildung von technischen Fertigkeiten allein kein wirksames sicherheitsförderndes Mittel, solange die individuellen Fähigkeiten nicht in einen direkten handlungsorientierten Bezug gesetzt werden können. Die Ausbildung von technischen Fertigkeiten in , risikoreduzierten Handlungssituationen' (z.B. auf stehenden Gewässern als Vorbereitung für das Kanu-Wildwasserfahren) erscheint demnach nur bedingt sinnvoll, da die kognitiven und emotionalen Bewertungsprozesse hierbei keinerlei Berücksichtigung finden. Dem Lernenden sollten daher möglichst früh umfangreiche Möglichkeiten für alternative und kompensatorische Handlungsmöglichkeiten aufgezeigt und entsprechend trainiert werden. Da das Unfallgeschehen z.B. beim Kanufahren nach dem Verlassen des Bootes - also nach einem Störfall - maßgeblich von den Möglichkeiten der Systemanpassung abhängig ist, müssen hier vor allem mögliche Handlungsalternativen z.B. im Wildwasser ausführlich eingeübt werden. Das kontrollierte Verlassen des Bootes oder das gezielte Schwimmen nach einer Kenterung mit Boot und Paddel gehören hier gleichermaßen dazu, wie das frühe Lesen und Erkennen von Strömungsformen. Natürlich ist ein derartiger Einstieg in das Element Wildwasser an eine geeignete Flussstelle, hinreichende Materialen und entsprechende Witterungsbedingungen gekoppelt.

Da das situative Risiko eine individuelle Dimension beinhaltet, sollte die normierte Bewegung in der Ausbildung zudem eine untergeordnete Rolle im Kontext des Risikomanagements spielen. Im Mittelpunkt steht die Auseinandersetzung mit einer individuellen Aufgabe und nicht das fixierte Endziel. Risikomanagement am Beispiel des Wildwasser-Unterrichts impliziert 
damit, dem Kanuschüler eine Vielzahl von verschiedenen Bewältigungsmöglichkeiten aufzuzeigen und diese im Lernprozess zu verfestigen. Dem KanuSchüler sollten folglich möglichst umfangreiche Handlungsstrategien angeboten werden, die eine Handlungsregulierung im Sinne einer individuellen Herausforderung ermöglichen. Hierzu gehören Risiko-MinimierungsStrategien, wie die Möglichkeit des Umtragens oder die Befahrung alternativer und leichterer Fahrrouten. Gleichzeitig müssen dem Kanu-Schüler jedoch auch Risiko-Optimierungs-Strategien, wie das Spielen mit verschiedenen Strömungsformen oder die Wahl schwierigerer Taktiken bzw. komplizierterer Routen, eröffnet werden. Sinnvoll erscheint in diesem Zusammenhang zudem die Kombination mehrerer (risiko-)sportlicher Aktivitäten, um dem Individuum möglichst verschiedene Lernfelder $\mathrm{zu}$ präsentieren und ihm somit die Möglichkeit zu geben, sein individuelles Risiko- und Erlebnismoment frei auszuwählen.

In diesem Zusammenhang ist im Hinblick auf die Unfallprophylaxe auch das zentrale Anliegen RUCKENBAUERs (1992, 94f) zu unterstützen, dem Lernenden ausnehmend viele Selbsterfahrungsgelegenheiten im Lernprozess zu ermöglichen. Zwar spielt im Risikoport auch das Lernen am Modell also die theoretische Aufarbeitung fachspezifischer Zusammenhänge - eine nicht unbedeutende Rolle, allerdings erscheint die Kopplung des Modelllernens mit eigenen, möglichst unmittelbaren Erfahrung wesentlich wirksamer. Insbesondere Sicherheitsmaßnahmen müssen vorwiegend das Ziel verfolgen, die Selbstverantwortung und das Selbstbewusstsein der Lernenden zu stärken, damit Probleme der Sicherheit nicht auf das Material oder an dritte abgegeben werden. Der von BAUER \& SCHULTE (1997, 106f) explizierte didaktische Rahmen für den Einstieg ins Kanu-Wildwasserfahren ist insofern auch unter unfallprophylaktischen Gesichtspunkten für andere Risikosportarten zu unterstützen ${ }^{267}$. Dies gilt insbesondere für die Sicherungs-, Rettungs- und Bergeübungen, also den Möglichkeiten, aktiv von außen in ein erdenkliches Unfallereignis einzugreifen. Erfahrungen spielen aber auch in der Fähigkeit zur Selbsteinschätzung eine entscheidende Rolle. SCHWIERSCH $(1991 b, 49)$ formuliert hierzu: „Erfahrung ist nichts als die Summe der vergangenen Irrtümer". Die Risikosituationen sollten vom Übungsleiter folgerichtig so ausgewählt werden, dass die Situation einerseits realistische Bewältigungsmöglichkeiten besitzt, andererseits aber ein Scheitern keine gravierenden Konsequenzen im Sinne von Verletzungen nach sich zieht. Der Misserfolg einer Handlung sollte dabei nicht nur in Kauf genommen, sondern je nach Persönlichkeitsdispositionen auch bewusst provoziert werden:

„Der Misserfolg wird natürlicher Teil des Lemprozesses, bei dem weniger ein fertiges Produkt im Auge liegt, sondern vielmehr das Erproben und Üben. Dies entspricht grundlegenden pädagogisch-didaktischen Kategorien und ermöglicht das Herantasten an die eigenen Grenzen" (ERDMANN 1990, 110). 
Hierzu gehört desgleichen die schon angedeutete „Entwicklung einer Fehlerkultur" (MUNTER 1999, 116), die durch einen bewusster Umgang mit Fehlern charakterisiert ist. Eine kontinuierliche Selbstreflexion sollte zudem forciert werden, die neben technischen und taktischen Fehlern auch persönliche und soziale Fehler, aber auch persönliche Stärken und Schwächen berücksichtigt. Gespräche dienen hierbei nicht nur als Mitteilung des Erlebten. Anregungen, Bedenken, Wünsche und Ängste der Lernenden lassen sich aufgreifen und in den Lernprozess integrieren. Im Erzählen, in der Verinnerlichung und der Reproduktion liegen neben unfallprophylaktischen Aspekten auch wesentliche pädagogische Anknüpfungspunkte (vgl. NEUMANN 1999, 149).

Entscheidend für das Interventionskonzept des Risikomanagements ist jedoch die sicherheitsvermittelnde Position und Funktion des Übungsleiters. Da die Handlung in Risikosituationen maßgeblich über das Gefühl der subjektiven Sicherheit reguliert wird, ist diese sicherheitsvermittelnde Position des Übungsleiters im Lernprozess bewusst einzusetzen. Um dem Anfänger einen relativ angst- und stressfreien Einstieg z.B. ins Wildwasser zu ermöglichen, ist die Vermittlung von Sicherheit zu Beginn des Lemprozesses durchaus anzuregen (vgl. BAUER \& SCHULTE 1997, 46). Im weiteren Verlauf sollte der Übungsleiter mit dieser Position aber organisiert umgehen und die sicherheitsvermittelnde Funktion gezielt einsetzen. Die Fähigkeit, beim KanuWildwasserfahren einen Fluss zu lesen oder die Notwendigkeit sich umfassende Informationen zu beschaffen und seine individuellen Bewältigungsmöglichkeiten in Abhängigkeit der situativen Anforderungen zu bewerten, wird insbesondere dann erfahren, wenn der ,natürliche' Regulationsmechanismus ausdrücklich gefordert wird. Die Funktion von sicherheitsdienlichen Aktivitäten, wie das Besichtigen vom Ufer aus oder auch das Anfahren von Kehrwässern, kann dem Kanuschüler als Beispiel für den lernenden Risikosportler daher nur eingeschränkt deduktiv vermittelt werden. Er sollte die Notwendigkeit derartigen Verhaltens real erfahren können und die Funktion sicherheitsdienlicher Aktivitäten bewusst erfassen.

Gleichzeitig kann über das Gefühl der subjektiven Sicherheit aber auch die Motivbefriedigung intensiviert werden, denn das Empfinden der Selbstbestätigung und des Triumphes ist umso größer, je eigenständiger das Handlungsziel erreicht wird. Für die Kanu-Wildwasserschulung bedeutet dies: Kleinere Gruppen sollten schon möglichst früh vorsätzlich ausgewählte Flussabschnitte allein - also ohne Übungsleiter befahren. Zu empfehlen ist auch von altgedienten Gruppenstrukturen (Übungsleiter fährt vor - Gruppe folgt hinterher) Abstand zu nehmen und die Gruppenpositionen und Ubungsleiter-Funktionen zu variieren. Natürlich kann und sollte sich der Übungsleiter in derartigen Situationen im Hintergrund aufhalten, um im Notfall einzugreifen. Vor allem weniger schwierige Abschnitte können aber infolgedessen zu einer echten Herausforderung werden, die zugleich in einem hohen Maße verhaltensverstärkend wirken.

Angst sollte im Lehrbetrieb des Risikosports gleichfalls akzeptiert und in ihrer Funktion als Warnsignal erkannt werden. Angstvermeidungsstrategien 
seitens der Lernenden müssen in diesem Zusammenhang thematisiert und diskutiert werden. Es ist in diesem Kontext anzuraten, in spezifischen Reflexionen die individuelle Bedeutung der Angst bei allen Gruppenmitgliedern hervorzuheben.

Der Übungsleiter sollte sich selbst - im Sinne des Risikomanagements als eine Art Vermittler von Risikosituationen verstehen, der entsprechend der konkreten Bedingungen die Risikosituation bewusst arrangiert und die Lernenden je nach Persönlichkeitsdispositionen in die Situation ,integriert'. Seine sicherheitsvermittelnde Funktion kann er dabei spielerisch und situativ variieren. Risikomanagement im Kanu-Wildwasser-Unterricht bedeutet damit sowohl für den Kanuschüler als auch für den Übungsleiter eine Abwendung bekannter Unterrichtsmuster. Vor allem für den Übungsleiter stellt dies eine Umstellung in seinem Verhalten dar. Die vom Kanuschüler auf ihn projizierte Sicherheitsfunktion zurückzuweisen und sich in bestimmten Situationen passiv zu verhalten, ohne die Kanu-Schüler allein zu lassen, fällt dem Lehrenden erfahrungsgemäß schwer. Einerseits genießt der Übungsleiter diese Position, die für ihn Anerkennung und Bewunderung bedeutet, andererseits zwingt ihn die generelle Verantwortung zu allgemeiner Vorsicht. Die bewusste ,Sicherheitsregulierung' durch den Übungsleiter stellt jedoch den entscheidenden Schlüssel zu einer risikobewussten Erziehung im Risikosport allgemein dar.

Risikomanagement im Risikosport sollte überdies auch eine möglichst umfangreiche Transparenz für die Lernenden bieten. Methodische und didaktische Schritte sollten vor dem Hintergrund des Risikobewusstseins offen vorgestellt und im Hinblick auf ihre Zielerreichung reflektiert werden. Angehende Risikosportler, die aufgrund ihrer ,Sicherheitssozialisation' die Vermittlung von Sicherheit gewohnt sind, werden ein Konzept des Risikomanagements letztlich eher akzeptieren, wenn sie die theoretischen Hintergründe begreifen.

\subsection{Beispiele für die Anwendung des Risikomanage- ments in der Praxis}

Will man das vorgestellte Konzept des sportlichen Risikomanagements in der Praxis etablieren, dann ist dafür mehr erforderlich als die umfangreiche Darstellung von theoretischen Zusammenhängen und ableitenden Überlegungen. Schließlich ist davon auszugehen, dass sich nur einige wenige Personen mit den dargestellten Gedankenfolgen auseinandersetzen werden, um diese schließlich in der Praxis anzuwenden. Für die zweckdienliche Verwendung bedarf es somit einer konzeptionellen Hilfestellung, die die beschriebenen Ideen des Risikomanagements wiedergibt und die entsprechenden Verhaltensdispositionen verstärkt. Ein derartige Entwurf sollte dabei vor allem zur Selbstständigkeit anleiten bzw. kommunikative und reflektorische Prozesse fördern. Gleichzeitig erscheint es sinnvoll, ein solches Vorhaben möglichst einfach zu gestalten, so dass es für möglichst alle Personen leicht 
verständlich bleibt. Praktische Anwendungen dieser Art sind insbesondere für das Touren-Skifahren entwickelt worden. Vereinzelt finden sich rudimentäre Ansätze auch im Bereich des alpinen Kletterns oder Kanufahrens.

Allgemeingültige und allumfassende Konzeptionen sind in diesem $\mathrm{Zu}$ sammenhang nicht $\mathrm{zu}$ erwarten. Die folgenden anwendungsbezogenen Entwürfe verstehen sich daher als eine Art Werkzeng, die im Hinblick auf eine bestimmte Zielsetzung ihren Zweck im Sinne eines Risikomanagements erfüllen sollen. Diese bis dato entwickelten Instrumente zum Risikomanagement werden im Folgenden in ihrer grundlegenden methodischen Konzeption vorgestellt und auf der Basis des hier angeführten Interventionsansatzes hinsichtlich ihrer Zweckdienlichkeit bewertet.

\subsubsection{3x3 Zoom-System und Risikoreduktionsmethode (MUNTER 1999)}

Das von MUNTER 1997 erstmals der Öffentlichkeit vorgestellte Modell der Risikoreduktionsmethode und des 3x3 Zoomsystems war das erste umfassende Risikomanagement-Konzept für das alpine Skifahren auf Varianten und das Ski-Tourengehen im alpinen Gelände. Es löste in der Folge eine breite Diskussion über Prinzipien und Paradigmen der Lawinenkunde aus und initiierte zahlreiche Folgeprojekte.

Bei den von MUNTER veröffentlichten Methoden handelt es letztlich um zwei prinzipiell unterschiedliche Ansätze, die zwar aufeinander bezogen sind und gleichsam zusammen publiziert wurden, aber in ihrer inhaltlichen Konzeption einschneidende Differenzen aufweisen.

Die dabei am stärksten diskutierte Methode stellt zweifelsfrei die so genannte Risikoreduktionsmethode dar. Diese elementare Methode beruht darauf, dass Lawinenrisiko mit Hilfe weniger handhabbarer Parameter zu berechnen und vor allem in unterschiedlichen Handlungssituationen anzuwenden. MUNTERs (1997) Ausgangspunkt für diese Methode war eine umfassende Berechung und Analyse von Lawinenabgängen im Bezug auf die Hangneigung, Hangexposition und deren Kombination mit Lawinenlageberichten. Mit Hilfe einfacher Vektorenrechnung lässt sich demnach das Lawinenrisiko individuell abgestimmt berechnen. Durch entsprechende Maßnahmen lässt sich das individuelle Risiko letztlich minimieren und damit eine allgemeine Handlungsfähigkeit aufrechterhalten. Die grundlegende Zielsetzung MUNTERs war es, mit Hilfe einfacher Überlegungen und kognitiven Kombinationen („Nachdenken statt Schaufeln“) eine neuartige Beurteilung der Lawinengefahr vorzunehmen.

Die andere von MUNTER entwickelte Methode, das 3x3 Zoomsystem ist bisweilen in der anwendungsbezogenen Diskussion vernachlässigt worden. Das Konzept stellt jedoch einen systemischen Ansatz dar, der durch eine Vernetzung von subjektiven und objektiven Systemkomponente gekennzeichnet ist. Die Methode repräsentiert damit den Versuch, die wesentlichen Kriterien zur Beurteilung der Situation in allen Phasen des Bewer- 
tungsprozesses heranzuziehen und in eine direkte Wechselbeziehung zu stellen.

MUNTER verbindet diese Aufgabe mit einer graphische Darstellung und einem stichpunktartigen Fragenkatalog, wodurch eine spezifische und intensive Auseinandersetzung mit den situativen Komponenten akzentuiert und eine Hilfestellung für flexibles, vernetztes und selbständiges Denken gegeben werden soll. Die Bedeutung eines Filtersystems wird durch die graphische Darstellung hervorgehoben. Vorausgehende Beurteilungen werden dadurch mit den aktuellen Situationseinschätzungen verglichen und Diskrepanzen zwischen situativen Konditionen und vorgefassten Bewertungen auf diese Weise entgegengewirkt. Bestimmte Faktoren können zudem „zoomartig heraus vergrößert" (ebd., 117) und die Beurteilungen auf verschiedene Ebenen verteilt werden. Durch die ständige Überprüfung vorausgehender Antizipationen wird ein regelrechtes ,Beurteilungs-Netz' aufgestellt, dass die Kontrolle der situativen Faktoren intensiviert.

MUNTER's methodische Instrumente sind, obwohl dies wenig praktiziert wird, getrennt voneinander zu bewerten. Während die Risikoreduktionmethode eher ein Instrument eines Gefahrenmanagements darstellt, ist die 3x3 Filtermethode ein pragmatisch anwendbares Risikomanagementkonzept für das Tourenski-Gehen. Dabei ist zunächst darauf hinzuweisen, dass es sich bei den von MUNTER fokussierten Risiken eher um die Kernhandlung beeinflussende Gefahren handelt: Lawinen stellen weder beim SkiTourengehen oder Variantenskifahren ein explizites Handlungselement dar, durch dessen Bewältigung eine Motivbefriedigung erfolgen wird. Sie werden vielmehr in Kauf genommen, um andere Motive in der Handlungssituation zu befriedigen. Lawinen sind deshalb eher als Gefahren zu bezeichnen, weil sie - von außerhalb kommend - vom handelnden Menschen höchstens durch einen präventiven Verzicht auf bestimmte Handlungsräume in ihren gefährlichen Wirkungen auf den Menschen verhindert werden können. Dies ist insofern von Bedeutung, als dass im Bezug auf Lawinen keine Risikooptimierung stattfindet, sondern eine klar strukturierte Gefahrenminimierung. Insofern stellt die Reduktionsmethode zwar ein anscheinend sehr probates Mittel zur Minimierung der Lawinengefahr dar ${ }^{268}$, eine Methode im Rahmen der verfolgten Risikomanagement-Konzeption ist sie allerdings nicht. Vor dem Hintergrund einer grundsätzlichen Abkehr von Sicherheitsdogmen kann sich die Anwendung sogar kontradiktorisch auswirken. Denn obwohl der Autor nachdrücklich darauf hinweist, dass es hier um ein Risikoinstrument handelt, kann die systematische Berechnung des individuellen Risikos auch als Sicherheitslegitimation eingesetzt werden ${ }^{269}$. Allgemeine Kritik richtet sich auch an die von MUNTER durchgeführte Statistik. Zwar konstatiert der Autor folgerichtig, dass die Unfallzahlen auf Hängen mit Nordexposition wesentlich höher sind als auf anderen Hangausrichtungen, eine expositionsbereinigte Berechnung auf Grundlage der tatsächlichen Begehungszahlen findet aber nicht statt (vgl. auch HÖLLER 2004).

Gänzlich anders ist hingegen die 3x3-Filter Methode zu bewerten. Durch die systemische Konzeption wird ein ganzheitlicher Zugang gewählt, der die 
Handelnden zu den hier geforderten Prozessen der Informationsaufnahme, der Reflexion und der Kommunikation zwingt. Die Methode wirkt dabei keinesfalls restriktiv, sondern lässt dem Individuum und der Gruppe alle Spielräume für eine eigene Entscheidung. Die Methode gibt letztlich die erforderlichen Hilfestellungen, um eine Entscheidung unter Risikobedingungen in Anbetracht der notwendigen Informationen durchzuführen. Durch die Unterteilung in die objektiven Systemfaktoren wird die Bedeutung der Bewertungsprozesse und des Informationsbedarfes pointiert, während die subjektiven Faktoren eine Verhaltensreflexionen und kommunikative Prozesse forcieren. Die durch das Filtersystem provozierte Fokussierung auf den Handlungsprozess ermöglicht zudem eine dynamische Auseinandersetzung, die mögliche Fehler durch ad-hoc Entscheidungen verhindert. Es bleibt allerdings fraglich, inwieweit diese Methode in der Praxis zum Einsatz kommt, da sie ein hohes $\mathrm{Maß}$ an Reflexions- und Kommunikationsfähigkeit von den Protagonisten fordert.

\subsubsection{Stop or go (LARCHER 1999)}

Das von LARCHER (1999) konzipierte Konzept ,Stop or go' ist ebenfalls eine kombinierte Methode zum ,Risikomanagement im alpinen Schneesport', wobei auch hier das Hauptaugenmerk auf der situativen Lawinengefahr liegt. Auch dieses Konzept ist durch zwei unterschiedliche Stufe gekennzeichnet, die enge Verwandtschaft zu MUNTERs methodischen Ansatz aufweist. Neben einer der Risikoreduktionsmethode ähnlichen Vorgehensweise wird im ersten Teil des methodischen Konzeptes durch Hinweise auf Hangexpositionen und Hangneigungen eine Entscheidungshilfe zur aktuellen Lawinengefahr in Abhängigkeit der offiziellen Lawinenwarnstufe gegeben. Im zweiten Teil werden anschließend fünf Wahrnehmungsaufgaben gestellt, die an bestimmten Gefahrenzeichnen für eine akute Lawinengefahr kennzeichnend sind. Die Methode überlässt die Handlungsentscheidung schließlich dem Handelnden, der - im Sinne eines ,stop or go' - eine Entscheidung herbeiführen muss.

Wie erwähnt ähnelt die Methode von LARCHER inhaltlich und konzeptionell sehr der von MUNTER. Auch hierbei handelt es sich streng genommen um ein Gefahrenmanagement, wobei - durch die Integration des zweiten Teils - eine Erweiterung zu einem Risikomanagement-Instrument durchaus gelingt. Die hierbei durchzuführende Fokussierung auf Gefahrenzeichnen erfasst aber nicht die Reflexion von persönlichen oder sozialen Einflüssen auf die Entscheidung, wie dies beispielhaft bei MUNTER's 3x3 Zoomsystem geschieht. Insgesamt erscheint das ,Stop or go'-System für die Beurteilung der Lawinengefahr zwar durchaus praktikabel, durch das Fehlen wesentlicher Reflexions- und Kommunikationsimpulse kann es aber nur bedingt als hilfreiches Risikomanagement-Instrument bezeichnet werden. 


\subsubsection{SnowCard (ENGLER \& MERSCH 2000)}

Die SnowCard von ENGLER \& MERSCH (2000) ist ähnlich wie die Methode von LARCHER (1999) aufgebaut. An der Stelle der einfachen Berechnung wird bei ENGLER \& MERSCH ein buntes Kärtchen eingesetzt, an welchem sich ablesen lässt, welche Hangneigung in Abhängigkeit von der Gefahrenstufe im grünen, gelben oder gar roten Bereich liegt, wobei die Grenzziehung zwischen den Bereichen bewusst verschwommen dargestellt ist. Durch Kippen der SnowCard erhält der Akteur die entsprechenden Werte sowohl für günstige als auch für ungünstige Expositionen ${ }^{270}$. Im zweiten Teil der Methode ist ein Faktorencheck angelegt, der - ähnlich wie bei LARCHER - die Wahrnehmung von bestimmten Gefahrenindikatoren abfragt.

Wenngleich die graphische Darstellung und Umsetzung der SnowCard eine wesentliche Erweiterung der ,stop or go'-Methode darstellt, ist der mangelnde Reflexionsimpuls, der von der SnowCard ausgeht, ebenfalls zu kritisieren. Zwar dient die Methode recht anschaulich dazu die Lawinengefährdung am einzelnen Hang abzuschätzen, eine Anregung seine eigenen Ziele und Wünsche und evt. die Bedürfnisse der anderen Akteure zu überdenken, wird aber nicht gegeben.

\subsubsection{Nivo Test (BOLOGNESI 2000)}

Der wenig praktizierte Nivo-Test von BOLOGNESI (2000) stellt eine in der Praxis weniger gebräuchliche Methode für das Risikomanagement im alpinen Schneesport dar. Auch hierbei wird das Ziel einer genauen Einschätzung der Lawinengefahr mit Hilfe von 25 Fragen verfolgt, die neben meteorologischen Einflussfaktoren auf Gefahrenmerkmale der Schneebeschaffenheit konzentrieren. Dabei wird die Berechnung der Antwortpunkte über eine Karte ermöglicht, so dass keinerlei weitere Hilfsmittel benötigt werden. Ist der somit summierte Wert kleiner als 8, so werden allgemein günstige Bedingungen postuliert, bei Werten größer als 23 wird die aktuelle Situation als ungünstig beschrieben und von einer Tour abgeraten.

Als Hilfestellung zur Erkennung möglicher Gefahrenindikatoren ist der Nivo-Test im Sinne eines lawinenspezifischen Gefahrenmanagements durchaus als Mittel der aufmerksamen Informationsvermittlung sinnvoll. Allerdings fehlen die für ein Risikomanagement wesentlichen Elemente der Reflexion auf eigenes Verhalten und Kommunikationsanregungen. Insofern ist auch der Nivo Test nur eingeschränkt als Risikomanagementmethode zu betrachten.

\subsubsection{3x3-Methode (GRAU 2004)}

GRAU (2004) adaptiert die von MUNTER (1999) für den Wintersport konzipierte 3x3 Filtermethode für den Bereich Wildwassersport. Hierbei wird das Konzept nahezu vollständig adaptiert, so dass die Methode allgemein ähnlich zu bewerten ist. Im Sinne einer Risikomanagement-Konzeption ist 
die Methode auch für den Wildwassersport durchaus sinnvoll angewendet, wenngleich wichtige Komponenten fehlen. So ist vor allem die Reflexion in einer Post-Aktionsphase vernachlässigt worden. Insgesamt stellt die von GRAU publizierte Methode aber mehr einen Diskussionsentwurf dar als eine fertige Konzeption.

Es muss also abschließend postuliert werden, dass bis dato nur wenige Methoden im Praxisfeld des Risikosports existieren, die die im Rahmen dieser Arbeit deduzierten Vorgaben eines gezielten Risikomanagements erfüllen. Lediglich MUNTER (1999), auf den sich GRAU (2004) bezieht, stellt mit seinem 3x3-Zoomsystem ein methodisches Konzept vor, welches über reine Wahrnehmungs- und Entscheidungshilfen hinaus, ganzheitliche Reflexions- und Kommunikationsprozesse initiiert. Die vernetzte Trennung von Beobachtungsebenen ermöglicht zudem eine dynamischen Entscheidungsund Reflexionsprozess, der sich entlang der Phasenstruktur einer risikosportlichen Handlung vollzieht. Es bleibt aber weiterführenden Forschungsansätzen überlassen, inwieweit die Anwendung dieser Methode zur erfolgreichen Initiierung von Prozessen der Informationssuche, der Reflexion und der Kommunikation führt. Zudem muss überprüft werden, ob eine dauerhafte Anwendung wirklich möglich ist, insofern die Methode mit einem hohen Zeitaufwand verbunden ist.

\subsection{Risikobewusstsein und Restrisiko: Eine kritische Schlussbetrachtung}

Das in diesem Kapitel dargestellte Interventionsmodell des Risikomanagements bedeutet die Abwendung konventionellen Sicherheitsdenkens zugunsten eines bewussten Umgangs mit Risiko und Gefahr in (risiko-)sportlichen Aktivitäten. Es repräsentiert die umfassende Idee, Sicherheit selbst zu erzeugen, in dem Risiko und Gefahr zu bedeutenden Verhaltenselementen der Handlungsregulation werden. Das dabei zu respektierende ,Recht auf Irrtum' verweist auf einen Paradigmenwechsel, der auch ein Umdenken im Bereich des Unfallgeschehens einschließt. Akzeptiert man, dass das Risiko ein essentieller Bestandteil unseres Lebens ist, dann müssen - zumindest in einem gewissen Umfang - auch Unfälle und Verletzungen als ,zum Leben gehörend' anerkannt werden. Eine Maxime der Fehlerfreundlichkeit bedeutet, Fehler zu akzeptieren und aus ihnen zu lernen, um sie langfristig zu verhindern. Eine bewusste ,Fehlerkultur' bedeutet jedoch gleichermaßen, dass sich Fehler nie völlig ausschließen lassen. Mit diesem Risiko jedoch verantwortungsvoll umzugehen und es bewusst zu kalkulieren, ist der eigentliche Reiz des Risikosports. Eine Erweiterung dieser Interventionsgedanken zu einem Risikomanagement-Modell im Sport könnte somit helfen, eine Vielzahl von Unfällen prophylaktisch zu vermeiden. Letztlich können Schäden und Verletzungen aber nie vollständig vermieden werden.

Zudem bleibt fraglich, inwieweit sich die in Kapitel 8 registrierten Unfälle praktisch durch das beschriebene Modell des Risikomanagements verhin- 
dern ließen. Es ist zu vermuten, dass allein die Auseinandersetzung mit Konzepten des Risikomanagements an ein gewisses Risikobewusstsein gebunden ist. $\mathrm{Zu}$ vermuten ist, dass insbesondere die Personen Unfälle erleiden, die sich kognitiv nur wenig mit Risiko und Sicherheit im Rahmen ihrer Sportbetätigung beschäftigen. Eine Aufklärung und Anwendung der hier vorgestellten Interventionsinhalten wird aber - so ist zu vermuten - gerade die Personen erreichen, die ohnehin schon über grundsätzliche Erfahrungen mit Risiko, Sicherheit und Gefahr sowie deren kognitiver Repräsentation verfügen. Die durch das Denken in Sicherheitsgarantien verursachten Unfälle im Kanusport oder alpinen Bergsport ließen sich insofern umfassend nur über ein generelles Umdenken in der Gesellschaft vermeiden. Es wäre in diesem Sinne hilfreich, Risiko und Sicherheit nicht mehr als unvereinbare Gegensätze zu verstehen, sondern als bedeutungsvolle und sich wechselseitig beeinflussende Elemente unseres Lebens zu begreifen. Zugegeben, dieses Interventionsziel bleibt wahrscheinlich Utopie, selbst wenn wissenschaftliche Forschungsansätze tiefer in diese Materie eindringen würden.

Es bleibt daneben ungewiss, inwieweit sich die vorgestellten Eckpfeiler des Risikomanagements - Information, Kommunikation, Reflexion - in eine Alltagspraxis des Risikosport integrieren lassen. Die Anwendung derartiger Konzepte ist letztlich ebenso an ein grundlegendes Risikobewusstsein gekoppelt, so dass ein Einsatz insbesondere bei den Personen zu vermuten ist, die ohnehin über entsprechende Regulations- und Verhaltensmechanismen verfügen.

Darüber hinaus birgt die Verwendung von praktischen Risikomanagement-Konzeptionen in sich die Gefahr einer inhärenten Risikokompensation. Eine Umdefinition einer Risikomanagement-Methode in eine Sicherheitsgarantie (,Wir halten uns streng an das Konzept, dann kann nichts passieren') würde einen entgegengesetzten Effekt verursachen, der der grundsätzlichen Idee des Risikomanagements widerspricht. Der eigentliche Schlüssel für ein Modell des Risikomanagements im Sport liegt somit in einer gezielten und strukturierten Ausbildung (s.o.). Der Umgang mit Risiko und Gefahr im Risikosport muss letztlich genauso erlernt werden, wie die Auseinandersetzung mit Boot, Paddel, Seil oder Haken. Im Sinne des sportlichen Risikomanagements kann sich dieser Lernprozess vielleicht manchmal unangenehm und mühsam gestalten, letztlich wird aber, wenn man den Protagonisten glauben darf, jede strapaziöse Minute mit beeindruckenden und intensivsten Erlebnissen mehrfach belohnt. 


\section{Resümee und Ausblick}

Jedes Ende ist tröstlich; trostlos ist nur Endlosigkeit.

(Ich komme deshalb zum Schluss. Danke für Ihre Geduld.).

(Peter Benary (*1931), dt. Musikwissenschaftler u. Aphoristiker)

Das Ziel der vorliegenden Arbeit war es, Risikosport als gesellschaftliches Phänomen und individuelles Handlungsfeld unter einer sportwissenschaftlichen Fokussierung zu untersuchen. Der gewählte Forschungszugang, der in einer Kombination aus interdisziplinärer Annäherung und empirischer Untersuchung bestand, sollte dabei Perspektiven eröffnen, das Phänomen Risikosport in seinen interdependenten Kontexten zu erklären. Dies ist - soweit es der Forschungsstand der einzelnen Disziplinen zuließ - auch gelungen. Risikosport als sportwissenschaftliches Phänomen konnte damit erstmals einer interdisziplinären Analyse unterzogen werden. Durch die intensive Auseinandersetzung mit dem Forschungsstand und einer Erweitung durch angrenzende wissenschaftliche Disziplinen haben sich dabei im Laufe der Arbeit eine Reihe von Schlussfolgerungen und Einsichten ergeben, die bereits im Anschluss an die einzelnen Kapitel sowie im Zwischenfazit intensiv diskutiert wurden. Eine Wiederholung dieser Erkenntnisse soll dem Leser nicht zugemutet werden, vielmehr wird auf die zusammenfassenden Ergebnisse der einzelnen Kapitel verwiesen. Zudem liegt der Erkenntnisgewinn dieser Arbeit nicht in einer zu diskutierenden Hypothese oder einer kausalen 
Theorie sondern in der Deduktion und Abduktion multidimensionaler Bezüge, wissenschaftlicher Zusammenhänge und interdisziplinärer Verweise. Eine Schlussbetrachtung, die ein einfaches abschließendes Fazit beinhaltet, ist demnach kaum möglich.

Freilich darf aus der interdisziplinären Betrachtung eines Phänomens kein wissenschaftliches Kaleidoskop entstehen, dennoch ist die sportwissenschaftliche Kodierung des Risikosports im synchronen und diachronen $\mathrm{Zu}$ sammenhang der unterschiedlichsten wissenschaftlichen Zugänge kein einfaches Unterfangen. Risikosport - so viel kann hier zweifelsfrei geschlussfolgert werden - lässt sich keinesfalls monokausal erklären oder gar in eine einheitliche Theorie drängen. Im Gegenteil: Risikosport offenbart sich nach Abschluss dieser Arbeit als ein Phänomen, welches sich nur durch intensives ,Querdenken' und der Suche nach alternativen Antworten nachvollziehen lässt. Zum Teil konterkariert es dabei klassische, gesellschaftlich relevante Erklärungsansätze ohne jedoch zu wesentlichen sportwissenschaftlichen Dogmen auf Distanz zu gehen.

Die theoretischen Ausführungen im ersten Teil der Arbeit haben offenbart, dass die in der Sportwissenschaft bisweilen wenig rezipierte Differenzierung von Unsicherheit im Verständnis LUHMANNs einen zentraler Ansatzpunkt zum Verständnis des Risikosports darstellt. Der hierbei vollzogene Zugang zur Thematik geht von einem subjektzentrierten Definitionsansatz von Sicherheit und Unsicherheit aus. Risiko - so kann hier zusammengefasst werden - wird erst dann zu einem solchen, wenn die Gefahr als Gegenstand und Folge einer eigenen Entscheidung zu sehen ist. Damit rückt das handelnde Individuum in das Zentrum der wissenschaftlichen Auseinandersetzung, wenngleich durch die inhaltliche Zuweisung von Merkmalen und Charakteristika auch das Handlungsfeld Risikosport im Rahmen dieser Arbeit eingegrenzt werden konnte. Der sich dadurch konstituierende Forschungszugang begründete letztlich den mehrperspektivischen Ansatz dieser Arbeit, der sich in der interdisziplinären theoretischen Konfrontation ebenso niederschlägt wie in der ergänzenden empirischen Analyse. Nur wenn Risiko und Sicherheit in ihren kollektiven Dimensionen dem individuellen Handeln im Risikosport gegenübergestellt werden, erschließt sich der Risikosport als Handlungsfeld auch unter rational-theoretischen Gesichtspunkten. Im Risikosport werden damit die gesellschaftlich relevanten Vorstellung von Sicherheit und Risiko außer Kraft gesetzt. Dieser Argumentationsfigur folgend, werden die verschiedenen Formen risikosportlicher Aktivitäten als eine spezifische Form des Unsicherheitserlebens gedeutet, in der das Bestreben nach einem Ausbruch aus den gewohnten kollektiven Sicherheitssystem deutlich wird und gleichzeitig der Wunsch nach einer sekundär selbst hergestellten Sicherheit zum Ausdruck kommt.

Die Erweiterung des Forschungsstands über das subjektive Risiko im Sport und das Phänomen Risikosport schließt damit nicht nur eine Forschungslücke, sondern eröffnet auch völlig neue Perspektiven für den wissenschaftlichen Risikodiskurs. In keiner anderen Risikothematisierung wird die Ambivalenz der öffentlichen Diskussion über Risiko und Sicherheit glei- 
chermaßen ersichtlich wie im Risikosport. Während Unsicherheit unter gesellschaftlicher Perspektive als kollektive Gefahr diskutiert und damit negativ konnotiert wird, tritt Unsicherheit im Risikosport als eine individuelle Chance auf. Die positiven Merkmale dieser Unsicherheitserfahrung äußern sich darin, dass das individuelle Handlungsrisiko stets auch den Reiz von persönlicher Bewährung und authentischer Selbsterfahrung einschließt. Durch die Bewältigung von unsicheren - aus dem Schema kollektiver Sicherheiten ausbrechenden - Handlungssituationen werden persönlichkeitsstabilisierende Prozesse initiiert, die den Spielraum für die Bewältigung von neuartigen, kritischen Situationen ausweitet. Somit bildet das Eingehen von Unsicherheit auch die Basis einer Verbesserung des individuellen Sicherheitszustandes.

Zugegeben: Risikosport und Gesellschaft stehen über die Vermittlungsinstanz des Individuums in einem durchaus ambivalenten Verhältnis, denn in der Grundfiguration des Abenteuerlichen offenbart sich im Risikosport ein gesellschaftlicher Kontrapunkt: Die Befunde der kulturhistorischen sowie soziologischen Teiluntersuchungen konnten deutlich machen, dass die im Risikosport erlebte und bewältigte Unsicherheit als ein besonders intensives und authentisches Erlebnis zu identifizieren ist, welches einer mit der Moderne einhergehenden Domestizierung des Alltags entgegensteht. Risikosport - so kann hier geschlussfolgert werden - argumentiert diesbezüglich aus der Moderne heraus und in die Moderne hinein. Risikosportler vollziehen eine Art ,Typifikation' im Sinne HUSSERLs: Sich machen auf das Abwesende im Anwesenden ihres Handelns aufmerksam.

Sicherheit und Risiko - so die zentrale These dieser Arbeit - stehen also im Risikosport in einer Art natürlichen Gleichgewicht, welches sich nur durch ein Umdenken relevanter Sicherheitsvorstellungen verstehen lässt. Aus den unterschiedlichen Teiluntersuchungen dieser Arbeit wird deutlich, dass nicht eine maximale, sondern eine optimale Sicherheit im Risikosport angestrebt wird und sich im Handeln niederschlägt. In risikosportlichen Aktivitäten wird also ein Spannungsfeld von Sicherheitsbedürfnis und Risikosuche wiederhergestellt, welches als Fließgleichgewicht weit über das Teilsystem Sport hinaus in die Gesellschaft zurückwirkt.

Es wird die Aufgabe weiterer inter- und transdisziplinärer sowie konservativ sportwissenschaftlicher Forschungsarbeiten sein, diese Erkenntnisse weitergehend zu untersuchen. Es muss dabei eine vordergründige Aufgabe der Sportwissenschaften sein, Sicherheit und Risiko zu sportwissenschaftlichen Kategorien zu erheben und damit einen Risikodiskurs zu eröffnen, der die bestehende Diskussion um Unsicherheit in der Gesellschaft erweitert. Die vorliegende Arbeit konnte einen wesentlichen Beitrag dazu leisten, das sportwissenschaftliche Desiderat zur Thematik des Risikosports zu füllen, wenngleich selbstkritisch angemerkt werden muss, dass mit dem gewählten Forschungszugang nur jeweils Ausschnitte eines Ganzen thematisiert werden konnten. Eine vertiefte Auseinandersetzung ist aber insbesondere im Bezug auf die psychologischen Rahmenbedingungen des Risikosports unbedingt notwendig, denn bis dato scheint nicht eindeutig geklärt, welche Rolle 
z.B. genetische oder auch sozialisationspragmatische Disposition für das Engagement in Risikosportarten spielen. Diese Erkenntnisse könnten aber wesentlich dazu beitragen das Verständnis dieser Aktivitäten zu verbessern und die praktische Intervention im Risikosport unter der Zielsetzung einer Unfallprävention zu berichtigen. Diese Intervention setzt am Verhalten der einzelnen Sportler an und verlangt über die vorliegenden psychologischen Forschungsansätze hinaus weitere handlungstheoretische Untersuchungen, die die Verhaltensregulation im Risikosport eingehend prüfen. Zwar konnte mit der empirischen Untersuchung dieser Arbeit ein erster Beitrag zum korrelativen Zusammenhang zwischen Risiko und Sicherheit während der Handlungsregulation im Risikosport geleistet werden, angesichts der existierenden methodischen Probleme, sind aber weitere Studien erforderlich. Einen wesentlichen Ansatzpunkt stellen diesbezüglich multidimensionale Forschungsansätze dar, die neben experimentellen Laboruntersuchungen auch qualitative Methoden berücksichtigen, denn die Handlungswirklichkeit der handelnden Akteure erschließt sich letztlich nur bedingt über die intensive Auseinandersetzung mit Individuen, so dass beispielsweise themenzentrierte oder auch biographische Interviews durchaus fruchtbare Erkenntnisse liefern könnten.

Abschließend bleibt anzumerken, dass mit dem gewählten Forschungsthema und dem damit verbundenen Forschungszugang neue Perspektiven für die Sportwissenschaften eröffnet werden konnten. Auf den gewonnen Erkenntnissen müssen nun weitere Studie folgen, denn es ist davon auszugehen, dass Risikosportarten auch in Zukunft einen bedeutenden Platz in der bisweilen bunten Sportlandschaft westlicher Gesellschaften einnehmen werden. Zugegeben: Die wissenschaftliche Bearbeitung derart komplexer Phänomene stellt bisweilen ein Risiko dar. Die sportwissenschaftlichen Forschungseinrichtungen wären allerdings gut beraten, diese Unsicherheit als Herausforderung zu begreifen und die noch existierenden Forschungslücken mit zahlreichen Untersuchungen und Studien zu füllen. 


\section{Literatur}

ABELE, A. \& BREHM, W. (1990): Sportliche Aktivität als gesundheitsbezogenes Handeln: Auswirkungen, Vorraussetzungen und Förderungsmöglichkeiten. In: SCHWARZER, R. (Hrsg.): Gesundheitspsychologie (S. 131 - 150). Göttingen.

ABRAHAM, A. (1998): Geschlecht als Strukturdimension sozialer Ungleichheit - auch im Sport. In: CACHAY, K. \& HARTMANN-TEWS, I. (1998): Sport und soziale Ungleichheit: Theoretische Überlegungen und empirische Befunde (S. 27 - 48). Stuttgart.

ADORNO, T.W. \& HORKHEIMER, M. (1992): Dialektik der Aufklärung. Frankfurt am Main.

ADORNO, T.W. (1969): Freizeit, in: ADORNO, T.W: (Hrsg.): Stichworte. Kritische Modelle 2 (S. 57-68). Frankfurt a. Main.

ALHEIT,P. (1995): Aufbruch in die ,Erlebniskultur'? In: Das Argument, 208(1995), S. 91-104.

ALKEMEYER, T. (1995): Sport, die Sorge um den Körper und die Suche nach Erlebnissen im Kontext gesellschaftlicher Modernisierung. In: HINSCHING, J. \& BORKENHAGEN, F. (Hrsg.): Modernisierung und Sport, S. 29-64. Sankt Augustin

ALLMER, H. (1998): ,No risk - no fun’ - Zur psychologischen Erklärung von Extremund Risikosport. In ALLMER, H. \& SCHULZ, N. (1998): Erlebnissport - Erlebnis Sport. In: ALLMER, H. \& SCHULZ, N. (Hrsg.): Erlebnissport - Erlebnis Sport (S.60-91). Sankt Augustin.

ALLMER, H. (2002): Sportengagement im Lebensverlauf - Forschungsstand und Forschungsperspektive. In: ALMMER, H. (Hrsg.): Sportengagement im Lebensverlauf (Brennpunkte der Sportwissenschaft)(S. 7-20).Sankt Augustin.

ALPINER-KAJAK-CLUB (Hrsg.) (1987): Kanugefahren. München. 
ALT, W. \& SCHAFF, P. \& SCHUMANN, H. (2000)(Hrsg.): Neue Wege der Unfallverhütung im Sport. Köln.

ALTENBERGER, H. (1994): Einzelfallanalysen von Schulsportunfällen unter psychologischen Aspekten. In: Pluspunkt 20 (1994), 4, S. 6-7.

ALTKIRCH, E. (1905): Weib und Alpinismus. In: Österreichische Alpenzeitung, 695 (1905), S. 217-220.

AMBROSI, C. (2004): Sport und Faschismen. Innsbruck.

AMD (2001) (Hrsg.): Standards zur Qualitätssicherung für Online-Befragungen. Ausgabe 2001. Frankfurt.

AMELANG, M. \& BARTUCCEK, D. (2001): Differentielle Psychologie und Persönlichkeitsforschung. (5.Auflage). Stuttgart.

AMESBERGER, G. (1992): Persönlichkeitsentwicklung durch Outdooraktivitäten? Untersuchung zur Persönlichkeitsentwicklung und Realitätsbewältigung bei sozial Benachteiligten. Frankfurt a. Main.

AMESBERGER, G. (2002): Warum die Unfallforschung im Sport in Österreich kaum ein Thema der Sportwissenschaften ist - Pragmatische und methodische Überlegungen. In: STRAUß, B. et al. (Hrsg.): Expertise im Sport (S. 102-103). Köln.

AMESBERGER, G. (2004): Entscheiden in Risikosituationen. In: KURATORIUM FÜR ALPINE SICHERHEIT (Hrsg.): Sicherheit im Bergland. Jahrbuch 2004. Innsbruck.

AMSTÄDTER, R. (1996): Der Alpinismus. Kultur Organisation Politik. Wien.

ANDERSON, B. (2000): Six Basic Dimensions of Personality and a Seventh Factor of Generalized Dysfunctional Personality: A Diathesis System Covering all Personality. In: Neuropsychobiology, 41 (2000), S. 5-23.

ANDRECS, H. (1987): Erleben und Wagen im Sport. In REDL, S. (Hrsg.): Sport für Morgen (S.124 - 124). Wien.

APTER, M. (1994): Im Rausch der Gefahr. München.

ATESLANDER, P. (1995): Methoden der empirischen Sozialforschung. Berlin.

ATKINSON, J. (1957): Motivational determinants of risktaking behavior. In: Psychological Review, 64 (1957) ,S.359-372.

AUFMUTH, U. (1983): Risikosport und Identitätsproblematik. Überlegungen am Beispiel des Extrem-Alpinismus. In: Sportwissenschaft, 13 (3), 249-270.

AUFMUTH, U. (1988): Zur Psychologie des Bergsteigens. Frankfurt a. Main.

AUFMUTH, U. (1989): Die Lust am Risiko. Gedanken über Extremformen der Bergsteigerleidenschaft. In DIETRICH, K. \& HEINEMANN, K. (Hrsg.): Der nichtsportliche Sport (S. 120- 135). Schorndorf.

AWA (1999): Freizeitsport. Allensbacher Markt- und Werbeträgeranalysen. Allensbach.

BADINTER. E. (1993): XY Die Identität des Mannes. Zürich.

BAHNERS, P. \& HANSEN, P.H. (1996): Als der Berg die Viktorianer rief. In: ZEBHAUSEN, H. / TRENTIN-MEYER, M. (Hrsg.): Zwischen Idylle und Tummelplatz (S. 70-73). München. 
BÄHR, I. (2003): Erleben Frauen das Sportklettern anders als Männer? Eine Studie zur Geschlechtstypik des Bewegungshandelns. In: GISSEL, N. \& SCHWIER, J. (Hrsg.): Abenteuer, Erlebnis und Wagnis: Perspektiven für den Sport in Schule und Verein? (S.65-70). Hamburg.

BAILLETTE, F. (1995): Mourir in extremis. In : BAILLETTE, F. \& BROHM, J.M. (Hrsg): Quel Corps? Critique de la modernité sportive (S. 321-332). Paris.

BALINT, M (1994): Angstlust und Regression (vierte Auflage). Stuttgart.

BALINT, M. (1973): Therapeutische Aspekte der Regression. Hamburg.

BALZ, E. \& NEUMANN, P. (1994): Mit dem Wagnis unterwegs. In: Sportpädagogik 18(3), S. 49-52.

BALZ, E. (1994): Warum Spaß nicht als Leitidee für den Schulsport taugt. In: Sportunterricht, 43(11), S. $468-47$.

BALZ, E. (1995): Gesundheitserziehung im Schulsport. Grundlagen und Möglichkeiten einer diätetischen Praxis. Schorndorf.

BÄNI, P. (1991a): Risiko als Faszination. In: Magglingen, 48 (6), S. 2-6.

BÄNI, P. (1991b): Risiko als Faszination - Ist Sicherheit lernbar?. Kanusport, 60 (6), 257-259.

BANSE, G. \& BECHMANN, G. (1998): Interdisziplinäre Risikoforschung. Opladen / Wiesbaden.

BANSE, G. (1996a)(Hrsg.): Risikoforschung zwischen Disziplinarität und Interdisziplinarität. Von der Illusion der Sicherheit zum Umgang mit Unsicherheit. Berlin.

BANSE, G. (1996b): Herkunft und Anspruch der Risikoforschung. In BANSE, G. (Hrsg.): Risikoforschung zwischen Disziplinarität und Interdisziplinarität (S. 16-73). Berlin.

BATINIC, B. (2001): Fragebogenuntersuchungen im Internet. Aachen.

BAUDRY, P. (1986): Le Corps Extreme. Approche sociologique des conduit à risque. Paris.

BAUER, A. \& SCHULTE, S. (1997): Handbuch für den Kanusport. Aachen.

BAUER, A. (1991): Belastung und Beanspruchung beim Wildwasserfahren - Eine empirische Untersuchung zur Analyse physiologischer Leistungsparameter. Dissertation Göttingen.

BAUER, H.G. (1983):Erlebnis- und Abenteuerpädagogik. Eine Literaturstudie. 3. erw. Auflage. München.

BAUER, K. (1995): Sicherheitserziehung und Unfallverhütung im Sportunterricht weiterführender Schulen - allgemeine Grundsätze. München.

BAUMANN, N. (1995): Ist Sicherheit messbar? In: Sportpädagogik, 19 (5), 6-8.

BAUMANN, S. (1992): Psychologische Unfallvermeidung (1). In: Sichere Schule 5 (1992), 2, S. 9-17.

BAUR, J \& HAHN, H. \& HOLZ, P. (1977): Grundlagen des Kanusports. Stuttgart.

BAUR, J. \& BRAUN, F. (2000): Freiwilliges Engagement und Partizipation in ostdeutschen Sportvereinen. Köln. 
BAUR, J. (1982): Erlernen von Strategien des Wildwasserfahrens. In: BAUR, J. (Hrsg.): Lehren und Lernen im Kanusport. Schriftenreihe des Deutschen Kanu-Verbandes e.V. (Band 1) (S. 65-73). Duisburg.

BECHMANN, G. (1993): Risiko und Gesellschaft. Grundlagen und Ergebnisse interdisziplinärer Risikoforschung. Opladen.

BECK, U. \& BECK-GERNSHEIM, E. (1994): Individualisierung in modernen Gesellschaften - Perspektiven und Kontroversen einer subjektorientierten Soziologie. In: BECK, U. \& BECK-GERNSHEIM, E. (Hrsg.): Riskante Freiheiten (S.10-49). Frankfurt a. Main.

BECK, U. \& GIDDENS, A \& LASH, S. (1997): Reflexive Modernisierung: Eine Kontroverse. Frankfurt am Main.

BECK, U. (1983): Jenseits von Stand und Klasse? In: KRECKEL, R. (Hrsg.): Soziale Ungleichheiten. (S. 35-74). Göttingen.

BECK, U. (1986): Risikogesellschaft. Auf dem Weg in eine andere Moderne. Frankfurt a. Main.

BECK, U. (1997): Individualisierung und Integration. Neue Konfliktlinien und neuer Integrationsmodus. Opladen.

BECKENBACH, N. (1994): Risikosoziologie. In KERBER, H. \& SCHMIEDER, A. (Hrsg.): Spezielle Soziologien (S.149-167). Hamburg.

BECKER, E. (1999): Spaß, Fun und Wohlbefinden - Wider die Kultur der Sprachlosigkeit. In: Spectrum der Sportwissenschaft, 11 (2); (S. 29 - 43).

BECKER, P. (1991): Theoretische Grundlagen. In: ABELE, A. \& BECKER, P. (Hrsg.): Wohlbefinden. Theorie-Empirie-Diagnostik. (S. 13 - 50). Weinheim und München.

BECKER, P. (1994): Offene Zukunft und riskante Entscheidungen. Gesellschaftliche und pädagogische Aspekte des Umgangs mit Unsicherheiten in der Moderne. In: $O$ lympische Jugend, 39 (12), 4-17.

BECKER, P. (1995): Auf der Suche nach dem Paradies: zur aktuellen Attraktivität von Erlebnis und Abenteuer. In PAWELKE, R. (Hrsg.): Neue Sportkultur (S.325-334). Lichtenau.

BECK-GERNSHEIM, E. (1983): Vom „Dasein für andere“ zum Anspruch auf ein Stück ,eigenes Leben“. Individualisierungsprozesse im weiblichen Zusammenhang. In: Soziale Welt 34, 307-340.

BEIER, K. (2001): Anreizstrukturen im Outdoorsport. Schorndorf.

BEIERWALTER, W. (1996): Petrarca und Augustinus: „Aufstieg des Herzens“. In: ZEBHAUSEN, H. / TRENTIN-MEYER, M. (Hrsg.): Zwischen Idylle und Tummelplatz (S. 394 - 395). München.

BERGER, P.A. \& HRADIL, S. (Hrsg.)(1990): Lebenslagen, Lebensläufe, Lebensstile. Sonderband 7 der Zeitschrift soziale Welt. Göttingen.

BERGER, P.A. (1986): Klassen und Klassifikationen. Zur neuen Unübersichtlichkeit in der soziologischen Ungleichheitsdiskussion. In: Kölner Zeitschrift für Soziologie und Sozialpsychologie, 1986, (39), S. 138-153.

BERGER, P.A. (1996): Individualisierung. Opladen. 
BERGER, P.A: \& SOPP, P. (1995)(Hrsg): Sozialstruktur und Lebenslauf. Opladen.

BERGHOLD, F. (1986): Der plötzliche Tod im Sport. Sportpraxis, 3 (2),35-38.

BERGHOLD, F. (1988): Sicheres Bergsteigen: Alpine Unfälle - und wie man sie vermeidet. München.

BERLYNE, D.E. (1974): Konflikt, Erregung, Neugier. Stuttgart.

BERNETT, H. (1981): Wehrsport - ein Pseudosport. In: Sportwissenschaft 11 (1981), S. 295-308.

BERNSTEIN, L. (1997): Wider die Götter - die Geschichte von Risiko und Risikomanagement von der Antike bis heute. München.

BETTE, K.H. (1989): Körperspuren. Berlin.

BETTE, K.H. (1993): Sport und Individualisierung. In: Spectrum der Sportwissenschaften, 5 (1), (S. $34-55)$.

BETTE, K.H. (1999): Systemtheorie und Sport. Frankfurt / Main.

BET'TE, K.H. (2004): Zur Soziologie des Abenteuer und Risikosports. Bielefeld.

BFU [Schweizer Beratungsstelle für Unfallverhütung](2004)(Hrsg.): Sport - mit Sicherheit mehr Spaß. Magglingen.

BILDEN, H. (1989): Geschlechterverhältnis und Individualität im gesellschaftlichen Umbruch. In: BILDEN, H. / KEUPP, H. (Hrsg.): Verunsicherungen. Das Subjekt im gesellschaftlichen Wandel, S. 19 - 46. Göttingen.

BIRNBACHER, D.(1996): Risiko und Sicherheit, Philosophische Aspekte. In BANSE, G. (Hrsg.): Risikoforschung zwischen Disziplinarität und Interdisziplinarität (S.193211). Berlin.

BÖHNUNG, L. \& WINTER, R. (1993): Männliche Sozialisation. Bewältigungsprobleme männlicher Geschlechtsidentität im Lebenslauf. Weihnheim.

BÖLLERT, K. (1995): Zwischen Intervention und Prävention. Eine andere Funktionsbestimmung Sozialer Arbeit. Neuwied.

BOLLNOW, O. (1959): Existenzphilosophie und Pädagogik. Stuttgart.

BOLOGNESI, R. (2000): Nivo Test. A pocket tool for avalanche risk assessing. Proc.Intnernational Snow Science Workshop, Big Sky, MT, USA, S. 554 - 557.

BONNINGTON, C. (2000): Triumph in Fels und Eis. Die Geschichte des Alpinismus. Stuttgart.

BONß, W. (1995): Vom Risiko: Unsicherheit und Ungewissheit in der Moderne. Hamburg.

BONB, W. (1997):Die gesellschaftliche Konstruktion von Sicherheit. In LIPPERT, E (Hrsg.): Sicherheit in der unsicheren Gesellschaft (S.21-43). Opladen.

BOPP, J. (1987): Die Tyrannei des Körpers. In: MICHEL, K.M. \& SPRENGLER, T. (Hrsg.): Kursbuch Gesundheit 88 (S. 49 - 66). Berlin.

BORNEMANN, J.J.W. (1812): Der Turnplatz in der Hasenheide. Originalabdruck in: BERLINER TURNERBUND (o.J.): Turnplatz Hasenheide 175 Jahre. Berlin.

BORSCHEID, P. (1997): Mit Sicherheit leben. Geschichte der deutschen Versicherungswirtschaft. In: Vierteljahrschrift für Sozial- und Wirtschaftsgeschichte, Band 84 (1997), S. 450. 
BORTZ, J. \& DÖRING, N. (2003). Forschungsmethoden und Evaluation. Für Human- und Sozialwissenschaftler. Berlin: Springer.

BORTZ, J. \& DÖRING, N. (2003): Forschungsmethoden und Evaluation. Berlin.

BORTZ, J. (1999) Statistik für Sozialwissenschaftler. 5. Auflage. Berlin.

BOUET, M. (1969): Les motivation des sportifs. Paris.

BOURDIEU, P. (1982). Die feinen Unterschiede. Kritik der gesellschaftlichen Urteilskraft. Frankfurt / Main.

BOYES, M (1991): The concept of risk for outdoor teachers. New Zealand journal of health, physical education and recreation 24 (4), 14-17.

BRAND, F. \& SCHALLER, F. \& VÖLKER, H. (2004)(Hrsg.): Transdisziplinarität. Bestandsaufnahme und Perspektiven. Beiträge zur THESIS-Arbeitstagung im Oktober 2003 in Göttingen. Göttingen.

BRÄUTIGAM, M. (1994): Spaß als Leitidee jugendlichen Sportengagements. In: Sportunterricht, 43(6), (S. 236 - 244).

BRÄUTIGAMM, M. (1993): Vereinskarrieren von Jugendlichen. Köln.

BRENGELMANN, J.C. (1988): Risiko - Lustaktivitäten. Valduz.

BRETTSCHNEIDER, W.D. \& BRÄUTIGAMM, M. (1990): Sport in der Alltagswelt von Jugendlichen. Frechen.

BREUER, C. \& MICHELS, H. (2003): Trendsport - Modelle, Orientierungen und Konsequenzen. In: BREUER, C. \& MICHELS, H. (Hrsg.), Trendsport: Modelle, Orientierungen und Konsequenzen (Edition Sport und Freizeit, 14, S. 11-17). Aachen.

BREUER, C. (2000): Distinktions, Stilisierungs- und Erlebnispotenziale als zentrale Erfolgsgrößen von Trendsportarten? Eine empirische Analyse von MarathonSkatern. In: ESCHER, A. \& EGNER, H. \& KLEINHANS, M. (Hrsg.): Trend- und Natursportarten in den Wissenschaften: Forschungsstand - Methoden - Perspektiven $(\mathrm{S} .41-54)$. Hamburg.

BREUER, C. (2002): Sozialer Wandel und Sportengagement im Lebenslauf. In: ALMMER, H. (Hrsg.): Sportengagement im Lebensverlauf (Brennpunkte der Sportwissenschaft)(S. 61-82). Sankt Augustin.

BRINKHOFF, K.P. (1998): Sport und Sozialisation im Jugendalter. Weinheim.

BROCKHAUS (1983): Kompakt Wissen in 5 Bänden. Wiesbaden.

BROGIATO, H.P. (2003): Die Anden - geographische Erforschung und künstlerische Darstellung. München.

BROHM, J.M. (1995) : La violence suicidaire du sport de competetion. In :

BAILLETTE, F. \& BROHM, J.M. (Hrsg): Quel Corps? Critique de la modernité sportive (S. 305-320). Paris.

BRUCKHEIMER, H. (1990): Homo adventurus oder das Bedürfnis nach Abenteuer. Zeitschrift für Erlebnispädagogik, 10 (8/9), 86-101. 
BRÜGGER, O. (2004): Sportunfallgeschehen in der Schweiz sowie in Deutschland und Österreich. In: «Sport - mit Sicherheit mehr Spass», Kongressband 3. Dreiländerkongress Schweiz Deutschland - Österreich vom 19.-21. September 2004 (s. 13 22). Magglingen.

BUBB, H. (1990): Bewertung und Vorhersagen der Systemzuverlässigkeit. In: HOYOS, C. GRAF \& ZIMOLONG, B. (Hrsg.): Ingenieurspsychologie, Band D, Serie III (S.285-311). Göttingen.

BUBITZ, G. (2000): Entwicklung, Sozialisation und Identität im Jugend- und Erwachsenenalter. Berlin.

BUCHMANN, M. (1989): Subkulturen und gesellschaftliche Individualisierungsprozesse. In: Haller, M. / Hoffmann-Nowotny, H.-J. / Zapf, W. (Hrsg.): Kultur und Gesellschaft. Frankfurt / Main, S. 627 - 638.

BUCK, R. (1999): The biological affects: A typology. In: Psychological Review, 106(1999), S. 301-336.

BURTSCHER, M. (2003): Österreichische Skiunfallerhebung im Winter 2002 / 2003 In: KURATORIUM FÜR ALPINE SICHERHEIT (Hrsg.): Sicherheit im Bergland Jahrbuch 2003 (S. 83 - 92). Innsbruck.

BUSS, W. (1985): Sport und Revanchismus in der Weimarer Republik - eine Studie zur Instrumentalisierung des Sports im Sinne militanter kriegsvorbereitender Politik am Beispielen aus dem Bereich der Hochschulen. In: BECKER, H. (Red.): Sport im Spannungsfeld von Krieg und Frieden (S. 80-96). Clausthal-Zellerfeld.

BYE, A. \& HOLLNAGEL E. \& BRENEFORD, T.S. (1998): Human-Machine Function Allocation: A Functional Modelling Approach, In: Reliabilty Engineering and System Safety 59 (1998) (S. 243-252). New York

CACHAY, K. \& THIEL, A. (2000): Soziologie des Sports. Zur Ausdifferenzierung und Entwicklungsdynamik des Sports in der modernen Gesellschaft. Weinheim, München.

CACHAY, K. (1988): Sport und Gesellschaft. Schorndorf.

CAILLOIS, R. (1964): Die Spiele und die Menschen. München.

CAPPEL, C. (1993): Belastungssituationen im Risikosport. Unveröffentlichte Diplomarbeit / Köln.

CARLSON, W.L. \& KAPLAN, R. L. (1975): Case studies considered as retroactive experiment. Accident Analysis \& Prevention, 7, 73-80.

CARTER, J.M. (2000): Sportgeschichte in mittelalterlichen Biographien: William Marshal (ca. 1146-1219). In: KRÜGER, A. \& WEDEMEYER, B. (Hrsg.): Aus Biographien Sportgeschichte lernen. Festschrift zum 90. Geburtstag von Prof. Dr. Wilhelm Henze (S.67-78). Hoya.

CHAKRABORTY, S. (1991): Ganzheitliche Risikobetrachtung: technische, ethische und soziale Aspekte. Köln.

CHODOROW, N. (1986): Das Erbe der Mütter. Psychoanalyse und Soziologie der Geschlechter. München.

CLAUSEN, S.A. (2003): Religiöse Dimensionen im Extremsport. Hamburg. 
CLONINGER, R.C. (1987): A Systematic Method for Clinical Description and Classifikation of Personality Variants. A Proposal. In: Archieves of General Psychiatry, 44, S. $573-586$.

COHEN, J. \& HANSEL, M. (1961): Glück und Risiko. Frankfurt.

COLEMAN, J. (1986): Die assymetrische Gesellschaft. Vom Aufwachsen mit unpersönlichen Systemen. Weinheim.

CONWAY, W.M. (1900): Mountaineering. In: EARL OF SUFFOLK AND

BERKSHIRE et.al. (Hrsg.): Encyclopdaedia of sport, Leo-Samb (S. 23-58). London.

COURNELOUP, J. (1997): Risque, opinion publique et pratiques d'escalade. In: Société, 55(1), S. 23-40.

CSIKSZENTMIHALYI, M. \& RATHUNDE, K. (1993): The measurment of flow in everyday life: Toward a theory of emergent motivation. In: DIENSTBIER, R.A. \& JACOBS, J.E. (Hrsg.): Nebraska Symposium on Motivation (S. 57 - 97). Lincoln.

CSIKSZENTMIHALYI, M. (1987): Das Flow-Erlebnis. Jenseits von Angst und Langeweile: Im Tun aufgehen. Stuttgart.

CSIKSZENTMIHALYI, M. (1992): Flow. Die sieben Elemente des Glücks. In: Psychologie beute, 19 (1), 20-29.

CSIKSZENTMIHALYI, M. (1993): Das Flow-Erlebnis (5. Auflage). Stuttgart.

CUBE v., F. (1995): Gefährliche Sicherheit - Verhaltensbiologie des Risikos. München.

DAHRENDORF, R. (1979): Lebenschancen. Anläufe zur sozialen und politischen Theorie. Frankfurt / M.

DAHRENDORF, R. (1992): Der moderne soziale Konflikt. Essays zur Politik der Freiheit. Stuttgart.

DAHRENDORF, R. (1994): Das Zerbrechen der Ligaturen und die Utopie der Weltbürgergesellschaft. In BECK, U. \& BECK-GERNSHEIM, E. (Hrsg.): Riskante Freiheiten (S. 421-437). Frankfurt a. Main.

DEUTSCHER UND ÖSTERREICHISCHER ALPENVEREIN (1933)(Hrsg.): Taschenbuch für Alpenvereinsmitglieder. München, Innsbruck, Wien.

DEUTSCHER-KANU-VERBAND (Hrsg.) (2000): Auslandsführer Band 1 (5. Auflage). Duisburg.

DIECKERT, J (1973): Freizeit versus Leistungssport. In: GRUBE, F. / RICHTER, M. (Hrsg.): Leistungssport in der Erfolgsgesellschaft (S. 147 - 168). Hamburg.

DIECKERT, J. \& WOPP, C. (2002): Handbuch Freizeitsport. Schorndorf.

DIECKERT, J. (2002): Geschichte und Entwicklung: Freizeitsport in Deutschland. In: DIECKERT, J. / WOPP, C. (Hrsg.): Handbuch Freizeitsport (S. 25 - 32). Schorndorf.

DIEHL, P. (2000): Darstellung von traditionellen und neuen Wintersportarten im kommerziellen Fernsehen. Unveröffentlichte Examensarbeit / Göttingen.

DIEM, C. (1923): Sport ist Kampf. Glücksstunden im Sport und anderes. Berlin.

DIEM, C. (1960): Weltgeschichte des Sports und der Leibeserziehung. Stuttgart. 
DIETRICH, K. \& HEINEMANN, K. \& SCHUBERT, M. (1990): Kommerzielle Sportanbieter - eine empirische Studie zu Nachfrage, Angebot und Beschäftigungschancen im privaten Sportmarkt. Schorndorf.

DIETRICH, K. / HEINEMANN, K. / SCHUBERT, M. (1990): Kommerzielle Sportanbieter - eine empirische Studie zu Nachfrage, Angebot und Beschäftigungschancen im privaten Sportmarkt. Schorndorf.

DIETRICH; K \& LANDAU, G. (1990): Sportpädagogik. Grundlagen - Positionen Tendenzen. Reinbek bei Hamburg.

DIGEL, H. (1984): Gesellschaftliche Entwicklung und Auftrag des Sportvereins. In Kultusministerium des Landes Nordrhein-Westfalen (Hrsg.): Sportentwicklung Einflüsse und Rahmenbedingungen (S. 52 - 67). Düsseldorf.

DIGEL, H. (1986): Über den Wandel der Werte in Gesellschaft, Freizeit und Sport. In: Deutscher Sportbund (Hrsg.): Die Zukunft des Sports. Materialien zum Kongress „Menschen im Sport 2000“ (S. 14 - 43). Schorndorf.

DIGEL, H. (1990): Die Versportlichung unserer Kultur und deren Folgen für den Sport - ein Beitrag zur Uneigentlichkeit des Sports. In: GABLER, H. / GÖHNER, U. (Hrsg.): Für einen besseren Sport... . Themen, Entwicklungen und Perspektiven aus Sport und Sportwissenschaft (S. 73 - 96). Schorndorf.

DILICH, W. (1986): Ritterspiele anno 1596. Kassel.

DILTHEY, W. (1971): Schriften zur Pädagogik. Paderborn.

DODERLEIN, J.M (1987): Introduction. In: SINGLETON, W.T. \& HOVDEN, J. (Hrsg.): Risk and Decisions (S. 1-8). Chichester.

DOEHLEMANN, M. (1991): Langeweile? Deutung eines verbreiteten Phänomens. Frankfurt a. Main.

DOLLARD, J. \& MILLER, N.E. (1950): Personality and psychotherapie. New York.

DONNELLY, P. (1993): Subcultures in sport: Resilience and transformation. In: INGHAM, A.G. \& LOY, J.W. (Hrsg.): Sport in Social Development - Traditions, Transitions and Transformations. Illinois.

DÖRNER, D \& KREUZIG, H. \& REITHER, F. \& STÄUDEL, T. (Hrsg.) (1983): Lohhausen: Vom Umgang mit Unbestimmtheit und Komplexität. Bern.

DÖRNER, D. (1992): Die Logik des Misslingens - Strategisches Denken in komplexen Situationen (2. Auflage). Reinbeck bei Hamburg.

DORSCH, F. (Hrsg.) (1998): PSYCHOLOGISCHES WÖRTERBUCH (13. völlig überarbeitete Auflage). Stuttgart.

DOUGLAS, M. \& WILDAVSKY, A. (1982): How can we know the risk we face? Why risk-selection is a social process. Risk Analysis, 2, 49-51.

DOUGLAS, M. (1990): Risk as a forensic resource. In: Schriftenreihe Daedalus (1990), S. $1-16$.

DRAGUNS, J.G. (1981): Kulturvergleichende Psychologie und Neugierforschung. In VOSS, H.G. \& KELLER, H. (Hrsg.): Neugierforschung. Grundlagen, Theorien, Anwendungen (S. 309-329). Weinheim.

DREYER, A. \& DREYER, D. \& OBIEGLO, D. (2001): Krisenmanagement im Tourismus. München. 
DSB Deutscher Sport Bund (2000) : Bestandserhebung 2000. Frankfurt / Main.

DÜPPE, A. (2004): Outdor-Training als betriebliche Weiterbildungsmaßnahme - unter besonderer Berücksichtigung seiner historischen Entwicklung. München.

DURKHEIM (1977): Über die Teilung der sozialen Arbeit. Frankfurt am Main.

EARL OF SUFFOLK AND AND BERKSHIRE (1900)(Hrsg): The Encyclopaedia of Sport. The Standard Edition. London.

EBERLE, T. (1995): Begriff Erlebnissport. [ONLINE-RESSOURCE: http://paed.unimuenchen.de/-eberle/erlebnissport/bgrerlsport.html Zugriff am 25.11.2004]

EBERS, N. (1995): Individualisierung. Georg Simmel - Norbert Elias - Ulrich Beck. Würzburg.

EBERSPÄCHER, H. (1988): Individuelle Handlungsregulation. Schorndorf.

EGNER, H. \& KLEINHANS, M. (2000): Trend- und Natursportarten - Ein Strukturierungsversuch. In: ESCHER, A./EGNER, H./KLEINHANS, M. (Hrsg.): Trendund Natursportarten in den Wissenschaften: Forschungsstand - Methoden - Perspektiven. Hamburg.

EGNER, H. (2000): Trend- und Natursportarten und Gesellschaft. In: ESCHER, A. / EGNER, H. / KLEINHANS, M. (Hrsg.): Trend- und Natursportarten in den Wissenschaften: Forschungsstand - Methoden - Perspektiven (S.7 - 20). Hamburg.

EIBL-EIBESFELD, I. (1986): Die Biologie des menschlichen Verhaltens (2. Auflage). München.

EIBL-EIBESFLED, I. (1988): Der Mensch - Das riskierte Wesen. München.

EICHBERG, H. (1983): Stimmung in der Heide - vom romantischen Blick zur Kolonisierung des Raumes. In: GROBKLAUS, G. \& OLDEMEYER, E. (Hrsg.): Natur als Gegenwelt. Beiträge zur Kulturgeschichte des Natur (S. 36-63). Karlsruhe.

EIMEREN, B. \& GERHARD, H. \& FREES, B. (2001): Entwicklung der Onlinenutzung in Deutschland: Mehr Routine, weniger Entdeckerfreude. ARD/ZDF-OnlineStudie 2001. In. Media Perspektiven 7 (2001), S. 382-397.

EIMEREN, B. \& GERHARD, H. \& FREES, B. (2002): Entwicklung der Onlinenutzung in Deutschland: Mehr Routine, weniger Entdeckerfreude. ARD/ZDF-OnlineStudie 2002. In. Media Perspektiven 8 (2002), S. 346-362.

ELIAN, N. (1976): Wandlung der Gesellschaft. Entwurf zu einer Theorie der Zivilisation. Band 2. Frankfurt am Main.

ELIAS, N. \& DUNNING, E. (1986): Quest for Excitment. Sport and Leisure in the civilizing process. Oxford.

ELIAS, N. \& DUNNING, E. (o.J.): Sport im Zivilisationsprozess. In HOPF, W. (Hrsg.): Studien zur Figurationssoziologie. Münster.

ELIAS, N. (1991): Über den Prozess der Zivilisation (16. Auflage). Frankfurt a. Main.

ELSÄSSER, A. (1979): Risiko im Sport. In RÖSCH, J. \& JAKOBI, H. (Hrsg.): Sport ohne Ethos (S. 64-85). Mainz.

ELZER, P.F. \& KLUWE, R.H., \& BOUSSOFFARA, B. (Hrsg.) (2000): Human error and system design and management. Lecture notes in control and information sciences 253. London 
EMBREY, D.E. \& HUMPHREYS, E.A., \&ROSA, E.A., \&KIRWAN, B., \& REA, K.(1984): SLIM-MAUD: An Approach to Assessing Human Error Probabilities Using Structured Expert Judgement, NUREGCR-3518. Washington DC.

ENDRUWEIT, G. \& TROMMSDORF, G. (1989): Wörterbuch der Soziologie. Band 1-3. Stuttgart.

ENGLER, M. \& MERSCH, J. (2000): SnowCard: Lawinen-Risiko-Check. Sulzberg.

ENGLER, M. (2001): SnowCard und Faktorencheck. In: bergundsteigen, 4(2001), S. 37 41.

EPSTEIN, S. (1977): Versuch einer Theorie der Angst. In: BIRBAUMER, N. (Hrsg.): Psychologie der Angst, S. 208-266. München.

ERDMANN, R. (1990): Sicherheit und Risikoerleben - Widerspruch? In KÖRNDLE, H. \& LUT'TER, H. \& THOMAS, A. (Hrsg.): Der Beitrag der Sportpsychologie zur Zielbestimmung einer modernen Erziehung und Ausbildung im Sport (S. 110 - 115). Köln.

ERDMANN, R. (1997): Leisten und pädagogische Verantwortung. In BALZ, E. \& NEUMANN, P. (Hrsg.): Wie pädagogisch soll der Schulsport sein? Auf der Suche nach fachdidaktischen Antworten (S. 79-92). Schorndorf.

EUERLING, J. (1990a): Suchet der Stadt Bestes. In: GABLER, H. / GÖHNER, U. (Hsrg.): Für einen besseren Sport. Festschrift für Ommo Grupe (S. 148 - 163). Schorndorf.

EULERING, J. (1990b): Sportgelegenheiten. In: Kultusministerium des Landes Nordrhein-Westfalen (Hrsg.): Sportgelegenheiten (S. 7 - 10). Düsseldorf.

EVERS, A. \& NOWOTNY, H. (1987): Über den Umgang mit Unsicherheit. Die Entdeckung der Gestaltbarkeit von Gesellschaft. Frankfurt a. Main.

EWALD, F. (1989): Die Versicherungs-Gesellschaft. In: Kritische Justiz, 22, S. 385-393.

FABRY, S. (1990): Die Angstlust als Phänomen der Gefühlsmischung und ihr Erleben über die Lebensspanne hinweg. Giessen.

FARLEY, E. (1991): The Type T personality with some implication for practice. In: The California Psychologist, 5 (1991), S. 381-188.

FAULHAMMER, E. (1994): Sicherheit und Risiko - verhaltenssteuernde Faktoren beim Bergsteigen? In JENNY, E. (Hrsg.): Jahrbuch der österreichischen Gesellschaft für Alpin- und Höhenmedizin 1994 (S. 83 - 91). Innsbruck.

FENICHEL, O. (1974): Psychoanalytische Neurosenlehre. Olten.

FENSKE, L. (1986): Adel und Rittertum im Spiegel früher heraldischer Formen ud deren Entwicklung. In: FLECKENSTEIN, J. (Hrsg.): Das ritterliche Turnier im Mittelalter (S. 75-162). Göttingen.

FERCHOFF, W. (1999).: Jugend an der Wende vom 20. zum 21. Jahrhundert. Opladen.

FINK, M. (1994): Angst als limitierender Faktor der Handlungsfähigkeit im Sport - am Beispiel des Kanu-Wildwasser-Unterrichts. Unveröffentlichte Examensarbeit / Göttingen.

FINNEY, B. \& HONSTON, J.D. (1996): Surfin: A History of the ancient Hawaiian Sport. San Francisco. 
FISCHER, E.P. (1991): Auf der Suche nach der verlorenen Sicherheit. München.

FISCHER, T. \& ZIEGENSPECK, J. (2000a)(Hrsg.): Handbuch Erlebnispädagogik. Von den Ursprüngen bis zur Gegenwart. Bad Heilbrunn.

FISCHER, T. \& ZIEGENSPECK, J.W. (2000b): Handbuch Erlebnispädagogik. Bad Heilbronn.

FLECKENSTEIN, J. (1986)(Hrsg.): Das ritterliche Turnier im Mittelalter. Göttingen.

FRANK, H. (1998): Die Entwicklung von Alpinistik und Wintersport in Österreich. In: BRUCKMÜLLER, E. \& STROHMEYER, H. (Hrsg.): Turnen und Sport in der Geschichte Österreichs (S. 86-104). Wien.

FRANKL, V.E. (1973a): Sport und Konflikt. In GRUPPE, O. (Hrsg.): Sport in unserer Welt (S. 85-89). Heidelberg.

FRANKL, V.E. (1973b): Symposium. In GRUPPE, O. (Hrsg.): Sport in unserer Welt (S. 28-37). Heidelberg.

FREY, J.H. (1991): Social Risk and the meaning if sport. In: Sociology of Sport Journal, 8, $136-145$.

FREY, N. (1987): Der Führerstaat. München.

FRIEDELL, E. (1982) : Kulturgeschichte der Neuzeit. Die Krisis der europäischen Seele von der schwarzen Pest bis zum ersten Weltkrieg 1. München.

FRITSCH, W. (1998): Geselligkeit. In: GRUPE, O. / MIETH, D. (Hrsg.): Lexikon der Ethik im Sport. (S. 201 - 204). Schorndorf.

FRÖHLICH, L. (1991): Die wahren Abenteuer erleben nur die Jungen. Mädchen stehen dem Helden bei. In: BRENNER, G. \& GRUBAUER, F. (Hrsg.): Typisch Mädchen? Typisch Junge? (S.120-122). Weinheim

FRY, C.B. (1905): C.B. Fry's Magazin of Sport and Outdoor Life. Vol. I. London.

FUCHS, W. et.al. (1988)(Hrsg.): Lexikon zur Soziologie. Opladen.

FUHRER, U. (1984): Mehrfachhandlungen in dynamischen Umfeldern. Göttingen.

GABLER, H. (2002): Motive im Sport. Motivationspsychologische Analysen und empirische Studien. Schorndorf.

GALLIAN, J. (1937): Held der Berge. In: Austria Nachrichten, 2 (1937), S. 24.

GEBAUER, G. \& ALKEMEYER, T. (2004): Treue zum Stil - die aufgeführte Gesellschaft. Bielefeld,

GEBKEN, U. (2001): Erziehung zum sozialen Handeln im Schulsport. Oldenburg.

GEBKEN, U. (2002): Geselligkeit und Gemeinschaft. In: DIECKERT, J. / WOPP, C. (Hrsg.): Handbuch Freizeitsport (S. 252 - 269). Schorndorf.

GEHLEN, A. (1950): Der Mensch. Seine Natur und seine Stellung in der Welt (4. Auflage). Frankfurt a. Main.

GEIGER, (1949): Die Klassengesellschaft im Schmelztiegel. Köln.

GEISS, P.H. (1991): Das Leben des Turnpädagogen Adolf Spieß. Ein Beitrag zur Geschichte des deutschen und schweizer Schulturnens. Idstein.

GEISSLER, R. (1994): Soziale Schichtung und Lebenschancen in Deutschland. Stuttgart. 
GELLERMANN, C. (1985): Der Sport in der ehemaligen deutschen Wehrmacht unter besonderer Berücksichtigung seiner historischen Entwicklung. In: BECKER, H. (Red.): Sport im Spannungsfeld von Krieg und Frieden (S. 122-144). ClausthalZellerfeld.

GETHMANN, C. \& KLOEPFER, M. (1993): Handeln unter Risiko im Umweltstaat. Berlin.

GIDDENS, A (1989): Sociology. Oxford.

GIDDENS, A. (1995): Konsequenzen der Moderne. Frankfurt / M.

GIDDENS, A. (1999): Der dritte Weg. Die Erneuerung der sozialen Demokratie.

Frankfurt / Main.

GIESEN, B. (1992): Die Postmoderne als Herausforderung der Gesellschaftstheorie", in: Wohin treibt die Moderne? Vorträge im Wintersemester 1991/92. Studium Generale an der Ruprecht-Karls-Universität Heidelberg (S. 23-36). Heidelberg.

GIEß-STÜBER, P. (1998): Unsicherheit macht Schule - Erlebnis, Abenteuer, Risiko im Schulsport? In ALLMER, H. \& SCHULZ, N. (Hrsg.): Erlebnissport - Erlebnis Sport (S.132-149). Sankt Augustin.

GIRTLER, R. (1995): Bergsteigen als Initiationsritus und die Suche nach dem Außeralltäglichen. In: KÖNIG, E. \& LUTZ, R. (Hrsg.): Bewegungskulturen. Ansätze zu einer kritischen Anthropologie des Körpers, S. 141-150. Sankt Augustin.

GLAS, B. (1995): Sportunterricht ohne Unfälle. Wiesbaden.

GLENDON, A. I. (1987): Risk Cognition. In: SINGLETON, W.T. \& HOVDEN, J. (Hrsg.): Risk and Decisions (S. 87 - 107). Chichester.

GLUCHOWSKI, P. (1988): Freizeit und Lebensstile: Plädoyer für eine integrative Analyse des Freizeitverhaltens. Erkrath.

GOFFMANN, E. (1994): Interaktionsrituale (3. Auflage). Frankfurt a. Main.

GÖTZ, J. (1996): Der Weltkrieg und meine Berge. In: ZEBHAUSEN, H. \& TRENTIN-MEYER, M. (Hrsg.): Zwischen Idylle und Tummelplatz (S.351-352). München.

GRAU, O. (2004): Richtig Wildwasserfahren. Riedering.

GREITBAUER, K. (1973): Das Ganze der alpinen Idee. Stuttgart.

GREITBAUER, K. (1977): Das unverstandene Bergsteigen. Zum geistigen Umfeld des Bergsteigens. Wien.

GRIESWELLE, D. (1978): Sportsoziologie. Stuttgart.

GROENEMEYER, A. (2001): Risikosoziologie und gesundheitsbezogenes Risikoverhalten - zwischen ,Empowerment' und ,Lifestyle Correctness'. In: RAITHEL, J. (Hrsg.): Risikoverhaltensweisen Jugendlicher Formen, Erklärungen und Prävention (S.31-60). Opladen.

GROPPE, H.H. (1987): Abenteuer und Eroberung. Was treibt die Männer in die Welt. In: MUSEUMSPÄDAGOGISCHER DIENST HAMBURG (Hrsg.): Männerbilder (S. 58-75). Hamburg.

GROSS, P. (1994): Die Multioptionsgesellschaft. Frankfurt / M.

GROSS, P. (1999): Ich-Jagd. Frankfurt am Main. 
GROßKLAUS, G. \& OLDEMEYER, E. (1983)(Hrsg.): Natur als Gegenwelt. Beiträge zur Kulturgeschichte der Natur. Karlsruhe.

GROßKLAUS, G. (1983): Der Naturraum des Kulturbürgers. In: GROßKLAUS, G. \& OLDEMEYER, E. (Hrsg.): Natur als Gegenwelt. Beiträge zur Kulturgeschichte der Natur (S. 169 -196). Karlsruhe.

GRUPE, O. \& KRÜGER, M (1997): Einführung in die Sportpädagogik. Schorndorf.

GRUPE, O. \& KRÜGER, M. (1998): Sport. In: GRUPE, O. \& MIETH, D. (Hrsg.): Lexikon der Ethik des Sports. Schorndorf.

GRUPE, O. (1988): Menschen im Sport 2000. Von der Verantwortung der Person und der Verpflichtung der Organisation. In: GIESELER, K.; GRUPE, O. \& HEINEMANN, K. (Hrsg.): Menschen im Sport 2000. Dokumentation des Kongresses „Menschen im Sport 2000“ (S. 44 - 66). Schorndorf.

GRUPE, O. (2000): Vom Sinn des Sports. Kulturelle, pädagogische und ethische Aspekte. Schorndorf.

GRYMER, H. (1989): Anmerkungen zum Begriff des Risikos in den Gesellschaftswissenschaften. In: COMPES, P.C. (Hrsg.): Risiko - subjektiv und objektiv (S. 133 171). Wuppertal.

GÜNTHER, D. (2003): Wandern und Sozialismus: Zur Geschichte des Touristenvereins die Naturfreunde im Kaiserreich und in der Weimarer Republik. Hamburg.

GUTTMANN, A. (1979) : Vom Ritual zum Rekord. Das Wesen des modernen Sports. Schorndorf.

HABERMAS, J. (1967): Soziologische Notizen zum Verhältnis von Arbeit und Freizeit. In: PLESSNER, H. / BOCK, H.E. / GRUPE, O. (Hrsg.): Sport und Leibeserziehung.: Sozialwissenschaftliche, pädagogische und medizinische Beiträge (S. 28 - 46). München.

HABERMAS, J. (1973): Arbeit, Freizeit, Konsum. Frühe Aufsätze. Gravenhage.

HABERMAS, J. (1980): Die Moderne - ein unvollendetes Projekt. Rede aus Anlass der Verleihung des Adorno-Preises der Stadt Frankfurt 1980. In: WELSCH, W. (Hrsg.): Wege aus der Moderne. Schlüsseltexte der Postmoderne. Berlin 21994, 177-192

HACKER, W. (1973): Allgemeine Arbeits- und Ingenieurpsychologie. Berlin.

HACKER, W. (1986): Arbeitspsychologie. Berlin.

HACKER, W. (1998): Arbeitspsychologie - psychische Regulation von Arbeitstätigkeiten. Bern.

HACKFORT, D. \& SCHWENKMEZGER, P. (1985): Angst und Angstkontrolle im Sport (2. Auflage). Köln.

HACKFORTH, J. (1993): Psychologie im Freizeitsport. In: GABLER, H. \& NITSCH, J.R. \& SINGER, R. (Hrsg.): Einführung in die Sportpsychologie. Teil 2: Anwendungsfehler (S. 188 - 203). Schorndorf.

HACKL, W. (2004): Eingeborene im Paradies. Die literarische Wahrnehmung des alpinen Tourismus im 19. und 20. Jahrhundert. Tübingen.

HAGEMANN-WHITE; C. (1984): Sozialisation: weiblich-männlich? Opladen. 
HAHN, H. (1983): Entstehung von Unfallsituationen im Wildwasser. In BAUR, J. (Hrsg.): Sicherheit im Kanusport. Schriftenreihe des Deutschen-Kanu-Verbandes e.V. (Band 2) (S.49-74). Duisburg.

HAHN, K. (1957): Hoffnung und Sorgen eines Landeserziehungsheimes. In HAHN, K.: Erziehung zur Verantwortung. Stuttgart; zit. nach SCHLESKE, W. (1977): Abenteuer - Wagnis - Risiko im Sport. Schorndorf.

HAHN, K. (1958): Erziehung zur Verantwortung. Stuttgart.

HALE, A.R. \& GLENDON, A. (1987): Individual Behavior in the Control of Damger. Amsterdam.

HALLER, M. (1998): Gesellschaft als Risiko? Zur Rolle der Versicherer in der gesellschaftlichen Risikodebatte. In: GESAMTVERBAND DER DEUTSCHEN VERSICHERUNGSWIRTSCHAFT (Hrsg.): Wieviel Risiko braucht die Gesellschaft? (S. 221 - 266). Berlin.

HANSEN, P.H. (1991): British Mountaineering 1850-1914. Cambridge.

HANSEN, P.H. (1995): Albert Smith, the Alpine Club and the invention of Mountaineering in Mid-Victorian Britain. In: Journal of British Studies, 34 (1), S. 300 - 324.

HARTGEN, H.-P. (1997): Unfall ist kein Zufall - Eine Studie über den Zusammenhang von Lebensereignissen und Lebensläufen mit Unfällen. Dissertation München.

HARTMANN, H. \& HAUBL, R. (1996): ,Erlebe Dein Leben'. In HARTMANN \& HAUBL (Hrsg.): Freizeit in der Erlebnisgesellschaft: Amüsement zwischen Selbstverwirklichung und Kommerz (S. 7-19). Opladen.

HARTMANN, H.A. (1995): „Nicht das ganze Leben lang im Bett liegen bleiben...“: Zur Analyse und Bewertung von Fun- und Extremsport. In: HARTMANN, H.A. \& HEYDENREICH, K. (Hrsg.): Verdammt viel Vergnügen! Beiträge zum Freizeitverhalten Jugendlicher, S. 34-51. Frankfurt am Main.

HARTMANN, H.A. (1996): The thrilling field, oder: „Bis ans Ende und dann noch weiter“. In HARTMANN, H. \& HAUBL, R. (Hrsg.): Freizeit in der Erlebnisgesellschaft: Amüsement zwischen Selbstverwirklichung und Kommerz (S.67-94). Opladen.

HARTMANN-TEWS, I. \& CACHAY, K. (1998): Soziale Ungleichheit und Sport eine Einführung. In: CACHAY, K. \& HARTMANN-TEWS, I. (Hrsg.): Sport und soziale Ungleichheit: Theoretische Überlegungen und empirische Befunde (S. 1 - 8). Stuttgart.

HAUBL, R. (1998): Des Kaisers neue Kleider? Struktur und Dynamik der Erlebnisgesellschaft. In ALLMER, H. \& SCHULZ, N. (Hrsg.): Erlebnissport - Erlebnis Sport (S.5-28). Sankt Augustin.

HAUPTMANNS, T. \& KNETSCH, M. (2004): Gefährdungsbäume zur Analyse von Unfällen und Gefährdungen. Bremerhaven.

HEBB, D.O. (1955): Drives and the C.N.S. In: Psychological Review, 62, S. 243-254.

HECKER, G. (1989): Abenteuer und Wagnis im Sport - Sinn oder Unsinn? Deutsche Zeitschrift für Sportmedizin, 40 (9), 328-331.

HECKER, G. (1991): Dosiertes Risiko im Sport als Sicherheitserziehung. In REDL, S. \& SOBOTKA, R. \& RUSS, A. (Hrsg.): Sport an der Wende (S. 251-231). Wien. 
HECKER, G. (1993): Vom guten Sinn der Unsicherheit. In PROHL, R. (Hrsg.): Facetten der Sportpädagogik (S.49-55). Schorndorf.

HECKHAUSEN, H. (1977): Entwurf einer Psychologie des Spielens. In HECKHAUSEN, H \& GRAUMANN, H. (Hrsg.): Pädagogische Psychologie, Band 1 (4. Auflage) (S.155-175). Frankfurt a. Main.

HECKHAUSEN, H. (1989): Motivation und Handeln. Berlin, Heidelberg. 2. Auflage.

HECKMEIER, B. \& MICHL, W. (2002): Erleben und Lernen. Einstieg in die Erlebnispädagogik. 4. überarbeitete Auflage. Neuwied.

HEEKERENS, H.-P. (1995): Risiko und Gefahr. In KÖLSCH, H. (Hrsg.): Wege moderner Erlebnispädagogik (S. 54-76). München.

HEGEL, G.W.F. (1986): Phänomenologie des Geistes. Frankfurt.

HEICHEL, W. (2003): Chronik der Erschließung des Karakorum. München.

HEIL, J. \& HENSCHEN, K. (1996): Assessment in Sport and Exercise Psychology. In: VAN RAALTE, J.L. \& BREWER, B.W. (Hrsg.): Sport and Exercise Psychology (S.229-256). Washington D.C.

HEINEMANN, K. \& SCHUBERT, K. (2001)(Hrsg.): Sport und Gesellschaften. Schorndorf.

HEINEMANN, K. (1983): Einführung in die Soziologie des Sports. 2. überarbeitete Auflage. Schorndorf.

HEINEMANN, K. (1989): Der „Nicht-Sportliche Sport“. In: DIETRICH, K. \& HEINEMANN, K. (Hrsg.): Der nicht-sportliche Sport (S. 11 - 28). Schorndorf.

HEINEMANN, K. (1990): Einführung in die Soziologie des Sports (3. Auflage). Schorndorf.

HEITZELHOFER, K. (1979a): Appetenz-Aversions-Konfliktverhalten bei Anfängern in Risikosportarten. In BÄUMLER, G. (Hrsg.): Aktuelle Probleme der Sportpsychologie (S. 123-126). Schorndorf.

HEITZELHOFER, K. (1979b): Zur Bewältigung der Angst in Risikosportarten. In GABLER; H. \& EBERSPÄCHER, H. \& HAHN, E. \& KERN, J. \& SCHILLING, G. (Hrsg.): Praxis der Sportpsychologie im Leistungssport (S. 329- 336). Berlin, München, Frankfurt a. Main.

HELFFERICH, C. (2001): Jugendliches Risikoverhalten aus geschlechtspezifischer Sicht. In: RAITHEL, J. (Hrsg.): Risikoverhaltensweisen Jugendlicher Formen, Erklärungen und Prävention (S.331-348). Opladen.

HENKE, T.: Sportunfälle im Vereinssport - Epidemiologie in verschiedenen Lebensabschnitten. In: «Sport - mit Sicherheit mehr Spass», Kongressband 3. Dreiländerkongress Schweiz Deutschland - Österreich vom 19.-21. September 2004 (S. 22 28). Magglingen.

HENKEL, O. (1987): Sportjahrbuch 1987/1988: Sportstatistik-Jahrbuch; das Sportjahr von A-Z. Rodgau.

HENKEL, O. (1998): Sportjahrbuch 1998/1999: Sportstatistik-Jahrbuch; das Sportjahr von A-Z. Rodgau.

HENKEL, O. (2000) :Sportjahrbuch 2000: Sportstatistik-Jahrbuch; das Sportjahr von A-Z. Rodgau. 
HENNING, C. (1997): Reiselust. Touristen, Tourismus und Urlaubskultur. Frankfurt a.M.

HENZE, W. (1998): Pflichtsport und Wehrsport an den deutschen Hochschulen in der Zeit des Nationalsozialismus. Die Gratwanderung eines Systemgegners zwischen Fremdbestimmung und Eigenständigkeit (Dr. Bernhard Zimmermann, Universität Göttingen). In: LANGENFELD, H. \& NIELSEN, S. (Hrsg.): Beiträge zur Sportgeschichte Niedersachsens (S. 149-163). Hoya.

HERBART, J. F. (1965): Allgemeine Pädagogik aus dem Zweck der Erziehung abgeleitet. Bochum.

HERZBERG, P.Y. \& SCHLAG, B. (2003): Sensation Seeking und Verhalten im Strassenverkehr. In: ROTH, M. \& HAMMELSTEIN, P. (Hrsg.): Sensation Seeking Konzeption, Diagnostik und Anwendung (S. 162 - 182). Göttingen.

HINRICH, S. \& WORTHMUTH, L. \& MUSAHL, H.P. (2002): Gefährdungsbeurteilung - objektive Analyse oder subjektive Gefährdungseinschätzung. In: TRIMPOP, R. (Hrsg):: Psychologie der Arbeitssicherheit und Gesundheit. Neue Welten - Alte Welten. Heidelberg.

HINRICHS, S. \& WORTMUTH, L. \& MUSHAL, H.P. (2002): Gefährdungsbeurteilung - Objektive Analyse oder subjektive Gefährdungseinschätzung? In: R. TRIMPOP, B.; ZIMOLONG \& A. KALVERAM (Hrsg.). Psychologie der Arbeitssicherheit und Gesundheit. Neue Welten - Alte Welten. 11. Workshop 2001. Heidelberg.

HINSCHING, J. (1995): Zur Einführung in das Thema. In: HINSCHING, J. \& BORKENHAGEN, F. (Hrsg.): Modernisierung und Sport, S. 7-12. Sankt Augustin. HIRN, A. (1936): Ursprung und Wesen des Sports. Berlin.

HIRSCHBICHLER, A.A. (1987): Aspekte der Persönlichkeit im Risikosport. Salzburg. HITZLER, R. \& HONER, A. (1994): Bastelexistenz. Über subjektive Konsequenzen von Individualisierung. In: BECK, U. \& BECK-GERNSHEIM, E. (Hrsg.): Riskante Freiheiten (S.307-315). Frankfurt a. Main.

HLAVAC, C. \& BAUMGARTNER, C. (2000): Trend- und Extremsportarten in Österreich. Reihe „Verkehr und Infrastruktur“ Heft Nr. 8, Arbeiterkammer Wien.

HOBERMAN, J. (2005): Testosteron Dreams. Berkeley.

HÖLLER, P. (2004): Strategische Methoden und Lawinenrisiko. In: KURATORIUM FÜR ALPINE SICHERHEIT (Hrsg.): Sicherheit im Bergland. Jahrbuch 2004 (S: 215-224). Innsbruck.

HOLLNAGEL, E. (1998): CREAM - Cognitive Reliability and Error Analysis Method, Elsevier Science. Oxford.

HÖRNING, K.H. \& GERHARD, A. \& MICHAILOW, M. (1990): Zeitpioniere: flexible Arbeitzeiten - neuer Lebensstil. Frankfurt am Main.

HORX, M. (1991): Trendbuch. München.

HORX, M. (1999): Die acht Sphären der Zukunft. München.

HÖRZ, H. (1980): Zufall, eine philosophische Untersuchung. Berlin.

HOYOS, C.G. (1965):Verkehrsverhalten und Persönlichkeit. In HOYOS, C.G. (Hrsg.): Psychologie des Straßenverkehrs (s. 77- 135). Bern. 
HOYOS, C.G. (1980): Psychologische Unfall- und Sicherheitsforschung. Stuttgart, Berlin, Köln, Mainz.

HOYOS, C.G. (1987): Verhalten in gefährlichen Arbeitssituationen. In KLEINBECK, U. \& RUTENFRANZ, J. (Hrsg.): Enzyklopädie der Psychologie. Band 1: Arbeitspsychologie (S. 577- 627). Göttingen.

HRADIL, S. (1992): Zwischen Bewusstsein und Sein. Die Vermittlung objektiver Lebensbedingungen und subjektiver Lebensweisen. Opladen.

HRADIL, S. (1995): Die Single-Gesellschaft. München.

HRADIl, S. (1996)(Hrsg.): Zwischen Differenz und Integration. Zur Zukunft modernen Gesellschaften. Opladen.

HUBER, A. (1994): Das Leben als Thriller: Nervenkitzel oder Glücksache? In: Psychologie beute, 21(6), 64-69.

HUBER, O. \& BEUTTER, C. \& MONTAYA, J. \& HUBER, O.W. (2001): Risk defusing behavior: towards an understanding of risky decsion making. In: The European Journal of Cognitive Psychology, 13 (409-426).

HÜBNER, H. \& HUNDELOH, H. (1997): Zehn Jahr „Mehr Sicherheit im Schulsport": Erfahrungen und Erkenntnisse zum Unfallgeschehen im Schulsport des Landes Nordrhein-Westfahlen. Münster.

HÜBNER, H. \& MIRBACH, A. (1991): Das Unfallgeschehen im Schulsport allgemeinbildender Schule in Westfalen-Lippe. Ein Zwischenbericht eines Forschungsprojektes - Ein Beitrag zur Landesinitiative ,Mehr Sicherheit im Schulsport'. Münster.

HÜBNER, H. (1997): Neue Ergebnisse aus handlungsorientierten Analysen zu Schulsportunfällen - ein Überblick. In: HÜBNER, H. \& HUNDELOH, H. (Hrsg.): Zehn Jahr „Mehr Sicherheit im Schulsport“: Erfahrungen und Erkenntnisse zum Unfallgeschehen im Schulsport des Landes Nordrhein-Westfahlen (S. 52-72). Münster.

HUGGER, P (1991): Pubertätsriten - einst und jetzt - aus der Sicht des Volkskundlers. In: KLOSINSKI, G. (Hrsg.): Pupertätsriten. Äquivalente und Defizite in unserer Gesellschaft. Bern.

HUMPHREYS, D. (1997): ,Shreadheads go mainstream'? Snowboarding and alternative youth. In: International Review for the sociology of sport, 32 (2), S. 147-160.

HÜRLIMANN, F.W. \& HEBENSTREIT, B. (1987): Verkehrssicherheit in der Praxis. Stuttgart.

HURRELMANN, K. (1989): Entwicklung, Sozialisation und Gesundheit - Überlegungen zu einer integrativen Theoriebildung. In: BRET'TSCHNEIDER, W.D. et.at. (Red.): Bewegungswelt von Kindern und Jugendlichen (S.18-30). Schorndorf.

HURRELMANN, K. (1999): Lebensphase Jugend. Eine Einführung in die sozialwissenschaftliche Jugendforschung. Weinheim.

HURRELMANN, K. (2000): Entwicklungsprobleme und Problemverhalten aus sozialwissenschaftlicher Perspektive. In: Pädagogikunterricht 1/20 (S. 8-14).

INGLEHART, R. (1995): Kultureller Umbruch: Wertewandel in der westlichen Welt. Frankfurt am Main. 
INGLEHART, R. (1997): Modernisierung und Postmodernisierung. Kultureller, wirtschaftlicher und politischer Wandel in 43 Gesellschaften. Frankfurt am Main.

INSTITUT FÜR INTEGRATIVEN TOURISMUS UND FREIZEITFORSCHUNG (Autoren: HLAVAC, C. \& BAUMGARTNER, C.): Trend- und Extremsportarten in Österreich. In: Verkehr und Infrastruktur Nr. 8. Wien.

INSTITUT FÜR SPORTSOZIOLOGIE / KVR (2000): Trendsport im Ruhrgebiet. [Online-Ressource: http://www.rvr-online.de/freizeit/marketing/bindata/Trendsport_2000.pdf Zugriff am 12.04.2003].

JAEGER, J. \& SCHERINGER, M. (1998): Transdisziplinarität: Problemorientierung ohne Methodenzwang. In: GALA 7 (1998), S. 10-25.

JAHN, G. (1992): Friedrich Ludwig Jahn. Volkserzieher und Vorkämpfer für Deutschlands Einigung. Göttingen.

JAMES, W. (1911): The moral Equivalent of war. In: JAMES, W. (Hrsg.): Memories and studies (S. 265 - 296). London.

JANTZEN, R. (1988): Montagne et symboles. Lyon.

JAPP, P. (2000): Risiko. Bielefeld.

JELDEN, E. (1998): Risiken sind gewusstes Nichtswissen. In KÖRBER STIFTUNG (Hrsg.): Risiko, Umgang mit Sicherheit, Chance und Wagnis (S.14-16). Hamburg.

JERUSALEM, M. \& PEKRUN, M. (1999)(Hrsg.): Emotion, Motivation und Leistung. Göttingen.

JESSOR, R. (2001): Problem-Behavior Theory. In: RAITHEL, J. (Hrsg).: Risikoverhaltensweisen Jugendlicher. Formen, Erklärungen und Prävention (S. 61-78). Opladen.

JOSI, W. (1991): Tourenplanung und Tourenauswertung. In: Magglingen, 48 (6), 5-8.

JUNGE, M. (2002): Individualisierung. Frankfurt am Main.

JUNGERMAN, H. \& PFISTER, H. R. \& FISCHER, K. (1998): Die Psychologie der Entscheidung: Eine Einführung. Heidelberg; Berlin.

JUNGERMANN, H. \& ROHRMANN, B. \& WIEDEMANN, P.M. (1991): RisikoKontroversen. Berlin.

JUNGERMANN, H. \& SLOVIC, P. (1987): Charakteristika individueller Risikowahrnehmung. In: BAYRISCHE RÜCK (Hrsg.): Gesellschaft und Unsicherheit (S.89107). Karlsruhe.

JÜT'TING, D.H. (1987): Sportentwicklung und Alltagskultur. In: DSB (Hrsg.): Sportentwicklung und Alltagskultur (S. 9 - 20). Frankfurt am M.

KAMINSKI, G. (1973): Bewegungshandlungen als Bewältigung von Mehrfachaufgaben. In: Sportwissenschaft 3 (1973), 2, S. 233-250.

KAMPER, D. (1992): Sport als extreme Erfahrung. In ALKEMEYER, H. u.a. (Hrsg.): Aspekte einer zukünftigen Anthropologie des Sports (S.100-107). ClausthalZellerfeld.

KARGE, K. (1993): Angst und Angstverarbeitung. Eine Untersuchung an Fallschirmspringern. Hannover.

KASCHUBA, W. (1989): Sportivität: Karriere eines neuen Leitwertes. In: Sportwissenschaft 19, (2), S. $154-171$. 
KATTERMANN, V. (1999): Stierkampf in Spanien. Männlichkeit zwischen Inszenierung und Alltag. In: Psychologie und Gesellschaftskritike, 23,1-2, S. 29-52.

KAUFMANN, F.X. (1973): Sicherheit als soziologisches und sozialpolitisches Problem (2. Auflage). Stuttgart.

KENNEDY, A.L. (2001): Stierkampf. Berlin.

KERR, J. (1991): Arousal-Seeking in Risks Sport Participants. In: Personality and Individual Differences (GB), 12 (6), 613-616.

KEUPP, H. \& HOBELSBERG, H. (1999): Kann man heute noch erwachsen werden? Identitätssuche und Identitätsentwicklung. In: Katechistische Blätter 6/99, S. 375-380. München.

KEUPP, H. \& HÖFER, R. (Hrsg.)(1997): Identitätsarbeit heute. Frankfurt.

KEUPP, H. (1994a): Ambivalenzen postmoderner Identität. In BECK, U. \& BECKGERNSHEIM, E. (Hrsg.): Riskante Freiheiten (S.336-353). Frankfurt a. Main.

KEUPP, H. (1994b): Psychologisches Handeln in der Risikogesellschaft. Gemeindepsychologische Perspektiven. München.

KEUPP, H. (2000): Eine Gesellschaft der Ich-Linge? Zum bürgerschaftlichen Engagement von Heranwachsenden. München.

KIPPELE, F. (1998): Was heißt Individualisierung? Die Antworten soziologischer Klassiker. Opladen.

KIPPHARDT, E.J. (1995): Ungewöhnliche Körpererfahrungen als Nervenkitzel und Abenteuer. In PAWELKE, R. (Hrsg.): Neue Sportkultur (S. 334-344). Lichtenau.

KIRCHNER, H.J: (1996): Gefahr, Gefährdung, Unfall. In: WENNINGER, G. / HOYOS, C. GRAF (Hrsg.): Arbeits-, Gesundheits- und Umweltschutz - Handwörterbuch verhaltenswissenschaftlicher Grundbegriffe (S. 397 - 406). Heidelberg.

KLAGES, H. (1981): Überlasteter Staat - verdrossene Bürger? Zu den Dissonanzen der Wohlfahrtsgesellschaft. Frankfurt / Main.

KLAGES, H. (1984): Wertorientierungen im Wandel. Rückblick, Gegenwartsanalysen, Prognosen. Frankfurt am Main.

KLAGES, H. (1988)(Hrsg.): Wertedynamik. Über die Wandelbarkeit des Selbstverständlichen. Frankfurt am Main.

KLAGES, H. (1999): Wertewandel und bürgerliches Engagement an der Schwelle zum 21. Jahrhundert. Speyer.

KLEBELSBERG, D. (1969): Risikoverhalten als Persönlichkeitsmerkmal. Bern

KLEBELSBERG, D. (1982): Verkehrspsychologie. Heidelberg.

KLEBELSBERG; D. (1965): Analyse des Verkehrsverhaltens. In HOYOS, C.G. (Hrsg.): Psychologie des Straßenverkehrs (S. 15- 77). Bern.

KLEIN, M. \& PÖTSCH, M. (2000): Wertewandel - gibt es einen Wertewandel hin zum reinen Postmaterialismus? In: Zeitschrift für Soziologie, 29 (2000), 3, S. 202 - 216.

KLEINBECK, U. (1987): Gestaltung von Motivationsbedingungen der Arbeit. In: KLEINBECK, U. \& RUTENFRANZ, J. (Hrsg.): Arbeitspsychologie. Enzyklopädie der Psychologie, Themenbereich D, Serie III, Bd. 1 (S. 440 - 492). Göttingen.

KLEINE, W. \& FRITSCHE, W. (Hrsg.) (1990): Sport und Geselligkeit. Aachen. 
KLEINER, K. \& AMESBERGER, G. \& SOBOTKA, R. \& SCHMIDT, M. (1990): Mehrdimensionale Analyse schwerer Unfälle im Unterricht Leibesübungen. In: Spectrum der Sportwissenschaft 2 (1990), 1 (S. 41-84).

KLEINHEMPEL, F. (1996): Die biopsychosoziale Einheit Mensch. Bielefeld.

KLOCKE, A. (1993): Sozialer Wandel, Sozialstruktur und Lebensstile in der Bundesrepublik Deutschland. Frankfurt am Main.

KNEISSL, O. (1984): Bemerkungen zur Vorbeugung von Sportverletzungen und Sportschäden im Schulsportbetrieb. In: Leibesübungen - Leibeserziehung, 38 (1984), 6 (S.144-149).

KOCH, J. (1994): Abenteuer und Risiko als pädagogische Kategorie. In: Sportpädagogik, 18, (5). S. 23-37.

KOCH, P. (1979): Was bringt die Beschäftigung mit Versicherungsgeschichte? In: I.VW (Hrsg.): Beträge zur Sicherheitsökonomik. Heft 3 (S. 11). St. Gallen.

KÖCK, C. (1990): Sehnsucht Abenteuer. Berlin.

KOGAN, N. \& WALLACH, M.A. (1964): Risk taking. New York.

KÖHLER, A. (1995): Frauenbergsteigen. Auf der Suche nach einer vergessenen Seite der alpinen Geschichte. In: Berg'95 Alpenvereinsjahrbuch, Jahrgang 1995, S. 161 168.

KÖHLER, E. (1970): Ideal und Wirklichkeit der höfischen Epik. Tübingen.

KÖHLER, T. (1995): Freuds Psychoanalyse: eine Einführung. Stuttgart.

KOHLI, M. (1989): Institutionalisierung und Individualisierung der Erwerbsbiographie. Aktuelle Veränderungstendenzen und ihre Folgen. In: BROCK, D. (Hrsg.): Subjektivität im gesellschaftlichen Wandel, S.?-? München.

KÖHNE, C.I. (2003): Familiäre Erziehungsziele zu Beginn des 21. Jahrhunderts. Eine internetbasierte Befragung von Müttern. Diss. Essen.

KOHUT, H. (1981): Narzissmus (3. Auflage). Frankfurt am Main.

KOLB, M. (1994): Methodische Prinzipien zur Entwicklung der Körperwahrnehmung. In: SCHIERZ, M. / HUMMEL, A. / BALZ, E. (Hrsg.): Sportpädagogik. Orientierungen - Leitideen - Konzepte (S: 239 - 260). Sankt Augustin.

KOLB, M. (2002): Körperzentrierung und Selbsterfahrung. In: DIECKERT, J. \& WOPP, C. (Hrsg.): Handbuch Freizeitsport. (S. 278-289). Schorndorf.

KONIETZKA, D. (1994): Individualisierung, Entstrukturierung und Lebensstile. Zu einigen konzeptionellen Fragen der Analyse von Lebensstilen. In: DANGSCHAT, J. \& BLASIUS, J. (Hrsg.): Lebensstile in den Städten. Konzepte und Methoden. Opladen, S. $150-168$.

KÖNIG, R. (1973): Handbuch der empirischen Sozialforschung (Geschichte und Grundprobleme Bd. 1). Stuttgart.

KRAFT, S. (1992): Modernisierung und Individualisierung. Eine kritische Analyse ihrer Bestimmungen. Regensburg.

KRÄMER, W. (1998): Risiko-Homöostase oder warum sichere Autos gefährlich sind. In KÖRBER STIFTUNG (Hrsg.): Risiko, Umgang mit Sicherheit, Chance und Wagnis, (S. 23-24). Hamburg. 
KRAUSE, D \& SCHÄUBLE, G. (1988): Jenseits von Klasse und Schicht. Stuttgart. KRIPPENDORF, J \& KRAMER, B. \& KREBS, R. (1984): Arbeitsgesellschaft im Umbruch - Konsequenzen für die Freizeit und Reisen. Bern.

KROHNE, H.W. (1996): Angst und Angstbewältigung. Stuttgart.

KROMREY, H. (1998): Empirische Sozialforschung : Modelle und Methoden der

Datenerhebung und Datenauswertung. 8. überarbeitete Auflage. Opladen.

KROMREY, H. (1998): Empirische Sozialforschung, 8.Auflage. Opladen.

KRÜGER, A. \& CARTER, E. (1990)(Hrsg.): Ritual and Record. New York.

KRÜGER, A. \& McCELLAND, G. (1984)(Hrsg.): Die Anfänge des modernen Sports in der Renaissance. London.

KRÜGER, A. \& VON LOJEWSKI, F. (1998): Ausgewählte Aspekte des Wehsports in Niedersachsen in der Weimarer Zeit. In: LANGENFELD, H. \& NIELSEN, S. (Hrsg.): Beiträge zur Sportgeschichte Niedersachsens (S. 124-148). Hoya.

KRÜGER, A. (1983): Die Rolle des Sports bei den Kriegsvorbereitungen des nationalsozialistischen Deutschlands. In: GÜLENPFENNIG, S. \& MEYER, H. (Hrsg.): Sportler für den Frieden (S. 137-152). Köln.

KRÜGER, A. (1996): Sport, Kommerzialisierung und Postmoderne. In: H. SARKOWICZ (Hrsg.): Schneller, Höher, Weiter. Eine Geschichte des Sports (S.390 - 406). Frankfurt am Main.

KRÜGER, A. (2000): Wilhelm Henze. Eine Würdigung. In: KRÜGER, A. \& WEDEMEYER, B. (Hrsg.): Aus Biographien Sportgeschichte lernen. Festschrift zum 90. Geburtstag von Prof. Dr. Wilhelm Henze (S.18-25). Hoya.

KRÜGER, M. (1993): Einführung in die Geschichte der Leibeserziehung und des Sports. Teil 2: Leibeserziehung im 19. Jahrhundert. Turnen fürs Vaterland. Schorndorf.

KRÜGER, M. (2004): Einführung in die Geschichte der Leibeserziehung und des Sports. Teil 1: Von den Anfängen bis ins 18. Jahrhundert. Schorndorf.

KRUPKA, K. (1999): Wildwasser-Geschichten aus dem Tessin und anderswo. In: Kanusport, 68 (11), 484- 487.

KUHLMANN, D. (2000): Methoden qualitativer Sozialforschung in der Sportwissenschaft. In: Sportwissenschaft, 30, 1 (2000), S. 20-39.

KUHN, G. \& TODT, E. (2003): Physiologische und psychologische Aspekte des Risikoverhaltens und des Risikoerlebens. In: GISSEL, N. \& SCHWIER, J. (Hrsg.): Abenteuer, Erlebnis und Wagnis. Perspektiven für den Sport in Schule und Verein? (S. 11-25). Gießen.

KUHN, G. (2001): Sportinteresse in der 2. Lebensdekade. Ergebnisse einer Retrospektivuntersuchung. In: SEILER, D. \& BIRRER, D. \& SCHMID, J. \& VALKANOVER, S. (Hrsg.): Sportpsychologie. Anforderungen-AnwendungenAuswirkungen (S.69-71). Köln.

KUNZ, T. (1992): Psychomotorische Förderung - ein neuer Weg der Unfallverhütung im Kindes- und Jugendalter. In: ZIMOLONG, B. \& TRIMPOP, R. (Hrsg.): Psychologie der Arbeitssicherheit 6. Workshop 1991 (S. 319 - 331). Heidelberg.

KUNZ, V. (2004): Rational Choice. Frankfurt am Main. 
KURRAS, L. (1992): Ritter und Turniere. Stuttgart.

KURZ, D. (1986): Vom Sinn des Sports. In DSB (Hrsg.): Die Zukunft des Sports. Materialien zum Kongress Menschen im Sport 2000 (S. 44 - 68). Schorndorf.

KURZ, D. (1988): Was suchen Menschen im Sport? Erwartungen und Bedürfnisse der Zukunft. In: BECKER, H. / GRUPE, O. (Red.): Menschen im Sport 2000 (S. 126 139). Schorndorf.

KUSZ, K. (2004): Extreme America: the cultural politics of extreme sports in 1990's America. In: WHEATIN, B. (Hrsg.): Understanding lifestyle sports (S.197-214). London

KUT'TER, P. (2000): Moderne Psychoanalyse: eine Einführung in die Psychologie unbewusster Prozesse (3. Auflage). Stuttgart.

LAASER, U. \& HURRELMANN, K. \& WOLTERS, P. (1993): Prävention, Gesundheitsförderung und Gesundheitserziehung. In: HURRELMANN, K. \& LAASER, U. (Hrsg.): Gesundheitswissenschaften. Handbuch für Lehre, Forschung und Praxis (S. 176 - 206). Weinheim.

LAMMER, E.G. (1907): Von alpiner Tat und alpinem Stil. In: Mitteilungen des Österreichischen Alpenvereins, o.J. (1907), ohne Seitenangaben.

LAMMER, E.G. (1908): Zur Psychologie des Alpinisten. In: Mitteilungen des Österreichischen Alpenvereins, o.J. (1908), ohne Seitenangaben.

LAMMER, E.G. (1925): Alpinismus und Sport. In: Der Bergsteiger, 50 (1925), ohne Seitenangaben.

LAMPRECHT, M. \& STAMM, H. (2002): Sport zwischen Kultur, Kult und Kommerz. Zürich.

LAMPRECHT, M. \& STAMM, H. (1994): Die soziale Ordnung der Freizeit. Zürich.

LAMPRECHT, M. \& STAMM, H. (1998): „Vom avantgardistischen Lebensstil zur Massenfreizeit: Eine Analyse des Entwicklungsmusters von Trendsportarten“. In: Sportwissenschaft 28(3-4): 370-387.

LAMPRECHT, M. \& MURER, K. \& STAMM, H. (2003): Die Genese von Trendsportarten - zur Wirkung von Institutionalisierungs- und Kommerzialisierungsprozessen. In: BREUER, C. / MICHELS, H. (Hrsg.): Trendsport: Modelle, Orientierungen und Konsequenzen (S. 33 - 50). Aachen.

LAMPRECHT, M. \& STAMM, H. (2003): Die Genese von Trendsportarten - Zur Wirkung von Institutionalisierungs- und Kommerzialisierungsprozessen". In: BREUER, C. \& MICHELS, H (Hrsg.):Trendsport. Modelle, Orientierungen und Konsequenzen (S. 33-50). Aachen.

LANGE, T. (1976): Idyllische und exotische Sehnsucht. Formen bürgerlicher Nostalgie in der deutschen Literatur des 18. Jahrhunderts. Kronberg/Ts.

LARCHER, M. (1999): Stop or Go - Entscheidungsstrategien für Tourengeher. In: bergundsteigen 4 (1999), S. 18-23).

LASH, S. (1991): Sociology of postmodernism. London.

LAZARUS, R.S. \& AVERILL, J.R. \& OPTON, E.M. (1977): Ansatz zu einer kognitiven Gefühlstheorie. In BIERBAUMER, N. (Hrsg.): Psychophysiologie der Angst (2. Auflage) (S. 182-205). München. 
LAZARUS, R.S. \& AVERILL, J.R. (1972): Emotion and cognition. With special refernde to anxiety. In: SPIELBERGER, C.D. (Hrsg.): Anxiety: Current trends in theory and research, S. $242-283$. New York.

LAZARUS, R.S. \& LAUNIER, R. (1981) : Streßbezogene Transaktionen zwischen Personen und Umwelt. In NITSCH, J.R. \& HACKFORT, D. (Hrsg.): Streß, Theorien, Untersuchungen, Maßnahmen (S.213-259). Bern.

LE BRETON, D. (1995): Lust am Risiko : Vom Bungee-jumping, U-Bahn-Surfen und anderen Arten das Schicksal herauszufordern. Frankfurt am Main.

LE BRETON, D. (2001): Riskantes Verhalten Jugendlicher als individueller Übergangsritus. In: RAITHEL, J. (Hrsg.): Risikoverhaltensweisen Jugendlicher Formen, Erklärungen und Prävention (S.11-130). Opladen.

LEHR, U. (1994): Der demographische Wandel: Langlebigkeit verpflichtet. In: BÖS, K. / WOLL, A. / BÖSING, L. / HUBER, G. (Hrsg.): Gesundheitsförderung in der Gemeinde. Schorndorf.

LEISERING, L. (1999): Eine Frage der Gerechtigkeit. Verteilung von Arm und Reich in der Bundesrepublik Deutschland. In: Aus Politik und Zeitgeschichte, 49 (1999), 18, S. 10-17.

LEISI, E. (1979): Ist Verhütung von Gefahren immer gut? Prävention als Kulturproblem. Neue Züricher Zeitung vom 20.10.1979; zit. nach HIRSCHBLICHLER A.A. (1987): Aspekte der Persönlichkeit im Risikosport. Salzburg.

LEJEUNE, D. (1988): Les “Alpinistes” en France (1875-1919). Paris.

LEMKE, A. (2000): Imagination kolonialer Helden am Beispiel westlicher Großwildjäger in Afrika. Abschlussarbeit der Philosophischen Fakultät der Universität zu Köln.

LENK, H. (1983): Die Macher der Natur? In: GROßKLAUS, G. \& OLDEMEYER, E. (Hrsg.): Natur als Gegenwelt. Beiträge zur Kulturgeschichte der Natur (S. 59 - 86). Karlsruhe.

LENK, K . (1985) : „Den wirklichen Gipfel werde ich nie erreichen“. Kulturphilosophische Bemerkungen zu Erlebnis und Erorberung im extremen Alpinismus. In: DEUTSCHER ALPENVEREIN (Hrsg.): Berg 85 (Alpenvereinsjahrbuch), S. 103 114. München.

LERCHER, A. (2001): Gruppen \& Dynamik. Das Spannungsfeld zwischen Individuum und Gruppe. In: bergundsteigen, 3/2001 (S. 29-32).

LEY, K. (1984): Von der Normal zur Wahlbiographie. In: KOHLI, M. \& ROBERT, G. (Hrsg.): Biographie und soziale Wirklichkeit (S. 239-260). Stuttgart.

LIENERT, G.A. \& RAATZ, U. (1994): Testaufbau und Testanalyse. 5.Auflage. Weinheim.

LÖFGREN, O. (1986): Natur, Tiere und Moral. Zur Entwicklung der bürgerlichen Naturauffassung. In: JEGGLE, U. et.al. (Hrsg.): Volkskultur in der Moderne. Probleme und Perspektiven empirischer Kulturforschung (S. 122 -144). Reinbek bei Hamburg.

LORCH, J. (1995): Trendsportarten in den Alpen, Konflikte, rechtliche Reglementierungen, Lösungen. Vaduz. 
LÜBBE, H. (1993): Sicherheit. Risikowahrnehmung im Zivilisationsprozess. In: BAYRISCHE RÜCK (Hrsg.): Risiko ist ein Konstrukt (S. 23-42). München.

LUHMANN, N. (1984): Soziale Systeme. Grundriss einer allgemeinen Theorie. Frankfurt a. Main.

LUHMANN, N. (1988): Die Moral des Risikos und das Risiko der Moral. Bielefeld.

LUHMANN, N. (1990): Risiko und Gefahr. In: LUHMANN, N. (Hrsg.): Soziologische Aufklärung 5. Konstruktivistische Perspektiven (S. 131 - 169). Opladen.

LUHMANN, N. (1991): Soziologie des Risikos. Berlin.

LUHMANN, N. (1993): Die Moral des Risikos und das Risiko der Moral. In: BECHMANN, G. (Hrsg.): Risiko und Gesellschaft. Grundlagen und Ergebnisse interdisziplinärer Risikoforschung, S.327 - 338. Opladen.

LUTZ, R. (1999a): Duelle mit dem Berg. In: HESSICHE VEREINIGUNG FÜR VOLKSKUNDE (Hrsg.): Berg-Bilder: Gebirge in Symbolen - Perspektiven -P Projektionen, S. 29 - 45. Marburg.

LUTZ, R. (1999b): Traumzeit Berg. Zur Philosophie der Natursportreisen. TourismusJournal, 1, S.?- ?.

MACHATSCHEK, H (1979): Kajakabenteuer bei Tag und Nacht. Kanusport 48 (9), 172.

MACKENSEN (1983): Deutsches Wörterbuch (10. Auflage). Köln.

MADLENER-SIEGRIST, G. (1996): Tradition im Wandel - Frauen im AlpinenVerband. In: EVANGELISCHE AKADEMIE BAD BOLL (Hrsg.): Alpinismus heute (S.17-22). Bad Boll.

MAGAKIS, M. \& WILLTAL, G. H. (1992): Der Schulsport als Vorbereitung für den Freizeitsport - Schulsportunfälle. In: AGDE, G. (Hrsg.): Frezeitunfälle im Kindesund Jugendalter (S. 235 - 253). Stuttgart.

MÄGDEFRAU, H. (1980): Bergsteigen - das freiwillig eingegangene Risiko ? In DEUTSCHER ALPEN VEREIN (Hrsg.): DAV - Jahrbuch ,Berg 1980’ (245 251). München.

MAHLER, B. (1921): Die Leibesübungen in den Ritterakademien. Erlangen.

MANDELL, R. C. (1984): Sport: A Cultural History. New York.

MANGAN, J.A. \& McKENZIE, C. (2003): ,Pig Sticking ist the Greatest Fun’: Material Conditioning on the Hunting Fields of Empire. In: MANGAN, J.A. (Hrsg): Militarism, Sport, Europe. War without weapons (S. 97-119). London.

MANGAN, J.A. (2003)(Hrsg): Militarism, Sport, Europe. War without weapons. London.

MANGAN, J.A. (2003): Prologue: Combative Sports and Combative Societies. In: MANGAN, J.A. (Hrsg): Militarism, Sport, Europe. War without weapons (S.1-9). London.

MANZENREITER, W. (2000): Die soziale Konstruktion des japanischen Alpinismus: Kultur, Ideologie und Sport im modernen Bergsteigen. Wien.

MARKOWITZ, J. (1990): Kommunikation über Risiken. Eine Theorie-Skizze. In: Schweizer Zeitschrift für Soziologie, 3 (1990), S. 385-429. 
MARTIN, P. \& PRIEST, S. (1986): Understanding the adventure experience. In: Journal of Adventure Education, 3(1), 18-21.

MASLOW, A. (1954): Motivation and Personality. New York.

MASLOW, A. (1981): Psychologie des Seins. München.

Mc CELLAND, D.C. (1978): Macht als Motiv. Stuttgart.

MEHL, K. (1989): Fehler und Unfälle sind keine homogenen Ereignisse. In

RÜMMELE, E. (Hrsg.): Sicherheit im Sport - eine Herausforderung für die Sportwissenschaft. Köln.

MEHR, A. (1997): Risikosport - Suche nach Nervenkitzel. In: Unimagazin-Zeitschrift der Universität Zürich, Nr. 264, 1-4.

MERLET, R. (1990): Abenteuerlust und Risikobereitschaft im Wildwasser-Kanusport. Unveröffentlichte Diplomarbeit / Köln.

MERSCH, J. \& TRENKWALDER, P. \& SCHWIERCH, M. \& STOPPER, D. (2005): Hallenklettern - narrensicher oder fehlerträchtig. In: bergundsteigen 2(2005), S. 52-58.

MESSNER, R. (1987): Überlebt - Alle 14 Achttausender. München 1987.

MESSNER, R. (1996): Nie zurück. München.

MEYER, O.E. (1907): Geist und Kleid. Zur Psychologie des Alpinismus und des alpinen Stils. In: Österreichische Alpenzeitung 28, (1907), S. 743.

MEYER, W. (1986): Turniergesellschaften. Bemerkungen zur sozialgeschichtlichen Bedeutung der Turniere im Spätmittelalter. In: FLECKENSTEIN, J. (Hrsg.): Das ritterliche Turnier im Mittelalter (S.500-512). Göttingen

MICHAILOW, M.(1990): Lebensstil. Konzeptualisierung einer neuen sozialen Integrationsform. Dissertation Aachen.

MICHAILOW,M. (1996): Individualisierung und Lebensstilbildung. In: SCHWENK, O.G. (Hrsg.): Lebensstil zwischen Sozialstrukturanalyse und Kulturwissenschaft (S. 71-98). Opladen.

MICHELS, H. (2002): Ausdruck und Gestaltung. In: DIECKERT, J. \& WOPP, C. (Hrsg.): Handbuch Freizeitsport (S. 270-278). Schorndorf.

MICHL, W. (1995): Anthropologische Grundlagen der Erlebnispädagogik. In: HOMFELD, H.G. (Hrsg.): Erlebnispädagogik (S. 203-218). 2. korrigierte Auflage. Hohengehren.

MIRBACH, A. (1995): Schulsportunfälle an allgemeinbildenden Schulen in WestfalenLippe - eine emprische Studie zu Rahmenbedingungen von Schulsportunfällen und Möglichkeiten einer gezielten Prophylaxe. Münster.

MITCHEL, T. (1991): Blood Sport. A social History of Spanish Bullfighting. Philadelphia.

MIT'TENECKER, E. (1981): Methoden und Ergebnisse der psychologischen Unfallforschung. Wien.

MÖLLER, A. \& HUBER, M. (2003): Sensation Seeking - Konzeptbildung und entwicklung. In: ROTH, M. \& HAMMELSTEIN, P. (Hrsg.): Sensation Seeking Konzeption, Diagnostik und Anwendung (S. 5-29). Göttingen. 
MONTEAU, M. \& PHAM, D. \& DAVILLERD, C. \& WENNIGER, G. (1999): Unfall und Sicherheitspsychologie. In: ASANGER, R. \& WENNIGER, G. (Hrsg.): Handwörterbuch Psychologie (S. 812-817). Weinheim.

MOOSER, J (1984): Arbeiterleben in Deutschland 1900 - 1970. Frankfurt.

MOTSCH, A. (1995): Entscheidung bei partieller Information. Vergleich entscheidungstheoretischer Modellkonzeptionen. Wiesbaden.

MÜCKE, F. (1982): Handlungsstruktur und Unfallursachen beim Wildwasserpaddeln. In BAUR, J. (Hrsg.): Lehren und Lernen im Kanusport. Schriftenreihe des Deutschen Kanu-Verbandes e.V. (Band 1) (S. 77 - 102). Duisburg.

MÜCKE, F. (1983): Wildwasserpaddeln und Kanuunterricht im Spannungsfeld von Risikohandeln und Sicherheitserziehung. In BAUR, J. (Hrsg.): Sicherheit im Kanusport. Schriftenreihe des Deutschen-Kanu-Verbandes e.V. (Band 2) (S. 7-48). Duisburg.

MÜCKE, F. (1985): Zur psychischen Regulation von Sporthandlungen am Beispiel des Wildwasserpaddelns. In SPERLE, N. \& SCHULKE, H.G. (Hrsg.): Handeln im Hochschulsport (S. 149-87). Ahrensburg.

MÜCKE, F. (1986): Dimensionsanalyse der Sicherheit im Kanusport. In DKV (Hrsg.): Sicherheit im Kanusport II. Schriftenreihe des Deutschen-Kanu-Verbandes e.V. (Band 5) (S. 50-71). Duisburg.

MÜLLER, E. (1991): Risikoverhalten in komplexen Problemsituationen. Dissertation Berlin.

MÜLLER, H.P. \& SCHMID, M. (2003) (Hrsg.): Hauptwerke der Ungleichheitsforschung. Wiesbaden.

MÜLLER, H.P. (1998): Sozialstrukturanaylse und soziale Ungleichheit. Theorien und Trends. In: CACHAY, K. \& HARTMANN-TEWS, I. (1998): Sport und soziale Ungleichheit: Theoretische Überlegungen und empirische Befunde (S. 9 - 26). Stuttgart.

MÜLLER; H.P. \& SCHMID, M. (1995)(Hrsg.): Sozialer Wandel: Modellbildung und theoretische Ansätze. Frankfurt am Main.

MÜLLER-GETHMANN, H. (2000): Der Effekt zeitlicher Unsicherheit bei der Vorbereitung auf Handlungen. München.

MÜLLER-SCHNEIDER, T. (1994): Schichten und Erlebnismilieus. Wandel der Milieustruktur in der Bundesrepublik Deutschland. Wiesbaden.

MÜLLER-SCHNEIDER, T. (2000): Stabilität subjektorientierter Strukturen. Das Lebensstilmodell von Schulze im Zeitvergleich. In: Zeitschrift für Soziologie 29 (2000), S. 361-374.

MUNTER, W. (1999): 3 \# 3 Lawinen: Entscheiden in kritischen Situationen (2. Auflage). Garmisch Partenkirchen.

MUSAHL, H.P. \& ALVESLEBEN, K. (1990): Gefahrenkognition bei Bergleuten. Ergebnisse und Perspektiven einer empirischen Studie. In: HOYOS, C.Graf (Hrsg.): Psychologie der Arbeitssicherheit. 5. Workshop 1989 (S. 60-68). Heidelberg

MUSAHL, H.P. (1992): Arbeitssicherheit im Steinkohlebergbau: Unfallanalysen, Ausbildungsmaßnahmen und deren Evaluation. Duisburg. 
MUSAHL, H.P. (1994): Arbeitssicherheit im Steinkohlebergbau: Unfallanalysen, Ausbildungsmaßnahmen und deren Evaluation II. Duisburg.

MUSAHL, H.P. (1996): Lernen. In: WENNINGER, G. \& HOYOS, C.G. (Hrsg.): Arbeits-, Gesundheits- und Umweltschutz. Handwörterbuch verhaltenswissenschaftlicher Begriffe (S. 438-448). Heidelberg.

MUSAHL, H.P. (1997): Gefahrenkognition. Theoretische Grundlagen, empirische Befunde und Anwendungsbezüge zur subjektiven Gefahrenkenntnis. Heidelberg.

NEDBAL, D. (1999): Ist der Berg männlich? In: Berg'99 Alpenvereinsjabrbuch, Jahrgang 1999, S. 263-272.

NERLICH, M. (1977): Kritik der Abenteuerideologie. Teil 1 \& 2. München.

NERLICH, M. (1990): Abenteuer. In: SANDKÜHLER, J. (Hrsg.): Europäische Enzyklopädie zu Philosophie und Wissenschaften (S. 25-30). Band 1. Hamburg.

NERLICH, M. (1995): Abenteuer oder das verlorene Selbstverständnis der Moderne von der Unaufhebbarkeit experimentellen Handelns. München.

NERLICH, M. (1998): Zur abenteuerlichen Moderne oder von Risiko und westlichen Zivilisationen. In: GDV (Hrsg.): Wieviel Risiko braucht die Gesellschaft? (S. 72-101). Berlin.

NERLICH, M. (1999): Die Geburt des Risikos. In: Politische Ökologie, 60 (1999), S. 14.

NETZER, H. (2001): Wer bin ich? Über Image, Gruppendruck und Erwartungshaltung(en). In: bergundsteigen, 3/2001 (S. 33-34).

NEULOH, O. \& GRAF, O. \& MAUSLOFF, A. \& RÜSSEL, A. \& RUHE, H. (1957): Der Arbeitsunfall und seine Ursachen. Stuttgart / Düsseldorf.

NEUMANN, P. (1998): No risk - no fun oder Wagniserziehung im Schulsport. Sportunterricht 47 (1), 4- 13.

NEUMANN, P. (1999): Das Wagnis im Sport. Schorndorf.

NEUMANN, P. (2002): Wagnis und Abenteuer. In: DIECKERT, J. / WOPP, C. (Hrsg.): Handbuch Freizeitsport (S. 235 - 241). Schorndorf.

NEUMANN, P. (2003): If life gets boring - risk it! Zur pädagogischen Ambivalenz wagnissportlicher Aktivitäten. In: GISSEL, N. \& SCHWIER, J. (Hrsg.): Abenteuer, Erlebnis und Wagnis. Perspektiven für den Sport in Schule und Verein? (S.25-34). Hamburg.

NICKEL, U. (1994): Etwas riskieren. Sportpädagogik, 18 (5), 42-45.

NIERMANN, J. \& ENGEL, R. (Hrsg.) (1992): Erlebnispädagogik und Abenteuersport. Wuppertal.

NIETZSCHE, F. (1955): Werke in drei Bänden (Band 2). München.

NITSCH, J. \& HACKFORT, D. (1981): Streß in der Schule und Hochschule - eine handlungspsychologische Funktionsanalyse. In: NITSCH, J. (Hrsg.): Streß. Theorien, Untersuchungen und Maßnahmen (S. 263 - 311). Bern.

NITSCH, J. (1986): Zur handlungstheoretischen Grundlegung der Sportpsychologie. In: GABLER, H. / NITSCH, J. \& SINGER, R. (Hrsg.): Einführung in die Sportpsychologie, Teil 1. Schorndorf. 
NITSCH, J. (1991): Handlungstheorie und empirische Forschung. In Singer, R. (Hrsg.): Sportpsychologische Forschungsmethodik - Grundlagen, Probleme, Ansätze (S.2642). Köln.

NORDEN, G. \& SCHULZ, W. (1988): Sport in der modernen Gesellschaft. Linz.

NUREG 1624 (1996)(Hrsg.): Technical Basis and Implementation Guidelines for A Technique for Human Event Analysis (ATHEANA), NRC. Washington DC.

OELKERS, J. (1992): Unmittelbarkeit als Programm. Zur Aktualität der Reformpädagogik. In: BEDACHT, A. (Hrsg.): Erlebnispädagogik: Mode, Methode oder mehr? (S. 96-118). München.

OERTER, R. \& MONTADA, L. (1995): Entwicklungspsychologie (3. Auflage). Weinheim.

OFFE, C. (1984): Arbeitsgesellschaft: Strukturprobleme und Zukunftsperspektiven. Frankfurt am Main.

OLDEMEYER, E. (1983): Entwirf einer Typologie des menschlichen Verhältnisses zur Natur. In: GROßKLAUS, G. \& OLDEMEYER, E. (Hrsg.): Natur als Gegenwelt. Beiträge zur Kulturgeschichte der Natur (S. 15- 42). Karlsruhe.

OPASCHOWSKI, H. (1987): Sport in der Freizeit. Mehr Lust als Leistung. Auf dem Weg zu einem neuen Sportverständnis. Hamburg.

OPASCHOWSKI, H. (1988): Psychologie und Soziologie der Freizeit. Hamburg.

OPASCHOWSKI, H. (1996): Die Zukunft des Sports. Zwischen Inszenierung und Vermarktung. Hamburg, BAT-Freizeit-Forschungsinstitut.

OPASCHOWSKI, H. (2000): Xtrem - Der kalkulierte Wahnsinn: Extremsport als Zeitphänomen. Hamburg.

OPITZ, U. (1991): „Mädchen-Malboro. In: BRENNER, G. \& GRUBAUER, F. (Hrsg.): Typisch Mädchen? Typisch Junge? (S.105-110). Weinheim.

OPPERMANN, H.-P. (1998): Sind Ballspiele im Schulsport gefährlich? In: Körpererziebung 48 (1998), 8 (S. 298 - 301).

ÖSTERREICHISCHER ALPENKLUB (1902)(Hrsg.): Österreichische Alpenzeitung, 23 (1902), S.181.

PALM, J. (1991): Sport for All. Approaches from Utopia to Reality. Schorndorf.

PARLEBAS, P. (1986): Éléments de sociologie du sport. Paris.

PEINER, W. (1937): Das Gesicht Ostafrikas. Frankfurt am Main.

PER HINRICHS (vom 29.09.1999): Kajakfahrt in die Katastrophe. Hamburger Abendblatt.

PETIN, J. (1992): Versicherung und gesellschaftliche Risikoproblematik. In: I.VW. (Hrsg.): I.VW - Schriftenreihe, Band 27 (S. 73ff). St.Gallen.

PFANNL, H. (1914): Der Alpinismus und der Krieg - dieser eine, wahrhaftige Krieg! In: Österreichische Alpenzeitung. 911 (1914), S. 250-252.

PFANNL, H. (1928): 50 Jahre Österreichischer Alpenklub. In: Österreichische Alpenzeitung 1080, S.218-219. Wien.

PFEIFFER-KARABIN, M. (2000): Sportvereine im Vergleich zu anderen Formen des Freizeitsports. Eine qualitative Analyse auch Sicht junger Erwachsener. Zürich. 
PFISTER, G. (2002): Frauen und Männer. In: DIECKERT, J. \& WOPP, C. (Hrsg.): Handbuch Freizeitsport (S. 197-208). Schorndorf.

PFITZNER, M. (2001): Das Risiko im Schulsport. Analysen zur Ambivalenz schulsportlicher Handlungen und Folgerungen für die Sicherheitsförderung in den Sportspielen. Münster.

PICHL, E. (1927): Wiens Bergsteigertum. Wien.

PILS, M. (1994): Berg frei. 100 Jahre Naturfreunde. Wien.

PLESSNER, H. (1956): Die verspätete Nation. Über die politische Verfügbarkeit des bürgerlichen Geistes. Stuttgart.

PÖHLMANN, R. (1986): Motorisches Lernen. Berlin.

PONGS, A. (1999): In welcher Gesellschaft leben wir eigentlich? Individuen und Gesellschaft in Zeiten der Globalisierung. Band 1. München.

PONGS, A. (2000): In welcher Gesellschaft leben wir eigentlich? Gesellschaftskonzepte im Vergleich. Band 2. München.

PÖSCHL, A. (1962): Fremdenverkehr und Fremdenverkehrspolitik. Berlin.

POSTMANN, N. (1988): Wir amüsieren uns zu Tode. Urteilsbildung im Zeitalter der Unterhaltungsindustrie. Frankfurt / Main.

PÖTCHKE, M. \& SIMONSON, J. (2001): Online-Erhebungen in der empirischen Sozialforschung: Erfahrungen mit einer Umfrage unter Sozial-, Markt- und Meinungsforschern. In: Zentralarchiv für empirische Sozialforschung, Universtät zu Köln. (ZA-Information) 49 (2001), S. 6-29.

POTEMPA, K. (1980): Unfälle und Gefährdungseinschätzung von Jugendlichen in der technischen Berufsausbildung. Duisburg.

PRAHL, H.W. \& STEINECKE, A. (1989): Der Millionen-Urlaub. Bielefeld.

PRAHL, H.W.(2002): Soziologie der Freizeit. Paderborn.

PREUSS, S. (1996): Gefahrenkognition und Risikobeurteilung. In: WENNIGER, G. \& HOYOS, C.G. (Hrsg).: Arbeits-, Gesundheits- und Umweltschutz. Handwörterbuch verhaltenswissenschaftlicher Grundbegriffe (S. 407 - 415). Heidelberg.

PRIEST, S (1999): Forschung in Outdoor-Adventure. In: PAFFRATH, H. et.al. (Hrsg.): Wissenschaftliche Forschung in der Erlebnispädagogik: Erleben, Forschen, Evaluieren (S.25-37). Augsburg.

PRIEST, S. \& CARPENTER G. (1993): Changes in Perceived Risk an Competence During Adventurous Leisure Experiences. Journal of Applied Recreation Research 18 (1), 51-71.

PRIEST, S. \& GASS, M. (1997): Effective Leadership in Adventure Programming. New Hamsphire.

PRIEST, S. (1990): Outdoor Leadership Components. In MILES, B. / PRIEST, S.: Adventure Education (S. 142-166). State College, Pa.

PRIVETTE, G. (1997): Psychological process of Peak, Average and Failing Performance in Sport. In: International review of sport psychology 28 (1997), S. 323-334.

PRUITT, D.G. (1961): Informational requirements in risky decisions. In: American Journal of Psychology, 74 (1961), S.433-439. 
RADLINGER, L. \& SEILER, R. (1984): Situationswahrnehmung und Angstentstehung im Bergsport und didaktische Konsequenzen für das Training. In: Zeitschrift des Schweizer Alpen Clubs: Die Alpen, 1, 15-21.

RAITHEL, J. (2001): Risikoverhaltensweisen Jugendlicher - ein Überblick. In: RAITHEL, J. (Hrsg).: Risikoverhaltensweisen Jugendlicher. Formen, Erklärungen und Prävention (S. 237-248). Opladen.

RAITHEL, J. (2001a): Risikoverhaltensweisen Jugendlicher - ein Überblick. In: RAITHEL, J. (Hrsg.): Risikoverhaltensweisen Jugendlicher Formen, Erklärungen und Prävention (S.11-30). Opladen.

RAITHEL, J. (2001b): Explizit risiko-konnotative Aktivitäten und riskante Mutproben. In: RAITHEL, J. (Hrsg.): Risikoverhaltensweisen Jugendlicher Formen, Erklärungen und Prävention (S.237-248). Opladen.

RAITHEL, J. (2004): Jugendliches Risikoverhalten. Eine Einführung. Wiesbaden.

RANDELZHOFER, P. \& SEMMEL, C. \& STOPPER, D. (2004): Unfallentwicklung im Jahrhundertsommer. In: Panorama, 4 (2004), S. 70 - 73.

RANDELZHOFER, P. (2002): Bergunfallstatistik 2000-2001. Die Bergunfallstatistik des Deutschen Alpenvereins. München.

RANDELZHOFER, P. (2004): Bergunfallstatistik 2002-2003. Die Bergunfallstatistik des Deutschen Alpenvereins. München.

REESE-SCHÄFER, W. (1997): Grenzgötter der Moral: der neue europäischamerikanische Diskurs zur politischen Ethik. Frankfurt.

REEVE, J. (1992): Understanding motivation and emotion. New York.

REICHEL, P. (1993): Der schöne Schein des Dritten Reiches. Frankfurt.

REINDERS, A. (2000): Erlebnis und Pädagogik: praktische Erlebnispädagogik; Ziele; Didaktik, Methoden, Wirkungen. München.

REITHMEIER, P. (1993): Sicherheit im Wildwasser (3. Auflage). Wien.

RENN, O. (1984): Risikowahrnehmung der Kernenergie. Frankfurt am Main.

RENN, O. (1989): Risikowahrnehmung - Psychologische Determinanten bei der intuitiven Erfassung und Bewertung von technischen Risiken. In: HOSEMANN, G. (Hrsg.): Risiko in der Industriegesellschaft (S. 167 - 192). Erlangen.

RENN, O. (2002): Zwischen Über- und Unterschätzung. Die Wahrnehmung technischer Risiken. In: Forschung und Lehre 9 (2), 68 - 70.

RHEINBERG, F. \& SCHNEIDER, K. (1996): Erlebnissuche und Risikomotivation. In AMELANG, M.: Temperaments- und Persönlichkeitsunterschiede (Band 3). (S. 407-439). Göttingen.

RHEINBERG, F. (1987): The Motivational Analysis of High-Risk Sport. In: HALISCH, F./KUHL,J. (Hrsg.): Motivation, Intention and Volition, S. 249 - 259. Berlin, Heidelberg, New York.

RHEINBERG, F. (1989): Zweck und Tätigkeit. Göttingen.

RHEINBERG, F. (1996): Flow-Erleben, Freude an riskantem Sport und anderen ,unvernünftigen' Motivationen. In HECKHAUSEN, H. \& KUHL, J. (Hrsg.): Motivation, Volition und Handlung (Band 4) (S. 101-118). Göttingen. 
RHEINBERG, F. (1997): Motivation (2. erweiterte Auflage). Stuttgart.

RHEINBERG, F. (2002): Motivation (4. erweiterte Auflage). Stuttgart.

RIDDER-SYMOENS, H. (1989): Die Kavaliertour im 16. und 17. Jahrhundert. In: BRENNER, P.J. (Hrsg.): Der Reisebericht (S. 197-223). Frankfurt am Main.

RIEDEL, U.E. (2004): Großwildjagd in Deutsch-Ostafrika Im Zeitraum 1891-1916:

Eine Untersuchung aus sozialwissenschaftlicher Perspektive. Dissertation Paderborn.

RINEHART, R. \& SYDNOR (2003): To the extreme, alternative sport, inside and out. Albany.

RINKE, H. \& MUSAHL, H.-P. (2003).Unfälle im Schulsport - was wissen die verantwortlichen Akteure? Sicherheitspsychologische Studie zur Gefahrenkenntnis von Sportlehrern und Schülern im Sportunterricht. In N. Gissel \& J. Schwier (Hrsg.), Abenteuer, Erlebnis und Wagnis. Perspektiven für den Sport in Schule und Verein. Jahrestagung der dvs-Sektion Sportpädagogik 2002, S. 57-65. Hamburg.

RINKE, H. (2002). Unfälle im Schulsport - Eine sicherheitspsychologische Reanalyse. Duisburg: Unveröffentlichte schriftliche Hausarbeit im Rahmen der Ersten Staatsprüfung für das Lehramt für die Sekundarstufe II/I.

RINKE, H. (2004).Unfälle im Schulsport - was wissen die verantwortlichen Akteure? Sicherheitspsychologische Studie zur Gefahrenkenntnis von Sportlehrern und Schülern im Sportunterricht. In H.-P. MUSAHL, C. SCHWENNEN \& S. HINRICHS (Hrsg.), Arbeitssicherheit - Führung - Qualität, S. 103-129. Hamburg.

RITTER, J. (1963): Landschaft. Zur Funktion des Ästethischen in der modernen Gesellschaft. In: RIT'TER, J. (Hrsg.): Subjektivität (S. 141-163). Frankfurt am Main.

RITTNER, V. \& BREUER, C. (2000): Soziale Bedeutung und Gemeinwohlorientierung des Sports. Köln.

RIT'TNER, V. (1989): Körperbezug, Sport und Ästhetik. In: Sportwissenschaft 19, (1989), S. $359-377$.

RITTNER, V. (1995): Zur Erweiterung des ästhetischen Vokabulars im Sport. Die Inszenierung von Individualität im Sport und der Untergang traditioneller Pathosformeln. In: GERHARD, V.\& WIRKUS, B. (Hrsg): Schriften der Deutschen Vereinigung für Sportwissenschaft, 63. Sport und Ästhetik: Tagung der dvs-Sektion Sportphilosophie im Juni 1992 in Köln. St. Augustin.

RITTNER, V. (1998): Sport in der Erlebnisgesellschaft. In ALLMER, H. / SCHULZ, N. (Hrsg.): Erlebnissport - Erlebnis Sport (S. 28 - 45). Sankt Augustin.

RITTNER, V. (2001): Risikoverhalten im Sport. In: RAITHEL, J. (Hrsg.): Risikoverhaltensweisen Jugendlicher Formen, Erklärungen und Prävention (S.217-236). Opladen.

ROBINSON, V. (2004): Taking risks: identity, masculinities and rock climbing. In: WHEATIN, B. (Hrsg.): Understanding lifestyle sports (S.113-130). London.

ROESE,L. (1993): Zur Bedeutung der Abenteuerlust im weiblichen und männlichen Individuationsprozess. In: Hans-Günther Homfeldt (Hg.): Erlebnispädagogik (S. 7081). Hohengehren.

RÖHRS, H. (1966): Bildung als Wagnis und Bewährung. Eine Darstellung des Lebenswerkes von Kurt Hahn. Heidelberg. 
ROMMELFANGER, H. \& EICKEMEIER, S. (2002): Entscheidungstheorie. Klassische Konzepte und Fuzzy-Erweiterung. Berlin, Heidelberg.

ROOSEVELT, T. (1886a): Ranch Life and Game-Shooting in the west. In: Outing, 7 (1886), S. 611-616.

ROOSEVELT, T. (1886b): Hunting Trips of a Ranchman. New York.

ROSE, L. \& MÜLLER, R. (1995): Hammer, Fräse, Sägespäne. Hand-Werk in der Jugendarbeit. Butzbach-Griedel.

ROSE, L. (1991): Kunstturnen - eine Risikosportart. In: ROSE, L. (Hrsg.): Das Drama des begabten Mädchens. Lebensgeschichten junger Kunstturnerinnen (S. 85-121). Weinheim.

ROSE, L. (1995): Sport - Männersache? Frauensache. In: BUNDESAUSSCHUSS FRAUEN IM SPORT DES DSB (Hrsg.): Fair Play? Für Mädchen und Frauen im Sport (S. 16-25). Frankfurt am Main.

ROSE; L (1997).: Abenteuer - nur für Jungen. Bausteine zur Aufarbeitung der Mädchenfrage im erlebnis- und abenteuerpädagogischen Diskurs. In: Friebertshäuser, Barbara u. a. (Hrsg.): Sozialpädagogik im Blick der Frauenforschung (S. 171 - 183). Weinheim.

ROUSSEAU, J.-J. (1975) : Emile oder über die Erziehung. Paderborn.

RUCH, W. \& ZUCKERMAN, M. (2001): Sensation Seeking and Adolescence. In: RAITHEL, J. (Hrsg.): Risikoverhaltensweisen Jugendlicher Formen, Erklärungen und Prävention (S.97-110). Opladen.

RUCKENBAUER, K. (1992): Psychosoziale Einflüsse auf das alpinistische Leistungsverhalten. In JENNY, E. (Hrsg.): Jahrbuch der österreichischen Gesellschaft für Alpin- und Höhenmedizin 1992 (S. 91-100). Innsbruck.

RÜMMELE, E. \& KAISER, D. (1989): Sicherheit im Sport - eine Herausforderung für die Sportwissenschaft. Köln.

RÜMMELE, E. (1988): Unfallforschung und Unfallverhütung im Schulsport. Frankfurt a.M.

RÜMMELE, E. (1993)(Hrsg.): Kognitive Repräsentation über Unfälle und Sicherheitsunterweisungen im Sport. Köln.

RÜMMELE, E. \& KAYSER, D. (1989)(Red.): Sicherheit im Sport - eine Herausforderung für die Sportwissenschaft. Köln.

RUMMELT, P. (2003): Risiko-Sport in der Risiko-Gesellschaft - semantischer Zufall oder logische Konsequenz? In: GISSEL, N. \& SCHWIER, J. (Hrsg.): Abenteuer, Erlebnis und Wagnis. Persepktiven für den Sport in Schule und Verein? (S.203 220). Hamburg.

RUPE, C. (2000): Trends im Abenteuersport: Touristische Vermarktung von Abenteuerlust und Risikofreude. Hamburg.

RUPPERT, F. \& HIRSCH, C. \& WALDHERR, B. (1985): Wahrnehmen und Erkennen von Gefahren. Schriftenreihe der Bundesanstalt für Arbeitsschutz (Nr. 426). Bremerhaven. 
RUPPERT, F. \& HIRSCH, C. \& WALDHERR, B. (1985): Wahrnehmen und Erkennen von Gefahren. Schriftenreihe der Bundesanstalt für Arbeitsschutz. Forschungsbericht Nr. 426). Bremerhaven.

RUSSELL, B. (1930): Wissen und Wahn. Skeptische Essays. München.

RÜT'TEN, A. (1998): Public Health und Sport - Prolegomena für einen transdiszipliniären Ansatz. In: RÜTTEN, A. (Hrsg.): Public Health und Sport (S. 5 - 35). Stuttgart.

RÜTTEN, A. (2002): Inhalte und Sportarten: Aktivitäten und Sportarten. In: DIECKERT, J. / WOPP, C. (Hrsg.): Handbuch Freizeitsport (S. 111 - 122). Schorndorf.

SAYERS, G.F. (1930): The handbook of Tanganyika. London.

SCHÄFERS, B. (1979): Sozialstruktur und Wandel der Bundesrepublik Deutschland. Ein Studienbuch. Stuttgart.

SCHALLBERGER, U. \& PFISTER, R. (2001): Flow-Erleben in Arbeit und Freizeit. Eine Untersuchung zum Paradox der Arbeit mit der Experience Sampling Method. In: Zeitschrift für Arbeits- und Organisationspsychologie, 45 (S. 176-187).

SCHALLBERGER, U. (1995): Risikosport. Zum Verhältnis von Risikosuche und Sicherheitsbedürfnis. In MUSEUM FÜR GESTALTUNG ZÜRICH (Hrsg.): Handbuch über Sicherheit und Zusammenarbeit (S. 76-85). Basel.

SCHARFFE, U. (1999): Risiko Schulsportalltag. Eine explorative Studie zur Risikoabschätzung im schulsportlichen Unfallgeschehen auf der Basis einer empirischen Analyse von Unterrichtsdokumenten. Stuttgart.

SCHELER, M. (1928): Die Stellung des Menschen im Kosmos. Darmstadt.

SCHELSKY, H. (1979): Auf der Suche nach Wirklichkeit. Gesammelte Aufsätze zur Soziologie der Bundesrepublik Deutschland. München.

SCHIEDEK, S. (2003): Angst und Leistung im Rahmen der Katastrophentheorie Untersuchungen zum optimalen Erregungsniveau bei Fallschirmspringern. Dissertation Göttingen.

SCHIERZ, M. (1993): Sportpädagogik. Sankt Augustin.

SCHIESTL, L. (1873): Das Wirken der Alpinvereine. In: Jabrbuch des Österreichischen Alpenvereins, 9 (1873), S. 328.

SCHILDMACHER, A.: Trends und Moden im Sport. In: dvs-Informationen 13 (1998) 2, 14-19

SCHILDWACH, B. (1999): Erneut mehrere tödliche Unfälle. Kanusport, 69 (3), 122 124.

SCHILDWACH, B. (2000): Gefahren richtig einschätzen. Kanusport, 70 (4), 176-178.

SCHILLING, J. (1989): Freizeit und Geselligkeit. Inhalte der Geselligkeitspädagogik. München.

SCHIMANK, U. \& VOLKMANN, U. (2000): Sozilogische Gegenwartsdiagnosen. Opladen.

SCHIMANK, U. \& VOLKMANN, U. (2002): Sozilogische Gegenwartsdiagnosen. Vergleichende Sekundäranalysen. Opladen 
SCHIMANK, U. (1992): Größenwachstum oder soziale Schließung? Das Inklusionsdilemma des Breitensports. In: Sportwissenschaft 22(1992), S. 32-45.

SCHLESKE, W. (1977): Abenteuer, Wagnis, Risiko im Sport. Schorndorf.

SCHLESKE, W. (1991): Grenzerfahrungen in den Erlebnissportarten - gesteigertes Leben konstruktive Selbstdarstellung und aktive Selbstermutigung. In: REDL, S. et.al. (Hrsg.): Sport an der Wende (S. 84-93). Wien.

SCHLESKE, W. (1998): Abenteuer. In:

SCHLICHT, W. \& PETERS JANSEN, J. \& SIEVERS, M. (1993): Kontrollüberzeugung und Verhaltensstile als personale Determinanten der Verletzungsschwere. In: RÜMMELE, E. (1993): Kognitive Repräsentation über Unfälle und Sicherheitsunterweisungen im Sport (S: 13-26). Köln.

SCHMITZ, S. (2003): Men the Hunter - Women the Gatherer? Dimensionen der Gender-Forschung am Beispiel biologischer Theoriebildung. In: Freiburger Frauenstudien, 13 (2003), S. 151-174.

SCHNEIDER, K. \& SCHMALT, H.D. (2000): Motivation. Stuttgart.

SCHNEIDER, K. (1996): Intrinsisch (autotelisch) motiviertes Verhalten - dargestellt an den Beispielen des Neugierverhaltens sowie verwandter Verhaltenssysteme. In KUHL, J. \& HECKHAUSEN, H. (Hrsg.): Motivation, Volition und Handlung. Enzyklopädie der Psychologie (Band 4). (S 101 -118). Göttingen.

SCHNEIDER-WOHLFAHRT, U. \& WACK, O.G. (Hrsg.) (1994): Entspannt sein Energie haben: achtzehn Methoden der Körpererfahrung. München.

SCHNOOR, W. (1987): Unfallanalyse - Wo lauern die Gefahren im Kanusport? Kanusport 57 (23), 559- 562.

SCHÖNHAMMER, R. (1991): In Bewegung. München.

SCHÖNPFLUG, W. (1989): Psychologie : allgemeine Psychologie und ihre Verzweigungen in die Entwicklungs-, Persönlichkeits- und Sozialpsychologie. München.

SCHÖNPFLUG, W. (2004): Geschichte und Systematik der Psychologie. Weinheim.

SCHÖTTLER, B. (1994): Statements zur Podiumsdisukussion: „Erlebnispädagogik Mode, Methode oder mehr?" In: BEDACHT, A. et.al. (Hrsg.): Erlebnispädagogik: Mode, Methode oder mehr (S. 22-27). München.

SCHRÖDER, J.U. (2004): Datenerfassung bei Unfallursachen und begünstigte Faktoren für Unfälle in der Seeschiffart. Dortmund.Berlin.

SCHROER, M. (2001): Das Individuum der Gesellschaft. Synchrone und diachrone Theorieperspektiven. Frankfurt / Main.

SCHULKE, H.J. (1988): Alltagslauf als Aufbruch. Aachen.

SCHULZ, N. (1994): Mit der Zeit gehen - zur Aktualisierung von Schulsportinhalten. Sportunterricht 18 (12), 492-503.

SCHULZE, G. (2003): Die beste aller Welten. Wohin bewegt sich die Gesellschaft im 21. Jahrhundert.

SCHULZE, G.(1995): Die Erlebnisgesellschaft. Kultursoziologie der Gegenwart (5. Auflage). Frankfurt a. Main. 
SCHULZE, J. (1995): Action und Abenteuer - ein Weg zu rechten Jugendlichen? Möglichkeiten und Grenzen einer erlebnisbezogenen Pädagogik. In: HOMFELDT, H.G. (Hrsg.): Erlebnispädagogik: Geschichtliches, Räume und Adressat(inn)en, erziehungswissenschaftliche Facetten, Kritisches (S. 92-100). Hohengehren.

SCHULZ-HARDT, S. \& FREY, D. \& LÜTHGENS, C. (1996): Sorglosigkeit und Risikoakzeptanz. . In: WENNINGER, G. \& HOYOS, C.G. (Hrsg.): Arbeits-, Gesundheits- und Umweltschutz. Handwörterbuch verhaltenswissenschaftlicher Begriffe (S. 468-477). Heidelberg.

SCHUMACHER, J. \& HAMMELSTEIN, P. (2003): Sensation Seeking und gesundheitsbezogenes Risikoverhalten. Eine Betrachtung aus gesundheitspsychologischer Sicht. In: ROTH, M. \& HAMMELSTEIN, P. (Hrsg.): Sensation Seeking - Konzeption, Diagnostik und Anwendung (S. 138 - 161). Göttingen.

SCHÜTZ, H. \& WIEDEMANN, P.M. \& GRAY, P.C.R. (2000): Risk Perception Beyond the Psychometric Paradigm. Jülich.

SCHWARZ, K. (1968): Die Kurzschulen Kurt Hahns. Ihre pädagogische Theorie und Praxis. Ratingen b. Düsseldorf.

SCHWIER, J. (1998a): „Do the right things“ - Trends im Feld des Sports. In: dvsInformationen 13 (1998), 2, (S. 7 - 13).

SCHWIER, J. (1998b): Erlebnis. In: GRUPE, O. \& MIETH, D. (Hrsg.): Lexikon der Ethik des Sports (128-131). Schorndorf.

SCHWIER, J. (2000): Sport als populäre Kultur. Sport, Medien und Cultural Studies. Hamburg.

SCHWIER, J. (2003): Was ist Trendsport? In: BREUER, C. \& MICHELS, H. (Hrsg.): Trendsport: Modelle, Orientierungen und Konsequenzen (S. 18 - 32). Aachen.

SCHWIERCH, M. (2003): Dem Fein ein Freund sein - vom Umgang mit Fehlern. IN: KURATORIUM FÜR ALPINE SICHERHEIT (Hrsg.): Sicherheit im Bergland. Jahrbuch 2003 (S. 18- 26). Innsbruck.

SCHWIERCH, M. (2004): Die verflixte Basisrate. In: bergundsteigen, 1/2004 (S. 17-21).

SCHWIERSCH, M. (1991a): Erlebnispädagogische Aktionen als Chance zur Persönlichkeitsentwicklung - Hinweise aus einer Betrachtung von Risikosportlern. Zeitschrift für Erlebnispädagogik, 11 (4), 38- 43.

SCHWIERSCH, M. (1991b): Wahrnehmungsfehler beim Bergsteigen, Teil 1: Tödliche Täuschung. Bergwelt Alpin, 3 (1), 48-51.

SCHWIERSCH, M. (1991c): Wahrnehmungsfehler beim Bergsteigen, Teil 2: Gemeinsamkeit macht schwach. Bergwelt Alpin, 3 (2), 56-59.

SCOT'T, C. (2000): Pushing the limit. The story of Canadian Moutnaineering. Calgary.

SEIFERT, M. (2005): Outdoor- und Erlebnissport in der betrieblichen und außerbetrieblichen Weiterbildung. Unveröffentlichte Hausarbeit zur Erlangung des Magistergrades an der Sozialwissenschaftlichen Fakultät der Georg-August-Universität Göttingen.

SEITZ, G. (1987): Wo Europa den Himmel berührt. Die Entdeckung der Alpen. München.

SEMMLER, G. (1994): Die Lust an der Angst. München. 
SENNETT, R. (1998): Der flexible Mensch. Berlin.

SHEDLETSKY, R. \& ENDLER, N.S. (1974): Anxiety: the state-trait model and the interactional model. In: Journal of Personality 42(1974) S. 511 - 527.

SHORT, J.F. (1984): The social fabric at risk: Toward the social transformation of risk analysis. In: American Sociological Review, 49 (1984), S. 711 - 725.

SIEGRIST, D. (1998): Extremtrends im Naturtourismus. Eine kultuwissenschaftliche Annäherung an den Risikonatursport. In: Tourismus-Journal 2, S. 237-252.

SIELAND, P. (2003): Trendsportarten in der Schule? Gießen.

SIMMEL, G. (1923): Philosophische Kultur. Über das Abenteuer, die Geschlechter und die Krise der Moderne. Berlin.

SIXTL, F. (1967): Meßmethoden der Psychologie. Theoretische Grundlagen und Probleme. Weinheim.

SKIBA, R. (1973): Die Gefahrenträgertheorie. Forschungsbericht Nr. 106. Dortmund: Bundesanstalt für Arbeitsschutz und Unfallforschung.

SLADEK, F. (2003): Alpinunfallbericht 2002. In: KURATORIUM FÜR ALPINE SICHERHEIT (Hrsg.): Sicherheit im Bergland. Jahrbuch 2003 (S. 38 - 48). Innsbruck.

SLADEK, F. (2004): Alpinunfallbericht 2003. In: KURATORIUM FÜR ALPINE SICHERHEIT (Hrsg.): Sicherheit im Bergland. Jahrbuch 2004 (S. 159 - 168). Innsbruck.

SLOVIC, P. (1987): Perception of risk. In: Science, 236 (1987), (S.2880 - 285).

SLUSARCZIK, J. (2000) : Die Annalen des polnischen Bergtourismus bis zum Jahre 1873. In: KRÜGER, A. \& TJA, A. \& TRANGBAEK, E. (Hrsg.): Europäische Perspektiven zur Geschichte von Sport, Kultur und Tourismus (S. 64-76). Berlin.

SLUSHER, H.S. (1967) : Man, Sport and Existence. Philadelphia.

SOMBART, W. (1916): Der moderne Kapitalismus, 2 Bände. (2. Auflage). München.

SPELLERBERG, A. (1998): Lebensstile im Zeitvergleich. Berlin.

SPIELBERGER, C. \& FRAIN, F. \& PETERS, R. (1981): Neugier und Angst. In VOSS, H.-G. \& KELLER, H. (Hrsg.): Neugierforschung. Grundlagen, Theorien und Anwendungen (S. 197-226). Weinheim.

SPIELBERGER, C.D. (1966)(Hrsg.): Anxiety and behavior. New York.

SPIES, K. \& HESSE, F.W. (1986): Interaktion von Emotion und Kognition. In: Psychologische Rundschau 1986, 57-80.

STEINHART, E. (2001): Safari Hunting. Black Poachers, White Hunters. New York.

STEINITZER, A. (1917): Der Deutsche und der Österreichische Alpenverein und der Krieg. In: Mitteilungen des Deutschen und Österreichischen Alpenvereins, 43 (1917), S. 102.

STEINS, G. (1986): Wo das Turnen erfunden wurde... Friedrich Ludwig Jahn und die 175jährige Geschichte der Hasenheide. Schriftenreihe Berliner Forum 6/86. Berlin.

STENGEL, M. (1995): Wertewandel. In: ROSENSTIEL, L. et al. (Hrsg.): Führung von Mitarbeitern. Handbuch für erfolgreiches Personalmanagement (S. 789-790). Stuttgart. 
STERN, M. (2003a): Wagnissportarten - Strukturelles Anforderungsprofil und WertHaltungen wagnisreicher Handlungspraxen. In: GISSEL, N. / SCHWIER, J. (Hrsg.): Abenteuer, Erlebnis und Wagnis. Persepktiven für den Sport in Schule und Verein? (S.189 - 202). Hamburg.

STERN, M. (2003b): Heldenfiguren im Wagnissport. Zur medialen Inszenierung wagnissportlicher Handlungspraxen. In: ALKEMEYER, T. \& BOSCHERT, B. \& SCHMIDT, R. \& GEBAUER, G. (Hrsg.): Aufs Spiel gesetzte Körper. Aufführungen des Sozialen in Sport und populärer Kultur. Konstanz.

STICHWEH, R. (1995): Sport und Moderne. In: HINSCHING, J. \& BORKENHAGEN, F. (Hrsg.): Modernisierung und Sport, S. 13 - 28. Sankt Augustin.

STIEHR, K. (1992): Risikokonflikte und der Streit um das Rauchen. Wiesbaden.

STRASSER, H. \& DEDERICHS, A. (2000): Die Restrukturierung der Klassengesellschaft - Elemente einer zeitgenössischen Ungleichheitstheorie. In: Berliner Journal für Soziologie, Band 10 (2000), 1, S.79-98.

STRASSER, J. (1986): Sicherheit als destruktives Element. Psychologie heute, 13 (5), 28-36.

STUMM, P. (2004): Sport und Globalisierung. Trendsportarten in Deutschland, Italien und Spanien. Wiesbaden.

SUDGEN, J. (1998): Boxing and society. An international Analysis. Manchester.

SWAIN, A.D. \& GUTMAN, H.E. (1983): Handbook of Human Reliability Analysis with Emphasis on Nuclear Power Plant Applications, Final Report. NUREGCR1278, Washington DC,

SZCZESNY-FRIEDMANN, C. (1982): Lohn der Angst. Dissertation München.

TAYLOR, D.H. (1976): Accidents, risks and models of explanation. Human Factors, $18,371-280$.

TELCHOW, S. (2000): Informelle Sportengagements Jugendlicher. Köln.

TENBRUCK, F.H. (1978): Zur Anthropologie des Handelns. In LENK, H. (Hrsg.): Handlungstheorien interdisziplinär (Band II, Teilband 2) (S. 89-139). München.

THEIS, I. (2002): Das Steer by Wire-System. Analyse der menschlichen Zuverlässigkeit. München.

THEOBALD, A. (2000): Das World Wide Web als Befragungsinstrument. Wiesbaden.

THIERSCH , H. (1995) : Abenteuer als Exempel der Erlebnispädagogik. In: HOMFELDT, H.G. (Hrsg.): Erlebnispädagogik: Geschichtliches, Räume und Adressat(inn)en, erziehungswissenschaftliche Facetten, Kritisches (S. 38-54). Hohengehren.

THIERSCH, H. (1996): Natur- und Erlebnispädagogik. In: EVANGELISCHE AKADEMIE BAD BOLL (Hrsg.): Alpinismus heute (S.4-14). Bad Boll.

THOMAS, A. (1995): Einführung in die Sportpsychologie. Göttingen.

THOREAU, D. (1971): Walden oder Leben in den Wäldern. Zürich.

TOMLINSON, J. (1997): Extreme Sports: das ultimative Buch! Augsburg.

TÖNNIES (1965): Einführung in die Soziologie. Stuttgart. 
TREBELS, A.H. (1988): Wagnis und Sicherheit. Sportpädagogik, 12 (5), 25-27.

TREBELS, A.H. (1991): Die Dialektik von Naturbeherrschung und Natursehnsucht. Sportpädagogik, 15(3), 25-28.

TRENTIN-MEYER, M. (1996): Skivereinigungen der Sektion Austria und Alpiner SkiClub München. In: ZEBHAUSER, H. \& TRENTIN-MEYER, M. (Hrsg.): Zwischen Idylle und Tummelplatz (S.317-319). München.

TREUTLEIN, G. \& FUNKE, J. \& SPERLE, N. (1992): Körpererfahrungen im Sport (2. Auflage). Aachen.

TRIMPOP, R.M. (1994): The psychology of Risk Taking Behavior. Amsterdam.

TRÜMPER, T. (1995): Die touristische Entwicklung der Risiko- und Abenteuersportarten. In: KRÜGER, A. \& DREYER, A. (Hrsg.): Sporttourismus: Management und Marketing-Handbuch (S. 203-237). München.

TSCHOFEN, B. (1999): Berg, Kultur, Moderne. Volkskundliches aus den Alpen. Wien.

TVERSKY, A. \& KAHNEMAN, D. (1974): Judgement under uncertainty: Heuristics an biases. In: Science, 185 (S. $1124-1131)$.

UETZINGER, C. (2004): Human Factors usa (2). In: bergundsteigen, 1/2004 (S. 51-57).

UNGERER, D. \& MORGENROTH, U. (2001): Analyse des menschlichen Fehlverhaltens in Gefahrensituationen. Empfehlungen für die Ausbildung. Bonn.

VAN DER LOO, H. \& VAN REIJEN, W. (1992): Modernisierung. Projekt und Paradox. München.

VESTER, M. (1992)(Hrsg.): Neue soziale Milieus und pluralisierte Klassengesellschaften. Hannover.

VESTER, M. (2001): Soziale Milieus im gesellschaftlichen Strukturwandel. Zwischen Integration und Ausgrenzung. Frankfurt am Main.

VIRILIO, P. (1989): Der negative Horizont. München.

VIRILIO, P. (1992): Rasender Stillstand. München.

VON DEM BUSCHE, A. (1989): Die Ritterakademien zu Brandenburg. Frankfurt am Main.

VON HALLER, A. (1729 / 1956): Die Alpen. Berlin.

VON HENTIG, H. (1966): Kurt Hahn und die Pädagogik. In: RÖHRS, H. (Hrsg.): Bildung als Wagnis und Bewährung. Eine Darstellung des Lebenswerkes von Kurt Hahn (S. 44-56). Heidelberg.

VOSS, H.-G. (1981): Kognition und Exploratives Handeln. In VOSS, H.-G. \& KELLER, H. (Hrsg.): Neugierforschung. Grundlagen, Theorien und Anwendungen (S. 175-197). Weinheim.

WAGNER, M. (1981): Die Alpen: Faszination unwirtschaftlicher Gegenden. In: Mit dem Auge des Touristen. Zur Geschichte des Reisebildes. Eine Ausstellung des Kunsthistorischen Instituts der Universität Tübingen (S. 67 - 79). Tübingen.

WAGNER, M. (1983): Das Gletschererlebnis - visuelle Naturaneignung im frühen Tourismus. In: GROßKLAUS, G. \& OLDEMEYER, E. (Hrsg.): Natur als Gegenwelt. Beiträge zur Kulturgeschichte der Natur (S. 235 - 264). Karlsruhe. 
WALLNER, M. (1977): Betriebliche Unfallstatistik. In BUNDESANSTALT FÜR ARBEITSSCHUTZ UND UNFALLFORSCHUNG (Hrsg.): Sicherheitsfachkräfte - Grundlehrgang B (S. 9- 31). Köln.

WANG, C. (1994): Entscheidungsfähigkeit in sportbezogenen Risikosituationen. Dissertation Hildesheim.

WANG, M. \& FISCHBECK, P. (2004): Incoporating framing into prospect theory modeling: a mixture-model approach. In: Journal of risk and uncertainty, 29 (2004), 2, S. 181-197.

WARWITZ, S. (2001): Sinnsuche im Wagnitz: Leben in wachsenden Ringen; Erklärungsmodell für grenzüberschreitendes Verhalten. Hohengrehen.

WEBER et. al. ( ?) (1994) :

WEBER, M. (1919): Vom inneren Beruf zur Wissenschaft. In: WINKELMANN, J. (Hrsg.): Max Weber - Soziologie, weltgeschichtliche Analysen, Politik (S. 311- 340). Stuttgart 1968.

WEBER, M. (1923): Wirtschaftsgeschichte. Abriß der universalen Sozial- und Wirtschaftsgeschichte. (4. Aufl.). Berlin 1981.

WEBER, M. (19765): Wirtschaft und Gesellschaft. Grundriß der verstehenden Soziologie. Tübingen [9.-13. Tausend].

WEBER, W. (1995): die wirtschaftliche Bedeutung des Sports. Schorndorf.

WEHMEYER, K. (1992): Stichwort Schulsportunfall. In: RÖTHIG, P. (Hsrg.): Sportwissenschaftliches Lexikon (S. 464). Schorndorf.

WEIS, K. (1994): Menschenbilder in der Erlebnispädagogik. In: BEDACHT, A. et.al. (Hrsg.): Erlebnispädagogik. Mode, Methode oder mehr? (S. 49-71). München.

WEIS, K. (1995): Vom gesellschaftlichen Erleben zum Erlebnisverlust in der Erlebnisgesellschaft. Individualisierung und Suche nach Wirklichkeit. In HECKMAIR, B. (Hrsg.): Die Wiederentdeckung der Wirklichkeit (S. 41- 73) Alling.

WEIß, O. (1999): Einführung in die Sportsoziologie. Wien.

WERTHEIMER, M. (1921)(Hrsg.): Turnvater Jahn. Eine Auswahl seiner Schriften. Offenbach am Main.

WESSELER, B. (1997): Neugier, Angst und Risikoverhalten im Sport. Unveröffentlichte Dissertation Vechta.

WESSLER, B. (1997): Neugier, Angst und Risikoverhalten im Sport. Eine exemplarische Untersuchung im Gleitschirmfliegen und sportpädagogische Konsequenzen. Jena.

WEST, C. \& ZIMMERMANN, D.H. (1987): Doing gender. In: Gender and Society, 1 (S. 125-151).

WETZSTEIN, T.A. \& WÜRTZ, S. (2001): Gruppenzugehörigkeit und das Risikoverhalten Jugendlicher. In: RAITHEL, J. (Hrsg.): Risikoverhaltensweisen Jugendlicher Formen, Erklärungen und Prävention (S.349-364). Opladen.

WHEATON, B. (2004): Understanding Lifestyle Sports. Consumption, identity and difference. London / New York. 
WHYMPER, E. (1872/1990): Berg- und Gletscherfahrten in den Alpen in den Jahren 1860 bis 1869. Autorisierte deutsche Bearbeitung von Dr. Friedrich Steger. Braunschweig.

WIEDEMANN, P. (1993): Tabu, Sünde, Risiko: Veränderungen der gesellschaftlichen Wahrnehmung von Gefährdungen. In: BAYRISCHE RÜCK (Hrsg.): Risiko ist ein Konstrukt (S. 43-68). München.

WIELAND, K. (1993): Eine empirische Studie zum Fallschirmspringen. Pfaffenweiler.

WIEMEYER, J. (1997): Bewegungslernen im Sport. Motorische, kognitive und emotionale Aspektte. Darmstadt.

WILDE, G.J.S. (1978): Theorie der Risikokompensation der Unfallverursachung und praktische Folgerungen für die Unfallverhütung. Hefte der Unfallheilkunde, Nr. 130, 130-156.

WILKEN, T. (1994): Abenteuer und ökologische Verantwortung. Sportpädagogik 18 (5), 38- 41.

WILLIMCZIK, K. (1997): Statistik im Sport: Grundlagen, Verfahren, Anwendung. 3.Auflage. Hamburg.

WILLKE, H. (1993) Systemtheorie: eine Einführung in die Grundprobleme der Theorie sozialer Systeme. Stuttgart/Jena.

WINKLER, J. (1995): Soziologie des Sports. Theorieansätze, Forschungsansätze und Forschungsperspektiven. Opladen.

WINKLER, J. (1998): Schichtspezifische Varianten des Sportverhaltens in den neuen und alten Bundesländern. In: HARTMANN-TEWS, I. \& CACHAY, K. (Hrsg.): Sport und soziale Ungleichheit (S. 121-141). Stuttgart.

WINKLER, S. (1989): Motivation - Risiko. Geschlechtsspezifische Unterschiede am Beispiel des Sportkletterns. Diplomarbeit an der Deutschen Sporthochschule Köln.

WIT'TE, D. (2002): Das Erlebnis - zur philosophischen, soziologischen, psychologischen und pädagogischen Dimension des Erlebnisses. In: Zeitschrift für Erlebnispädagogik, 3\&4, S.10-21.

WOLL, A. \& BÖS, K. (2000): Gesundheitserziehung. In: HAAG, H. /\&HUMMEL, A. (Hrsg.): Handbuch Sportpädagogik. Schorndorf.

WOLL, A. (1996): Gesundheitsförderung in der Gemeinde. Neu-Isenburg.

WOLL; A. \& BÖS, K. (2002): Fitness und Gesundheit. In: DIECKERT; J \& WOPP, C. (Hrsg.): Handbuch Freizeitsport (S. 229 - 234). Schorndorf.

WOLL; A. \& BÖS, K. (2002): Fitness und Gesundheit. In: DIECKERT; J \& WOPP, C. (Hrsg.): Handbuch Freizeitsport (S. 229 - 234). Schorndorf.

WOPP, C. (1995): Entwicklungen und Perspektiven des Freizeitsports. Aachen.

WOPP, C. (2002): Gesellschaft und Freizeitsport. In: DIECKERT, J. \& WOPP, C. (Hrsg.): Handbuch Freizeitsport. (S. 45-62). Schorndorf.

WORTHMUTH, L. \& HINRICHS, S. \& BAILEY, A. \& CRANEY, S. \& RINKE, H. (2001): Das Konzept der Gefahrenkenntnis in kleinen und mittleren Unternehmen. In: R. TRIMPOP, B. ZIMOLONG \& A. KALVERAM (Hrsg.). Psychologie der Arbeitssicherheit und Gesundheit. Neue Welten - Alte Welten. 11. Workshop 2001. Heidelberg. 
WOZNIAKOWSKI, J. (1987): Die Wildness. Zur Deutungsgeschichte des Berges in der europäischen Neuzeit. Frankfurt am Main.

WOZNIAKOWSKI, J. (1987): Die Wildnis, Zur Deutungsgeschichte des Berges in der europäischen Neuzeit, Frankfurt.

WÜRTL, W. (2003): Lawinenereignisse in Österreich im Winter 2002 / 2003. In KURATORIUM FÜR ALPINE SICHERHEIT (Hrsg.): Sicherheit im Bergland Jahrbuch 2003 (S. 49-82). Innsbruck.

WUST, P. (1937): Ungewissheit und Wagnis. Leipzig.

YATES, F.Y. (1992): Risk taking behavior. Chichester.

ZAPF, W. (1994): Modernisierung, Wohlfahrtsentwicklung und Transformation. Berlin.

ZAPF, W. (1998): Modernisierung und Transformation. In: SCHÄFER, B. \& ZAPF, W. (Hrsg.): Handwörterbuch zur Gesellschaft Deutschlands (S.472 - 481). Opladen.

ZEBHAUSER, H. \& TRENTIN-MEYER, M. (Hrsg.)(1996): Zwischen Idylle und Tummelplatz. München.

ZEBHAUSER, H. (1998): Alpinismus im Hitlerstaat. Gedanken, Erinnerungen, Dokumente. München.

ZEBHAUSER, H. (1999): Sehnsucht nach Wildnis. Der Wandel des Naturverständnis. In: Berg'99 Alpenvereinsjahrbuch, Jahrgang 1999, S. 273-282.

ZELINKA, U. (1997): Sicherheit - ein Grundbedürfnis des Menschen? In LIPPERT, E. (Hrsg.): Sicherheit in der unsicheren Gesellschaft (S. 43-59). Opladen.

ZERGER, F. (2000): Klassen, Milieus und Individualisierung. Eine empirische Untersuchung. Frankfurt am Main.

ZIAK, K. (1965): Der Mensch und die Berge. Eine Weltgeschichte des Alpinismus. Salzburg.

ZIEGENSPECK, J. (1987): Kurt Hahn. Erinnerungen - Gedanken - Aufforderungen. Lüneburg.

ZIEGENSPECK, J. (1999): Erlebnispädagogik. In: REINHOLD, G. et.al. (Hrsg.): Pädagogik-Lexikon (S.135-139). München.

ZIMMER, B. (1995): Geschichte und Entwicklung des Tourismus. Trier.

ZIMOLONG, B. (1978): Gefährdungseinschätzung beim Rangieren. Bremerhaven.

ZIMOLONG, B. (1984): Psychologische Untersuchungen der Arbeitssicherheit in absturzgefährdeten Situationen. In: Psychologie und Praxis - Zeitschrift für Arbeits- und Organisationspsychologie, 28, (S.50 - 56).

ZIMOLONG, B. (1990): Fehler und Zuverlässigkeit. In: HOYOS, C. GRAF \& ZIMOLONG, B. (Hrsg.): Ingenieurspsychologie, Band D, Serie III (S.313-344). Göttingen.

ZIMOLONG, B. (1996): Systemsicherheit und Sicherheitskultur. In: WENNINGER, G \& GRAF HOYOS, C. (Hrsg.): Arbeits, Gesundheits- und Umweltschutz. Handwörterbuch verhaltenswissenschaftlicher Grundbegriffe (S.24-34). Heidelberg.

ZOBL, N. \& LADSTÄDTER, E. (2001): Erkenntnisse zu Werner Munter aus der Sicht der Alpingendarmerie. In: KURATORIUM FÜR ALPINE SICHERHEIT (Hrsg.): Sicherheit im Bergland. Jahrbuch 2001, S. 157-163. Innsbruck. 
ZUCKERMAN, M. \& KUHLMANN, D.M. (2000): Personality and risk-taking:

Common biosocial factors. In: Journal of Personality, 68, (S. 999-1028).

ZUCKERMAN, M. (1978): Warum manche Menschen den Nervenkitzel brauchen. In: Psychologie heute 5/1978 (S.15-21). 


\section{Anhang:}

Anhang A: Ergebnistabellen der empirischen Untersuchung

Anhang B: ASS-Schadensanzeige (Unfallberichtsbogen des DAV)

Anhang C: Wissenschaftlicher Bildungsgang

Anhang D: Eidesstattliche Erklärung 


\section{Anhang A: Ergebnistabellen der empirischen Untersuchung}

Tabelle Anhang 1: Verunfallte Personen der untersuchten Aktivitäten im alpinen Bergsport in den Jahren 2000 - 2003 (statistische Berücksichtigung aller beim DAV eingegangenen Unfallbögen):

\begin{tabular}{|c|c|c|c|c|c|c|}
\hline & Bergwandern & Hochtouren & Felsklettern & Tour & Variante/Piste & Gesamt 2000 \\
\hline 2000 (gesamt) & 125 & 64 & 63 & 32 & 84 & 368 \\
\hline tot / vermisst & 21 & 7 & 4 & & & 32 \\
\hline schwer verletzt & 7 & 5 & 11 & 2 & 7 & 32 \\
\hline leicht verletzt & 79 & 39 & 33 & 26 & 75 & 252 \\
\hline unverletzt & 16 & 11 & 14 & 4 & 1 & 46 \\
\hline \multirow[t]{2}{*}{ unbekannt } & 2 & 2 & 1 & & 1 & 6 \\
\hline & Bergwandern & Hochtouren & Felsklettern & Tour & Variante/Piste & Gesamt 2001 \\
\hline 2001 (gesamt) & 128 & 81 & 87 & 44 & 117 & 457 \\
\hline tot / vermisst & 8 & 9 & 6 & 4 & 2 & 29 \\
\hline schwer verletzt & 12 & 4 & 10 & & 4 & 30 \\
\hline leicht verletzt & 89 & 44 & 57 & 35 & 107 & 332 \\
\hline unverletzt & 19 & 23 & 13 & 4 & 1 & 60 \\
\hline \multirow[t]{2}{*}{ unbekannt } & & 1 & 1 & 1 & 3 & 6 \\
\hline & Bergwandern & Hochtouren & Felsklettern & Tour & Variante/Piste & Gesamt 2002 \\
\hline 2002 (gesamt) & 176 & 75 & 78 & 60 & 114 & 503 \\
\hline tot / vermisst & 14 & 8 & 9 & 4 & 1 & 36 \\
\hline schwer verletzt & 13 & 10 & 11 & 1 & 8 & 43 \\
\hline leicht verletzt & 134 & 38 & 49 & 43 & 102 & 366 \\
\hline unverletzt & 14 & 15 & 9 & 12 & 1 & 51 \\
\hline \multirow[t]{2}{*}{ unbekannt } & 1 & 4 & & & 2 & 7 \\
\hline & Bergwandern & \begin{tabular}{|l|} 
Hochtouren \\
\end{tabular} & Felsklettern & Tour & Variante/Piste & Gesamt 2003 \\
\hline 2003 (gesamt) & 206 & 158 & 100 & 67 & 176 & 707 \\
\hline tot / vermisst & 23 & 18 & 10 & 6 & & 57 \\
\hline schwer verletzt & 8 & 16 & 21 & 2 & 9 & 56 \\
\hline leicht verletzt & 148 & 86 & 53 & 47 & 165 & 499 \\
\hline unverletzt & 27 & 38 & 16 & 12 & 2 & 95 \\
\hline unbekannt & & & & & & \\
\hline
\end{tabular}

Tabelle Anhang 2: Subjektive Einschätzung der Unfallhäufigkeit der untersuchten Aktivitäten im alpinen Bergsport (alle Befragten):

\begin{tabular}{|l|l|l|l|l|l|}
\hline & Bergwandern & Hochtouren & Felsklettern & Ski Touren & $\begin{array}{l}\text { Ski Piste \& } \\
\text { Variante }\end{array}$ \\
\hline Mittelwert (Unfall-Häufigkeit) & 3,7 & 3,26 & 3,09 & 3,03 & 3,95 \\
\hline Standardabweichung & 1,09 & 0,95 & 0,95 & 0,9 & 1,01 \\
\hline z-Wert (Unfall-Häufigkeit) & 0,818614457 & 0,406522825 & 0,879871321 & 1,046935495 & 1,514715185 \\
\hline z-Wert-Differenz & - & - & - & & \\
\hline
\end{tabular}


Tabelle Anhang 3: Subjektive Einschätzung der Unfallfolgen der untersuchten Aktivitäten im alpinen Bergsport (alle Befragten):

\begin{tabular}{|c|c|c|c|c|c|}
\hline & Bergwandern & Hochtouren & Felsklettern & Ski Touren & $\begin{array}{l}\text { Ski Piste \& } \\
\text { Variante }\end{array}$ \\
\hline Mittelwert (Unfallschwere) & 3,61 & 4,42 & 4,47 & 4,4 & 4,09 \\
\hline Standardabweichung & 0,84 & 0,81 & 0,93 & 1,03 & 0,87 \\
\hline z-Wert (Unfallschwere) & \begin{tabular}{|l}
- \\
1,820721413
\end{tabular} & 0,687415227 & 0,842238477 & 0,625485928 & $-0,334418219$ \\
\hline z-Wert (Differenz) & $\begin{array}{l}- \\
3,010721413\end{array}$ & 0,167415227 & 0,202238477 & 2,065485928 & 0,575581781 \\
\hline
\end{tabular}

Tabelle Anhang 4: Mittelwerte der Ausübungshäufigkeiten der untersuchten Aktivitäten im alpinen Bergsport:

\begin{tabular}{|l|l|l|l|l|l|}
\hline & & & & \multicolumn{2}{l|}{ Ski Piste \& Varian- } \\
& Bergwandern & Hochtouren & Felsklettern & Ski Touren \\
\hline Mittelwert (Ausübungshäufigkeit) & 4,33 & 3,7 & 4,23 & 3,42 & 3,12 \\
\hline
\end{tabular}

Tabelle Anhang 5: Subjektive Einschätzung der Unfallhäufigkeit der untersuchten Aktivitäten im alpinen Bergsport (nur Männer):

\begin{tabular}{|l|l|l|l|l|l|}
\hline \multirow{2}{*}{ Männlich } & Bergwandern & Hochtouren & Felsklettern & $\begin{array}{l}\text { Ski Tou- } \\
\text { ren }\end{array}$ & Ski Piste \& Variante \\
\hline Mittelwert Unfallhäufigkeit & 3,75 & 3,27 & 3,1 & 2,96 & 3,905 \\
\hline Z-Wert & 0,95863055 & $-0,34488975$ & $-0,80655318$ & $-1,186746$ & 1,37955898 \\
\hline Z-Wert Differenz & $-0,49136945$ & $-0,23488975$ & $-0,34655318$ & 0,3232534 & 0,74955898 \\
\hline
\end{tabular}

Tabelle Anhang 6: Subjektive Einschätzung der Unfallhäufigkeit der untersuchten Aktivitäten im alpinen Bergsport (nur Frauen):

\begin{tabular}{|l|l|l|l|l|l|}
\hline weiblich & Bergwandern & Hochtouren & Felsklettern & Ski Touren & Ski Piste \& Variante \\
\hline Mittelwert Unfallhäufigkeit & 3,47 & 3,2 & 3,06 & 3,29 & 4,135 \\
\hline Z-Wert & 0,10363987 & $-0,6138669$ & $-0,98590745$ & 0,37469798 & 1,87083247 \\
\hline z-Wert Differenz & $-1,34636013$ & $-0,5038669$ & $-0,52590745$ & 1,13530202 & 1,24083247 \\
\hline
\end{tabular}

Tabelle Anhang 7: Subjektive Einschätzung der Unfallfolgen für die untersuchten Aktivitäten im alpinen Bergsport (nur Männer):

\begin{tabular}{|l|l|l|l|l|l|}
\hline Männlich & Bergwandern & Hochtouren & Felsklettern & Ski Touren & Ski Piste \& Variante \\
\hline Mittelwert Unfallschwere & 3,64 & 4,42 & 4,47 & 4,4 & 4,095 \\
\hline Z-Wert & $-1,81261135$ & 0,68975476 & 0,85016284 & 0,62559153 & $-0,35289778$ \\
\hline z-Wert Differenz & $-3,00261135$ & 0,16975476 & 0,21016284 & 2,06559153 & 0,55710222 \\
\hline
\end{tabular}


Tabelle Anhang 8: Subjektive Einschätzung der Unfallfolgen für die untersuchten Aktivitäten im alpinen Bergsport (nur Frauen):

\begin{tabular}{|l|l|l|l|l|l|}
\hline weiblich & Bergwandern & Hochtouren & Felsklettern & Ski Touren & Ski Piste \& Variante \\
\hline Mittelwert Unfallschwere & 3,63 & 4,4 & 4,45 & 4,38 & 4,03 \\
\hline Z-Wert & $-1,7572047$ & 0,7118603 & 0,8721892 & 0,64772874 & $-0,47457353$ \\
\hline Z-Wert Differenz & $-2,9472047$ & 0,1918603 & 0,2321892 & 2,08772874 & 0,43542647 \\
\hline
\end{tabular}

Tabelle Anhang 9: Subjektive Einschätzung der Unfallhäufigkeiten und Unfallfolgen für die untersuchten Aktivitäten im alpinen Bergsport in Abhängigkeit der alpinen Erfahrung (Männer und Frauen):

\begin{tabular}{|c|c|c|c|c|c|}
\hline$<4$ Jahre & Bergwandern & Hochtouren & Felsklettern & Ski Touren & Ski Piste \& Variante \\
\hline U.Häufigkeit & 3,86 & 3,39 & 3,21 & 3 & 3,985 \\
\hline Z-Wert & 0,98424071 & $-0,26264105$ & $-0,74017023$ & $-1,297287$ & 1,31585819 \\
\hline Z-Wert Diff. & $-0,46575929$ & $-0,15264105$ & $-0,28017023$ & 0,21271239 & 0,68585819 \\
\hline U.Schwere & 3,65 & 4,65 & 4,56 & 4 & 3,935 \\
\hline Z-Wert & $-1,32629459$ & 1,27939222 & 1,04488041 & $-0,4143042$ & $-0,58367385$ \\
\hline z-Wert Diff. & $-2,51629459$ & 0,75939222 & 0,40488041 & 1,0256958 & 0,32632615 \\
\hline Ausüb.Häuf. & 4,43 & 3,69 & 3,95 & 1,95 & 2,665 \\
\hline 5-12 Jahre & Bergwandern & Hochtouren & Felsklettern & Ski Touren & Ski Piste \& Variante \\
\hline U.Häufigkeit & 3,59 & 3,22 & 3,04 & 3,08 & 4,03 \\
\hline Z-Wert & 0,53028444 & $-0,46065113$ & $-0,94272789$ & $-0,835599$ & 1,7086943 \\
\hline z-Wert-Diff. & $-0,91971556$ & $-0,35065113$ & $-0,48272789$ & 0,67440028 & 1,0786943 \\
\hline U.Schwere & 3,58 & 4,29 & 4,45 & 4,39 & 4,055 \\
\hline Z-Wert & $-1,81003132$ & 0,43276491 & 0,93818377 & 0,7486517 & $-0,30956906$ \\
\hline z-Wert Diff. & $-3,00003132$ & $-0,08723509$ & 0,29818377 & 2,1886517 & 0,60043094 \\
\hline Ausüb.Häuf. & 4,37 & 3,66 & 4,01 & 2,81 & 2,985 \\
\hline 13-20 Jahre & Bergwandern & Hochtouren & Felsklettern & Ski Touren & Ski Piste \& Variante \\
\hline U.Häufigkeit & 3,75 & 3,24 & 3,168 & 3 & 3,995 \\
\hline Z-Wert & 0,84684421 & $-0,50534911$ & $-0,69624699$ & $-1,141675$ & 1,49642728 \\
\hline Z-Wert Diff. & $-0,60315579$ & $-0,39534911$ & $-0,23624699$ & 0,36832462 & 0,86642728 \\
\hline U.Schwere & 3,56 & 4,46 & 4,41 & 4,49 & 4,115 \\
\hline Z-Wert & $-1,84875254$ & 0,72292796 & 0,58005682 & 0,80865065 & $-0,2628829$ \\
\hline Z-Wert Diff. & $-3,03875254$ & 0,20292796 & $-0,05994318$ & 2,24865065 & 0,6471171 \\
\hline Ausüb.Häuf. & 4,33 & 3,64 & 4,27 & 3,56 & 3,155 \\
\hline 20-28 Jahre & Bergwandern & Hochtouren & Felsklettern & Ski Touren & Ski Piste \& Variante \\
\hline U.Häufigkeit & 3,68 & 3,32 & 3,15 & 3,01 & 4,04 \\
\hline Z-Wert & 0,64096931 & $-0,32048465$ & $-0,77450458$ & $-1,148403$ & 1,60242327 \\
\hline z-Wert Diff. & $-0,80903069$ & $-0,21048465$ & $-0,31450458$ & 0,36159666 & 0,97242327 \\
\hline U.Schwere & 3,58 & 4,43 & 4,52 & 4,49 & 4,1 \\
\hline Z-Wert & $-1,81294121$ & 0,57991598 & 0,83327733 & 0,74882355 & $-0,34907564$ \\
\hline z-Wert Diff. & $-3,00294121$ & 0,05991598 & 0,19327733 & 2,18882355 & 0,56092436 \\
\hline Ausüb.Häuf. & 4,33 & 3,82 & 4,23 & 3,83 & 3,22 \\
\hline$>28$ Jahre & Bergwandern & Hochtouren & Felsklettern & Ski Touren & Ski Piste \& Variante \\
\hline U.Häufigkeit & 3,8 & 3,24 & 2,97 & 3,02 & 3,77 \\
\hline Z-Wert & 1,22620249 & $-0,33441886$ & $-1,08686129$ & $-0,9475201$ & 1,14259777 \\
\hline z-Wert Diff. & $-0,22379751$ & $-0,22441886$ & $-0,62686129$ & 0,5624799 & 0,51259777 \\
\hline
\end{tabular}




\begin{tabular}{|l|l|l|l|l|l|} 
U.Schwere & 3,72 & 4,5 & 4,51 & 4,24 & 4,085 \\
\hline Z-Wert & $-1,67289612$ & 0,9846578 & 1,018729 & 0,09880649 & $-0,42929717$ \\
\hline Z-Wert Diff. & $-2,86289612$ & 0,4646578 & 0,378729 & 1,53880649 & 0,48070283 \\
\hline Ausüb.Häuf. & 4,27 & 3,75 & 4,52 & 3,75 & 3,23 \\
\hline
\end{tabular}

Tabelle Anhang 10: Subjektive Einschätzung der Unfallhäufigkeiten und Unfallfolgen für die untersuchten Aktivitäten im alpinen Bergsport in Abhängigkeit des Alters (Männer und Frauen):

\begin{tabular}{|c|c|c|c|c|c|}
\hline 21-30 Jahre & Bergwandern & Hochtouren & Felsklettern & Ski Touren & Ski Piste \& Variante \\
\hline U.Häufigkeit & 3,63 & 3,19 & 3,08 & 3,02 & 4 \\
\hline Z-Wert & 0,65628241 & $-0,51755605$ & $-0,81101566$ & $-0,9710845$ & 1,64337383 \\
\hline z-Wert Diff. & $-0,79371759$ & $-0,40755605$ & $-0,35101566$ & 0,53891546 & 1,01337383 \\
\hline U.Schwere & 3,69 & 4,42 & 4,407 & 4,24 & 4,02 \\
\hline Z-Wert & $-1,69848766$ & 0,96566359 & 0,9182198 & 0,30874958 & $-0,49414531$ \\
\hline z-Wert Diff. & $-2,88848766$ & 0,44566359 & 0,2782198 & 1,74874958 & 0,41585469 \\
\hline Ausüb.Häuf. & 4,36 & 3,63 & 4,16 & 3,75 & 3,18 \\
\hline 31-40 Jahre & Bergwandern & Hochtouren & Felsklettern & Ski Touren & Ski Piste \& Variante \\
\hline U.Häufigkeit & 3,71 & 3,29 & 3,1 & 2,96 & 3,95 \\
\hline Z-Wert & 0,82654859 & $-0,30056312$ & $-0,810447$ & $-1,1861509$ & 1,47061243 \\
\hline z-Wert Diff. & $-0,62345141$ & $-0,19056312$ & $-0,350447$ & 0,3238491 & 0,84061243 \\
\hline U.Schwere & 3,55 & 4,37 & 4,5 & 4,47 & 4,07 \\
\hline Z-Wert & $-1,80736826$ & 0,50110833 & 0,86708633 & 0,78262987 & $-0,34345627$ \\
\hline z-Wert Diff. & $-2,99736826$ & $-0,01889167$ & 0,22708633 & 2,22262987 & 0,56654373 \\
\hline Ausüb.Häuf. & 4,23 & 3,59 & 4,16 & 3,58 & 3,13 \\
\hline 41-50 Jahre & Bergwandern & Hochtouren & Felsklettern & Ski Touren & Ski Piste \& Variante \\
\hline U.Häufigkeit & 3,77 & 3,37 & 3,25 & 3,03 & 3,95 \\
\hline Z-Wert & 0,87489765 & $-0,30739647$ & $-0,66208471$ & $-1,312346$ & 1,40693001 \\
\hline z-Wert Diff. & $-0,57510235$ & $-0,19739647$ & $-0,20208471$ & 0,19765352 & 0,77693001 \\
\hline U.Schwere & 3,64 & 4,63 & 4,45 & 4,38 & 4,19 \\
\hline Z-Wert & $-1,81972613$ & 1,09536913 & 0,56535181 & 0,35923396 & $-0,20022877$ \\
\hline z-Wert Diff. & $-3,00972613$ & 0,57536913 & $-0,07464819$ & 1,79923396 & 0,70977123 \\
\hline Ausüb.Häuf. & 4,3 & 3,79 & 4,2 & 3,53 & 3,06 \\
\hline 51-60 Jahre & Bergwandern & Hochtouren & Felsklettern & Ski Touren & Ski Piste \& Variante \\
\hline U.Häufigkeit & 3,53 & 3,14 & 2,85 & 3,07 & 3,91 \\
\hline Z-Wert & 0,61208324 & $-0,42579704$ & $-1,19755416$ & $-0,612083$ & 1,6233512 \\
\hline z-Wert Diff. & $-0,83791676$ & $-0,31579704$ & $-0,73755416$ & 0,89791676 & 0,9933512 \\
\hline U.Schwere & 3,52 & 4,48 & 4,49 & 4,3 & 4,08 \\
\hline Z-Wert & $-1,81958934$ & 0,85136749 & 0,87918996 & 0,35056308 & $-0,26153119$ \\
\hline z-Wert Diff. & $-3,00958934$ & 0,33136749 & 0,23918996 & 1,79056308 & 0,64846881 \\
\hline Ausüb.Häuf. & 4,26 & 3,88 & 4,73 & 3,46 & 3,29 \\
\hline$>61$ Jahre & Bergwandern & Hochtouren & Felsklettern & Ski Touren & Ski Piste \& Variante \\
\hline U.Häufigkeit & 4 & 3,17 & 2,89 & 2,93 & 3,72 \\
\hline Z-Wert & 1,48666497 & $-0,38861151$ & $-1,02123491$ & $-0,930860$ & 0,85404158 \\
\hline z-Wert Diff. & 0,03666497 & $-0,27861151$ & $-0,56123491$ & 0,57913986 & 0,22404158 \\
\hline U.Schwere & 3,72 & 4,45 & 4,55 & 4,1 & 4,1 \\
\hline Z-Wert & $-1,57470094$ & 0,90273804 & 1,24211324 & $-0,285075$ & $-0,28507517$ \\
\hline z-Wert Diff. & $-2,76470094$ & 0,38273804 & 0,60211324 & 1,15492483 & 0,62492483 \\
\hline Ausüb.Häuf. & 4,31 & 4,03 & 4,58 & 3,27 & 3,01 \\
\hline
\end{tabular}


Tabelle Anhang 11: Subjektive Einschätzung der Unfallhäufigkeiten und Unfallfolgen für die untersuchten Aktivitäten im alpinen Bergsport in Abhängigkeit der Übungsleiterlizenz (Männer und Frauen):

\begin{tabular}{|c|c|c|c|c|c|}
\hline ÜL Bergsteigen & Bergwandern & Hochtouren & Felsklettern & Ski Touren & Ski Piste \& Variante \\
\hline U.Häufigkeit & 4,03 & 3,28 & 3,15 & 3,08 & 3,98 \\
\hline Z-Wert & 1,26939136 & $-0,54057731$ & $-0,85430521$ & $-1,023235$ & 1,14872678 \\
\hline Z-Wert-Diff. & $-0,18060864$ & $-0,43057731$ & $-0,39430521$ & 0,48676438 & 0,51872678 \\
\hline U.Schwere & 3,74 & 4,44 & 4,6 & 4,48 & 4,17 \\
\hline Z-Wert & $-1,77798292$ & 0,50148236 & 1,022503 & 0,63173752 & $-0,37773996$ \\
\hline z-Wert Diff & $-2,96798292$ & $-0,01851764$ & 0,382503 & 2,07173752 & 0,53226004 \\
\hline Ausüb.Häuf. & 4,6 & 4,25 & 4,13 & 3,25 & 2,815 \\
\hline ÜL Alpinklettern & Bergwandern & Hochtouren & Felsklettern & Ski Touren & Ski Piste \& Variante \\
\hline U.Häufigkeit & 3,76 & 3,23 & 2,91 & 3,07 & 3,845 \\
\hline Z-Wert & 1,06202894 & $-0,35579307$ & $-1,21183655$ & $-0,783814$ & 1,28941549 \\
\hline Z-Wert Diff. & $-0,38797106$ & $-0,24579307$ & $-0,75183655$ & 0,72618519 & 0,65941549 \\
\hline U.Schwere & 3,49 & 4,31 & 4,42 & 4,81 & 4,08 \\
\hline Z-Wert & $-1,68049108$ & 0,20202625 & 0,45455906 & 1,34990267 & $-0,3259969$ \\
\hline z-Wert Diff. & $-2,87049108$ & $-0,31797375$ & $-0,18544094$ & 2,78990267 & 0,5840031 \\
\hline Ausüb.Häuf. & 4,28 & 3,97 & 5,38 & 4,13 & 3,39 \\
\hline ÜL Hochtouren & Bergwandern & Hochtouren & Felsklettern & Ski Touren & Ski Piste \& Variante \\
\hline U.Häufigkeit & 3,81 & 3,29 & 3,16 & 2,94 & 3,77 \\
\hline Z-Wert & 1,21502458 & $-0,30375614$ & $-0,68345132$ & $-1,3260124$ & 1,09819529 \\
\hline z-Wert-Diff. & $-0,23497542$ & $-0,19375614$ & $-0,22345132$ & 0,1839876 & 0,46819529 \\
\hline U.Schwere & 3,57 & 4,4 & 4,45 & 4,67 & 4,085 \\
\hline Z-Wert & $-1,74349216$ & 0,4325958 & 0,56368544 & 1,14047983 & $-0,39326891$ \\
\hline z-Wert Diff. & $-2,93349216$ & $-0,0874042$ & $-0,07631456$ & 2,58047983 & 0,51673109 \\
\hline Ausüb.Häuf. & 4,36 & 4,64 & 4,79 & 4,34 & 3,355 \\
\hline ÜL Klettersport & Bergwandern & Hochtouren & Felsklettern & Ski Touren & Ski Piste \& Variante \\
\hline U.Häufigkeit & 3,77 & 3,21 & 2,95 & 2,96 & 3,985 \\
\hline Z-Wert & 0,92681136 & $-0,38714905$ & $-0,9972021$ & $-0,973738$ & 1,43127831 \\
\hline z-Wert-Diff. & $-0,52318864$ & $-0,27714905$ & $-0,5372021$ & 0,53626148 & 0,80127831 \\
\hline U.Schwere & 3,65 & 4,42 & 4,25 & 4,37 & 4,12 \\
\hline Z-Wert & $-1,85361304$ & 0,9340472 & 0,31858974 & 0,7530303 & $-0,15205419$ \\
\hline Z-Wert Diff. & $-3,04361304$ & 0,4140472 & $-0,32141026$ & 2,1930303 & 0,75794581 \\
\hline Ausüb.Häuf. & 3,81 & 2,77 & 5,39 & 2,69 & 2,955 \\
\hline ÜL Ski & Bergwandern & Hochtouren & Felsklettern & Ski Touren & Ski Piste \& Variante \\
\hline U.Häufigkeit & 3,63 & 3,37 & 3,22 & 3,13 & 3,86 \\
\hline Z-Wert & 0,69881233 & $-0,26763025$ & $-0,82519329$ & $-1,1597311$ & 1,55374231 \\
\hline z-Wert Diff. & $-0,75118767$ & $-0,15763025$ & $-0,36519329$ & 0,3502689 & 0,92374231 \\
\hline U.Schwere & 3,57 & 4,58 & 4,67 & 4,17 & 4,125 \\
\hline Z-Wert & $-1,66824995$ & 0,91204477 & 1,14197202 & $-0,1354016$ & $-0,25036523$ \\
\hline z-Wert Diff. & $-2,85824995$ & 0,39204477 & 0,50197202 & 1,3045984 & 0,65963477 \\
\hline Ausüb.Häuf. & 4,2 & 3,28 & 3,32 & 3,26 & 5,1 \\
\hline
\end{tabular}




\begin{tabular}{|l|l|l|l|l|l|}
\hline Ohne Lizenz & Bergwandern & Hochtouren & Felsklettern & Ski Touren & Ski Piste \& Variante \\
\hline U.Häufigkeit & 3,42 & 3,24 & 3,1 & 2,93 & 3,955 \\
\hline Z-Wert & 0,25848101 & $-0,25280011$ & $-0,6504632$ & $-1,1333398$ & 1,7781221 \\
\hline Z-Wert Diff. & $-1,19151899$ & $-0,14280011$ & $-0,1904632$ & 0,3766602 & 1,1481221 \\
\hline U.Schwere & 3,22 & 4,4 & 4,45 & 4,13 & 4,005 \\
\hline Z-Wert & $-1,85499898$ & 0,81113841 & 0,92411033 & 0,20109002 & $-0,08133978$ \\
\hline Z-Wert Diff. & $-3,04499898$ & 0,29113841 & 0,28411033 & 1,64109002 & 0,82866022 \\
\hline Ausüb.Häuf. & 4,56 & 3,38 & 3,42 & 2,3 & 2,535 \\
\hline
\end{tabular}

Tabelle Anhang 12: Verunfallte Personen der untersuchten Aktivitäten im Kanusport in den Jahren 1998 - 2003 (statistische Berücksichtigung aller registrierter Unfälle mit eindeutigen Folgen):

\begin{tabular}{|l|l|l|l|l|l|l|}
\hline Jahrgang & $\begin{array}{l}\text { Kanu- } \\
\text { Wandern } \\
\text { Fluss }\end{array}$ & $\begin{array}{l}\text { Kanu- } \\
\text { Wandern } \\
\text { See }\end{array}$ & See-Kajak & $\begin{array}{l}\text { Kanu-WW } \\
(1-2)\end{array}$ & $\begin{array}{l}\text { Kanu-WW } \\
(3-4)\end{array}$ & $\begin{array}{l}\text { Kanu-WW } \\
(5-6)\end{array}$ \\
\hline 1998 & 6 & 0 & 2 & 2 & 4 & \\
\hline 1999 & 6 & 4 & 1 & 8 & 1 & 0 \\
\hline 2000 & 10 & 2 & 3 & 8 & 3 & 0 \\
\hline 2001 & 4 & 0 & 0 & 0 & 3 & 1 \\
\hline 2002 & 7 & 0 & 0 & 1 & 0 & 0 \\
\hline \\
2003
\end{tabular}

Tabelle Anhang 13: Subjektive Einschätzung der Unfallhäufigkeit der untersuchten Aktivitäten im Kanusport (alle Befragten):

\begin{tabular}{|l|l|l|l|l|l|l|}
\hline & $\begin{array}{l}\text { Kanu- } \\
\text { Wandern } \\
\text { Fluss }\end{array}$ & $\begin{array}{l}\text { Kanu- } \\
\text { Wandern } \\
\text { See }\end{array}$ & Seekajak & WW 1-2 & WW 3-4 & WW 5-6 \\
\hline $\begin{array}{l}\text { Mittelwert } \\
\text { (U.Häufigkeit) }\end{array}$ & 3,04 & 2,45 & 3,05 & 3,13 & 3,53 & 3,56 \\
\hline $\begin{array}{l}\text { Standarda- } \\
\text { bweichung }\end{array}$ & 0,98 & 0,8 & 0,9 & 0,92 & 0,98 & 1,27 \\
\hline $\begin{array}{l}\text { z-Wert } \\
\text { (U.Häufigkeit) }\end{array}$ & $-0,2341867$ & $-1,82845786$ & $-0,20716518$ & 0,00900718 & 1,08986897 & 1,17093361 \\
\hline $\begin{array}{l}\text { z-Wert- } \\
\text { Differenz }\end{array}$ & - & & & & & \\
\hline
\end{tabular}


Tabelle Anhang 14: Subjektive Einschätzung der Unfallfolgen der untersuchten Aktivitäten im Kanusport (alle Befragten):

\begin{tabular}{|c|c|c|c|c|c|c|}
\hline & \begin{tabular}{|l} 
Kanu- \\
Wandern \\
Fluss \\
\end{tabular} & $\begin{array}{l}\text { Kanu- } \\
\text { Wandern } \\
\text { See } \\
\end{array}$ & Seekajak & WW 1-2 & WW 3-4 & WW 5-6 \\
\hline $\begin{array}{l}\text { Mittelwert } \\
\text { (U.Schwere) }\end{array}$ & 3,34 & 3,2 & 4,11 & 3,14 & 3,97 & 4,97 \\
\hline $\begin{array}{l}\text { Standarda- } \\
\text { bweichung }\end{array}$ & 1,15 & 1,26 & 1,08 & 0,83 & 0,95 & 0,91 \\
\hline $\begin{array}{l}\text { z-Wert } \\
\text { (U.Schwere) }\end{array}$ & $\begin{array}{l}- \\
0,694515659 \\
\end{array}$ & $-0,91139044$ & 0,49829562 & $-1,00433677$ & 0,28142084 & 1,8305264 \\
\hline z-Wert (Diff.) & $\begin{array}{l}- \\
2,514515659\end{array}$ & $-0,14139044$ & 0,56829562 & $-1,59433677$ & 0,56142084 & 3,1205264 \\
\hline
\end{tabular}

Tabelle Anhang 15: Mittelwerte der Ausübungshäufigkeiten der untersuchten Aktivitäten im Kanusport:

\begin{tabular}{|l|l|l|l|l|l|l|}
\hline & $\begin{array}{l}\text { Kanu- } \\
\text { Wandern } \\
\text { Fluss }\end{array}$ & $\begin{array}{l}\text { Kanu- } \\
\text { Wandern } \\
\text { See }\end{array}$ & Seekajak & WW 1-2 & WW 3-4 & WW 5-6 \\
\hline $\begin{array}{l}\text { Mittelwert } \\
\text { (Ausübung) }\end{array}$ & 3,97 & 3,14 & 1,77 & 3,84 & 3,78 & 1,88 \\
\hline
\end{tabular}

Tabelle Anhang 16: Subjektive Einschätzung der Unfallhäufigkeit der untersuchten Aktivitäten im Kanusport (nur Männer):

\begin{tabular}{|l|l|l|l|l|l|l|}
\hline Männlich & $\begin{array}{l}\text { Kanu- } \\
\text { Wandern } \\
\text { Fluss }\end{array}$ & $\begin{array}{l}\text { Kanu- } \\
\text { Wandern } \\
\text { See }\end{array}$ & Seekajak & WW 1-2 & WW 3-4 & WW 5-6 \\
\hline $\begin{array}{l}\text { Mittelwert } \\
\text { Unfallhäufigkeit }\end{array}$ & 3,04 & 2,47 & 3,01 & 3,11 & 3,5 & 3,5 \\
\hline Z-Wert & $-0,18653456$ & $-1,82229914$ & $-0,27262743$ & 0,01434881 & 1,13355616 & 1,13355616 \\
\hline & & & & & & \\
Z-Wert Diff. & $-1,97653456$ & $-1,22229914$ & 0,32737257 & $-0,68565119$ & 1,17355616 & 2,39355616 \\
\hline
\end{tabular}

Tabelle Anhang 17: Subjektive Einschätzung der Unfallhäufigkeit der untersuchten Aktivitäten im Kanusport (nur Frauen):

\begin{tabular}{|l|l|l|l|l|l|l|}
\hline weiblich & $\begin{array}{l}\text { Kanu- } \\
\text { Wandern } \\
\text { Fluss }\end{array}$ & $\begin{array}{l}\text { Kanu- } \\
\text { Wandern } \\
\text { See }\end{array}$ & Seekajak & WW 1-2 & WW 3-4 & WW 5-6 \\
\hline $\begin{array}{l}\text { Mittelwert } \\
\text { Unfallhäufigkeit }\end{array}$ & 3,03 & 2,38 & 3,22 & 3,19 & 3,69 & 3,84 \\
\hline Z-Wert & $-0,4114391$ & $-1,78290279$ & $-0,01054972$ & $-0,07384804$ & 0,98112402 & 1,29761564 \\
\hline & & & & & & \\
Z-Wert Diff. & $-2,2014391$ & $-1,18290279$ & 0,58945028 & $-0,77384804$ & 1,02112402 & 2,55761564 \\
\hline
\end{tabular}


Tabelle Anhang 18: Subjektive Einschätzung der Unfallfolgen der untersuchten Aktivitäten im Kanusport (nur Männer):

\begin{tabular}{|l|l|l|l|l|l|l|}
\hline Männlich & $\begin{array}{l}\text { Kanu- } \\
\text { Wandern } \\
\text { Fluss }\end{array}$ & $\begin{array}{l}\text { Kanu- } \\
\text { Wandern } \\
\text { See }\end{array}$ & Seekajak & WW 1-2 & WW 3-4 & WW 5-6 \\
\hline $\begin{array}{l}\text { Mittelwert } \\
\text { Unfallschwere }\end{array}$ & 3,36 & 3,23 & 4,13 & 3,15 & 3,97 & 5,01 \\
\hline Z-Wert & $-0,68872777$ & $-0,88843323$ & 0,49414298 & $-1,01132889$ & 0,24835165 & 1,84599526 \\
\hline & & & & & & \\
Z-Wert Diff. & $-1,87872777$ & $-1,40843323$ & $-0,14585702$ & 0,42867111 & 1,15835165 & 1,84599526 \\
\hline
\end{tabular}

Tabelle Anhang 19: Subjektive Einschätzung der Unfallfolgen der untersuchten Aktivitäten im Kanusport (nur Frauen):

\begin{tabular}{l|l|l|l|l|l|l}
\hline & $\begin{array}{l}\text { Kanu- } \\
\text { Wandern } \\
\text { weiblich }\end{array}$ & $\begin{array}{l}\text { Kanu- } \\
\text { Wandern } \\
\text { See }\end{array}$ & Seekajak & WW 1-2 & WW 3-4 & WW 5-6 \\
\hline $\begin{array}{lllllll}\text { Mittelwert } \\
\text { Unfallschwere }\end{array}$ & 3,28 & 3,06 & 4,03 & 3,09 & 3,94 & 4,78 \\
Z-Wert & $-0,67430776$ & $-1,03034226$ & 0,53944621 & $-0,9817921$ & 0,39379573 & 1,75320018 \\
Z-Wert Diff. & $-1,86430776$ & $-1,55034226$ & $-0,10055379$ & 0,4582079 & 1,30379573 & 1,75320018
\end{tabular}


Tabelle Anhang 20: Subjektive Einschätzung der Unfallhäufigkeiten und Unfallfolgen für die untersuchten Aktivitäten im Kanusport in Abhängigkeit der Erfahrung (Männer und Frauen):

\begin{tabular}{|c|c|c|c|c|c|c|}
\hline$<4$ Jahre & $\begin{array}{l}\text { Kanu- } \\
\text { Wandern } \\
\text { Fluss } \\
\end{array}$ & $\begin{array}{l}\text { Kanu- } \\
\text { Wandern } \\
\text { See }\end{array}$ & Seekajak & WW 1-2 & WW 3-4 & WW 5-6 \\
\hline U.Häufigkeit & 2,69 & 2,28 & 2,94 & 2,81 & 3,53 & 3,59 \\
\hline Z-Wert & $-0,61369042$ & $-1,50173655$ & $-0,07219887$ & $-0,35377448$ & 1,20572118 & 1,33567915 \\
\hline z-Wert Diff. & $-2,40369042$ & $-0,90173655$ & 0,52780113 & $-1,05377448$ & 1,24572118 & 2,59567915 \\
\hline U.Schwere & 3,06 & 2,97 & 3,91 & 2,91 & 3,84 & 4,69 \\
\hline Z-Wert & $-0,7800207$ & $-0,9194946$ & 0,5372328 & $-1,0124772$ & 0,4287531 & 1,74600659 \\
\hline z-Wert Diff. & $-1,9700207$ & $-1,4394946$ & $-0,1027672$ & 0,4275228 & 1,3387531 & 1,74600659 \\
\hline Ausüb.Häuf. & 3,13 & 2,34 & 1,5 & 3,58 & 3,25 & 1,35 \\
\hline 5-12 Jahre & $\begin{array}{l}\text { Kanu- } \\
\text { Wandern } \\
\text { Fluss }\end{array}$ & $\begin{array}{l}\text { Kanu- } \\
\text { Wandern } \\
\text { See }\end{array}$ & Seekajak & WW 1-2 & WW 3-4 & WW 5-6 \\
\hline U.Häufigkeit & 2,97 & 2,35 & 3,16 & 3,1 & 3,57 & 3,64 \\
\hline Z-Wert & $-0,37938994$ & $-1,83436993$ & 0,06649102 & $-0,07431349$ & 1,0286552 & 1,19292714 \\
\hline z-Wert-Diff. & $-2,16938994$ & $-1,23436993$ & 0,66649102 & $-0,77431349$ & 1,0686552 & 2,45292714 \\
\hline U.Schwere & 3,28 & 3,01 & 4,12 & 3,1 & 3,91 & 5,01 \\
\hline Z-Wert & $-0,65473036$ & $-1,04042606$ & 0,54521182 & $-0,91186082$ & 0,24522628 & 1,81657914 \\
\hline z-Wert Diff. & $-1,84473036$ & $-1,56042606$ & $-0,09478818$ & 0,52813918 & 1,15522628 & 1,81657914 \\
\hline Ausüb.Häuf. & 3,9 & 2,9 & 1,83 & 3,77 & 3,97 & 1,9 \\
\hline 13-20 Jahre & $\begin{array}{l}\text { Kanu- } \\
\text { Wandern } \\
\text { Fluss } \\
\end{array}$ & $\begin{array}{l}\text { Kanu- } \\
\text { Wandern } \\
\text { See }\end{array}$ & Seekajak & WW 1-2 & WW 3-4 & WW 5-6 \\
\hline U.Häufigkeit & 3,07 & 2,48 & 3,05 & 3,33 & 3,71 & 3,74 \\
\hline Z-Wert & $-0,37016283$ & $-1,73513824$ & $-0,41643318$ & 0,23135177 & 1,11048848 & 1,17989401 \\
\hline Z-Wert Diff. & $-2,16016283$ & $-1,13513824$ & 0,18356682 & $-0,46864823$ & 1,15048848 & 2,43989401 \\
\hline U.Schwere & 3,31 & 3,12 & 4,02 & 3,21 & 4,12 & 5,12 \\
\hline Z-Wert & $-0,72354706$ & $-0,99487721$ & 0,29037086 & $-0,8663524$ & 0,4331762 & 1,86122961 \\
\hline Z-Wert Diff. & $-1,91354706$ & $-1,51487721$ & $-0,34962914$ & 0,5736476 & 1,3431762 & 1,86122961 \\
\hline Ausüb.Häuf. & 4,29 & 3,5 & 1,78 & 3,8 & 3,78 & 1,95 \\
\hline 21-28 Jahre & $\begin{array}{l}\text { Kanu- } \\
\text { Wandern } \\
\text { Fluss }\end{array}$ & $\begin{array}{l}\text { Kanu- } \\
\text { Wandern } \\
\text { See }\end{array}$ & Seekajak & WW 1-2 & WW 3-4 & WW 5-6 \\
\hline U.Häufigkeit & 3,47 & 2,67 & 3,07 & 3,2 & 3,33 & 3,47 \\
\hline Z-Wert & 0,96859739 & $-1,91914637$ & $-0,47527449$ & $-0,00601613$ & 0,46324223 & 0,96859739 \\
\hline z-Wert Diff. & $-0,82140261$ & $-1,31914637$ & 0,12472551 & $-0,70601613$ & 0,50324223 & 2,22859739 \\
\hline U.Schwere & 3,73 & 3,6 & 4,47 & 3,4 & 3,93 & 5,33 \\
\hline Z-Wert & $-0,53151927$ & $-0,730839$ & 0,60306994 & $-1,03748473$ & $-0,22487354$ & 1,92164659 \\
\hline z-Wert Diff. & $-1,72151927$ & $-1,250839$ & $-0,03693006$ & 0,40251527 & 0,68512646 & 1,92164659 \\
\hline Ausüb.Häuf. & 4 & 3,73 & 1,67 & 3,73 & 3,53 & 2,07 \\
\hline$>28$ Jahre & $\begin{array}{l}\text { Kanu- } \\
\text { Wandern } \\
\text { Fluss }\end{array}$ & $\begin{array}{l}\text { Kanu- } \\
\text { Wandern } \\
\text { See }\end{array}$ & Seekajak & WW 1-2 & WW 3-4 & WW 5-6 \\
\hline U.Häufigkeit & 3,32 & 2,8 & 2,92 & 3,2 & 3,32 & 3,08 \\
\hline Z-Wert & 1,09119094 & $-1,56858698$ & $-0,95479208$ & 0,47739604 & 1,09119094 & $-0,13639887$ \\
\hline
\end{tabular}




\begin{tabular}{|l|l|l|l|l|l|l|} 
z-Wert Diff. & $-0,69880906$ & $-0,96858698$ & $-0,35479208$ & $-0,22260396$ & 1,13119094 & 1,12360113 \\
\hline U.Schwere & 3,68 & 3,84 & 4,24 & 3,29 & 4,12 & 4,68 \\
\hline Z-Wert & $-0,67012291$ & $-0,30666642$ & 0,60197482 & $-1,55604812$ & 0,32938245 & 1,60148018 \\
\hline z-Wert Diff. & $-1,86012291$ & $-0,82666642$ & $-0,03802518$ & $-0,11604812$ & 1,23938245 & 1,60148018 \\
\hline Ausüb.Häuf. & 4,64 & 3,84 & 2 & 4,44 & 4,04 & 2,24 \\
\hline
\end{tabular}

Tabelle Anhang 21: Subjektive Einschätzung der Unfallhäufigkeiten und Unfallfolgen für die untersuchten Aktivitäten im Kanusport in Abhängigkeit des Alters (Männer und Frauen):

\begin{tabular}{|c|c|c|c|c|c|c|}
\hline$<20$ Jahre & $\begin{array}{l}\text { Kanu- } \\
\text { Wandern } \\
\text { Fluss } \\
\end{array}$ & $\begin{array}{l}\text { Kanu- } \\
\text { Wandern } \\
\text { See }\end{array}$ & Seekajak & WW 1-2 & WW 3-4 & WW 5-6 \\
\hline U.Häufigkeit & 2,93 & 2,36 & 3,57 & 3,5 & 4,36 & 4,5 \\
\hline Z-Wert & $-0,81057986$ & $-1,57216863$ & 0,04453736 & $-0,04899109$ & 1,10007267 & 1,28712956 \\
\hline z-Wert Diff. & $-2,60057986$ & $-0,97216863$ & 0,64453736 & $-0,74899109$ & 1,14007267 & 2,54712956 \\
\hline U.Schwere & 3,07 & 3,07 & 3,93 & 3,14 & 4,21 & 5,21 \\
\hline Z-Wert & $-0,89720601$ & $-0,89720601$ & 0,20245741 & $-0,80769853$ & 0,56048737 & 1,83916577 \\
\hline z-Wert Diff. & $-2,08720601$ & $-1,41720601$ & $-0,43754259$ & 0,63230147 & 1,47048737 & 1,83916577 \\
\hline Ausüb.Häuf. & 3,64 & 2,93 & 1,64 & 3,93 & 4,07 & 2,07 \\
\hline 21-30 jahre & $\begin{array}{l}\text { Kanu- } \\
\text { Wandern } \\
\text { Fluss }\end{array}$ & $\begin{array}{l}\text { Kanu- } \\
\text { Wandern } \\
\text { See }\end{array}$ & Seekajak & WW 1-2 & WW 3-4 & WW 5-6 \\
\hline U.Häufigkeit & 3,12 & 2,49 & 3,02 & 3,18 & 3,57 & 3,43 \\
\hline Z-Wert & $-0,0436744$ & $-1,87799919$ & $-0,33483707$ & 0,1310232 & 1,26655759 & 0,85892986 \\
\hline z-Wert Diff. & $-1,8336744$ & $-1,27799919$ & 0,26516293 & $-0,5689768$ & 1,30655759 & 2,11892986 \\
\hline U.Schwere & 3,43 & 3,22 & 3,91 & 3,18 & 4,03 & 5,06 \\
\hline Z-Wert & $-0,57977176$ & $-0,90444394$ & 0,16233609 & $-0,96628626$ & 0,34786305 & 1,94030281 \\
\hline z-Wert Diff. & $-1,76977176$ & $-1,42444394$ & $-0,47766391$ & 0,47371374 & 1,25786305 & 1,94030281 \\
\hline Ausüb.Häuf. & 3,7 & 2,63 & 1,69 & 3,57 & 3,4 & 1,68 \\
\hline 31-40 Jahre & $\begin{array}{l}\text { Kanu- } \\
\text { Wandern } \\
\text { Fluss }\end{array}$ & $\begin{array}{l}\text { Kanu- } \\
\text { Wandern } \\
\text { See }\end{array}$ & Seekajak & WW 1-2 & WW 3-4 & WW 5-6 \\
\hline U.Häufigkeit & 2,86 & 2,29 & 3 & 3,02 & 3,31 & 3,37 \\
\hline Z-Wert & $-0,32454511$ & $-1,93316$ & 0,07055328 & 0,12699591 & 0,94541401 & 1,1147419 \\
\hline z-Wert Diff. & $-2,11454511$ & $-1,33316$ & 0,67055328 & $-0,57300409$ & 0,98541401 & 2,3747419 \\
\hline U.Schwere & 3,29 & 3,06 & 4,16 & 3,04 & 3,84 & 4,73 \\
\hline Z-Wert & $-0,64007178$ & $-1,01120584$ & 0,76378313 & $-1,04347837$ & 0,24742271 & 1,68355015 \\
\hline z-Wert Diff. & $-1,83007178$ & $-1,53120584$ & 0,12378313 & 0,39652163 & 1,15742271 & 1,68355015 \\
\hline Ausüb.Häuf. & 4 & 3,12 & 1,73 & 4,08 & 4,23 & 2,06 \\
\hline 41-50 Jahre & $\begin{array}{l}\text { Kanu- } \\
\text { Wandern } \\
\text { Fluss }\end{array}$ & $\begin{array}{l}\text { Kanu- } \\
\text { Wandern } \\
\text { See } \\
\end{array}$ & Seekajak & WW 1-2 & WW 3-4 & WW 5-6 \\
\hline U.Häufigkeit & 3,03 & 2,57 & 2,89 & 3 & 3,35 & 3,59 \\
\hline Z-Wert & $-0,12780912$ & $-1,53882183$ & $-0,55724777$ & $-0,21983169$ & 0,85376493 & 1,58994547 \\
\hline z-Wert Diff. & $-1,91780912$ & $-0,93882183$ & 0,04275223 & $-0,91983169$ & 0,89376493 & 2,84994547 \\
\hline U.Schwere & 3,27 & 3,24 & 4,3 & 3,14 & 3,95 & 5,08 \\
\hline Z-Wert & $-0,80059931$ & $-0,84348856$ & 0,67193157 & $-0,98645272$ & 0,171557 & 1,78705204 \\
\hline z-Wert Diff. & $-1,99059931$ & $-1,36348856$ & 0,03193157 & 0,45354728 & 1,081557 & 1,78705204 \\
\hline Ausüb.Häuf. & 4,43 & 3,95 & 1,89 & 3,92 & 3,89 & 2,19 \\
\hline
\end{tabular}


Tabelle Anhang 22: Subjektive Einschätzung der Unfallhäufigkeiten und Unfallfolgen für die untersuchten Aktivitäten im Kanusport in Abhängigkeit der Übungsleiterlizenz (Männer und Frauen):

\begin{tabular}{|c|c|c|c|c|c|c|}
\hline $\begin{array}{l}\text { ÜL Freizeit } \\
\text { (Wandern) }\end{array}$ & $\begin{array}{l}\text { Kanu- } \\
\text { Wandern } \\
\text { Fluss }\end{array}$ & $\begin{array}{l}\text { Kanu- } \\
\text { Wandern } \\
\text { See }\end{array}$ & Seekajak & WW 1-2 & WW 3-4 & WW 5-6 \\
\hline U.Häufigkeit & 3,07 & 2,33 & 2,96 & 3,33 & 3,48 & 3,33 \\
\hline Z-Wert & $-0,03517852$ & $-1,98758619$ & $-0,32540128$ & 0,65080256 & 1,04656087 & 0,65080256 \\
\hline z-Wert-Diff. & $-1,82517852$ & $-1,38758619$ & 0,27459872 & $-0,04919744$ & 1,08656087 & 1,91080256 \\
\hline U.Schwere & 3,52 & 3,74 & 4,26 & 3,11 & 4,07 & 5,3 \\
\hline Z-Wert & $-0,69613974$ & $-0,37707569$ & 0,37707569 & $-1,29075909$ & 0,10152038 & 1,88537845 \\
\hline Z-Wert Diff & $-1,88613974$ & $-0,89707569$ & $-0,26292431$ & 0,14924091 & 1,01152038 & 1,88537845 \\
\hline Ausüb.Häuf. & 4,93 & 3,89 & 1,96 & 4,12 & 3,67 & 1,67 \\
\hline $\begin{array}{l}\text { ÜL Freizeit } \\
\text { (WW) }\end{array}$ & $\begin{array}{l}\text { Kanu- } \\
\text { Wandern } \\
\text { Fluss }\end{array}$ & $\begin{array}{l}\text { Kanu- } \\
\text { Wandern } \\
\text { See }\end{array}$ & Seekajak & WW 1-2 & WW 3-4 & WW 5-6 \\
\hline U.Häufigkeit & 3,09 & 2,48 & 2,88 & 3,07 & 3,23 & 3,07 \\
\hline Z-Wert & 0,49670531 & $-2,02821334$ & $-0,37252898$ & 0,41392109 & 1,07619483 & 0,41392109 \\
\hline z-Wert Diff. & $-1,29329469$ & $-1,42821334$ & 0,22747102 & $-0,28607891$ & 1,11619483 & 1,67392109 \\
\hline U.Schwere & 3,48 & 3,41 & 4,28 & 2,98 & 3,98 & 5,14 \\
\hline Z-Wert & $-0,5682284$ & $-0,66808444$ & 0,57298345 & $-1,28148581$ & 0,14502901 & 1,79978619 \\
\hline z-Wert Diff. & $-1,7582284$ & $-1,18808444$ & $-0,06701655$ & 0,15851419 & 1,05502901 & 1,79978619 \\
\hline Ausüb.Häuf. & 4 & 2,98 & 1,65 & 4,65 & 4,67 & 2,43 \\
\hline ohne Lizenz & $\begin{array}{l}\text { Kanu- } \\
\text { Wandern } \\
\text { Fluss }\end{array}$ & $\begin{array}{l}\text { Kanu- } \\
\text { Wandern } \\
\text { See }\end{array}$ & Seekajak & WW 1-2 & WW 3-4 & WW 5-6 \\
\hline U.Häufigkeit & 2,95 & 2,42 & 3,08 & 3,11 & 3,67 & 3,79 \\
\hline Z-Wert & $-0,4806519$ & $-1,63858604$ & $-0,19663032$ & $-0,13108688$ & 1,09239069 & 1,35456446 \\
\hline z-Wert-Diff. & $-2,2706519$ & $-1,03858604$ & 0,40336968 & $-0,83108688$ & 1,13239069 & 2,61456446 \\
\hline U.Schwere & 3,17 & 2,98 & 4,09 & 3,15 & 3,95 & 4,87 \\
\hline Z-Wert & $-0,79473072$ & $-1,07874107$ & 0,5804773 & $-0,82462654$ & 0,37120651 & 1,74641452 \\
\hline z-Wert Diff. & $-1,98473072$ & $-1,59874107$ & $-0,0595227$ & 0,61537346 & 1,28120651 & 1,74641452 \\
\hline Ausüb.Häuf. & 3,85 & 3,04 & 1,81 & 3,61 & 3,55 & 1,73 \\
\hline
\end{tabular}




\section{Anhang B: ASS-Schadensanzeige (Unfallbogen des DAV):}

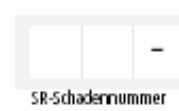

\section{Durchschrift für die}

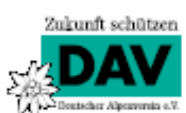

ELVIA Reiseversicherungs -Gesellschaft AG · Schadenabteilung

Ludmillastrafe $26 \cdot 81543$ München - Telefon 089/6 2424-0

Telefax 089/62424-222. E-Mail: service@elviade

\section{Sicherheitsforschung des Deutschen Alpenvereins ASS 2004}

Bitte mit an ELVIA einsenden, wird weitergeleitet!

1. Angaben zur Person (Bitte legen Sie eine Kopie des DAV-Mitgliedsausweises bei!)

Name und Vorname
Straße
PLZ/Ort
Telefon tagsüber
Mitglied der Sektion

Name und Anschrift der Krankenkasse/Krankenversicherung

Wurde eine private Auslandskrankenversicherung/private Zusatzkrankenversicherung abgeschlossen?

Name und Anschrift der Gesellschaft

Versicherungs-Nr.

\section{Angaben zum Schaden}

Wann war der Unfall/die Notlage/die Suche?

Genaue Ortsangabe (mit Talort/Gebiet)

Bitte schildern Sie den Hergang der Notlage (ggf. auch auf einem Beiblatt)

Welche Verletzungen, welche Erkrankungen hat das DAV-Mitglied erlitten?

Stationare Behandlung vor Ort oder untenwegs? nein

Anschrift Krankenhaus

Krankenhausaufenthalt nach der Rückkehr?

nein

Anschrift Krankenhaus

Ambulante arztlich Behandlung? ja, von

bis

ja, von

bis

3. Angaben zu Begleitpersonen

Name und Anschrift von Begleitpersonen

Ist ein Begleiter verunglückt/gesucht/gerettet? nein

Ist (sind) der (die) Begleitperson(en) Sektionsmitglied des DAV

Gibt es weitere Zeugen für das Ereignis? nein 


\section{SICHERHEITSFORSCHUNG DES DAV}

SR-Schadernumm

Sehr geehrtes Mitglied,

wir bitten die nachfolgenden Fragen ergănzend - in freiwilliger Weise - zu beantworten.

Die Auskünfte dienen ausschließlich der Sicherheitsforschung des DAV. Herzlichen Dank!

Welche Stelle hat den Unfall aufgenommen?

Sind Sie lediglich Begleiter oder Kletterpartner des Verunfallten? Dann bitte keine weiteren Fragen beantworten.

Sind Sie verunfalt oder in Bergnot geraten? Dann bitte folgende Fragen beantworten.

Bei verstorbenen oder vermissten Personen fülen bitte die Angehorigen oder ein Begleiter die Fragen aus.

Allgemeine Daten

Alte

Geschlecht mannlich weiblich $\square$

Unfallfolgen

unverletzt

verletzt

verstorben

vermisst

behelfsmaßige Bergung/Suche

organisierte Bergrettung

Alarmierung durch Handy

anders, durch

Tatigkeit beim Unfall

Aufstieg

Abstieg/Abfahrt

Abseilen

bei Familienwanderung

als Alleingeher

als Geführter

mit gleichwertigem Partner(n)

als FÖ/Bergüuhrer mit Gruppe

Art der Tour

Wanderung/Bergwanderung

Hochtour

Radtour

Skilauf alpin

Skitour

Klettersteig

künstliche Kletteranlage

Klettergarten

alpine Sportkettertour

klassische alpine Klettertour

Eis-/mixed Route

Hohentrecking

Hohenbergsteigen (ab $7000 \mathrm{~m}$ )

sonstiges
Besondere Verhăltnisse

nass

verschneit

vereist

sehr schlechte Sicht

plotzlicher Wettersturz

Unfallgelănde

Meereshohe

Alpen

auBerhalb der Alpen

Wanderweg/Pfad

wegloses Gelande

Schneefeld

Gletscher

kombiniertes Gelande

steiles Felskettergelynde

steiles Eisklettergelande

Piste

Loipe

Skivariante

freies Skitourengelande

Sicherung

seilfrei

gleichzeitiges Gehen am Seil

gesichertes Klettern

angeseilt mit

- Hũftgurt

- Hũft- und Brustgurt

- Komplettgurt

- behelfsmaßig

- Klettersteigbremse

Helm

Seit wie vielen Jahren betreiben Sie diese Bergsportdisziplin?

An wie vielen Tagen im Jahr üben Sie diese Disziplin ungefahr aus?

Herz-/Kreislaufversagen

Erschopfung

Erkrankung

Verirren/Versteigen

Sturz wegen

- Ausrutschen/Stolpem

- Skisturz/Kollision

- Kletterschwierigkeit

- Schwierigkeitsgrad

- Tritt-/Griffausbruch

- Materialversagen

was hat versage?

- Kommunikationsproblem

sonstiges

Steinschlag

Eisschlag

Blitzschlag

Wechtenbruch

Spaltensturz

Lawine

- Schneebrett

- Lockerschneelawine

- teilverschüttet

- ganzverschüttet

- Tiefe

- Verschüttungsdauer

- Hangrichtung

- Steilheit Grad

- Gefahrenstufe LIB

sonstiges

Genaue Bezeichnung?

Vielen Dank für Ihre Mitarbeit! 


\section{Anhang C: Wissenschaftlicher Bildungsgang}

Persönliche Daten:

Staatsangehörigkeit: Bildungsgang:

Berufliche Tätigkeiten

Lehrtätigkeiten

Referententätigkeiten

Fremdsprachen
Arne Göring, Steinweg 7, 37120 Bovenden, geb. am 18.02.1975 in Wolfenbüttel, verheiratet, ein Kind.

Deutsch

Mai 1994 Abitur am Robert-Koch-Gymnasium in ClausthalZellerfeld

1995 - 2001 Studium der Sportwissenschaften und Biologie an der Georg-August-Universität Göttingen.

1. Staatsexamen mit der Note: 1,1

1998 - 2002 Studium der Soziologie, Politikwissenschaften und Volkswirtschaftslehre an der Georg-August-Universität Göttingen. 1. Staatsexamen mit der Note 1,7

Seit 2002: Promotion im Bereich Sportwissenschaften bei Prof. Dr. Arnd Krüger am Institut für Sportwissenschaften der Georg-August-Universität Göttingen

2001 -2003 Geschäftsführer des Fitness- und Gesundheitszentrums an der Georg-August-Universität Göttingen

Seit 2003 wiss. Mitarbeiter am Institut für Sportwissenschaften, gleichzeitig stellv. Leiter des Hochschulsports der Universität Göttingen

Seit 2001 Lehrbeauftragter für die Wasser- und Wintersportausbildung am Institut für Sportwissenschaften

Seit 2002 verschiedene Einführungs- und Vertiefungsveranstaltung in den Bereichen Sport und Gesellschaft (Schwerpunkt Sportsoziologie \& Sportpsychologie), Sportmanagement sowie Sport und Erziehung (Schwerpunkt Erlebnispädagogik)

Seit 2003 Fachleiter für den Bereich Wintersport

Seit 2001 Referent des Deutschen Sportärztebundes

Seit 2003 Referent des Allgemeinen Deutschen Hochschulsportverbandes

Englisch, Französisch 


\section{Anhang D: Eidesstattliche Erklärung}

Ich versichere, dass ich die eingereichte Dissertation

Risikosport - interdisziplinäre Annäherung, empirische Befunde und Anwendungsbezüge

selbstständig und ohne unerlaubte Hilfsmittel verfasst habe. Andere als der von mir angegebenen Hilfsmittel und Schriften habe ich mich nicht bedient. Alle wörtlich oder sinngemäß den Schriften anderer Autoren entnommenen Stellen habe ich kenntlich gemacht.

Göttingen, den 22.10.2005 
